\title{
Dimensionsstabile und pilzresistente Furnierwerkstoffe durch Zellwandmodifizierung mit niedermolekularem Phenol- Formaldehyd
}

\author{
Dissertation
}

zur Erlangung des Doktorgrades (Dr. rer. nat.) der Fakultät für Forstwissenschaften und Waldökologie der Georg-August-Universität Göttingen

\author{
vorgelegt von
}

Sascha Bicke

geboren am 03. Mai 1983 in Aalen

Göttingen, Juni 2019 
1. Gutachter: Prof. Dr. Holger Militz

2. Gutachter: Prof. Dr. Carsten Mai

Tag der mündlichen Prüfung: 16.09.19 



\section{Danksagung}

Ich bedanke mich für die Anstellung an der Abteilung Holzbiologie und Holzprodukte der Universität Göttingen und das mir entgegen gebrachte Vertrauen durch Herrn Prof. Dr. Holger Militz, welcher mir damit die Erstellung der vorliegenden Arbeit ermöglichte.

Für die Impulse und Finanzierung zu Beginn der Untersuchungen mit Fokus auf dem LVL sowie die stete Bereitstellung von Buchenfurnieren danke ich Ralf Pollmeier und seinen Mitarbeitern.

Besonderer Dank gilt auch den Herstellern der eingesetzten Phenol-Formaldehyd-Harze.

Für die Finanzierung im Rahmen des Verbundvorhabens „Spitzencluster-BioEconomy: TG1, Dauerhafte Buchenholzprodukte (VP1.10b/DauerBuche) - Teilprojekt A“ danke ich dem BioEconomy Cluster e.V. und dem Bundesministerium für Bildung und Forschung.

Für die Durchführung der UMSP-Messungen am Institut für Holzforschung in Hamburg bedanke ich mich bei Dr. habil. Gerald Koch und seinen Mitarbeitern vielmals.

Für die Durchführung der DLS-Messungen habe ich Herrn Dr. Marco Fleckenstein zu danken.

Zuletzt danke ich herzlichst allen Kollegen, technischen Mitarbeitern und studentischen Hilfskräften die mich unterstützt haben, denn ohne sie wäre die Umsetzung der umfangreichen Versuche nicht möglich gewesen. 


\section{Inhaltsverzeichnis}

Danksagung

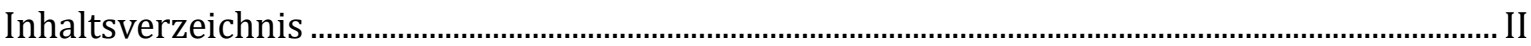

Abkürzungsverzeichnis............................................................................................................................IV

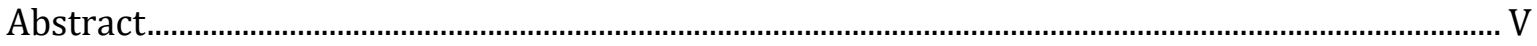

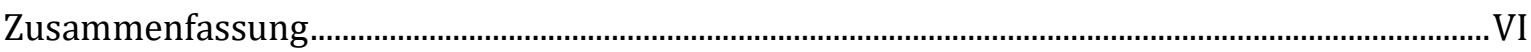

1 Einleitung

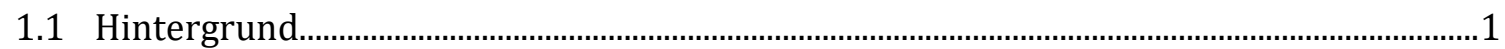

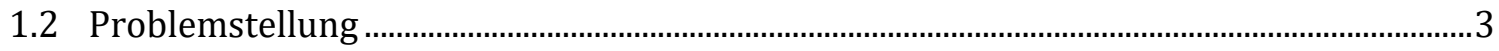

1.3 Zielstellung und Lösungsansatz ............................................................................................

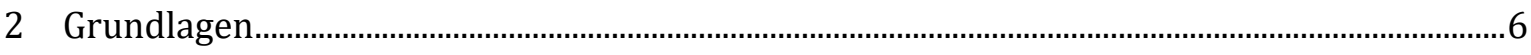

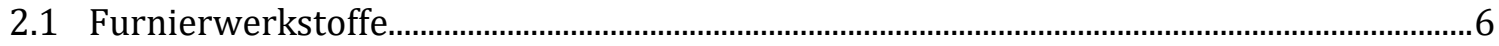

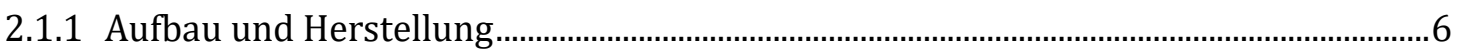

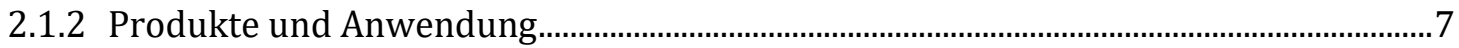

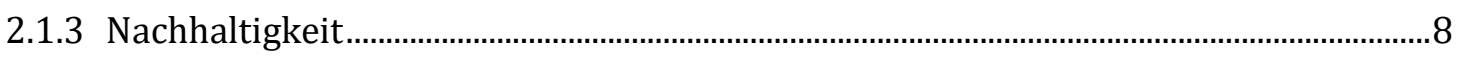

2.2 Holzmodifizierung mit Phenol-Formaldehyden ..................................................................... 10

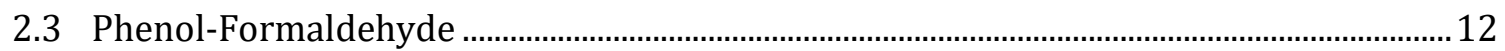

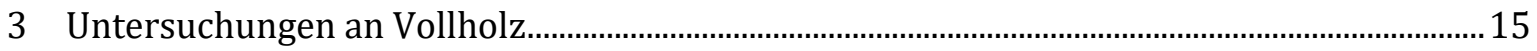

3.1 Material und Methoden ............................................................................................... 15

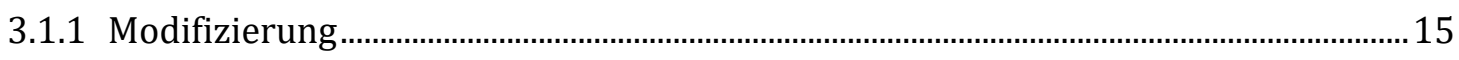

3.1.2 Bestimmung des Weight Percent Gain (WPG) ……………………………................. 16

3.1.3 Bestimmung des Bulking...................................................................................... 16

3.1.4 Bestimmung der Anti-Swell-Efficiency (ASE)...............................................................16

3.1.5 Nachweis der Verteilung der Phenol-Formaldehyde mittels UV-

Mikrospektrophotometrie (UMSP)...................................................................................... 17

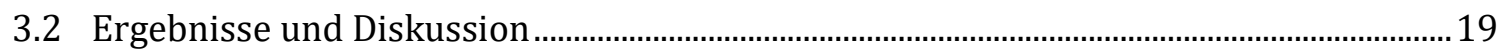

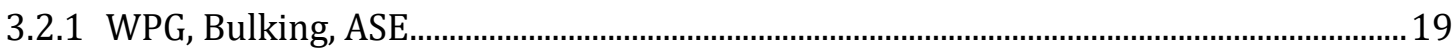

3.2.2 Nachweis der Verteilung der Phenol-Formaldehyde mittels UVMikrospektrophotometrie (UMSP)......................................................................................... 21

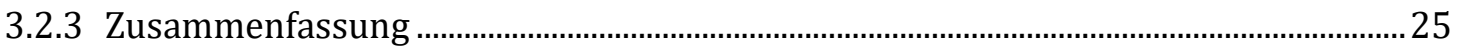

4 Untersuchung bestimmter Eigenschaften von Phenol-Formaldehyden ..................................26

4.1 Alterung von Phenol-Formaldehyd und Auswirkung auf Quellvergütung von

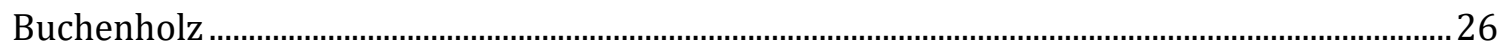

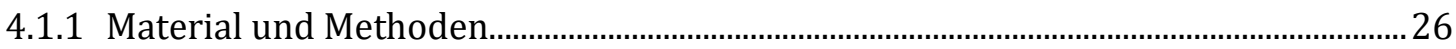

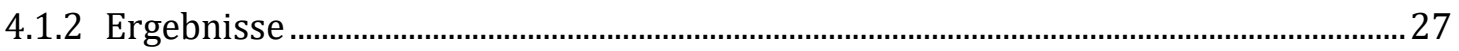

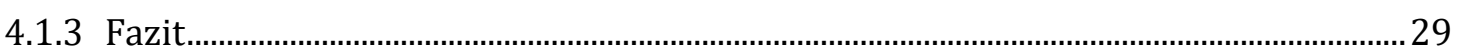

4.2 Bestimmung der Molekülgrößenverteilung mittels DLS....................................................... 30

4.2.1 Material und Methoden.............................................................................................. 30

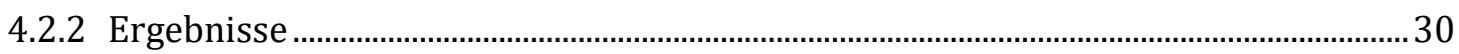

4.3 Untersuchung der Reaktionskinetik mittels DSC .............................................................. 31 
4.3.1 Material und Methoden.............................................................................................. 31

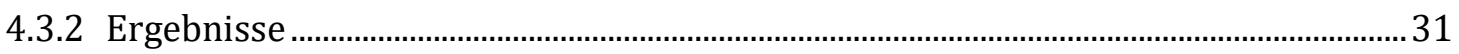

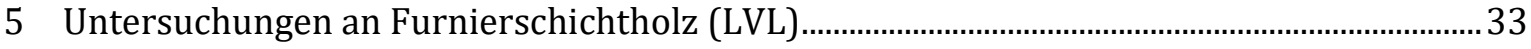

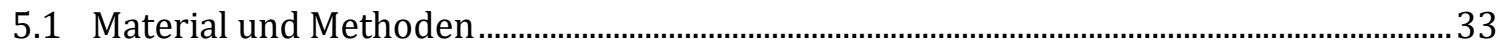

5.1.1 Bestimmung der Lösungsaufnahme von Buchenfurnieren ........................................... 33

5.1.2 PF-Modifizierung der Furniere .................................................................................... 33

5.1.3 Plattenproduktion und Prüfkörperherstellung ………………………….................... 35

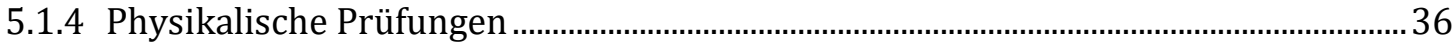

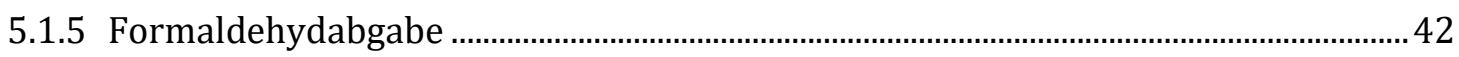

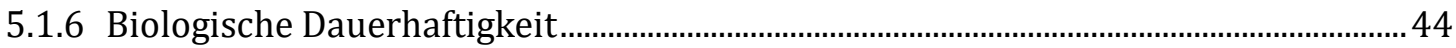

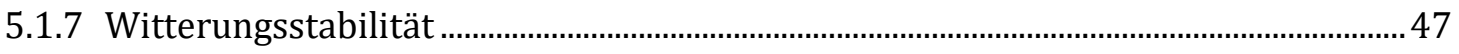

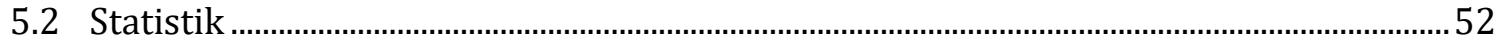

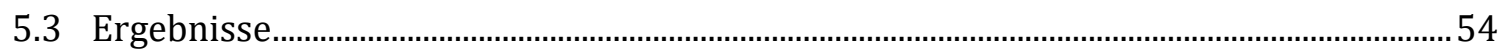

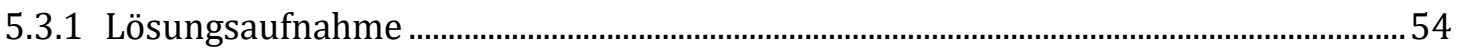

5.3.2 Physikalische Prüfungen ........................................................................................... 57

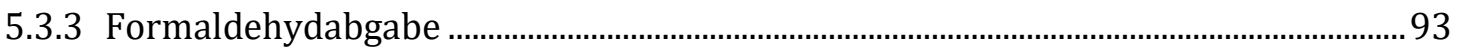

5.3.4 Biologische Dauerhaftigkeit................................................................................... 95

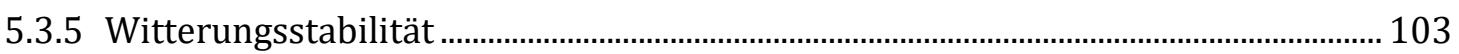

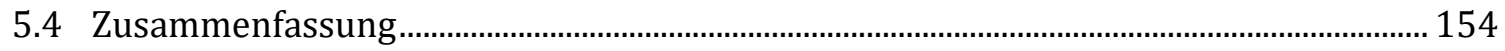

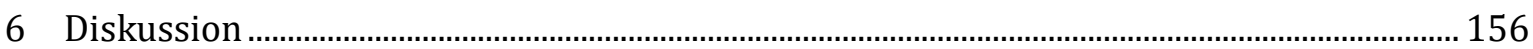

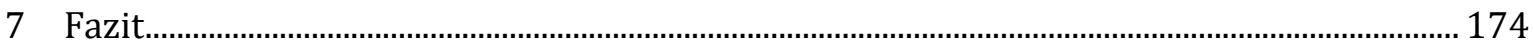

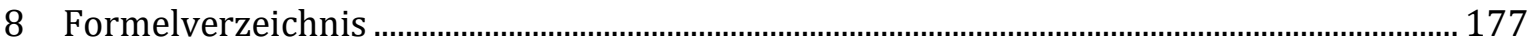

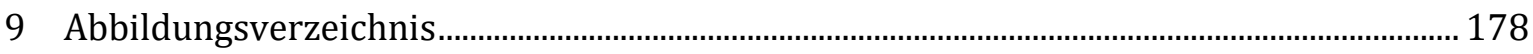

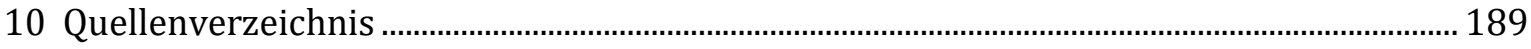

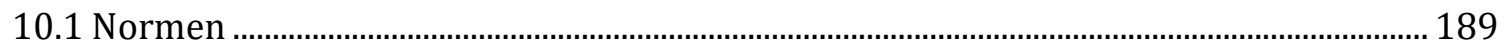

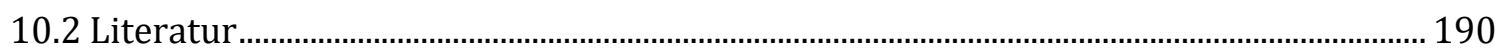

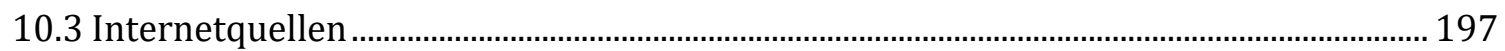

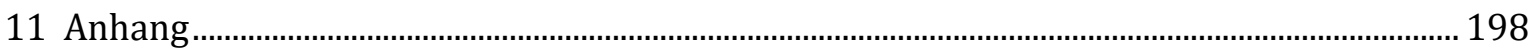

11.1 Abbildungen zur Untersuchung der Zellwandeindringung mit UMSP ............................ 198

11.2 Abbildungen zur Freilandbewitterung von Buchen-LVL ................................................. 201 


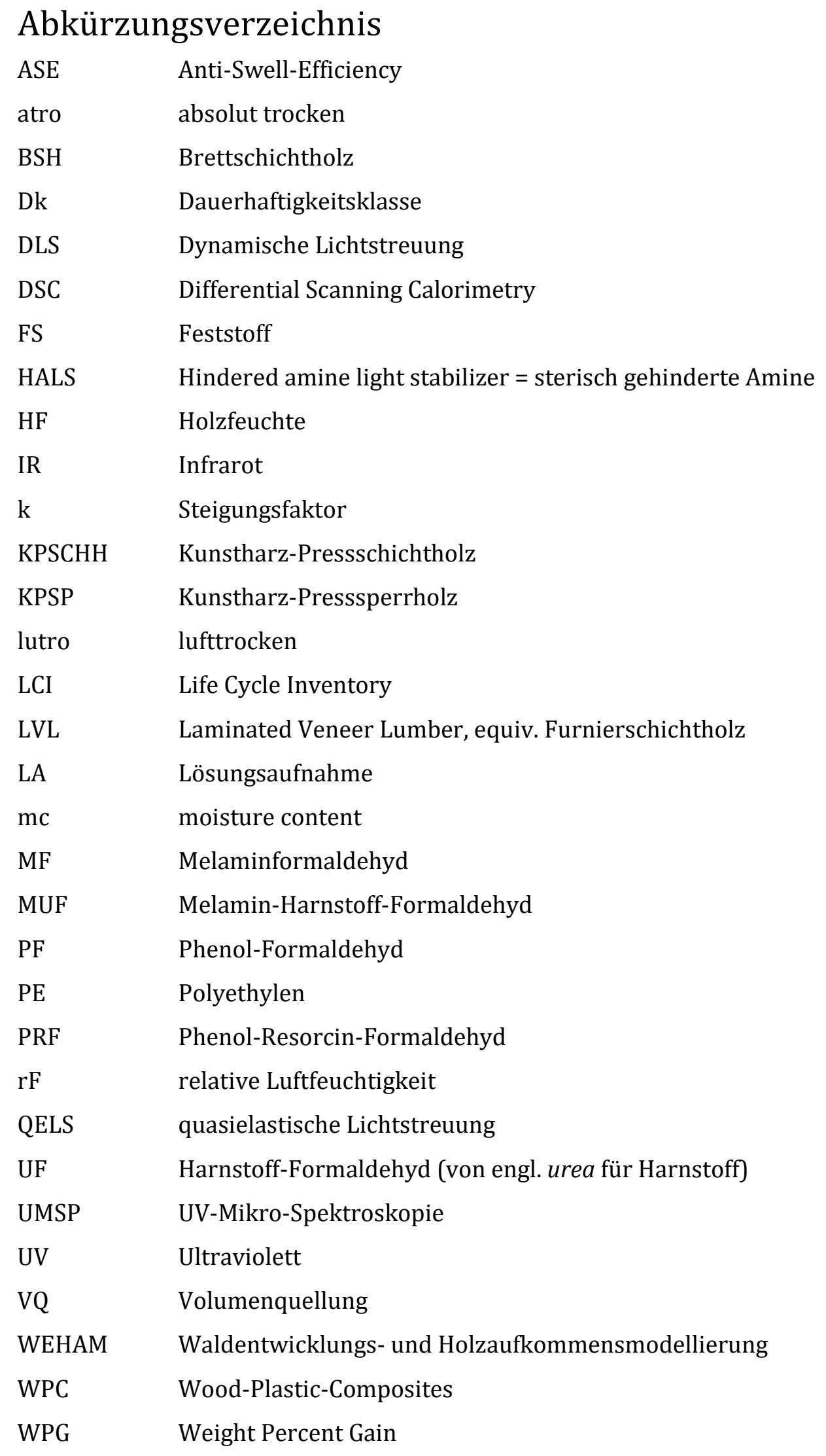




\section{Abstract}

The sorption and desorption of water within the cell wall of wood lead to swelling and shrinkage. Severe stresses occur under varying climatic conditions and damage of the wood structure can result, which eventually causes face checking and pronounced deterioration. Generally, high moisture contents lead to degradation of the wood by basidiomycetes. To protect wood and wood products against these corrupting influences, the modification of the wooden cell wall to inhibit the water uptake, is the best way to provide dimensional stability and durability. In this work rotary cut beech (Fagus sylvatica L.) veneers, $3.7 \mathrm{~mm}$ and $2.1 \mathrm{~mm}$ thick, were treated in with low molecular weight phenol formaldehyde in aqueous solution. The process contained a two-step impregnation, within a vacuum was followed by atmospheric or over-pressure and a pre-drying. The final curing took place while gluing the veneers in a heated press to form a laminated veneer lumber (LVL) of eight layers resp. 15 layers. It was the aim of the treatment to decrease the water uptake and the extent of dimensional changes by incorporating the phenol formaldehyde resin inside the cell wall and to achieve a permanent bulking. The used resins were water-soluble alkaline types. Related to the used PFconcentration in solution, medium, middle and high dry weight gains were achieved. The fixation was proven in a leaching test according to EN84 with an initial water impregnation under vacuum and with that a dimensional stability that was reached by the modification was found. The swelling in radial direction was reduced by $57 \%$ at moderate WPG and was fully eliminated at highest WPG. The durability of the LVL against white rot fungi has been tested for 16 weeks according to the standard ENV 12038. This test revealed that the mass loss had been decreased to almost $1 \%$ by the PF-treatment. Because the moisture content of the samples was above the fibre saturation point, fungal growth had been possible, by the way no constrains of fungal growth could be observed. As it is known that cell wall modification has an influence on the mechanical properties of wood, the modulus of rupture (MOR), modulus of elasticity (MOE) and impact bending strength have been assessed. It came out that the MOR and MOE can be increased by the modification and the pressing regime. Concerning the impact bending strength the energy that was absorbed by sample during the test was considerably lower. The weathering performance was tested both in a self-created lab test for LVL and in a field test. Both tests showed that weathering stability can be achieved, but under consideration of economical aspects, a coating is seen to be beneficial. Furthermore, an in-ground test over two years appears the modified LVL to be resistant against soft rot. It can be concluded that an appropriate process exists to produce LVL from the PF modified beech veneers, which is highly durable against white rot fungus Trametes versicolor, dimensional stable, weatherproof, usable in ground contact and with adequate mechanical properties. 


\section{Zusammenfassung}

Die vorliegende Arbeit beschäftigt sich mit der Entwicklung eines dimensionsstabilen, witterungsbeständigen und gegen Pilzabbau resistenten Furnierschichtholzes, beziehungsweise Laminated Veneer Lumber (LVL), auf Basis von PhenolFormaldehydmodifizierten Buchenschälfurnieren.

Es wurde dabei auf die Entwicklungen zu Beginn des 20sten Jahrhunderts zurückgegriffen, die darauf beruhten, dass sehr dünne Furniere unter einem Millimeter Dicke mit reichlich PhenolFormaldehydharz beaufschlagt und in einer Etagenpresse unter hohem Druck und hoher Temperatur ausgehärtet wurden. Unter Berücksichtigung einiger jüngerer wissenschaftlicher Erkenntnisse zu dem Penetrationsvermögen von niedermolekularen Phenol-Formaldehyden und ihrer notwendigen Beschaffenheit, in Verbindung mit einer weiterentwickelten Schäl- und Pressentechnologie, wurde ein Prozess zur Herstellung der phenolmodifizierten LVLs entworfen. In Verbindung mit verschiedenen etablierten Herstellern für Kleb- und Imprägnierharze wurden kommerzielle Produkte auf Basis von niedermolekularem PhenolFormaldehyd ausgewählt und damit in diversen Prozessen Buchenholzproben und Buchenfurniere imprägniert und die Zellwandeindringung der Harze sowie deren Fixierung untersucht. Dabei wurden drucklose (Atmosphärendruck) Verfahren sowie Vakuum-DruckVerfahren im Autoklav angewendet. Es zeigte sich, dass sich in einen optimalen Prozess an eine Vakuum-Druck-Imprägnierung ein Trocknungsprozess anschließt, der die Weiterverarbeitung der Furniere ermöglicht ohne eine Aushärtung des Oligomers zu bewirken. Aus den behandelten Furnieren stellte man dann LVL mit und ohne Sperrlagen unter verschiedenen Drücken her, die zu unterschiedlichen Verdichtungen führten. Es zeigte sich, dass ein einstufiger Herstellungsprozess, welcher die Aushärtung der Reaktionschemikalie und die Verklebung vereint, geeignet war.

Die auf diese Weise im Labor erzeugten Werkstoffe wurden einer Vielzahl physikalischer, chemischer und biologischer Prüfungen unterzogen. Unter anderem wurden sie durch die Bestimmung der Bruchschlagarbeit, Biegefestigkeit und des Biegeelastizitätsmoduls hinsichtlich ihrer mechanisch-technologischen Eigenschaften charakterisiert. Dabei zeigte sich, dass die PF-Modifizierung zu einer weitgehenden Erhöhung dieser Eigenschaften führen konnte - mit Ausnahme der Bruchschlagarbeit, welche einer Abnahme unterlag. Die Kriterien einer Verwendung für tragende Zwecke wurden damit jedoch erfüllt. Auswaschversuche zeigten eine sehr gute Fixierung. Die dabei ermittelte Langzeitquellung erwies eine drastisch verbesserte Dimensionstabilität mit ASE-Werten von bis zu 70\%. Die Untersuchung der biologischen Dauerhaftigkeit (Resistenz gegenüber holzzerstörenden Pilzen) zeigte, dass mit niedrigen Beladungsgraden (WPG) im Bereich zwischen 15\% (Gewichts-\%) und 30\% eine mit dauerhaftem Tropenholz vergleichbare Dauerhaftigkeit (DK 1 statt DK5) erreicht wurde. Wobei der Standard CEN/TS 15083-1 zur Einteilung genutzt wurde. In den Bewitterungsversuchen im Labor und Freiland bestätigte sich eine hohe Dimensionsstabilität. Durch UV-Mikro-Spektroskopie (UMSP) konnte gezeigt werden, dass die entwickelten Verfahren zu einer deutlich Zunahme phenolischer Strukturen in der Zellwand, bei gleichzeitig freibleibenden Zelllumen, führen. Diese Beobachtungen gingen mit signifikanten Messwerten 
für die dauerhafte Volumenzunahme (Bulking) infolge einer im wasserfreien Zustand gequollenen Zellwand einher.

Es wird geschlussfolgert, dass die Phenolmodifizierung von Buchenfurnieren zum Zwecke der Herstellung dauerhaften LVLs aus 3,7 mm dicken Furnieren ohne Sperrlagen gut geeignet und industriell umsetzbar ist. Somit wird ein Ersatz für Baustoffe auf Basis rein fossiler Rohstoffe selbst in tragenden Außenanwendungen sowie eine sinnvolle stoffliche Nutzung des Buchenholzpotenzials in Deutschland ermöglicht. 


\section{Einleitung}

\subsection{Hintergrund}

„Der Baustoff Holz erlebt in jüngster Zeit eine verdiente Renaissance. Seine Vielseitigkeit als Werkstoff ist schon lange bekannt - seine Bedeutung als erneuerbare Ressource wurde erst in den letzten Jahren wiederentdeckt." Mit diesen verheißungsvollen Worten beginnt Ralf Pollmeier, geschäftsführender Gesellschafter der Pollmeier Unternehmensgruppe, seine BauBuche als Ingenious Hardwood - zu Deutsch: ausgeklügeltes Laubholz - auf seiner Internetpräsenz vorzustellen. Gemeint ist damit ein aus der Buche hergestelltes Furnierschichtholz, welches im Thüringischen Creuzburg hergestellt und $\mathrm{zu}$ Trägern weiterverarbeitet wird. Damit will man den konstruktiven Holzbau beflügeln, welcher bislang fast ausschließlich von Nadelholz dominiert wurde. Die Bedeutung dieses Vorhabens wird unterstrichen durch die Investition von rund 100 Mio. € (DWORSCHAK 2014) in die modernste Fertigungstechnologie für das weltweit erste Buchen-Furnierschichtholzwerk seiner Art mit einer Kapazität von $180.000 \mathrm{~m}^{3}$ pro Jahr.

Die Rundholzversorgung oben genannter und zukünftiger Vorhaben dürfte gesichert sein. Von insgesamt 10,1 Mio. ha bestockter Holzbodenfläche in Deutschland, steht die Buche (Fagus sylvatica L.) laut (OEHMichen 2011) auf 17\% bzw. nach dem Ergebnis der dritten Bundeswaldinventur (BMEL 2012) auf mindestens 15\% und ist somit nach der Fichte (Picea abies L.) und der Kiefer (Pinus sylvestris L.) die dritthäufigste Baumart in Deutschland. Der Vorrat an Buchenholz wird auf 635 Mio. $\mathrm{m}^{3}$ im Jahr 2012 beziffert (BMEL 2012). Somit ist der Vorrat seit der letzten Inventur 2001/2002 um 10\% gestiegen.

SEINTSCH UND WEIMAR (2012) stellten heraus, dass derzeit durch Einschlag nur $60 \%$ des jährlichen Zuwachses der Buche genutzt werden. Im Gegensatz dazu wird damit gerechnet, dass ein Beibehalt des durchschnittlichen jährlichen Einschnittes bei der Fichte, wie er im Zeitraum zwischen 2002 und 2008 stattfand, nach der Waldentwicklungs- und Holzaufkommensmodellierung (WEHAM) nur unter einem Vorratsabbau möglich sein wird. Um diese Schere abzubauen, wird es hilfreich und notwendig sein, Buchenholz vermehrt stofflich, z.B. in Form von Bauprodukten, einzusetzen.

Die Wirklichkeit sieht jedoch noch anders aus.

In Deutschland wurde im Jahr 2010 erstmals mehr Holz energetisch (50,5 \%) als stofflich $(49,5 \%)$ genutzt (MANTAU 2012) - allein der Verbrauch für den Hausbrand stieg von 12 Mio. $\mathrm{m}^{3}$ im Jahr 2000 auf 34 Mio. $\mathrm{m}^{3}$ im Jahr 2010. Nach DöRING ET AL. (2016) verbrauchten im Jahr 2014 die Haushalte zu 62,4 \% Laubholz und zu 37,6 \% Nadelholz.

Neben der Verfügbarkeit von Laub- und Nadelholz stellt die Vermeidung von Treibhausgasen bei der Errichtung von Gebäuden einen weiteren, wesentlichen Anreiz für die Verwendung von Holz und Holzwerkstoffen in Form von Bauprodukten dar. Die deutschen Treibhausgasemissionen würden ohne die Senkenwirkung von Forstwirtschaft und Holzverwendung um 14\% höher ausfallen (BAUHUS ET AL. 2017).

HAFNER ET AL. (2017) zeigen in dem Abschlussbericht zur Treibhausgasbilanzierung von Holzgebäuden (FKZ28W-B-3-054-01), dass in den nächsten Jahren ein großes Potenzial für 
Kohlenstoffspeicherung im Wohnungsneubau, sowie zur Reduktion von TreibhausgasEmissionen durch Substitution mineralischer Baustoffe besteht. Bei der Berechnung vergleichender Ökobilanzen, d.h. im Vergleich bestimmter Gebäude, bei denen die mineralischen Baustoffe für die Konstruktion hauptsächlich durch Holz ersetzt wurden, konnten Substitutionsfaktoren zwischen 0,09 und 0,56 errechnet werden. Demnach ließen sich bis zu 56\% der Treibhausgase für die Errichtung eines Einfamilienhauses einsparen. Die untersuchten Mehrfamilienhäuser erzielten aufgrund der Brandschutzbestimmungen geringere Werte. Ein großes Hemmnis, welches aufgezeigt wurde, ist der Ausschluss brennbarer Baustoffe für tragende Zwecke bei der Gebäudeklasse 5 mit einer Höhe $>13 \mathrm{~m}$ und Nutzungseinheiten $>400 \mathrm{~m}^{2}$ entsprechend der Landesbauordnungen, die Abweichungen nur durch Erstellung objektbezogener Brandschutzkonzepte ausnahmsweise ermöglichen (HAFNER, RÜTER ET AL. 2017).

Durch die bauaufsichtliche Zulassung von Buchen-Brettschicht-Holz (BSH) im Oktober 2009 durch das Deutsche Institut für Bautechnik sind für die Substitution die notwendigen rechtlichen Voraussetzungen auch geschaffen. Jedoch gilt die Verarbeitung von Laubholz generell als aufwendiger und kostenintensiver als die des Nadelholzes. Und so darf es nicht wundern, dass derzeit Brettschichtholz (BSH) zwar das wichtigste Bauprodukt für den Ingenieurholzbau darstellt, jedoch zu 95\% aus Fichte hergestellt wird (KNORZ 2012).

Eine Alternative zum BSH stellen Furnierschichtholzträger für tragende Zwecke dar. Diese werden ebenfalls vornehmlich aus Fichte und Kiefer hergestellt. Ein bekannter Hersteller ist die finnische Firma Metsä Wood, welche diese unter dem Markennamen Kerto vertreibt. Vergleicht man jedoch die Festigkeitseigenschaften von Laubhölzern mit denen von Nadelhölzern, sind ihnen vornehmlich Laubhölzer mit einer höheren Dichte $\left(\rho>550 \mathrm{~kg} / \mathrm{m}^{3}\right)$ hinsichtlich der elasto-mechanischen Eigenschaften deutlich überlegen. Die Zugfestigkeit der Buche ist um 100\% höher als die der Fichte (SCHMIDT, KNORZ \& TORNo 2014).

Furnierschichtholzträger für tragendende Zwecke aus Buchenfurnieren herzustellen, stellt somit den logischen nächsten Schritt in der Weiterentwicklung von Bauprodukten aus Holz in Deutschland dar. Dabei kann Deutschland an eine lange Tradition der Werkstoffherstellung mit Buchenfurnieren anknüpfen. So stellte die Blomberger Holzindustrie B. Hausmann GmbH \& Co. KG die erste Sperrholzplatte aus drei bis fünf Buchenfurnieren 1893 in Blomberg her ein Unternehmen, welches heute Bestandteil der Delignit Gruppe ist. Deren Spezialsperrhölzer aus Buchenholz werden weltweit für verschiedenste Produkte im Automobil-, Anlagen-, Industrie-, Hoch- und Werkzeugbau eingesetzt (DELIGNIT 2014 \& LOHMANN 2008). Im Gegensatz zum Sperrholz mit abwechselnd 90 Grad zueinander verdrehten Lagen, bestehen Furnierschichthölzer (LVL) für tragende Zwecke aus überwiegend gleichgerichteten Furnieren. Dies ermöglicht den Einsatz von kontinuierlichen Pressensystemen, ähnlich der Span- und Faserplattenindustrie, anstelle von Mehretagen-Takt-Pressen. Diese Form der Herstellung ist wirtschaftlicher und ermöglicht die Produktion von sehr langen Platten. Die faserparallele Ausrichtung aller Furnierlagen führt dazu, dass LVL in eine Richtung sehr viel belastbarer ist als Sperrholz. 


\subsection{Problemstellung}

Während die Herstellung und Verarbeitung von Nadelholzprodukten seit Jahrzenten als ausgereift bezeichnet werden kann, müssen bei der Produktion von Buchenholzprodukten Herausforderungen gemeistert und Hemmnisse abgebaut werden. Dazu zählen neben dem wuchstypischen Merkmal von Laubbäumen, der Krummschäftigkeit, das Trocknungsverhalten, das Quell- und Schwindverhalten sowie die äußerst geringe Dauerhaftigkeit (SCHMIDT, KNORZ \& TORNO 2014). Ersteres ist durch waldbauliche Konzepte, d.h. Sortenauswahl (Saatgutherkunft) und Bestandespflege, oder später durch die Rundholzsortierung und den gezielten Einkauf beherrschbar. Dass das spezifische Trocknungsverhalten der Buche beherrschbar ist, zeigen Buchenschnittholzerzeuger, ebenso wie die Buchensperrholzhersteller in der Praxis anschaulich. Ein wichtiges Problem bei der Verwendung von Buchenholz besteht jedoch in dem relativ starken Schwinden und Quellen bei Klimawechseln sowie in der mangelnden Resistenz gegen holzzerstörende Pilze (Dauerhaftigkeitsklasse 5 nach DIN EN 350, 2016). Dadurch versagen hinsichtlich eines Wetterschutzes auch die üblichen Beschichtungssysteme in Form von Lacken und Lasuren. Gegen holzzerstörende Pilze könnten biozidhaltige Holzschutzmittel eingesetzt werden, welche jedoch zunehmend im Hinblick auf Auswaschung, Ökotoxizität und Ökopersistenz kritisch gesehen werden.

Aufgrund der mangelnden natürlichen Dauerhaftigkeit und der schlechten Dimensionsstabilität des Buchenholzes, kann auch das Furnierschichtholz bisher nicht für Gebrauchsklassen tauglich sein, in denen eine Materialauffeuchtung stattfinden kann. Die andauernde Exposition des Produktes gegenüber Witterungseinflüssen ist damit auszuschließen. Daher werden in der Anwendung für tragende Zwecke mit potenziellem Witterungseinfluss häufig Nichtholz-Bauprodukte wie Beton und Stahl vorgezogen.

Im Garten- und Landschaftsbau werden neben den üblichen mit Holzschutzmitteln Kesseldruck-imprägnierten Nadelhölzern, bisher vor allem Tropenhölzer wie z.B. Bankirai (Shorea laevis RIDL.) für optisch höherwertige Produkte im Garten- und Living-Bereich eingesetzt. Dazu kommt seit einigen Jahren ein stetig zunehmender Anteil an Wood-PlasticComposites (WPC) als Terrassenbelag.

Die Motivation für diese Forschungsarbeit ergibt sich aus dem zunehmenden Aufkommen an Buchenholz und Mangel an Verarbeitungstechnologien, welche die stoffliche Nutzung dieses wertvollen Rohstoffes, auch in Außenanwendungen, weiter vorantreiben.

Bisher wurden zwei Arten von Furnierwerkstoffen kommerziell angeboten, bei denen eine Holzmodifizierung zur Verbesserung der Eigenschaften Anwendung findet und deren Herstellungsprozess dem hier angestrebten ähnelt. Auf der einen Seite produzierte die Fritz Becker KG (Brakel) Formteile aus DMDHEU-modifizierten Buchenholzfurnieren für wetterfeste Möbel. Dazu wurden die Furniere zunächst modifiziert und dann wie bei der konventionellen Sperrholzherstellung weiterverarbeitet. Auf der anderen Seite bietet die Firma Blomberger (Delignit AG), ebenso wie einige andere Anbieter (z. B. Pagholz Formteile und Röchling), noch weltweit Formteile und Sperrholz mit hohem Phenol-Formaldehydgehalt an. Phenol-Formaldehyd dient bei der Sperrholzherstellung üblicherweise als Klebstoff. Bei diesen Produkten wird mehr Harz, als für die Verklebung notwendig, durch Tauchen oder 
Gießen aufgetragen. Sofern die beleimten Furniere getrocknet werden, erfolgt dies bei niedrigen Temperaturen, damit das Harz nicht voraushärtet. Ohne erneuten Leimauftrag werden die Furniere in der Heißpresse verklebt und verdichtet. Das in das Holz eingedrungene Harz kann dabei möglicherweise eine Modifizierung der Zellwand bewirken, bewiesen ist dies aber nicht. Dass o.g. Produkte bereits kommerziell hergestellt werden, beweist zumindest, dass es einen Markt und eine Nachfrage nach solchen Produkten gibt.

Das Problem besteht jedoch darin, dass alle heute nach diesem Verfahren hergestellten Produkte auf Furnierbasis, aufgrund hoher Beladungsgrade (=Harzanteil) und Verdichtung beim Pressen, extrem hohe Dichten von $1200 \mathrm{~kg} / \mathrm{m}^{3}$ bis $1450 \mathrm{~kg} / \mathrm{m}^{3}$ aufweisen. Durch eine Erhöhung der Dichte werden die mechanischen Kennwerte positiv beeinflusst sowie die Wasseraufnahme vermindert. Jedoch stellt die Erhöhung der Dichte eine Erhöhung des Ressourcenverbrauchs dar, der bewirkt, dass das absetzbare Volumen bei gleichem Materialund Kostenaufwand sinkt. Eigene Untersuchungen zur Dauerhaftigkeit dieser Produkte durch Holzabbauversuche mit Basidiomyceten nach ENV 12038 (2002) haben zudem gezeigt, dass diese Form der Behandlung zur Erzielung einer sehr hohen Pilzresistenz unzureichend ist, da signifikante Masseverluste festgestellt werden konnten (BICKE, BIZIKS ET AL. 2016). Eine mittelfristig verringerte Wasseraufnahme versagte zudem im Langzeitversuch. Die hohe Dichte bewirkte zusätzlich ein überproportional hohes Quellen.

Verschiedene Untersuchungen zeigen, dass durch die Modifizierung mit Kondensationsharzen auf Basis von Phenol-Formaldehyd die Dauerhaftigkeit von Holz ohne Einsatz von Bioziden deutlich erhöht wird (STAMm, SEBorg ET AL. 1962; FuRUno, IMAMURA ET AL. 2004; BicKe, MAI ET AL. 2012). Außerdem ist modifiziertes Holz dimensionsstabiler, härter und weist höhere Druckfestigkeiten auf (STAMm AND SEBORg 1942; GALPERIN, KulESHOV ET AL. 1995; RoWELl 1999; DEKA AND SAIKIA 2000; EPMEIER, WESTIN ET AL. 2004).

\subsection{Zielstellung und Lösungsansatz}

Die der vorliegenden Dissertationsschrift zugrundeliegende Forschung hatte das Ziel, Buchenvollholz und Buchenfurniere für die Herstellung von Furnierschichtholz (FSH), bzw. Laminated Veneer Lumber (LVL), biozidfrei zu modifizieren, um eine verringerte Feuchteaufnahme, verbesserte Dimensionsstabilität und erhöhte Resistenz gegen holzzerstörende Pilze zu erreichen. Daraus resultierende Produkte sollten im Außenbereich mit und ohne Erd- oder Süßwasserkontakt (Gebrauchsklassen 3-4 nach EN 335 (2013)) als Baustoff für tragende und nichttragende Zwecke geeignet sein.

Die wesentliche Aufgabe einer wirkungsvollen Holzmodifizierung besteht in der Eindringung des Stoffes, nicht nur in das Zelllumen, sondern auch in die Zellwand und der dortigen Fixierung (HILl 2006). Der Lösungsansatz umfasste den Einsatz kommerziell erhältlicher, niedermolekularer Phenol-Formaldehyd-Harze zum Zweck der Zellwandmodifizierung. Diese sollten eine definierte Molmassenverteilung aufweisen, welche bewirken sollte, dass der größtmögliche Teil des eingesetzten Harzes unter bestimmten Prozessbedingungen in die Zellwand eindringt und dort polymerisiert. Die Vorkondensate sollten zudem einen niedrigeren Feststoffgehalt als übliche Tränk- und Klebharze aufweisen und verschiedene Lösungsmittel beinhalten. Dies sollte gewährleisten, dass mit einer hohen Ressourceneffizienz und geringer Steigerung der Dichte, die Resistenz gegenüber holzabbauenden Basidiomyceten 
sowie eine stark verminderte Quellung unter Erhalt der charakteristischen elastomechanischen Eigenschaften erreicht werden.

Zu den Hauptzielen dieser Forschungsarbeit zählten somit die Entwicklung eines geeigneten Imprägnierprozesses, eines angepassten Trocknungsprozesses, einer optimierten Verklebung sowie die Vorbereitung eines dem Herstellungsprozess des Standardfurnierschichtholzes ähnlichen semi-kontinuierlichem Prozesses. Teilziele umfassten den Nachweis der Einbringung und der Fixierung der Modifizierungschemikalie in der Zellwand, den Nachweis der erhöhten biologischen Dauerhaftigkeit durch in vitro-Abbauversuche sowie das Erreichen einer hohen Dimensionsstabilität. Des Weiteren waren gute mechanische Festigkeiten der Werkstoffe Voraussetzung für den Einsatz im konstruktiven Ingenieurholzbau, welche mit Hilfe von Standardprüfverfahren untersucht wurden.

$\mathrm{Zu}$ diesem Zweck wurden Buchenholzfurniere unter Einsatz von Vakuum und Druck in einer Labortränkanlage mit Phenol-Formaldehyd imprägniert. Hierbei sollten für verschiedene Harze mit unterschiedlichen Molekülgrößen und Konzentrationen die optimalen Prozessbedingungen zur vollständigen und effizienten Tränkung der Furniere gefunden werden. Kommerziell erhältliche PF-Imprägnierharze sollten dafür charakterisiert und der Einfluss verschiedener Prozessparameter auf die Werkstoffeigenschaften im Labor-und Technikumsmaßstab untersucht werden. Dazu gehörten der Entwurf eines an das Material angepassten Bewitterungstests im Labormaßstab und eine adaptierte Freilandbewitterung sowie eine Dauerhaftigkeitsuntersuchung im Freiland mit Erdkontakt.

Unter Berücksichtigung der Ergebnisse von MAI (2009) und WEPNER (2006), wurde für die Herstellung des Furnierschichtholzes (LVL) ein einstufiger Prozess gewählt, bei dem die Verklebung und Aushärtung der Modifizierungschemikalie zeitgleich in einer Heißpresse erfolgten, da hier die höchste Verklebungsqualität zu erwarten war. 


\section{Grundlagen}

\subsection{Furnierwerkstoffe}

\subsubsection{Aufbau und Herstellung}

Furnierwerkstoffe sind mehrlagige plattenförmige Werkstoffe aus miteinander verklebten Schälfurnieren. Je nach der Faserrichtung der Furniere unterscheidet man zwischen Sperrholz und Furnierschichtholz bzw. Laminated Veneer Lumber (LVL). Beim Furniersperrholz werden alle Furniere kreuzweise verleimt, so dass sich ein Faserwinkel zwischen den Furnieren von $90^{\circ}$ ergibt. LVL hingegen besteht aus mehreren Lagen faserparallel orientierter Furniere. Durch bis zu 15\% zusätzliche querorientierte Furnierlagen wird es zudem deutlich dimensions- und formstabiler. Die Furniere werden überwiegend im Schälverfahren hergestellt, bei dem ein Stammabschnitt in Rotation um die Längsachse versetzt wird und ein Schälmesser mit Druckbalken daran geführt wird. In diesem Verfahren hergestellte Furniere werden dann als Schälfurniere bezeichnet. Geschälte Furniere durchlaufen dann eine kontinuierliche Trocknung die nur wenige Min dauert bei Temperaturen von bis zu $130^{\circ} \mathrm{C}$. Daran schließt sich eine Qualitätssortierung und ggf. ein Ausschneiden von Holzfehlern an. Um ein bestimmtes Plattenmaß zu erzielen, werden Furniere zum Teil in einem separaten Verfahren wieder zusammengefügt. Bei der Verwendung kontinuierlicher Pressen wird jedoch darauf verzichtet. Hier werden die Furniere in Produktionsrichtung abgeschrägt und mit einer entsprechenden Überlappung abgelegt. An kontinuierliche Pressen schließt sich eine Diagonalkreissäge an. Zur Verklebung der Furniere werden derzeit noch am häufigsten Phenol-Formaldehyd (PF)-Klebstoffe eingesetzt. Dabei sind Auftragsmengen zwischen 190 $\mathrm{g} / \mathrm{m}^{2}$ (BORTOLETTO 2006) und $200 \mathrm{~g} / \mathrm{m}^{2}$ (ADERHOLD 2006) üblich. PF-Klebstoffe zeichnen sich durch Kochfestigkeit und niedrige Formaldehydemmissionen aus, jedoch ist die dunkelbraune Leimfuge deutlich erkennbar. PF gebundene Platten werden heiß eingestapelt, um ein Nachreifen zu ermöglichen. Harnstoffformaldehyd(HF)-Klebstoff gebundene Platten müssten gekühlt werden, um eine Hydrolyse zu verhindern. HF-Klebstoffe kommen aufgrund ihrer Unbeständigkeit gegen Feuchte nur selten zum Einsatz. Eine Alternative bieten die ebenfalls feuchtebeständigen hellen Melaminformaldehyd-Klebstoffe. Neben reinen PF-, MF- und HFKlebstoffen werden auch Mischkondensate mit Melamin eingesetzt. Seit mehr als 10 Jahren kommen auch die etwas teureren Polyurethan- und Isocyanat-basierten Klebstoffe zum Einsatz (YEH 2006). Sie kommen ohne Formaldehyd aus, stellen aber neue Anforderungen an die Arbeitssicherheit bei der Herstellung, aufgrund der akuten Toxizität der Cyanatgruppen. Je nach Produkt können die Oberflächen nach der Verklebung geschliffen und beschichtet, sowie auf Format geschnitten werden.

Bei Furnierwerkstoffen bleibt das günstige Verhältnis von Festigkeiten und Gewicht des Holzes erhalten. Im Vergleich zum Massivholz ist das Schichtholz aber deutlich homogener hinsichtlich seiner Festigkeitseigenschaften, da Holzfehler im Werkstoff verstreut werden. Die Abmessungen der Platten sind allein durch die Anlagen des Herstellers begrenzt. Furnierwerkstoffe sind insgesamt hochwertiger und teurer als andere, übliche plattenförmige Holzwerkstoffe (insbes. Span- und Faserplatten) und wird daher in besonders anspruchsvollen Bereichen eingesetzt. Darüber hinaus gibt es zahlreiche Sonderausführungen für spezielle Anwendungen (KolLMANN 1951, NiEMZ 1993). 


\subsubsection{Produkte und Anwendung}

Furnierwerkstoffe sind in der Regel plattenförmige Werkstoffe. Sie finden ihre Anwendung im Bauwesen, im Möbel- und Instrumentenbau sowie im Transportwesen - häufig werden sie im Innen-und Messebau verwendet. „Sperrholz folgt der Spanplatte als dem mengenmäßig weltweit bedeutendsten Holzwerkstoff dicht auf dem zweiten Platz" (MARUTZKY \& SCHWAB 2008).

Im Bauwesen dienen vor allem Sperrhölzer als Schalungsplatten oder als aussteifende Wandund Deckenelemente. Im Möbelbau dienen Sperrhölzer als flächige Teile von Kastenmöbeln, aber auch als Böden von Schubladen und als Rückwand. Unter Verwendung dünner Furniere und spezieller Pressformen lassen sich jedoch auch Formsperrhölzer, z.B. für freischwingende Stühle, herstellen. Ein weiteres wichtiges Einsatzgebiet sind auch die Böden und Innenausbauten von Schiffen und Kraftfahrzeugen, insbesondere im Güterverkehr. In der Industrie dienen sie dem Modell- und Werkzeugbau, z.B. als Vorrichtung zum Umformen von Blechen.

Das LVL findet man überwiegend im Bauwesen und es wird überall dort eingesetzt, wo auch Brettschichtholz eingesetzt wird (MARUTZKY \& SCHWAB 2008). Wie bereits erwähnt, sind die Dimensionen nur durch die Anlagengröße des Herstellers begrenzt. Furniere lassen sich zu nahezu beliebig großen Blättern zusammenfügen und mit etwas Versatz der Fügestelle zwischen den Lagen können negative Einflüsse auf die Festigkeitseigenschaften vermieden werden. Abbildung 1 veranschaulicht, wie typische Produkte aus Buchen-LVL aufgebaut sein können. Es wird deutlich, dass das LVL nicht ausschließlich als Platte Anwendung findet, sondern auch durch ein Auftrennen und erneutes Zusammenfügen zu Trägern weiterverarbeitet wird. Dabei werden häufig kalthärtende hydrolysestabile Phenol-ResorcinHarze (PRF) genutzt. Ein Anwendungsbeispiel von Trägern im konstruktiven Ingenieurholzbau zeigt Abbildung 2.

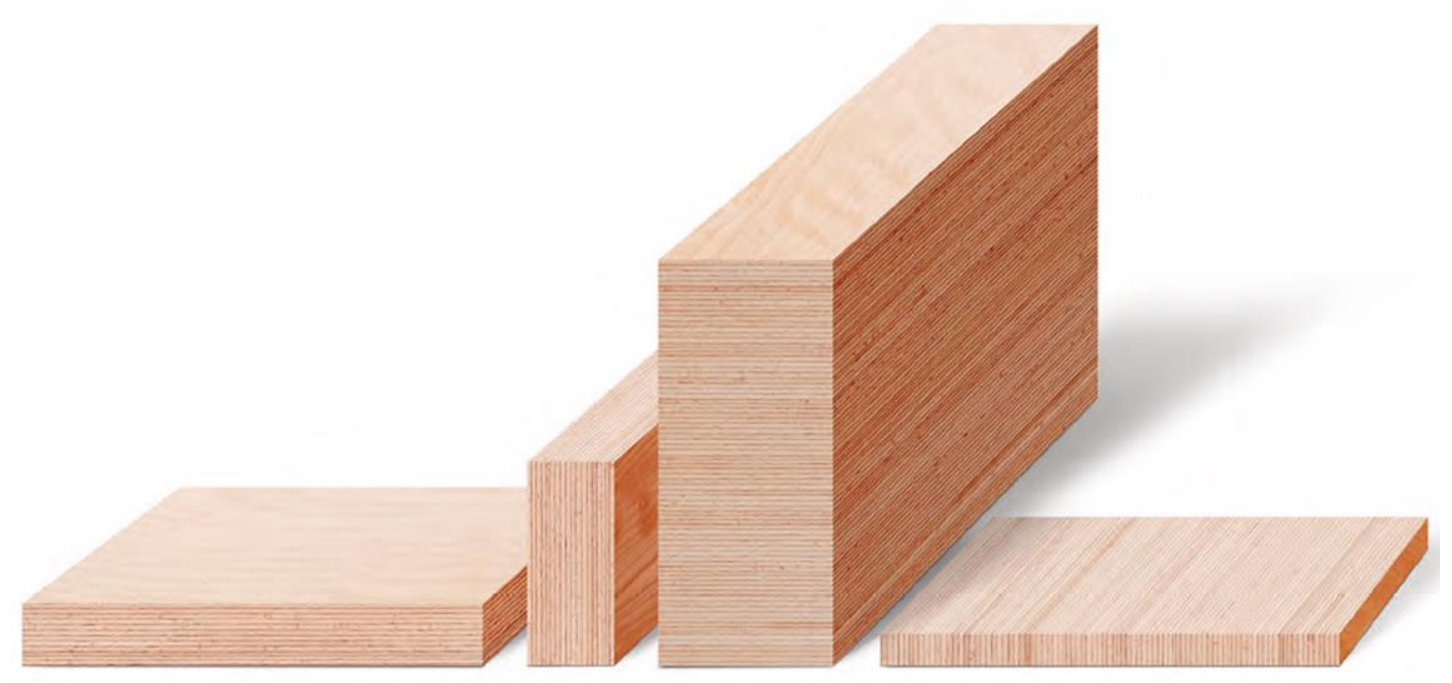

Abbildung 1: Platten, Träger und Paneele auf Basis von Furnieren - faserparallel oder mit bis zu $20 \%$ Querlagen verklebt (Pollmeier 2014). 


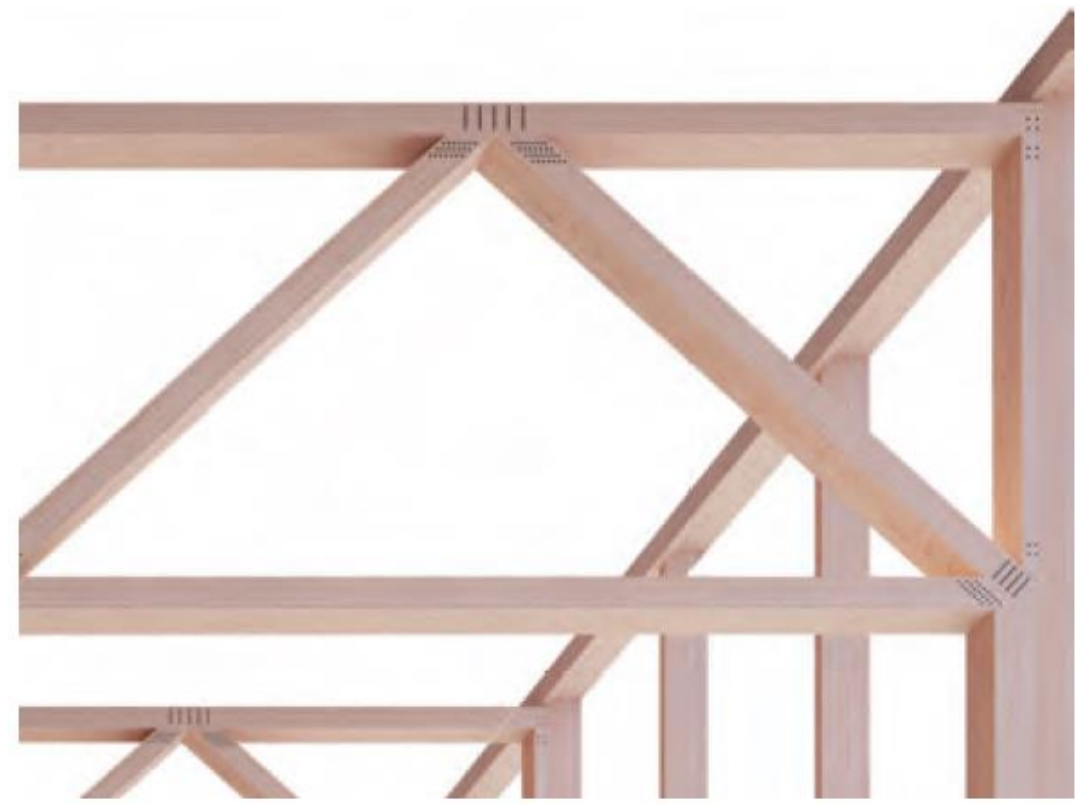

Abbildung 2: Verwendung von Furnierschichtholz als Fachwerksträger (Pollmeier 2014).

\subsubsection{Nachhaltigkeit}

Mit der Entwicklung dauerhafter Bauprodukte aus Buchenfurnieren als eine natürlich nachwachsende Ressource, wird ein Beitrag zu dem Problem der Verknappung fossiler Ressourcen geleistet. Wie in Abbildung 3 dargestellt wird, verbraucht die Herstellung von 1 Tonne Beton und Stahl die 3- und 17-fache Menge an Energie wie für die Herstellung von Holzprodukten. In dieser Betrachtung findet das relativ günstigere Verhältnis von Festigkeit zu Gewicht bei Holzprodukten jedoch noch keine Beachtung. Somit können Energiebilanzen für definierte Anwendungen, z.B. Autobahnbrücken, noch günstiger ausfallen.

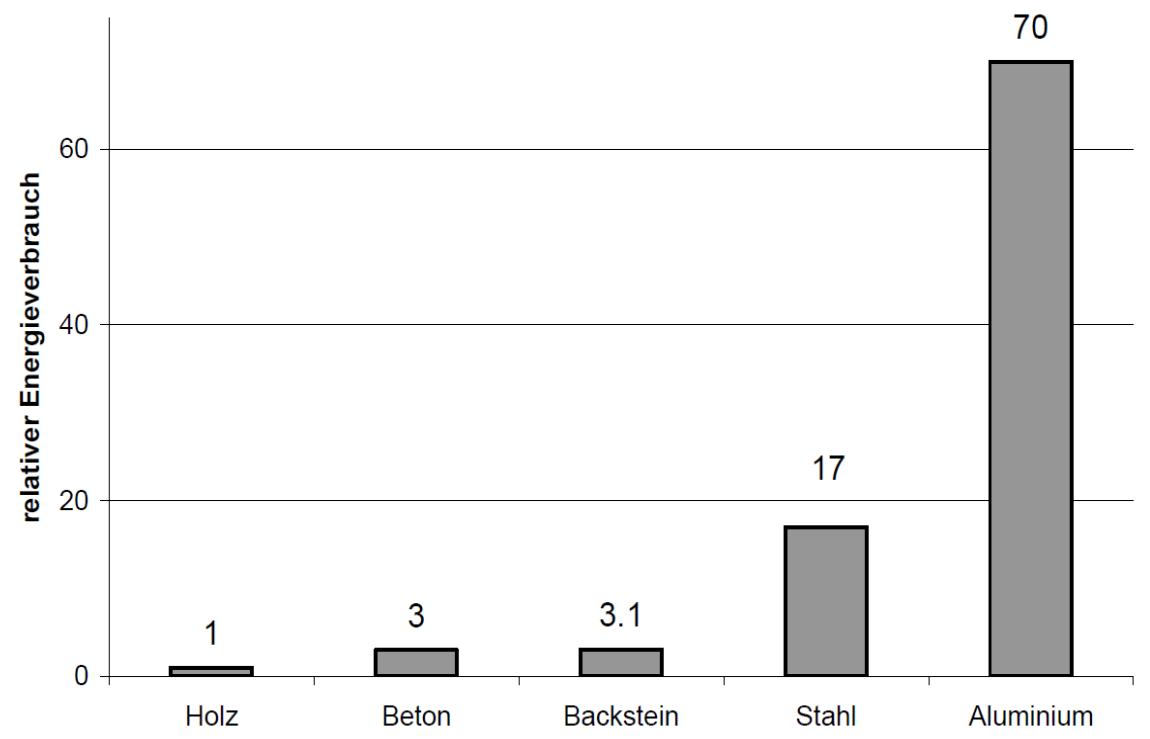

Abbildung 3: Mittlerer relativer Energieverbrauch $(\mathrm{Holz=1})$ ausgewählter Rohstoffe zur Herstellung pro Tonne Fertigprodukt (Lippke, Wilson et al. 2004).

Der generellen Betrachtung des relativen Energieverbrauches sei nun eine differenziertere Betrachtung der Holzwerkstoffe hinzugefügt. (PuETTMANN \& WiLSON 2005) untersuchten in 
einer Life-Cycle-Inventory (LCI) den kumulativen Energieverbrauch bei der Herstellung (Cradle-to-Gate) üblicher Holzbauprodukte in den Vereinigten Staaten von Amerika.

Abbildung 4 zeigt, dass der Energieverbauch für die Furnierwerkstoffe LVL, Sperrholz und Glulam, die im Südosten der USA hergestellt wurden, im Bereich von $6000 \mathrm{MJ} / \mathrm{m}^{3}$ lagen. Im Vergleich zu kammergetrockneten Schnittholz liegen sie um ca. 72\% höher. Dies ist auch zum großen Teil auf die Verwendung von Klebstoffen zurückzuführen. Bei der Herstellung von LVL entfielen $1048 \mathrm{MJ} / \mathrm{m}^{3}$ und damit ein Anteil von 17\% des kumulativen Energieverbauchs auf die Herstellung des PF-Klebstoffes. Eine detaillierte Aufschlüsselung aller eingesetzten Materialien, Treibstoffe und Elektrizität zur Produktion von LVL und Nebenprodukten sowie Emissionen geben (WILSON \& DANCER 2005).

WILSON (2009) zeigte, dass in den USA für die Produktion von UF (65 \%ig) 29,4 MJ/kg, für MUF (60 \%) 31,7 MJ/kg, für PF (47,4 \%) 40,4 MJ/kg und PRF (60\%) 40,5 MJ/kg nötig waren. Diese Betrachtung schloss alle Treibstoffe, Ausgangsstoffe und Elektrizität ein. Dabei basierten alle chemischen Bestandteile auf Gas oder Erdöl. In der Betrachtung von LVL aus 3,7 mm starken Buchenfurnieren folgt daraus, dass bei einer PF-Harz basierten Verklebung bei einem Beleimungsgrad von $150 \mathrm{~g} / \mathrm{m}^{2} 1638 \mathrm{MJ} / \mathrm{m}^{3}$ auf das fertige Produkt entfallen. Bei einem vermehrten Einsatz von PF, wie im Falle einer Imprägnierung der Furniere, erhöht sich dieser Anteil entsprechend.

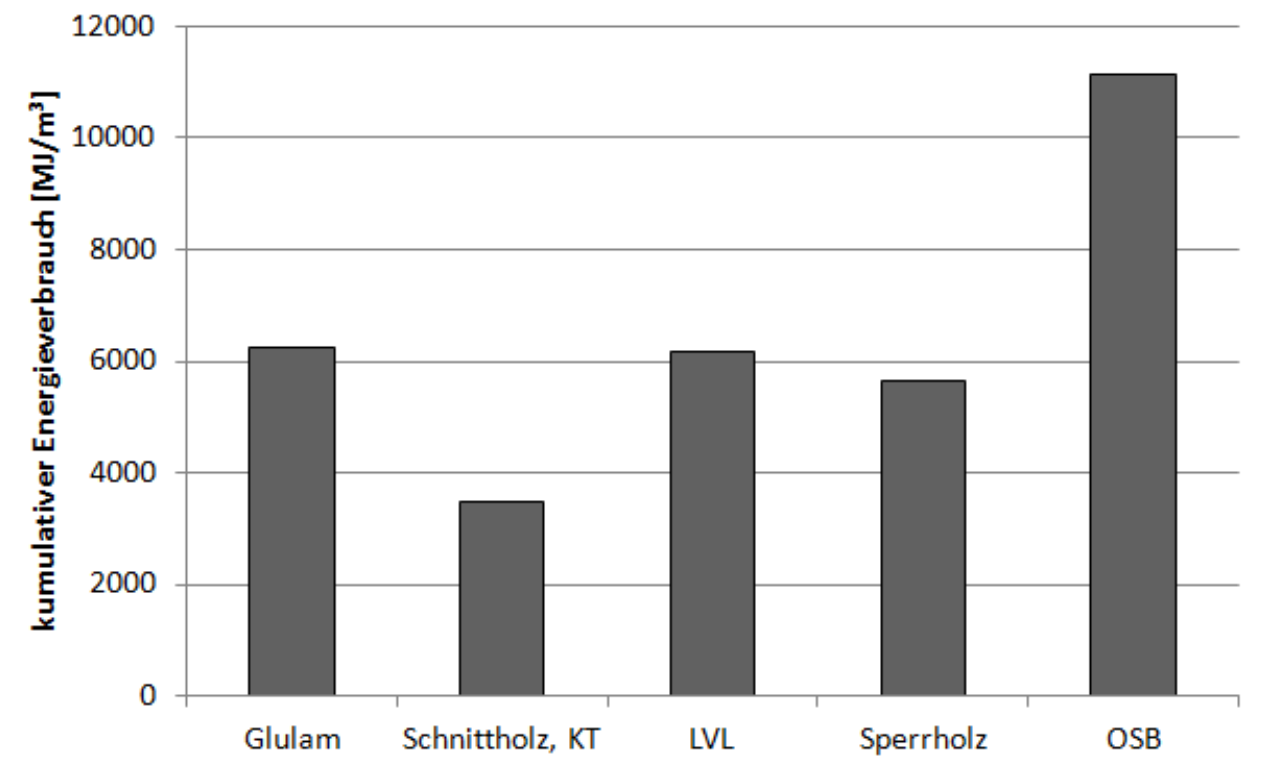

Abbildung 4: Absoluter Energieverbrauch bei Holzwerkstoffherstellung (KT=kammergetrocknet) (Puettmann \& Wilson 2005). 


\subsection{Holzmodifizierung mit Phenol-Formaldehyden}

Die Holzmodifizierung ist eine chemisch-technische Vergütung, welche ohne den Einsatz von herkömmlichen biozidhaltigen Holzschutzmitteln die Resistenz gegenüber holzzerstörenden Organismen erhöht. Des Weiteren führt die Modifizierung zu einer Reduzierung der Wasseraufnahme und den damit verbundenen negativen Eigenschaften wie Dimensionsänderungen und Rissbildung. Auf diese Weise lassen sich bei einheimischen Holzarten vergleichbare Eigenschaften wie bei Tropenhölzern schaffen.

Klassische Verfahren um die Resistenz gegenüber Schadorganismen zu erhöhen, erforderten bisher den Einsatz von Bioziden mit ökotoxischer Wirkung. Um eine Hydrophobierung zu erreichen, wurden bisher technische Paraffine auf der Holzoberfläche oder im Zelllumen eingesetzt, die wenig langzeitstabil sind und die Verklebung negativ beeinflussen. Die Erfahrungen auf dem Gebiet der chemischen Holzmodifizierung, durch Arbeiten von H. Militz, C. Hill und weiteren, haben gezeigt, dass eine wirksame dauerhafte Holzmodifizierung nur in der Zellwand stattfinden kann, da dies der Ort ist, an dem sich Wasser anlagert, zu Quellung führt und Pilze ihren Abbau verursachen. Daraus lässt sich schließen, dass für eine effektive Modifizierung grundsätzlich andere Anforderungen an ein Harz erfüllt sein müssen, als in der Verwendung als Klebstoff.

Bereits seit den 1930er Jahren werden Imprägnierungen von Holz mit verschiedenen Harzen wissenschaftlich erforscht. Die potentiell geeignetsten und am genauesten untersuchten Harze basieren auf Phenol-Formaldehyd (PF), Melaminformaldehyd (MF), 1,3-Dimethylol-4,5dihydroxyethylenharnstoff (DMDHEU) und Furfurylalkohol (FA) (HILL 2006). Prozesse zur Vollholzmodifizierung mit DMDHEU und FA wurden bereits kommerzialisiert; ebenso die Behandlung von Sperrholzfurnieren mit DMDHEU und Phenol-Formaldehyd. Die komplizierte Prozessführung bei der Furfurylierung und die starke Versprödung des Holzes durch DMDHEU sind Schwachpunkte für die angestrebte Entwicklung. Die eigene Forschung hat sich daher auf die Untersuchung von PF-Harzen zur Modifizierung konzentriert.

Die Modifizierung mit PF-Harz verbessert die Resistenz gegenüber holzzerstörenden Pilzen (Dauerhaftigkeitsklasse 1...2) deutlich (LUKOWSKI 1999; YUSUF, SUDIYANI ET AL. 1999). Hierbei wirken verschiedene Schutzmechanismen zusammen; u.a. verringert das in die Zellwand eingelagerte Harz die Zugänglichkeit für Feuchtigkeit und Abbaureagenzien. Dazu verbessert die Behandlung die Dimensionsstabilität, Härte und Druckfestigkeit des Holzes. Die Zugfestigkeit verschlechtert sich gewöhnlich etwas, während die Biegefestigkeit und der Biege-E-Modul unbeeinflusst bleiben oder leicht zunehmen (STAMM \& SEBORG 1942; GALPERIN, Kuleshov et Al. 1995; Rowell 1999; Deka \& SAiKia 2000; Epmeier, WeStin ET AL. 2004). Das Ausmaß dieser Veränderungen wird durch den Harztyp und den Beladungsgrad bestimmt. Mit zunehmendem Beladungsgrad verändern sich die Eigenschaften dabei unterschiedlich schnell. Während Beladungsgrade von 10...15 \% oft einen Grenzwert für die Abbaubarkeit gegen Pilze darstellen (TAKAHASHI \& IMAMURA 1990), entwickeln sich die mechanischen Eigenschaften eher linear. Andererseits wirken auch die Prozessparameter entscheidend auf die Festigkeitseigenschaften (RowELl 1999). Durch die Abstimmung aller Faktoren könnten die späteren Holz(-Werkstoff)-Eigenschaften an die Verwendung angepasst werden. 
Es ist bekannt, dass das Harz für eine effiziente Modifizierung in die Zellwand eingelagert und fixiert werden muss (Furuno, ImAMura et AL. 2004; Bicke, MAI ET AL. 2012). Dimere und Oligomere sollten bei der Imprägnierung daher entsprechend klein sein, so dass diese in die Zellwand eindringen können. RowELl (2005) gab an, dass dies mit einer Molmasse von 290$480 \mathrm{~g} / \mathrm{mol}$ der Fall ist, während mit einer Molmasse von $820 \mathrm{~g} / \mathrm{mol}$ der überwiegende Teil im Zelllumen verbleibt (Furuno, IMAMURA ET AL. 2004). BIZIKS ET AL. (2016) zeigten, dass ein Zusammenhang zwischen der Resistenz gegenüber Holzabbau durch Braun- und Weißfäulen mit der Molmasse der Harze und ihrer Fähigkeit in die Zellwand einzudringen, besteht. Neben einer verminderten Wasseraufnahme der Zellwand infolge des Bulking wurde eine physikalische Blockierung der Mikroporen der Zellwand mit einhergehender Unwegsamkeit für Enzyme und nicht-enzymatische Metabolite der Pilze als Wirkmechanismus vermutet. HoSSEINPOURPIA \& MAI (2016) befanden, dass mit zunehmendem WPG bei der PF-Modifzierung von Mikrofurnieren aus Pinus sylvestris L. die Fenton-Reaktion (Oxidativer Abbau der Zellwandpolymere) zunehmend unterbunden werden konnte. Maßgeblich verantwortlichen dafür machten sie die verminderte Fähigkeit der Eisenionen $\left(\mathrm{Fe}^{2+}\right)$ in die Zellwand zu diffundieren und mit Wasserstoffperoxid $\left(\mathrm{H}_{2} \mathrm{O}_{2}\right)$ Hydroxyradikale $(\cdot \mathrm{OH}) \mathrm{zu}$ bilden. Dies bewiesen sie indirekt auch bei steigendem WPG durch einen verminderten Verbrauch an $\mathrm{H}_{2} \mathrm{O}_{2}$ sowie eine verringerte Festigkeitsabnahme (Zugfestigkeit) in Faserrichtung. Chemische Reaktionen des PF-Harzes mit Holzbestandteilen sind möglich, aber nicht notwendig für eine erfolgreiche Modifizierung (HILL 2006). Die wesentliche Aufgabe bei einer wirkungsvollen Holzmodifizierung besteht somit in der Eindringung des Wirkstoffes in die Zellwand und der dortigen Fixierung. Zu diesem Zweck sind in dieser Arbeit vorwiegend basische PhenolFormaldehyd-Harze mit niedriger Molmasse vorgesehen. Bestimmte Harze werden hinsichtlich ihrer Molekülgrößen (-verteilung) und Reaktionskinetik charakterisiert. Dies soll ermöglichen, dass der größtmögliche Teil des eingesetzten Harzes unter bestimmten Prozessbedingungen in die Zellwand eindringt und dort polymerisiert. Angewendet werden sollen die Harze zudem mit einem niedrigeren Feststoffgehalt als üblich. Der in der Lösung enthaltende Feststoffgehalt liegt je nach Anforderung zwischen $6 \%, 25 \%$ und $55 \%$ (Gewichts-\%). Die Lösungsmittel können Wasser und verschiedene Alkohole sein (z.B. Ethanol und Methanol) bzw. Mischungen von beiden.

Bisher wurden zwei Arten von Produkten kommerziell angeboten, deren Herstellungsprozess dem hier angestrebten ähneln. Auf der einen Seite produzierte die Fritz Becker KG (Brakel) Formteile aus DMDHEU-modifizierten Buchenholzfurnieren für wetterfeste Möbel. Dazu werden die Furniere zunächst modifiziert und anschließend wie bei einer konventionellen Sperrholzherstellung weiterverarbeitet. Auf der anderen Seite bietet die Firma Blomberger (Delignit AG) ebenso wie einige andere Anbieter (z.B. Pagholz Formteile oder Röchling) Formteile und Sperrholz mit einem sehr hohen Phenol-Formaldehydgehalt an. PhenolFormaldehyd dient bei der Sperrholzherstellung üblicherweise als Klebstoff. Bei diesen Produkten wird durch Tauchen oder Gießen mehr Harz als für die Verklebung allein notwendig aufgetragen.

Soweit bekannt, weisen alle heute nach diesem Verfahren hergestellten Produkte auf Furnierbasis aufgrund hoher Beladungsgrade (=Harzanteil) und der Verdichtung beim Pressen extrem hohe Dichten von $1200 \ldots 1450 \mathrm{~kg} / \mathrm{m}^{3}$ auf. Durch eine Erhöhung der Dichte werden die 
elasto-mechanischen Kennwerte sowie die Wasseraufnahme zwar positiv beeinflusst, jedoch ist damit ein erhöhter Ressourcenverbrauch verbunden. In der vorliegenden Arbeit werden insbesondere wenig verdichtete Produkte angestrebt, die durch den effektiveren Einsatz mit deutlich geringeren Beladungsgraden unterschiedlicher Harzsysteme, und damit geringerem Material- und Kostenaufwand, hergestellt werden können. Dabei muss das Harz für eine effektive Modifizierung grundsätzlich in die Zellwand eindringen können. Da deren Porengröße unter 4nm beträgt, muss das Harz somit andere Anforderungen erfüllen als in der Verwendung als Klebstoff. Hier wird daher ein Verfahren angestrebt, bei dem - ähnlich der Modifizierung mit DMDHEU - die Modifizierung und die Verklebung getrennt sind.

Aufgrund des Verzichts auf biozide und schwermetallhaltige Holzschutzmittel entfällt daraufhin die Einordnung in die Altholzklasse IV, welche eine Wiederverwertung ausschließen und nur noch die energetische Nutzung vorsehen würde. Es besteht somit die Möglichkeit der Kaskadennutzung durch Aufbereitung von Altholz zu Holzhackschnitzeln und Holzspänen für die Herstellung von Holzwerkstoffen, wie z.B. Spanplatten. Dauerhafte Buchenholzprodukte können nahezu energieneutral produziert werden, da entstehende Rinden-, Schleif- und Sägeabfälle der standorteigenen Wärmeenergieerzeugung des Produktionsbetriebes dienen können.

\subsection{Phenol-Formaldehyde}

Phenol-Formaldehyde, die auch Phenoplaste genannt werden, sind Polymerverbindungen auf Basis von Phenol und Formaldehyd (HABENICHT 2009). Erste Versuche zur Herstellung von Phenol-Formaldehyd-Kondensaten gehen auf das Jahr 1872 zurück. Als erstes synthetisches PF-Harz wird das sog. Laccain als Schellack-Ersatz im Jahr 1902 erwähnt, aber als Geburtsstunde des Duroplasten bezeichnet GrunWALD (2002) das auf das Jahr 1907 datierte „Hitze und Druck“-Patent von L.H. Baekeland. Phenol-Formaldehyde sind grundsätzlich nach ihrem Syntheseweg und dem pH-Milieu in dem sie reagieren zu unterscheiden. So werden PFHarze, die durch alkalische Kondensation hergestellt werden, als Resole bezeichnet, während Produkte der sauren Kondensation als Novolake bezeichnet werden (GRunWALD 2002). Resole sind im Anfangsstadium löslich und schmelzbar und reagieren zu duroplastischen Netzwerken, sog. Resiten, aus, wohingegen Novolake auch nach dem Härten, z.B. mit Hexamethylentetramin, eine weitgehend lineare Struktur aufweisen (HABENICHT 2009). Novolake finden in dieser Arbeit keine Anwendung.

Die Herstellung von Phenol-Formaldehyden basiert in der Regel auf dem Einsatz unsubstituierten Phenols - nur selten werden substituierte Phenole wie Kresol, Resorcin oder Bisphenol A eingesetzt. Resole werden unter Einsatz von wässrigen Formaldehydlösungen (30-50\%) und basischer Katalysatoren in Rührkesselreaktoren diskontinuierlich synthetisiert. Katalysatoren haben nicht nur Einfluss auf die Reaktionsgeschwindigkeit, sie können die Wahrscheinlichkeit der Addition von Formaldehyd in der ortho-Position in folgender Reihe begünstigen: $\mathrm{K}<\mathrm{Na}<\mathrm{Li}<\mathrm{Ba}<\mathrm{Sr}<\mathrm{Ca}<\mathrm{Mg}$ (SCHROD 2002). Daneben können auch organische Katalysatoren, die Trialkylamine, eingesetzt werden. Diese sind, bis auf das Triethylamin, jedoch schwächer als $\mathrm{NaOH}$. Für Standardharze wird am häufigsten die Verwendung von $\mathrm{NaOH}$ als Katalysator genannt. Die Synthese verläuft in zwei Stufen. Primär kommt es zu einer Methylolierung durch nucleophile Addition von 1 bis 3 Molekülen 
Formaldehyd an ein Phenolation bei $\mathrm{T}<60^{\circ} \mathrm{C}$. Da diese Reaktion leicht exotherm verläuft, kann das Abführen von Energie sinnvoll sein oder die Energie für die Fortführung der Synthese genutzt weden. Sekundär kommt es bei $\mathrm{T}>60^{\circ} \mathrm{C}$ zu einer Kondensation (Abbildung 6) von Methylolphenolen (hydroxymethylierten Phenolen) - untereinander oder mit Phenol - unter Abgabe von Wasser. Demnach weisen die entstehenden Netzwerkstrukturen Methylenbrücken (- $\left.\mathrm{CH}_{2}-\right)$ und Methylenetherbrücken $\left(-\mathrm{CH}_{2}-\mathrm{O}-\mathrm{H}_{2} \mathrm{C}-\right.$ ) auf (GRUNWALD 2002 \& HABENICHT 2009). In allen Schritten sind die Temperaturkontrolle sowie die Zeit entscheidend für die spätere Zusammensetzung des Harzes hinsichtlich seiner Molekülgrößenverteilung.

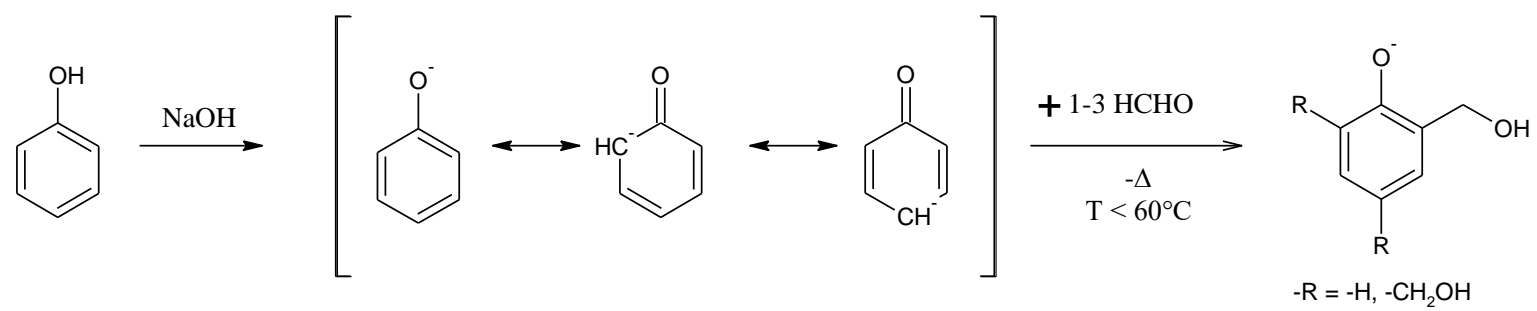

Abbildung 5: Methylolierung von Phenolationen. Gezeichnet in ACD/ChemSketch V.14.01.

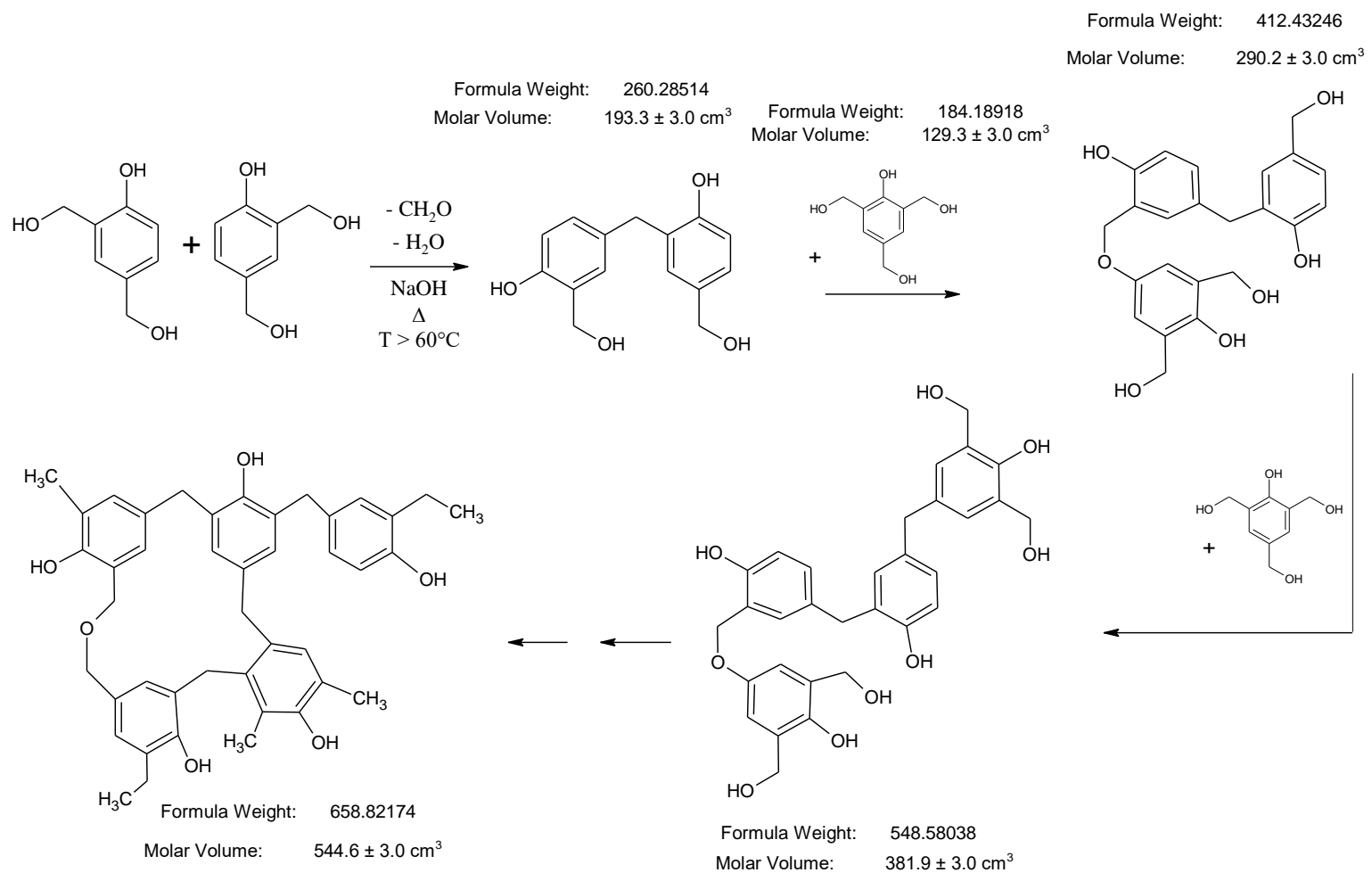

Abbildung 6: Kondensationsreaktion eines Resols. Gezeichnet und berechnet in ACD/ChemSketch V.14.01.

Aufgrund der chemischen (C-C-)Bindung des Phenols an das Formaldehyd, sind PF-Harze thermisch sehr stabil und neigen nicht zur Rückreaktion. Aufgrund dessen kommen sie heute überwiegend als Klebstoffe mit niedrigem Formaldehydabgabepotenzial zum Einsatz, wenn besonders feuchte- und wetterfeste Verklebungen benötigt werden (GRUNWALD 2002 \& HABENICHT 2009). Am häufigsten werden sie derzeit in der Sperrholz- und Furnierschichtholzindustrie eingesetzt, wo ihre charakteristische dunkelbraune Leimfuge nicht als störend empfunden wird. Im Bereich der Span-und OSB-Plattenproduktion kommen sie nicht zum Einsatz, bei ersterem dominiert das Harnstoffharz (UF-Harz) und bei letzterem 
dominieren das polymere Isocyanat (PMDI) und das Melamin-Harnstoff-Formaldehyd (MUF). Bei der Faserplattenproduktion (MDF und HDF), wo ebenfalls vornehmlich das PMDI Verwendung findet, kommen PF-Harze nur bei Nischenprodukten, wie z.B. der Faserformteilproduktion, zum Einsatz. Hier werden die relativ gute Lagerstabilität und Kaltklebrigkeit geschätzt, die es ermöglichen Fasern zu beleimen und das Faservlies als Halbzeug zwischenzulagern und zum Endkunden $\mathrm{zu}$ versenden, welcher in einer Formteilpresse unter Hitzeeinwirkung Bauteile für den Automobilsektor herstellt. Neben Verklebungen im Holzwerkstoffbereich finden sie auch Anwendung im Bereich nichtorganischer Materialien, wie z.B. Metallen. Der allgemein hohe Vernetzungsgrad der entstehenden Resite (bis zu drei Bindungen pro Phenolkern) ermöglicht ihren Einsatz auch überall dort, wo hohe Temperaturfestigkeiten, z.B. bei Brems- und Kupplungsbelägen, gefordert werden (HABENICHT 2009). Die damit verbundene Sprödigkeit führt jedoch zu einer geringen Schlagfestigkeit. Verschiedene Modifikationen der Resole werden von HABENICHT (2009) genannt: Polyvinylformal, Polyvinylbutyral, Elastomere (z.B. Polychloropren), Polyamide und Epoxide. Er führt dazu aus, dass der Einsatz dieser Stoffe durch sterische Hinderung einen geringeren dreidimensionalen Vernetzungsgrad und einen höheren Anteil kettenförmiger Strukturen, teilweise mit thermoplastischen Eigenschaften, verursacht.

Hinsichtlich der in dieser Arbeit verwendeten Phenol-Formaldehyde ist nach ihrem genauen Verwendungszweck zu unterscheiden. Die einen Harze dienen mit besonders niedriger Viskosität als Imprägnierharze, die anderen dienen mit höherer Viskosität als Klebharze. Die Imprägnierharze werden, wie später noch ausführlich beschrieben, auf deutlich niedrigere Feststoffgehalte verdünnt, im Gegensatz zu den Klebharzen werden ihnen auch keine Füllstoffe zugesetzt. Der Vernetzungsgrad bzw. der Grad der Vorkondensation ist bei den Imprägnierharzen deutlich geringer als bei den Klebharzen. Daraus resultierend weisen die Imprägnierharze geringere Molmassen und molare (hydrodynamische) Volumen auf. Abbildung 6 veranschaulicht mit Hilfe der Software ChemSketch V.14.01 die Struktur verschieden stark vernetzter Moleküle. Die darin dargestellten Werte wurden durch einen Literaturvergleich validiert. DURCHSCHLAG UND ZIPPER (1994) errechneten für Phenol ein molares Volumen von $84,6 \mathrm{~cm}^{3} / \mathrm{mol}$ und verglichen diesen Wert mit einem experimentell ermittelten Ergebnis von HøILAND (1986 IN HINZ (ED.)) von 86,2 cm³ $/ \mathrm{mol}$.

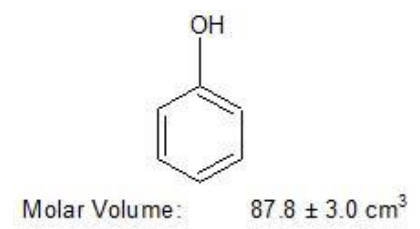

Abbildung 7: Molares Volumen von Phenol berechnet in ACD/ChemSketch V.14.01.

Das mit ChemSketch ermittelte molare Volumen von $87,8(+/-3,0) \mathrm{cm}^{3} / \mathrm{mol}$ für Phenol liegt nur knapp über $(+3,7 \%$ und $+1,9 \%)$ den in der Literatur gefundenen Werten, welche im Bereich der angegebenen Standardabweichung liegen. Die weiteren in Abbildung 6 dargestellten Werte werden somit als hinreichend belastbar für weitere Überlegungen zum Zusammenhang von Molekülgröße und der Zellwandeindringung erachtet. 


\section{Untersuchungen an Vollholz}

\subsection{Material und Methoden}

Die Untersuchungen an Vollholz dienten der Abschätzung des Potenzials einer imprägnierenden Modifizierung von Buchenholz (Fagus sylvatica L.) mit basischen Kondensationsharzen auf Basis von Phenol-Formaldehyd zur Verbesserung der Dimensionsstabilität. Zu diesem Zweck wurde die zyklische Quell-/Schwindbeanspruchung, nachfolgend ASE (Abkürzung von Anti-Swell-Efficiency) genannt, durchgeführt. Als Nachweis der Zellwandeindringung wurden zusätzlich aus den ASE-Prüfkörpern Proben gewonnen, die mit Hilfe der UV-Mikrospektrophotometrie (UMSP) untersucht wurden. Die Ergebnisse wurden in Verbindung zum Weight-Percent-Gain (WPG) und Bulking (dauerhafte Quellung der trockenen Zellwand) gesetzt.

\subsubsection{Modifizierung}

Ausgangsmaterial für die Herstellung der Vollholzprüfkörper war Buchenschnittholz (Fagus sylvatica L.) aus Deutschland mit einer mittleren Darrdichte von $695 \mathrm{~kg} / \mathrm{m}^{3}$. Für die ASEPrüfung wiesen die Prüfköper folgende Dimension auf: $25 \mathrm{~mm} * 25 \mathrm{~mm} * 10 \mathrm{~mm}$ (radial*tangential*longitudinal). Für die Bestimmung der Bruchschlagarbeit wiesen die Prüfköper folgende Dimensionen auf: $10 \mathrm{~mm} * 10 \mathrm{~mm} * 150 \mathrm{~mm}$ (radial*tangential*longitudinal).

Zur Modifizierung der ASE-Prüfkörper dienten die Phenol-Formaldehydtypen A, B und C (Tabelle 4), deren zahlenmäßig mittlere Molekülgewichte ähnlich waren und unter $440 \mathrm{~g} / \mathrm{mol}$ lagen. Diese PF-Harze wurden in den Feststoff- (FS-) Konzentrationen 6\%, $8 \%$ und $25 \%$ eingesetzt, um WPGs von 10\%, $15 \%$ und $30 \%$ zu erzielen. Zur Modifizierung der Bruchschlagarbeitsprüfkörper wurde nur der Harztyp A verwendet, um die WPGs 15\% und $30 \%$ zu erzielen. Der FS-Anteil einer Lösung wurde dabei durch Aushärten bei $140^{\circ} \mathrm{C}$ in einem Ofen kontrolliert. Die Prüfkörper für die ASE-Prüfung wurden in mit der Lösung befüllten Wannen und gegen ein Auftreiben gesichert in einem Laborautoklav imprägniert. Nach einem Vakuum (80mbar) für 45 Min folgte eine Zeit der Lösungsaufnahme unter Normaldruck für 60 Min. Daran schloss sich eine Trocknungsphase im Normalklima $\left(20^{\circ} \mathrm{C}^{\circ} / 65 \% \mathrm{rF}\right)$ an sowie weitere Phasen bei $40^{\circ} \mathrm{C}, 80^{\circ} \mathrm{C}, 103^{\circ} \mathrm{C}$ für jeweils $24 \mathrm{~h}$. Um die Härtung des Harzes sicherzustellen, schloss sich an diese Trocknung eine Phase mit erhöhter Temperatur von $120^{\circ} \mathrm{C}$ für $2 \mathrm{~h}$ an. Wie nachfolgend beschrieben, wurden zur Charakterisierung der modifizierten Proben das WPG und das Bulking bestimmt.

Für den Nachweis der Verteilung der PF-Harze mittels UV-Mikrospektroskopie (UMSP) wurden weitere ASE-Prüfkörper, wie oben beschrieben, imprägniert, jedoch auf unterschiedliche Weise getrocknet und ausgehärtet (vgl. 3.1.5). Ein Kollektiv erfuhr ein moderates Trocknungsprogramm. Es wurde normal getrocknet $\left(\mathrm{T}_{\max }=103^{\circ} \mathrm{C}\right)$, die Proben dann aber nicht der Härtungstemperatur von $120^{\circ} \mathrm{C}$ ausgesetzt. Das zweite Kollektiv wurde schneller getrocknet und dann für zwei $2 \mathrm{~h}$ bei $120^{\circ} \mathrm{C}$ ausgehärtet. Bei dieser schnelleren Trocknung wurde nach $72 \mathrm{~h}$ bei $20^{\circ} \mathrm{C}$ die Temperatur innerhalb einer Stunde auf $103{ }^{\circ} \mathrm{C}$ erhöht und für $24 \mathrm{~h}$ gehalten, während sonst erst $40{ }^{\circ} \mathrm{C}$ und $80^{\circ} \mathrm{C}$ für jeweils $24 \mathrm{~h}$ eingestellt wurden. 


\subsubsection{Bestimmung des Weight Percent Gain (WPG)}

Das Weight-Percent-Gain (WPG) gibt die Zunahme der absolut trockenen Prüfkörpermasse durch die Modifizierung, bezogen auf die Ausgangsmasse, an und ist abhängig von der Lösungsaufnahme und Konzentration, sowie von der Fixierung des Harzes im Holz. Das WPG ergibt sich wie folgt:

Formel 1: Berechnung des Weight-Percent-Gain (WPG)

$W P G=\frac{\left(m_{b}-m_{u}\right)}{m_{u}} * 100 \%$

$m_{u} \quad$ absolut trockene Prüfkörpermasse vor der Behandlung [g]

$m_{b} \quad$ absolut trockene Prüfkörpermasse nach der Behandlung [g]

\subsubsection{Bestimmung des Bulking}

Wesentliches Kriterium für den Erfolg einer Modifizierung ist die Eindringung des PF in die Zellwand. Ist die Zellwand im absolut-trockenen Zustand nach der Behandlung gequollen, wird dies als Bulking $\beta$ in Prozent ausgedrückt und nachfolgender Gleichung berechnet:

Formel 2: Berechnung des Bulking (dauerhafte Quellung) der Zellwand

$\beta=\frac{\left(l_{b}-l_{u}\right)}{l_{u}} * 100 \%$

$\beta \quad$ Bulking; Dimensionsänderung in Prozent

$l_{u} \quad$ Dimension im absolut trockenen Zustand vor der Behandlung [mm]

$l_{b} \quad$ Dimension im absolut trockenen Zustand nach der Behandlung [mm]

Dabei kann die Dimension entweder die tangentiale oder radiale anatomische Richtung des Holzes sein.

\subsubsection{Bestimmung der Anti-Swell-Efficiency (ASE)}

Als das Maß für die Dimensionsstabilisierung wurde die Anti-Swell-Efficiency (ASE) bestimmt. Dabei wurde die verminderte Quellung des Querschnitts behandelter Proben auf die Quellung unbehandelter Proben bezogen.

Die Quellung $Q$ wurde dabei durch Imprägnieren kleiner Vollholzprüfkörper in den Dimensionen $25 \mathrm{~mm}^{*} 25 \mathrm{~mm} * 10 \mathrm{~mm}$ (radial*tangential*longitudinal) mit Wasser im Vakuum-Druck-Verfahren und anschließender Wasserlagerung für $24 \mathrm{~h}$ herbeigeführt. Bei der ASE-Prüfung handelt es sich generell um einen Zyklustest, welcher 3-12 Mal wiederholt wird und immer im absolut-trockenen Zustand der Prüfkörper beginnt. Pro Serie wurden 8 Prüfkörper getestet und das Ergebnis als arithmetischer Mittelwert mit Standardabweichung dargestellt. 
Formel 3: Berechnung der maximalen Quellung des Querschnittes

$Q_{u, b}=\frac{\left(A_{1}-A_{0}\right)}{A_{0}} * 100 \%$

$Q_{u} \quad$ maximale Quellung des unbehandelten Holzes [\%]

$Q_{b} \quad$ maximale Quellung des behandelten Holzes [\%]

$A_{0} \quad$ Querschnittsfläche im absolut trockenen Zustand $\left[\mathrm{mm}^{2}\right]$

$A_{l} \quad$ Querschnittsfläche im wassergesättigten Zustand $\left[\mathrm{mm}^{2}\right]$

Formel 4: Berechnung der Anti-Swell-Effiiency (ASE)

$$
A S E=\frac{\left(Q_{u}-Q_{b}\right)}{Q_{u}} * 100 \%
$$

ASE relative Verminderung der maximalen Quellung [\%]

$Q_{u} \quad$ maximale Quellung des unbehandelten Holzes [\%]

$Q_{b} \quad$ maximale Quellung des behandelten Holzes [\%]

\subsubsection{Nachweis der Verteilung der Phenol-Formaldehyde mittels UV- Mikrospektrophotometrie (UMSP)}

Der Nachweis der Verteilung der Phenol-Formaldehyde wurde indirekt über den Nachweis phenolischer Strukturen auf subzellulärer Ebene mit Hilfe eines Raster-UMSP (ZEISS UMSP Mikrospektralphotometer 80) nach КосH \& KLEIST (2001) und КосH \& GRÜNWALD (2004) erbracht. JAFFÉ \& ORCHIN (1962), HERGERT, SARKANEN ET Al. (1971), HESSE, MEIER ET AL. (1991), zitiert nach KOCH \& GRÜNWALD (2004), geben an, dass chromophore Strukturelemente, wie die Phenylpropaneinheiten des Lignins, zu einem charakteristischen Absorptionsspektrum in einem Wellenlängenbereich zwischen $212 \mathrm{~nm}$ und $280 \mathrm{~nm}$ führen. Im adulten Holz gibt es daneben keine anderen Strukturen, die eine Strahlungsabsorption in der gleichen Region aufweisen (КосH \& GRÜNWALD 2004). Aufgrund der strukturellen Ähnlichkeit des Lignins mit Phenol, resp. der chromophoren Gruppen, ist es auf diese Weise möglich, über die Intensität der Absorption der UV-Strahlung die Konzentration von Lignin und Phenol in der Zellwand verholzten Gewebes zu bestimmen.

Zur Probenvorbereitung wurden kleine Holzblöcke ( $1 \mathrm{~mm} * 1 \mathrm{~mm} * 5 \mathrm{~mm}$ ) aus verschiedenen ASE-Prüfkörpern entnommen und direkt in Spurr-Epoxidharz (SPURR 1969) unter mildem Vakuum mit mehreren Zyklen der Evakuierung und Belüftung eingebettet (KLEIST UND SCHMITT 1999), um chemische Veränderungen durch Reaktion mit Lösungsmitteln zu vermeiden. Abschnitte mit einer Dicke von $1 \mu \mathrm{m}$ wurden mit einem Diamantmesser angefertigt, auf Quarzglasobjektträger übertragen und in Glycerin eingebettet. 
Das ZEISS UMSP 80 Mikrospektralphotometer war mit einem Scantisch ausgestattet, um die Bestimmung der Bildprofile bei konstanten Wellenlängen mit dem Scan-Programm APAMOS (Zeiss) zu ermöglichen. Die Phenole und Lignine wurden bei einer Wellenlänge von $278 \mathrm{~nm}$ detektiert. Das Scan-Programm digitalisierte rechteckige Felder, mit einer lokalen geometrischen Auflösung von 0,25 $\mu \mathrm{m} * 0,25 \mu \mathrm{m}$ und liefert eine photometrische Auflösung von 4096 Graustufen, welche in 14 Grundfarben, resp. Klassen, umgewandelt werden, die die Absorptionsintensitäten visualisieren. Die Scans können als zwei - oder dreidimensionale Bildprofile dargestellt werden, einschließlich einer statistischen Auswertung (als Histogramm) der UV-Absorptionswerte. 


\subsection{Ergebnisse und Diskussion}

\subsubsection{WPG, Bulking, ASE}

Die Behandlung von ASE-Prüfkörpern aus Buchenvollholz hat gezeigt, dass sich mit den oben genannten PF-Harzen unterschiedliche WPGs erzielen ließen. Diese führten zu einem unterschiedlichen Bulking, also zu unterschiedlicher Eindringung und dauerhafter Quellung der Zellwand. Daraus resultiert dann eine entsprechende ASE, wie in Abbildung 8, mit Ergebnissen nach dem 1. Zyklus der Prüfung, gezeigt.

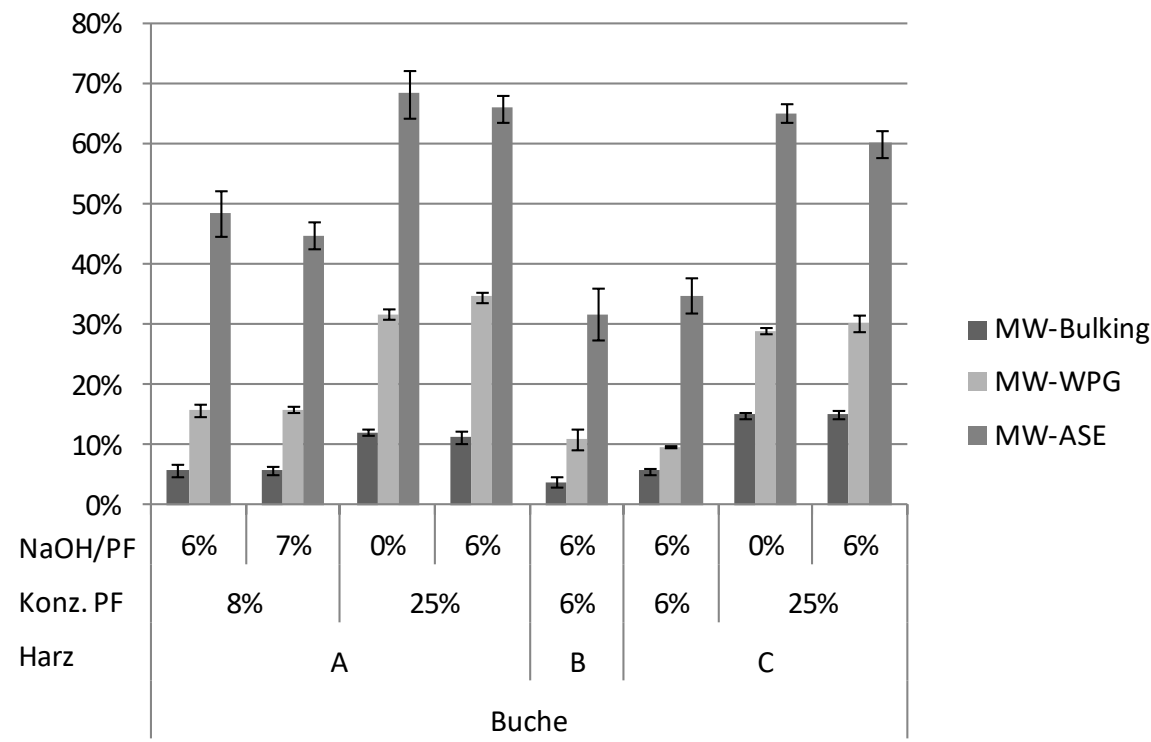

Abbildung 8: WPG, Bulking und resultierende ASE für modifiziertes Buchenvollholz (Mittelwerte und Standardabweichungen).

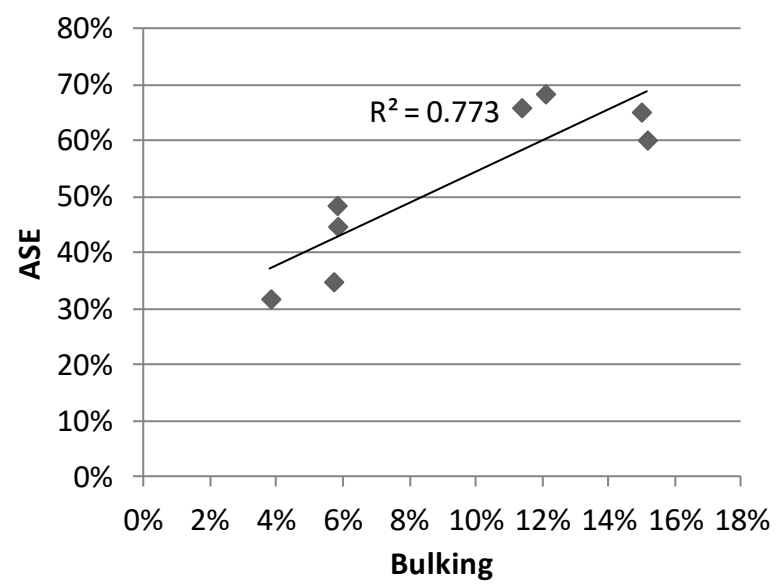

Abbildung 9: Zusammenhang von Bulking und ASE für alle Harze, Harzkonzentrationen und Katalysatorzugaben.

Wie Abbildung 9 zeigt, bestand eine positive Korrelation zwischen dem Bulking und der ASE, welche darauf beruht, dass der Bezugsquerschnitt infolge der dauerhaften Quellung der Zellwand auch im trockenen Zustand größer war als vor der Modifizierung. Die maximale Quellung, welche sich aus Bulking und nachträglicher Quellung (Sorption von Wasser) zusammensetzt, hat hingegen wenig oder gar nicht zugenommen. Die nachträgliche Quellung durch Sorption stand somit zum Bulking in einem umgekehrt proportionalen Zusammenhang. 
Das Bulking zeigte noch einen weiteren Effekt, indem es das Volumen des Holzkörpers änderte und somit Einfluss auf die Darrdichte nahm (Tabelle 1).

Tabelle 1: Veränderung der Darrdichte $\rho$ infolge der Modifizierung und WPG.

\begin{tabular}{|c|c|c|c|c|c|}
\hline Harz & Konz. $\mathrm{m} / \mathrm{m}$ & $\rho$, atro unbehandelt & $\rho$, atro behandelt & delta-p [\%] & WPG [\%] \\
\hline Referenz & $0 \%$ & 0.689 & 0.689 & 0.0 & 0.0 \\
\hline \multirow[t]{2}{*}{ A } & $8 \%$ & 0.689 & 0.740 & 7.4 & 15.8 \\
\hline & $25 \%$ & 0.685 & 0.802 & 17.1 & 33.2 \\
\hline B & $6 \%$ & 0.695 & 0.730 & 5.1 & 10.9 \\
\hline \multirow[t]{2}{*}{ C } & $6 \%$ & 0.714 & 0.730 & 2.3 & 9.6 \\
\hline & $25 \%$ & 0.715 & 0.788 & 10.1 & 29.6 \\
\hline
\end{tabular}

In der Regel drückt das WPG die Zunahme an trockener Masse bezogen auf den Ausgangswert aus. Die Ergebnisse zeigen, dass dieser Wert alleine jedoch keinen Rückschluss auf die Zunahme der Darrdichte zulässt. Wie sich aus Tabelle 1 ableiten lässt, entsprach die Zunahme der Darrdichte für die Harze A und B durchschnittlich 48\% des WPGs, jedoch bei Harztyp C nur 29 \% des WPGs, welcher, wie zuvor gezeigt, das höchste Bulking aufwies. Aus wirtschaftlicher Sicht könnte bei der Herstellung eines Produktes mit dem Harztyp C bei gleichem Materialeinsatz ein höheres Volumen produziert werden.

Da es sich bei der ASE-Prüfung um einen zyklischen Test handelt, in dem sich gequollenes Stadium und trockenes Stadium abwechseln, ist auch die Entwicklung der Werte des Bulkings und der ASE von Interesse.

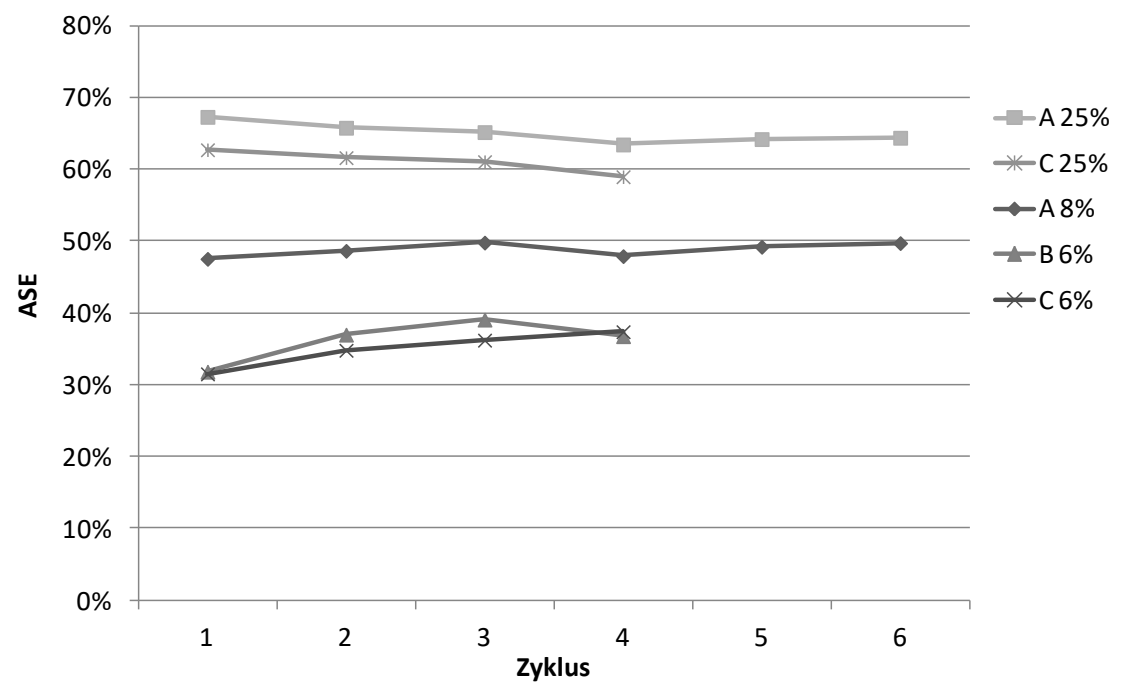

Abbildung 10: Ergebnisse der ASE-Prüfung für bis zu sechs Zyklen an PF-modifiziertem Buchenholz.

Abbildung 10 zeigt, dass auch bei wiederholtem Trocknen und Quellen des Holzes die ASE, resp. die Dimensionsstabilität, erhalten blieben und die Harze somit stabil im Holz verblieben.

Da zwischen den Harzen auch bei ähnlichem WPG hinsichtlich Bulking und ASE Unterschiede bestanden, wurden nachträglich Serien angefertigt, die mit vier im Labor eines Herstellers hergestellten Musterharzen imprägniert wurden, welche sich hinsichtlich ihrer zahlenmäßig mittleren Molekülgröße unterschieden. Wie Abbildung 11 zeigt, ließ sich ein deutlicher 
Einfluss der zahlenmäßig mittleren Molmasse $\left(\mathrm{M}_{\mathrm{n}}\right)$ feststellen. Bei einer Imprägnierung mit einer Konzentration von 27 \% PF und einem WPG von $23 \%$ lag die ASE bei $\mathrm{M}_{\mathrm{n}}$ von $297 \mathrm{~g} / \mathrm{mol}$ und $421 \mathrm{~g} / \mathrm{mol}$ auf dem hohen Niveau von 68\%, welches auch die kommerziellen Harze (Abbildung 8) zeigten. Bei einer $\mathrm{M}_{\mathrm{n}}$ von $655 \mathrm{~g} / \mathrm{mol}$ zeigte sich bereits eine deutliche Abnahme der Dimensionsstabilität. Bei $854 \mathrm{~g} / \mathrm{mol}$ betrug die ASE zwar immer noch 50\%, die Quellung wäre gegenüber unbehandeltem Buchenholz um die Hälfte reduziert gewesen, jedoch ist die ASE um 18\% gesunken und die gleiche Menge Harz hat deutlich weniger effizient gewirkt.

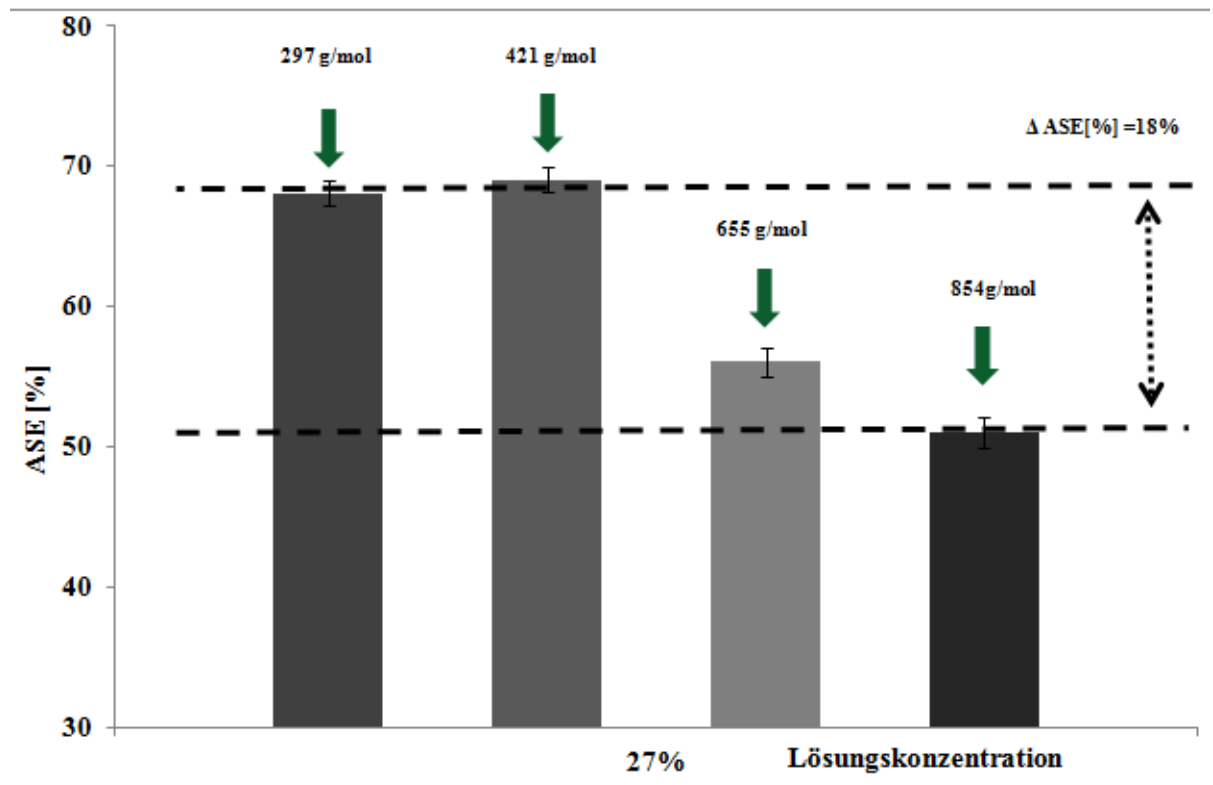

Abbildung 11: Anti-Swell-Efficiency in Abhängigkeit der Molekülgröße an Buchenvollholz

3.2.2 Nachweis der Verteilung der Phenol-Formaldehyde mittels UVMikrospektrophotometrie (UMSP)

Abbildung 12 zeigt die Absorption bei $278 \mathrm{~nm}$ an einer unbehandelten Buchenholzprobe im Bereich der Fasern. Die durchschnittliche Absorption, innerhalb der Grenzwerte, lag hier bei 0.227. Anhand des 3D-Linienscans wird die Zunahme der Absorption und mithin der Konzentration an Lignin in Richtung der Mittellamelle verdeutlicht. Laubholzlignin besteht im Wesentlichen aus Guaiacyl- und Syringyllignin - das Verhältnis zueinander hängt von dem Gewebetyp ab (HON \& SHIRAISHI 2000). Buchenholz enthält natürlich zwischen 22,6 \% und 28,3 \% Lignin bezogen auf die atro-Holzmasse (KolLMANN 1951). 


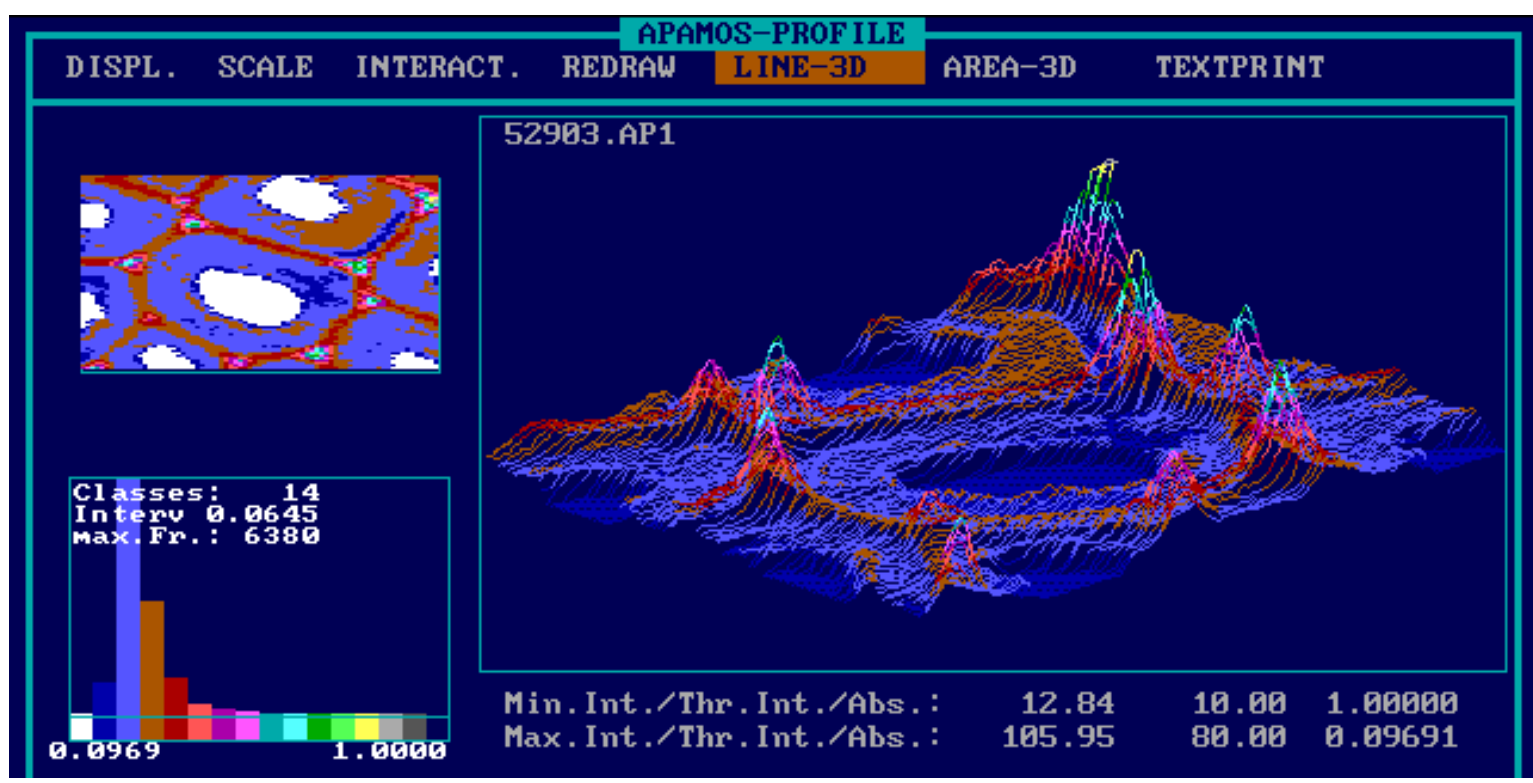

Abbildung 12: UV-Mikrospektrogramm, Häufigkeitsverteilung der Absorptionswerte und 3D-Linienscan von Fasern einer unbehandelten Buchenholzprobe.

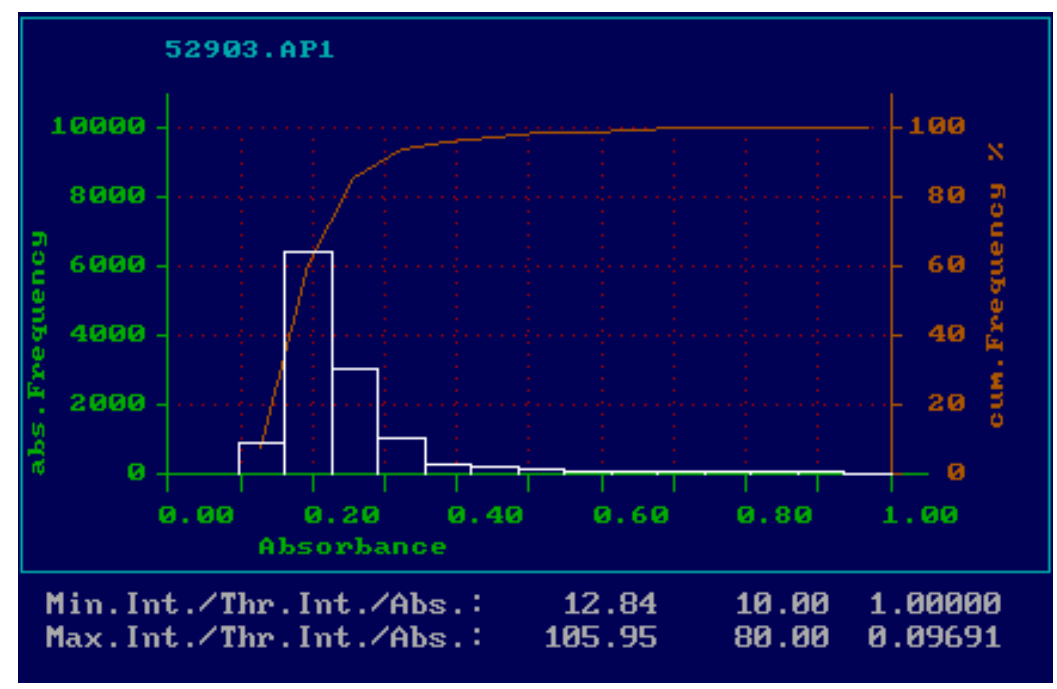

Abbildung 13: Kumulative Häufigkeit der 14 Klassen (resp. Grundfarben) der Absorption der Fasern einer unbehandelten Buchenholzprobe bei regulären Grenzwerten.

Aus Abbildung 13 wird eine diskrete Verteilung der Absorptionsintensitäten bei der Untersuchung der Fasern unbehandelter Referenzproben deutlich. Die Absorption der Fasern, bei denen die S2-Zellwände den flächenmäßig größten Anteil haben, zeigten bei zwei Messungen eine mittlere Absorption von 0.24 für unbehandelte Referenzen (R) und stimmten damit gut mit der Angabe von KoCH \& GRÜNWALD (2004) überein, welche Werte zwischen 0.16 und 0.29 angaben.

Die bei den Referenzen ermittelte, im Vergleich zu den Fasern höhere Absorption der Gefäßwände (Tabelle 2), stand im Einklang mit den Ausführungen von (КосH \& GRÜNWALD 2004). 


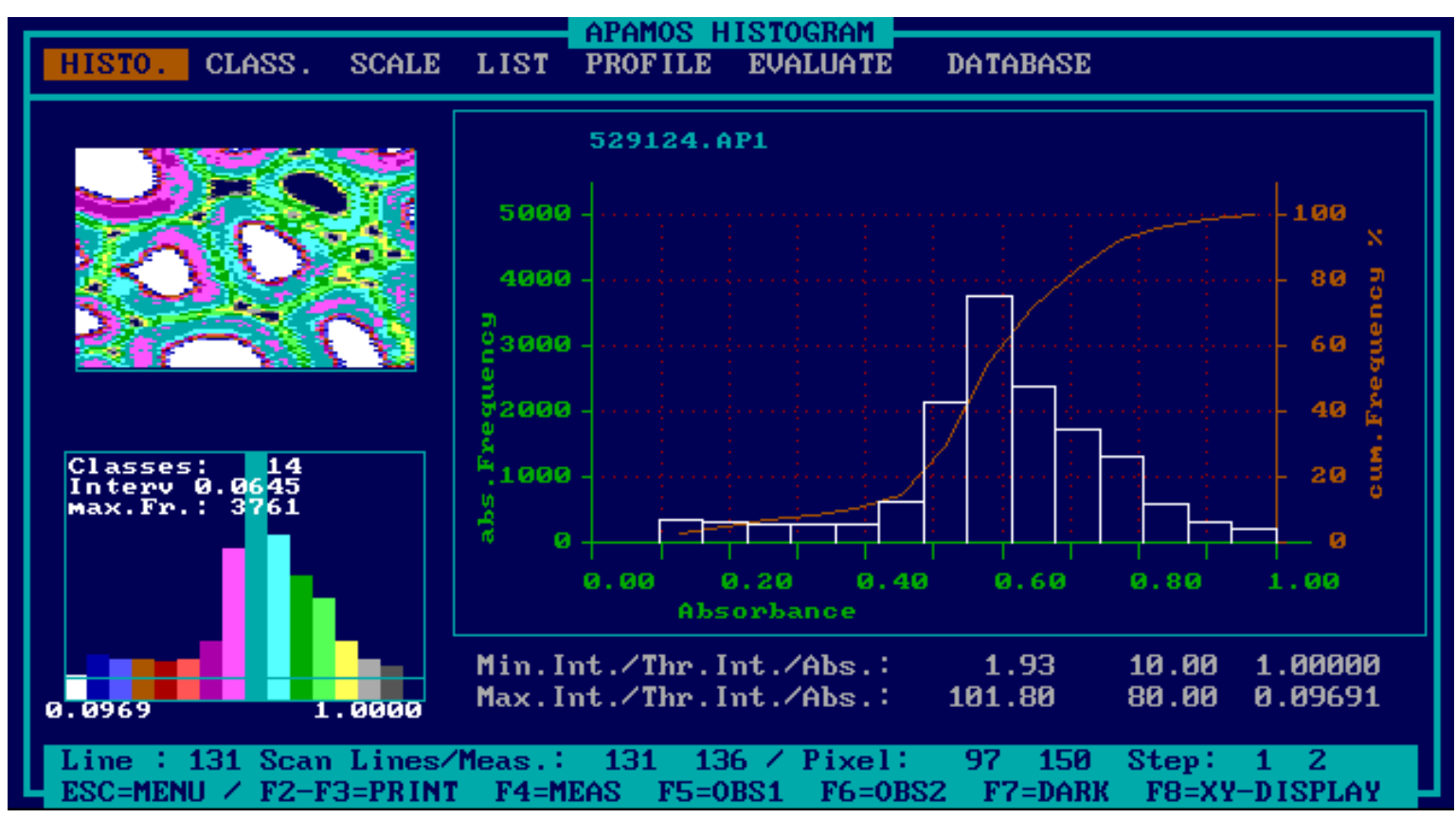

Abbildung 14: Harztyp A, 16\% WPG. Häufigkeitsverteilung der 14 Klassen der Absorption bei regulären Grenzwerten.

Infolge der Modifizierung mit Phenol-Formaldehyd kam es, wie in Abbildung 14 dargestellt, zu einer signifikanten Rechtsverschiebung der Häufigkeitsverteilung, mithin zu einer Erhöhung der durchschnittlichen Absorption.

Tabelle 2: Mittlere UV-Absorption der UV-Mikrospektrogramme bei $278 \mathrm{~nm}$ und einer Auflösung von $0,25 \mu \mathrm{m}^{2}$.

\begin{tabular}{lllll} 
Harz & $\mathbf{R}$ & $\mathbf{A}$ & $\mathbf{B}$ & $\mathbf{A}$ \\
\hline WPG & $0 \%$ & $16 \%$ & $12 \%$ & $31 \%$ \\
Bulking & $0 \%$ & $7 \%$ & $3 \%$ & $12 \%$ \\
& & Absorption & & \\
Gefäß & 0.284 & 0.527 & 0.458 & 0.578 \\
& 0.310 & 0.541 & 0.504 & 0.582 \\
Faser & 0.227 & 0.507 & 0.515 & 0.829 \\
& 0.243 & 0.565 & 0.366 & 0.678 \\
Parenchym & 0.272 & 0.517 & 0.434 & 0.722 \\
& 0.279 & 0.500 & 0.372 & 0.778 \\
Mittelwert & 0.269 & 0.526 & 0.441 & 0.694
\end{tabular}

Tabelle 2 zeigt die mittleren Absorptionswerte aller Proben unterteilt nach den in Laubholz vorkommenden Gewebetypen. Bei dem höheren WPG und Bulking wurden erhöhte Absorptionswerte in allen Gewebetypen gemessen, die auf eine Zunahme chromophorer Strukturen und damit auf die Anreicherung von Phenol-Formaldehyd in den Geweben hindeuten. Diese deutliche Zunahme der Absorption war positiv linear mit dem Bulking korreliert und ließ auf eine Abhängigkeit zwischen Zellwandeindringung und dem zahlenmäßig mittleren Molekülgewicht (Abbildung 15) schließen. 


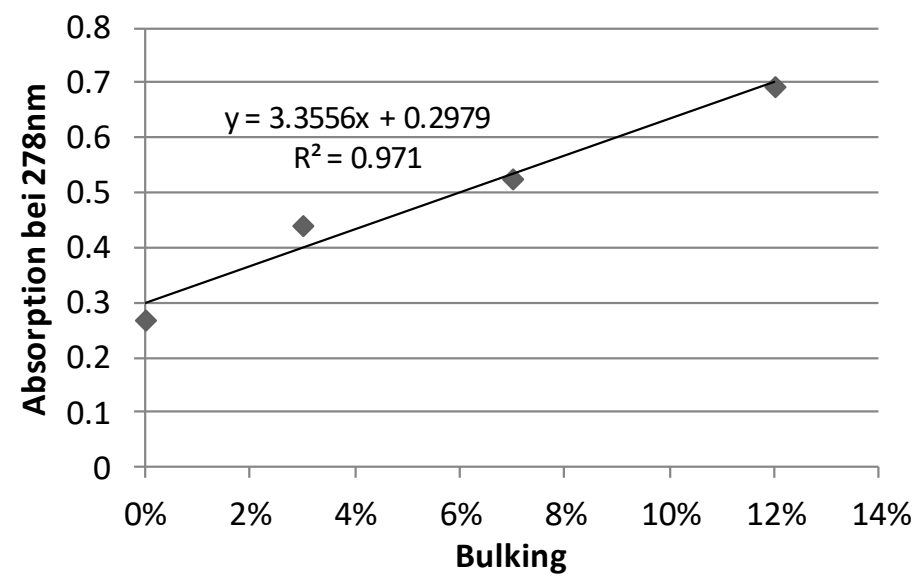

Abbildung 15: Zusammenhang zwischen UV-Absorption bei 278 nm und dem Bulking

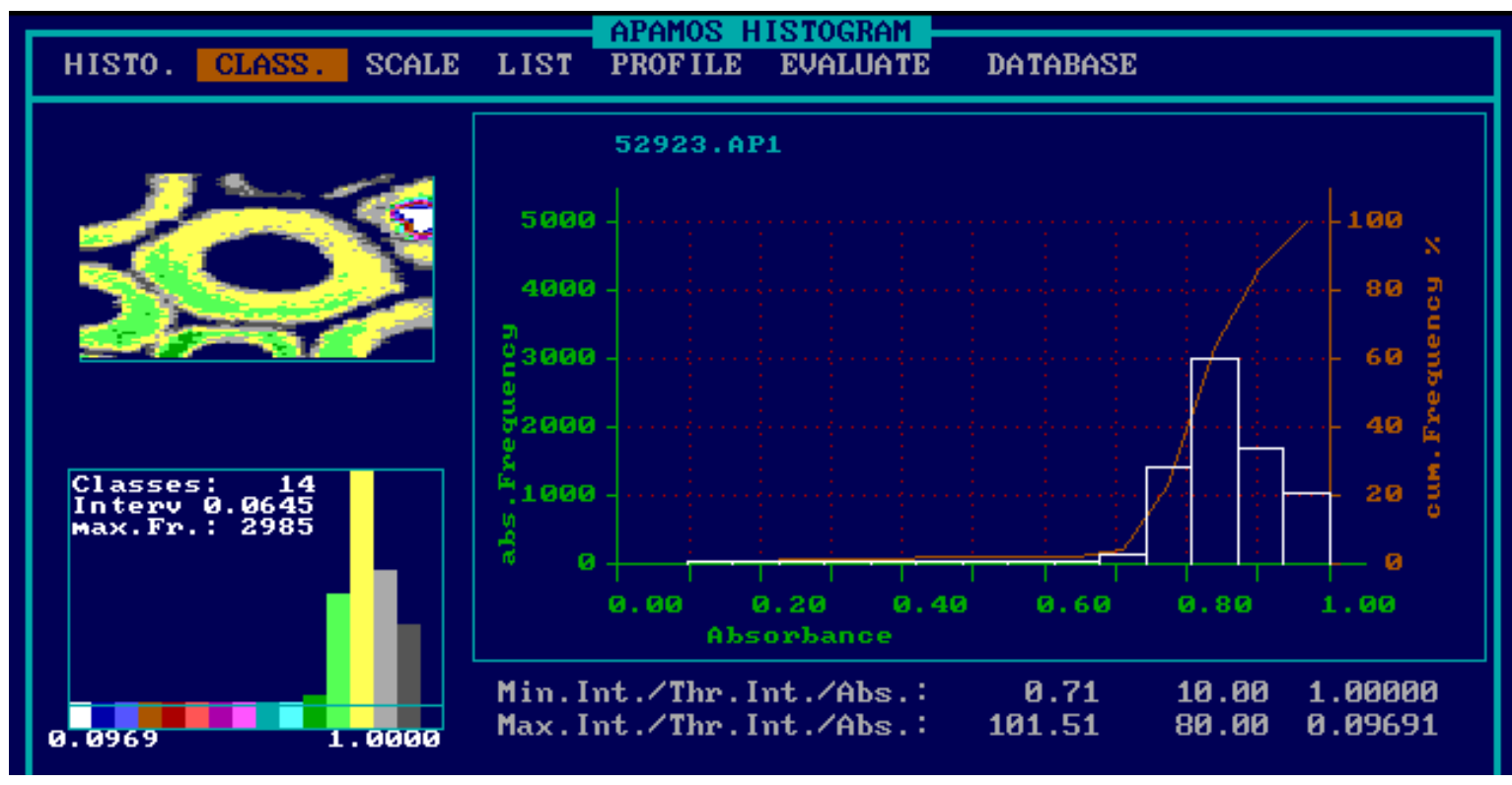

Abbildung 16: Harztyp A, 31\% WPG. Häufigkeitsverteilung der 14 Klassen der Absorption bei regulären Grenzwerten.

Wie Abbildung 16 zeigt, führte ein WPG von 31\% zu einer derart starken Absorption, dass alle Werte in nur vier von 14 Klassen eingeordnet und entsprechend dargestellt wurden. Dies erlaubt den direkten Vergleich zu allen anderen Messungen, bei denen die Klassenweite gleich war, d.h. die Klassen gleichmäßig zwischen der Intensität 10 und 80 verteilt waren. Dies führte jedoch zu einer erschwerten Differenzierung der Absorptionswerte auf Zellwandebene. Um die Bereiche hoher Absorption stärker auflösen zu können, konnte durch eine Veränderung der Grenzwerte und damit verbundenen Einengung des Messbereiches die Klassenweite verringert werden (Abbildung 17). Die Zunahme der Absorption im Vergleich zur Referenz blieb dabei erhalten, da sich der Mittelwert der Absorption nicht geändert hat. 


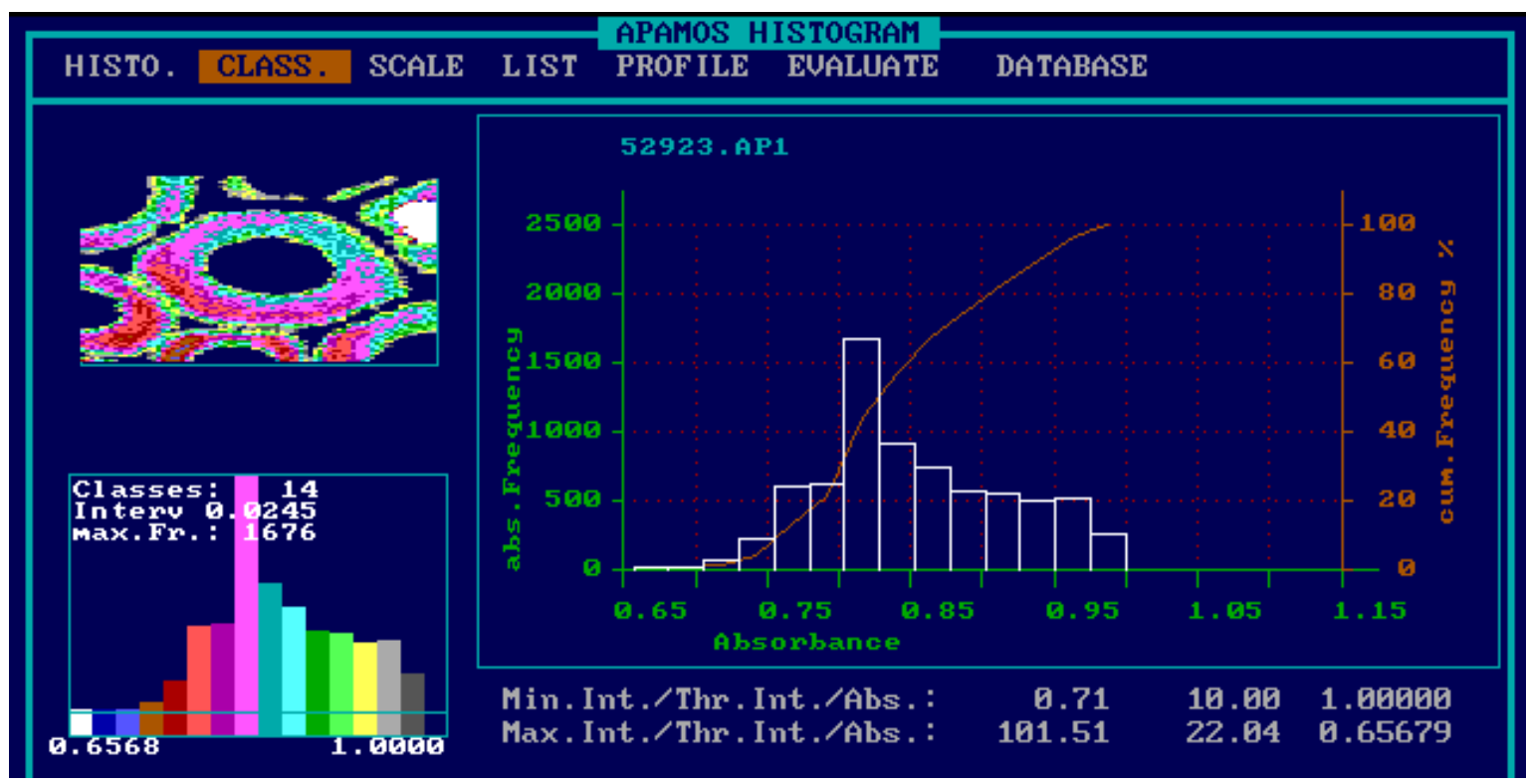

Abbildung 17: Harz A, 31\% WPG. Häufigkeitsverteilung der 14 Klassen der Absorption bei veränderten Grenzwerten.

In Abbildung 17 konnte nach der Verringerung des Klassenintervalls besser verdeutlicht werden, dass es weiterhin einen Absorptionsgradienten ausgehend von der Mitte der S2-Wand in Richtung Lumen und Mittellamelle gab. Dabei lagen die Bereiche das Zelllumens, ebenso wie Bereiche der Mittellamelle außerhalb des möglichen Messbereichs. Für zukünftige Untersuchungen der Zellwandeindringung von Phenol-Formaldehyd mittels UMSP beutet dies, dass sich WPGs unter $30 \%$ besser eignen.

\subsubsection{Zusammenfassung}

Die Überprüfung des Bulkings und der ASE hat gezeigt, dass sich zwischen den Harzen nur geringe Unterschiede zeigten, jedoch schon bei geringen WPG hohe ASE erzielen ließen. Insgesamt konnte die höhere ASE mit dem höheren WPG erzielt werden, die Zunahme viel jedoch unterproportional aus, was auf eine geringere Effizienz des Harzeinsatzes bei höheren WPG schließen lässt. Das höchste WPG lag jedoch bei 33\% und es wurden hier keine höheren WPGs untersucht, da insbesondere die Zellwandmodifizierung und der Einsatz niedrigerer Harzkonzentrationen im Fokus stand.

Als wichtigster Indikator für die erzielbare ASE konnte das Bulking identifiziert werden. Wie die UMSP-Messungen belegten, war das Bulking sehr gut mit der Absorption phenolischer Substanzen in der Zellwand korreliert und beweist damit, dass effektive Holzmodifizierung in der Zellwand stattfindet. 


\section{Untersuchung bestimmter Eigenschaften von Phenol- Formaldehyden}

\subsection{Alterung von Phenol-Formaldehyd und Auswirkung auf Quellvergütung von} Buchenholz

Alkalische Phenol-Formaldehydharze (Resole) und ihre wässrigen Verdünnungen unterliegen einer zeit- und temperaturabhängigen Alterung, die überwiegend auf einem stetigen Wachsen des Molekülnetzwerkes beruht. In Abhängigkeit des Lösungsalters werden somit die Wasserverdünnbarkeit sowie das Eindringvermögen in die Zellwand negativ beeinflusst.

Das Ziel einer Imprägnierung von Holz mit Phenol-Formaldehyd im Sinne einer Modifizierung, ist die Eindringung in die Zellwand. Damit soll eine dauerhafte Quellung (Bulking) hervorgerufen und das Anlagern von Wasser verhindert werden. Das Holz wird dadurch dimensionsstabil und dauerhaft geschützt gegen den Abbau von holzzerstörenden Pilzen.

In dieser Untersuchung wurde Buchenvollholz mit wässrigen Phenol-Formaldehydlösungen unterschiedlichen Alters modifiziert und der Einfluss des Lösungsalters auf das Eindringverhalten und die Dimensionsstabilität ermittelt.

\subsubsection{Material und Methoden}

Für die Untersuchung wurde ein bestimmtes alkalisches PF-Harz (Typ A, Tabelle 4) auf einen Feststoffgehalt von $25 \%$ unter Zugabe von Wasser verdünnt. Daraus wurden zwei Teilmengen gebildet, wovon eine bei einer Temperatur von $20^{\circ} \mathrm{C}$ und die andere bei $7{ }^{\circ} \mathrm{C}$ gelagert wurden. Damit wurden im Abstand von 7 Tagen jeweils 8 Buchenvollholzprüfkörper unter Vakuum imprägniert.

Die Prüfkörper wiesen die folgenden Dimensionen auf:

$25 \mathrm{~mm} * 25 \mathrm{~mm} * 10 \mathrm{~mm}$ (radial*tangential*longitudinal).

Zu Beginn wurden die Gewichte und Dimensionen im darr-trockenen (atro) Zustand ermittelt. Nach der Imprägnierung wurde die Lösungsaufnahme $d L$ gravimetrisch wie nachfolgend bestimmt:

Formel 5: Berechnung der Lösungsaufnahme nach dem Imprägnierprozess mit PF

$$
d L=\frac{\left(m_{1}-m_{0}\right)}{m_{0}} * 100 \%
$$

$d L$

$$
\text { Lösungsaufnahme [\%] }
$$

$m_{0} \quad$ darrtrockene Masse vor Imprägnieren [g]

$m_{1} \quad$ nasse Masse nach Imprägnieren $[\mathrm{g}]$

Die Prüfkörper wurden anschließend schonend getrocknet und ausgehärtet. Dabei durchliefen sie nacheinader mehrere Temperaturstufen: $72 \mathrm{~h}-20^{\circ} \mathrm{C} ; 24 \mathrm{~h}-80^{\circ} \mathrm{C} ; 48 \mathrm{~h}-103^{\circ} \mathrm{C}$. 
Im absolut trockenen Zustand wurden die atro-Gewichte und Dimensionen ermittelt. Aus der Zunahme an trockener Masse ergab sich, bezogen auf darr-trockene Anfangsmasse $m_{0, a t r o}$ (reine Holzmasse), der Weight-Percent-Gain (WPG) gemäß Formel 1.

Die Zellwandeindringung wird indirekt durch die dauerhafte Quellung (Bulking) infolge der Harzeinlagerung nachgewiesen. Zu diesem Zweck wurden die Dimensionen im darr-trockenen Zustand vor und nach der Behandlung verglichen. Da die Quellung in der tangentialen und radialen anatomischen Richtung deutlich stärker ausgeprägt ist, als in longitudinaler Richtung, wurde nur die Zunahme der Querschnittsfläche als Bulking $A B$ in Prozent berechnet:

Formel 6: Berechnung des Bulking der Querschnittfläche (rad*tang) an Vollholzproben

$$
A B=\frac{\left(A_{1, \text { atro }}-A_{0, \text { atro }}\right)}{A_{0, \text { atro }}} * 100 \%
$$
Zustand $\left[\mathrm{mm}^{2}\right]$

$A_{1, \text { atro }} \quad$ Querschnitt (rad.*tang.) der Probe nach der Behandlung im darr-trockenen Zustand $\left[\mathrm{mm}^{2}\right]$

\subsubsection{Ergebnisse}

Nachfolgend werden die Ergebnisse der Untersuchung zur Lagerstabilität eines wässrigen Phenol-Formaldehydharzes (Typ A) mit einem Feststoffanteil von 25 \% dargestellt.

Tabelle 3: Übersicht aller Ergebnisse der Untersuchung zur Lagerstabilität eines wässrigen PhenolFormaldehydharzes (Typ A) mit einem Feststoffanteil von $25 \%$.

\begin{tabular}{cccccc}
$\begin{array}{c}\text { Alter } \\
\text { [Wochen] }\end{array}$ & Lagertemp. & WPG & A-Bulking & A-Quellung & ASE1 \\
\hline 0 & $20^{\circ} \mathrm{C}$ & $39 \%$ & $10 \%$ & $6 \%$ & $69 \%$ \\
\hline 1 & $20^{\circ} \mathrm{C}$ & $31 \%$ & $10 \%$ & $7 \%$ & $61 \%$ \\
& $7^{\circ} \mathrm{C}$ & $28 \%$ & $11 \%$ & $7 \%$ & $61 \%$ \\
\hline 2 & $20^{\circ} \mathrm{C}$ & $24 \%$ & $9 \%$ & $9 \%$ & $50 \%$ \\
& $7^{\circ} \mathrm{C}$ & $33 \%$ & $11 \%$ & $7 \%$ & $62 \%$ \\
\hline 3 & $20^{\circ} \mathrm{C}$ & $17 \%$ & $7 \%$ & $10 \%$ & $44 \%$ \\
& $7^{\circ} \mathrm{C}$ & $22 \%$ & $9 \%$ & $8 \%$ & $54 \%$ \\
\hline 4 & $20^{\circ} \mathrm{C}$ & $16 \%$ & $6 \%$ & $11 \%$ & $43 \%$ \\
& $7^{\circ} \mathrm{C}$ & $24 \%$ & $10 \%$ & $8 \%$ & $55 \%$ \\
\hline & Referenzen & - & - & $19 \%$ & $0 \%$
\end{tabular}

Tabelle 3 gibt eine Übersicht über alle wesentlichen Ergebnisse dieser Untersuchung.

Im Weiteren sollen einzelne Abbildungen zu den untersuchten Kriterien die beobachteten Zusammenhänge erläutern. 


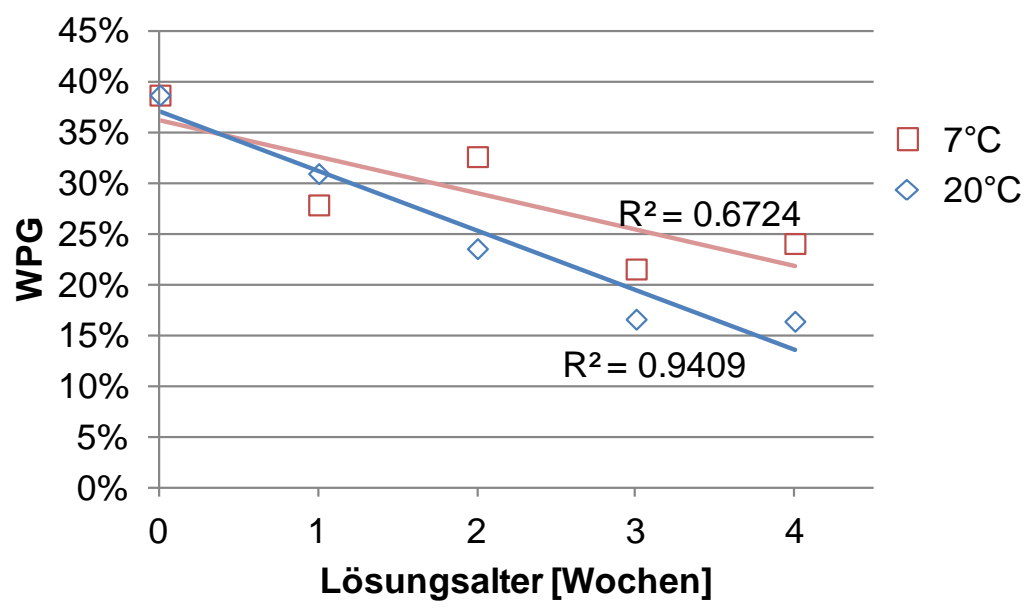

Abbildung 18: Abnahme des Weight-Percent-Gain (WPG) in Abhängigkeit von Lösungsalter und Lagerungstemperatur bei Imprägnierung von Buchenvollholz mit Phenol-Formaldehyd (Harztyp A, 25\%FS).

Abbildung 18 zeigt, dass mit zunehmenden Lösungsalter der WPG trotz vollständiger Imprägnierung der Proben abnahm. Diese Abnahme war bei einer Lagerung der Lösung bei $7^{\circ} \mathrm{C}$ deutlich geringer als bei $20^{\circ} \mathrm{C}$. Es ist zu bemerken, dass nach einer Woche praktisch kein Unterschied festzustellen war, während nach 2 Wochen der Unterschied am größten war.

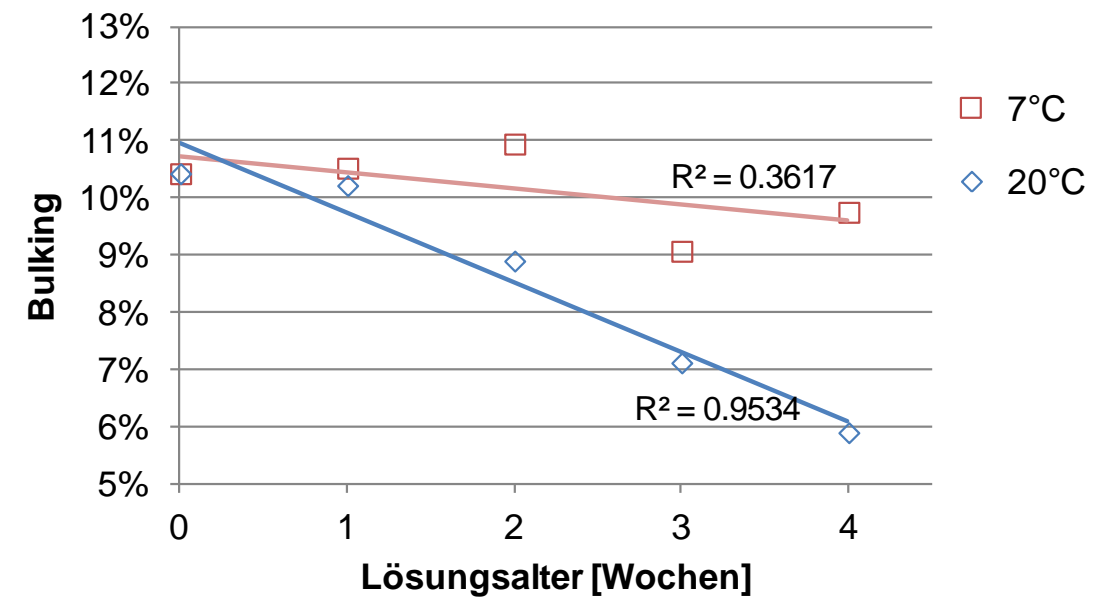

Abbildung 19: Bulking (Dauerhafte Quellung der Zellwand) in Abhängigkeit von Lösungsalter und Lagerungstemperatur bei Imprägnierung von Buchenvollholz mit Phenol-Formaldehyd (Harztyp A, 25\%FS).

Abbildung 19 zeigt, dass die Zelleindringung des Harzes stark von der Lagerungstemperatur der Lösung abhing. Die bei $7{ }^{\circ} \mathrm{C}$ gelagerte Lösung zeigte nach 4 Wochen nur eine geringe Abnahme - von 10,8\% auf 9,5\%. Bei der bei $20{ }^{\circ} \mathrm{C}$ gelagerten Lösung hatte das Bulking sehr viel stärker abgenommen - von $11 \%$ auf $6 \%$. 


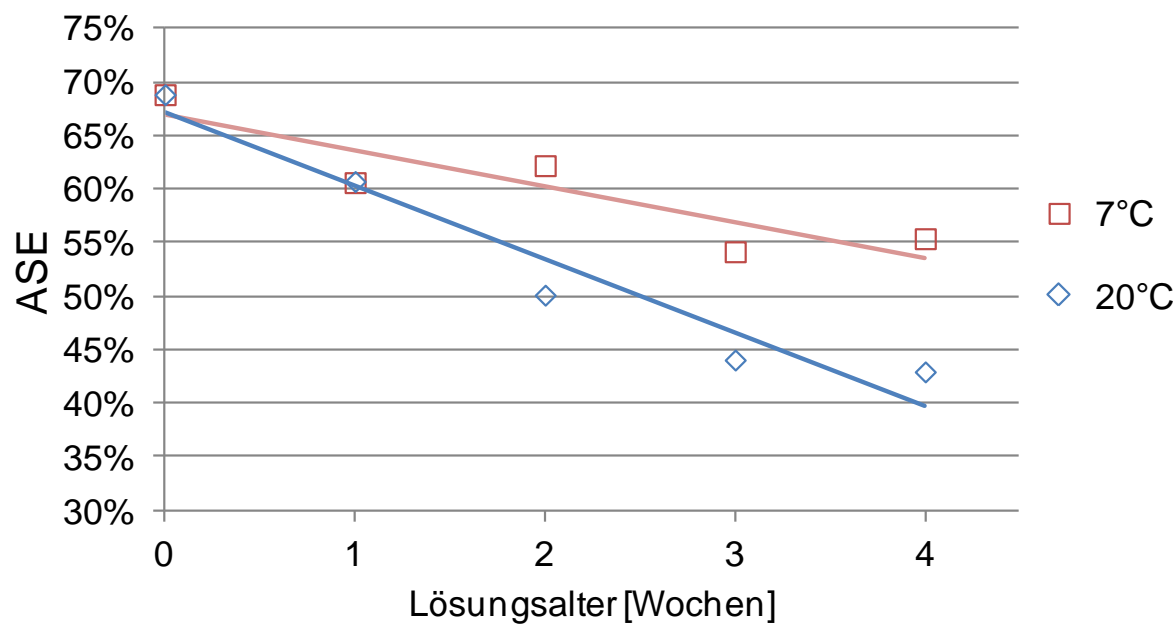

Abbildung 20: Anti-Swell-Efficiency (ASE) in Abhängigkeit von Lösungsalter und Lagerungstemperatur bei Imprägnierung von Buchenvollholz mit Phenol-Formaldehyd (25\%FS).

Abbildung 20 zeigt, dass genau wie das Bulking es schon vermuten ließ, das Maß für die Dimensionsstabilisierung (ASE) mit zunehmender Lagerungsdauer bei beiden Lagerungstemperaturen abnahm. Dieser Effekt wurde jedoch erst nach 2 Wochen Lagerung deutlich. Eine höhere Lagerungstemperatur verstärkte die Abnahme. Es ist zu beachten, dass die ASE der Prüfkörper, welche zu Beginn des Versuchs imprägniert wurden, außergewöhnlich hoch war. Dies ist wahrscheinlich auf ein hohes WPG zurückzuführen und widerspricht bisherigen Untersuchungen.

\subsubsection{Fazit}

Phenol-Formaldehydharze verändern ihre Eigenschaften mit zunehmendem Lösungsalter in Abhängigkeit von der Lagertemperatur. Wahrscheinlich führt ein stetiges Wachstum des Molekülgerüsts oder eine Aggregation zu einer verminderten Fähigkeit in die Zellwand einzudringen. Dies äußerte sich in einem mit der Zeit abnehmenden Bulking und damit einhergehender Abnahme der ASE. Höhere Temperaturen bei der Lagerung beschleunigen diesen Prozess. Es ist bekannt, dass tiefgefrorene PF-Harze keiner Alterung unterliegen. Da dies jedoch aufwendig und kostenintensiv ist, wird eine Lagerung bei möglichst tiefen Temperaturen empfohlen. Dies gewährleistet bis zu 2 Wochen gleiche Ergebnisse hinsichtlich der Modifizierung und in der Folge eine nur moderate Abnahme der ASE von 60\% (1Woche) auf 55\% (4Wochen). Ein Weiterverwenden der Lösung wird durch eine gleichfalls mit der Zeit abnehmende Wasserverdünnbarkeit/Lösungsstabilität zwar begrenzt, jedoch kann diesem Effekt wahrscheinlich mit der Zugabe frischen PF-Harzes und der damit verbundenen Abnahme des rechnerischen mittleren Lösungsalters entgegengewirkt werden. 


\subsection{Bestimmung der Molekülgrößenverteilung mittels DLS}

\subsubsection{Material und Methoden}

Bei den meisten der verwendeten Harze lagen die Informationen über Molmassen ( $\mathrm{g} / \mathrm{mol}$ ) vor. Für den Harztyp A lagen diese Informationen zu Beginn dieser Arbeit jedoch noch nicht vor. Da aber für die Zellwandeindringung direkt nur die Molekülgrößen (nm) ausschlaggebend sind, wurde für dieses Harz eine Charakterisierung nach der dem Prinzip der dynamischen Lichtstreuung (DLS) eingesetzt. Die Durchführung erfolgte in Zusammenarbeit mit $\mathrm{M}$. Fleckenstein, welcher dieses Ergebnis selbst nicht verwendete.

Für dieses Verfahren werden auch die Synonyme Photonenkorrelationsspektroskopie (PCS) oder quasielastische Lichtstreuung (QELS) verwendet. Zur Detektion von Molekülen im Nanometerbereich ist die dynamische Lichtstreuung eine geeignete Methode.

Das Messprinzip der dynamischen Lichtstreuung beruht auf der Tatsache, dass Moleküle der Brown'schen Molekularbewegung unterliegen. Kleine Moleküle bewegen sich schneller als große. Daraus resultiert, dass das gestreute Licht in seiner Intensität schwankt. Die direkte Korrelation zwischen Streulichtfrequenz und Partikelgröße wird bei der dynamischen Lichtstreuung genutzt, um einen Rückschluss auf die Partikelgrößen zu erlangen. An dieser Stelle wird erneut darauf hingewiesen, dass über die Partikelgrößenverteilung nicht auf die Molmassenverteilung geschlossen werden kann.

Ein Lichtstrahl ist auf die Probe gerichtet. Die Partikel in der Probe streuen je nach Größe das Licht in einem bestimmten Streuwinkel. Die Stärke der Streuung wird mit einem Detektor erfasst. Meist erfolgt die Auswertung mittels Autokorrelation. Dies bedeutet, dass die erfassten Signale verschiedenster Zeitpunkte miteinander verglichen werden.

\subsubsection{Ergebnisse}

Die Abbildung 21 zeigt die Molekülgrößenverteilung des Imprägnierharzes Typ A. Es wird ersichtlich, dass bei beiden Messungen Molekülgrößen zwischen $1 \mathrm{~nm}$ und $4 \mathrm{~nm}$ ermittelt wurden. HILL (2006) gibt die maximale Größe der Mikroporen verholzter Zellwände im wassergequollenen Zustand mit 2-4 nm an, somit ist der überwiegende Anteil dieses Harzes in der Lage in die Zellwand einzudringen. 


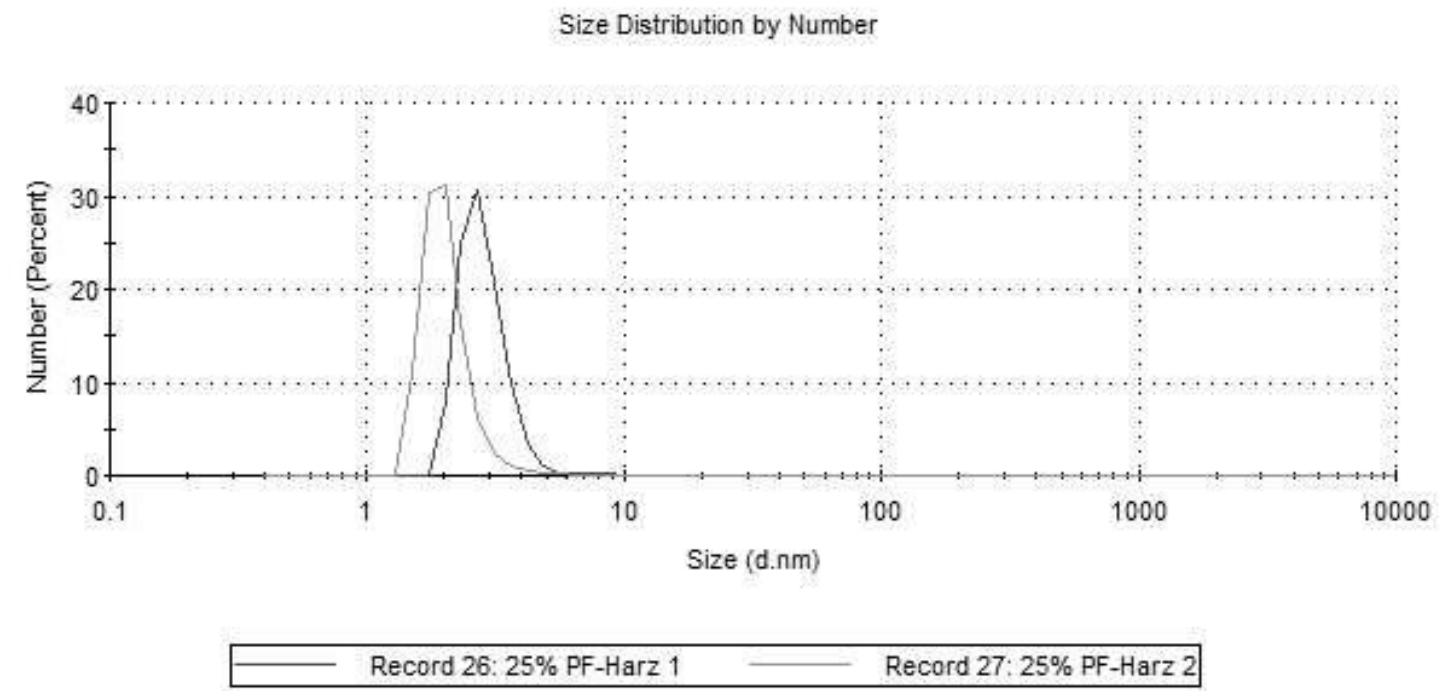

Abbildung 21: Molekülgrößenverteilung einer 25\%igen Lösung von Typ A (2 Wdh.) in Wasser, gemessen nach der DLS-Methode.

\subsection{Untersuchung der Reaktionskinetik mittels DSC}

\subsubsection{Material und Methoden}

Die Differential-Scanning-Calorimetry wurde genutzt, um die Reaktionstemperaturen des Imprägnierharzes TYP A zu untersuchen und Rückschlüsse auf die Aushärtebedingungen anzustellen. Zu diesem Zweck wurden Proben von $10 \mathrm{mg}-20 \mathrm{mg}$ in einem Tiegel eingewogen und bei einer Heizrate von $5 \mathrm{~K} / \mathrm{min}$ auf $180^{\circ} \mathrm{C}$ aufgeheizt. Durch Abgleich mit einem leeren Referenztiegel konnte die Starttemperatur der exothermen Polymerisationsreaktion des Harzes sowie dessen Enthalpie bestimmt werden.

\subsubsection{Ergebnisse}

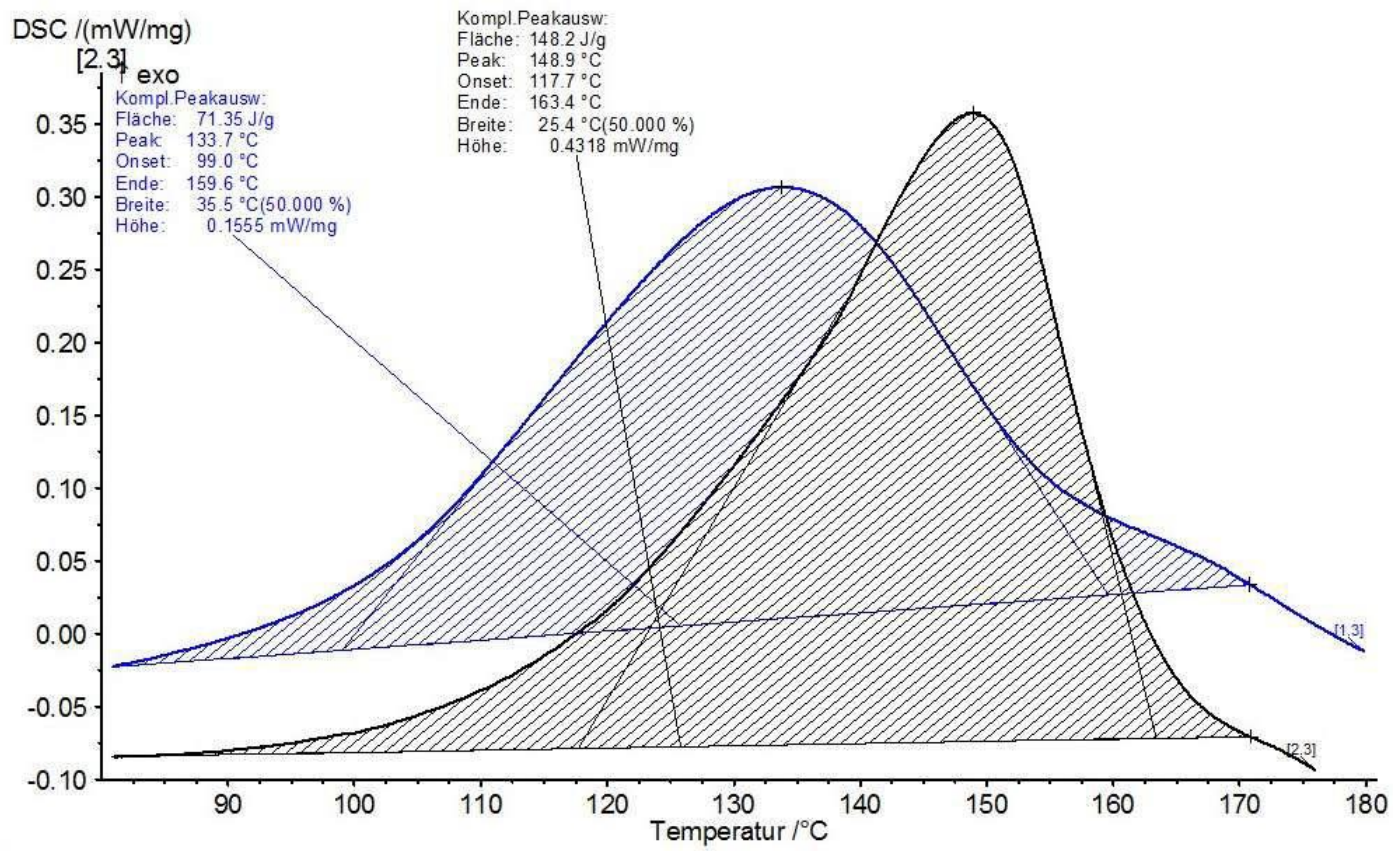

Abbildung 22: Reaktionsenthalpie bei der Aushärtung vom Klebharz 15J173 (Dynea) (blaue Linie) und dem Imprägnierharz TYP A (schwarze Linie). 
Bei der Untersuchung der Reaktionskinetik zeigte sich, dass das deutlich weniger vorkondensierte Imprägnierharz erst bei höherer Temperatur (Onset: $118^{\circ} \mathrm{C}$ ) mit einer exothermen Reaktion startet zu kondensieren und deutlich mehr Energie $(+177 \%)$ dabei frei wird, als bei einem bereits vorkondensierten Klebharz (Onset: $99^{\circ} \mathrm{C}$ ). Folglich müssen bei der Härtung der Imprägnierharze in der Heißpresse auch in der Mitte einer Furnierschichtplatte höhere Temperaturen erzielt werden, als dies nur für die Härtung des Klebstoffes nötig wäre. Eine Überwachung der Temperatur in der Plattenmitte ist zudem sinnvoll, da durch eine größere Energieabgabe infolge einer größeren Anzahl an Vernetzungsreaktionen wiederum die Temperatur der Platte beeinflusst wird. Um eine vollständige Vernetzung, bzw. Kondensation der Imprägnierharze zu gewährleisten, wurde, anders als bei der Verwendung von Klebharz, eine entsprechend längere Presszeit für die Herstellung der Versuchsplatten gewählt (vgl. Kapitel 5.1.3). 


\section{Untersuchungen an Furnierschichtholz (LVL)}

\subsection{Material und Methoden}

\subsubsection{Bestimmung der Lösungsaufnahme von Buchenfurnieren}

Um das Erreichen eines Ziel-WPG möglich zu machen, war die Kenntnis der Lösungsaufnahme bei bestimmter Ausgangsfeuchte notwendig. Zu diesem Zweck wurden in einem Vorversuch zunächst darr-trockene Buchenfurniere (Format $3,7 * 80^{*} 180 \mathrm{~mm}^{3}$ ) mit einer $15 \%$ igen und 30\%igen PF-Lösung unter Vakuum (45 min) und Atmosphärendruck (60 min) voll gesättigt. Die gravimetrisch ermittelte Lösungsaufnahme (bezogen auf die darrtrockene Holzmasse) diente als Basis für die Überprüfung der These, dass bei bekannter Holzfeuchte durch Anpassung der Lösungskonzentration das Ziel-WPG erreichbar ist. Hierzu wurden drei Gruppen von Furnieren (Format 3,7*80*180 $\mathrm{mm}^{3}$ ) bis zum Erreichen eines stabilen Gewichts unterschiedlich klimatisiert:

1. Darrofen mit $103^{\circ} \mathrm{C}$,

2. Klimaraum mit Normalklima $20^{\circ} \mathrm{C}$ und $65 \% \mathrm{rF}$,

3. Klimaschrank mit $20^{\circ} \mathrm{C}$ und $95 \% \mathrm{rF}$.

Nach Erreichen der Gewichtskonstanz wurden die Feuchten der klimatisierten Furniere berechnet und im Anschluss wie nachfolgend beschrieben mit Lösungen unterschiedlicher Konzentration imprägniert. Die Konzentration wurde entsprechend des Ziel-WPGs angepasst an die maximal mögliche Lösungsaufnahme. In Vorversuchen wurde diese ermittelt, da sie abhängig von der Darrdichte und der Holzfeuchte der Furniere war.

\subsubsection{PF-Modifizierung der Furniere}

Die Modifizierung der Furniere für die Produktion von LVL setzte sich aus einer Imprägnierung und einer Trocknung zusammen.

Die Imprägnierung der Furniere mit den Phenol-Formaldehydlösungen ist einer der wesentlichen Schritte der Modifizierung und kann auf verschiedene Weisen erzielt werden. Zum einen kann durch Vakuum - und ggf. Überdruck - eine Lösung beschleunigt in den Holzkörper gepresst werden, zum anderen sorgt der Kapillareffekt in Verbindung mit der Hygroskopizität der Holzpolymere zu einer langsamen Lösungsaufnahme beim Kontakt mit der wässrigen (polaren) Lösung. Aus diesem Grund wurden zwei Verfahren - ein Vakuumprozess im Autoklaven im Technikumsmaßstab und ein Tauchverfahren untersucht.

Im Vakuumverfahren wurden die Furniere je nach Menge entweder in eine mit der Imprägnierlösung gefüllten Wanne gelegt oder auf einer Lore in den unbefüllten Autoklaven gefahren. Es wurde ein Unterdruck von 50-100mbar erzeugt (30-45 Min.), darauf folgte die eigentliche Imprägnierung durch Einstellen von Atmosphärendruck und - je nach Menge und Größe der Furniere - mit einem Überdruck mit 5-8bar (30-45 Min.). Im Falle einer Imprägnierung ohne Wanne (gefluteter Autoklav) wurde die Lösung durch den Überdruck abgelassen bzw. abgepumpt. Nach der Entnahme der Furniere wurde ein Abtropfen der Furniere in Senkrechtstellung vollzogen (15-30 Min.) bevor die Lösungsaufnahme ermittelt wurde. 
Für die Furniermodifizierung nach dem oben genannten Verfahren wurden folgende kommerziell erhältliche Harze angewandt:

Tabelle 4: Übersicht verwendeter Imprägnierharze in Lieferform.

\begin{tabular}{|c|c|c|c|c|c|c|c|}
\hline Harztyp & $\begin{array}{l}\text { Feststoffgehalt } \\
\text { in Lieferform } \\
\left(2 \mathrm{~h}, 120^{\circ} \mathrm{C}\right)\end{array}$ & $\begin{array}{l}\text { Viskosität in } \\
\text { Lieferform }\end{array}$ & $\begin{array}{l}\mathrm{pH}- \\
\text { Wert }\end{array}$ & $\begin{array}{l}\text { Freies } \\
\text { Phenol } \\
\text { (Monomer) }\end{array}$ & $\begin{array}{l}\text { Mw } \\
{[\mathrm{g} / \mathrm{mol}]}\end{array}$ & $\begin{array}{l}\mathrm{Mn} \\
{[\mathrm{g} / \mathrm{mol}]}\end{array}$ & $\begin{array}{l}\text { Poly- } \\
\text { dispersität }\end{array}$ \\
\hline TYP A & $58 \%-61 \%$ & $34 \mathrm{mPas}$ & $8,4-8,8$ & $<1,5 \%$ & 555 & 440 & 1.26 \\
\hline TYP B & $52 \%-55,8 \%$ & $23-34 \mathrm{mPas}$ & 8,3 & $5,2 \%-6,0 \%$ & 618 & 397 & 1,56 \\
\hline TYP C & $71 \%$ & $433 \mathrm{mPas}$ & 8,08 & $3 \%-10 \%$ & 454 & 333 & 1,363 \\
\hline TYP D & $43 \%-44 \%$ & $23 \mathrm{mPas}$ & - & $10-15 \%$ & 521 & 335 & 1,555 \\
\hline TYPE & $55 \%$ & $66 \mathrm{mPas}$ & 5,2 & $3 \%$ & 394 & 302 & 1,305 \\
\hline TYP F & $56.0 \%-58.2 \%$ & $186-207 m P a s$ & 9.8 & $1,4 \%-2,5 \%$ & 691 & 452 & 1.529 \\
\hline TYP G & $63.5 \%-64.8 \%$ & 268 - 292mPas & 8-9 & $3-10 \%$ & 601 & 397 & 1.5139 \\
\hline
\end{tabular}

Beim Vergleich der Viskositäten fällt auf, dass insbesondere beim Typ C eine höhere Viskosität vorlag, als bei den übrigen Harzen. Häufig wird die Vsikosität mit der Molmasse in Verbindung gebracht (HAUPT \& SELLERS 1994). Beim Vergleich der Herstellerangaben dazu, wird insbesondere durch den Typ D deutlich, dass selbst bei höheren Molmassen, die Viskosität niedriger lag. Bei diesem Harz gibt der Hersteller einen Methanolgehalt in Lieferform von 20 $25 \%$ an. Da alle Harze in unterschiedlichen Feststoffkonzentrationen geliefert wurden und beim Typ C diese am höchsten war, liegt der Schluss nahe, dass diese einen größeren Einfluss auf das Ergebnis der Viskositätsmessung hatte als die mittlere Molmasse (Zahlenmittel Mn und Massenmittel $\mathrm{Mw}$ der Molmassen). Für das Imprägnierharz TYP A lagen anfangs keine Herstellerinformationen über die Molmasse und die Molekülgröße vor. Die Molekülgröße wurde dann über das Prinzip der dynamischen Lichtstreuung (DLS) ermittelt. HILL (2006) gibt die maximale Größe der Mikroporen verholzter Zellwände im wassergequollenen Zustand mit 2-4 nm an. Wie oben gezeigt, wiesen 30\% der gemessenen Partikel eine Größe unter $4 \mathrm{~nm}$ auf und waren damit in der Lage in die Zellwand einzudringen. Weitere Faktoren wie die Viskosität und der Feststoffgehalt sowie die Reaktionskinetik ließen das Harz vergleichbar zu den anderen erscheinen.

Um im Anschluss an die Imprägnierung die Lösungsmittel, bzw. das Wasser, zu entfernen und das Harz dabei in der Zellwand zu deponieren, folgte eine Trocknung. Diese erfolgte bei einer Temperatur von $50^{\circ} \mathrm{C}$ bis maximal $60^{\circ} \mathrm{C}$ und damit unterhalb der für Kondensationsharze kritischen Temperatur. Bei der Synthese von Phenol-Formaldehyden wird üblicherweise davon ausgegangen, dass ab $60^{\circ} \mathrm{C}$ die Kondensationsreaktion der dominierende Reaktionstyp ist (Schrod 2002). Auf diese Weise wird die Zielfeuchte des Holzes bzw. der Furniere von unter 10\% - i.d.R. 4-5\% - erreicht, ohne die Harze frühzeitig zu immobilisieren und eine Diffusion in die Zellwand zu verhindern. Das Erreichen der Zielfeuchte ist eine Bedingung für die optimale Weiterverarbeitung und Verklebung der Furniere. Eigene Untersuchungen zeigten, dass diese Niedrigtemperaturtrocknung einen bedeutenden Einfluss auf das Maß der Zellwandeindringung hat. Zudem zeigten die Ergebnisse von MAI (2009), dass diese Form der Trocknung in Verbindung mit einer nachgelagerten Aushärtung der Imprägnierchemikalie während der Verklebung in der Heißpresse, zu einer besseren Qualität der Verklebung führte. 


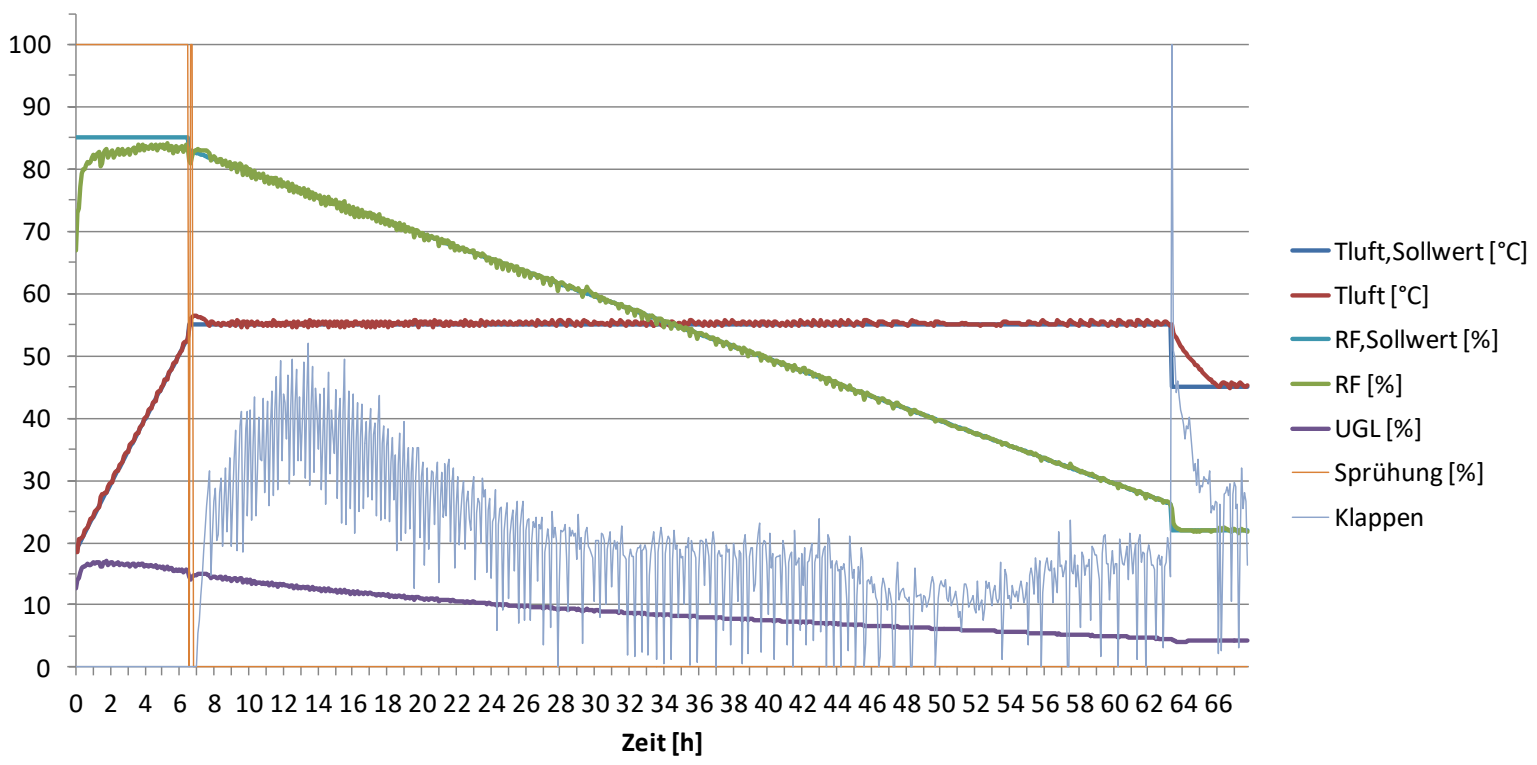

Abbildung 23: Trocknungsprozess der PF-Harzimprägnierten Buchenfurniere.

Wie Abbildung 23 zeigt, bestand der gesamte Trocknungsprozess aus den 3 Phasen: Aufheizen, Trocknen, Abkühlen.

Das Aufheizen erfolgte unter nicht trocknenden Bedingungen bei einer konstanten relativen Luftfeuchte von $85 \%$ auf eine Endtemperatur von $50-60^{\circ} \mathrm{C}$ innerhalb von $6 \mathrm{~h}$ bei einer Heizrate von $+5,3^{\circ} \mathrm{C} / \mathrm{h}$. Die Luftgeschwindigkeit im gesamten Trocknungsprozess lag bei $5 \mathrm{~m} / \mathrm{s}$. An das Aufheizen schloss sich die eigentliche Trocknung an, die bei konstanter Temperatur und einer sich absenkenden relativen Luftfeuchte erfolgte. Über einen Zeitraum von $57 \mathrm{~h}$ wurde die relative Luftfeuchte (r.F.) im Trockner um 1,05\%/h bis auf 25\% r.F. abgesenkt, um eine Ausgleichsfeuchte des Holzes von 4\% (+-0,5\%) zu erreichen. Daran schloss sich eine Kühlphase bei Aufrechterhalten der Ausgleichsfeuchte von 6-12h an, bevor der Trockner entladen wurde.

\subsubsection{Plattenproduktion und Prüfkörperherstellung}

Für die Herstellung von Furnierschichtholz kamen Buchenholzschälfurniere der Fa. Pollmeier (Creuzburg) mit den Dicken 2,13 mm und 3,7 mm zum Einsatz. Mit den 2,13 mm-Furnieren wurden 10-und 15-lagige Platten hergestellt, deren Furniere mit einem parallelen Faserverlauf orientiert waren. Bei den 3,7 mm-Furnieren wurden zwei 8-lagige Plattentypen hergestellt. Diese unterschieden sich darin, dass bei dem einen alle Furniere gleichgerichtet (paralleler Faserverlauf) und bei den anderen das 2. und 7. Furnier als Sperrlage um $90^{\circ} \mathrm{C}$ gedreht waren.

Die Beleimung der modifizierten Furniere erfolgte mit einem heißhärtenden basischen PhenolFormaldehyd-Klebstoff (Resol) vom Typ Prefere 15J173 in Verbindung mit dem Härter Prefere 25J400 - bereitgestellt durch die Dynea Erkner GmbH. Der Klebstoffauftrag betrug $180 \mathrm{~g} / \mathrm{m}^{2}$. Für erhöhte Zieldichten wurde eine Zwischentrocknung (30-45 min bei $60^{\circ} \mathrm{C}$ ) des Klebstoffes durchgeführt, bevor die Furniere zusammengelegt und in die Heißpresse eingelegt wurden, um das Imprägnierharz und den Klebstoff simultan auszuhärten. Die Presszeit wurde variiert, betrug aber mindestens $30 \mathrm{~min}\left(1 \mathrm{~min} / 1 \mathrm{~mm}\right.$-Plattendicke) bei $145^{\circ} \mathrm{C}$ und wurde aufgrund von ersten Ergebnissen zur Formaldehydabgabe auf $45 \mathrm{~min}$ (1,5 min / 1mm-Plattendicke) 
erhöht. Da eine Erhöhung der Heißpresszeit für einen Hersteller von Furnierwerkstoffen zu einer Erhöhung der Produktionskosten führt (Yazaki, Collins et al. 1994), ist dies bei einer ökonomischen Betrachtung eines Produktes aus modifizierten Furnieren $\mathrm{zu}$ beachten. Alternativ besteht für zukünftige Untersuchungen oder die industrielle Herstellung die Möglichkeit die Temperatur anzuheben, um schneller die erforderlichen Aushärtebedingungen in der Plattenmitte zu erzielen und eine verlängerte Presszeit zu vermeiden. Nach dem Heißpressen wurde für alle Platten eine Reifelagerung im Stapel durchgeführt, die ein langsames Abkühlen über 18-24 h ermöglichte. Nach dem Abkühlen erfolgten das Formatieren durch Besäumen und den Prüfkörperzuschnitt.

Die im Laufe der Arbeit verwendeten Pressdrücke lagen im Bereich von 1,5 - $6 \mathrm{~N} / \mathrm{mm}^{2}$. Auf diese Weise wurden zum einen Standarddrücke der Furnierindustrie für unverdichtete Furnierwerkstoffe (LVL und Sperrholz) angewandt und zum anderen höhere Drücke untersucht, die auf eine Verdichtung abzielten.

Tabelle 5: Untersuchte Kombinationen von Herstellungsparametern

\begin{tabular}{|c|c|c|c|c|}
\hline $\begin{array}{c}\text { Furnierstärke u. } \\
\text { Lagenzahl }\end{array}$ & Furnierausrichtung & WPGs & $\begin{array}{c}\text { Drücke } \\
{\left[\mathbf{N} / \mathbf{m m}^{2}\right]}\end{array}$ & Presstemperaturen \\
\hline $2,13 \mathrm{~mm} / 10 \mathrm{u} .15$ & parallel & $15 \%, 30 \%, 60 \%$ & $1,5,2,3,4,6$ & $145^{\circ} \mathrm{C}$ \\
\hline $3,7 \mathrm{~mm} / 8$ & parallel / 2-Sperrlagen & $15 \%, 30 \%, 60 \%$ & $2,4,6$ & $145^{\circ} \mathrm{C}$ \\
\hline
\end{tabular}

\subsubsection{Physikalische Prüfungen}

\subsubsection{Dichte}

Die Dichtebestimmung der Versuchsplatten wurde an den Prüfkörpern für die unterschiedlichen physikalischen und biologischen Prüfungen durchgeführt. In der Regel wurde die Dichte im darrtrockenen Zustand bestimmt. Dazu wurden die jeweiligen Prüfkörper nach einer Darrtrocknung bis zur Gewichtskonstanz bei $103^{\circ} \mathrm{C}$ getrocknet, im Exsikkator über Silikagel abgekühlt, vermessen und gewogen (Formel 7). Bei den Prüfkörpern für die mechanischen Festigkeiten erfolgte die Bestimmung der Rohdichte im klimatisierten Zustand $\left(20^{\circ} \mathrm{C}\right.$ und $\left.65 \% \mathrm{rF}\right)$, um einen Einfluss der Trocknung auf die mechanischen Eigenschaften auszuschließen. Nach erfolgter mechanischer Prüfung konnte anhand von Reststücken die Feuchte und Darrdichte ermittelt werden.

Formel 7: Berechnung der Darrdichte.

$$
\begin{array}{ll}
\sigma_{\text {atro }}=\frac{m_{\text {atro }}}{r_{\text {atro }} * t_{\text {atro }} * l_{\text {atro }}}=\frac{m_{\text {atro }}}{V_{\text {atro }}} \\
\begin{array}{ll}
\text { Dichte }\left[\mathrm{g} / \mathrm{cm}^{3} ; \mathrm{kg} / \mathrm{m}^{3}\right] \\
m_{\text {atro }} & \text { darrtrockene Masse }[\mathrm{g}] \\
r, t, l_{\text {atro }} & \text { Abmessung in longitudinaler, tangentialer und radialer Richtung }[\mathrm{cm} ; \mathrm{m}] \\
V_{\text {atro }} & \text { Volumen }\left[\mathrm{cm}^{3} ; \mathrm{m}^{3}\right]
\end{array}
\end{array}
$$


Die Verdichtung deltaD des LVL wurde anhand der Summe der Dicken der einzelnen Furniere vor dem Pressen sowie der Dicke der gepressten Platte $d_{\text {Platte }}$ im abgekühlten Zustand vor einer Weiterverarbeitung ermittelt. Sie unterscheidet sich demnach von der Dichte, da sie nur über die Dimensionen und nicht über das Gewicht ermittelt wurde.

Formel 8: Berechnung der Verdichtung.

deltaD $=\frac{\left(\sum d_{i}-d_{\text {Platte }}\right)}{\sum d_{i}} \times 100 \%$

deltaD Verdichtung [\%]

$d_{i} \quad$ Dicke der einzelnen Furniere vor dem Pressen [mm]

$d_{\text {Platte }} \quad$ Dicke der abgekühlten Platte nach dem Pressen [mm]

\subsubsection{Fixierung und Dimensionsstabilität}

Die Bestimmung der Wasseraufnahme, des Quellens sowie der Fixierung des PF-Harzes wurde, in Anlehnung an die vor Abbauversuchen obligatorische Auswaschbeanspruchung DIN EN 84 (1997), mit den Prüfkörpern durchgeführt, die später anschließend in den Abbauversuch überführt wurden. Dabei wurden Plattenprüfkörper des Formates $50 \mathrm{~mm} * 50 \mathrm{~mm} *$ Plattendicke unter Vakuum (80mbar, $1 \mathrm{~h}$ ) mit Leitungswasser imprägniert und für 14 Tage im Wasser bei regelmäßigem Wasserwechsel belassen. Zuvor wurden die Gewichte und Dimensionen (longitudinal, radial und tangential) im absolut-trockenen ( atro $_{0}$ ) Zustand ermittelt. Nach dieser Zeit wurden die Proben im nassen Zustand erneut gemessen, bevor sie sukzessive getrocknet und dann erneut darr-trocken (atro ${ }_{1}$ ) gemessen wurden. Die Wasseraufnahme wurde anhand der Massenänderung ermittelt.

Formel 9: Berechnung der Wasseraufnahme.

$W=\frac{\left(m_{\text {wet }}-m_{\text {atro0 }}\right)}{m_{\text {atro } 0}} * 100 \%$

$W \quad$ Wasseraufnahme [\%]

$m_{\text {wet }} \quad$ wassergesättigte Masse $[\mathrm{g}]$

$m_{\text {atro0 }} \quad$ darrtrockene Masse vor der Auswaschbeanspruchung [g]

Die Quellung wurde, für jede anatomische Holzrichtung (longitudinal, radial und tangential) getrennt, anhand der jeweiligen Dimensionsänderung berechnet (Formel 10). Dabei diente die longitudinale Dimension nur der Bestimmung der Volumenquellung $Q_{V}$ entsprechend Formel 13. 
Formel 10: Berechnung der Quellrate für eine anatomische Holzrichtung.

$Q_{\text {long,rad,tan }}=\frac{\left(l_{1}-l_{0}\right)}{l_{0}} * 100 \%$

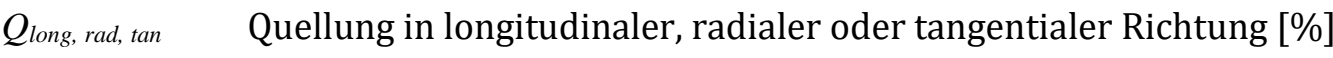

$l_{0} \quad$ absolut-trockene Dimension [mm $]$

$l_{l} \quad$ wassergesättigte Dimension [mm]

Neben der Berechnung der Dickenquellung (senkrecht zur Plattenebene) wurde, ebenfalls an den Prüfkörpern für den Pilzabbau, zusätzlich eine radiale Anti-Swell-Efficiency (ASE ${ }_{\text {rad}}$ ) kalkuliert. Diese drückt die Verminderung der Dickenquellung der modifizierten Proben in Bezug zur Dickenquellung der jeweiligen Referenz mit gleichem Pressdruck aus.

Formel 11: Berechnung der ASErad.

$A S E_{r a d}=\frac{\left(Q_{r a d_{\mathrm{Ref}}}-Q_{\text {rad }_{i}}\right)}{Q_{\text {rad }_{i}}} * 100 \%$

$Q_{\text {rad }}$ Dickenquellung in Prozent in radialer holzanatomischer Richtung bzw. orthogonal zur Plattenebene

Ref Index für die Referenzserie

$i \quad$ Index für die modifizierte Serie

Die Fixierung wurde über die Auswaschung $L$, welche auf den darr-trockenen Massen vor und nach der Auswaschbeanspruchung beruht, festgestellt.

Formel 12: Berechnung der Auswaschung.

$L=\frac{\left(m_{\text {atro0 }}-m_{\text {atrol }}\right)}{m_{\text {atro } 0}} * 100 \%$

$m_{\text {atro }}$ darrtrockene Masse vor der Auswaschbeanspruchung [g]

$m_{\text {atrol }} \quad$ darrtrockene Masse nach der Auswaschbeanspruchung [g]

Zusätzlich erfolgte die Bestimmung der Volumenquellung $V Q$ nach einer 4-stündigen Kochung, d.h. Wasserlagerung bei $100^{\circ} \mathrm{C}$, mit anschließendem Abkühlen in $20^{\circ} \mathrm{C}$ warmem Wasser. $\mathrm{Zu}$ diesem Zweck wurden jedem Biegeprüfkörper einer Platte zwei Proben vom Format $100 * 50 \mathrm{~mm}^{2}$ entnommen. Davon wurde jeweils einer zur Dichtebestimmung und einer für die Volumenquellung verwendet. Durch eine 4-stündige Lagerung der Prüfkörper in Wasser bei $100^{\circ} \mathrm{C}$ und Messung der Dimensionen (longitudinal, tangential, radial), vorher im lufttrockenen Zustand und nachher wassergesättigt, wurde die Volumenquellung ermittelt. 
Formel 13: Berechnung der Volumenquellung.

$Q_{V}=\frac{\left(l_{\text {long1 }}-l_{\text {long } 0}\right) *\left(l_{\text {rad } 1}-l_{\text {rad } 0}\right) *\left(l_{\tan 1}-l_{\tan 0}\right)}{l_{0} * l_{\text {rad } 0} * l_{\tan 0}} * 100 \%$

$Q_{V} \quad$ Volumenquellung [\%]

$l_{0} \quad$ absolut-trockene Dimension $[\mathrm{mm}]$

$l_{l} \quad$ wassergesättigte Dimension $[\mathrm{mm}]$

\subsubsection{Holzfeuchte}

Die Bestimmung der Ausgleichsfeuchte im Normalklima $\left(20^{\circ} \mathrm{C} / 65 \% \mathrm{rF}\right)$ erfolgte an den Prüfkörpern die für den Abbauversuch gemäß ENV 12038 (2002) vorgesehen waren und der zuvor beschriebenen Auswaschbeanspruchung gemäß DIN EN 84 (1997) unterzogen wurden. Die Plattenprüfkörper wurden nach der gravimetrischen Feststellung ihres absolut-trockenen Gewichts für 5 Wochen im Normalklima gelagert und regelmäßig gewogen bis sich die Massenänderung innerhalb von $6 \mathrm{~h}$ kleiner als 0,1\% war. Die reguläre Holzfeuchte der Platten wurde gemäß DIN EN 322 (1993) berechnet, wobei die Bezugsgröße (Nenner) die PF enthaltende Gesamtmasse nach der Modifizierung war (Formel 14).

Formel 14: Berechnung der regulären Holzfeuchte.

$u=\frac{\left(m_{\text {lutro }}-m_{\text {atro }}\right)}{m_{\text {atro }}} * 100 \%$

$u \quad$ Holzfeuchte [\%]

$m_{\text {lutro }} \quad$ lufttrockene Masse $[\mathrm{g}]$

$m_{\text {atro }} \quad$ darrtrockene Masse $[\mathrm{g}]$

Um die wahre Holzfeuchte zu schätzen, wurde das in dem Prüfkörper enthaltene Wasser auf die reine Holzmasse, ohne den Anteil Imprägnierharz, bezogen. Dafür wurde die reguläre Formel abgewandelt und das atro-Probengewicht um den WPG korrigiert.

Formel 15: Berechnung der korrigierten Holzfeuchte.

$u_{\text {korr }}=\frac{\left(m_{\text {lutro }}-m_{\text {atro }}\right) *(1+W P G)}{m_{\text {atro }}} * 100 \%$

$u_{\text {korr }} \quad$ korrigierte Holzfeuchte [\%]

$m_{\text {lutro }} \quad$ lufttrockene Masse [g]

$m_{\text {atro }} \quad$ darrtrockene Masse $[\mathrm{g}]$

WPG Weight-Percent-Gain [\%] 


\subsubsection{Biegefestigkeit und Elastizitätsmodul}

Die Biegefestigkeit (MOR) und das Elastizitätsmodul (MOE) wurden an LVL-Versuchsplatten ermittelt, welche nach dem Vakuumverfahren PF-modifiziert wurden.

Es wurden anhand eines parallelen Plattenaufbaus die Auswirkung der Modifizierung mit dem Harztyp A an 3,7 mm und 2,13 mm dicken Furnieren mit 8 Lagen bzw. 15 Lagen untersucht. Dabei wurden die Pressdrücke $2 \mathrm{~N} / \mathrm{mm}^{2}, 4 \mathrm{~N} / \mathrm{mm}^{2}$ und $6 \mathrm{~N} / \mathrm{mm}^{2}$ eingesetzt, die zu unterschiedlichen Verdichtungen führten. Die Auswirkung der Modifizierung mit unterschiedlichen Harzen wurden an 8-lagiegm Buchen-LVL mit und ohne Sperrlagen untersucht. Es wurden dazu die Platten mit zwei Sperrlagen geprüft, deren Furniere mit den Harzen A, B, C, D und E mit WPGs von 30\% und 60\% behandelt waren. Des Weiteren wurden Platten verwendet, die näher an der industriellen Realität lagen und einen parallelen Aufbau aufwiesen, deren Furniere mit den Harzen A, F und G in der Art modifiziert waren, dass sie WPGs von 15\% und 30\% aufwiesen. Die Prüfung erfolgte nach DIN EN 310 (1993) „Holzwerkstoffe- Bestimmung des Biege-Elastizitätsmoduls und der Biegefestigkeit“ im 3Punkt-Biegversuch. Abweichend von der Norm musste die Stützweite auf das 14-fache der Nennplattendicke (30 mm) und somit auf $420 \mathrm{~mm}$ reduziert werden, da die Versuchsplatten auf einer Laborpresse im Format $500 \mathrm{~mm} * 500 \mathrm{~mm}$ hergestellt wurden, welches durch ein notwendiges Besäumen auf $480 \mathrm{~mm} * 480 \mathrm{~mm}$ reduziert wurde. Alle Prüfkörper wurden vorher im Normalklima $\left(20^{\circ} \mathrm{C} / 65 \% \mathrm{rF}\right)$ bis zur Gewichtskonstanz gelagert.

Formel 16: Berechnung des Elastizitätsmodul

$E_{m}=\frac{l_{1}^{3} *\left(F_{2}-F_{1}\right)}{4 * b^{*} t^{3} *\left(a_{2}-a_{1}\right)}$

$E_{m} \quad$ Elastizitätsmodul $\left[\mathrm{N} / \mathrm{mm}^{2}\right]$

$l_{l} \quad$ Abstand zwischen den Mitten der Auflager [mm]

$b \quad$ Prüfkörperbreite [mm]

$t \quad$ Prüfkörperdicke [mm]

$F_{2}-F_{1} \quad$ Kraftzunahme im linearen Bereich [N]

$a_{2}-a_{1} \quad$ Lageänderung bzw. Durchbiegung in Prüfkörpermitte [mm]

Formel 17: Berechnung der Biegefestigkeit

$f_{m}=\frac{3 * F_{\max } * l_{1}}{2 * b^{*} t^{2}}$

$f_{m} \quad$ Biegefestigkeit $\left[\mathrm{N} / \mathrm{mm}^{2}\right]$

$F_{\max } \quad$ Maximalkraft [N]

$l_{l} \quad$ Abstand zwischen den Mitten der Auflager [mm]

$b \quad$ Prüfkörperbreite $[\mathrm{mm}]$

$t \quad$ Prüfkörperdicke [mm] 


\subsubsection{Bruchschlagarbeit}

Die Bestimmung der Bruchschlagarbeit $w$ erfolgte in Anlehnung an die DIN 52189 (1981) im Schlagbiegeversuch. Zusätzlich wurde vor der Schlagbiegeprüfung der jeweilige statische Elastizitätsmodul bestimmt.

Abweichend von der Norm wurden, statt Vollholzproben, fehlerfreie LVL-Proben in 8-lagigem und 15-lagigem Aufbau mit 3,7 mm und 2,13 mm dicken Furnieren im ausschließlich parallelen Aufbau verwendet, die unter einem Pressdruck von $2 \mathrm{~N} / \mathrm{mm}^{2}, 4 \mathrm{~N} / \mathrm{mm}^{2}$ und 6 $\mathrm{N} / \mathrm{mm}^{2}$ verklebt wurden. Bei den Referenzplatten waren die Furniere unbehandelt und bei den modifizierten Serien wurde der Harztyp A eingesetzt. Die Imprägnierung erfolgte ausschließlich im Vakuumverfahren und es wurden WPGs von 15\%, 30\% und 60\% erzielt. Die Kantenlängen der Prüfkörper betrugen tangential $20 \mathrm{~mm}$ und longitudinal mindestens $300 \mathrm{~mm}$ - die Kantenlänge in radialer Richtung entsprach der Plattendicke und lag somit in Abhängigkeit von der Verdichtung zwischen $20 \mathrm{~mm}$ und $30 \mathrm{~mm}$. Die Bestimmung des Elastizitätsmoduls erfolgte auf einer Universalprüfmaschine (Zwick 10kN), im zuvor ermittelten linearen reversiblen Bereich der Verformung, mit einer Maximalkraft von $151 \mathrm{~N}$ bei einer Stützweite von $400 \mathrm{~mm}$. Bei der Schlagbiegepüfung war die Schlagrichtung des Pendelschlagwerkes (150J) tangential, also auf die stehenden Furniere bzw. die stehende Leimfuge. So sollten ggf. auftretende Spaltbrüche und unvollständig durchtrennte Proben vermieden werden. KolLMANN (1951) sah den radialen Schlag für diese Form der Holzprüfung ebenfalls als ungeeignet an. Zudem wurde auf diese Weise eine immer gleiche Prüfkörperhöhe erzielt, welche im Einklang mit der normgerechten Stützweite von $240 \mathrm{~mm}$ (210 mm lichte Weite) stand. Vor der Prüfung erfolgte die Lagerung der Proben im Normalklima $\left(20^{\circ} \mathrm{C} / 65 \%\right.$ r.F.) bis zum Erreichen der Ausgleichsfeuchte. Nach der Prüfung erfolgten die Bestimmung der Rohdichte, der Darrdichte und der Feuchte zum Zeitpunkt der Prüfung. Für jede Variante wurden 8 Prüfkörper getestet. Die Berechnung erfolgte entsprechend Formel 18.

Formel 18: Berechnung der Bruchschlagarbeit

$$
w=\frac{1000 * W}{b * h}=\frac{1000 * W}{A}
$$

$w \quad$ Bruchschlagarbeit $\left[\mathrm{kJ} / \mathrm{m}^{2}\right]$

W $\quad$ Arbeit zum Durchschlagen der Probe [J]

$b, h \quad$ Querschnittsmaße [m]

A Querschnittsfläche $\left[\mathrm{m}^{2}\right]$ 


\subsubsection{Formaldehydabgabe}

\subsubsection{Bestimmung der Formaldehydabgabe nach der Prüfkammer-Methode}

Die Bestimmung der Formaldehydabgabe nach der Prüfkammer-Methode, gemäß DIN EN 717-1 (2005), erfolgte zu Beginn der Arbeit an Buchen-LVL, welches 10-lagig aus 2,13mm dicken Furnieren mit einem WPG von 30\% hergestellt wurde. Bei der Herstellung in der Heißpresse wurden zwei unterschiedlich lange Presszeiten eingesetzt, um deren Einfluss auf die Formaldehydabgabe zu untersuchen. Für den Vergleich unterschiedlicher Harze wurde, aufgrund einer relativ kürzeren Versuchsdauer, die nachstehende Gasanalyse-Methode bevorzugt.

Für die Prüfkammer kamen jeweils zwei identische Platten zum Einsatz, deren Schmalflächen derart abgeklebt waren, dass sich eine gesamte Oberfläche von einem $1 \mathrm{~m}^{2} / \mathrm{m}^{3}$ Kammer ergab. Die in der Norm festgesetzten Bedingungen in der Kammer hinsichtlich der Temperatur, der relativen Luftfeuchte, dem Beladungsfaktor, dem Luftwechsel und der Luftwechsel wurden eingehalten. Die Probenentnahme erfolgte immer werktags über einen Zeitraum von 4 Wochen.

Die Bestimmung des Formaldehydgehaltes im Absorbens der Gaswaschflaschen erfolgte photometrisch über das Acetyl-Aceton-Verfahren, wobei zwei Parallelproben für jede Flasche resp. Messkolben angesetzt wurden. Die Messung erfolgte mit einem Spektrophotometer „Specord 205“ der Analytik Jena AG, Jena, gemäß den Vorgaben der DIN EN 717-1 (2005) und bei einer Wellenlänge von $412 \mathrm{~nm}$ gegenüber Reinstwasser als Referenz. Dabei wurde die Konzentration des entstandenen Di-acetyldihydrolutidin (DDL) gemessen und unter Verwendung einer Kalibrierkurve (Formaldehyd-Standardlösung) die Formaldehydkonzentration der Lösung berechnet. Zusätzlich zu den Proben aus den Gaswaschflaschen wurden auch die Blindwerte der verwendeten Chemikalien bestimmt und das Messergebnis entsprechend korrigiert.

Formel 19: Berechnung der absorbierten Formaldehydmenge bei der Prüfkammer-Methode

$G[m g]=\left(A_{s}-A_{b}\right) * f * V_{\text {sol }}$

G Gehalt an Formaldehyd in jeder Absorptionslösung in mg

$A_{s} \quad$ Extinktion der Blindwerte [-]

$A_{b} \quad$ Extinktion der Lösung aus der Gaswaschflasche [-]

$f \quad$ Steigungsfaktor der Kalibrierkurve $[\mathrm{mg} / \mathrm{ml}]$

$V \quad$ Volumen der Absorptionslösung [ml]

Die Einzelwerte $G$ an einem Tag wurden zu einem Gesamtgehalt an Formaldehyd $G_{t o t}$ addiert. Die Konzentration an Formaldehyd in der Prüfkammer ergab sich nach:

$c\left[\mathrm{mg} / \mathrm{m}^{3}\right]=\frac{G_{t o t}}{V_{L u}}$

c Formaldehydkonzentration $\left[\mathrm{mg} / \mathrm{m}^{3}\right]$ 
$G_{\text {tot }} \quad$ Gesamtgehalt an Formaldehyd in den Absorptionslösungen [mg]

$V_{L u} \quad$ Volumen der Luftprobe $\left[\mathrm{m}^{3}\right]$

\subsubsection{Bestimmung der Formaldehydabgabe nach der Gasanalyse-Methode}

Bei der Bestimmung der Formaldehydabgabe von Buchen-LVL unter Anwendung der Gasanalyse-Methode, gemäß der europäischen Norm (EN) 717-2 (1994), wurden immer zwei Prüfkörper pro Variante mit einer Länge von $400 \mathrm{~mm}$ und einer Breite von $50 \mathrm{~mm}$ verwendet. Deren Schmalflächen (Schnittkanten) wurden mit einem Aluminiumklebeband versiegelt. Eine Zwischenlagerung der Prüfkörper erfolgte durch Einschweißen in Folie unter Vakuum und Lichtabschluss. Das zur Extraktion genutzte Gerät „GreCon GA 5000“ der Firma Grecon, AlfeldHannover verfügte über zwei parallel zu betreibende Kammern mit angeschlossener Waschflaschenbatterie. Die Kammern wurden auf eine Temperatur von $60^{\circ} \mathrm{C}\left( \pm 0,5^{\circ} \mathrm{C}\right)$ erhitzt. Die Gaswaschflaschen (8 Stk. pro Kammer) waren gefüllt mit Reinstwasser. Die Prüfkörper wurden erst nach Erreichen der vorgegebenen Temperatur in die Kammern gestellt. Nach dem Startvorgang regelte das Gerät den Luftdruck und Luftwechsel automatisch und leitete das Gasgemisch durch die Waschflaschen, wobei stündlich ein anderes Flaschenpaar zugeschaltet wurde. Das in dem Gasgemisch enthaltene Formaldehyd wurde dann von dem Reinstwasser in den Flaschen absorbiert. Die Extraktion dauerte 4 Stunden. Nach der Entnahme der Prüfkörper wurden sie gewogen, bei $103{ }^{\circ} \mathrm{C}$ darrgetrocknet und wieder gewogen, um die Feuchte und Darrdichte zu bestimmen. Die Inhalte der Waschflaschen wurden getrennt für jede Stunde der Messung in Messkolben überführt, welche auf $250 \mathrm{ml}$ aufgefüllt wurden. Eine Zwischenlagerung erfolgte lichtgeschützt bei $7^{\circ} \mathrm{C}$ für höchstens zwei Tage.

Die Bestimmung des Formaldehydgehaltes im Absorbens der Gasflaschen erfolgte, wie bei der Prüfkammer-Methode beschrieben, photometrisch über das Acetyl-Aceton-Verfahren, wobei zwei Parallelproben für jeden Messkolben angesetzt wurden.

Der Gasanalysewert $G_{i}$ für jede Stunde der Extraktion ergab sich durch folgende Berechnung:

Formel 20: Berechnung des Gasanalysewert Gi für jede Stunde der Extraktion

$G_{i}\left[\frac{m g}{m^{2} h}\right]=\frac{\left(A_{S}-A_{B}\right) * f * V}{F}$

$G_{i} \quad$ Gasanalysewert $G_{i}$ für eine Stunde der Extraktion $\left[\mathrm{mg} / \mathrm{m}^{2} \mathrm{~h}^{-1}\right]$

$A_{S} \quad$ Extinktion der Lösung aus den Waschflaschen [-]

$A_{B} \quad$ Extinktion der Blindwerte [-]

f Steigungsfaktor der Kalibrierkurve $[\mathrm{mg} / \mathrm{ml}]$

$V \quad$ Volumen des Messkolbens [ml]

F Oberfläche des Prüfkörpers $\left[\mathrm{m}^{2}\right]$

Die Berechnung des mittleren Gasanalysewert $G_{m}$ erfolgte, bei einem geringeren Formaldehydgehalt der ersten Stunde, durch Mittelwertbildung der zweiten bis vierten Stunde, sonst wurde der Mittelwert für alle vier Stunden gebildet. Die Ergebnisse beider Kammern, bzw. Prüfköper, wurden wiederum gemittelt. 
Formel 21: Berechnung des mittleren Gasanalysewertes $G_{m}$ gemäß EN 717-2 (1994)

Für $\mathrm{G}_{1}<\mathrm{G}_{2}$ galt:

$G_{m}\left[\frac{m g}{m^{2} h}\right]=\frac{G_{2}+G_{3}+G_{4}}{3}$

Für $\mathrm{G}_{1}=\mathrm{G}_{2}$ und $\mathrm{G}_{1}>\mathrm{G}_{2}$ galt:

$G_{m}\left[\frac{m g}{m^{2} h}\right]=\frac{G_{1}+G_{2}+G_{3}+G_{4}}{4}$

\subsubsection{Biologische Dauerhaftigkeit}

\subsubsection{Basidiomyceten}

Die Prüfung der Resistenz gegenüber holzabbauenden Basidiomyceten erfolgte nach DIN ENV 12038 (2002). Als Prüfpilz wurde der Weißfäuleerreger Trametes versicolor L. (Stamm CTB 863A. Centre Technique du Bois et de l'Ameublement, 10 Avenue de Saint-Mandré, F75012 Paris) ausgewählt, da er gemäß Norm als obligatorischer Prüfpilz für Laubholz auszuwählen ist. Die Fähigkeit dieser Weißfäule neben Zellulose auch Lignin und andere phenolische Substanzen abzubauen (HoFrichter 2002, SCHLOSSER ET AL. 1997) qualifiziert ihn besonders, um den Erfolg einer PF-Modifizierung zu beurteilen. Neben den Virulenzen, welche anhand ihres erzielten Masseverlustes die Vitalität des Prüfpilzes anzeigen, dienten unbehandelte Plattenprüfkörper sowie Vollholzprüfkörper in den Abmessungen der Plattenprüfkörper, sog. Maß-Kontrollprüfkörper, engl. Size controls, als Referenzen. Sie wiesen eine Länge und Breite von jeweils $50 \mathrm{~mm}$ und eine Dicke von $30 \mathrm{~mm}$ im absolut trockenen Zustand auf. Alle Prüfkörper wurden vor Beginn des Abbauversuchs einer Auswaschbeanspruchung nach EN 84 (1997) unterzogen und anschließend 5 Wochen bei $20^{\circ} \mathrm{C} / 65 \% \mathrm{rF}$ klimatisiert. Abweichend von der Norm konnte bei der Klimatisierung der behandelten Serien keine Gleichgewichtsfeuchte erzielt werden, da infolge der Modifizierung eine stark verlangsamte Sorption $\mathrm{zu}$ beobachten war und es fortlaufend zu einer geringen Änderung kam. Die Ergebnisse der ermittelten Feuchten werden den Ergebnissen des Abbauversuchs vorangestellt. In diesem wurde jeweils ein Prüfkörper pro Schale auf T. versicolor im Prüfraum bei $22 \pm 1{ }^{\circ} \mathrm{C}$ und $70 \pm 5 \% \mathrm{rF}$ (Abbildung 24) inkubiert. Die Bestimmung des Masseverlustes erfolgte durch Vergleich der atro-Gewichte vor und nach dem Abbauversuch. Durch Einwaage der gereinigten Prüfkörper direkt nach Versuchsende wurde die Feuchte nach Ausbau ermittelt.

Um die Masseverluste verschiedener Plattentypen bzw. Serien zu vergleichen, wurde einerseits die Einteilung in Dauerhaftigkeitsklassen nach dem Masseverlust gemäß dem Standard CEN/TS 15083-1 (2005) und andererseits nach Berechnung des Befallsanfälligkeitsindex (engl. Decay Susceptibility Index = DSI) (Tabelle 6). Die Berechnung des DSI erfolgte gemäß Anhang E der ENV 12038 (2002) durch Bildung der Quotienten aus den Masseverlusten der Prüfköper einer Serie und dem mittleren Masseverlust der Size controls, resp. Maß-Kontrollprüfkörper, welche aus unbehandeltem Buchenvollholz in den oben genannten Abmessungen bestanden (Formel 22). Dieses Vorgehen wurde gewählt, da die ENV 12038 (2002) selbst keine Einteilung in Dauerhaftigkeitsklassen vorsieht. Stattdessen 
seien, ohne weitere Klassifizierung, alle Erzeugnisse, deren mittlerer Masseverlust der Prüfkörper kleiner als 3\% betrage, wobei nicht mehr als ein Prüfkörper maximal 5\% aufweisen dürfe, als voll beständig gegen holzzerstörende Basidiomyceten zu bezeichnen.

Formel 22: Berechnung des Befallsanfälligkeitsindex (DSI) nach Anhang E in ENV 12038 (2002).

$D S I[x]=\frac{T}{S}$

$T \quad$ Masseverlust eines einzelnen Prüfkörpers [\%]

$S \quad$ mittlerer Masseverlust der Maßkontrollprüfkörper [\%]

Tabelle 6: Dauerhaftigkeitsklassen nach EN 350 (2016) und zugehörige mediane Masseverluste in Anlehnung an CEN/TS 15083-1 (2005) bzw. relative mittlere Masseverluste (Decay susceptibility indices, DSI) nach Anhang E in ENV 12038 (2002).

\begin{tabular}{llll}
\hline Dauerhaftigkeitsklasse & Beschreibung & Median Masseverlust [\%] & DSI $[\mathbf{x}]$ \\
\hline 1 & Sehr dauerhaft & $\leq 5$ & $\mathrm{x} \leq 0.15$ \\
2 & Dauerhaft & $>5$ to $\leq 10$ & $\mathrm{x}>0.15$ to $\leq 0.30$ \\
3 & Mäßig dauerhaft & $>10$ to $\leq 15$ & $\mathrm{x}>0.30$ to $\leq 0.60$ \\
4 & Wenig dauerhaft & $>15$ to $\leq 30$ & $\mathrm{x}>0.60$ to $\leq 0.90$ \\
5 & Nicht dauerhaft & $>30$ & $\mathrm{x}>0.90$ \\
\hline
\end{tabular}

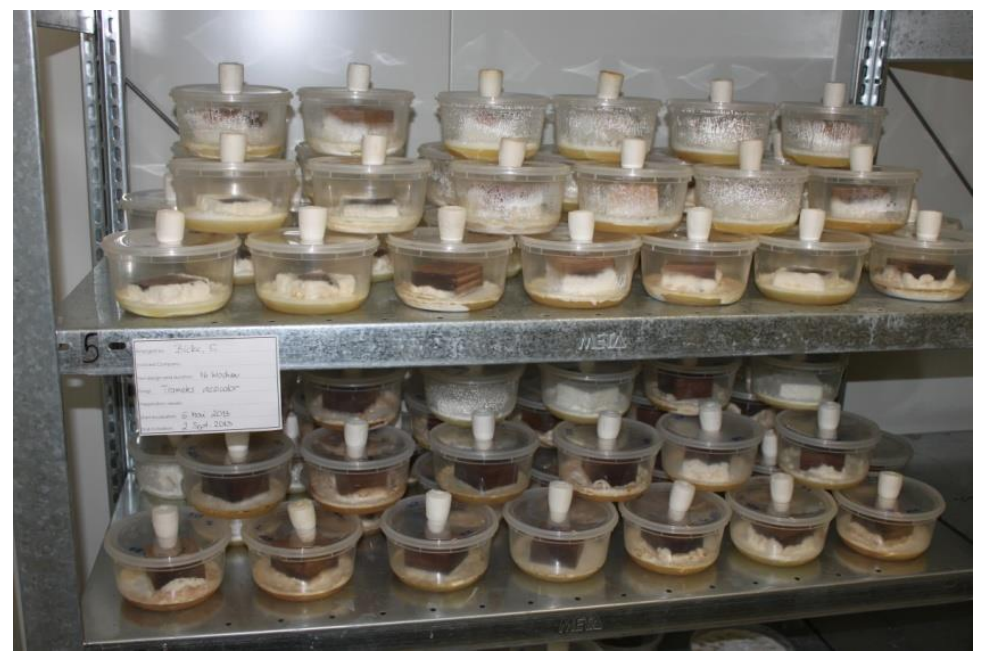

Abbildung 24: Buchen-LVL-Prüfkörper während der Inkubation auf Trametes versicolor im Prüfraum bei $22 \pm 1^{\circ} \mathrm{C}$ und $70 \pm 5 \%$ relativer Luftfeuchte. 


\subsubsection{Freilandprüfverfahren mit Erdkontakt}

Die Bestimmung der Schutzwirkung der Modifizierung mit PF-Harz im Erdkontakt erfolgte in Anlehnung an EN 252 (2015). Abweichend von der Norm wurden je Serie mindestens 8, i.d.R. 9, Prüfkörper mit einer Länge von $480 \mathrm{~mm}$, einer Breite von $25 \mathrm{~mm}$ und einer Dicke von durchschnittlich $25 \mathrm{~mm}$, entsprechend der Plattendicke, verwendet. Sie wurden mit einem Reihenabstand von $30 \mathrm{~cm}$ und einem Prüfkörperabstand in der Reihe von $30 \mathrm{~cm}$ auf einem Versuchsfeld entlang des Burckhardtwegs in Göttingen, Deutschland, $240 \mathrm{~mm}$ tief in den Erdboden gesteckt (Abbildung 25). Dichterer Pflanzenbewuchs wurde durch Ausbringung eines Vlieses sowie regelmäßiges manuelles Entfernen verhindert. Eine Evaluation der Prüfkörper erfolgte einmal im Jahr entsprechend den Normkriterien (EN 252, 2015). Die nachfolgende Tabelle 7 gibt einen Überblick über die Bewertungsziffern und die entsprechenden Restquerschnitte der Prüfkörper.

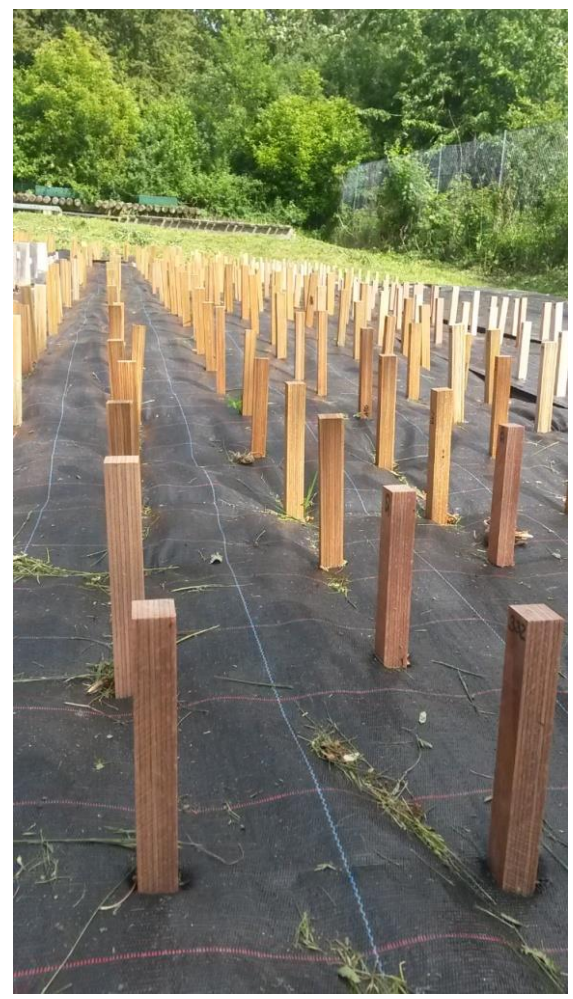

Abbildung 25: Freilandprüfung im Erdkontakt (EN 252, 2015) mit PF-modifiziertem Buchen-LVL in Göttingen.

Tabelle 7: Bewertungsziffern, Einstufung und Kriterien der Prüfkörper aus dem Freilandprüfverfahren mit Erdkontakt (EN 252, 2015) sowie verbleibender Restquerschnitt.

\begin{tabular}{|c|c|c|c|}
\hline $\begin{array}{c}\text { Bewertungsziffer } \\
\text { EN 252 }\end{array}$ & Einstufung & Bewertungskriterien & Restquerschnitt \\
\hline 0 & kein Angriff & keine Veränderungen & $625 \mathrm{~mm}^{2}=100 \%$ \\
\hline 1 & leichter Angriff & Weichwerden bis $1 \mathrm{~mm}$ Tiefe & $531 \mathrm{~mm}^{2}=85 \%$ \\
\hline 2 & mittlerer Angriff & Weichwerden bis $3 \mathrm{~mm}$ Tiefe & $363 \mathrm{~mm}^{2}=58 \%$ \\
\hline 3 & starker Angriff & $\begin{array}{c}\text { großflächiges Weichwerden bis } 5 \mathrm{~mm} \\
\text { Tiefe oder auf begrenztem Bereich bis } \\
15 \mathrm{~mm}\end{array}$ & $225 \mathrm{~mm}^{2}=36 \%$ \\
\hline 4 & Ausfall & Durchbrechen des Stabes & - \\
\hline
\end{tabular}




\subsubsection{Witterungsstabilität}

\subsubsection{Schnellbewitterung}

Ein häufiges Problem von Holz und Holzwerkstoffen, die der Witterung ausgesetzt sind, sind Feuchtewechsel. Dabei kann durch Regen Wasser auf dem Bauteil liegen oder darüber abfließen und so zu einer partiellen, zum Teil oberflächlichen Quellung führen. In der Regel schließt sich an eine Phase der Auffeuchtung, z.B. durch Wetterumschwung, eine Phase der Trocknung bei gleichzeitig höheren Temperaturen an. Bootdecks, Terrassendielen und Träger sind Anwendungen, in denen Staunässe, aufgrund einer horizontalen Bauteilanordnung, auftritt. Wiederholte Abfolgen von Quellen und Schwinden führen in der Regel zu Rissbildung, auch Beschichtungen werden in der Folge vom Wasser unterwandert und können abplatzen. Um diese Neigung zur Rissbildung unterschiedlich behandelter Furnierwerkstoffe zu untersuchen, wurde ein Zyklustest zur Simulation des oben beschriebenen Sachverhaltes entwickelt. Wie aus Abbildung 26 zu entnehmen ist, wurden hierbei die Proben über einem Wasserbassin horizontal gelagert. Ein Gestell darüber ermöglichte die Beregnung sowie die Bestrahlung mit Infrarot(IR)- und Ultraviolett(UV)- Lampen. Dabei stieg die Oberflächentemperatur um $40^{\circ} \mathrm{K}$ über die Umgebungstemperatur.

Bei der Infrarotquelle handelte es sich um eine Lampe vom Typ Osram Siccatherm mit $250 \mathrm{~W}$ Nennleistung. Als UV-Strahlungsquelle kam der Hochdruck-Ultraviolettstrahler Ultra-Vitalux der Firma Osram zum Einsatz, welcher mit einer Nennleistung von $300 \mathrm{~W}$ bei $230 \mathrm{~V}$ im Bereich der UVA-Strahlung (315-400 nm) eine Leistungsabgabe von 13,6 W und im Bereich der UVB-Strahlung (280 - $315 \mathrm{~nm}$ ) eine Leistungsabgabe von 3,0 W aufwies.
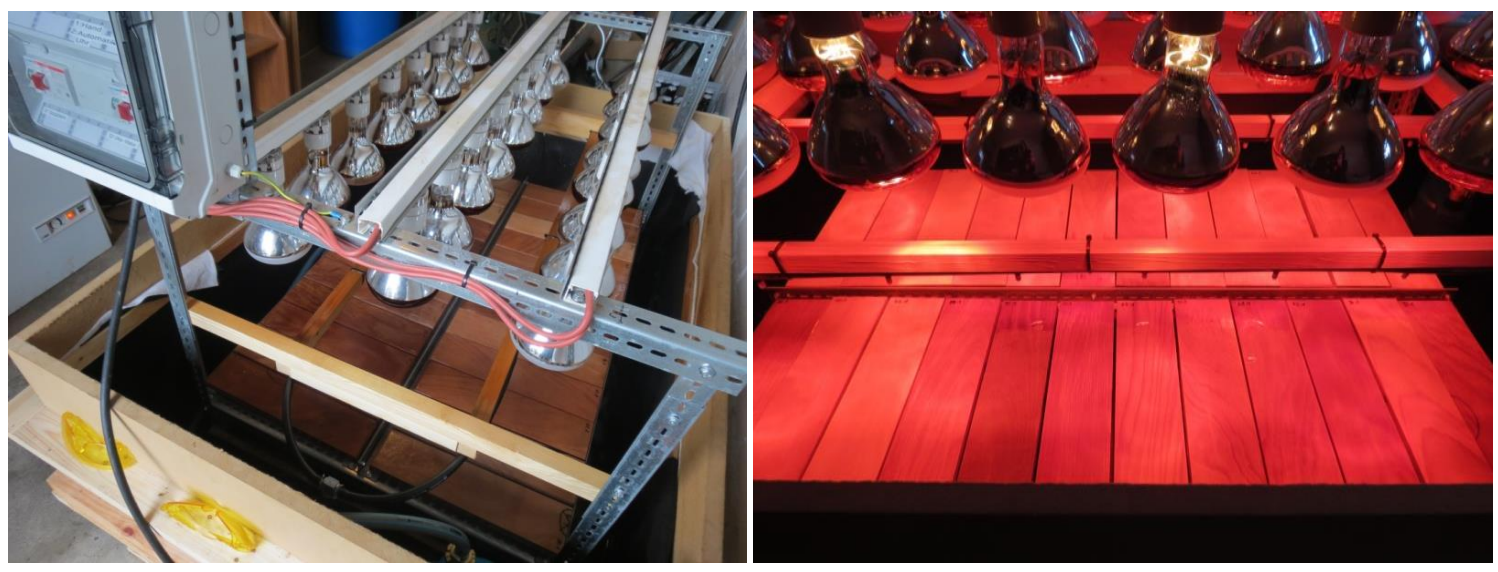

Abbildung 26: Aufbau der Schnellbewitterungsanlage (links), Prüfkörper unter infrarotem Licht (rechts). 

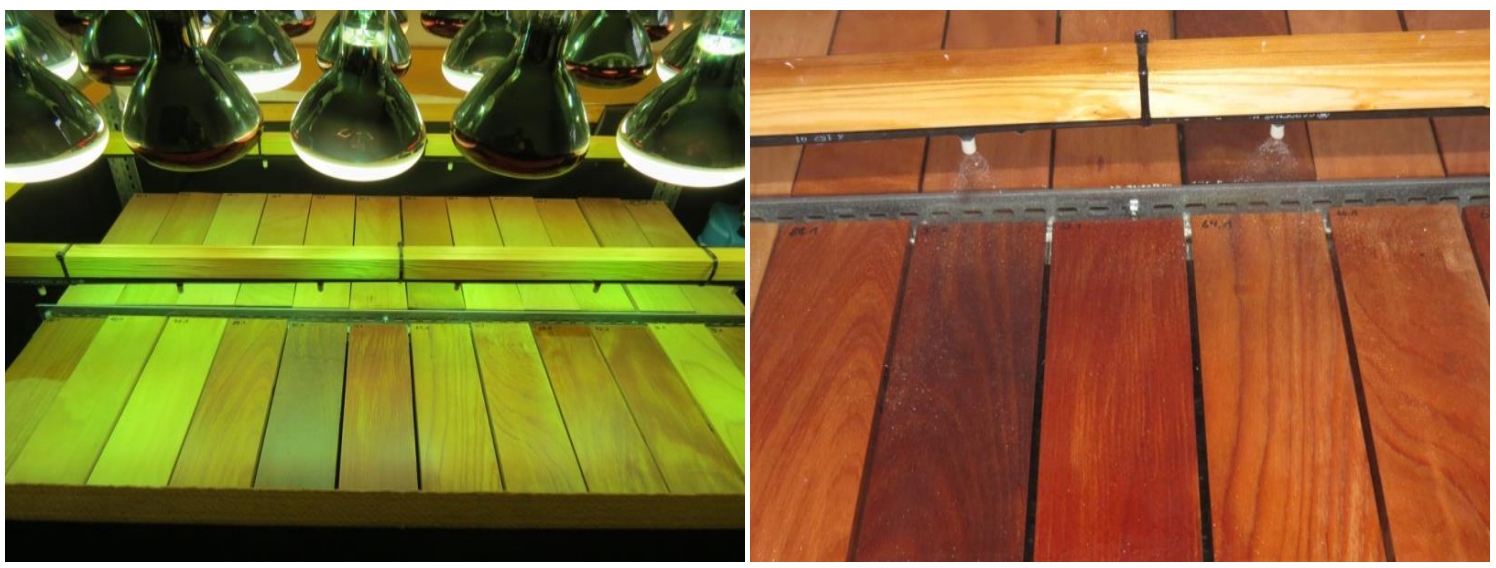

Abbildung 27: Prüfkörper unter UV-Licht (links)und während der Beregnung (rechts).

Bei einer Testdauer von 5 Wochen wurden so täglich für mehrere Stunden Niederschlag und später Sonneneinstrahlung simuliert werden (Abbildung 28).

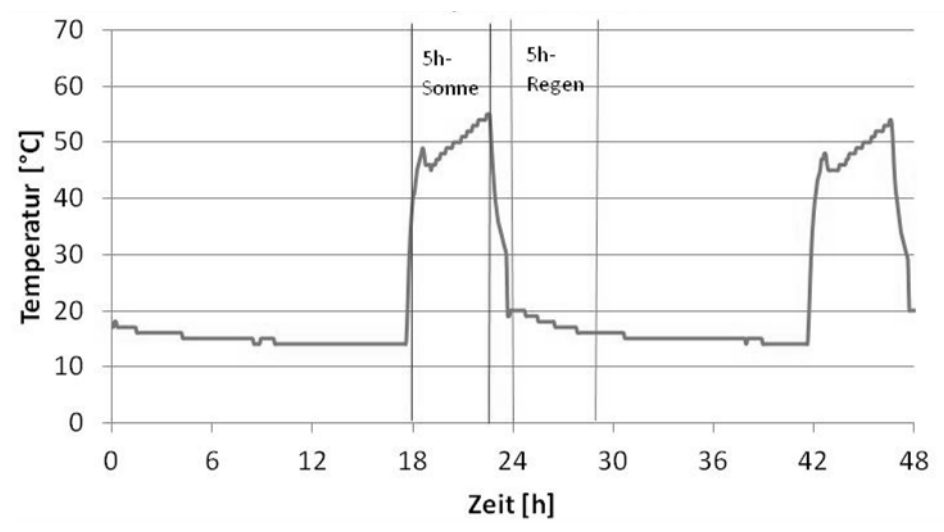

Abbildung 28: Temperaturverlauf der Buchen-LVL-Oberflächen in der Schnellbewitterungsanlage

Die Wasseraufnahme, die Quellung und das Rissverhalten sowie kalibrierte Farbscans wurden in wöchentlichem Turnus aufgenommen.

Die Rissevaluation orientierte sich an der DIN ISO 4628-4 (1997), deren Bestimmung eigentlich die Beurteilung von Beschichtungsschäden, und im Teil 4 insbesondere der Bewertung des Rissgrades, ist. Die Anwendung war jedoch auch für das Furnierschichtholz sinnvoll, da eine systematische Kategorisierung der Menge und der Breite der Risse der Oberfläche bzw. dem Deckfurnier zu erfolgen hatte, um eine Vergleichbarkeit der Serien zueinander zu ermöglichen.

Tabelle 8: Kennwerte zum Bewerten der Menge von Rissen nach DIN ISO 4628-4 (1997).

\begin{tabular}{|c|c|}
\hline Kennwert & Menge der Risse \\
\hline 0 & Keine, d.h. keine erkennbaren Risse \\
\hline 1 & Einzelne, d.h. einige sich gerade abzeichnende Risse \\
\hline 2 & Wenige, d.h. Risse von geringer, aber deutlich erkennbarer \\
Menge
\end{tabular}


Tabelle 9: Kennwerte zum Bewerten der Breite von Rissen nach DIN ISO 4628-4 (1997).

\begin{tabular}{|c|c|}
\hline Kennwert & Breite der Risse \\
\hline 0 & Keine sichtbaren Risse bei 10facher Vergrößerung \\
\hline 1 & Gerade sichtbare Risse bei bis zu 10facher Vergrößerung \\
\hline 2 & $\begin{array}{c}\text { Gerade sichtbare Risse mit bloßem Auge (auf Normalsichtigkeit korrigiertes } \\
\text { Sehvermögen) }\end{array}$ \\
\hline 3 & $\begin{array}{c}\text { Deutlich sichtbare Risse mit bloßem Auge (auf Normalsichtigkeit korrigiertes } \\
\text { Sehvermögen) }\end{array}$ \\
\hline 4 & Breite Risse, bis zu $1 \mathrm{~mm}$ breit \\
\hline 5 & Sehr breite Risse, mehr als $1 \mathrm{~mm}$ breit \\
\hline
\end{tabular}

\subsubsection{Freilandversuch}

Für PF-modifiziertes Buchen LVL wird die Widerstandskraft gegenüber Witterungseinflüssen auf einem Testfeld in Anlehnung an EN 927-3 (2006) untersucht. Diese Norm, die im, Grundsatz auf die Evaluierung von Beschichtungstoffen und Beschichtungssystemen für Holz im Außenbereich ausgelegt ist, wurde gewählt, da es in Europa keine gültige Norm zur Charakterisierung von unbeschichtetem $\mathrm{Holz}$ gegenüber Witterungseinflüssen gibt. Gleichzeitig bietet dieses Vorgehen die Möglichkeit Vergleiche zu den Arbeiten von WEPNER (2006) und MAI (2009) herzustellen. Zu diesem Zweck wurden je Versuchserie zwei Prüfkörper aus unterschiedlichen Platten (8-lagig, Furnierstärke 3,7mm) im Format $100 \mathrm{~mm}$ x $440 \mathrm{~mm}$ x Plattendicke gefertigt.

In einer ersten Phase des Versuchs wurde der Harztyp A mit den WPGs 15\%, 30\% und 60\% anhand von LVL mit und ohne Sperrlagen untersucht. Der Versuch startete im Dezember 2013. Zur gleichen Zeit wurde LVL mit zwei Sperrlagen und den Harztypen B, C, D und E, bei denen auf die Variante mit 15\% WPG verzichtet wurde, ausgebracht. Zu einem späteren Zeitpunkt erfolgte die Fertigstellung von Versuchsserien ohne Sperrlagen, die mit den Harztypen F und G mit WPGs von 15\%, 30\% und 60\% modifiziert waren. Deren Bewitterung im Freiland begann im Juli 2014. In der Auswertung werden die genauen Zeitpunkte der Evaluierung sowie die jeweiligen Versuchsdauern entsprechend berücksichtigt.

Alle oben genannten Varianten wurden ohne eine Kantenversiegelung und ohne eine Beschichtung oder weitere Bearbeitung verwendet.

Die Prüfkörperanordnung im Freiland war $45^{\circ}$ geneigt, auf Ständern, die Richtung Süden $\left(180^{\circ}\right)$ ausgerichtet waren (Abbildung 29). Ebenso wie bei der Schnellbewitterung, sollte anhand der Kriterien Menge und Breite von Rissen in der Oberfläche sowie der Wasseraufnahme und Dickenquellung das Verhalten der Versuchsserien beschrieben werden. $\mathrm{Zu}$ diesem Zweck wurden die Proben zu Beginn der Prüfung, nach einer Lagerung im Normalklima $\left(20^{\circ} \mathrm{C} / 65 \% \mathrm{rF}\right)$, gewogen, gescannt und vermessen, sowie die Rissbeurteilung durchgeführt. Die Rissbeurteilung und alle Messungen wurden im ersten Jahr alle drei Monate wiederholt. Dabei wurden die Proben ohne Wartezeit gewogen und vermessen, der Scan erfolgte jedoch erst im Anschluss, um eine trockene Oberfläche aufnehmen zu können. Die Ergebnisse wurden in Microsoft Excel 2016 ausgewertet und Mittelwerte in Balkendiagrammen und Liniendiagrammen dargestellt, da aufgrund der geringen Anzahl an Werten je Versuchsserie keine Boxdiagramme geeignet waren. 


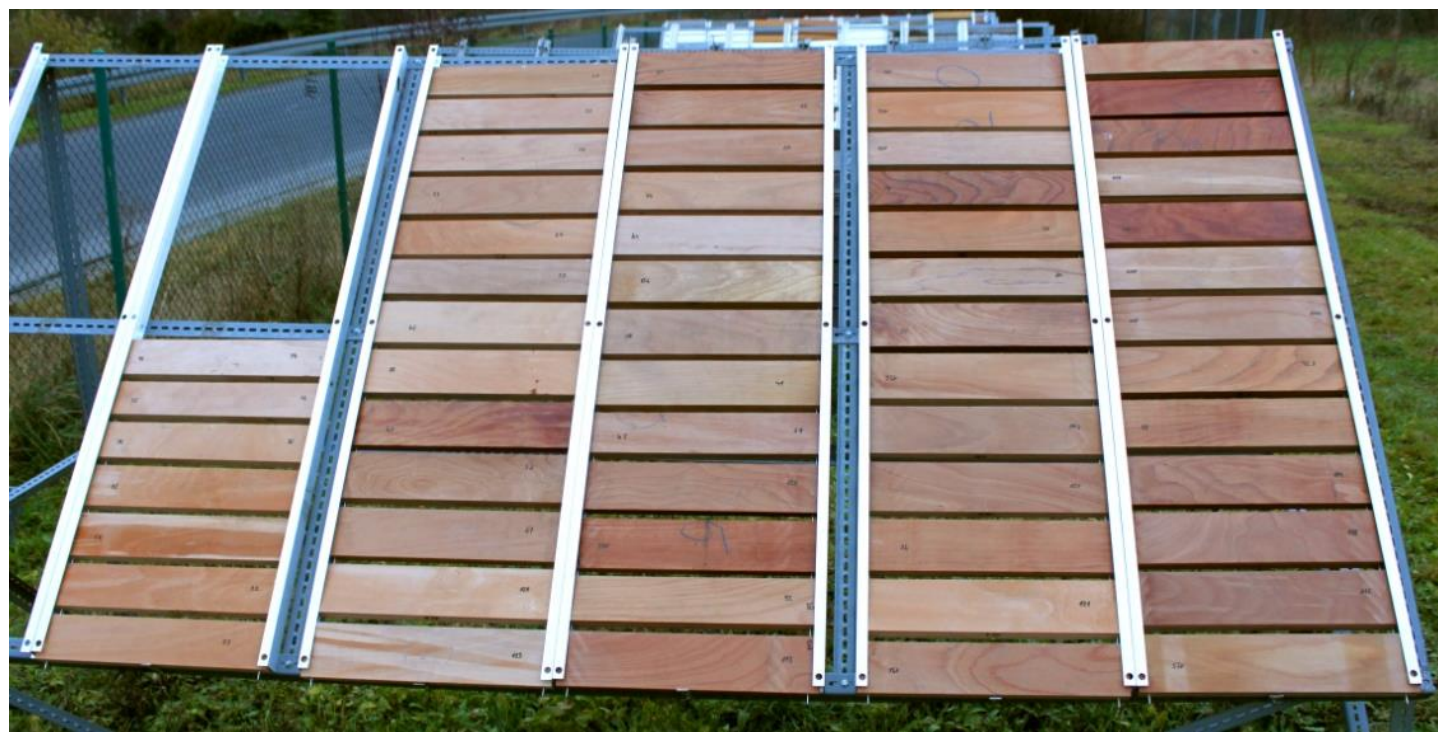

Abbildung 29: Versuchsaufbau Freilandbewitterung.

Für den Versuchszeitraum wurden die Klimadaten von der Abteilung Bioklimatologie der Universität Göttingen, welche $652 \mathrm{~m}$ entfernt in Richtung Ost-Süd-Ost (118) liegt, aufgezeichnet und zur Verfügung gestellt. Die Lufttemperatur und Luftfeuchte wurden in $2 \mathrm{~m}$ Höhe über dem Boden gemessen.

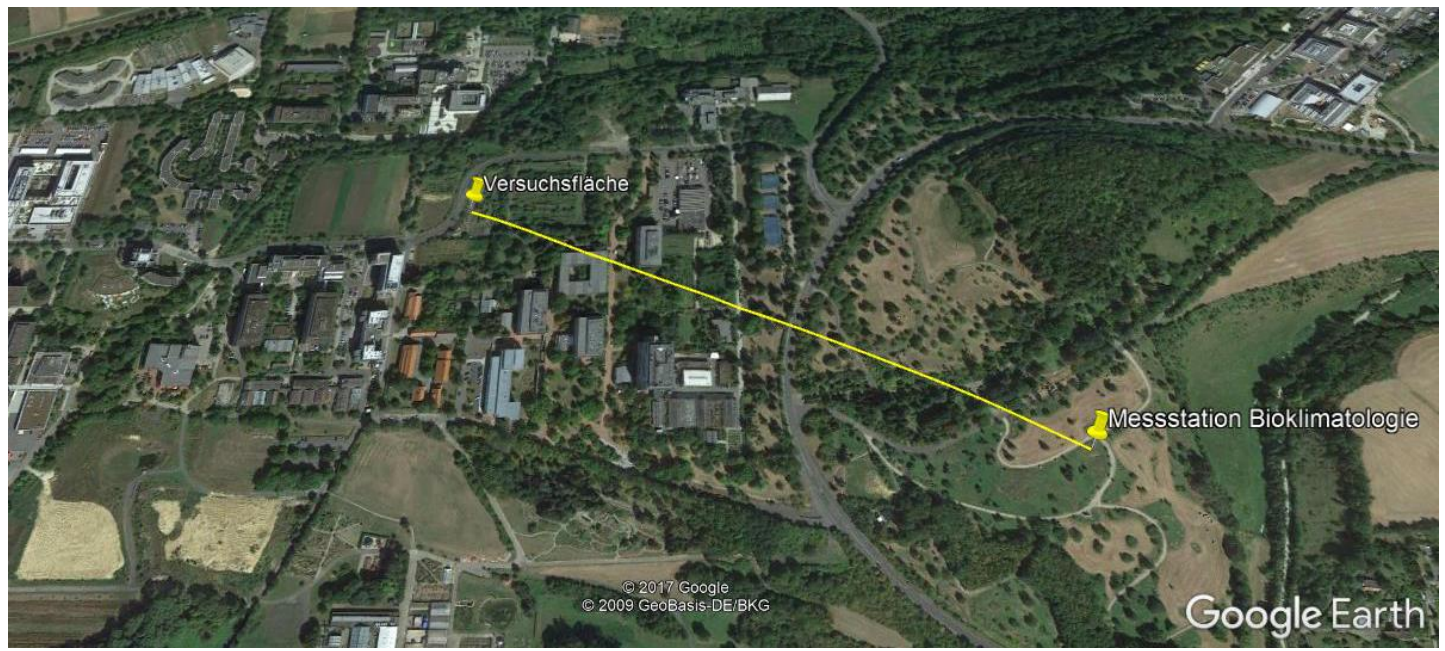

Abbildung 30: Satellitenbild mit Versuchsfläche der Abt. Holzbiologie und Holzprodukte und der meteorologischen Messstation der Abt. Bioklimatologie der Universität Göttingen. 


\subsubsection{Beschichtung}

Da die Modifizierung von Buchenfurnieren zur Herstellung von dauerhaftem LVL mit zusätzlichen Kosten verbunden ist, die proportional zur eingesetzten Menge an Imprägnierharz sind, wird die Beschichtung durch Lacke und Lasuren nur für Varianten mit niedrigen WPGs zwischen 5\% und 18\% untersucht und sich auf den Harztyp F konzentriert.

Als Beschichtung wurde ein zweistufiges System der Firma Remmers Baustofftechnik, Löningen, gewählt. Zuerst wurde die Grundierung IL-170 durch 60-sekündiges Tauchen aufgetragen. Nach erfolgter Zwischentrocknung (24 Std.) wurde der Wetterschutzlack UV+ mit einer Schichtdicke von $150 \mu \mathrm{m}$ durch Spritzen appliziert. Bei der Grundierung handelt es sich um eine lösemittelbasierte, nicht filmbildende Imprägnierung mit hydrophoben Eigenschaften. Bei dem Wetterschutzlack handelte es sich um eine wasserbasierte, transparente Acrylatdispersion mit UV-Absorbern.

Die Prüfkörper wurden dann in der Schnellbewitterung und im Freiland auf die gleiche Weise wie die unbeschichteten Varianten hinsichtlich Wasseraufnahme, Dickenquellung und Rissverhalten bewertet.

\subsubsection{Lab-Auswertung}

Um Farbveränderungen möglichst objektiv zu beschreiben und zu quantifizieren, wurden die Scans mit Hilfe eines kalibrierten Scanners im CIE-Lab-Modus durchgeführt. Nach 12 Monaten Versuchsdauer erfolgte eine Lab-Auswertung. Die Normfarbtafel von CIE geht darauf zurück, dass versucht wird, grundlegende Standards und Prozesse für metrische Erfassung von Licht und Beleuchtung aufzustellen. Dazu dienen die drei Messwerte L, a und b.

$L \quad$ lightness $=$ Helligkeit: wird dargestellt auf einer Skala von 0 (schwarz) bis 100 (weiß)

$a \quad$ Rot-Grün-Achse: $+\mathrm{a}=$ rot; $-\mathrm{a}=$ grün

$b \quad$ Gelb-Blau-Achse: $+\mathrm{b}=$ gelb; $-\mathrm{b}=$ blau

Da die Auswertung der Lab-Werte mit Adobe Photoshop durchgeführt wurde und die Ergebnisse im Wertebereich von 0 bis 255 ausgegeben wurden, erfolgten folgende rechnerische Korrekturen:

Für die Helligkeit L: Division durch 2,55 um Ergebnisse in einem Wertebereich von 0-100 zu erhalten.

Für die Farbachsen a und b: Subtraktion von 128.

Zusätzlich findet als errechnete Größe die Buntheit C (Chroma) Anwendung und stellt die Verschiedenheit einer Farbe zum gleichhellen Unbunt dar:

Formel 23: Berechnung der Buntheit $C$

$C=\sqrt{a^{2}+b^{2}}$

C Buntheit mit a: Rot-Grün-Achse und b: Gelb-Blau-Achse 
Die Änderung dieser Werte nach zwölf Monaten in der Freilandbewitterung wurde ausgewertet durch:

a) Darstellung von $\mathrm{L}$ und $\mathrm{C}$

b) Darstellung von $\Delta \mathrm{L}$ (Helligkeitsänderung) und $\Delta \mathrm{C}$ (Farbänderung)

Formel 24: Berechnung der Helligkeitsänderung $\Delta \mathrm{L}$

$\Delta L=L_{1}-L_{2}$

$\Delta \mathrm{L} \quad$ Helligkeitsänderung

Formel 25: Berechnung der Farbveränderung $\Delta \mathrm{C}$

$$
\Delta C=\sqrt{\left(a_{1}-a_{2}\right)^{2}+\left(b_{1}-b_{2}\right)^{2}}
$$

$\Delta \mathrm{C} \quad$ Farbänderung

c) Berechnung und Darstellung von $\Delta \mathrm{E}$ (Gesamtveränderung von Farbe und Helligkeit; beinhaltet alle Lab-Werte)

Formel 26: Berechnung der Gesamtveränderung von Farbe und Helligkeit $\Delta \mathrm{E}$

$\Delta E=\sqrt{\left(L_{1}-L_{2}\right)^{2}+\left(a_{1}-a_{2}\right)^{2}+\left(b_{1}-b_{2}\right)^{2}}$

$\Delta \mathrm{E} \quad$ Gesamtveränderung von Farbe und Helligkeit

\subsection{Statistik}

Die Auswertung und grafische Darstellung der Ergebnisse mit einem Stichprobenumfang ab acht Prüfkörpern pro Variante erfolgte mit OriginPro8.5 (2010) für Windows OriginLab Corporation (Northampton, MA, USA), in der Regel ist das bei physikalischen Prüfungen sowie der biologischen Dauerhaftigkeit der Fall. Für die Darstellung der Ergebnisse der jeweiligen Serien wurden Boxplots erstellt, wobei die Box den Wertebereich zwischen 5\% und 95\% der Werte darstellt. Zusätzliche Informationen liefern die in Abbildung 31 gezeigten Symbole.

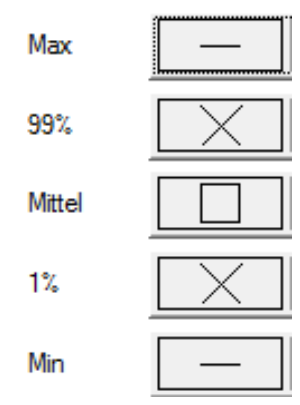

Abbildung 31: Statistische Angaben zum Wertebereich von Stichprobenergebnissen in Boxdiagrammen durch Symbole.

Wurden Versuche durchgeführt, die eine hohe Anzahl Varianten aufwiesen, jedoch nur geringe Wiederholungszahlen, dann wurden die Ergebnisse in Microsoft Excel 2016 ausgewertet und Mittelwerte in Balkendiagrammen, Punkt- und Liniendiagrammen dargestellt. Wenn nicht anderes angegeben sind Ergebnisse als Mittelwerte (arithmetisches Mittel) einer Stichprobe 
berechnet. In bestimmten Fällen ist der Median verwendet worden und entsprechend angegeben. Die Streuung der Werte erfolgt durch Angaben der Standardabweichung (Stabw.) und des Variationskoeffizenten (Varkoeff.). Die Untersuchung, ob die der Stichprobe zugrundeliegende Grundgesamtheit normalverteilt ist, erfolgte mit dem Shapiro-Wilk-Test für $\mathrm{n}<50$. Um signifikante Unterschiede zwischen Mittelwerten zu prüfen, wurden t-Tests durchgeführt. Lineare Korrelationen wurden nach der Methode der kleinsten Abweichungsquadrate berechnet (Chi-Quadrat-Minimierung). Als Maß für den Zusammenhang zwischen abhängiger und unabhängiger Variable wird der R-Wert (Pearson's $\mathrm{R})$ angegeben und die Güte einer Regression wird durch die Angabe des Bestimmtheitsmaßes $\mathrm{R}^{2}$ dargestellt. 


\subsection{Ergebnisse}

\subsubsection{Lösungsaufnahme}

Da angenommen wurde, dass die Dichte einen Einfluss auf die spätere Lösungsaufnahme haben würde, wurden die Furniere nach ihrer Dichte sortiert und gleichmäßig in die Gruppen bzw. Klimata aufgeteilt.

Tabelle 10: Darrdichten der Furniere, aufgeteilt nach den Klimata.

\begin{tabular}{lccccc} 
Klima & Min. $\left[\mathrm{kg} / \mathrm{m}^{3}\right]$ & Mittelwert $\left[\mathrm{kg} / \mathrm{m}^{3}\right]$ & Max. $\left[\mathrm{kg} / \mathrm{m}^{3}\right]$ & Stabw. $\left[\mathrm{kg} / \mathrm{m}^{3}\right]$ & Varkoeff. [\%] \\
\hline atro & 595 & 638 & 721 & 29 & 4.6 \\
$20 / 65$ & 600 & 643 & 728 & 26 & 4.0 \\
$20 / 95$ & 609 & 648 & 734 & 27 & 4.2
\end{tabular}

Tabelle 10 zeigt, dass die Verteilung der Darrdichten der verwendeten Furniere gleichmäßig war. Die Imprägnierung von 24 darrtrockenen Furnieren brachte die in Abbildung 32 dargestellten Lösungsaufnahmen hervor.

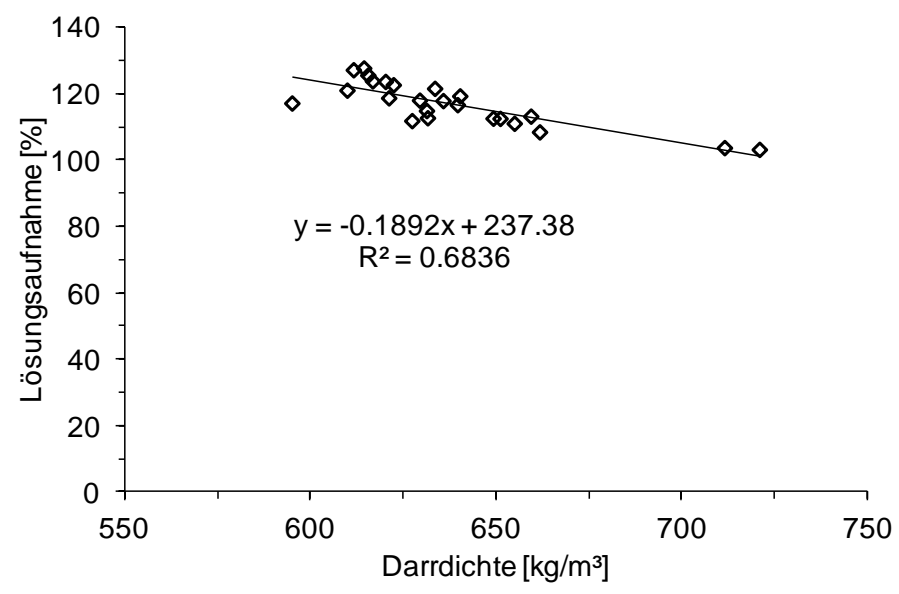

Abbildung 32: Lösungsaufnahme und Darrdichte von absolut trockenen Buchenfurnieren.

Es wurde ersichtlich, dass eine Zunahme der Dichte eine Abnahme der Lösungsaufnahme bedingt. Die Lösungsaufnahmen der darrtrockenen Furniere lagen zwischen 103\% und 128\% - im Mittel bei 117\% bei einer Dichte von $638 \mathrm{~kg} / \mathrm{m}^{3}$. Die Abnahme der Lösungsaufnahme in Abhängigkeit von der Dichte erfolgte hier jedoch unterproportional - das Verhältnis von Lösungsaufnahmeveränderung zu Dichteänderung lag bei 0,91. Dieser Einfluss der Dichte auf die Lösungsaufnahme musste für alle Versuchsserien bzw. Klimata gleich sein, da die Dichten ähnlich waren. Im Vergleich des auf empirisch ermittelten Werten basierenden Modells zu dem von Kollmann (1951) publizierten Modell zur maximalen Holzfeuchte, stellt sich eine gute Übereinstimmung heraus. 
Formel 27: Maximal möglicher Wassergehalt $\mathbf{u}_{\max }$ von Holz in Abhängigkeit vom Darrgewicht (Kollmann 1951).

$u_{\max }=0,28+\frac{1,50-r_{\text {atro }}}{1,50 * r_{\text {atro }}}$

$u_{\max } \quad$ Maximal möglicher Wassergehalt

ratro Darrdichte $\left[\mathrm{kg} / \mathrm{m}^{3}\right]$

Beispiel: $r_{\text {atro }}=0,65 \mathrm{~g} / \mathrm{cm}^{3}$

Kollmann: $\mathrm{u}_{\max }=1,15 \quad$ Eigene Regression aus Abbildung 32: $\mathrm{u}_{\max }=1,14$

Somit ließ sich bei absoluttrockenen Furnieren die Aufnahme wässriger Lösungen in Abhängigkeit von der Darrdichte der Furniere voraussagen.

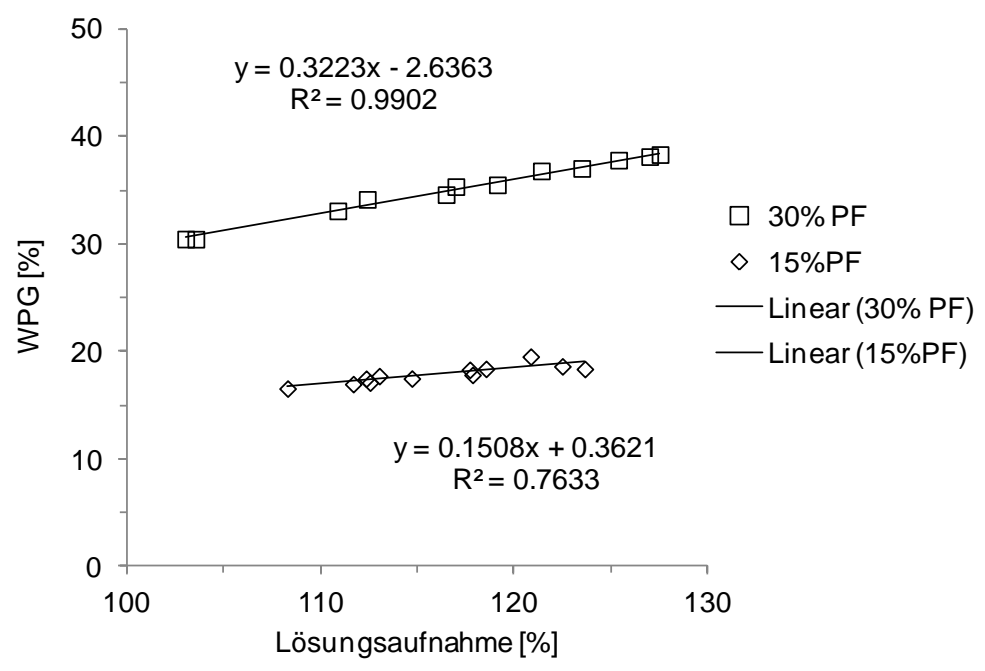

Abbildung 33: WPG in Abhängigkeit von Konzentration und Lösungsaufnahme, ermittelt an darrtrockenen Furnieren $(\mathrm{HF}=0 \%)$.

Abbildung 33 bestätigt, dass der WPG und die Lösungsaufnahme bei den PF-Konzentrationen $15 \%$ und $30 \%$ positiv korreliert waren.

Für die Überprüfung der Hypothese, dass die maximale Lösungsaufnahme bei gleicher Dichte durch die Holzfeuchte vorgegeben ist, wurden die Konzentrationen der Lösungen entsprechend der Ziel-WPG eingestellt und dann damit die unterschiedlich feuchten Furniere imprägniert. 


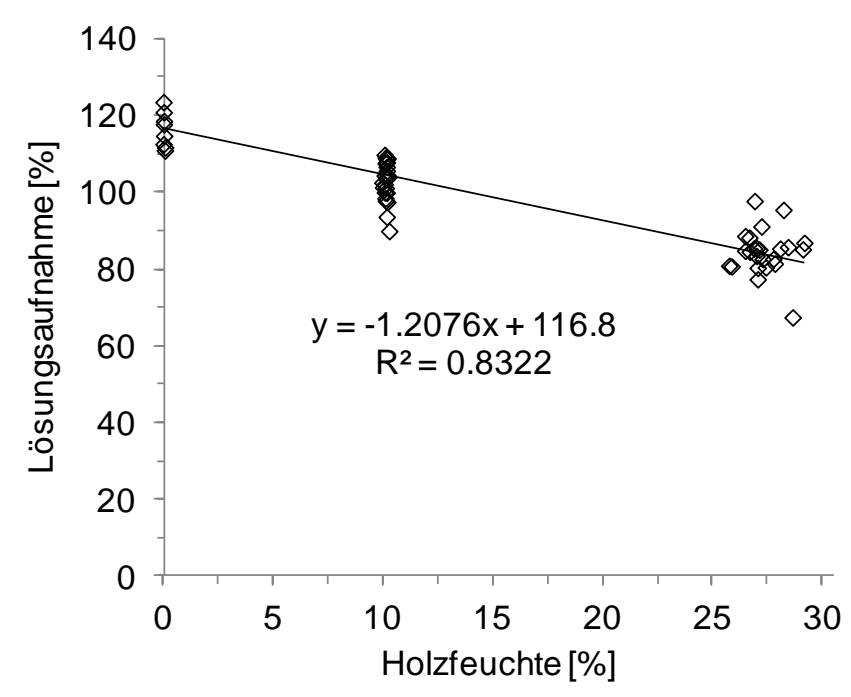

Abbildung 34: Darstellung der Lösungsaufnahmen in Abhängigkeit von der Holzfeuchte

Abbildung 34 zeigt die erzielten Lösungsaufnahmen aller Serien. Es wird ersichtlich, dass die erzielten Holzfeuchten im Normalklima sehr nahe beinander lagen, während bei $95 \% \mathrm{rF}$ eine deutliche Streuung erkennbar war. Dennoch wies das lineare Regressionsmodell mit einem Bestimmtheitsmaß $\left(\mathrm{r}^{2}\right)$ von 0,83 auf eine ausgeprägte negative Korrelation der Lösungsaufnahme und der Holzfeuchte. Dabei sank die Lösungsaufnahme mit zunehmender Holzfeuchte überproportional. Erhöhte sich die Holzfeuchte um 10\%, dann sank die Lösungsaufnahme um 12\%. Dafür sind insbesondere die Quellung der Zellwand und die damit verbundene Reduzierung des verfügbaren Raumes für aufzunehmende Flüssigkeiten verantwortlich. Ein zahlenmäßiger Aufschlag zur Darrdichte im Umfang der zur Holzfeuchte korrespondierenden Masse Wasser wäre somit fehlerbehaftet bei der Berechnung der Lösungsaufnahme.

Wird jedoch aus der linearen Regression von Darrdichte und Lösungsaufnahme und der Regression von Holzfeuchte und Lösungsaufnahme eine gemeinsame Funktion erstellt, die beide Einflussfaktoren berücksichtigt, ergibt sich Formel 28 bzw. Formel 29.

Formel 28: Herleitung der Funktion zur Berechnung der Lösungsaufnahme in Abhängigkeit von Darrdichte und Holzfeuchte

$$
L A=2,374+k_{r} * 1 \frac{m^{3}}{k g} * r+k_{H F} * H F * 100 \%
$$

LA Lösungsaufnahme [\%]

$r \quad$ Darrdichte $\left[\mathrm{kg} / \mathrm{m}^{3}\right]$

HF Holzfeuchte [\%] 
Mit den Steigungen $k_{r}=-0,00189$ und $k_{H F}=-0,01208$ aus Abbildung 32und Abbildung 34 ergab sich:

Formel 29: Funktion zur Berechnung der Lösungsaufnahme in Abhängigkeit von Darrdichte und Holzfeuchte

$$
L A=2,374-0,00189 * 1 \frac{m^{3}}{k g} * r-0,01208 * H F * 100 \%
$$

Unter Anwendung der hergeleiteten Formel konnte nachfolgendes Diagramm erstellt werden, aus dem sich die Lösungsaufnahme in Abhängigkeit von der Darrdichte und der Holzfeuchte ableiten lassen.

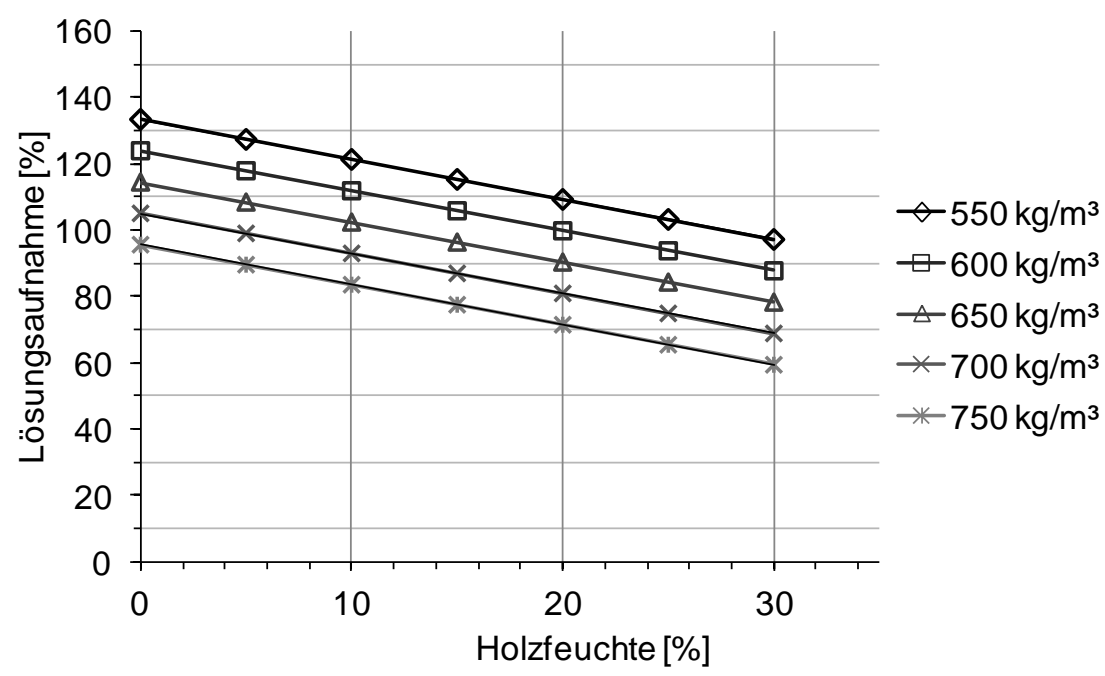

Abbildung 35: Theoretische Lösungsaufnahmen bei Buchenfurnieren in Abhängigkeit von der Holzfeuchte und der Darrdichte, berechnet nach Formel 17.

\subsubsection{Physikalische Prüfungen}

\subsubsection{Dichte}

Die Dichte des LVL, gemessen als Darrdichte, hängt sehr stark von den Parametern bei der Herstellung der Platten ab. Die Abbildung 36 zeigt, dass 10-lagiges Buchen-LVL aus unbehandelten Furnieren bei einem Pressdruck von $6 \mathrm{~N} / \mathrm{mm}^{2}(6 / 0)$ eine Darrdichte von $679 \mathrm{~kg} / \mathrm{m}^{3}$ aufwies. Infolge der Imprägnierung stieg die Darrdichte an. Bei einem WPG von $30 \%$ und einem Pressdruck von 1,5 N/ $\mathrm{mm}^{2}$ wurde eine Darrdichte ermittelt, die $10 \%$ und beim Pressdruck von $3 \mathrm{~N} / \mathrm{mm}^{2}$ eine die $28 \%$ über den Referenzen lag, obwohl diese mit einem höheren Pressdruck hergestellt wurden. Mit einem WPG von 60\% stieg die Darrdichte weiter an und erreichte beim höchsten Pressdruck eine Darrdichte von $1251 \mathrm{~kg} / \mathrm{m}^{3}$, was dem 1,8fachen Wert der Darrdichte der Referenzen entsprach. Der Einfluss des Pressdrucks auf die Darrdichte von modifizierten Buchen-LVL wird insbesondere durch die Serie 6/23, welche durch Tauchen ein WPG von 23\% erzielte, deutlich. Diese Serie wies die gleiche Darrdichte auf wie die Serie 3/60, welche einen WPG von $60 \%$ aufwies. 


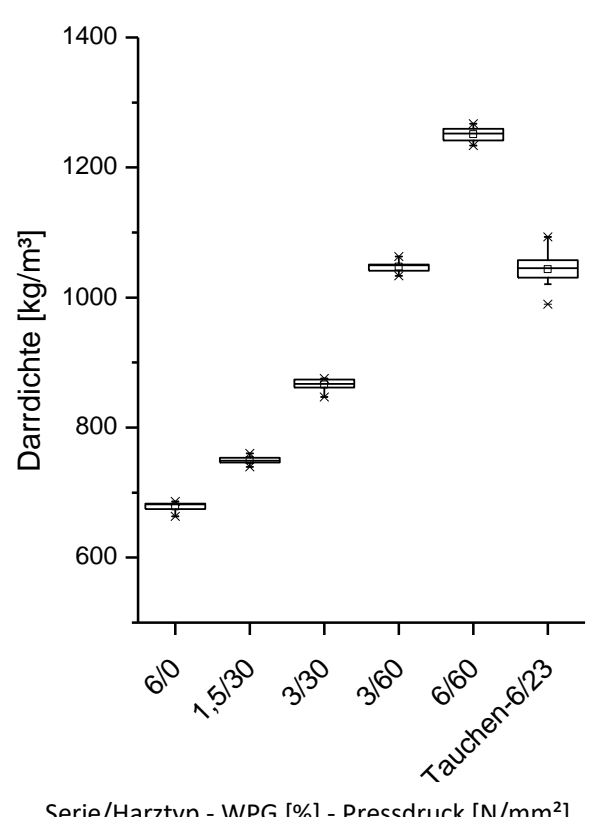

Abbildung 36: Darrdichte des 10-lagigen Buchen-LVL-Prüfkörper ohne Sperrlagen $(10 * 2,13 \mathrm{~mm})$, modifiziert mit dem Harztyp A. Pressdrücke: 1,5 N/mm , 3 N/mm $\mathrm{mm}^{2}$ und 6 N/mm $\mathrm{mm}^{2}$. WPG: 0\%, 23\%, 30\%, 60\%.

Nachfolgend werden die Darrdichten von Buchen-LVL mit und ohne Sperrlagen aus Furnieren mit einer Dicke von 3,7 mm dargestellt. Hierbei wird systematisch auf verschiedene Pressdrücke, WPG und Harztypen eingegangen.

Wie Abbildung 37 für LVL ohne Sperrlagen zeigt, lagen die Dichten aller Serien bei dem höchsten Druck $\left(6 \mathrm{~N} / \mathrm{mm}^{2}\right)$ deutlich höher als beim niedrigsten Druck $\left(2 \mathrm{~N} / \mathrm{mm}^{2}\right)$. Die modifizierten Serien zeigten auch beim mittleren Druck $\left(4 \mathrm{~N} / \mathrm{mm}^{2}\right)$ eine deutliche Dichtezunahme. Der Pressdruck hatte demnach einen großen Einfluss auf die Darrdichte. Bei den unbehandelten Referenzen war im Verhältnis keine Dichtezunahme beim mittleren Druck festzustellen. Im Vergleich der Versuchsserien beim jeweiligen Druck zeigte sich jedoch auch, dass Serien mit unterschiedlichen WPGs auch signifikant unterschiedliche Darrdichten aufwiesen. Der Harztyp hingegen hatte keinen erkennbaren Einfluss. 


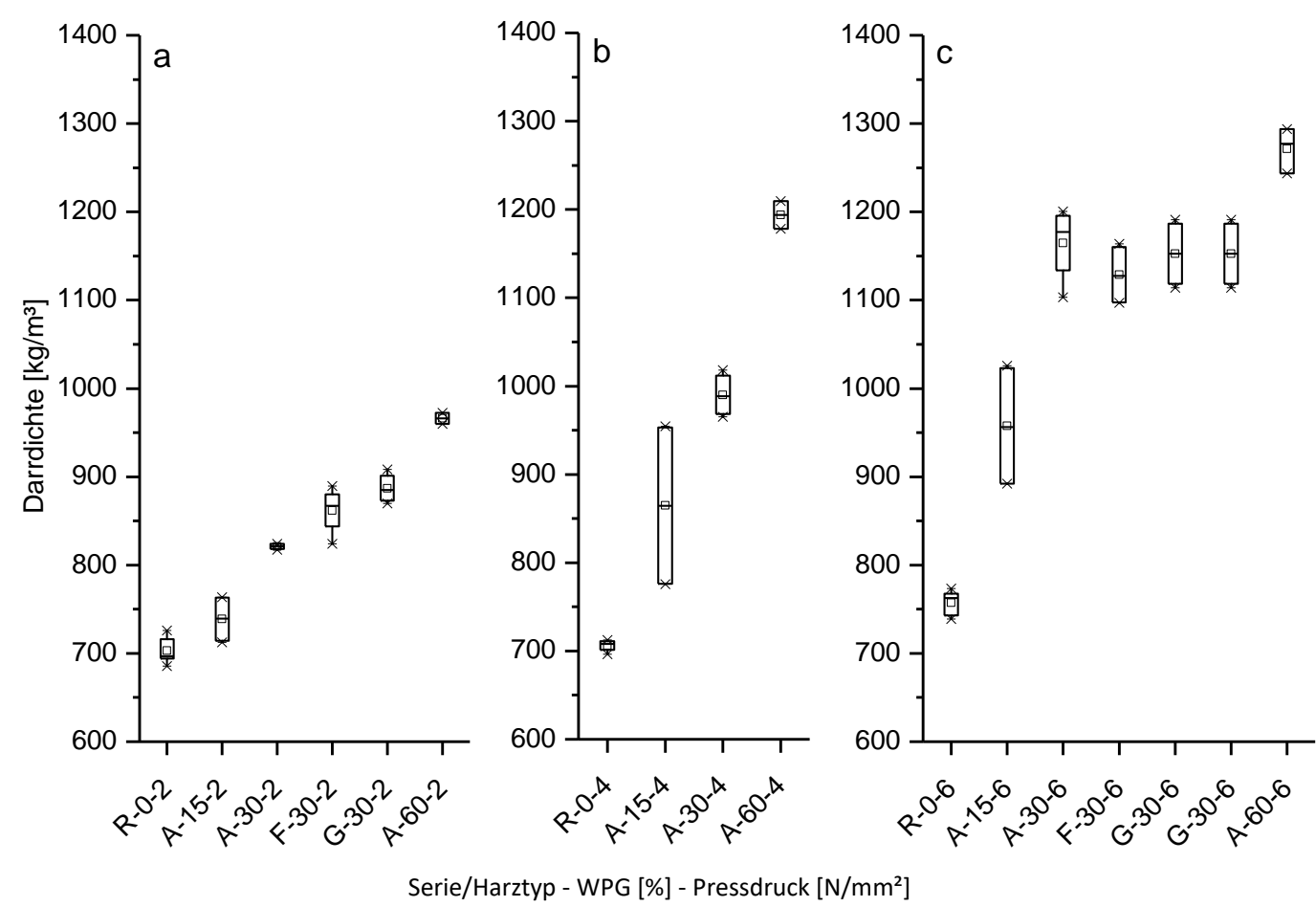

Abbildung 37: Darrdichte von Buchen-LVL ohne Sperrlagen $\left(8^{*} 3,7 \mathrm{~mm}\right.$ parallel) bei Verwendung der Imprägnierharze A, F und G, mit WPGs von $15 \%, 30 \%$ und $60 \%$, bei Pressdrücken von $2 \mathrm{~N} / \mathrm{mm}^{2}$ (a), $4 \mathrm{~N} / \mathrm{mm}^{2}$ (b) und $6 \mathrm{~N} / \mathrm{mm}^{2}$ (c) $\left(\mathrm{t}=45 \mathrm{Min}\right.$., $\left.\mathrm{T}=140^{\circ} \mathrm{C}\right)$.

Die oben beschriebenen Zusammenhänge konnten auch beim 8-lagigen LVL mit zwei Sperrlagen gefunden werden(Abbildung 39). Bei Anwendung des höchsten Pressdruckes von $6 \mathrm{~N} / \mathrm{mm}^{2}$ lagen alle Darrdichten erwartungsgemäß höher als beim niedrigeren Pressdruck. Diese Steigerung war bei den behandelten Serien wieder größer als bei der unbehandelten Referenz. Bei beiden Pressdrücken unterschieden sich die Dichten in Abhängigkeit des WPG. Die Unterschiede zwischen den Harztypen waren jedoch gering. 


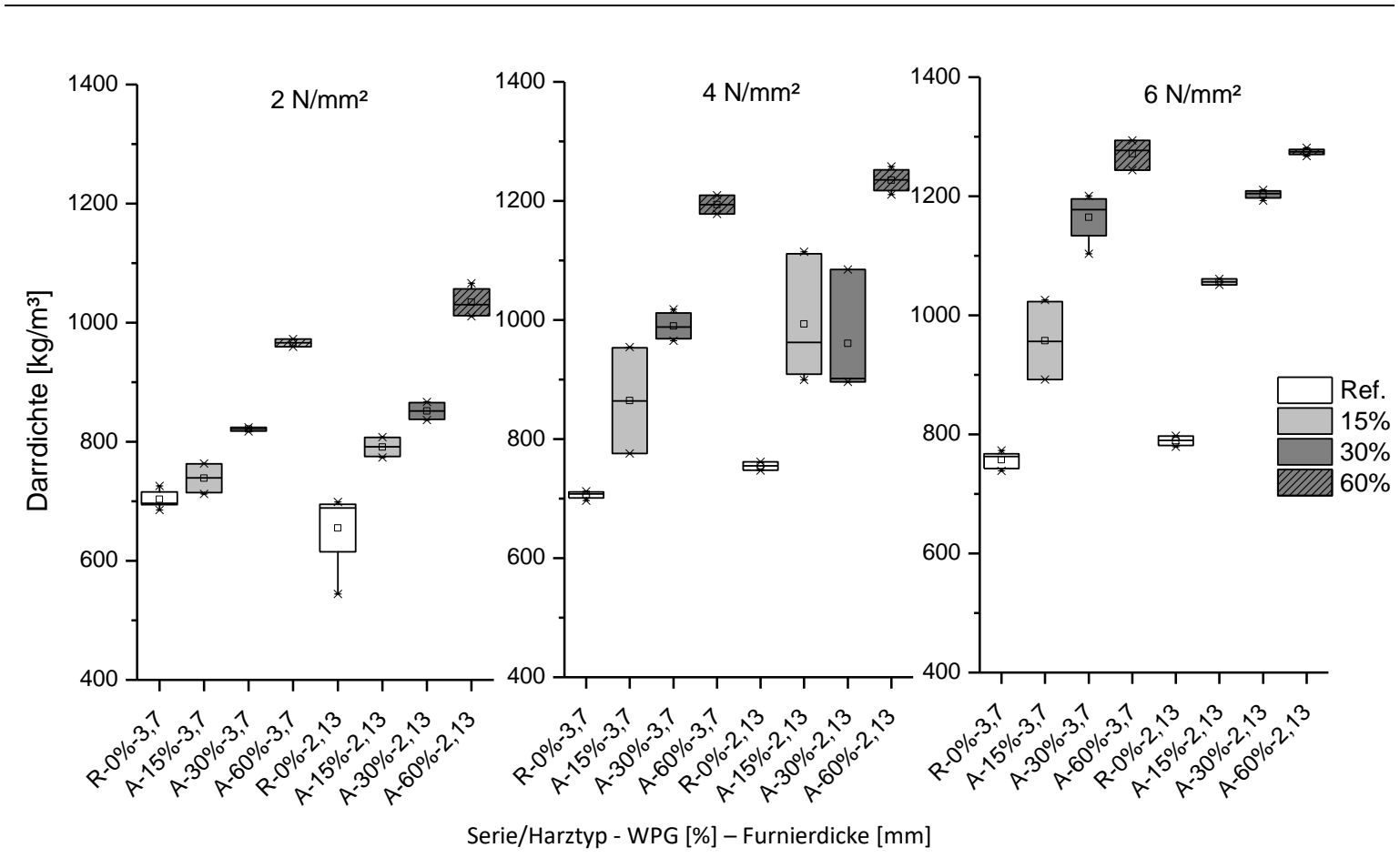

Abbildung 38: Darrdichten des Buchen-LVL ohne Sperrlagen mit 3,7 mm (8-lagig) und 2,13 mm (15-lagig) dicken Furnieren mit WPGs von 15\%, 30\% und 60\% des Harztyps A und den Pressdrücken 2 N/mm², $4 \mathrm{~N} / \mathrm{mm}^{2}$ und $6 \mathrm{~N} / \mathrm{mm}^{2}$.
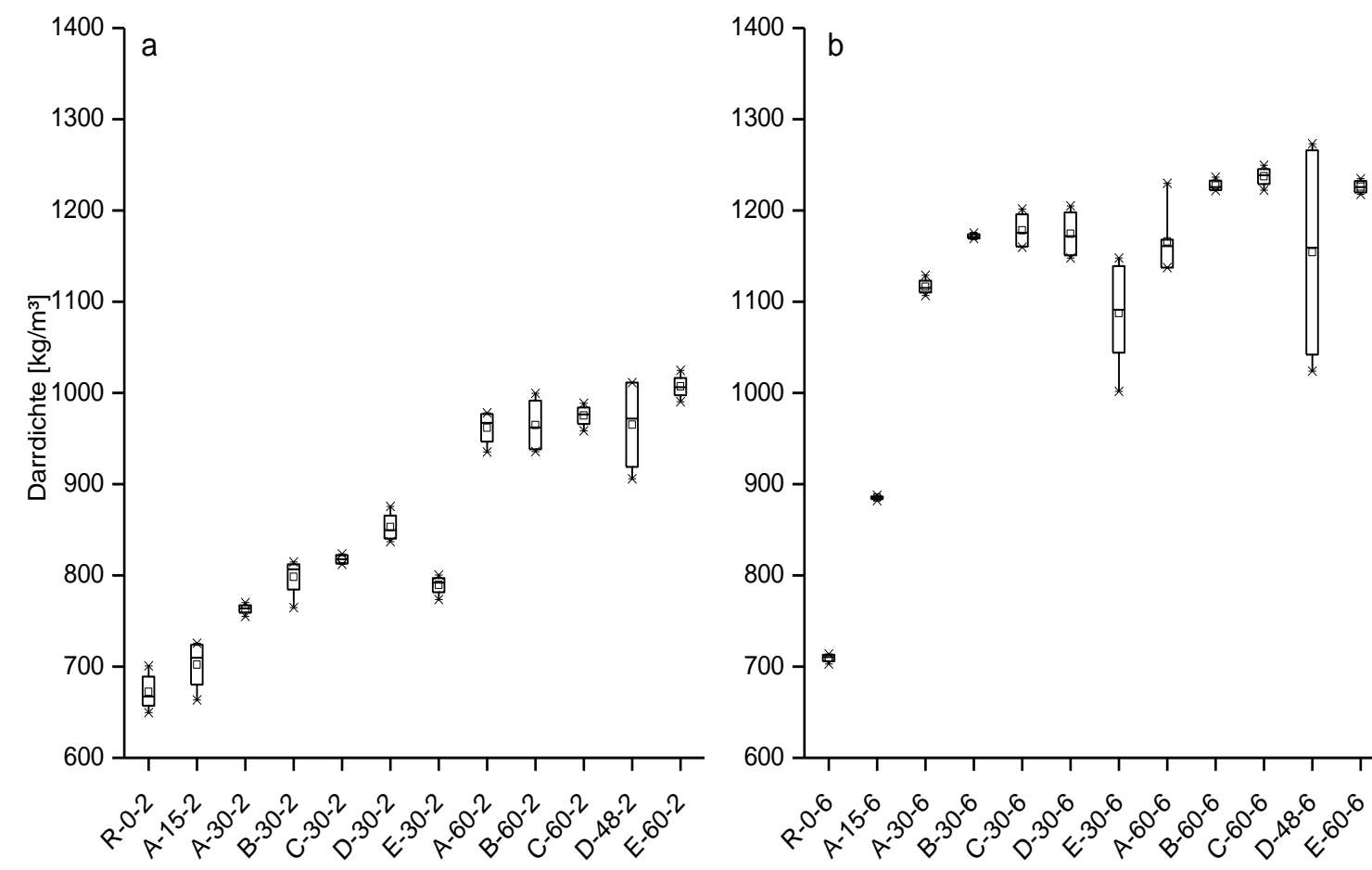

Serie/Harztyp - WPG [\%] - Pressdruck [N/mm²]

Abbildung 39: Darrdichte von Buchen-LVL mit 2 Sperrlagen $(8 * 3,7 \mathrm{~mm})$ bei Verwendung der Imprägnierharze A, B. C, D und E, bei WPGs von 15\%, 30\% und 60\%, bei Pressdrücken von $2 \mathrm{~N} / \mathrm{mm}^{2}$ (a) und $6 \mathrm{~N} / \mathrm{mm}^{2}$ (b). 
Durch Verwendung aller Ergebnisse in einer Matrix und Interpolation konnte ein Oberflächendiagramm erstellt werden, welches die Darrdichte in Graustufen in Abhängigkeit von Pressdruck und WPG zeigt (Abbildung 40).

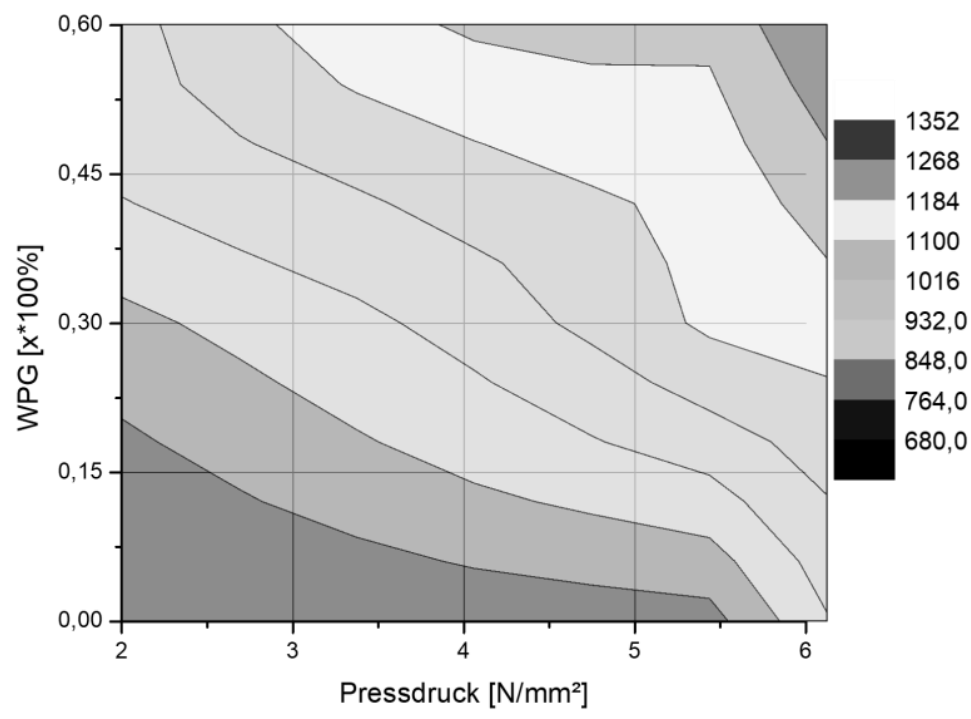

Abbildung 40: Oberflächendiagramm zur Darstellung der erzielten Darrdichte $\left[\mathrm{kg} / \mathrm{m}^{3}\right]$ bei unterschiedlichen Kombinationen aus Pressdruck und WPG. Ausgangsdaten: 4 mal 4 Wertepaare (WPG/Druck) mit Mittelwerten für die Darrdichte über alle Harze; In Matrix umgewandelt und interpoliert auf 9*11 Gitterpunkte nach dem Renka-Cline Verfahren.

Um den Einfluss des Pressdruckes auf die Darrdichte besser zu verdeutlichen, wurde mit Hilfe der Plattendicken nach dem Pressvorgang die Verdichtung berechnet. Am Beispiel der Platten des Harztyps A soll gezeigt werden, dass die modifizierten Platten bei gleichem Pressdruck zum einen deutlich stärker verdichtet wurden als die unbehandelte Referenz, zum anderen diese Verdichtung für beide WPGs gleich stark ausgeprägt war. Der absolut höhere Wert der Darrdichte bei 30\% WPG war somit auf die in das Holz verbrachte höhere Feststoffmenge im Vergleich zum WPG von 15\% zurückzuführen. 


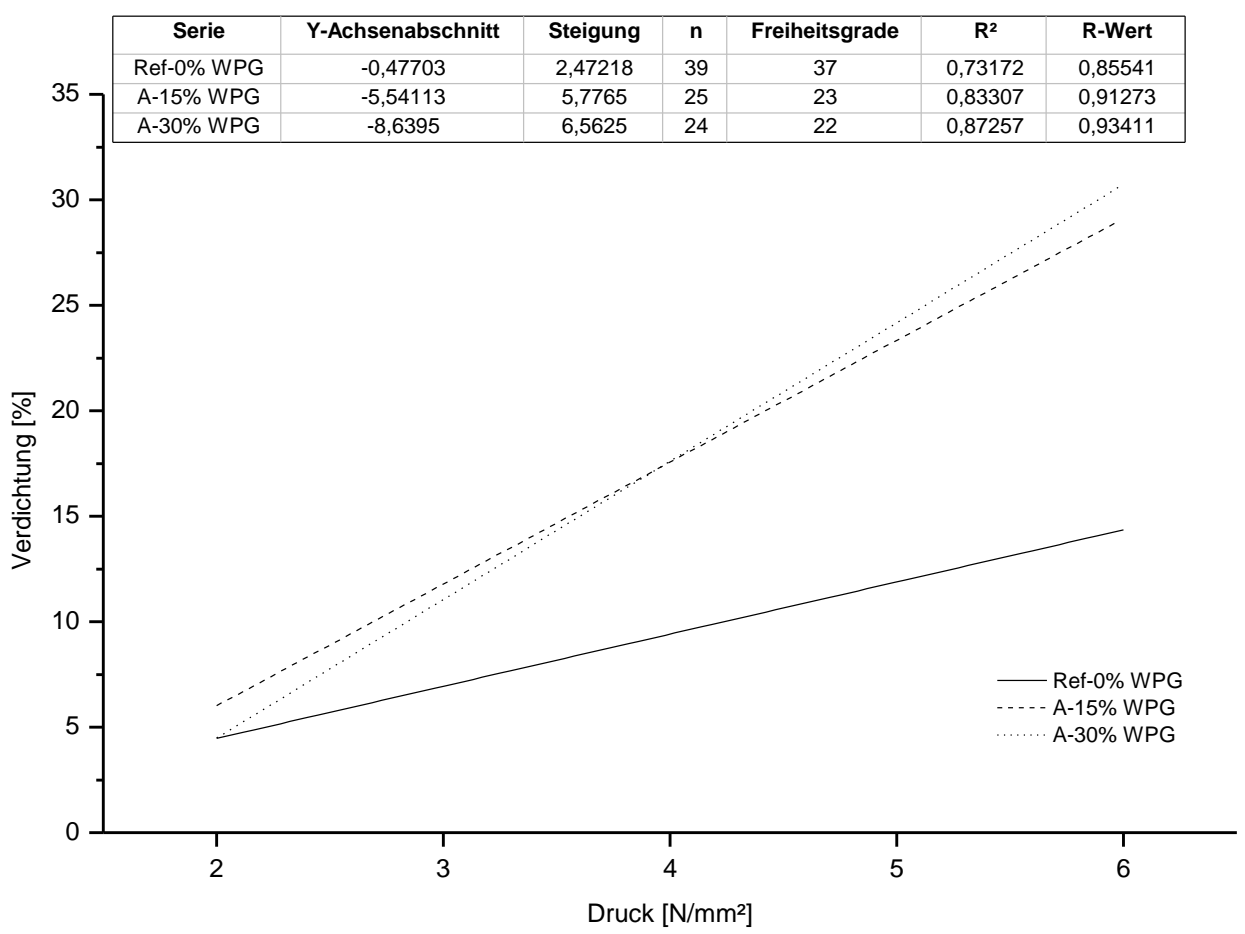

Abbildung 41: 8-lagiges Buchen-LVL ohne Sperrlagen. Untersuchung der Korrelation zwischen spezifischem Pressdruck und Verdichtung für unterschiedliche WPGs des Harztyps A durch lineare Regressionsmodelle.

Die Abbildung 41 stellt den Zusammenhang zwischen Pressdruck und Verdichtung beim 8lagigem Buchen LVL mit verschiedenen WPGs dar. Es wird ersichtlich, dass sich modifizierte Furniere leichter verdichten ließen als unbehandelte Furniere.

Unter Berücksichtigung aller Harze und Pressdrücke und WPGs ließ sich ein Oberflächendiagramm für die resultierende Verdichtung erstellen (Abbildung 40). 


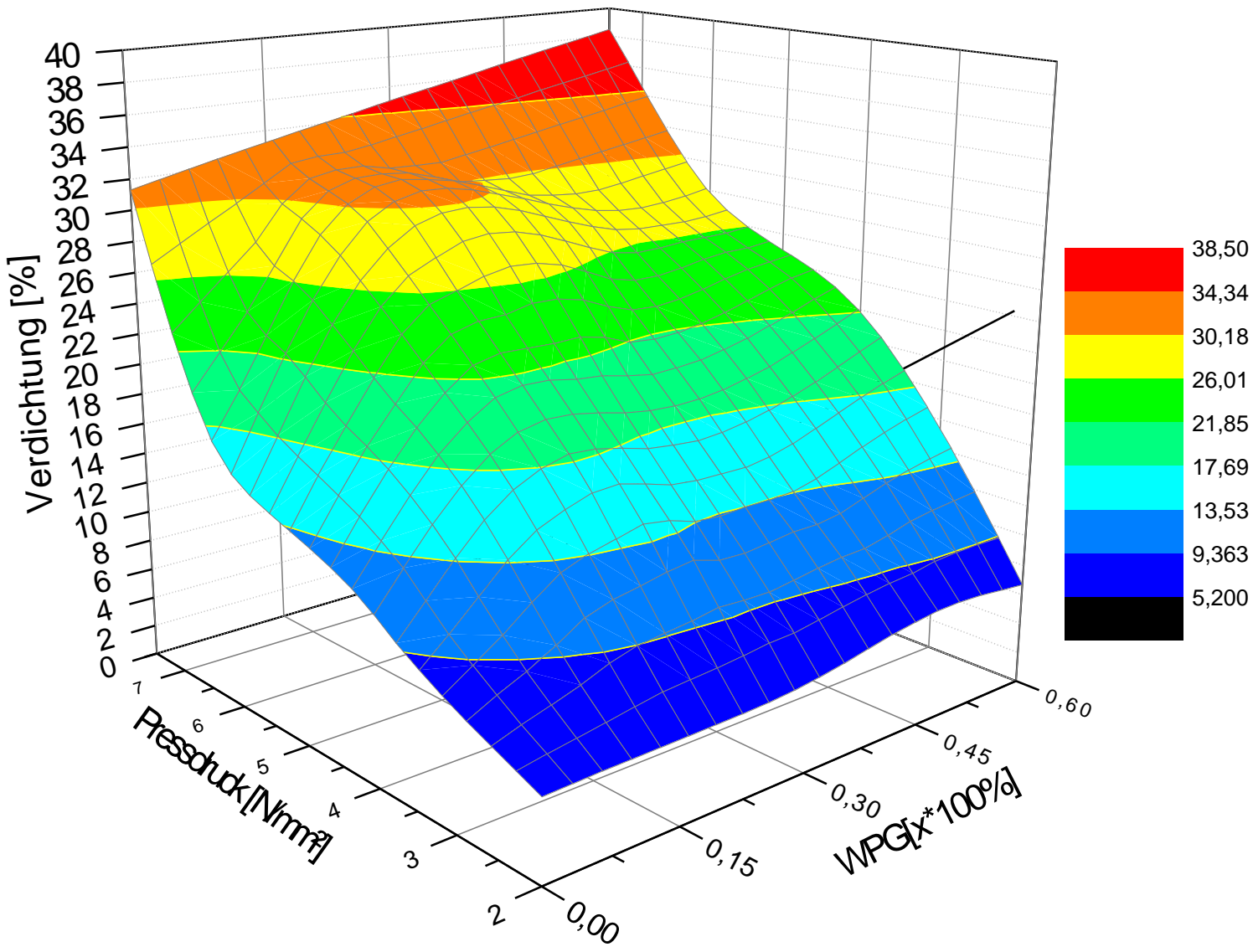

Abbildung 42: Verdichtung von Buchen-LVL in Abhängigkeit von Pressdruck und WPG.

Das Oberflächendiagramm für die Verdichtung in Abhängigkeit von Pressdruck und WPG zeigt, dass ein minimaler WPG von 15\% nötig ist um hohe Verdichtungen zu erzielen. Es zeigt auch, dass höhere WPGs als 30\% nicht immer eine höhere Verdichtung erzielen ließen. 
Untersuchungen an Furnierschichtholz (LVL)

Tabelle 11: Darrdichte und Verdichtung von 8-lagigem Buchen-LVL aus 3,7 mm dicken Furnieren mit zwei Sperrlagen.

\begin{tabular}{|c|c|c|c|c|c|c|c|}
\hline Furnierrichtung & WPG & Harztyp & $\begin{array}{c}\text { Druck } \\
{\left[\mathrm{N} / \mathrm{mm}^{2}\right]}\end{array}$ & $\begin{array}{c}\text { Verdichtung } \\
{[\%]}\end{array}$ & $\begin{array}{c}\text { Stabw. } \\
\text { Verdichtung } \\
{[\%]}\end{array}$ & $\begin{array}{c}\text { Darrdichte } \\
{\left[\mathrm{kg} / \mathrm{m}^{3}\right]}\end{array}$ & $\begin{array}{c}\text { Stabw. } \\
\text { Darrdichte } \\
{\left[\mathrm{kg} / \mathrm{m}^{3}\right]}\end{array}$ \\
\hline \multirow[t]{28}{*}{ mit 2Sperrlagen } & $0 \%$ & $\mathrm{R}$ & 2 & 5.2 & 1.2 & 756 & 119.5 \\
\hline & & & 4 & 9.4 & 1.1 & 652 & 6.6 \\
\hline & & & 6 & 19.9 & 0.5 & 709 & 4.2 \\
\hline & $15 \%$ & $A$ & 2 & 6.4 & 3.3 & 702 & 24.9 \\
\hline & & & 4 & 17.5 & 2.1 & 769 & 16.9 \\
\hline & & & 6 & 30.4 & 1.1 & 885 & 2.1 \\
\hline & $30 \%$ & $A$ & 2 & 7.0 & 0.5 & 763 & 5.3 \\
\hline & & & 4 & 21.5 & 0.5 & 924 & 8.6 \\
\hline & & & 6 & 31.9 & 0.6 & 1117 & 8.1 \\
\hline & & B & 2 & 9.3 & 2.3 & 798 & 19.7 \\
\hline & & & 6 & 34.8 & 1.2 & 1172 & 2.4 \\
\hline & & C & 2 & 8.7 & 1.3 & 818 & 4.8 \\
\hline & & & 6 & 33.1 & 0.2 & 1178 & 18.2 \\
\hline & & $\mathrm{D}$ & 2 & 7.0 & 2.7 & 853 & 14.7 \\
\hline & & & 6 & 28.6 & 0.5 & 1174 & 24.2 \\
\hline & & $E$ & 2 & 4.3 & 0.2 & 789 & 9.9 \\
\hline & & & 6 & 31.6 & 3.0 & 1087 & 53.5 \\
\hline & $48 \%$ & $D$ & 2 & 9.3 & 1.0 & 965 & 47.1 \\
\hline & & & 6 & 29.5 & 0.1 & 1154 & 112.8 \\
\hline & $60 \%$ & $A$ & 2 & 8.7 & 0.6 & 962 & 17.2 \\
\hline & & & 4 & 22.7 & 0.3 & 1157 & 13.3 \\
\hline & & & 6 & 28.1 & 1.3 & 1166 & 31.1 \\
\hline & & $B$ & 2 & 7.8 & 1.6 & 965 & 27.4 \\
\hline & & & 6 & 30.3 & 0.6 & 1227 & 5.9 \\
\hline & & C & 2 & 6.1 & 0.1 & 975 & 10.9 \\
\hline & & & 6 & 27.6 & 0.8 & 1237 & 9.9 \\
\hline & & $E$ & 2 & 5.6 & 2.1 & 1007 & 12.2 \\
\hline & & & 6 & 24.1 & 0.7 & 1226 & 6.7 \\
\hline
\end{tabular}


Tabelle 12: Darrdichte und Verdichtung von 8-lagigem Buchen-LVL aus 3,7 mm dicken Furnieren ohne Sperrlagen.

\begin{tabular}{|c|c|c|c|c|c|c|c|}
\hline Furnierrichtung & WPG & Harztyp & $\begin{array}{c}\text { Druck } \\
{\left[\mathrm{N} / \mathrm{mm}^{2}\right]}\end{array}$ & $\begin{array}{c}\text { Verdichtung } \\
{[\%]}\end{array}$ & $\begin{array}{c}\text { Stabw. } \\
\text { Verdichtung } \\
{[\%]}\end{array}$ & $\begin{array}{c}\text { Darrdichte } \\
{\left[\mathrm{kg} / \mathrm{m}^{3}\right]}\end{array}$ & $\begin{array}{c}\text { Stabw. } \\
\text { Darrdichte } \\
{\left[\mathrm{kg} / \mathrm{m}^{3}\right]}\end{array}$ \\
\hline \multirow[t]{24}{*}{ parallel } & $0 \%$ & $\mathrm{R}$ & 2 & 4.5 & 1.8 & 703 & 13.6 \\
\hline & & & 4 & 11.9 & 0.1 & 706 & 6.1 \\
\hline & & & 6 & 13.8 & 5.2 & 757 & 12.8 \\
\hline & $15 \%$ & $A$ & 2 & 6.1 & 2.1 & 739 & 24.4 \\
\hline & & & 4 & 20.0 & 8.0 & 865 & 88.7 \\
\hline & & & 6 & 29.0 & 4.6 & 958 & 65.4 \\
\hline & & $\mathrm{F}$ & 2 & 4.6 & 0.6 & 832 & 6.7 \\
\hline & & & 6 & 22.2 & 0.7 & 1060 & 7.0 \\
\hline & & G & 2 & 9.5 & 1.8 & 775 & 20.6 \\
\hline & & & 6 & 33.1 & 0.7 & 1061 & 21.8 \\
\hline & $30 \%$ & $A$ & 2 & 0.6 & 2.0 & 821 & 3.0 \\
\hline & & & 4 & 19.4 & 4.1 & 990 & 22.3 \\
\hline & & & 6 & 26.2 & 1.1 & 1165 & 37.9 \\
\hline & & $F$ & 2 & 4.8 & 0.6 & 862 & 23.9 \\
\hline & & & 6 & 23.7 & 1.3 & 1129 & 31.4 \\
\hline & & G & 2 & 5.3 & 0.7 & 887 & 15.2 \\
\hline & & & 6 & 27.3 & 1.5 & 1153 & 34.2 \\
\hline & $60 \%$ & $A$ & 2 & 7.0 & 0.1 & 966 & 6.3 \\
\hline & & & 4 & 23.8 & 0.1 & 1194 & 15.8 \\
\hline & & & 6 & 29.4 & 1.1 & 1271 & 20.8 \\
\hline & & $\mathrm{F}$ & 2 & 8.3 & 0.8 & 1031 & 19.9 \\
\hline & & & 6 & 19.1 & 0.4 & 1192 & 21.2 \\
\hline & & $G$ & 2 & 8.5 & 1.1 & 1027 & 15.7 \\
\hline & & & 6 & 21.6 & 0.6 & 1206 & 4.5 \\
\hline
\end{tabular}

Die Tabelle 11und Tabelle 12 zeigen, dass je nach Pressdruck und WPG unterschiedliche Verdichtungen und Darrdichten bei den Platten zu finden waren. Sie verdeutlichen auch, dass ähnliche Darrdichten infolge von Verdichtung bei unterschiedlichen WPGs erzielbar waren.

\subsubsection{Fixierung und Dimensionsstabilität}

Die Fixierung der Harze wurde über eine Auswaschbeanspruchung nach EN84 durchgeführt. In diesem Zusammenhang wurden die Holzfeuchte, die Dickenquellung und für Platten ohne Sperrlagen auch die Volumenquellung evaluiert. 


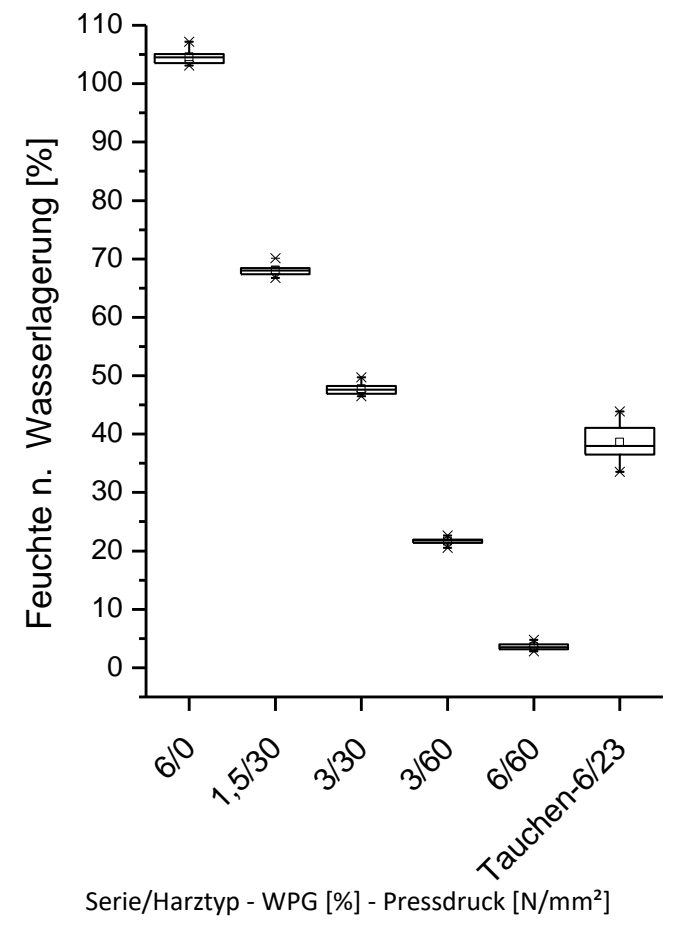

Abbildung 43: Feuchte des 10-lagigen Buchen-LVL-Prüfkörper ohne Sperrlagen $(10 * 2,13 \mathrm{~mm})$ nach Wasserlagerung gemäß EN84.

Die ersten Plattenserien, die aus 2,13 mm dicken Furnieren 10-lagig hergestellt wurden, wiesen eine mit zunehmenden WPG und Pressdruck abnehmende Wasseraufnahme auf gemessen durch die Feuchte nach der Wasserlagerung (Abbildung 43). Der Einfluss des Pressdruckes wird am deutlichsten zwischen den Serien mit einem WPG von 30\% und einem Pressdruck von 1,5 N/mm² bei der einen, sowie einem Pressdruck von $3 \mathrm{~N} / \mathrm{mm}^{2}$ bei der anderen. Im Wesentlichen ist das auf die Verdichtung zurückzuführen, die zuvor beschreiben wurde. Zellhohlräume, insbesondere die Zelllumen, wurden zusammengedrückt und infolge der Aushärtung des Imprägnierharzes weitestgehend in ihrer Form fixiert, wodurch der verfügbare Raum für Wasser verringert wurde. Bei dem höheren WPG von 60\% war infolge eines höheren Pressdruckes die Wasseraufnahme bzw. die Feuchte auf durchschnittlich 3,6\% reduziert.

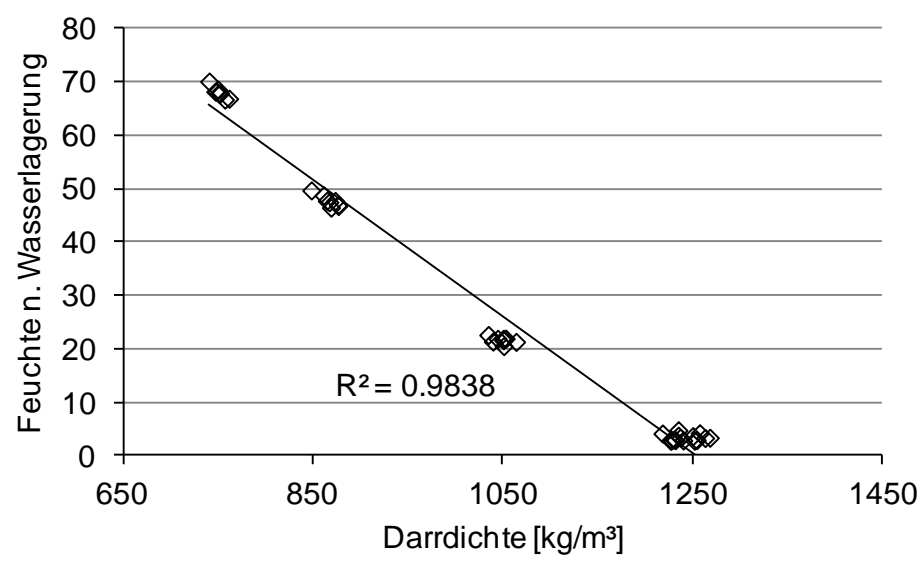

Abbildung 44: Korrelation der maximalen Feuchte nach Wasserlagerung (EN84) und der Darrdichte von modifiziertem Buchen-LVL (10*2,13 mm). 
Durch Erstellung eines linearen Regressionsmodells mit den Ergebnissen aller modifizierten Prüfkörper (Abbildung 44) wird der Zusammenhang zwischen der Darrdichte und der maximalen Holzfeuchte verdeutlicht.

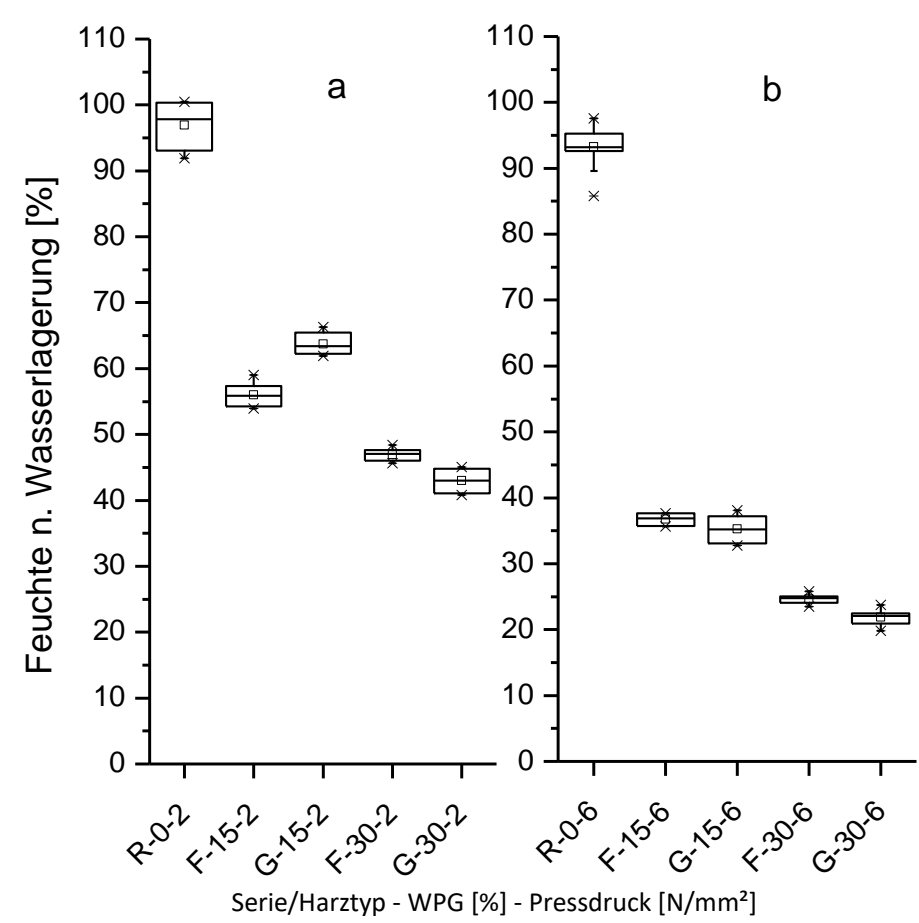

Abbildung 45: Feuchte der Buchen-LVL Prüfkörper ohne Sperrlagen (8-lagig) nach Wasserlagerung gemäß EN84- a) $2 \mathrm{~N} / \mathrm{mm}^{2}$ Pressdruck; b) $6 \mathrm{~N} / \mathrm{mm}^{2}$ Pressdruck.

Infolge der Harzimprägnierung und Verdichtung waren auch beim 8-lagigen Buchen-LVL die Wasseraufnahmen in Abhängigkeit von der Darrdichte reduziert. Da hier der niedrigste Pressdruck bei $2 \mathrm{~N} / \mathrm{mm}^{2}$ lag, wurde bereits bei einem WPG von 15\% eine ähnliche Dichte wie beim 10-lagigen LVL mit einem WPG von $30 \%$ bei $1,5 \mathrm{~N} / \mathrm{mm}^{2}$ erzielt. Aus diesem Grund lag auch die Feuchte nach der Wasserlagerung im gleichen Bereich bzw. etwas darunter. Mit zunehmendem WPG der Serien sank hier die Wasseraufnahme weiter. Alle Unterschiede zwischen den Harztypen ließen sich durch Unterschiede in den Darrdichten erklären. Beispielsweise war bei einem Pressdruck von $2 \mathrm{~N} / \mathrm{mm}^{2}$ und einem WPG von $30 \%$ die Wasseraufnahme beim Harztyp G niedriger als beim Harztyp F, jedoch wies der Harztyp G auch eine höhere Darrdichte auf (Abbildung 37). Beim Pressdruck von $6 \mathrm{~N} / \mathrm{mm}^{2}$, der eine noch stärkere Verdichtung zur Folge hatte, wurden niedrigere Holzfeuchten als bei $2 \mathrm{~N} / \mathrm{mm}^{2}$ erzielt, die Unterschiede zwischen den beiden WPGs waren ähnlich. Wird beim Buchenholz ein Fasersättigungspunkt von 28\% (KoLLMANN 1951) angenommen, so lagen die Serien mit einem WPG von $30 \%$ beim Pressdruck von $6 \mathrm{~N} / \mathrm{mm}^{2}$ deutlich darunter. An dieser Stelle ist anzumerken, dass die tatsächliche Holzfeuchte rechnerisch höher liegen könnte, da hier die aufgenommene Wassermenge auf den darrtrocknen Prüfkörper bezogen wurde, dessen Gewicht entsprechend seines WPG zugenommen hatte. Jedoch sind es gerade die Gewichtsund Dichtezunahmen, die die Verringerung der Wasseraufnahme herbeiführten. Deshalb ist die gewählte Form der Berechnung und Darstellung anschaulicher, sowie eine Unterscheidung zwischen der hier gezeigten „Feuchte n. Wasserlagerung“ und der Wasseraufnahme unnötig. 


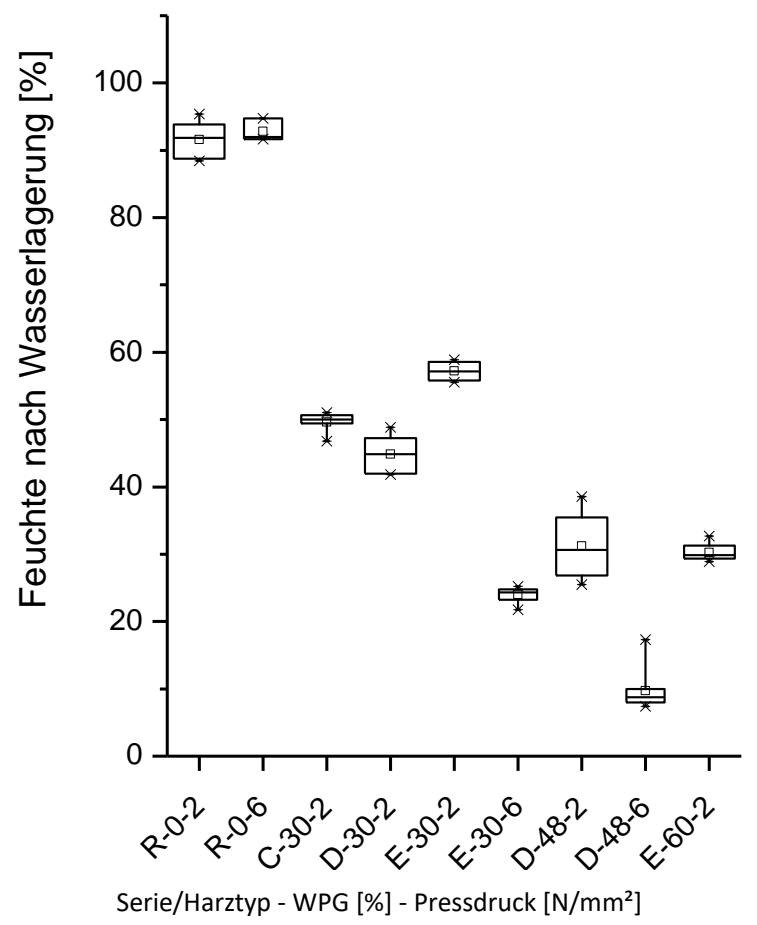

Abbildung 46: Feuchte der Buchen-LVL Prüfkörper mit Sperrlagen (8-lagig) nach Wasserlagerung gemäß EN84.

Das 8-lagige Buchen-LVL mit zwei Sperrlagen wies mit zunehmendem Pressdruck und WPG niedrigere Feuchten nach der Wasserlagerung auf. Zwischen den Harztypen gab es einige Unterschiede. Bei einem WPG von 30\% und einem Pressdruck von $2 \mathrm{~N} / \mathrm{mm}^{2}$ wies der Harztyp D eine höhere Feuchte und der Harztyp E eine niedrigere Feuchte als der Harztyp C auf. Betrachtet man dazu noch einmal die Ergebnisse der zugehörigen Darrdichten, stellt man fest, dass der Harztyp D eine höhere Darrdichte und der Harztyp E eine niedrigere Darrdichte als der Harztyp C aufwiesen.

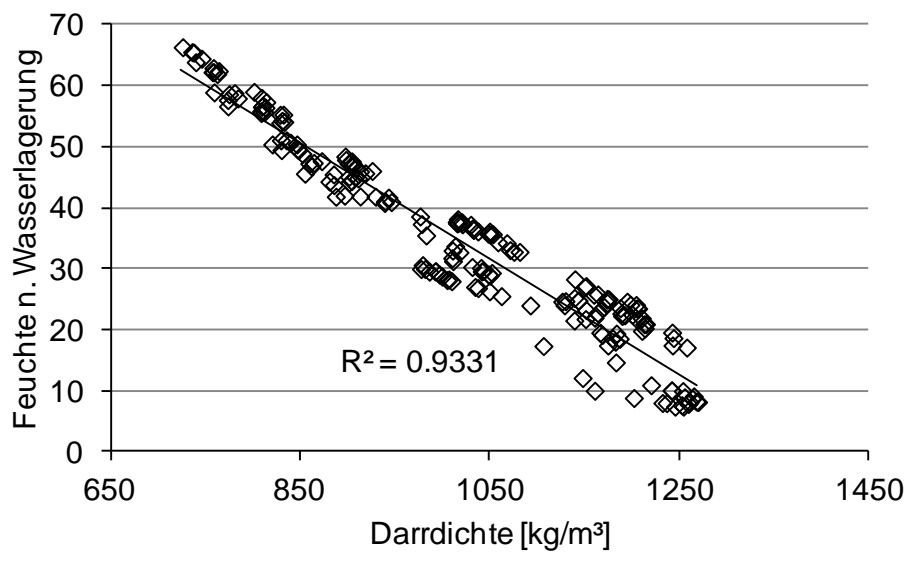

Abbildung 47: Korrelation der maximalen Feuchte nach Wasserlagerung (EN84) und der Darrdichte von modifiziertem Buchen-LVL $(8 * 3,7 \mathrm{~mm})$. 
Am oben genannten Beispiel der Harztypen C, D und E wurde bereits ersichtlich, dass die Wasseraufnahme und die Darrdichte korrelierten. In Abbildung 47 ist die lineare Regression der Darrdichten aller Prüfkörper modifizierten LVLs und der Wasseraufnahme bzw. Feuchte nach Wasserlagerung dargestellt. Diese zeigt den Zusammenhang, wie er sich bereits beim 10lagigen LVL mit dem Harztyp A zeigte (Abbildung 44), und es wird klar, dass eine unterschiedliche Wasseraufnahme bei einer Wasserlagerung gemäß EN84 nicht auf die Harztypen zurückzuführen war.

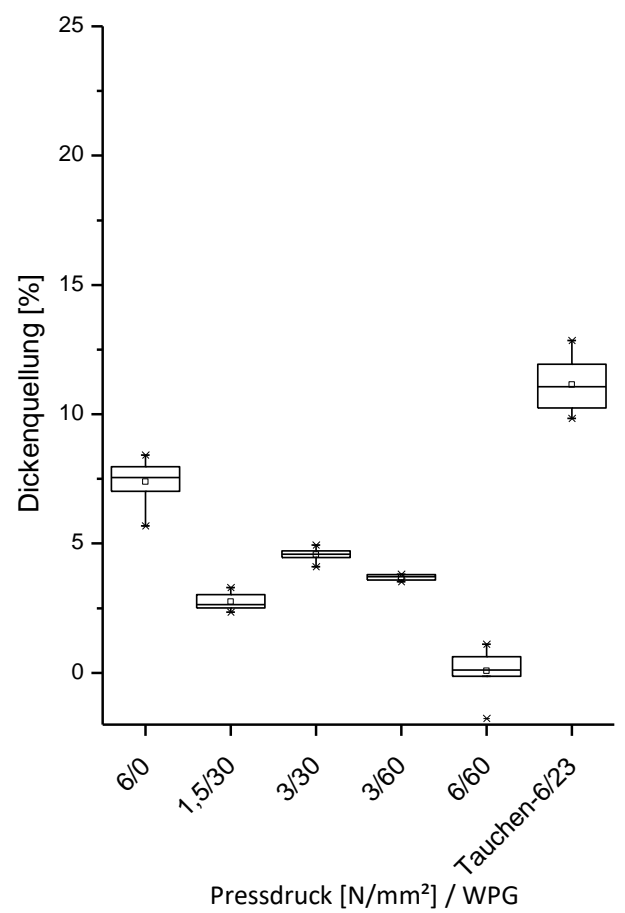

Abbildung 48: Dickenquellung des 10-lagigen Buchen-LVL-Prüfkörper ohne Sperrlagen (10*2,13mm) nach Wasserlagerung gemäß EN84.

Wie in Abbildung 48 zu sehen, war die Dickenquellung aller Versuchsserien aus dem 10lagigen Buchen-LVL ohne Sperrlagen unterschiedlich. Alle im Vakuumverfahren modifizierten Serien wiesen eine niedrigere Quellung als die Referenzserie auf. Bei einem WPG von 30\% zeigte sich, dass die höher verdichteten Proben auch die höhere Dickenquellung aufwiesen. Bei einem WPG von 60\% wiederum war es umgekehrt. Hier wurde bei einem Pressdruck von 6 $\mathrm{N} / \mathrm{mm}^{2}$ nur noch 1\% Dickenquellung gemessen. Dieses Ergebnis ging einher mit der höchsten Darrdichte und der niedrigsten Wasseraufnahme. Die Serie, welche im Tauchverfahren modifiziert wurde und ebenfalls beim höchsten Druck gepresst wurde, zeigte die höchste Dickenquellung aller Serien, die noch über den Referenzen lag. Bei dieser Variante zeigte sich ein Zusammenhang der, bereits oben gezeigten, großen Verdichtung in Verbindung mit einem relativ niedrigen WPG und einer deutlichen Wasseraufnahme. 


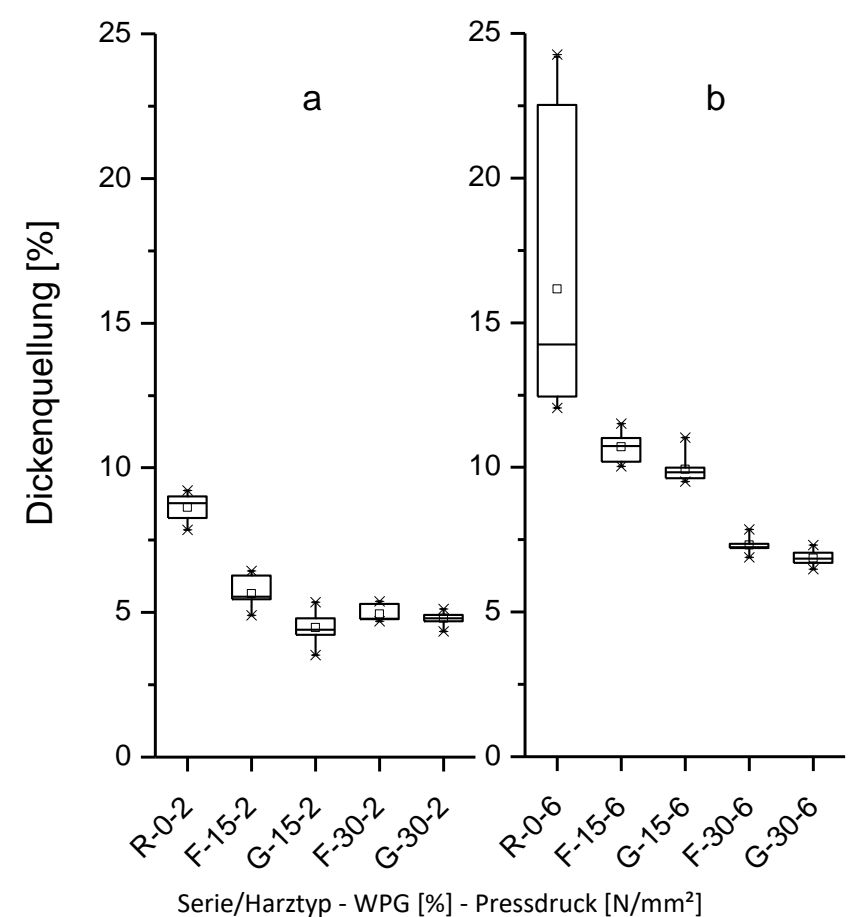

Abbildung 49: Dickenquellung der Buchen-LVL-Prüfkörper ohne Sperrlagen (8-lagig) nach Wasserlagerung gemäß EN84- a) 2 N/mm² Pressdruck; b) $6 \mathrm{~N} / \mathrm{mm}^{2}$ Pressdruck.

Die Abbildung 49 zeigt, dass bei einem Pressdruck von $2 \mathrm{~N} / \mathrm{mm}^{2}$ (a), der nur zu einer geringen Verdichtung führte, das modifizierte 8-lagige Buchen-LVL ohne Sperrlagen eine niedrigere Dickenquellung als die Referenz aufwies. Die Ergebnisse bei einem WPG von 15\% und von 30\% waren hier nah beieinander. Hinsichtlich der Harztypen wies der Harztyp G eine geringfügig niedrigere Dickenquellung auf, jedoch wies F im Vergleich zu G eine um 10\% höhere Darrdichte auf, was zu diesem Ergebnis geführt haben könnte. Dafür spricht auch, dass bei einem WPG von 30\% beim gleichen Pressdruck F und G gleiche Darrdichten und die gleiche Dickenquellung aufwiesen.

Die höhere Verdichtung bei einem Pressdruck von $6 \mathrm{~N} / \mathrm{mm}^{2}$ (b) führte bei Referenzen und modifizierten Serien zu einer höheren Dickenquellung bei der Wasserlagerung. Bei einem WPG von 15\% lagen beide Harztypen ( $G$ und F) über allen Ergebnissen der unverdichteten Serien. Die Varianten mit einem WPG von 30\% lagen auch noch oberhalb der modifizierten unverdichteten Serien, jedoch niedriger als die unverdichteten Referenzen. 


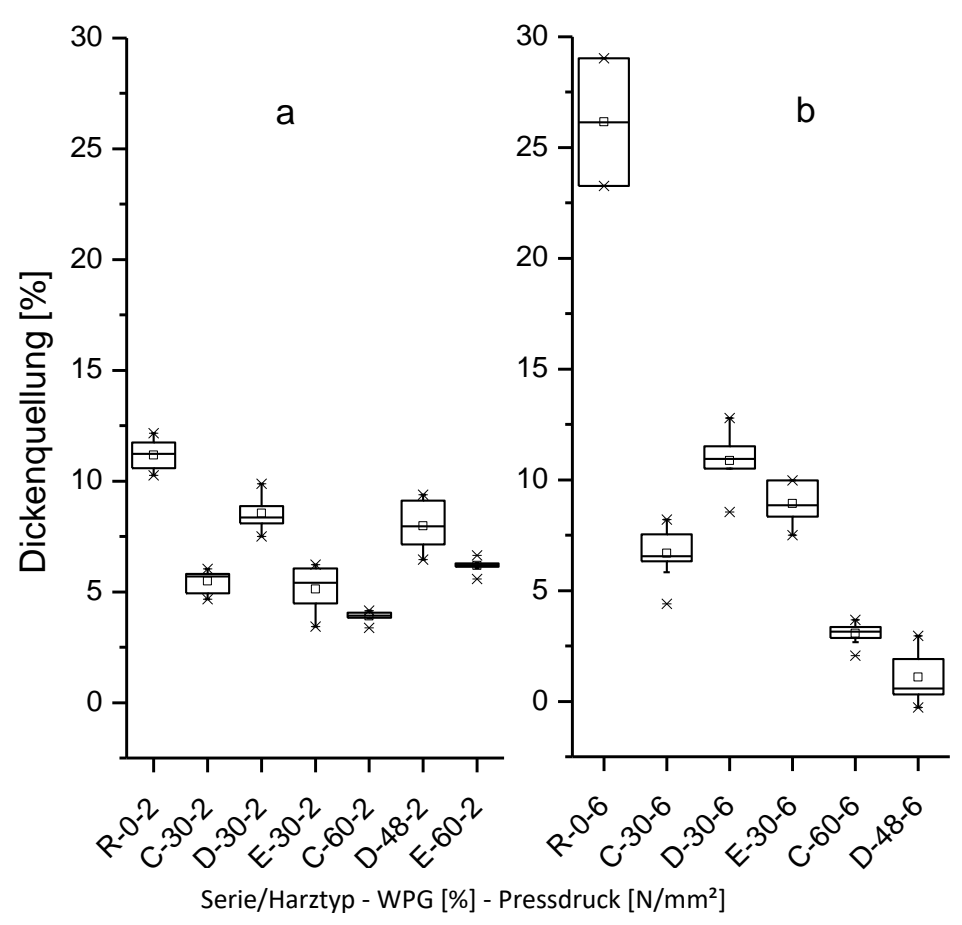

Abbildung 50: Dickenquellung der Buchen-LVL-Prüfkörper mit Sperrlagen (8-lagig) nach Wasserlagerung gemäß EN84- a) $2 \mathrm{~N} / \mathrm{mm}^{2}$ Pressdruck; b) $6 \mathrm{~N} / \mathrm{mm}^{2}$ Pressdruck.

In Abbildung 50 zeigte sich links (a) für 8-lagiges Buchen-LVL mit Sperrlagen ein zur Wasseraufnahme entgegengesetztes Bild für die Harztypen C, D und E bei 30\% WPG und 2 $\mathrm{N} / \mathrm{mm}^{2}$, denn hier quollen die Prüfkörper umso stärker je höher die Darrdichte war bzw. umso weniger je niedriger die Darrdichte war. Bei einem WPG von 60\% lag die Darrdichte zwar höher, jedoch nur aufgrund des höheren Harzanteils und nicht infolge einer höheren Verdichtung, die, wie bereits gezeigt, bei beiden WPGs bei einem Druck von $2 \mathrm{~N} / \mathrm{mm}^{2}$ gleich war. Somit quollen die Serien bei beiden WPGs ähnlich, obwohl die Feuchte nach Wasserlagerung bei 60\% WPG niedriger war. Es bleibt an dieser Stelle spekulativ anzunehmen, dass bei beiden WPGs ähnlich viel Harz in der Zellwand war und im gleichen Maße zur Reduzierung der Quellung der Zellwand beigetragen haben könnte. Bei den Referenzen, die bei einem Pressdruck von $6 \mathrm{~N} / \mathrm{mm}^{2}$ hergestellt wurden, zeigte sich wie beim 10-lagigen LVL, eine deutliche Zunahme der Dickenquellung infolge der reversiblen Verdichtung. Die modifizierten, verdichteten Serien wiesen dagegen wieder eine verringerte Dickenquellung auf, die umso niedriger war je höher der WPG war. 


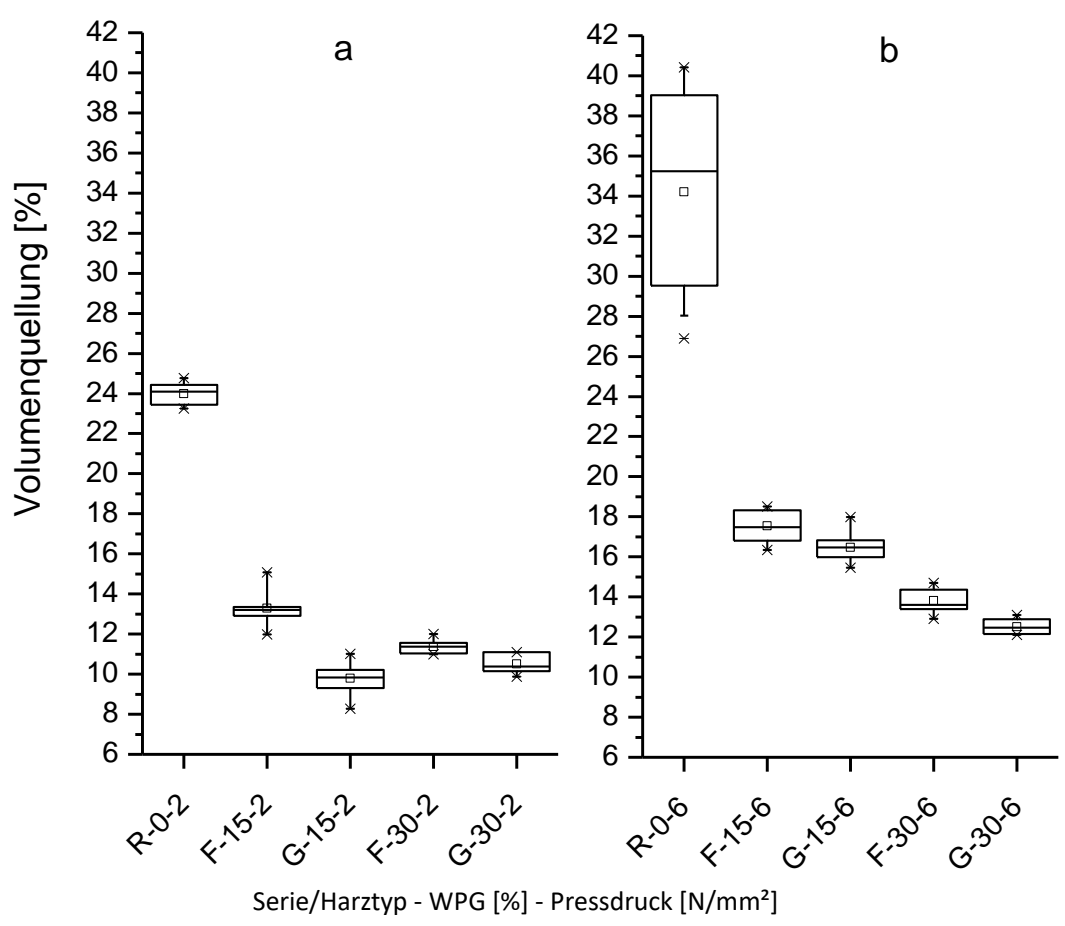

Abbildung 51: Volumenquellung der Buchen-LVL ohne Sperrlagen nach Wasserlagerung gemäß EN84- a) 2 N/mm² Pressdruck; b) $6 \mathrm{~N} / \mathrm{mm}^{2}$ Pressdruck.

In Abbildung 51 dargestellt ist die Volumenquellung, welche neben der Dickenquellung, d.h. der Quellung in radialer Richtung, auch die tangentiale und longitudinale Richtung berücksichtigt. Hier wird deutlich, dass das Volumen des ganzen Plattenprüfkörpers infolge von Wasseraufnahme und Quellung zunahm. Beim Vergleich der Serien zueinander zeigten sich hier, beim Buchen-LVL ohne Sperrlagen, die gleichen Verhältnisse wie bei der Dickenquellung. Es ist dabei erwähnenswert, dass die maximale Volumenzunahme der Referenzen mit dem höchsten Pressdruck bei 41\% lag, während die Variante mit dem Harztyp G bei 15\% WPG und $2 \mathrm{~N} / \mathrm{mm}^{2}$ Pressdruck die geringste Volumenzunahme aufwies.

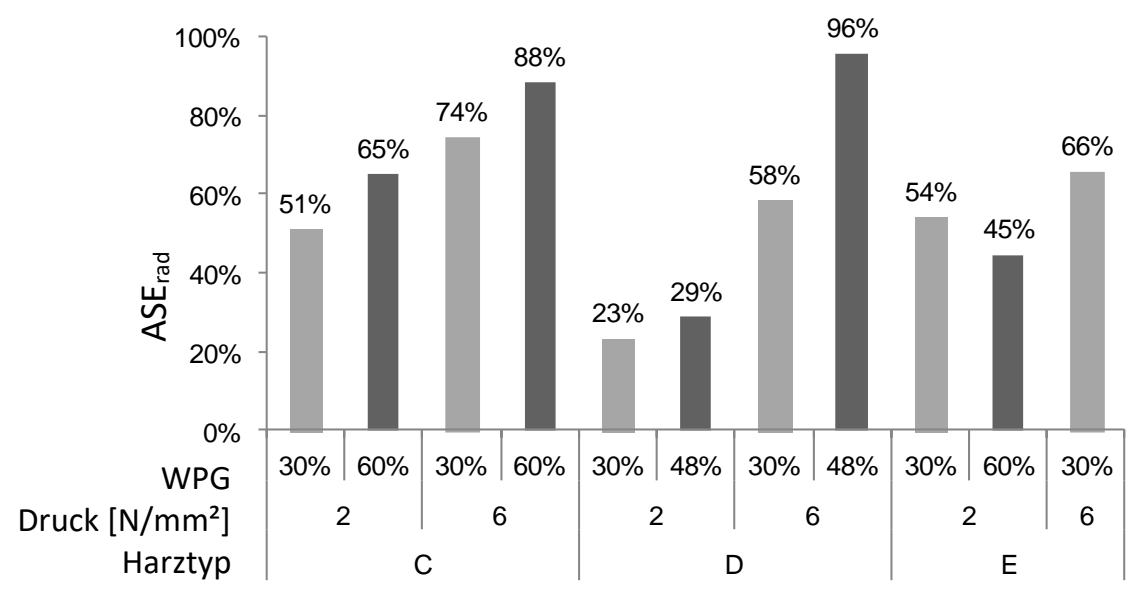

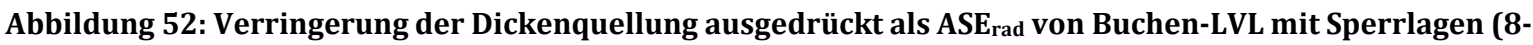
lagig) nach Wasserlagerung gemäß EN84. 
Die hier berechnete und dargestellte $\mathrm{ASE}_{\text {rad, }}$ welche sich nur auf die Dickenquellung der Referenzen und der modifizierten Serien bezieht, verdeutlicht die Verbesserung der Dimensionsstabilität von Buchen-LVL durch die PF-Modifizierung. Bei Buchen-LVL mit Sperrlagen (Abbildung 52), zeigte der höhere WPG häufig die höhere ASE $E_{\text {rad. Bei genauerer }}$

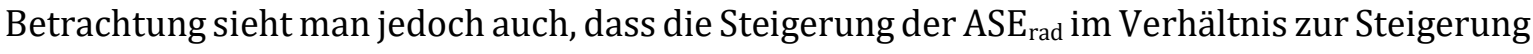
des WPG unterproportional ausfiel und eine, aus wirtschaftlichen Gesichtspunkten, schlechtere Aufwand-zu-Nutzen-Relation mit sich brachte. Einen besonderen Einfluss schien auch der Pressdruck zu haben, jedoch lag das auch an seiner Bezugsgröße bei der Berechnung, denn die Referenzen, die beim höheren Pressdruck hergestellt wurden, wiesen eine sehr stark erhöhte Dickenquellung auf.

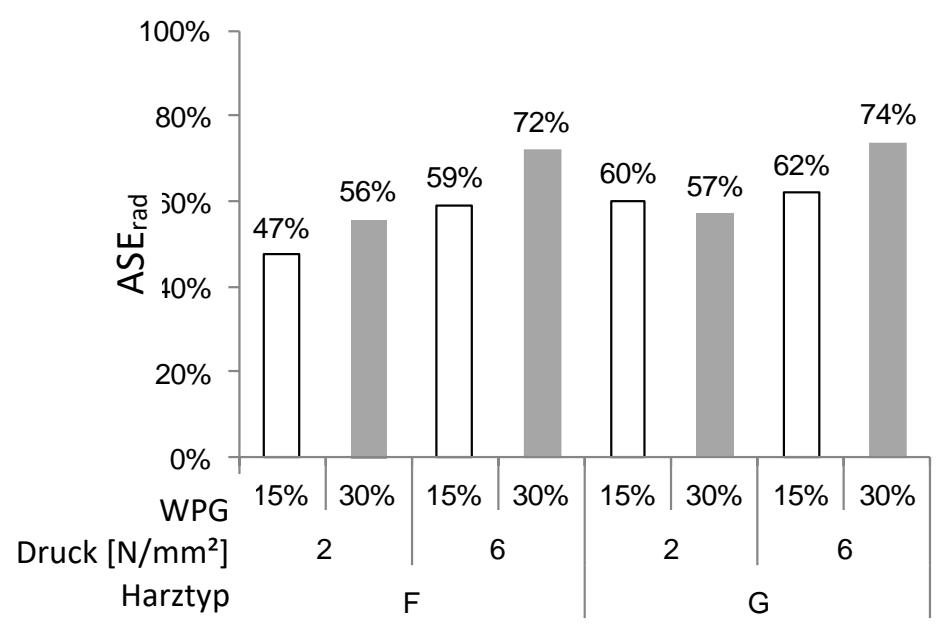

Abbildung 53: Verringerung der Dickenquellung ausgedrückt als ASErad von Buchen-LVL ohne Sperrlagen (8-lagig, parallel) nach Wasserlagerung gemäß EN84.

Aus Abbildung 53 wird ersichtlich, dass beim Buchen-LVL ohne Sperrlagen, je nach Harztyp und Pressdruck, eine Verminderung der Dickenquellung in Höhe $47 \%$ bis $62 \%$ bei einem WPG von $15 \%$ möglich war. Bei einem WPG von $30 \%$ waren in Abhängigkeit des Pressdruckes

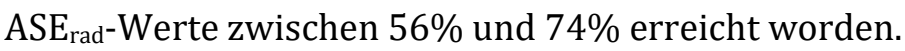


Tabelle 13: Abnahme der darrtrockenen Masse der Prüfkörper infolge von Auswaschung gemäß EN84.

\begin{tabular}{|c|c|c|c|c|c|c|}
\hline Furnierdicke & Furnierrichtung & Harztyp & WPG & Druck $\left[\mathrm{N} / \mathrm{mm}^{2}\right]$ & Mittelwert [\%] & Stabw $[\%]$ \\
\hline \multirow[t]{8}{*}{2.13} & parallel & $\mathrm{R}$ & $0 \%$ & 2 & 2.3 & 0.1 \\
\hline & & & & 4 & 2.5 & 0.1 \\
\hline & & & & 6 & 2.2 & 0.2 \\
\hline & & A & $30 \%$ & 1.5 & 1.6 & 0.1 \\
\hline & & & & 3 & 1.0 & 0.0 \\
\hline & & & & 6 & 0.0 & 0.1 \\
\hline & & & $60 \%$ & 3 & 0.2 & 0.1 \\
\hline & & & & 6 & 0.1 & 0.1 \\
\hline \multirow[t]{25}{*}{3.7} & mit 2Sperrlagen & $\mathrm{R}$ & $0 \%$ & 2 & 1.8 & 0.2 \\
\hline & & & & 4 & 2.0 & 0.0 \\
\hline & & & & 6 & 2.1 & 0.1 \\
\hline & & C & $30 \%$ & 2 & 1.3 & 0.2 \\
\hline & & & & 6 & 0.4 & 0.2 \\
\hline & & & $60 \%$ & 2 & 0.5 & 0.0 \\
\hline & & & & 6 & 0.2 & 0.2 \\
\hline & & $\mathrm{D}$ & $30 \%$ & 2 & 2.0 & 0.2 \\
\hline & & & & 6 & 0.8 & 0.2 \\
\hline & & & $48 \%$ & 2 & 1.7 & 0.2 \\
\hline & & & & 6 & 0.3 & 0.2 \\
\hline & & $\mathrm{E}$ & $30 \%$ & 2 & 1.3 & 0.2 \\
\hline & & & & 6 & 0.8 & 0.1 \\
\hline & & & $60 \%$ & 2 & 1.5 & 0.2 \\
\hline & parallel & $\mathrm{R}$ & $0 \%$ & 2 & 2.1 & 0.1 \\
\hline & & & & 4 & 2.1 & 0.1 \\
\hline & & & & 6 & 2.2 & 0.1 \\
\hline & & $\mathrm{F}$ & $15 \%$ & 2 & 1.6 & 0.1 \\
\hline & & & & 6 & 1.5 & 0.0 \\
\hline & & & $30 \%$ & 2 & 1.5 & 0.1 \\
\hline & & & & 6 & 1.2 & 0.1 \\
\hline & & G & $15 \%$ & 2 & 1.6 & 0.1 \\
\hline & & & & 6 & 1.6 & 0.1 \\
\hline & & & $30 \%$ & 2 & 1.2 & 0.1 \\
\hline & & & & 6 & 07 & 0.1 \\
\hline
\end{tabular}

Hinsichtlich der Fixierung der PF-Harze ließ sich mit den Ergebnissen der Auswaschbeanspruchung (Tabelle 13) feststellen, dass die Fixierung sehr gut gelang. Bei den Referenzen war die Auswaschung stets höher als bei den modifizierten Serien. Das lag auch daran, dass die Referenzen, da sie nicht imprägniert wurden, noch mehr lösliche Bestandteile enthielten als die modifizierten Serien, die durch die Imprägnierung und anschließende Trocknung eine leichte Vorauswaschung erfahren hatten. Somit waren es bei den modifizierten Serien vermutlich auch zu einem größeren Anteil anorganische Salze, z.B. NaOH, die als Katalysatoren im Harz eingesetzt und nun ausgewaschen wurden. 
Nachfolgend dargestellt sind die Ergebnisse zur Dimensionsstabilität nach Lagerung der von Buchen-LVL-Proben für 4 Stunden in kochendem Wasser.
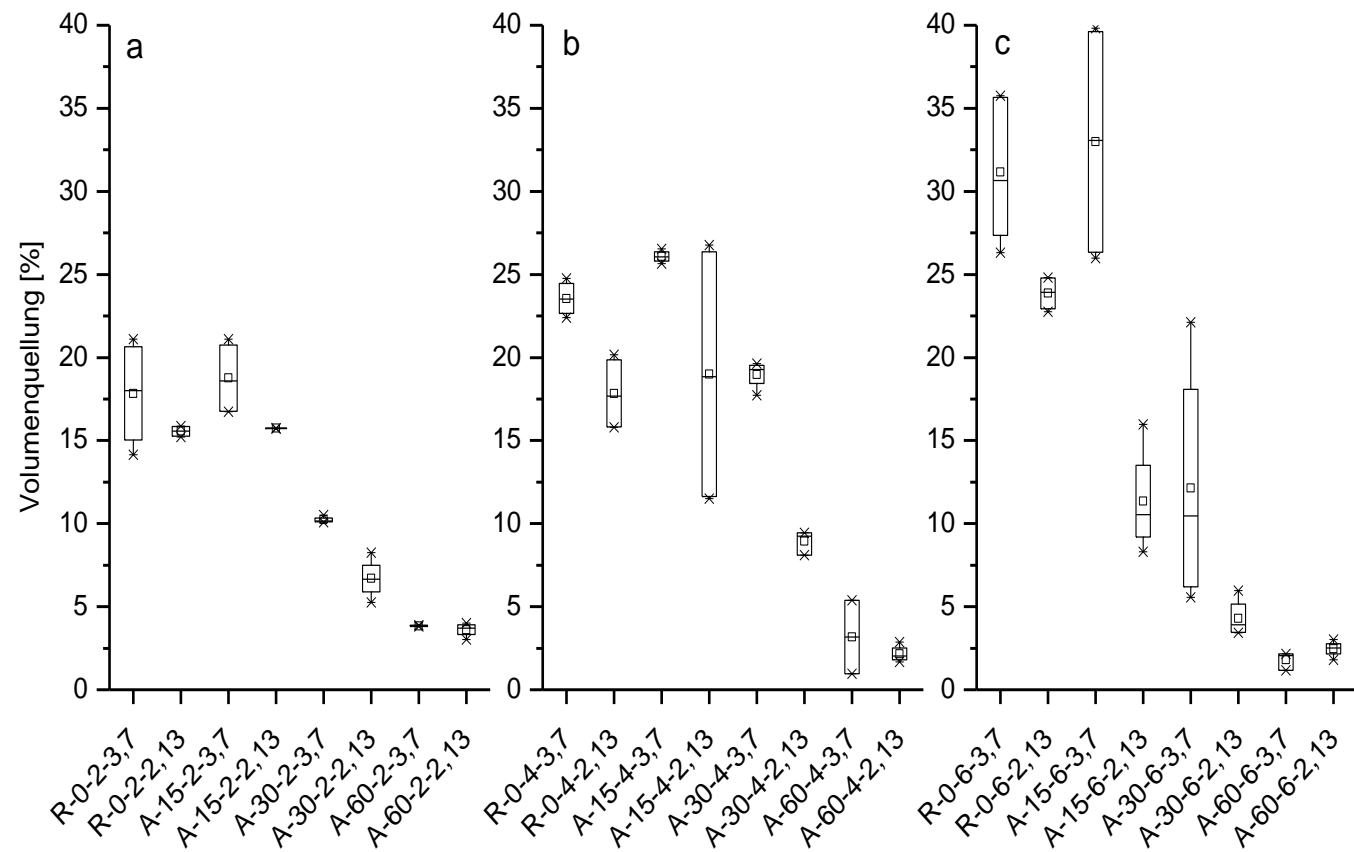

Serie/Harztyp - WPG [\%] - Pressdruck [N/mm²] - Furnierdicke [mm]

Abbildung 54:Volumenquellung nach 4-stündiger Kochung von Buchen-LVL ohne Sperrlagen mit den Furnierstärken 3,7 mm (8-lagig) und 2,13mm (15-lagig). Herstellung bei unterschiedlichen Pressdrücken; a) $p=2 \mathrm{~N} / \mathrm{mm}^{2}$, b) $p=4 \mathrm{~N} / \mathrm{mm}^{2}$ und c) $p=6 \mathrm{~N} / \mathrm{mm}^{2}$.

Bei der Untersuchung der Dimensionsstabilität mit Harztyp A zeigte sich durch Betrachtung der Ergebnisse zur Volumenquellung (Abbildung 54), dass bei unbehandelten Referenzen, trotz geringer Verdichtung, mit erhöhtem Pressdruck auch eine Erhöhung der Volumenquellung einherging. Hierbei zeigte sich ein Einfluss unterschiedlicher Furnierdicken. Die höhere Lagenzahl bei dünneren Furnieren und ein entsprechend höherer Klebharzanteil bewirkten, neben einer verminderten Wasseraufnahme, auch eine Reduzierung der Volumenquellung. Ein WPG von 15\% zeigte, für den niedrigsten und den erhöhten Druck, statt einer Reduzierung eine Erhöhung der Volumenquellung. Die Plattenserie mit dünneren Furnieren, welche hingegen beim höchsten Druck verpresst wurden, zeigte eine deutliche Reduzierung. Bei einem WPG von 30\% hatten alle Serien eine Verringerung der Volumenquellung erfahren, wenngleich die dünneren Furniere stets die niedrigeren Werte aufwiesen. Dieser Unterschied war beim höchsten WPG von 60\% nicht mehr erkennbar. Eine Verbesserung der Dimensionstabilität ist aufgrund dieser Ergebnisse somit zum einen durch dünnere Furniere und zum anderen durch Modifizierung bei einem WPG von mindestens 30\% erzielt worden, wobei Verdichtung eine weitere Verbesserung bewirkte. 


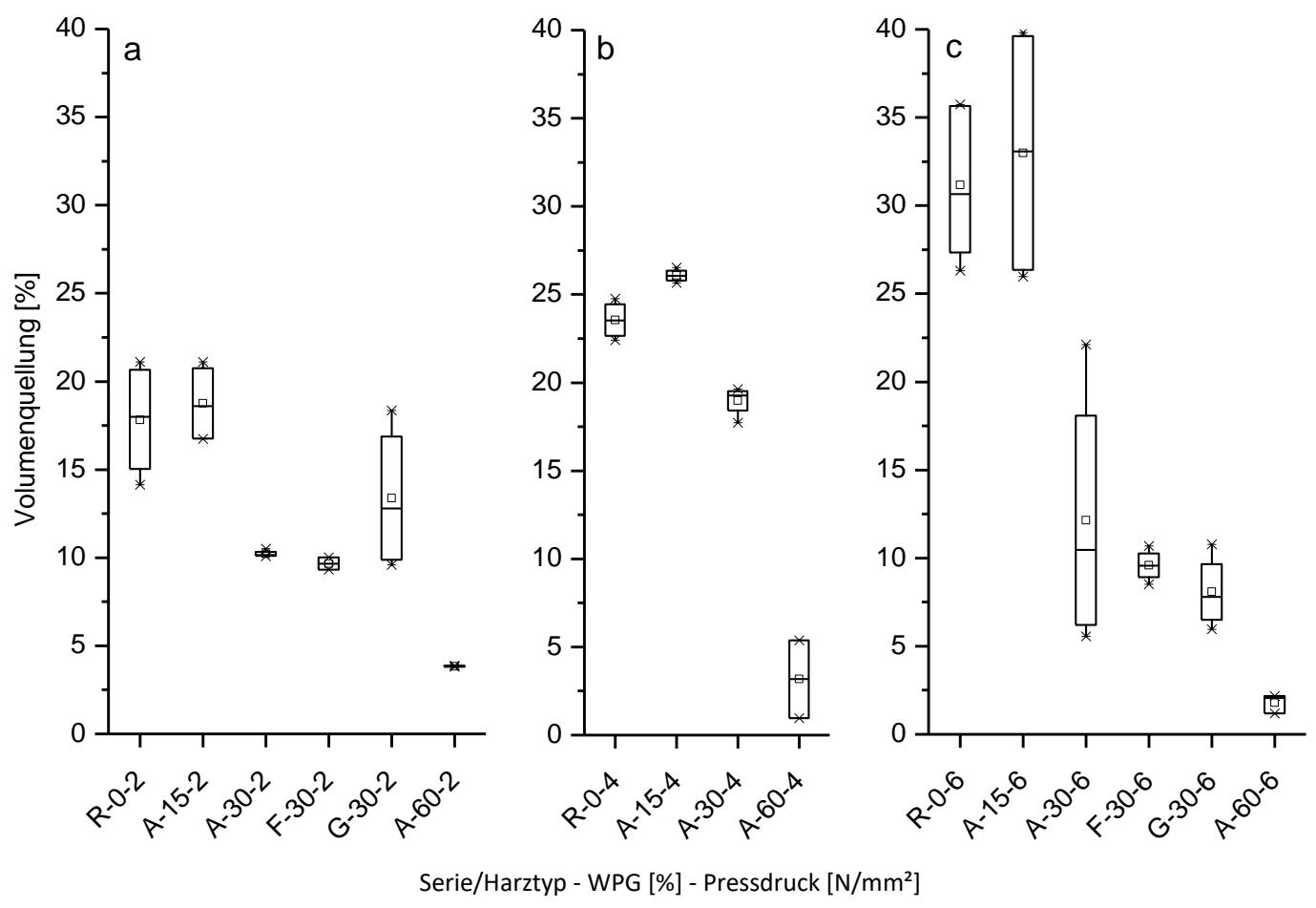

Abbildung 55: Volumenquellung nach 4-stündiger Kochung von Buchen-LVL ohne Sperrlagen unter Verwendung verschiedener Harze; 8-lagig, Furnierstärke $3,7 \mathrm{~mm}$. Hergestellt bei a) $\mathrm{p}=2 \mathrm{~N} / \mathrm{mm}^{2}$, b) $p=4 \mathrm{~N} / \mathrm{mm}^{2}$ und c) $\mathrm{p}=6 \mathrm{~N} / \mathrm{mm}^{2}$.

Die Untersuchung der Dimensionsstabilität von 8-lagigem Buchen-LVL ohne Sperrlagen mit verschiedenen Harzen zeigte, dass höhere WPG in Verbindung mit höherem Druck die Volumenquellung reduzierten (Abbildung 55). Allgemein fiel im Vergleich zur Dickenquellung nach Wasserlagerung gemäß EN84 die Streuung der Werte bei der Kochquellung größer aus. Das mag an der kürzeren Versuchsdauer oder auch an einer fehlenden Vakuumimprägnierung mit Wasser gelegen haben. Für den Harztyp A wurde hier auch eine 8-lagige Variante mit 3,7mm dicken Furnieren mit einem WPG 15\% untersucht. Diese führte zu keinerlei Verbesserung hinsichtlich der Volumenquellung. Je höher der Pressdruck bei diesem Harztyp und WPG, desto höher wurde die Volumenquellung, welche zum Teil die Referenzen übertraf. Alle übrigen Serien, d.h. mit einem WPG von $30 \%$ oder $60 \%$, ließen eine deutliche Verbesserung der Dimensionsstabilität feststellen. Aufgrund der hohen Streuung der Ergebnisse ließen sich Unterschiede zwischen den Harztypen nicht erklären. 

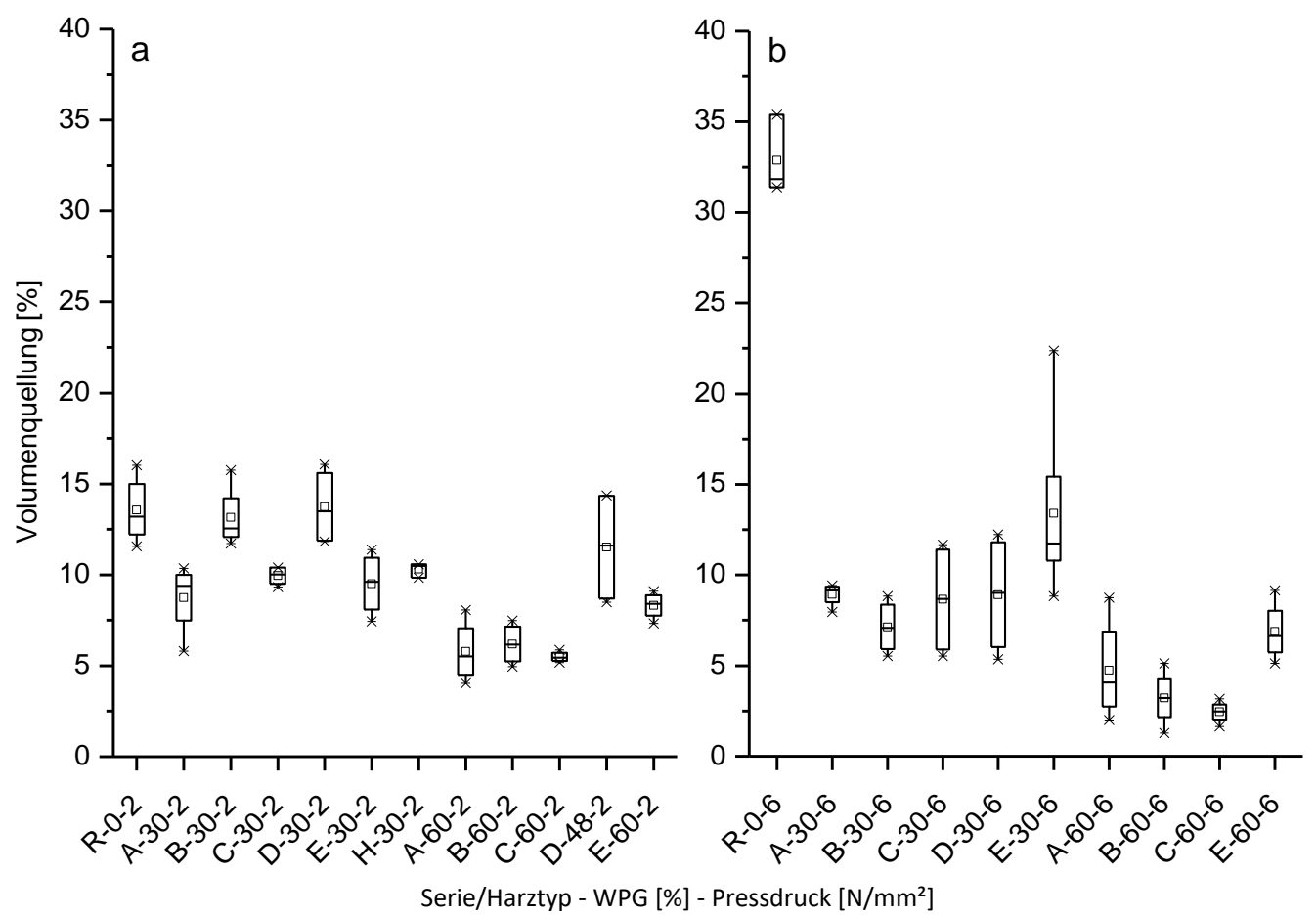

Abbildung 56: Volumenquellung nach 4-stündiger Kochung von Buchen-LVL mit 2 Sperrlagen unter Verwendung verschiedener Harze; 8-lagig, Furnierstärke 3,7 mm. Hergestellt bei a) $p=2 \mathrm{~N} / \mathrm{mm}^{2}$ und b) $p=6$ $\mathbf{N} / \mathbf{m m}^{2}$.

Zwischen den Harztypen konnten Unterschiede gefunden werden. So führte beim Pressdruck von $2 \mathrm{~N} / \mathrm{mm}^{2}$ der Harztyp A zu einer signifikanten Reduzierung der Volumenquellung, wohingegen die Harztypen B und D auf dem Niveau der Referenz lagen. Bei gleichem Pressdruck und dem WPG von 60\% wiesen alle Harze eine niedrigere Volumenquellung auf. Bei den Harzen A und B war sie gleichermaßen reduziert und kein Unterschied erkennbar. Die Anwendung des höheren Pressdruckes führte bei den Referenzen zu einer starken Zunahme der Volumenquellung. Alle modifizierten Serien wiesen im Vergleich eine niedrigere Volumenquellung auf. Mit Ausnahme von Harztyp E wiesen die Serien des höheren Pressdruckes bei gleichem WPG eine höhere Dimensionsstabilität auf als bei dem niedrigeren Pressdruck. 


\subsubsection{Biegefestigkeit und Elastizitätsmodul}

Im folgenden Abschnitt soll der Einfluss der Parameter bei der Plattenherstellung sowie der Einfluss der Modifizierung der Furniere auf die Biegefestigkeit und den Elastizitätsmodul erläutert werden. Die Holzfeuchte der Biegeproben nach Lagerung im Normalklima $\left(20^{\circ} \mathrm{C} / 65 \% \mathrm{rF}\right)$ wurde nicht ermittelt, jedoch zeigten die Prüfkörper, die für den Abbauversuch gemäß ENV 12038 (2002) bestimmt waren und unter gleichen Bedingungen gelagert wurden, im Durchschnitt eine korrigierte Holzfeuchte (HF) von 9,2\% für PFmodifizierte Serien und 10,7\% für die Referenzen. Signifikante Unterschiede zwischen den Harztypen ließen sich nicht erkennen, jedoch war die Feuchte bei einem WPG von 60\%, in Kombination mit einer Verdichtung bei $6 \mathrm{~N} / \mathrm{mm}^{2}$, niedriger als bei den übrigen Varianten.

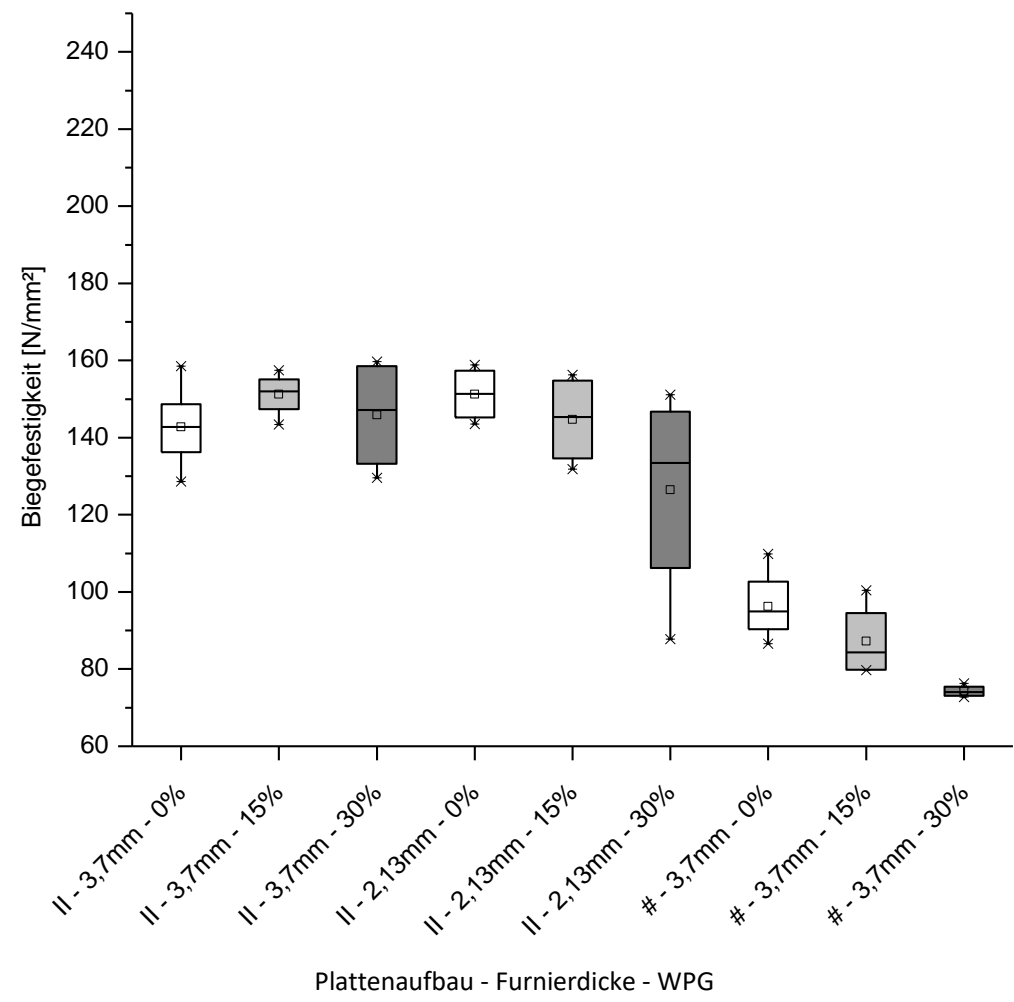

Abbildung 57: Biegefestigkeit von Buchen-Furnierschichtholz mit 2 Sperrlagen (\#) und ausschließlich parallelem Faserverlauf (II). Variation der Schichtdicke $(3,7 \mathrm{~mm}$ und 2,13 mm) und des WPG bei einem Pressdruck von $2 \mathrm{~N} / \mathrm{mm}^{2}$ unter Verwendung des Harztyp A.

Abbildung 57 zeigt, dass die Festigkeit des Buchenfurnierschichtholzes, welches bei einem Pressdruck von $2 \mathrm{~N} / \mathrm{mm}^{2}$ hergestellt wurde, stärker vom Plattenaufbau abhing als von der Furnierdicke und der Modifizierung. Bei ausschließlich parallelem Aufbau mit 3,7 mm und 2,13 mm dicken Furnieren gibt es keinen signifikanten Einfluss der Modifizierung auf die Festigkeit bei einem Alpha von 5\%. Bei den Serien die zwei Sperrlagen enthielten, ist bei Verwendung unbehandelter Furniere ein Rückgang der Festigkeit um 29\% festgestellt worden. Ein zunehmender WPG dieser Serien resultierte zudem in einer weiteren signifikanten Reduzierung der Biegefestigkeit $(\alpha=5 \%)$. 


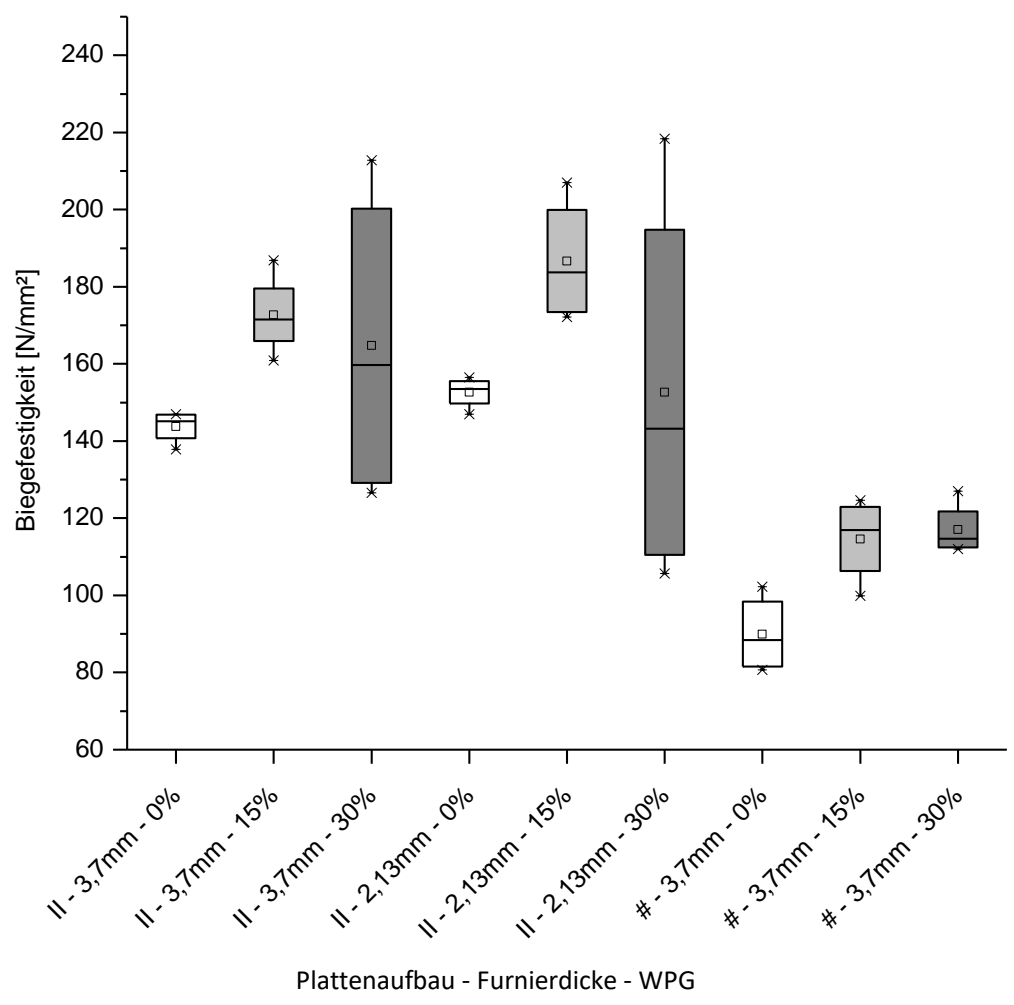

Abbildung 58: Biegefestigkeit von Buchen-Furnierschichtholz mit 2 Sperrlagen (\#) und ausschließlich parallelem Faserverlauf (II). Variation der Schichtdicke $(3,7 \mathrm{~mm}$ und 2,13 mm) und des WPG bei einem Pressdruck von $4 \mathrm{~N} / \mathrm{mm}^{2}$ unter Verwendung des Harztyp A.

Unter Verwendung eines Pressdruckes von $4 \mathrm{~N} / \mathrm{mm}^{2}$ kam es bei den Referenzen zu keiner Steigerung der Biegefestigkeit. Bei den meisten modifizierten Serien hingegen kam es zu einer Steigerung in unterschiedlichem Ausmaß. Am deutlichsten war sie für einen WPG von 15\% festzustellen. Bei dem parallel orientierten LVL mit einem WPG von 30\% stiegen zwar auch die Mediane, jedoch hatte insbesondere die Streuung zugenommen. Bei LVL mit zwei Sperrlagen lag die Variante mit 30\% auf dem gleichen Niveau wie die Variante mit 15\% und wies damit im Vergleich zu den Ergebnissen mit einem Pressdruck von $2 \mathrm{~N} / \mathrm{mm}^{2}$ eine relativ große Steigerung auf. 


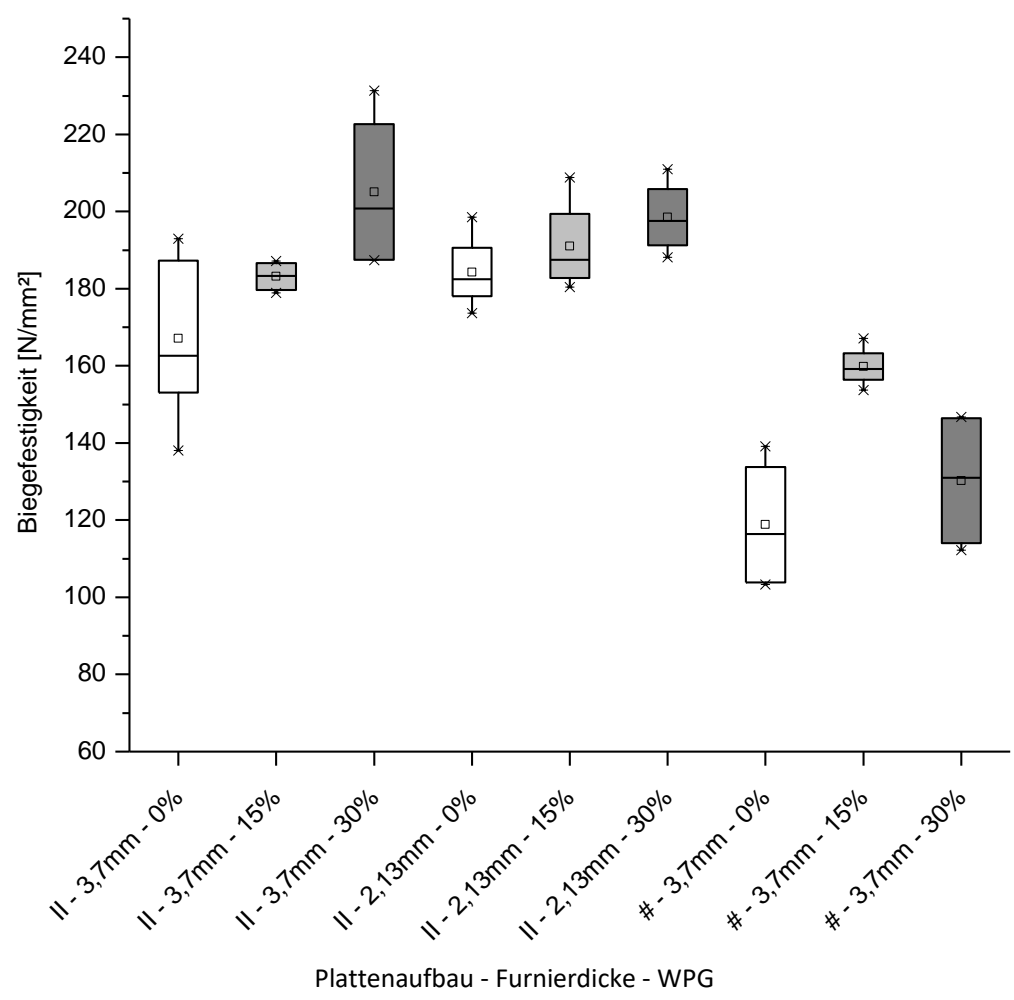

Abbildung 59: Biegefestigkeit von Buchen-Furnierschichtholz mit 2 Sperrlagen (\#) und ausschließlich parallelem Faserverlauf (II). Variation der Schichtdicke $(3,7 \mathrm{~mm}$ und 2,13 $\mathrm{mm}$ ) und des WPG bei einem Pressdruck von $6 \mathrm{~N} / \mathrm{mm}^{2}$ unter Verwendung des Harztyp A.

Die Herstellung des LVL bei einem Pressdruck von $6 \mathrm{~N} / \mathrm{mm}^{2}$ führte zu einer Erhöhung aller Ergebnisse für die Biegefestigkeit (Abbildung 59). Im Gegensatz zu dem LVL, welches bei 2 $\mathrm{N} / \mathrm{mm}^{2}$ hergestellt wurde, zeigte sich hier ein deutlicher Einfluss des WPG. Die Serie mit 3,7 mm-dicken Furnieren in ausschließlich paralleler Orientierung und einem WPG von 30\% wies eine um 23\% signifikant $(\alpha=5 \%)$ höhere Biegefestigkeit auf als die unbehandelte Referenz. Dies war insbesondere auf die gesteigerte Darrdichte zurückzuführen (Abbildung 38). Insbesondere die Zunahme der Biegefestigkeit bei den unbehandelten Referenzen verdeutlicht diesen Befund, da auch sie bei $6 \mathrm{~N} / \mathrm{mm}^{2}$ eine deutlich gesteigerte Darrdichte aufwiesen. Hinsichtlich der Furnierdicken zeigte sich, dass, wie zuvor beim niedrigeren Pressdruck, bei gleicher Orientierung der Furniere, kein signifikanter Unterschied bestand. Die Orientierung der Furniere zeigte erneut, dass bei einem Plattenaufbau mit Sperrlagen die Biegefestigkeit negativ beeinflusst war. Die unbehandelte Referenz mit Sperrlagen wies im Vergleich zu ausschließlich paralleler Orientierung einen um 29\% signifikant verringerten Mittelwert der Biegefestigkeit auf. 

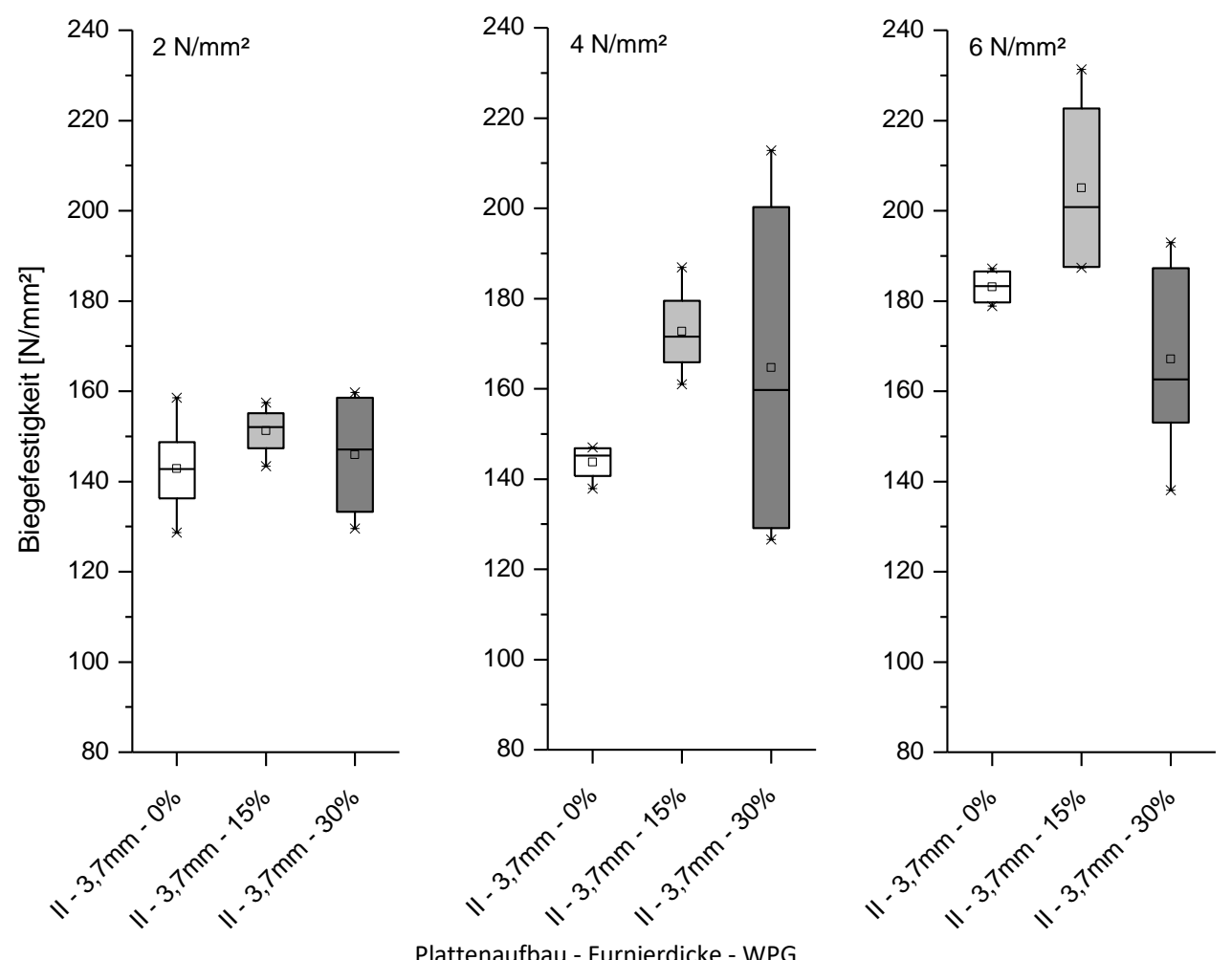

Abbildung 60: Biegefestigkeit von Buchen-Furnierschichtholz mit parallelem Faserverlauf (II) bei einer Furnierdicke von 3,7 mm unterschieden nach WPG (nur Harztyp A) und Pressdruck.

Die Abbildung 60 fasst noch einmal die Ergebnisse für das Buchen-LVL ohne Sperrlagen mit 3,7mm dicken Furnieren zusammen und stellt die Ergebnisse für die WPGs 15\% und 30\% bei den Pressdrücken von $2 \mathrm{~N} / \mathrm{mm}^{2}, 4 \mathrm{~N} / \mathrm{mm}^{2}$ und $6 \mathrm{~N} / \mathrm{mm}^{2}$ gegenüber. Es wird deutlich, dass es bei den modifizierten Serien zu einer Steigerung der Biegefestigkeit durch Erhöhung des Pressdruckes kommt. Insbesondere bei $4 \mathrm{~N} / \mathrm{mm}^{2}$ ist dies der Fall und begründet sich vor allem in einer Zunahme der Darrdichte infolge von Verdichtung. Bei den Referenzen kam es jedoch erst ab einem Pressdruck von $6 \mathrm{~N} / \mathrm{mm}^{2}$ zu einer nennenswerten Steigerung - was ebenso im Einklang mit den Ergebnissen der Darrdichte stand. 


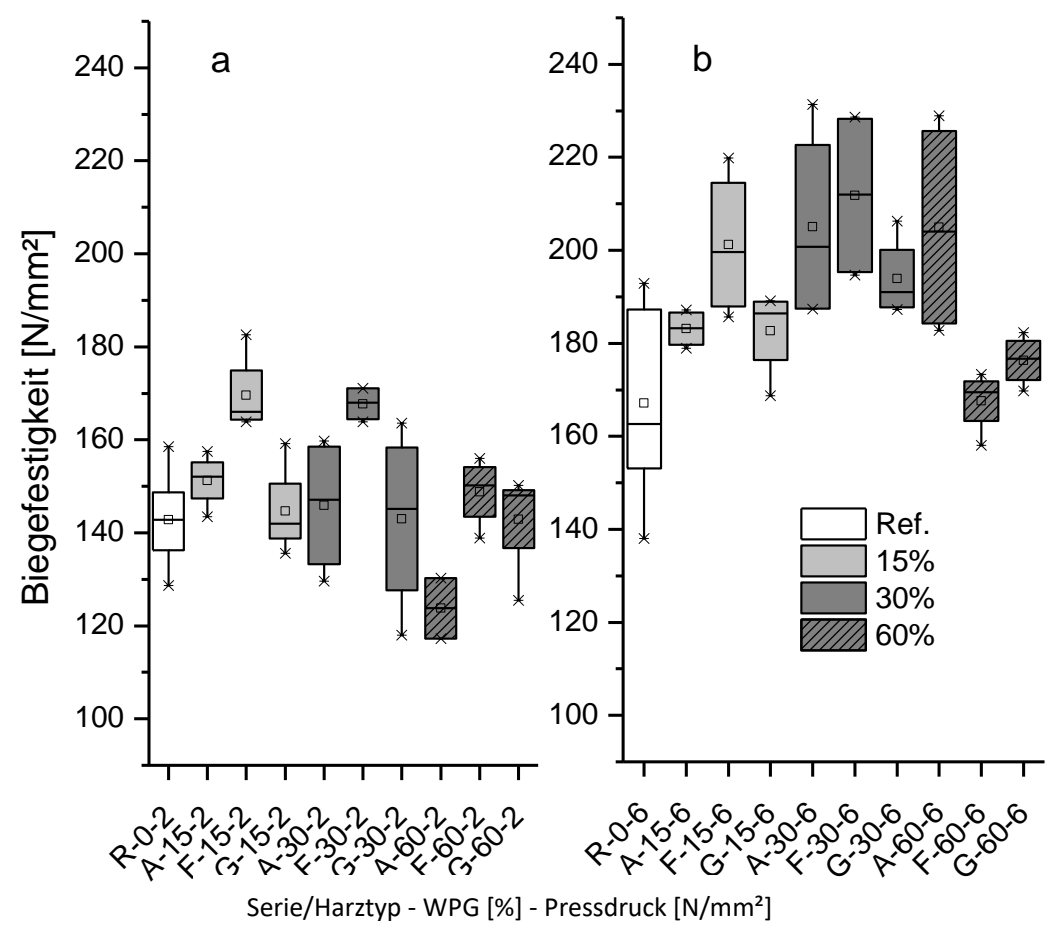

Abbildung 61: Biegefestigkeit von 8-lagigem Buchen-LVL ohne Sperrlagen bei unterschiedlichen WPGS unter Verwendung verschiedener Harze und Pressdrücke $\left(a=2 \mathrm{~N} / \mathrm{mm}^{2}, b=6 \mathrm{~N} / \mathrm{mm}^{2}\right)$.

Die Abbildung 61 zeigt, dass die Biegefestigkeit infolge der Modifikation der Pressparameter und der Verdichtung bei bestimmten Serien um bis zu 20\% gestiegen ist, jedoch vereinzelt auch eine Abnahme von bis zu 25\% stattfand. Bei Betrachtung der Ergebnisse für $2 \mathrm{~N} / \mathrm{mm}^{2}$ fällt auf, dass die Harze A, F und G bei einem WPG von 15\%, im Vergleich zur Referenz, erhöhte Biegefestigkeiten aufwiesen. Bei den gleichen Harzen mit einem WPG von 30\% kam es jedoch schon zu Ergebnissen, die unterhalb des Referenzniveaus lagen, wenngleich die Mittelwerte und der Median noch knapp darüber lagen. Bei einem WPG von 60\% war die Abnahme der Biegefestigkeit am deutlichsten bei den Harztypen A und F zu erkennen, während der Harztyp $G$ gleich dem Referenzwert war. Bei den Serien, die bei einem Pressdruck von $6 \mathrm{~N} / \mathrm{mm}^{2}$ hergestellt wurden, lagen alle Werte der modifizierten Serien mit einem WPG von 30\% höher als die der Referenz, die gegenüber dem niedrigeren Druck ebenfalls deutlich erhöht waren. Bei einem WPG von 60\% lagen die Harztypen F und G niedriger als die anderen Varianten, aber nicht unterschiedlich zu den Referenzen. 


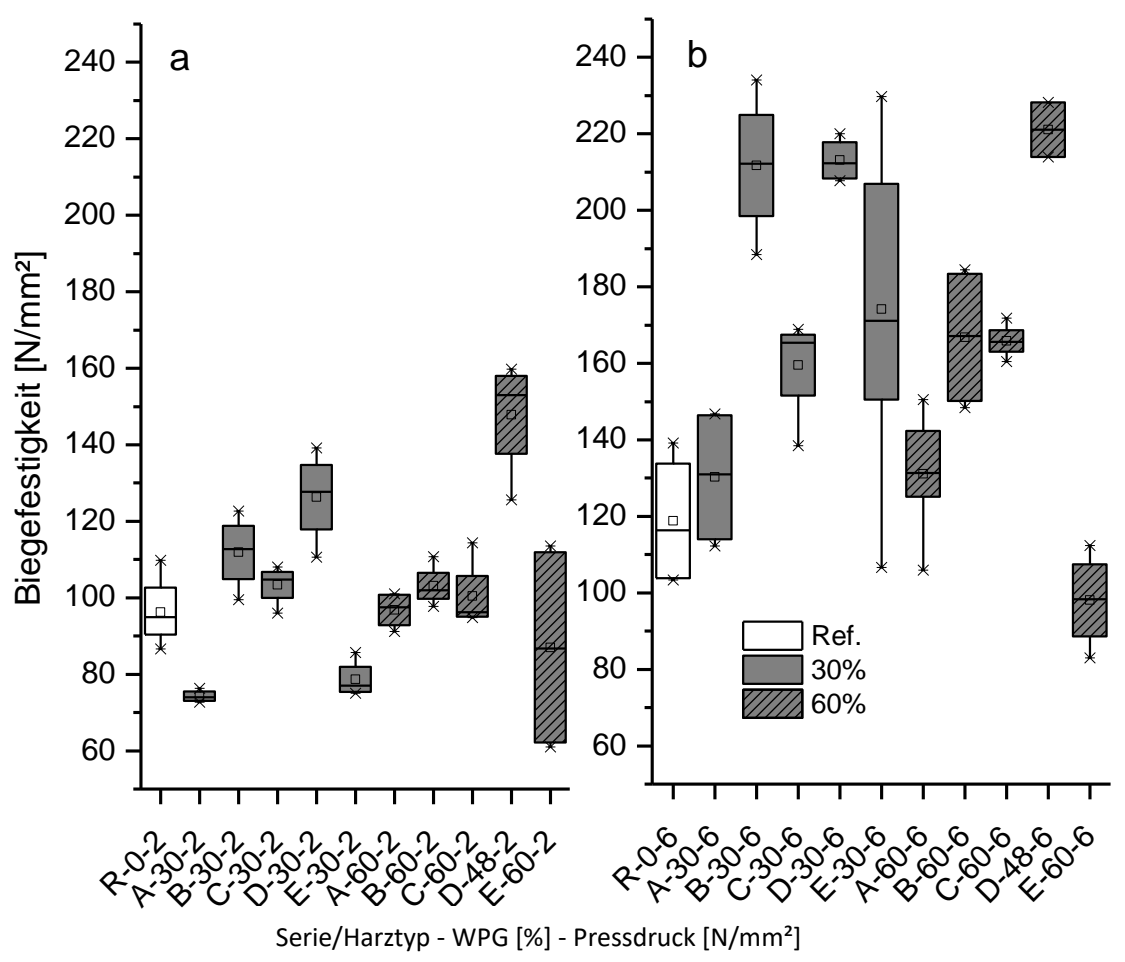

Abbildung 62: Biegefestigkeit von 8-lagigem Buchen-LVL mit zwei Sperrlagen bei unterschiedlichen WPGS unter Verwendung verschiedener Harze und Pressdrücke $\left(a=2 \mathrm{~N} / \mathrm{mm}^{2}, b=6 \mathrm{~N} / \mathrm{mm}^{2}\right)$.

Die Biegefestigkeiten von Buchen-LVL mit Sperrlagen waren, insbesondere bei einem Pressdruck von $2 \mathrm{~N} / \mathrm{mm}^{2}$, niedriger als beim LVL ohne Sperrlagen. Bei diesem Pressdruck fiel auf, dass ein WPG von 60\% schlechtere Werte lieferte als ein WPG von 30\%. Die Unterschiede zwischen den Harzen ergaben sich in der Form, dass die Harztypen A und E niedrigere Werte lieferten als die Harztypen B, C und D.

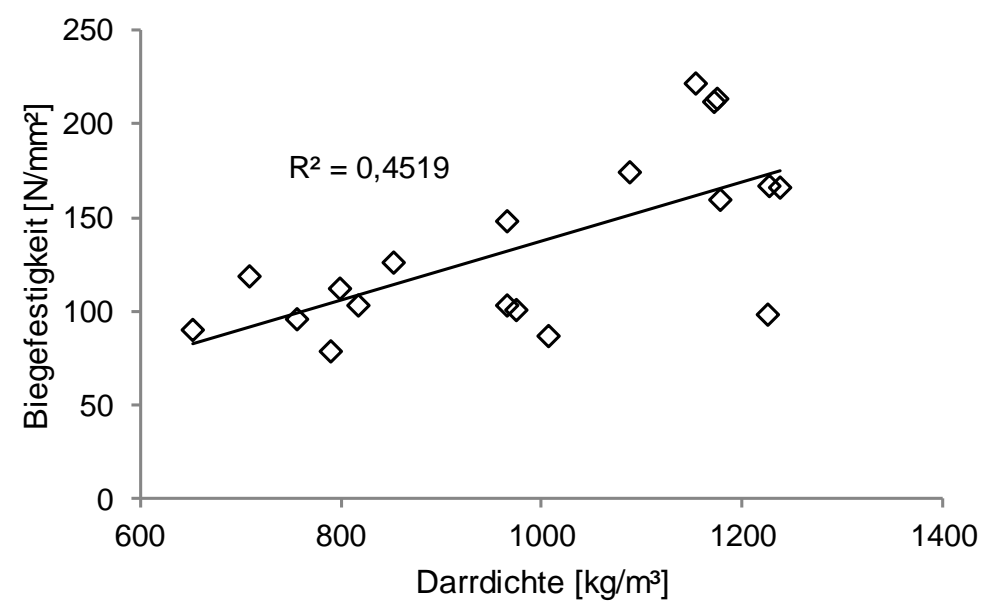

Abbildung 63: Korrelation von Biegefestigkeit und Darrdichte bei 8-lagigem Buchen-LVL mit Sperrlagen aus modifizierten und unbehandelten Furnieren. WPG: 0\%-60\%.

Um zu erklären, warum es zu höheren oder niedrigeren Biegefestigkeiten bei den unterschiedlichen Serien gekommen ist, wurden Korrelationen zwischen der Darrdichte und der Biegefestigkeit sowie der Verdichtung und der Biegefestigkeit erstellt. Dabei berücksichtigt wurde nur 8-lagiges Buchen-LVL mit 3,7 mm Furnierdicke. 
Bei unbehandeltem Holz und Holzwerkstoffen sind die Festigkeiten sehr eng mit der Rohdichte oder der Darrdichte korreliert (Kollmann 1951, Niemz 1993). Wie in Abbildung 63 und Abbildung $64 \mathrm{zu}$ erkennen, war bei den Versuchsserien die Korrelation zwar positiv vorhanden, jedoch mit einem niedrigen Bestimmtheitsmaß sehr unsicher.

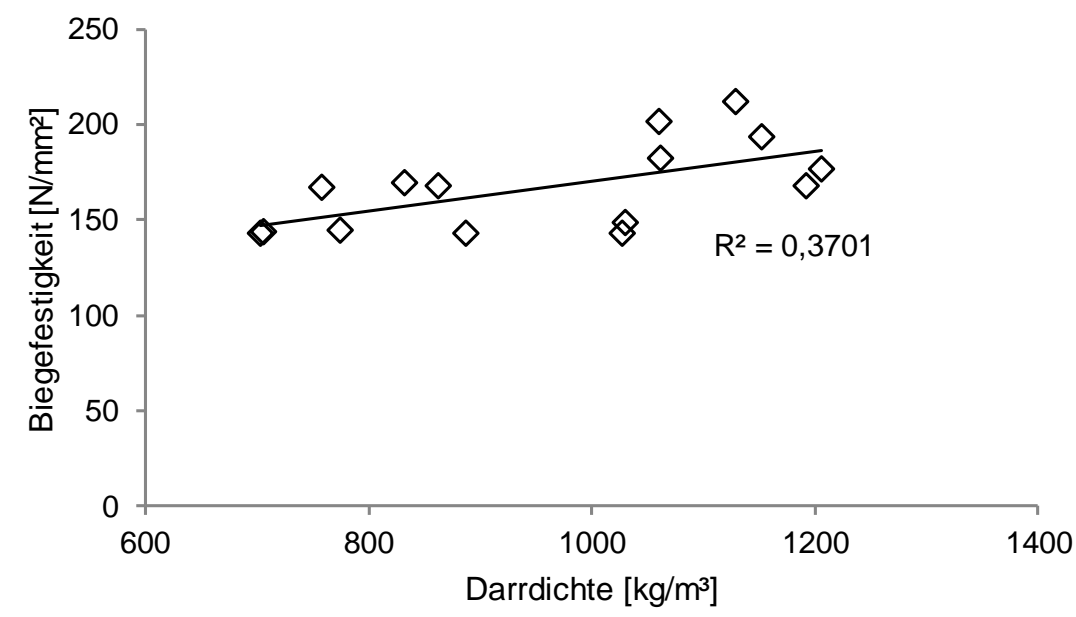

Abbildung 64: Korrelation von Biegefestigkeit und Darrdichte bei 8-lagigem Buchen-LVL ohne Sperrlagen aus modifizierten und unbehandelten Furnieren. WPG: 0\%-60\%.

Wie bereits bei den Ergebnissen zur Darrdichte erläutert, trug der WPG maßgeblich zu einer Dichteerhöhung bei, auch wenn die Platte bei einem niedrigen Pressdruck kaum eine Verdichtung erfuhr. Dadurch wird die Korrelation zwischen der Darrdichte und den Festigkeiten gestört. Um zu untersuchen wie die Verdichtung sich auf die Biegefestigkeit auswirkte, wurden die nachfolgenden Korrelationen erstellt.

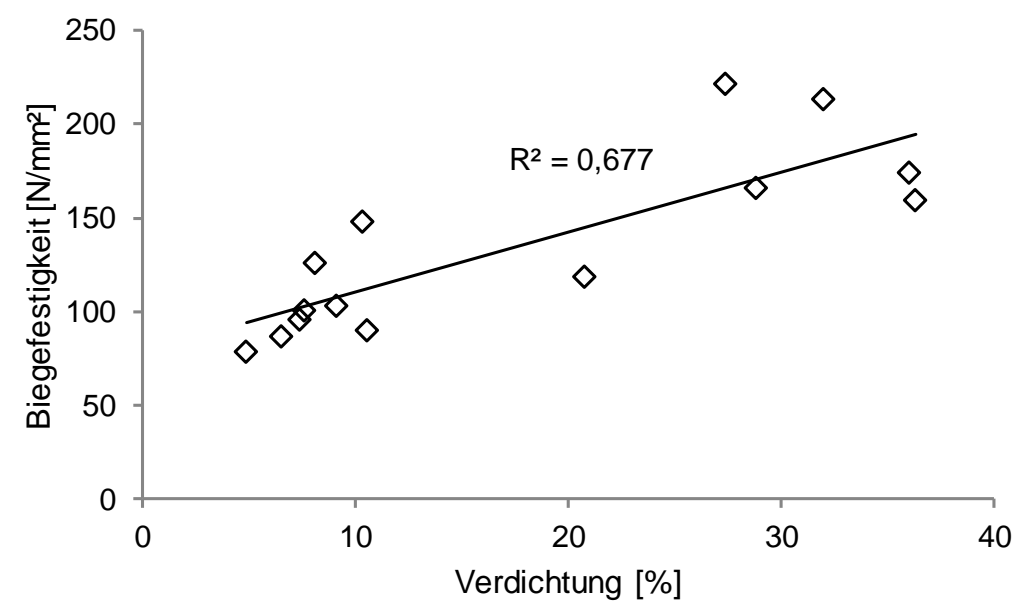

Abbildung 65: Korrelation von Biegefestigkeit und Verdichtung bei 8-lagigem Buchen-LVL mit Sperrlagen aus modifizierten und unbehandelten Furnieren. WPG: 0\%-60\%. 


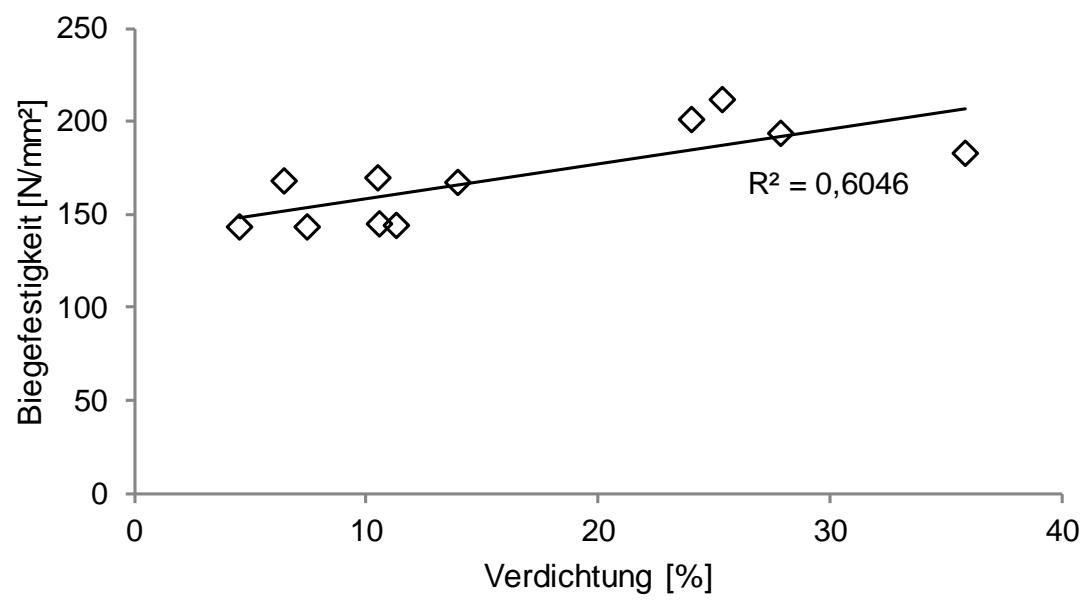

Abbildung 66: Korrelation von Biegefestigkeit und Verdichtung bei 8-lagigem Buchen-LVL ohne Sperrlagen aus modifizierten und unbehandelten Furnieren. WPG: 0\%-60\%.

Die Abbildung 65 und Abbildung 66 zeigen, dass die Korrelation zwischen der Biegefestigkeit und der Verdichtung nicht nur positiv, sondern auch deutlich sicherer ist (höheres Bestimmtheitsmaß) als die Korrelation mit der Darrdichte. Daraus ergibt sich die Schlussfolgerung, dass eine Steigerung der Biegefestigkeit vor allem durch die Verdichtung von Buchen-LVL zu erzielen ist. Wie bereits mit der Abbildung 42 gezeigt, hing diese Verdichtung wiederum davon ab, ob das Furnier mit PF-Harz imprägniert und welcher Pressdruck angewendet wurde.

Bei der Bestimmung der Biegefestigkeit erfolgte auch die Bestimmung des BiegeElastizitätsmoduls.

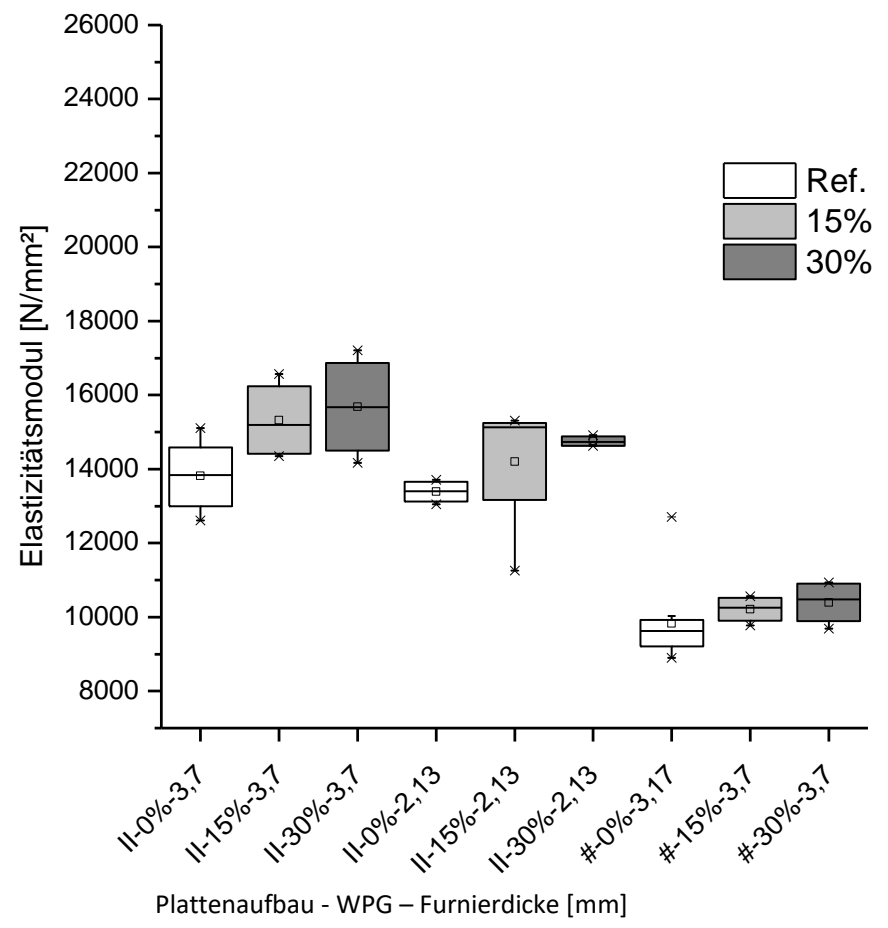

Abbildung 67: Biege-Elastizitätsmodul von Buchen-Furnierschichtholz mit 2 Sperrlagen (\#) und ausschließlich parallelem Faserverlauf (II). Variation der Schichtdicke $(3,7 \mathrm{~mm}$ und 2,13 mm) und des WPG bei einem Pressdruck von $2 \mathrm{~N} / \mathrm{mm}^{2}$ unter Verwendung des Harztyp A. 
Im Vergleich von Buchen-LVL, welches mit unterschiedlich dicken Furnieren mit einem WPG von maximal 30\% unter Verwendung des Harztyps A hergestellt wurde, zeigte sich eine Erhöhung des Elastizitätsmodul gegenüber der Referenz. Einen signifikanten Unterschied zwischen der Furnierdicke und dem WPG gab es nicht. Erwartungsgemäß lagen alle Serien, bei denen zwei von acht Furnieren als Sperrlagen ausgeführt wurden, niedriger als die Serien ohne Sperrlagen. Hier wiesen ebenso die modifizierten Serien die höheren Werte auf. Im Vergleich zur Biegefestigkeit bedeutet dies ein anderes Ergebnis, da dort keine Zunahme infolge der Modifizierung festgestellt wurde.

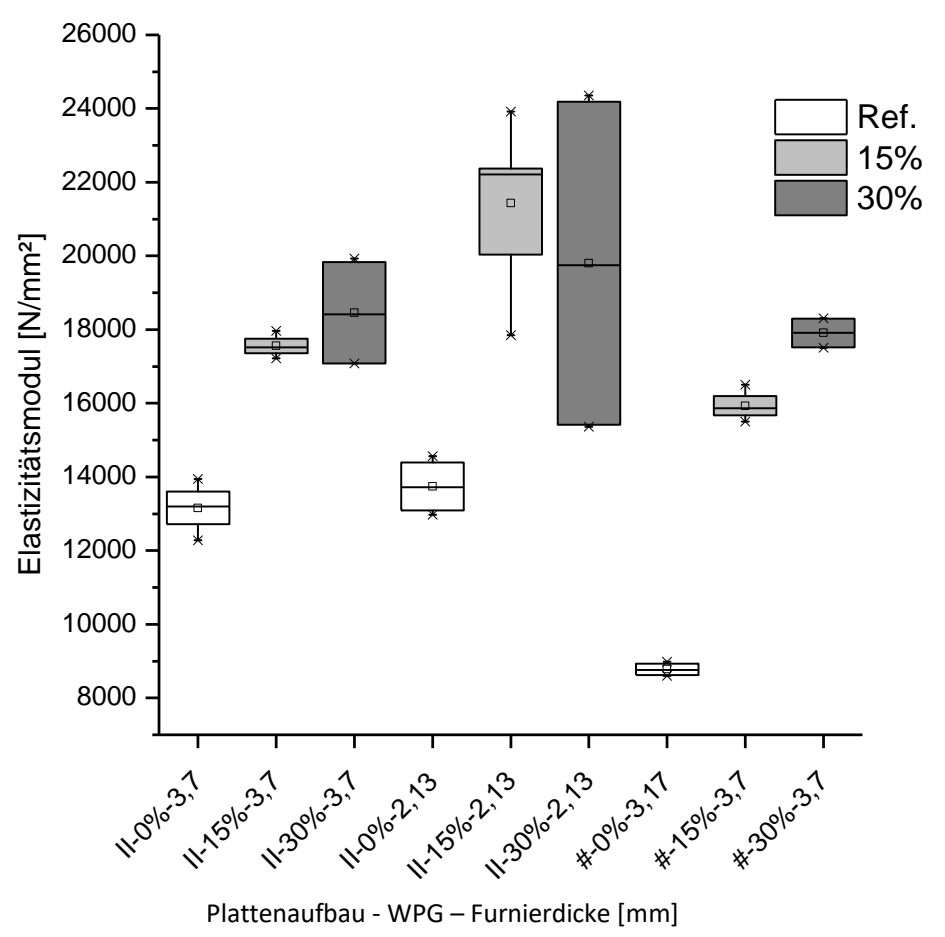

Abbildung 68: Biege-Elastizitätsmodul von Buchen-Furnierschichtholz mit 2 Sperrlagen (\#) und ausschließlich parallelem Faserverlauf (II). Variation der Schichtdicke $(3,7 \mathrm{~mm}$ und 2,13 mm) und des WPG bei einem Pressdruck von $4 \mathrm{~N} / \mathrm{mm}^{2}$ unter Verwendung des Harztyp A.

Das Buchen-LVL, welches mit einem höheren Pressdruck von $4 \mathrm{~N} / \mathrm{mm}^{2}$ hergestellt wurde und höhere Darrdichten aufwies, zeigte bei den modifizierten Serien deutlich höhere Werte für den Elastizitätsmodul. Die Referenzen blieben unverändert. Auch bei den Serien, die Sperrlagen enthielten, wiesen die Referenzen die niedrigsten Werte auf. Die modifizierten Serien hingegen zeigten eine deutliche Steigerung, die fast an das Ergebnis der Serien mit gleicher Furnierdicke ohne Sperrlagen heranreichte. Insgesamt wies die Entwicklung der Ergebnisse der Serien mit einem Pressdruck von $4 \mathrm{~N} / \mathrm{mm}^{2}$ eine größere Ähnlichkeit zu den Ergebnissen der Biegefestigkeit auf, als dies bei $2 \mathrm{~N} / \mathrm{mm}^{2}$ Pressdruck der Fall war. 


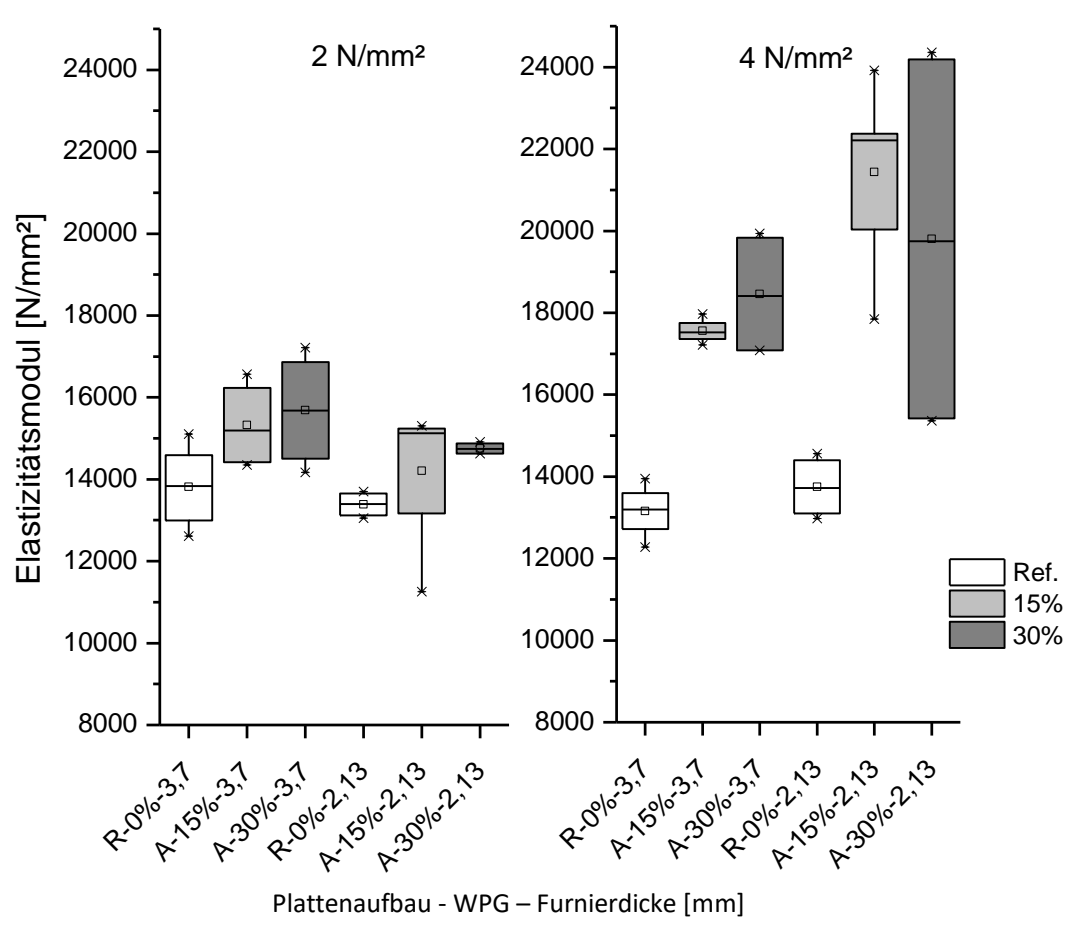

Abbildung 69: Biege-Elastizitätsmodul von Buchen-LVL ohne Sperrlagen im 3-Punkt-Biegeversuch mit 3,7 mm und 2,13 mm dicken Furnieren bei unterschiedlichen WPGs und Pressdrücken.

Die Abbildung 69 stellt noch einmal die Ergebnisse von Buchen-LVL ohne Sperrlagen mit ausschließlich parallelem Aufbau und unterschiedlichen Pressdrücken gegenüber. Es ist erkennbar, dass die Referenzen beider Furnierstärken ähnliche Werte lieferten und infolge eines höheren Pressdruckes keine Steigerung des Elastizitätsmoduls erfuhren. Die modifizierten Serien zeigten eine mit der Verdichtung im Einklang stehende Erhöhung auf, welche jedoch auch mit einer Erhöhung der Streuung der Werte einherging.

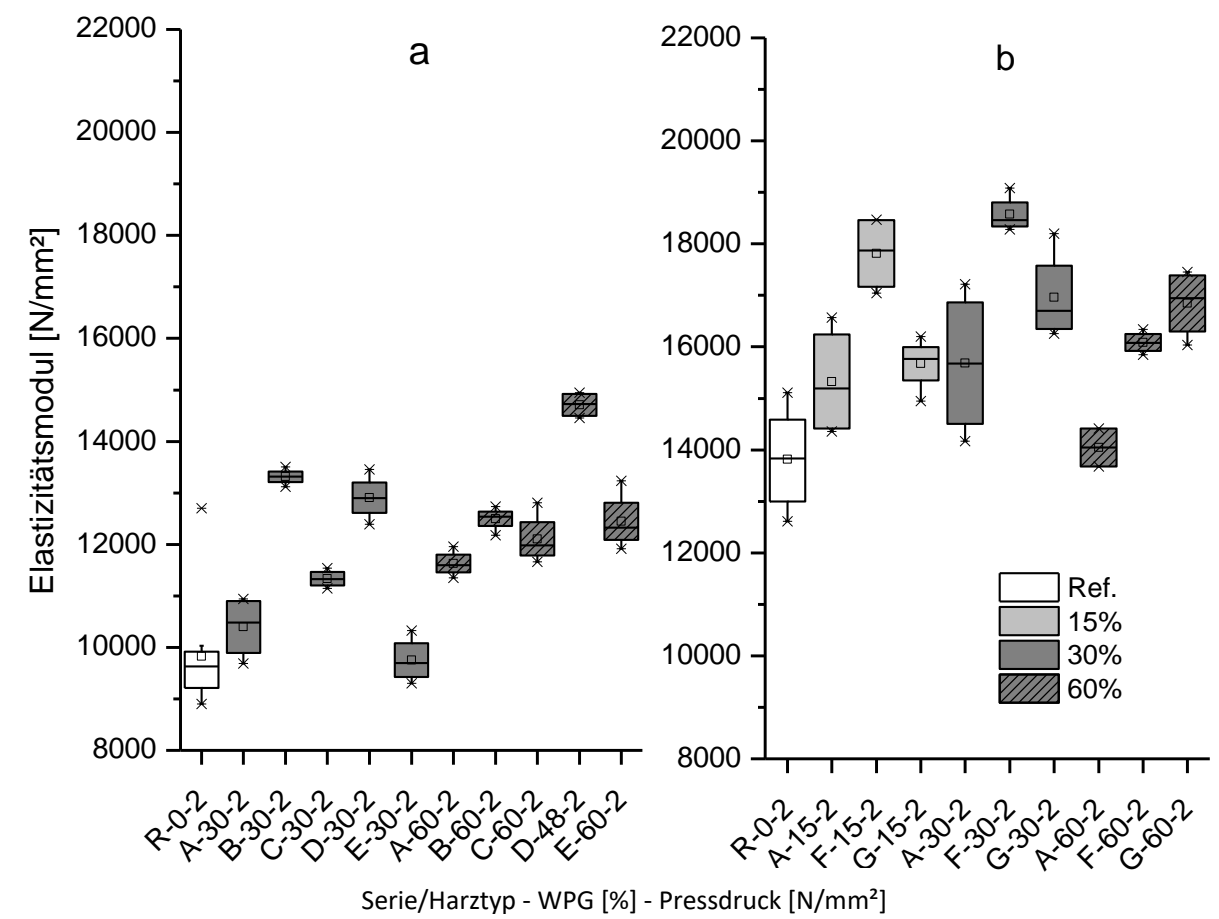

Abbildung 70 Biege-Elastizitätsmodul von 8-lagigem Buchen-LVL mit Sperrlagen (a) und ohne Sperrlagen (b) bei unterschiedlichen WPGs unter Verwendung verschiedener Harze beim Pressdruck von $2 \mathrm{~N} / \mathrm{mm}^{2}$. 
Mit dem Vergleich weiterer für die Modifizierung eingesetzter Harztypen zeigte sich eine größere Streuung der Ergebnisse für den Elastizitätsmodul. Die im linken Bereich unter (a) in der Abbildung 70 dargestellten Ergebnisse liegen aufgrund eines Plattenaufbaus mit zwei Sperrlagen niedriger als die auf der rechten Seite der Abbildung unter (b). Bei einem WPG von $30 \%$ wiesen die Harztypen zueinander signifikante Unterschiede auf, die nicht über die Dichte erklärt werden können. Mit einem WPG von $60 \%$ zeigen sich ähnliche Werte wie bei $30 \%$. Bis auf den Harztyp D liegen die Ergebnisse näher beieinander. Die Darrdichten dieser Prüfkörper lagen ebenso nah beieinander, aber auch deutlich höher als bei 30\% WPG. Der Umstand, dass hier insgesamt jedoch kein höherer Elastizitätsmodul festgestellt werden konnte, verdeutlicht, dass es keinen linearen Zusammenhang zwischen dem WPG und diesem Kennwert gab, sondern die Verdichtung den größeren Einfluss hatte.

Bei den modifizierten Serien ohne Sperrlagen auf der rechten Seite der Abbildung 70 (b) zeigten sich auch einige Unterschiede zwischen den Harztypen. Bei genauerer Betrachtung der jeweiligen Verdichtung und der erzielten Darrdichte erklären sich die Unterschiede. Bei einem WPG von 15\% wies der Harztyp A zwar eine Verdichtung von 16\% auf, jedoch nur eine Darrdichte von $739 \mathrm{~kg} / \mathrm{m}^{3}$. Mit geringerer Verdichtung, jedoch einer Darrdichte von $832 \mathrm{~kg} / \mathrm{m}^{3}$ bei gleichem WPG, konnte Harztyp F einen höheren Elastizitätsmodul erreichen. Ebenso ist bei dem WPG von 30\% der höhere Wert des Harztyps F, im Vergleich zum Harztyp A, auf dessen höhere Darrdichte zurückzuführen. Trotz höherer Darrdichten konnten hier wie beim LVL mit Sperrlagen die Serien mit einem WPG von 60\% keine höheren Werte erzielen. Es wird daraus geschlossen, dass beim PF-modifizierten LVL der Verdichtung und der damit erzielten Darrdichte eine größere Bedeutung hinsichtlich der mechanischen Eigenschaften zukommt, als der Höhe des WPG oder dem Harztyp, wenn es sich um niedermolekulare Harze handelt.

\subsubsection{Bruchschlagarbeit}

Für die Beurteilung der Ergebnisse der Bruchschlagarbeit wurden die Verdichtung, die Darrdichte, der Elastizitätsmodul sowie die Holzfeuchten (regulär und korrigiert) der verwendeten Proben der Auswertung der Bruchschlagarbeit vorangestellt. 

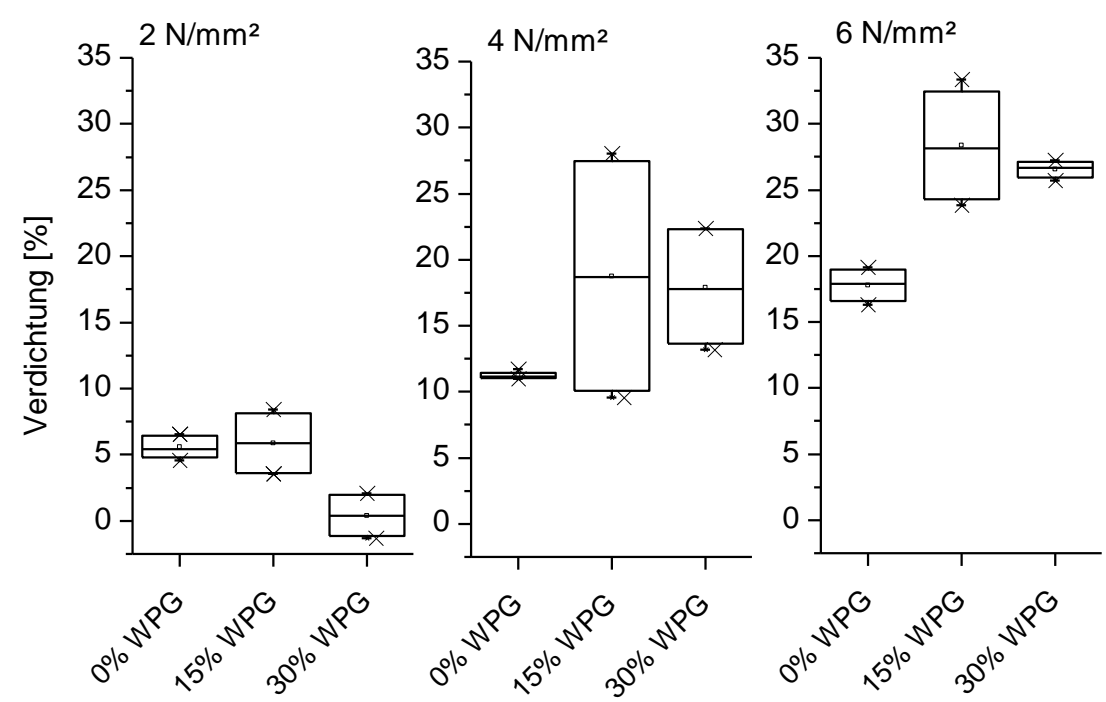

Abbildung 71: Verdichtung der Prüfkörper für die Ermittlung der Bruchschlagarbeit. 8-lagiges Buchen-LVL ohne Sperrlagen; Furnierdicke 3,7 mm, Harztyp A.

Entsprechend der vorangegangenen Untersuchungen zeigen die Prüfkörper der Bruchschlagarbeit eine größere Verdichtung bei höherem Pressdruck. Die Referenzen lagen in der Regel unter den modifizierten Serien, welche sich beim jeweiligen Pressdruck nicht voneinander unterschieden.
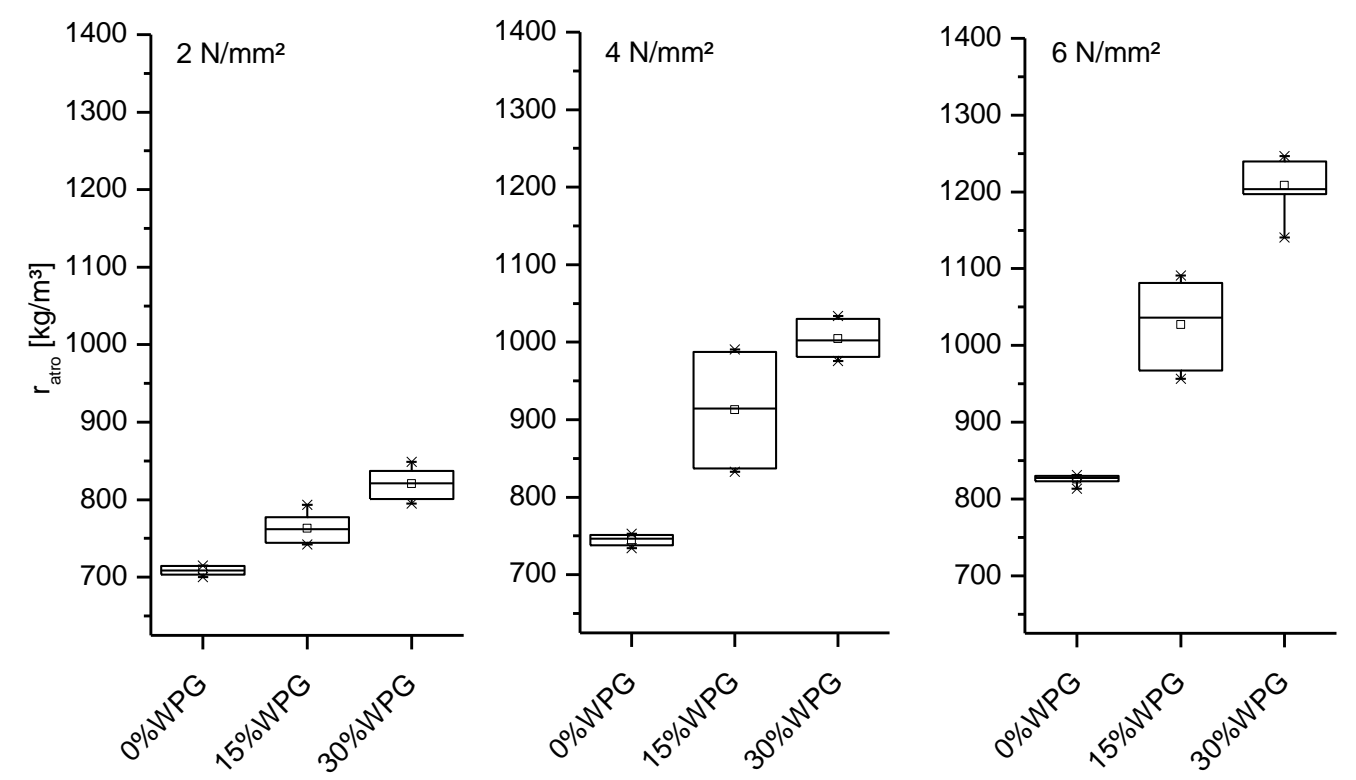

3

Abbildung 72: Darrdichte der Prüfkörper bei der Ermittlung der Bruchschlagarbeit an 8-lagigem BuchenFurnierschichtholz mit einer Furnierdicke von 3,7mm. Harztyp A.

In der Kombination von Verdichtung und dem WPG ergaben sich die in Abbildung 72 dargestellten Darrdichten. Sie wiesen mit zunehmenden WPG und Pressdruck gesteigerte Werte auf. 


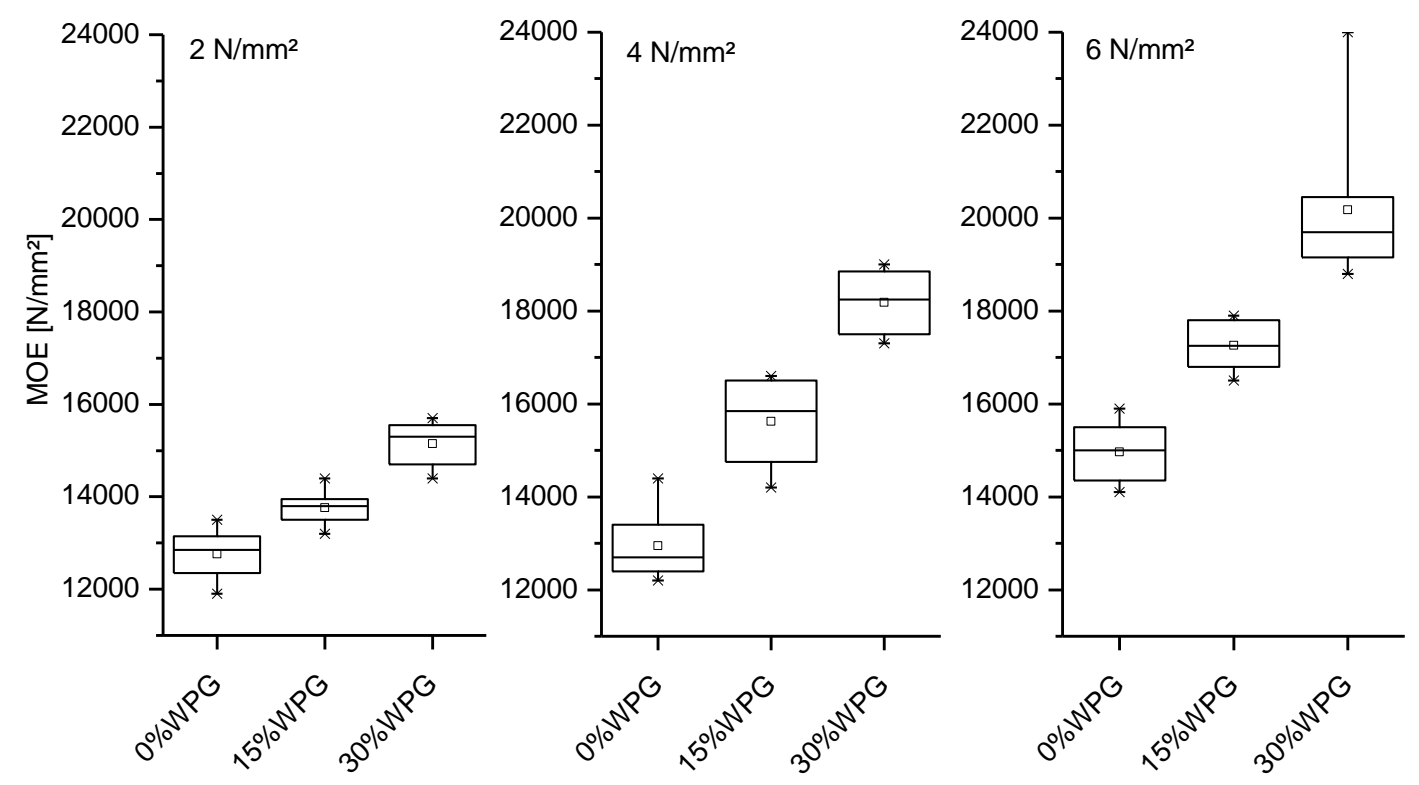

Abbildung 73: Biege-Elastizitätsmodul (MOE) der Prüfkörper bei der Ermittlung der Bruchschlagarbeit an 8-lagigem Buchen-Furnierschichtholz mit einer Furnierdicke von $3,7 \mathrm{~mm}$ bei unterschiedlichen Pressdrücken.

Der an den Prüfkörpern der Bruchschlagarbeit ermittelte Elastizitätsmodul wies für die WPGs 15\% und 30\% beim jeweiligen Pressdruck unterschiedliche Werte auf. Diese Beobachtung konnte auch bei den Ergebnissen der Biegeprüfkörper mit den gleichen WPGs gemacht werden, wenngleich sie weniger deutlich ausgeprägt war. Insgesamt wiesen die hier gezeigten Serien ein mit dem WPG und dem Pressdruck steigendes Elastizitätsmodul auf. Hinsichtlich der Holzfeuchten fiel auf, dass die PF- modifizierten Serien, auch nach einer Korrektur um den WPG (Formel 15), eine umso geringere Holzfeuchte aufwiesen je höher der WPG war.

Tabelle 14: Holzfeuchte (gesamt) und korrigierte Holzfeuchte der Proben bei der Ermittlung der Bruchschlagarbeit an 8-lagigem Buchen-Furnierschichtholz mit einer Furnierdicke von 3,7 mm bei unterschiedlichen Pressdrücken.

\begin{tabular}{|c|c|c|c|}
\hline Druck & $2 \mathrm{~N} / \mathrm{mm}^{2}$ & & \\
\hline WPG & $0 \%$ & $15 \%$ & $30 \%$ \\
\hline $\mathrm{N}$ gesamt & 8 & 16 & 8 \\
\hline Holzfeuchte[\%] & $8,7 \pm 0,1$ & $6,4 \pm 0,1$ & $4,8 \pm 0,1$ \\
\hline korr. Holzfeuchte [\%] & $8,7 \pm 0,1$ & $7,2 \pm 0,2$ & $6,2 \pm 0,1$ \\
\hline Druck & $4 \mathrm{~N} / \mathrm{mm}^{2}$ & & \\
\hline WPG & $0 \%$ & $15 \%$ & $30 \%$ \\
\hline $\mathrm{N}$ gesamt & 8 & 16 & 8 \\
\hline Holzfeuchte[\%] & $8,2 \pm 0,4$ & $7,0 \pm 1,0$ & $4,8 \pm 0,6$ \\
\hline korr. Holzfeuchte [\%] & $8,2 \pm 0,4$ & $7,8 \pm 1,1$ & $6,3 \pm 0,7$ \\
\hline Druck & $6 \mathrm{~N} / \mathrm{mm}^{2}$ & & \\
\hline WPG & $0 \%$ & $15 \%$ & $30 \%$ \\
\hline $\mathrm{N}$ gesamt & 8 & 16 & 8 \\
\hline Holzfeuchte[\%] & $8,0 \pm 0,3$ & $7,5 \pm 1,1$ & $5,2 \pm 0,5$ \\
\hline korr. Holzfeuchte [\%] & $8,0 \pm 0,3$ & $8,4 \pm 1,2$ & $6,7 \pm 0,7$ \\
\hline
\end{tabular}



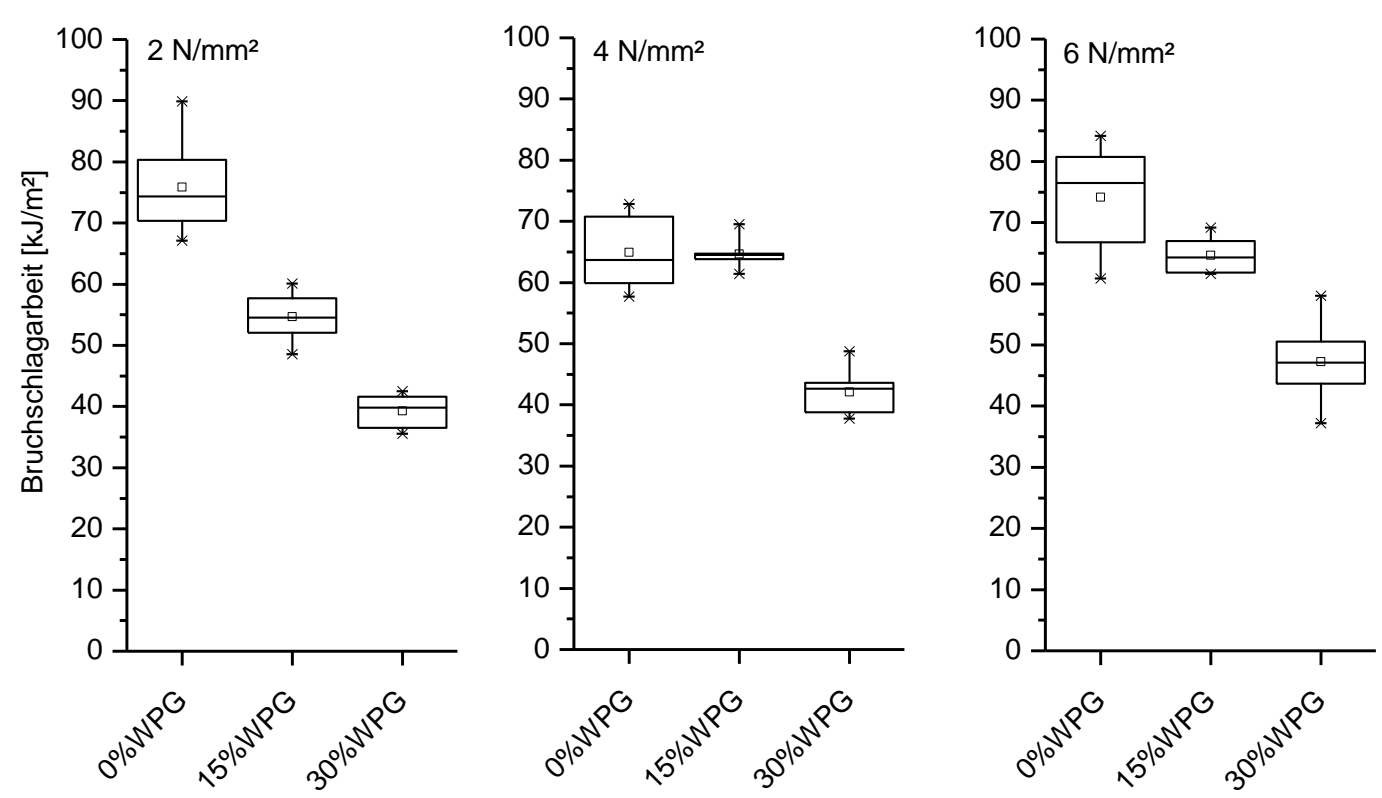
Abbildung 74: Ergebnisse der Bruchschlagarbeitsprüfung an 8-lagigem Buchen-Furnierschichtholz mit
einer Furnierdicke von 3,7 mm bei unterschiedlichen Pressdücken und WPGs mit dem Harztyp A.

Die in Abbildung 74 dargestellten Ergebnisse zur Bruchschlagarbeit zeigen, dass mit der Modifizierung eine Abnahme dieses Kennwertes verbunden war. Die Referenzen zeigten ein weitgehend vom Pressdruck unabhängiges Ergebnis, da auch ihre Darrdichten wenig Veränderung erfuhren. Auch bei den modifizierten Serien konnten zwischen den Pressdrücken keine Unterschiede festgestellt werden, wenngleich zwischen den WPGs jeweils ein signifikanter Unterschied vorlag - der höhere WPG erzielte stets niedrigere Werte. Einen Einfluss wird hier auch die geringere Holzfeuchte der modifizierten Serien gehabt haben, da die Dehnung von Holz positiv mit dessen Feuchte korreliert ist und somit niedrigere Holzfeuchten auch eine niedrigere Bruchdehnung herbeiführen.

Mit den Werten des Elastizitätsmoduls zeigte die Bruchschlagarbeit eine negative Korrelation (Abbildung 75, Abbildung 76 und Abbildung 77). Demnach hat die größere Steifigkeit einen negativen Einfluss gehabt. Das ergibt insoweit Sinn, da weniger Verformung bei gleicher Kraft stattfinden und somit deutlich weniger Energie des auftreffenden Hammers in Wärme umgewandelt werden konnte. 


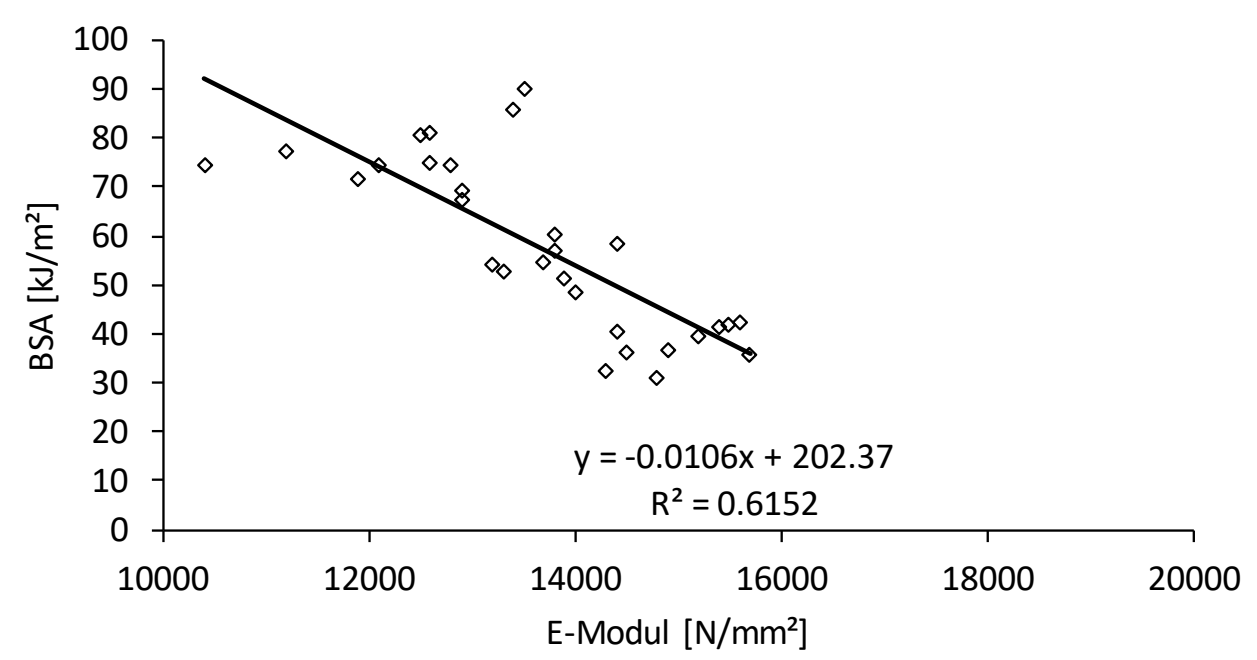

Abbildung 75: Korrelation zwischen Elastizitätsmodul und Bruchschlagarbeit von Buchen-LVL, modifziert mit Harztyp A mit WPGs von 0\%, 15\%, 30\% und 60\%, bei einem Pressdruck von 2 N/mm².

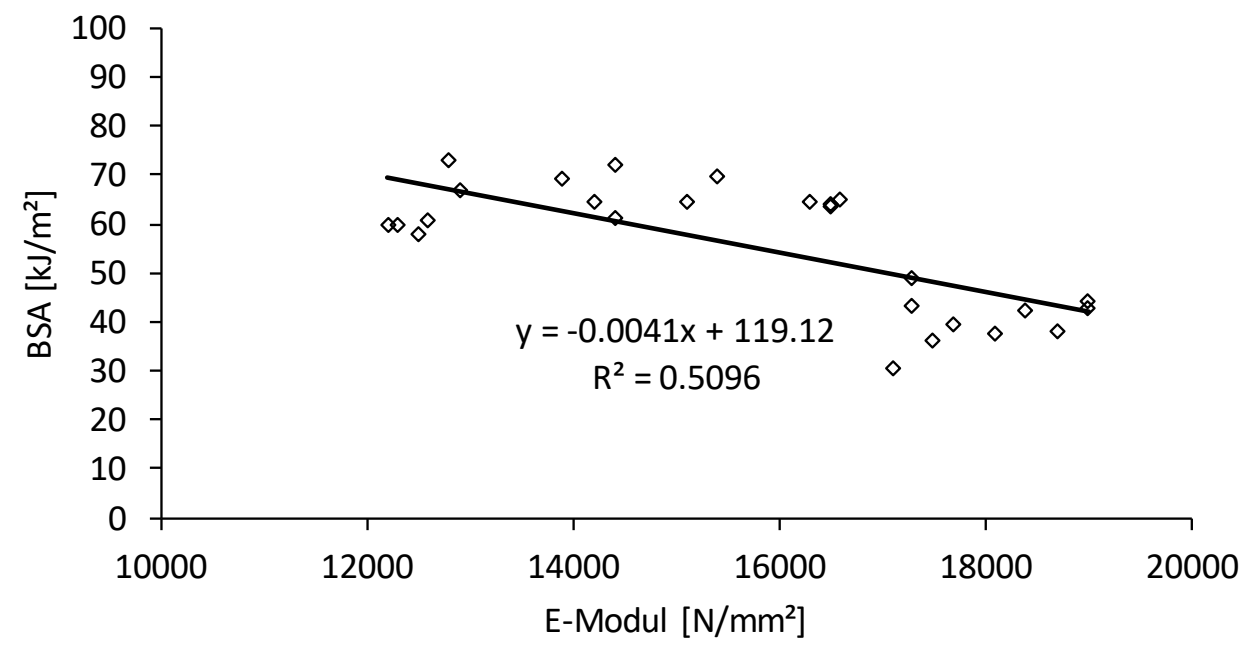

Abbildung 76: Korrelation zwischen Elastizitätsmodul und Bruchschlagarbeit von verdichtetem BuchenLVL, modifziert mit Harztyp A mit WPGs von 0\%, 15\%, 30\% und 60\%, bei einem Pressdruck von $4 \mathrm{~N} / \mathrm{mm}^{2}$. 


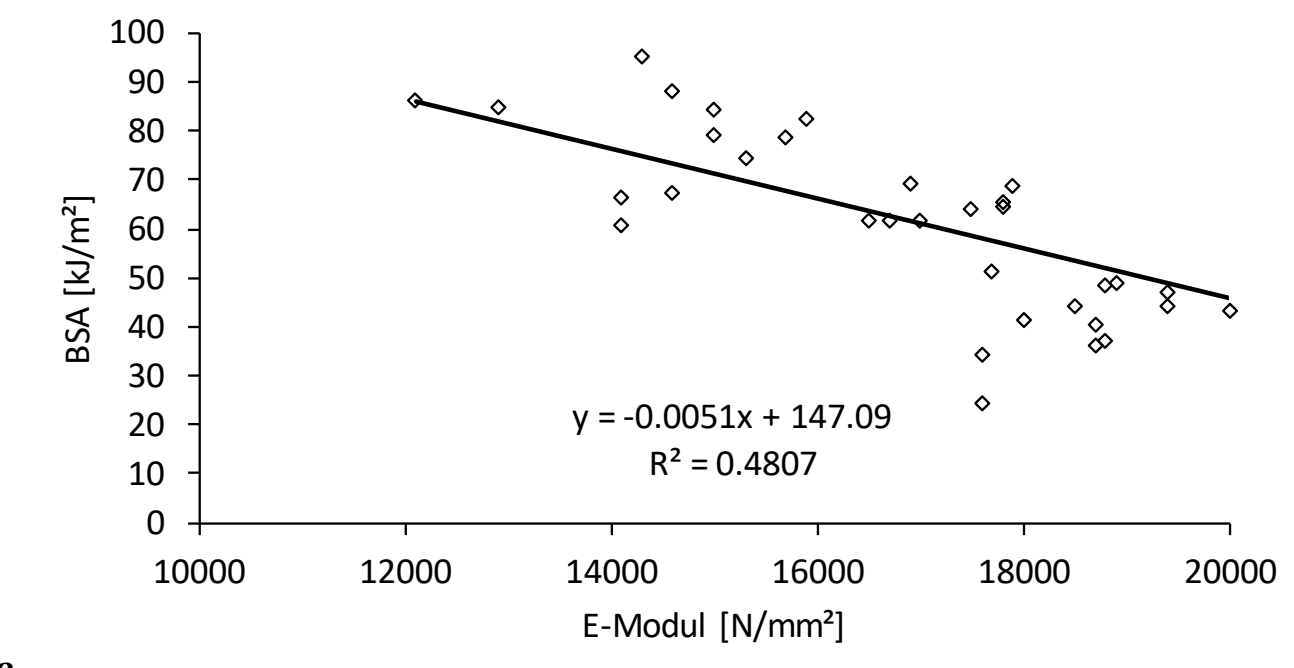

3

Abbildung 77: Korrelation zwischen Elastizitätsmodul und Bruchschlagarbeit von verdichtetem BuchenLVL, modifiziert mit Harztyp A mit WPGs von 0\%, 15\%, 30\% und 60\%, bei einem Pressdruck von 6 N/mm².

\subsubsection{Formaldehydabgabe}

Abbildung 78 zeigt die Ausgleichsfunktionen und Einzelwerte der Formaldehydabgabe von 10lagigem Buchen-LVL mit einem WPG von 30\%. Es wird ersichtlich, dass die Ausgleichsfunktion für die Platte mit einer Presszeit von 30 min deutlich oberhalb derer verläuft, die $45 \mathrm{~min}$ gepresst wurde. Bei beiden Platten lag die Pressentemperatur bei $145^{\circ} \mathrm{C}$, jedoch lag die erzielte Temperatur in Plattenmitte darunter, wie Abbildung 79 zeigt. Es wird auch ersichtlich, dass die Werte auch nach 28 Tagen noch fielen und nicht konstant waren. Beide berechneten Konzentrationskurven wiesen bis zuletzt innerhalb von 4 Tagen einen Abfall auf, der größer war als 5\%. Gemäß der DIN EN 717-1 (2005) wurde somit die Konzentration am 28. Tag (672h) der Messung als Ausgleichskonzentration bestimmt. Somit ergaben sich für das modifizierte Buchen LVL mit Presszeiten von 30 min und 45 min Ausgleichskonzentrationen von $0,14 \mathrm{mg} / \mathrm{m}^{3}$ und $0,08 \mathrm{mg} / \mathrm{m}^{3}$. Das LVL mit der längeren Presszeit unterschritt den E1Grenzwert $0,124 \mathrm{mg} / \mathrm{m}^{3}$ nach 13 Tagen, während das LVL der kürzeren Presszeit bis zuletzt darüber lag. 


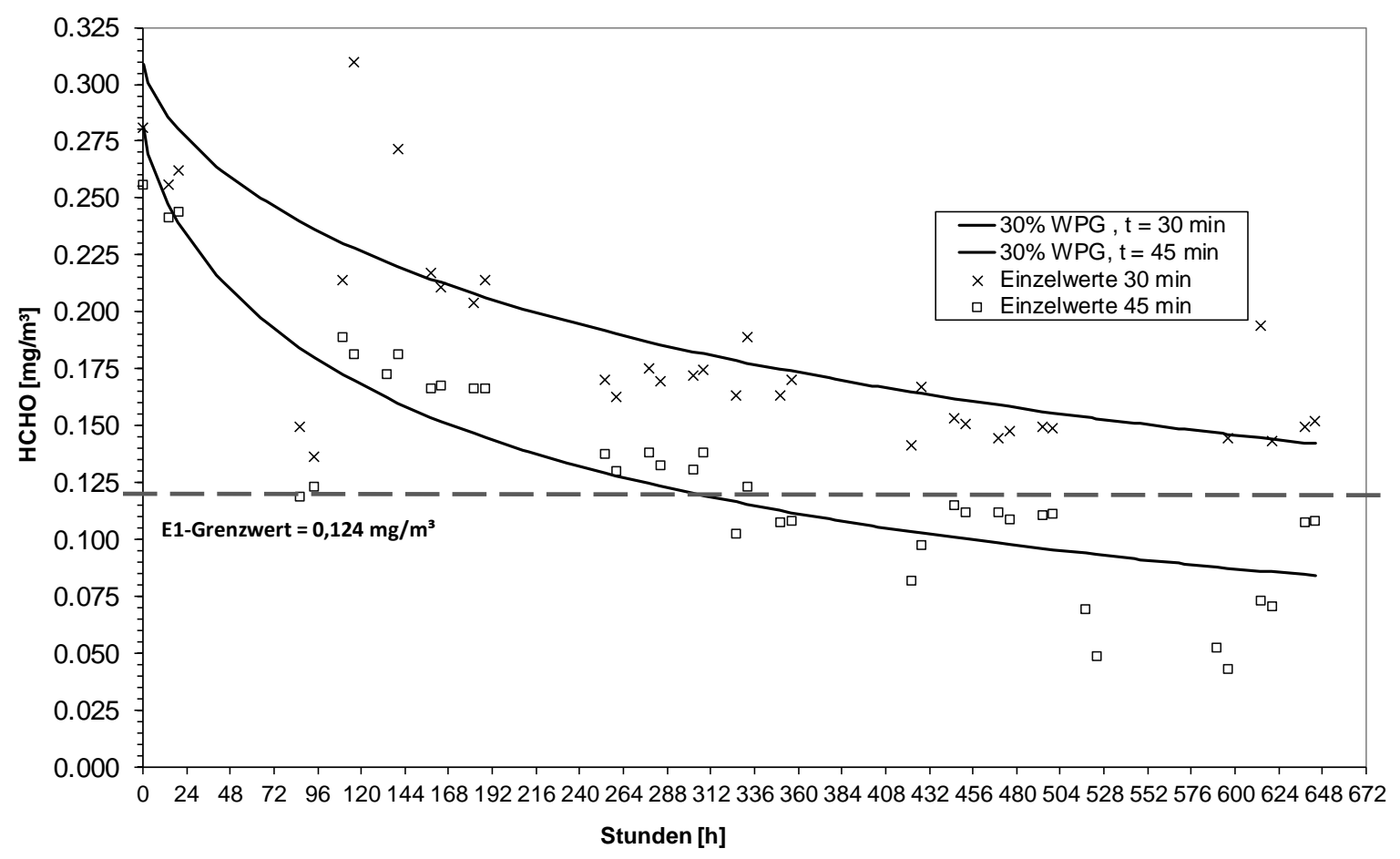

Abbildung 78: Formaldehydabgabe (Ausgleichsfunktion und Einzelwerte) von Buchen-LVL $(10 * 2,13 \mathrm{~mm})$ mit einem WPG von 30\% bei zwei unterschiedlichen Presszeiten, gemessen nach Prüfkammer-Methode (DIN EN 717-1: 2005).

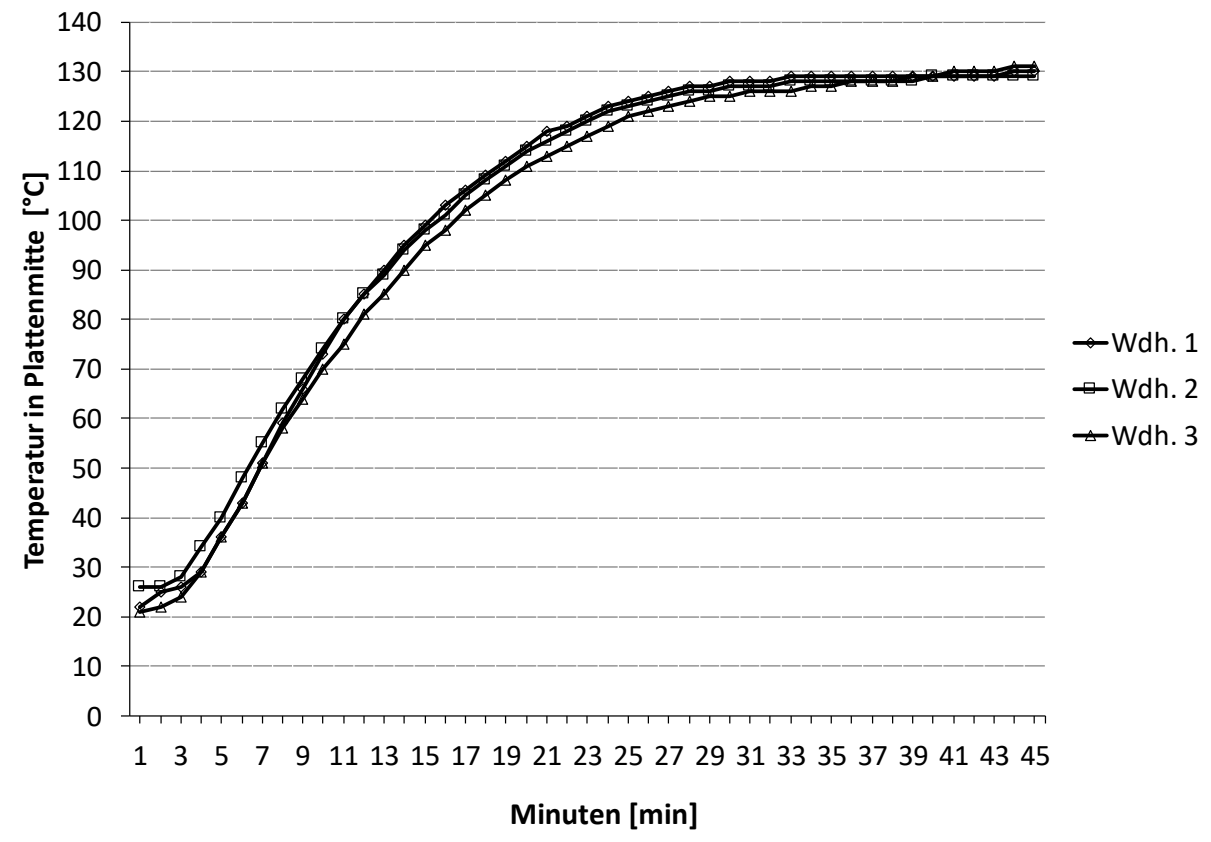

Abbildung 79: Temperaturverlauf in der Plattenmitte von Buchen-LVL mit einem WPG 30\% bei einem Pressdruck von $2 \mathrm{~N} / \mathrm{mm}^{2}$ und einer Presstemperatur von $145^{\circ} \mathrm{C}$.

In Abbildung 80 dargestellt, ist die Formaldehydabgabe von Buchen-LVL nach der GasanalyseMethode (DIN EN 717-2: 1994) für drei unterschiedliche Harztypen beim WPG von 30\% und einer unbehandelte Referenz. 


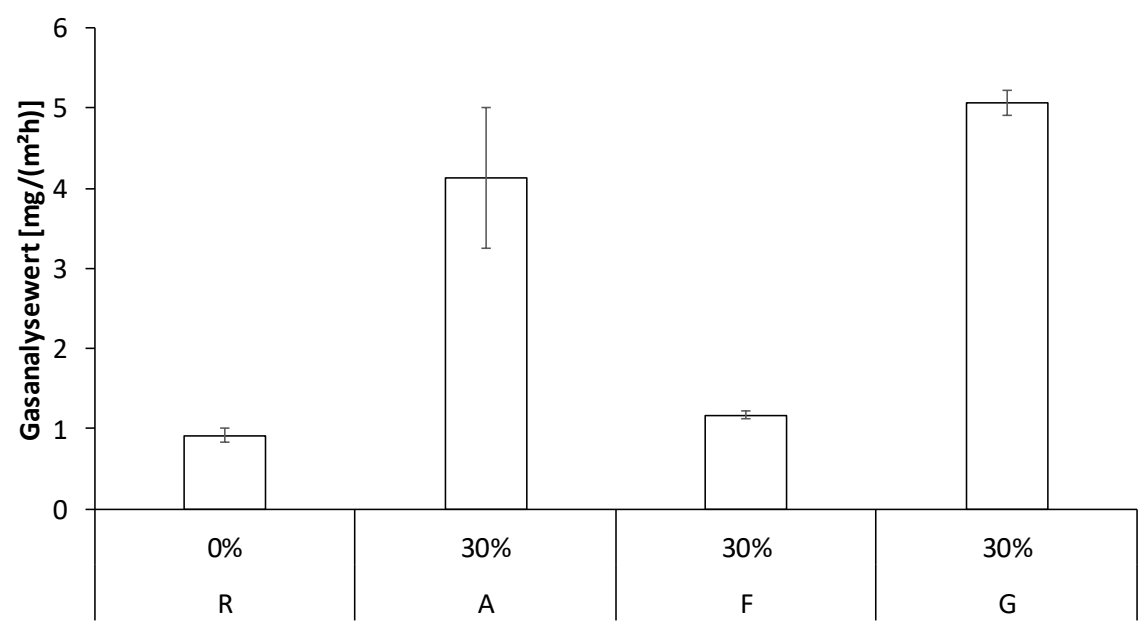

Abbildung 80: Formaldehydabgabe von 8-lagigem Buchen-LVL $(8 * 3,7 \mathrm{~mm})$ unter Verwendung unterschiedlicher Harztypen beim WPG von 30\% und einer Presszeit von $45 \mathrm{~min}(\sim$ 1,5 min / mm), gemessen nach der Gasanalyse-Methode (DIN EN 717-2: 1994).

Im Vergleich der Gasanalysewerte wird ersichtlich, dass auch bei ausschließlicher Anwendung der längeren Presszeit (45 min) einige Platten resp. Harztypen eine signifikant höhere Formaldehydabgabe aufwiesen, als die unbehandelte Referenz. Dies kann auf unterschiedliche Konzentrationen an freiem Formaldeyhd in den PF-Harzen hindeuten. Zum Teil kann das Ergebnis auch auf ein unterschiedliches Probenalter zurückgeführt werden. Aus den Ergebnissen der Kammermethode wurde ein starker Abfall der Formaldehydabgabe in den ersten Tagen ersichtlich. An Proben welche eine Woche gelagert wurden, konnte eine Halbierung des Messwertes stattgefunden haben.

Generell wird hinsichtlich der Formaldehydabgabe von PF-modifiziertem Buchen-LVL deutlich, dass ein Potenzial erhöhter Emissionen besteht, jedoch der Ausgangsgehalt freien Formaldehyds im Harz, die Presszeit, die Temperatur in der Plattenmitte und das Probenalter zum Zeitpunkt der Messung einen Einfluss auf das Ergebnis haben.

\subsubsection{Biologische Dauerhaftigkeit}

\subsubsection{Basidiomyceten}

In Tabelle 15 sind die mittleren Holzfeuchten und die unter Berücksichtigung des WPG korrigierten Holzfeuchten der Prüfköper vor Einbau in den Abbauversuch mit T. versicolor aufgelistet. Die normale Berechnung der Holzfeuchte, gemäß Formel 14, führte, ohne Berücksichtigung der im Holz eingelagerten Menge an PF, zu fallenden Ergebnissen mit steigendem WPG und Druck. Eine Korrektur der Bezugsgröße durch Reduzierung der absolut trockenen Masse (Formel 15), um den Anteil PF (WPG), führte zu vergleichsweise höheren Ergebnissen. Die modifizierten Serien mit dem niedrigeren Pressdruck lagen dabei näher an den unbehandelten Referenzen, als die modifizierten Serien mit dem höheren Pressdruck. Die Einlagerung des PF-Harzes in die Zellwand, hat somit die Ausgleichsfeuchte im Normalklima kaum beeinflusst, wenngleich die Gewichtskonstanz nach 5 Wochen der in der Lagerung im Normalklima noch nicht bei allen Proben erreicht war. Im Durchschnitt aller PF-modifizierter Serien lag die korr. Holzfeuchte (HF) bei 9,2\% und die der Referenzen bei 10,7\%. 
Tabelle 15: Mittelwerte und Standardabweichungen der Holzfeuchten und korrigierten Holzfeuchten (exkl. PF) des Buchen-LVLs für alle Harztypen gruppiert nach dem WPG und dem Pressdruck.

\begin{tabular}{cccccc}
$\begin{array}{c}\text { WPG } \\
{[\%]}\end{array}$ & $\begin{array}{c}\text { Druck } \\
{\left[\mathrm{N} / \mathrm{mm}^{2}\right]}\end{array}$ & $\begin{array}{c}\text { HF } \\
{[\%]}\end{array}$ & $\begin{array}{c}\text { Stabw. } \\
\text { HF [\%] }\end{array}$ & $\begin{array}{c}\text { korr. HF } \\
{[\%]}\end{array}$ & $\begin{array}{c}\text { Stabw. } \\
\text { korr. HF [\%] }\end{array}$ \\
\hline 0 & 2 & 10.7 & 0.2 & 10.7 & 0.2 \\
& 4 & 10.6 & 0.2 & 10.5 & 0.2 \\
& 6 & 10.7 & 0.3 & 10.7 & 0.4 \\
\hline 15 & 2 & 8.7 & 0.4 & 9.7 & 0.4 \\
& 6 & 7.9 & 0.4 & 9.4 & 0.2 \\
\hline 30 & 2 & 7.6 & 0.6 & 9.3 & 0.7 \\
& 6 & 6.3 & 0.8 & 8.2 & 1.2 \\
\hline 48 & 2 & 5.6 & 0.5 & 8.3 & 0.8 \\
& 6 & 2.2 & 0.4 & 3.2 & 0.7 \\
\hline 60 & 2 & 6.4 & 0.7 & 10.4 & 1.3 \\
& 6 & 4.7 & 1.3 & 5.6 & 0.5
\end{tabular}

Nachfolgend werden die Masseverluste von Buchen-LVL nach 16-wöchiger Inkubation mit dem Weißfäuleerreger Trametes versicolor, getrennt nach den Plattentypen bzw. der Dicke der eingesetzten Furniere, dargestellt.

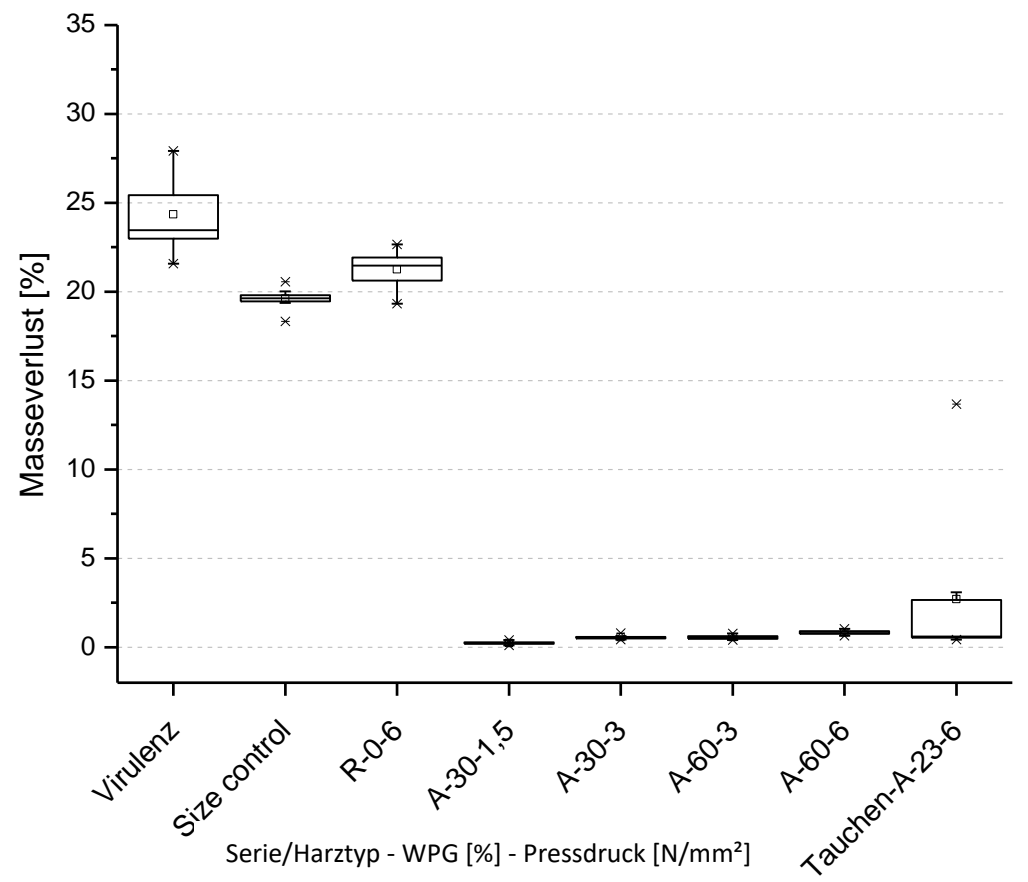

Abbildung 81: Masseverlust von Buchen-LVL ohne Sperrlagen $(10 * 2,13 \mathrm{~mm})$ im Abbauversuch mit Weißfäule (Trametes versicolor) nach ENV 12038 (2002). Modifizierung mit Harztyp A im Vakuum- und im Tauchverfahren.

In Abbildung 81 ist der Masseverlust von Buchen-LVL dargestellt, welches 10-lagig aus 2,13 mm-Furnieren ohne Sperrlagen hergestellt wurde. Neben unterschiedlichen WPGs und Pressdrücken, wurde auch eine Serie untersucht, die statt im Vakuumprozess im Tauchverfahren mit dem Harztyp A imprägniert wurde.

Die Ergebnisse des Abbauversuches zeigen, dass der Prüfpilz ausreichend virulent war, da der Masseverlust der Virulenzprüfkörper über dem geforderten Grenzwert von 20\% lag. Die MaßKontrollprüfkörper (engl. Size control), welche das Format der Plattenprüfkörper aufwiesen, 
dabei jedoch nur eine mittlere Plattendicke angenommen wurde, und aus Buchenvollholz bestanden, zeigten demgegenüber einen etwas geringeren Masseverlust. Auch die Referenzserie, welche aus unbehandelten Buchenfurnieren hergestellt wurde (Serie R-0-6 mit $6 \mathrm{~N} / \mathrm{mm}^{2}$ Pressdruck und 0\%WPG, $\rho=0,68 \mathrm{~g} / \mathrm{cm}^{3}$ ), lag im Ergebnis unter den Virulenzen, jedoch knapp über den Maß-Kontrollprüfkörpern. Aus diesen Ergebnissen lässt sich ein Einfluss der Prüfkörperabmessungen und Prüfkörpermassen ableiten. Da die MaßKontrollprüfkörper sowie die Plattenprüfkörper größere Abmessungen und eine höhere Masse aufwiesen als die Virulenzen, ist der relative Masseverlust kleiner. Somit ist die Berechnung des DSI, wie in Material und Methoden beschrieben, sinnvoll und notwendig um die Ergebnisse der Versuchsserien zu vergleichen. Bei allen Versuchsserien, die im Vakuumprozess imprägniert wurden und einen WPG von 30\% bzw. 60\% aufwiesen, konnte hingegen nur ein sehr geringer Masseverlust unter 1\% festgestellt werden. Somit konnte weder ein Einfluss der Darrdichte, welche bei einem WPG von 30\% mit 1,5 N/mm² Pressdruck bei $0,75 \mathrm{~g} / \mathrm{cm}^{3}$ und bei $3 \mathrm{~N} / \mathrm{mm}^{2}$ Pressdruck bei $0,87 \mathrm{~g} / \mathrm{cm}^{3}$ sowie bei $60 \%$ WPG und $6 \mathrm{~N} / \mathrm{mm}^{2}$ bei $1,2 \mathrm{~g} / \mathrm{cm}^{3}$ lag, noch ein Einfluss des WPG und des Pressdruckes gezeigt werden.

Die Serie, der kein Vakuumprozess zugrunde lag, sondern bei der die Furniere durch ein zeitlich begrenztes Tauchen imprägniert wurden, wiesen bei einem WPG von 23\% einen geringfügig höheren Masseverlust auf, einhergehend mit einer vergleichsweise stärkeren Streuung der Messwerte. Dennoch lag der Median des Masseverlustes dieser Variante unter $3 \%$ bzw. alle Werte unter $5 \%$.

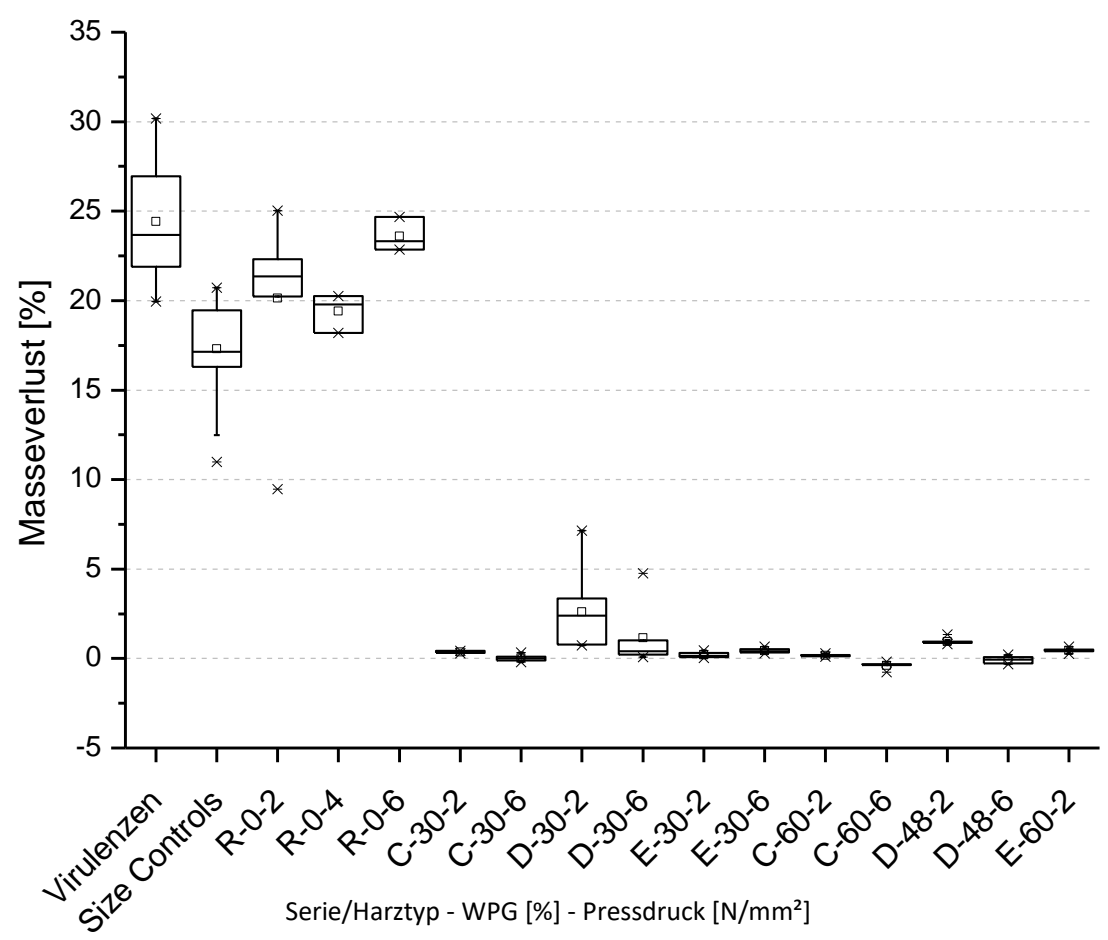

Abbildung 82: Masseverlust von Buchen-LVL mit Sperrlagen (8-lagig, Furnierdicke 3,7mm) im Abbauversuch mit Weißfäule (Trametes versicolor) nach EN 12038.

Buchen-LVL, welches aus 3,7 mm-Furnieren mit zwei Sperrlagen hergestellt wurde und keiner Modifizierung unterlag (R-), wurde im Abbauversuch mit der Weißfäule Trametes versicolor (Abbildung 82) stark abgebaut. Die Mediane der Masseverluste lagen dabei zwischen $20 \%$ und 
$25 \%$ und zwischen den Masseverlusten der Maß-Kontrollprüfkörper und der Virulenzen. Im Vergleich zum unbehandelten 10-lagigen Buchen-LVL mit 2,13 mm-Furnieren lässt sich somit kein Unterschied hinsichtlich des Abbaus feststellen. Bei den Ergebnissen der modifizierten Serien mit den Harztypen C, D und E zeigte sich für alle WPGs, die hier mindestens bei $30 \%$ lagen, dass der Masseverlust deutlich reduziert war. Mit Ausnahme des Harztyps D lag bei den modifizierten Serien der maximale Einzelwert für den Abbau bei 0,7 \% und der größte Median bei 0,44\%. Diese Ergebnisse ähneln somit auch denen des 10-lagigen LVLs aus 2,13 mm-Furnieren. Beim Harztyp $\mathrm{D}$ kam es jedoch $\mathrm{zu}$ größeren Masseverlusten, insbesondere bei einem WPG von $30 \%$ beim Pressdruck von $2 \mathrm{~N} / \mathrm{mm}^{2}$. Der größte Einzelwert lag bei dieser Variante bei einem Masseverlust von $7 \%$ und der Median bei 2,4\%. Mit dem Shapiro-Wilk-Test wurde beim Niveau 0,05 festgestellt, dass die Daten aus einer normalverteilten Grundgesamtheit gezogen wurden. Nach einem t-Test für verbundene Stichproben unterscheidet sich der Mittelwert des Harztyps D bei einem WPG von $30 \%$ und einem Pressdruck von $2 \mathrm{~N} / \mathrm{mm}^{2}$ von den Mittelwerten der Harztypen C und E signifikant $(\alpha=0,05)$. Da beim gleichen Harztyp D mit dem höheren Pressdruck von $6 \mathrm{~N} / \mathrm{mm}^{2}$ bei gleichem WPG kein signifikanter Unterschied zu den anderen Serien festgestellt wurde, hatte der Pressdruck hier einen negativen Einfluss auf den Abbau.

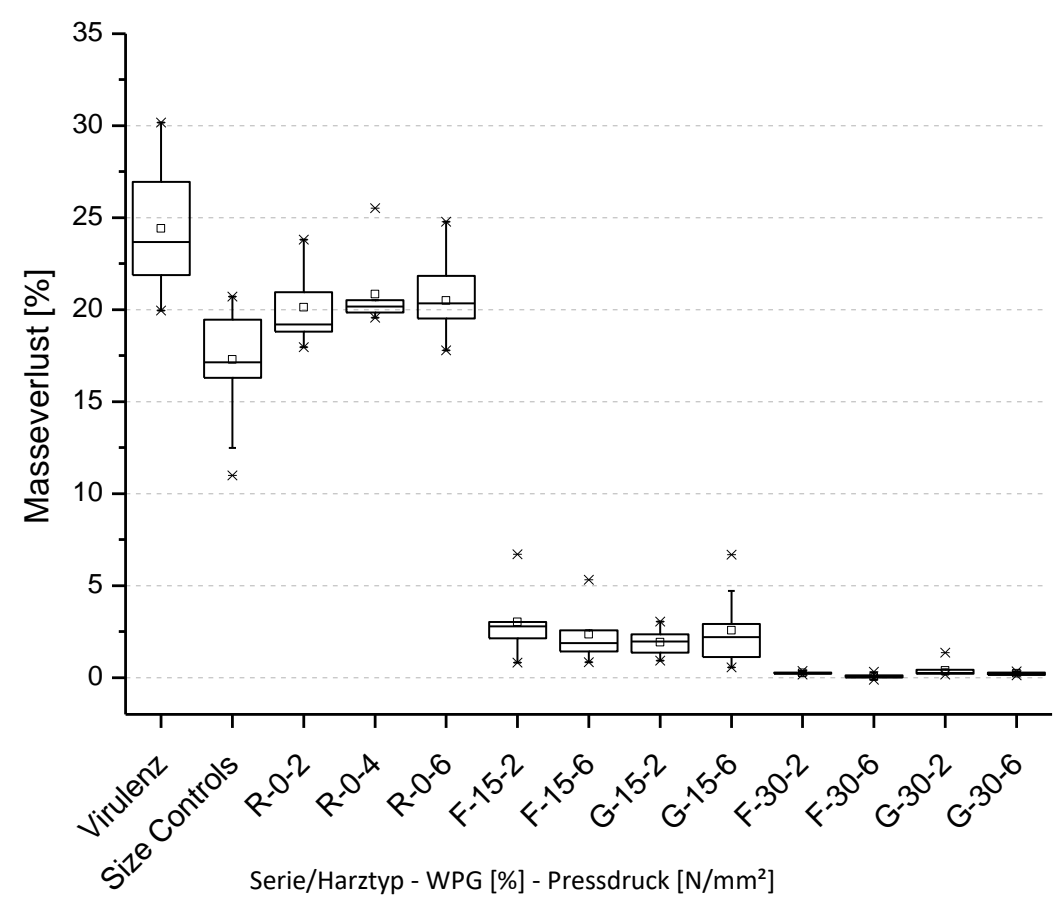

Abbildung 83: Masseverlust von Buchen-LVL ohne Sperrlagen (8-lagig, parallel, Furnierdicke 3,7mm) im Abbauversuch mit Weißfäule (Trametes versicolor) nach EN 12038.

Buchen-LVL, welches aus 3,7 mm-Furnieren ohne Sperrlagen hergestellt wurde und keiner Modifizierung unterlag (R-), wurde unabhängig vom Pressdruck von Trametes versicolor stark abgebaut (Abbildung 83). Die Mediane der Masseverluste lagen dabei knapp über $20 \%$ und zwischen den Masseverlusten der Maß-Kontrollprüfkörper (Size controls) und der Virulenzen. Im Vergleich zum 8-lagigen Buchen-LVL mit Sperrlagen wurde somit der gleiche Abbau festgestellt. Betrachtet man die Ergebnisse der modifizierten Serien mit den Harztypen F und G bei einem WPG von $15 \%$ zeigt sich, dass es hierbei zu einem deutlich reduzierten Abbau kam, welcher maximal bei $7 \%$ lag, die Mediane und die $95 \%$-Perzentile deutlich unter $5 \%$ 
lagen. Einzelwerte lagen hier jedoch über 5\% und bei alleiniger Anwendung der ENV 12038 (2002) wären diese Serien nicht mehr als voll beständig gegen holzerstörende Basidiomyceten zu klassifizieren. Die Berechnung des DSI und die Einteilung in Dauerhaftigkeitsklassen (Tabelle 6 und Tabelle 16) sollte hier Abhilfe schaffen, um eine einfache Beurteilung des Erfolgs des Modifizierungsprozesses zu ermöglichen. Wie im Versuch mit 10-lagigem LVL, konnte bei den Varianten mit einem WPG von 30 \% kein Abbau bzw. kein Median des Masseverlustes über 0,3 \% festgestellt werden. Im Vergleich der Mittelwerte für alle Prüfköper mit $30 \%$ und für alle Prüfkörper mit 15 \% liegt hier ein signifikant unterschiedliches Ergebnis zum Niveau 0,05 vor. Folglich hindert eine Phenolmodifizierung der Furniere die Weißfäule am Abbau, jedoch ist ähnlich wie bei der Wasseraufnahme und Quellung festzustellen, dass das Maß der Reduzierung vom WPG abhängig war und hinsichtlich der biologischen Dauerhaftigkeit beim Buchen-LVL erst ab einem WPG von 30 \% mit den Harztypen C, E, F und G keine Steigerung der Resistenz mehr auftrat.

Nachfolgend dargestellt wird einmal eine hypothetische Einteilung in Dauerhaftigkeitsklassen nach dem absoluten Masseverlust einerseits und dem relativen Masseverlust im Verhältnis zu den Maß-Kontrollprüfkörpern (engl. Size controls) aus Buchenvollholz.

Tabelle 16: Einteilung der Versuchsserien in Dauerhaftigkeitsklassen nach dem absoluten Masseverlust und nach dem berechneten DSI.

\begin{tabular}{|c|c|c|c|c|c|c|c|c|}
\hline $\begin{array}{c}\text { Furnier- } \\
\text { stärke } \\
\text { [mm] }\end{array}$ & Furnierrichtung & Harztyp & WPG & $\begin{array}{c}\text { Druck } \\
{\left[\mathrm{N} / \mathrm{mm}^{2}\right]}\end{array}$ & $\begin{array}{c}\text { Masse- } \\
\text { verlust } \\
{[\%]}\end{array}$ & $\begin{array}{l}\text { Dauerhaftig- } \\
\text { keitsklasse } \\
\text { Masseverlust }\end{array}$ & DSI & $\begin{array}{c}\text { Dauerhaftig- } \\
\text { keitsklasse } \\
\text { DSI }\end{array}$ \\
\hline \multirow[t]{2}{*}{2.13} & \multirow[t]{2}{*}{ parallel } & Maß-Kontroll. & $0 \%$ & - & 19.6 & 4 & 1.00 & 5 \\
\hline & & $\begin{array}{l}\mathrm{R} \\
\mathrm{A}\end{array}$ & $\begin{array}{c}0 \% \\
23 \% \\
30 \%\end{array}$ & $\begin{array}{c}6 \\
6 \\
1.5 \\
3 \\
6 \\
3 \\
6\end{array}$ & $\begin{array}{l}21.2 \\
2.7 \\
0.2 \\
0.6 \\
0.7 \\
0.5 \\
0.8\end{array}$ & $\begin{array}{l}4 \\
1 \\
1 \\
1 \\
1 \\
1 \\
1\end{array}$ & $\begin{array}{l}1.08 \\
0.14 \\
0.01 \\
0.03 \\
0.03 \\
0.03 \\
0.04\end{array}$ & $\begin{array}{l}5 \\
1 \\
1 \\
1 \\
1 \\
1 \\
1\end{array}$ \\
\hline \multirow[t]{26}{*}{3.7} & \multirow[t]{15}{*}{ mit 2Sperrlagen } & Maß-Kontroll. & $0 \%$ & - & 17.3 & 4 & 1.00 & 5 \\
\hline & & $R$ & $0 \%$ & 2 & 20.1 & 4 & 1.16 & 5 \\
\hline & & & & 4 & 19.4 & 4 & 1.12 & 5 \\
\hline & & & & 6 & 23.6 & 4 & 1.37 & 5 \\
\hline & & C & $30 \%$ & 2 & 0.4 & 1 & 0.02 & 1 \\
\hline & & & & 6 & 0.0 & 1 & 0.00 & 1 \\
\hline & & & $60 \%$ & 2 & 0.2 & 1 & 0.01 & 1 \\
\hline & & & & 6 & -0.4 & 1 & -0.02 & 1 \\
\hline & & D & $30 \%$ & 2 & 2.6 & 1 & 0.15 & 1 \\
\hline & & & & 6 & 1.2 & 1 & 0.07 & 1 \\
\hline & & & $48 \%$ & 2 & 0.9 & 1 & 0.05 & 1 \\
\hline & & & & 6 & -0.1 & 1 & 0.00 & 1 \\
\hline & & $E$ & $30 \%$ & 2 & 0.2 & 1 & 0.01 & 1 \\
\hline & & & & 6 & 0.4 & 1 & 0.03 & 1 \\
\hline & & & $60 \%$ & 2 & 0.4 & 1 & 0.03 & 1 \\
\hline & \multirow[t]{11}{*}{ parallel } & $\mathrm{R}$ & $0 \%$ & 2 & 20.1 & 4 & 1.16 & 5 \\
\hline & & & & 4 & 20.8 & 4 & 1.20 & 5 \\
\hline & & & & 6 & 20.5 & 4 & 1.18 & 5 \\
\hline & & $\mathrm{F}$ & $15 \%$ & 2 & 3.0 & 1 & 0.17 & 2 \\
\hline & & & & 6 & 2.4 & 1 & 0.14 & 1 \\
\hline & & & $30 \%$ & 2 & 0.2 & 1 & 0.01 & 1 \\
\hline & & & & 6 & 0.1 & 1 & 0.00 & 1 \\
\hline & & $G$ & $15 \%$ & 2 & 1.9 & 1 & 0.11 & 1 \\
\hline & & & & 6 & 2.6 & 1 & 0.15 & 1 \\
\hline & & & $30 \%$ & 2 & 0.4 & 1 & 0.02 & 1 \\
\hline & & & & 6 & 0.2 & 1 & 0.01 & 1 \\
\hline
\end{tabular}


Aus der Tabelle 16 wird ersichtlich, dass alle modifizierten Serien einen Masseverlust aufwiesen, der in die Einteilung in die Dauerhaftigkeitsklasse 1 führen könnte. Mit der Berechnung des DSI stellt sich das für die modifizierten Serien genauso dar, jedoch fällt nach der gewählten Einteilung die Variante mit dem Harztyp F bei einem WPG von 15\% und einem Pressdruck von $2 \mathrm{~N} / \mathrm{mm}^{2}$ in die Dauerhaftigkeitsklasse 2, obwohl sie einen Abbau von nur $3 \%$ Masseverlust erfahren hatte. Der Grund dafür ist, dass der errechnete DSI größer als 0,15 ausfiel, da die Maß-Kontrollprüfkörper, auf die sich bezogen wurde, nur einen Masseverlust von 17,3 \% aufwiesen. Auf diese Weise konnte eine durch das Prüfkörperformat geringere Abbaurate durch den Prüfpilz berücksichtigt werden. Die Referenzen wurden basierend auf dem DSI der Klasse 5 zugeordnet. Sie wiesen einen DSI auf, der größer als 1,0 war, was verdeutlicht, dass diese Serien stärker abgebaut wurden als reines Buchenvollholz. Auf diese Weise konnte, unabhängig von den Virulenzprüfkörpern, der Einfluss des Herstellungsprozesses (Dämpfen, Schälen, Trocknen, Verkleben, etc.) und die Effektivität der Modifizierung zum Ausdruck gebracht werden.

\subsubsection{Dauerhaftigkeit im Erdkontakt}

Die Bewertung der Freilandprüfkörper im Erdkontakt (EN 252, 2015) ergab, dass modifiziertes Buchen-LVL über einen Zeitraum von drei Jahren im Erdkontakt eine im Vergleich zur unbehandelten Referenz deutlich verbesserte Resistenz gegen holzzerstörende Mikroorganismen, wie z.B. Moderfäulen, aufwies. Im ersten Jahr wurde bei den Referenzen, d.h. bei Buchen-LVL aus unbehandelten Furnieren, ein mittlerer (Bewertungsziffer 2) bis starker (Bewertungsz. 3) Angriff festgestellt. Dabei war ein Weichwerden des Holzes von bis zu $3 \mathrm{~mm}$ Tiefe bzw. von bis zu $5 \mathrm{~mm}$ Tiefe (ganze Fläche) oder von bis zu $15 \mathrm{~mm}$ Tiefe (in begrenzten Bereichen) festgestellt worden. Im zweiten Jahr war es bereits zum kompletten Ausfall, d.h. einer Vergabe der Bewertungsziffer 4, aufgrund des Durchbrechens der Prüfkörper, gekommen (Abbildung 84).

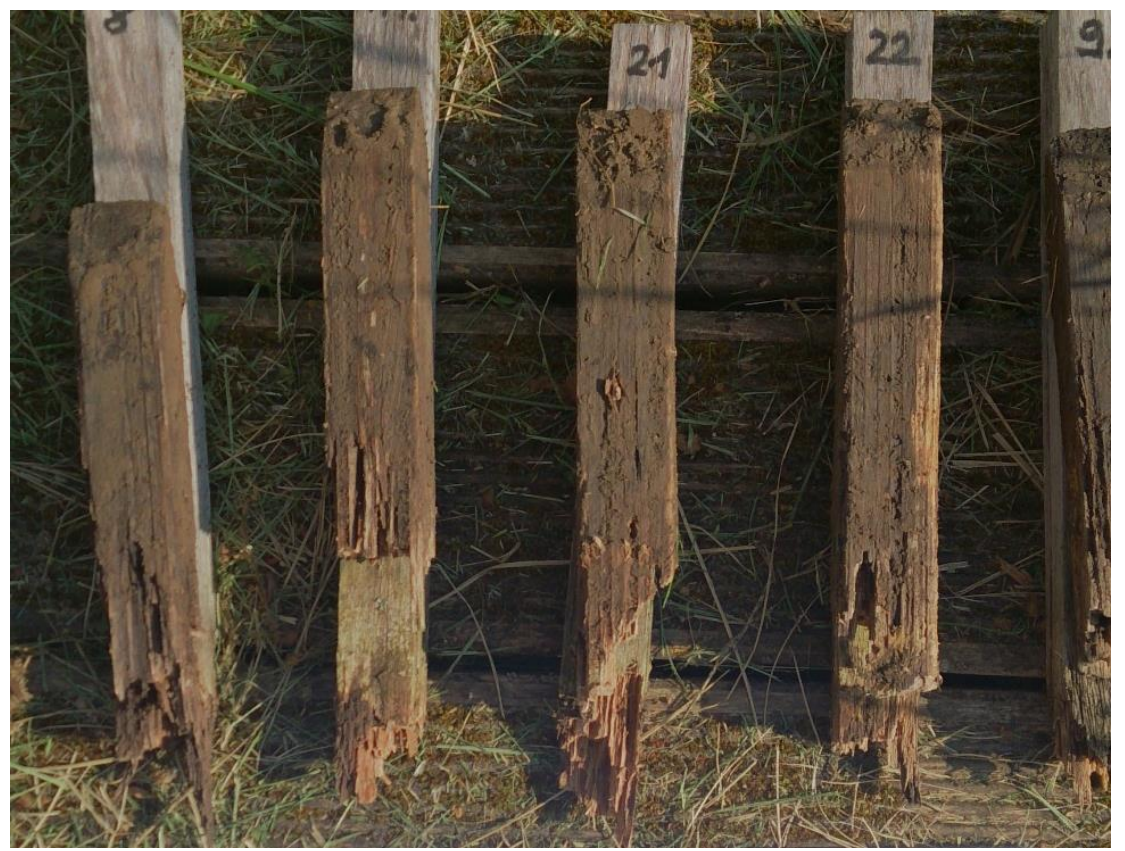

Abbildung 84: Unbehandelte Referenzprüfkörper (Buchen-LVL) nach 2 Jahren im Erdkontakt (EN 252, 2015). 
Anhand des Harztyps A wird in Abbildung 85 der Einfluss der Modifizierung, wie auch des WPG, ersichtlich. Alle modifizierten Serien zeigten nach drei Jahren signifikant niedrigere Bewertungsziffern als die unbehandelte Referenz. Bei den modifizierten Proben konnten im ersten Jahr an einigen Proben mit einem WPG von 15 \% Veränderungen im Sinne von weichen Stellen, mit einer Tiefe $<1 \mathrm{~mm}$, deren Verteilung vereinzelt war, beobachtet werden, jedoch im Durchschnitt noch die Bewertungsziffer 0 vergeben werden. Bei den höheren WPGs kam es, im ersten Jahr noch unabhängig vom Pressdruck bzw. der Dichte, noch seltener zu Proben, die Veränderungen feststellen ließen. Im zweiten Jahr zeigten die modifizierten Proben wieder signifikant weniger Veränderungen und niedrigere Bewertungsziffern als die Referenzen. Bei einem WPG von $15 \%$ war jedoch eine Zunahme der Veränderungen festzustellen und die Bewertungsziffer 1 wurde für alle Pressdrücke vergeben. Die Serien mit den höheren WPGs von $30 \%$ und auch $60 \%$ zeigten demgegenüber im Durchschnitt geringere bzw. keine Veränderungen, die bei den höheren Pressdrücken als $2 \mathrm{~N} / \mathrm{mm}^{2}$, signifikant unterschiedlich zum WPG von $15 \%$ waren. Nach drei Jahren fiel auf, dass es beim WPG von 15\% bei der unverdichteten Variante zu einem Sprung von der Bewertungsziffer 1 zur Bewertungsziffer 3 kam. Das bedeutet, dass ein starker Angriff mit einem großflächigen Weichwerden bis $5 \mathrm{~mm}$ Tiefe oder auf begrenztem Bereich bis $15 \mathrm{~mm}$ Tiefe stattgefunden hatte. Auch die unverdichteten Variante mit einem WPG von 30\% wies leichte Veränderungen auf, wobei noch die Bewertungsziffer 1 vergeben wurde. Die gleiche Variante zeigte beim höheren Pressdruck vergleichsweise geringere Veränderungen und wurde mit einem durchschnittlichen Wert von 0,6 noch der Klasse 1 zugeordnet. Beim höchsten Pressdruck jedoch konnten, ebenso wie bei $60 \%$ WPG, keine Veränderungen festgestellt und somit eindeutig die Bewertunsgziffer 0 vergeben werden.
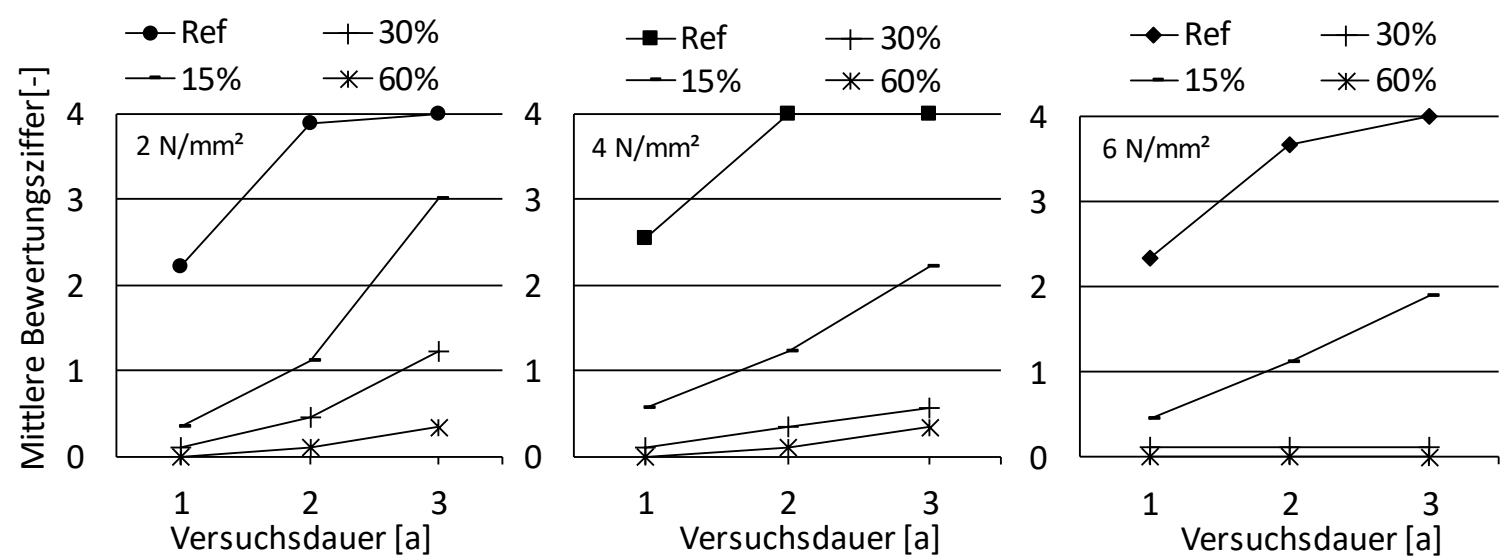

Abbildung 85: Ergebnisse der Evaluierung der Freilandprüfung mit Erdkontakt in Anlehnung an EN252 (2015). Mittlere Bewertungsziffern der modifizierten Buchen-LVL mit dem Harztyp A bei Pressdrücken von $2 \mathrm{~N} / \mathrm{mm}^{2}, 4 \mathrm{~N} / \mathrm{mm}^{2}$ und $6 \mathrm{~N} / \mathrm{mm}^{2}$ und WPGs von 15\%, 30\% und $60 \%(\mathrm{n}=9)$.

Bei den modifizierten Proben mit einem WPG von 15 \% und unterschiedlichen Harzen konnten im ersten Jahr für den Harztyp A, wie oben gezeigt, im Durchschnitt die mittlere Bewertungsziffer 0,3 vergeben werden. Bei den Harztypen F und $G$ waren hingegen an keinen Proben Veränderungen feststellbar (Abbildung 86). Im zweiten Jahr, als für den Harztyp A die Bewertungsziffern im Durchschnitt bei 1,1 lagen, näherte sich der Harztyp F mit einer Bewertungsziffer von 0,9 diesem Ergebnis an, wohingegen beim Harztyp G nur an einzelnen Proben eine Veränderung festgestellt wurde und im Durchschnitt die Bewertungsziffer 0,2 
betrug. Im dritten Jahr wird deutlich, was schon zuvor gezeigt wurde. Bei den Harztypen F und G kommt es mit der Zeit, wie auch beim Harztyp A, bei einem WPG von 15 \% zu stärkeren Veränderungen. Beim Vergleich von verdichteten zu unverdichteten Varianten fällt auf, dass nach zwei Jahren die Harztypen F und G zwar keine Veränderungen aufwiesen, jedoch nach drei Jahren nun auch leichte Veränderungen zu beobachten waren. Somit kann beim WPG von 15\%, unabhängig vom Harztyp und Pressdruck, darauf geschlossen werden, dass zwar eine Verzögerung des Abbaus, jedoch kein dauerhafter Schutz, erreicht wurde.

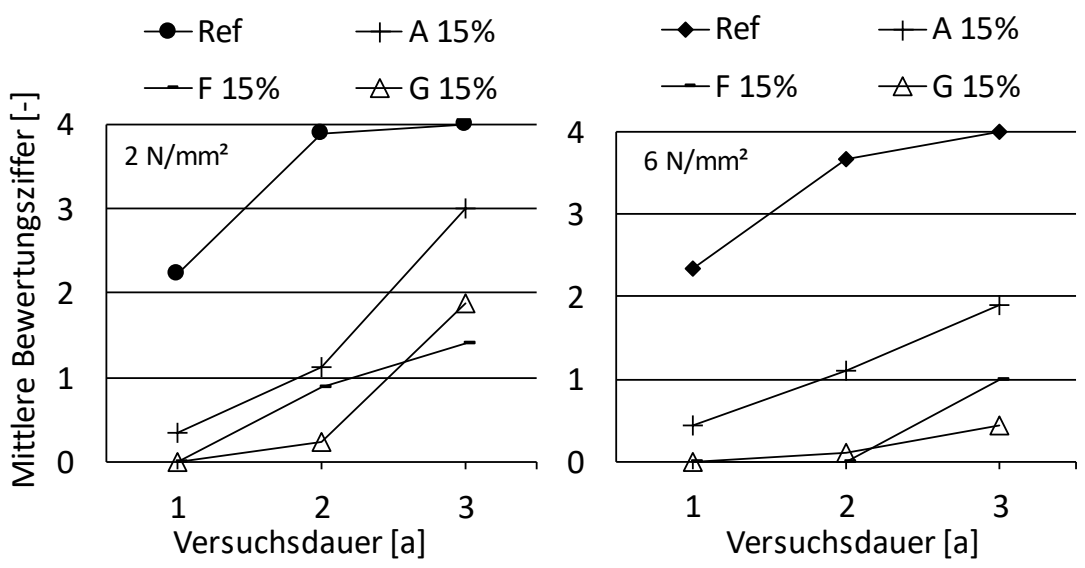

Abbildung 86: Ergebnisse der Evaluierung der Freilandprüfung mit Erdkontakt in Anlehnung an EN252 (2015). Mittlere Bewertungsziffern der modifizierten Buchen-LVL mit den Harztypen A, F und G bei den Pressdrücken $2 \mathrm{~N} / \mathrm{mm}^{2}$ und $6 \mathrm{~N} / \mathrm{mm}^{2}$ beim WPG von $15 \%(\mathrm{n}=9)$.

Abbildung 87 zeigt die Ergebnisse der Serien mit einem WPG von 30\%. Für alle modifizierten Serien wurde nach einem Jahr die Bewertungsziffer 0,1 im Durchschnitt vergeben, d.h., dass hier überwiegend noch keine Veränderungen festzustellen waren. Im zweiten Jahr zeigte Harztyp D leichte Veränderungen und erhielt bei $2 \mathrm{~N} / \mathrm{mm}^{2}$ und bei $6 \mathrm{~N} / \mathrm{mm}^{2}$ die durchschnittlichen Bewertungsziffern 0,7 und 0,8, wobei einzelne Proben bereits die Ziffer 2 erhielten. Bei den Harztypen E und A kam es weniger häufig zu leichten Veränderungen. Auffallend waren die durchgehend unveränderten Proben des Harztyps C nach ein und zwei Jahren. Nach drei Jahren waren alle unbehandelten Prüfkörper ausgefallen und zwischen den Pressdrücken wurde ein Unterschied ersichtlich. Bei dem niedrigeren Druck wurden die leichten Veränderungen für alle Harztypen häufiger, wohingegen beim höheren Druck die gleichen Bewertungsziffern vergeben wurden wie nach zwei Jahren. 


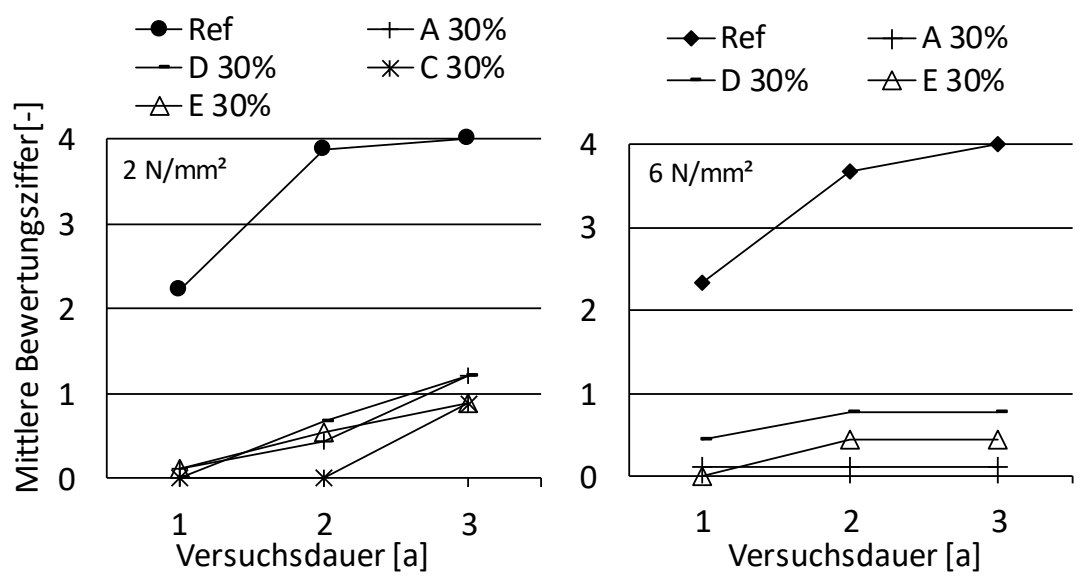

Abbildung 87: Ergebnisse der Evaluierung der Freilandprüfung mit Erdkontakt in Anlehnung an EN252 (2015). Mittlere Bewertungsziffern der modifizierten Buchen-LVL mit den Harztypen A, C, D und E bei den Pressdrücken $2 \mathrm{~N} / \mathrm{mm}^{2}$ und $6 \mathrm{~N} / \mathrm{mm}^{2}$ beim WPG von $30 \%(\mathrm{n}=9)$.

Der Harztyp A nimmt aufgrund dieser Ergebnisse eine intermediäre Stellung in Bezug auf eine Schutzwirkung von Buchen-LVL im Erdkontakt ein, da bei einem WPG von 15\% zwei andere Harztypen weniger angegriffen waren und bei einem WPG von 30\% zwei andere Harztypen schlechter bewertet wurden als der Harztyp A. In Bezug zur Prüfung von Holzschutzmitteln empfehlen Nicholas UND MILITZ (2008) einen Prüfzeitraum von 5 Jahren. Da für den überwiegenden Teil der Harze, Harztyp C ausgenommen, die Ergebnisse nach einem Jahr und nach zwei Jahren unterschiedlich waren, wird der Test mit dem modifizierten Buchen-LVL weitergeführt und die Standzeit der PF-modifizierten LVL ermittelt.

\subsubsection{Witterungsstabilität}

\subsubsection{Schnellbewitterung}

\subsection{Unbeschichtetes modifiziertes LVL}

Nachfolgend sind die Ergebnisse der Schnellbewitterung hinsichtlich Wasseraufnahme und Dickenquellung für den Harztyp A bei unterschiedlichen Drücken, WPGs und Plattenaufbauten (mit und ohne Sperrlagen) gezeigt.

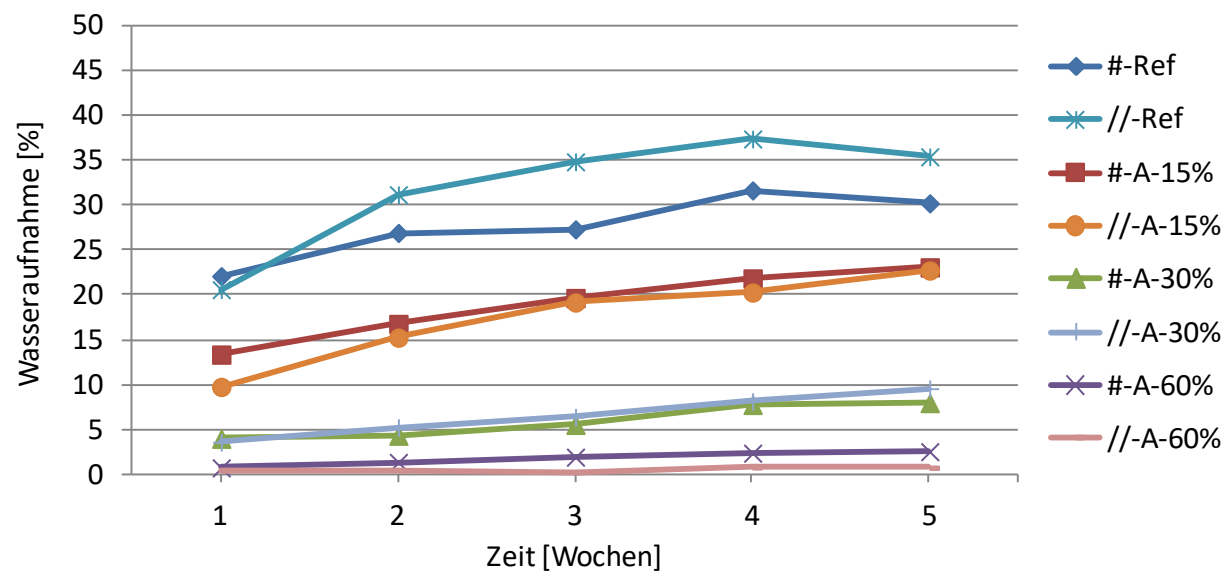

Abbildung 88: Wasseraufnahme bei Buchen-LVL $(8 * 3,7 \mathrm{~mm})$ mit (\#)und ohne Sperrlagen $(/ /)$, hergestellt bei $2 \mathrm{~N} / \mathrm{mm}^{2}$ mit unterschiedlichem WPG (Harztyp A). 
Bei der Schnellbewitterung von Buchen-LVL, welches mit einem Pressdruck von $2 \mathrm{~N} / \mathrm{mm}^{2}$ hergestellt wurde, zeigte sich, dass mit dem Harztyp A modifiziertes LVL weniger Wasser aufnahm, als die unmodifizierte Referenz (Abbildung 88). Dabei war die Wasseraufnahme umso niedriger, je größer der WPG war. Wenngleich zwischen den Referenzen mit unterschiedlichem Plattenaufbau, d.h. mit und ohne Sperrlagen, anscheinend ein Unterschied zu sehen war, so konnte dieser bei modifizierten Serien mit gleichem WPG nicht festgestellt werden.

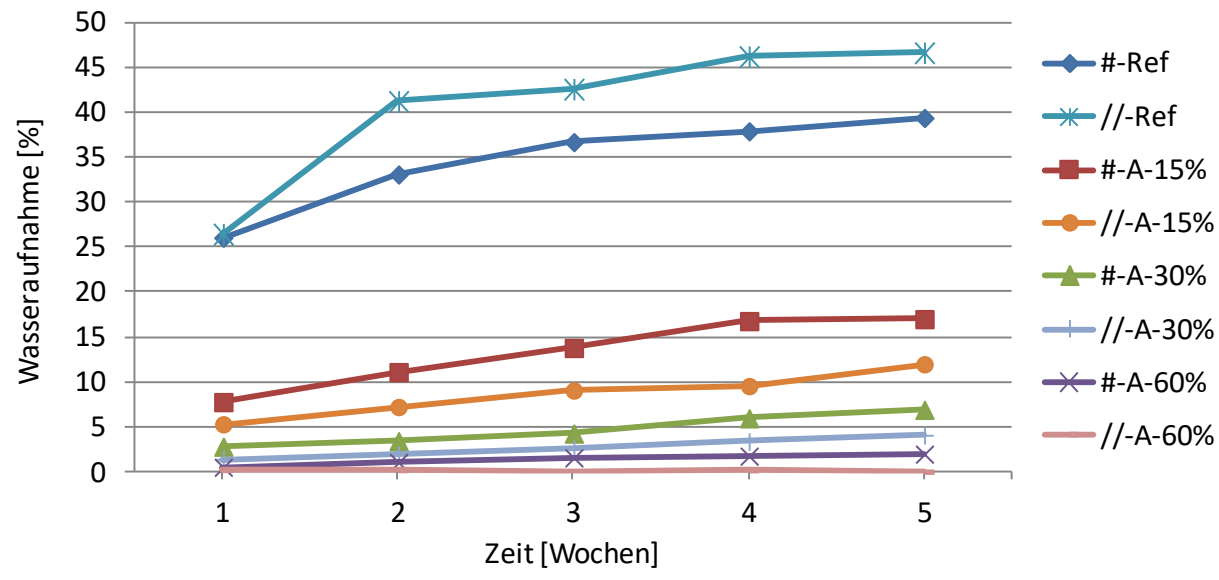

Abbildung 89: Wasseraufnahme bei Buchen-LVL $(8 * 3,7 \mathrm{~mm})$ mit (\#)und ohne Sperrlagen $(/ /)$, hergestellt bei $4 \mathrm{~N} / \mathrm{mm}^{2}$ mit unterschiedlichem WPG (Harztyp A).

Bei Versuchsplatten, die mit einem Pressdruck von $4 \mathrm{~N} / \mathrm{mm}^{2}$ hergestellt wurden, zeigten die unbehandelten Referenzen eine, im Vergleich $\mathrm{zu} 2 \mathrm{~N} / \mathrm{mm}^{2}$ Pressdruck, erhöhte Wasseraufnahme. Wieder war zwischen den Referenzen ein Unterschied in der Art, dass die Platten ohne Sperrlagen mehr Wasser aufnahmen als die Platten mit Sperrlagen. Da beide Referenzserien die gleichen Darrdichten aufwiesen, gibt es keine Erklärung dafür. Die modifizierten Serien wiesen im Vergleich zu denen mit dem niedrigeren Pressdruck eine weitere Reduzierung der Wasseraufnahme auf. Wobei auch hier beim höheren WPG die niedrigere Wasseraufnahme gemessen wurde. Hinsichtlich des Plattenaufbaus gab es bei einem WPG von 15\% den Unterschied, dass die Prüfkörper mit Sperrlagen eine höhere Wasseraufnahme aufwiesen als die Prüfkörper ohne Sperrlagen.

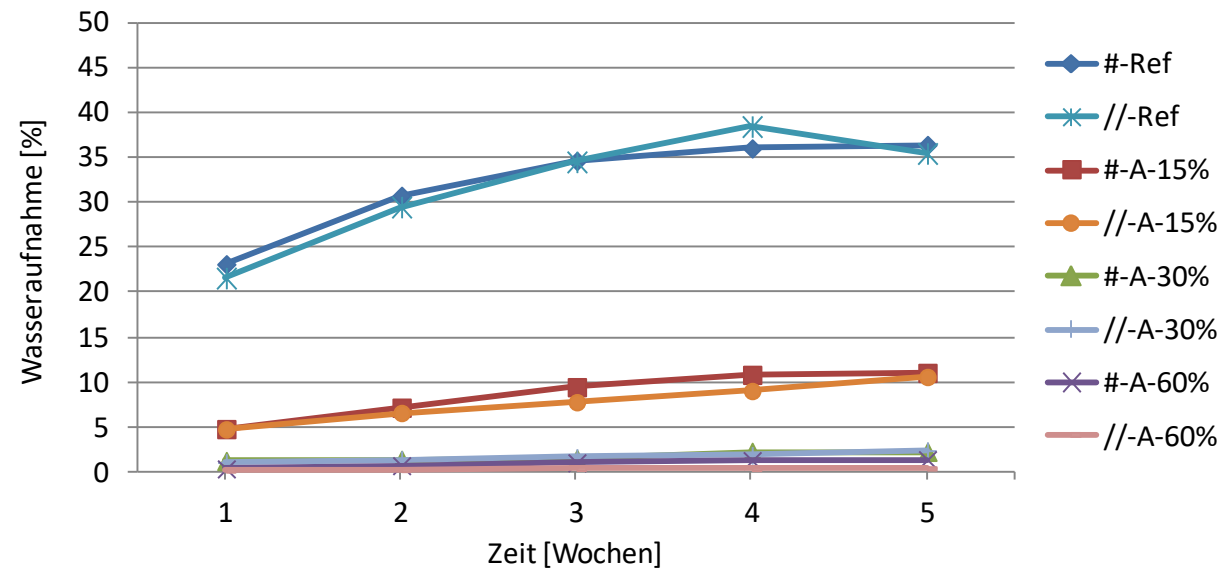

Abbildung 90: Wasseraufnahme bei Buchen-LVL $(8 * 3,7 \mathrm{~mm})$ mit (\#)und ohne Sperrlagen $(/ /)$, hergestellt bei $6 \mathrm{~N} / \mathrm{mm}^{2}$ mit unterschiedlichem WPG (Harztyp A). 
Wie aus Abbildung 90 ersichtlich wird, wiesen unter den Platten, die mit $6 \mathrm{~N} / \mathrm{mm}^{2}$ hergestellt wurden, erwartungsgemäß die Referenzen die höchste Wasseraufnahme auf. Mit maximal $38 \%$ lagen sie zwischen den Ergebnissen bei $2 \mathrm{~N} / \mathrm{mm}^{2}$ und $4 \mathrm{~N} / \mathrm{mm}^{2}$. Die Prüfkörper mit einem WPG von $15 \%$ wiesen eine maximale Wasseraufnahme von $11 \%$ auf. Diese war somit infolge der Verdichtung mehr als halbiert worden. Bei den höheren WPGs mit einem Maximalwert von 2,4\% kam es am Ende ebenso zu einer drastischen Reduzierung.

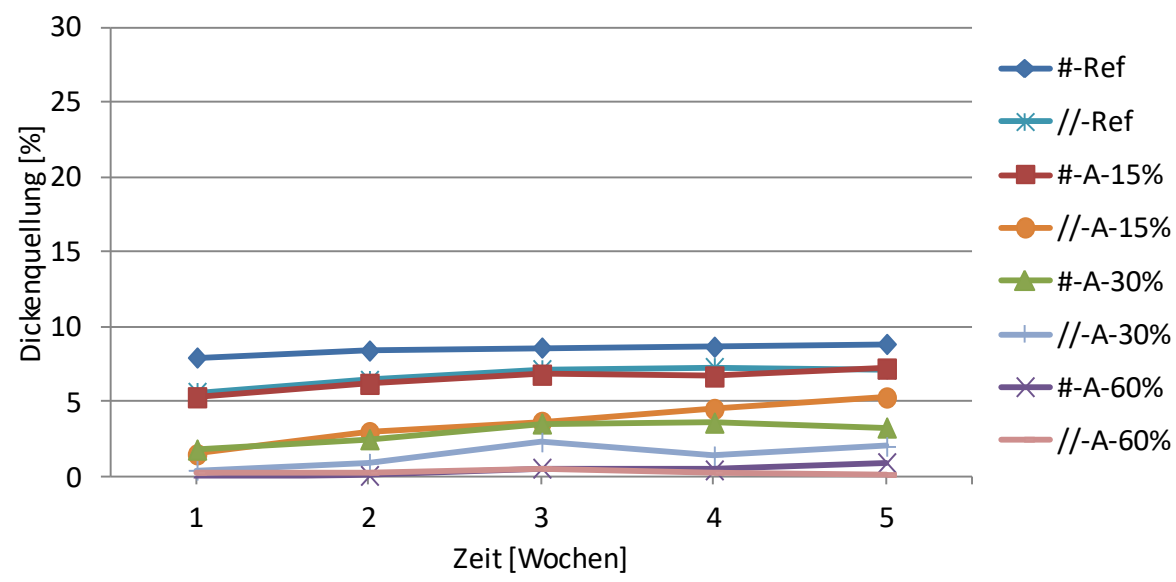

Abbildung 91: Dickenquellung bei Buchen-LVL $(8 * 3,7 \mathrm{~mm})$ mit (\#)und ohne Sperrlagen (//), hergestellt bei $2 \mathrm{~N} / \mathrm{mm}^{2}$ mit unterschiedlichem WPG (Harztyp A).

Bei Dickenquellung von modifiziertem LVL, welches bei einem Pressdruck von $2 \mathrm{~N} / \mathrm{mm}^{2}$ hergestellt wurde, zeigte sich eine umso stärker ausgeprägte Verringerung der Dickenquellung je höher der WPG der Platten war (Abbildung 91). Alle Ergebnisse wiesen dabei von Messintervall zu Messintervall nur eine geringe Zunahme auf. Keine der modifizierten Serien wies eine höhere Quellung auf als die Referenz. Hinsichtlich des Plattenaufbaus wurde festgestellt, dass mit Ausnahme der Serie mit 60\% WPG, die Platten mit Sperrlagen eine geringfügig höhere Dickenquellung aufwiesen. Besonders deutlich wurde dies bei den Referenzen und bei der Serie mit einem WPG von 15\%. Dies lässt sich nicht durch die Wasseraufnahme erklären, da diese, wie oben gezeigt, beim jeweiligen WPG für beide Plattentypen mit einem Pressdruck von $2 \mathrm{~N} / \mathrm{mm}^{2}$ identisch war.

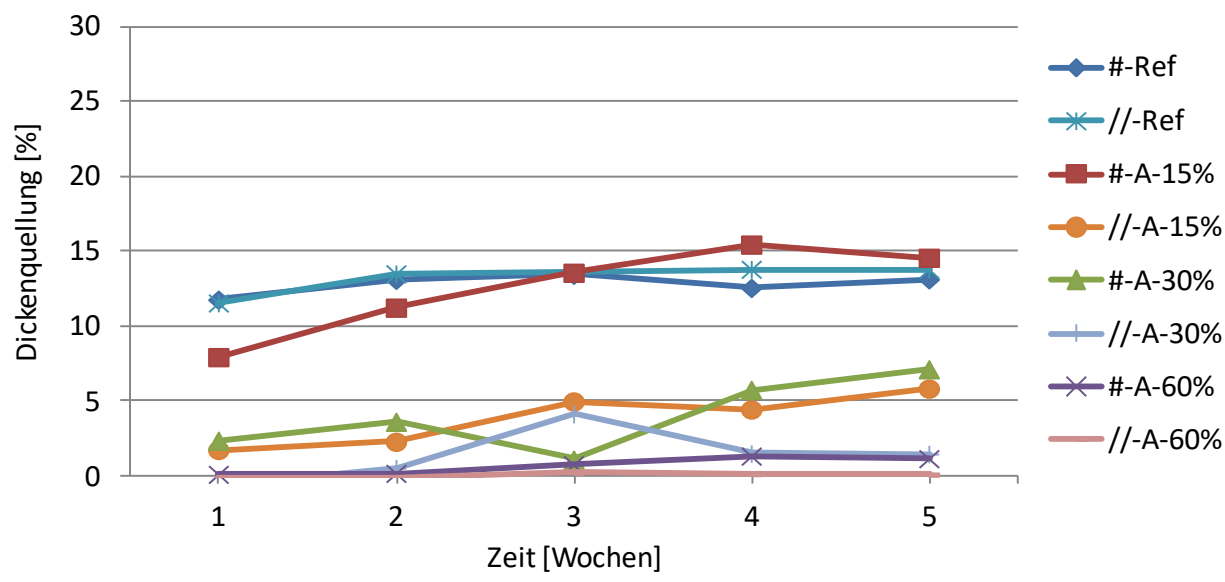

Abbildung 92: Dickenquellung bei Buchen-LVL $(8 * 3,7 \mathrm{~mm})$ mit (\#)und ohne Sperrlagen $(/ /)$, hergestellt bei $4 \mathrm{~N} / \mathrm{mm}^{2}$ mit unterschiedlichem WPG (Harztyp A). 
Im Unterschied zu einem Pressdruck von $2 \mathrm{~N} / \mathrm{mm}^{2}$ erfuhren die Platten mit $4 \mathrm{~N} / \mathrm{mm}^{2}$ eine gewisse Verdichtung. Bei der Dickenquellung äußerte sich dies nun darin, dass insbesondere die Referenzen, die am wenigsten verdichtet waren, anscheinend stärker quollen, weil hier die Rückquellung bzw. der Deformationsrückgang und die normale Quellung des Holzes zusammenwirkten. Bei den modifizierten Serien, konnte mit Ausnahme der Serie ohne Sperrlagen (//) und einem WPG von 60\%, auch eine Steigerung festgestellt werden. Insbesondere beim WPG von 15\% war die Steigerung beachtlich, wobei die Platten mit Sperrlagen sogar die Dickenquellung der Referenzen übertrafen.

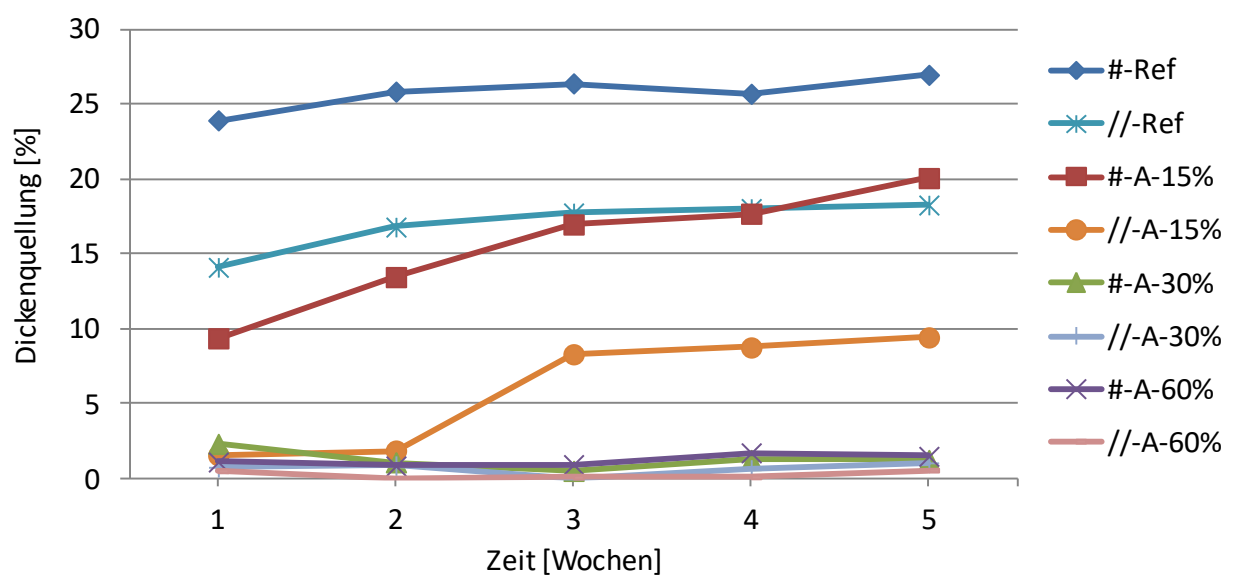

Abbildung 93: Dickenquellung bei Buchen-LVL $(8 * 3,7 \mathrm{~mm})$ mit (\#)und ohne Sperrlagen (//), hergestellt bei $6 \mathrm{~N} / \mathrm{mm}^{2}$ mit unterschiedlichem WPG (Harztyp A).

Bei einem Pressdruck von $6 \mathrm{~N} / \mathrm{mm}^{2}$ in der Herstellung kam es zu einer noch stärkeren Verdichtung bei allen Plattenserien. Die Referenzen quollen senkrecht zur Plattenebene noch stärker als bei $4 \mathrm{~N} / \mathrm{mm}^{2}$ und dabei erzielte die Serie mit Sperrlagen, trotz gleicher Wasseraufnahme, deutlich die höchste Dickenquellung mit maximal 27\% nach fünf Wochen. Beim niedrigsten WPG wurde ebenso eine deutliche Steigerung der Werte ersichtlich, wobei insbesondere erneut die höhere Dickenquellung der Platten mit Sperrlagen auffiel. Bei den WPG von 30\% und 60\% war die Quellung im Gegenteil dazu auf maximal 1,5\% nach 5 Wochen reduziert. Während diese starke Reduzierung bei einem Druck von $4 \mathrm{~N} / \mathrm{mm}^{2}$ nur bei $60 \%$ möglich war, konnte dies infolge der höchsten Verdichtung auch mit einem WPG von 30\% erzielt werden, da hier neben der Füllung der Zellwand durch das Harz auch die Zelllumen zusammengedrückt waren und der Flüssigwassertransport effektiv eingeschränkt wurde. Da bei den meisten untersuchten Serien in der Schnellbewitterung bereits innerhalb des ersten Messintervalls die stärkste Zunahme der Plattendicke $\mathrm{zu}$ verzeichnen war und weitere Zunahmen in den folgenden Wochen überwiegend gering ausfielen, kann auf eine ausreichende Messdauer für die Beurteilung von Unterschieden zwischen den zwei Plattenaufbauten, Pressdrücken und WPG geschlossen werden. 

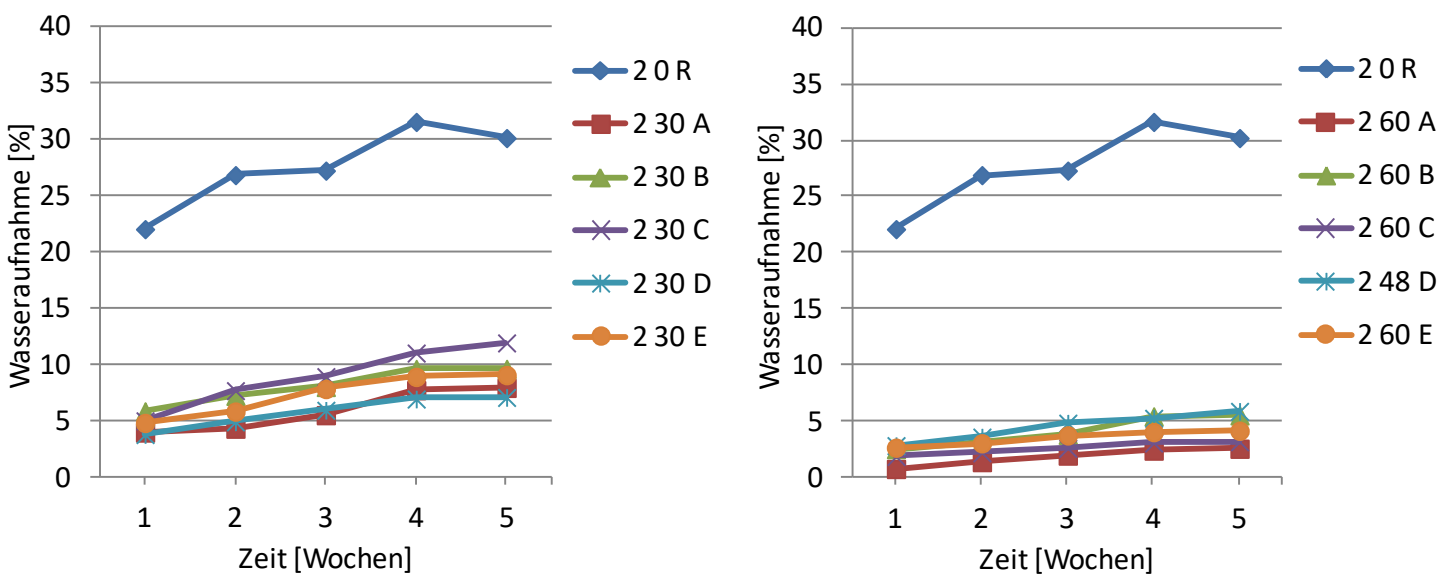

Abbildung 94: Wasseraufnahme in der Schnellbewitterung von Buchen-LVL $(8 * 3,7 \mathrm{~mm})$ mit Sperrlagen, hergestellt bei einem Pressdruck von $2 \mathrm{~N} / \mathrm{mm}^{2}$ mit einem WPG von 30\% (links) und 60\% (rechts) unter Verwendung verschiedener PF-Harze.

Aus Abbildung 94 wird ersichtlich, dass alle modifizierten Serien im Vergleich zur unbehandelten Referenz eine deutlich reduzierte Wasseraufnahme aufwiesen. Im linken Diagramm abgebildet sind die Serien mit einem WPG von 30\%. Alle Ergebnisse dieser modifizierten Serien lagen hier nah beieinander und zeigten von Messung zu Messung eine erkennbare Steigerung. Die Harztypen A und D wiesen die geringste Wasseraufnahme mit 8\% auf, die Harztypen C und B zeigten eine etwas höhere Wasseraufnahme mit maximal 12\%. Die im rechten Diagramm abgebildeten Serien mit einem WPG von 60\%, resp. 48\% beim Harztyp D, wiesen eine noch stärker reduzierte Wasseraufnahme auf, mit einem Maximum von 5,5\% beim Harztyp B.
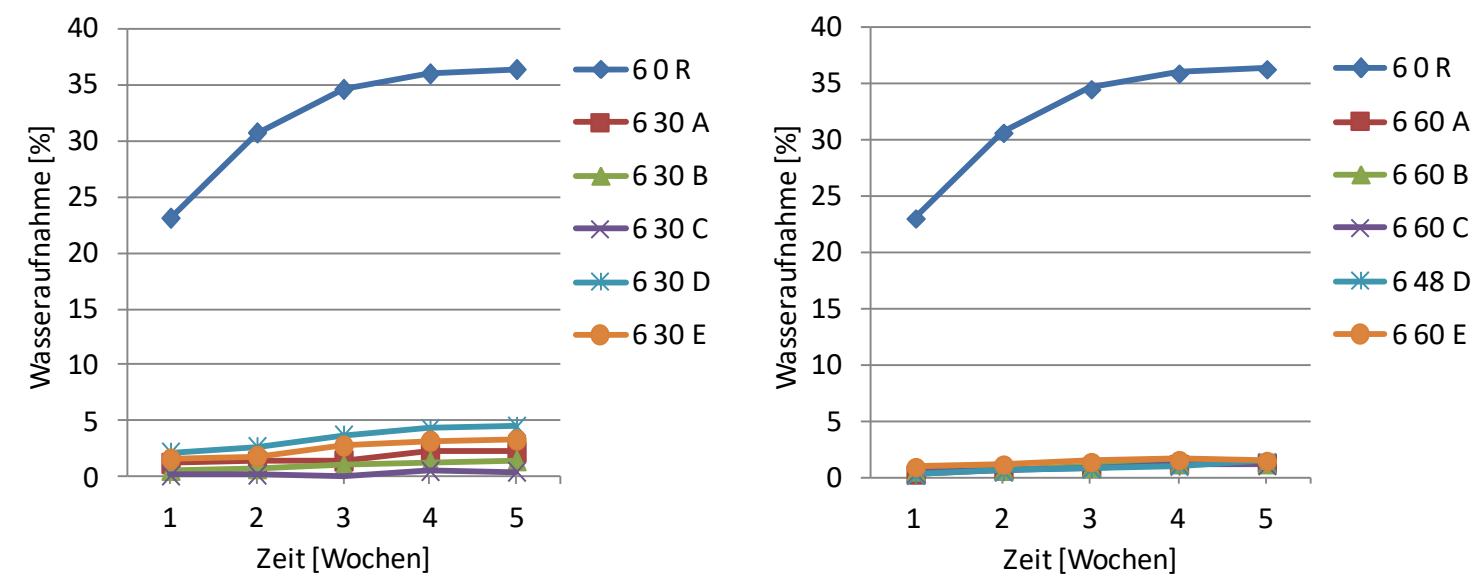

Abbildung 95: Wasseraufnahme in der Schnellbewitterung von Buchen-LVL $(8 * 3,7 \mathrm{~mm})$ mit Sperrlagen, hergestellt bei einem Pressdruck von $6 \mathrm{~N} / \mathrm{mm}^{2}$ mit einem WPG von 30\% (links) und 60\% (rechts) unter Verwendung verschiedener PF-Harze.

Die Wasseraufnahme von Buchen-LVL mit Sperrlagen, welches bei einem Pressdruck von $6 \mathrm{~N} / \mathrm{mm}^{2}$ hergestellt wurde, ist in Abbildung 95 dargestellt. Bei einem WPG von 30\% (links) ähneln die Messwerte jenen der Serien bei einem WPG von 60\% und einem Pressdruck von $2 \mathrm{~N} / \mathrm{mm}^{2}$. Aufgrund der höheren Verdichtung konnte die Wasseraufnahme hier also mit dem halben Harzeinsatz erzielt werden. Die geringste Wasseraufnahme wurde für die Variante mit dem Harztyp C ermittelt. Bei einem WPG von 60\% (rechts) und dem Pressdruck von 6 N/mm² kam es infolge der Verdichtung in Kombination mit dem hohen WPG zu der stärksten 
beobachteten Reduzierung der Wasseraufnahme, da für alle Harze der Maximalwert bei 1,5\% lag.
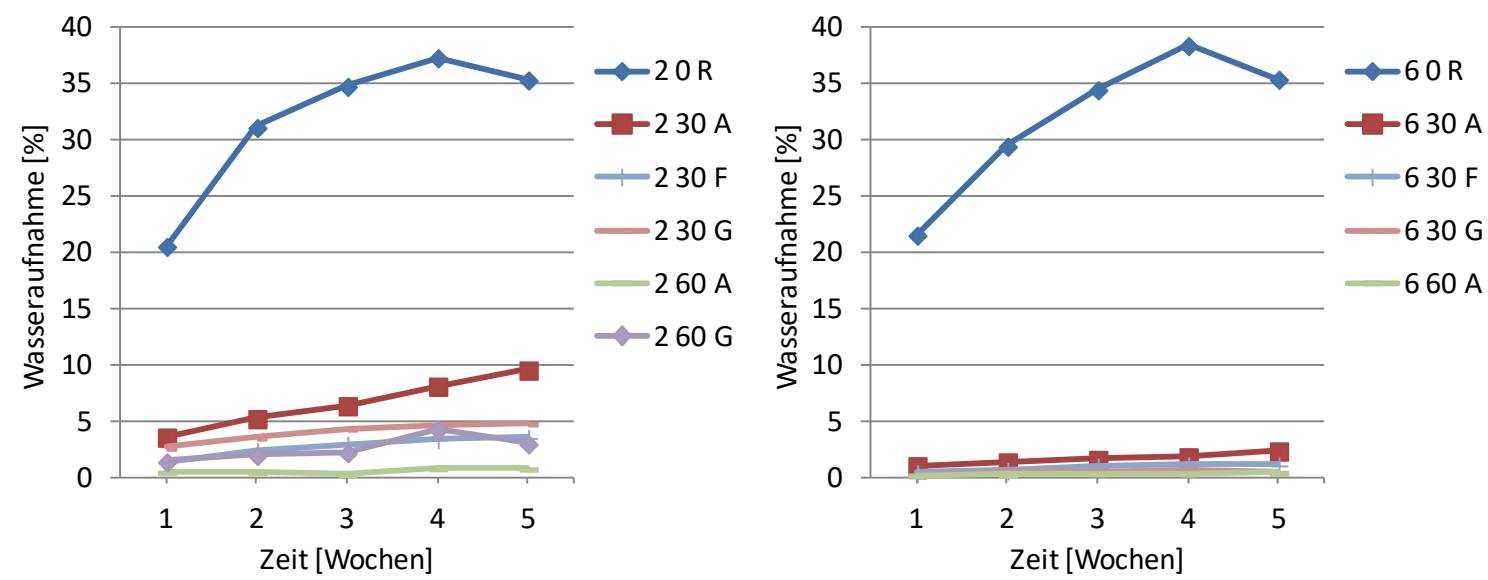

Abbildung 96: Wasseraufnahme in der Schnellbewitterung von Buchen-LVL $(8 * 3,7 \mathrm{~mm}$, parallel) ohne Sperrlagen, hergestellt bei einem Pressdruck von $2 \mathrm{~N} / \mathrm{mm}^{2}$ (links) und $6 \mathrm{~N} / \mathrm{mm}^{2}$ (rechts) bei WPGs von $30 \%$ und $60 \%$ unter Verwendung verschiedener PF-Harze.

In Abbildung 96 ist die Wasseraufnahme von Buchen-LVL in der Schnellbewitterung, welches ohne Sperrlagen, d.h. unter ausschließlich paralleler Anordnung der Furniere, hergestellt wurde, abgebildet. Bei den Serien mit einem Pressdruck von $2 \mathrm{~N} / \mathrm{mm}^{2}$ zeigte sich für alle modifizierten Serien eine deutlich verringerte Wasseraufnahme (links). Bei einem WPG von 30\% lag der Maximalwert nach 5 Wochen bei 10\% beim Harztyp A, während die Harztypen F und G nicht mehr als 4,8\% Wasser bezogen auf das Ausgangsgewicht aufnahmen. Beim gleichen Pressdruck mit einem WPG von 60\% lagen die Werte unter 4,3\% und für den Harztyp A sogar bei nur 0,8\%. Wie in Abbildung 96 auf der rechten Seite dargestellt, konnten die modifizierten Serien bei einem Pressdruck von $6 \mathrm{~N} / \mathrm{mm}^{2}$ nur maximal 2,4\% Wasser aufnehmen (Harztyp A) bei einem WPG von 30\% und nur 0,4\% bei einem WPG von 60\%. Der höhere Pressdruck konnte somit insbesondere bei einem WPG von 30\% effizient die Wasseraufnahme reduzieren.
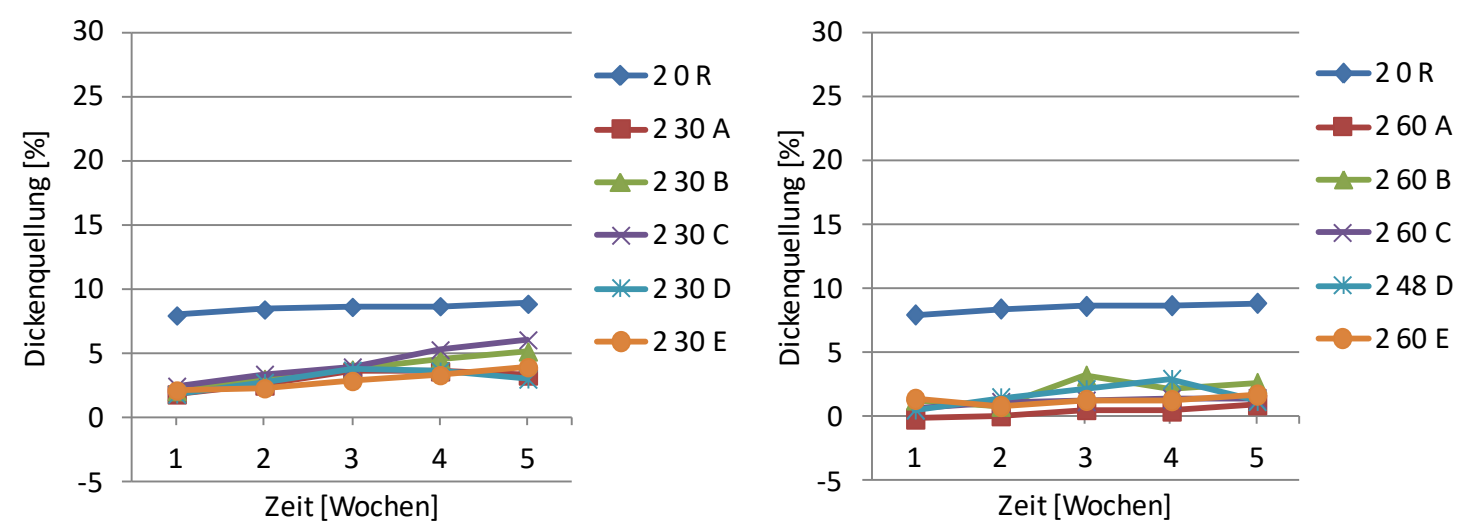

Abbildung 97: Dickenquellung in der Schnellbewitterung von Buchen-LVL $(8 * 3,7 \mathrm{~mm})$ mit Sperrlagen, hergestellt bei einem Pressdruck von $2 \mathrm{~N} / \mathrm{mm}^{2}$ mit einem WPG von 30\% (links) und 60\% (rechts) unter Verwendung verschiedener PF-Harze. 
Infolge der Wasseraufnahme der Proben während der Schnellbewitterung, kam es, wie Abbildung 97 zeigt, zu einer messbaren Dickenquellung. Bei dem Pressdruck von $2 \mathrm{~N} / \mathrm{mm}^{2}$ wiesen die Referenzen eine nahezu konstante Dickenquellung auf, beginnend mit 8,0\% und final mit 8,9\% nach 5 Wochen. Bei den modifizierten Serien mit einem WPG von 30\% fiel der Anstieg deutlich stärker aus, wenngleich die maximale Dickenquellung nur bei 6,1\% lag. Zwischen den Harzen war in den ersten 3 Wochen kein Unterschied feststellbar und von daher bedurfte es zwei weiterer Wochen bis Unterschiede erkennbar waren. Dann wiesen die Proben mit dem Harztyp C eine höhere Dickenquellung auf als bei den Harztypen A, D und E - Harztyp B lag dazwischen. Bei einem WPG von $60 \%$ beim gleichen Pressdruck konnte keine Differenzierung zwischen den Harzen stattfinden, da keines nach 5 Wochen eine Dickenquellung über 2,6\%, und selbst Harztyp D mit nur 48\% WPG (aufgrund niedrigerem Feststoffgehalts des Stammharzes) zuletzt nur 1,2\% Dickenquellung, aufwies.
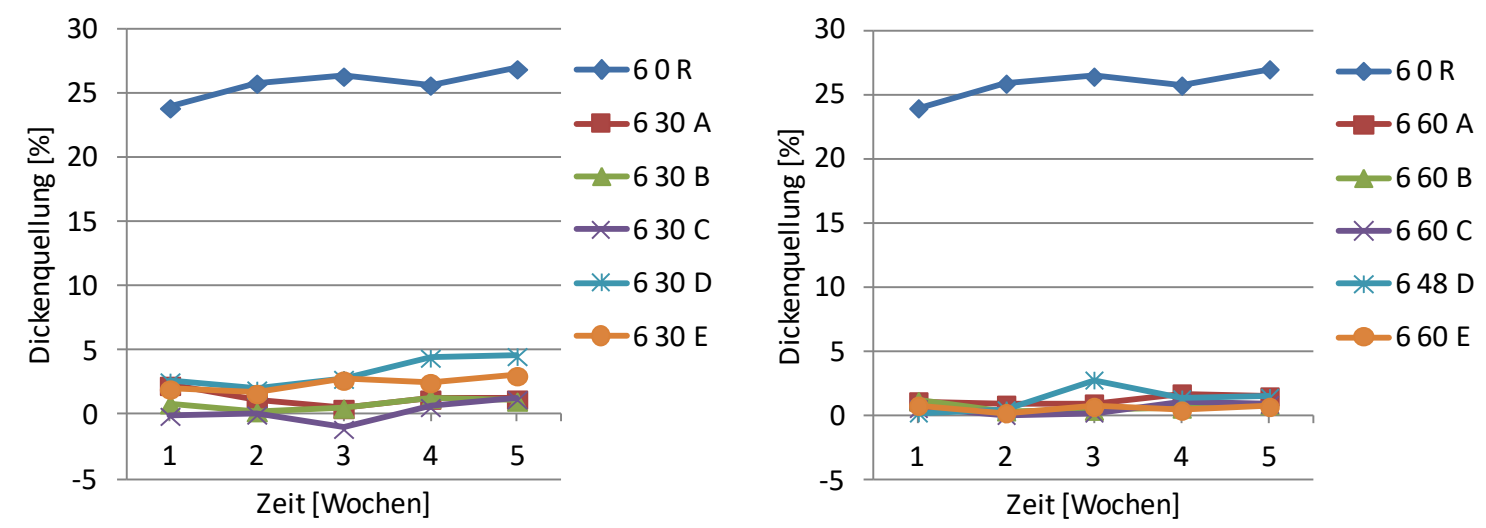

Abbildung 98: Dickenquellung in der Schnellbewitterung von Buchen-LVL $(8 * 3,7 \mathrm{~mm})$ mit Sperrlagen, hergestellt bei einem Pressdruck von $6 \mathrm{~N} / \mathrm{mm}^{2}$ mit einem WPG von 30\% (links) und 60\% (rechts) unter Verwendung verschiedener PF-Harze.

Mit der Anwendung eines Pressdruckes von $6 \mathrm{~N} / \mathrm{mm}^{2}$ und folglich einer hohen Verdichtung der Proben, stieg insbesondere bei den Referenzen die Dickenquellung durch die Schnellbewitterung. Von 9\% beim niedrigeren Pressdruck erhöhte sie sich auf maximal 27\% nach 5 Wochen. Bei den modifizierten Serien mit 30\% zeigten sich mehr Schwankungen als zuvor, jedoch war die Dickenquellung weiter reduziert, das Maximum bei 4,6\% lag und bis auf den Harztyp D die anderen Harztypen unter 3,1\% lagen. Mit dieser einen Ausnahme konnte nur durch den höheren Pressdruck ein ähnliches Ergebnis wie bei $2 \mathrm{~N} / \mathrm{mm}^{2}$ mit einem WPG von 60\% erzielt werden. Die Kombination des höchsten WPG mit dem höchsten Pressdruck (6 $\mathrm{N} / \mathrm{mm}^{2}$ ) wies durch die Kombination aus hohem Harzanteil und hoher Verdichtung die niedrigsten Werte hinsichtlich der Dickenquellung von modifiziertem Buchen-LVL mit Sperrlagen auf, denn, mit Ausnahme des Harztyp D, lag der Maximalwert bei 1,7\% für alle Harze. Folglich waren bei den unterschiedlichen Kombinationen aus Pressdruck, resp. Verdichtung, und WPG immer unterschiedliche Harze besser und daher eine eindeutige Aussage hinsichtlich des Harztyps nicht möglich. Es wurde jedoch ersichtlich, dass Serien mit einer reduzierten Wasseraufnahme auch eine reduzierte Dickenquellung aufwiesen. 

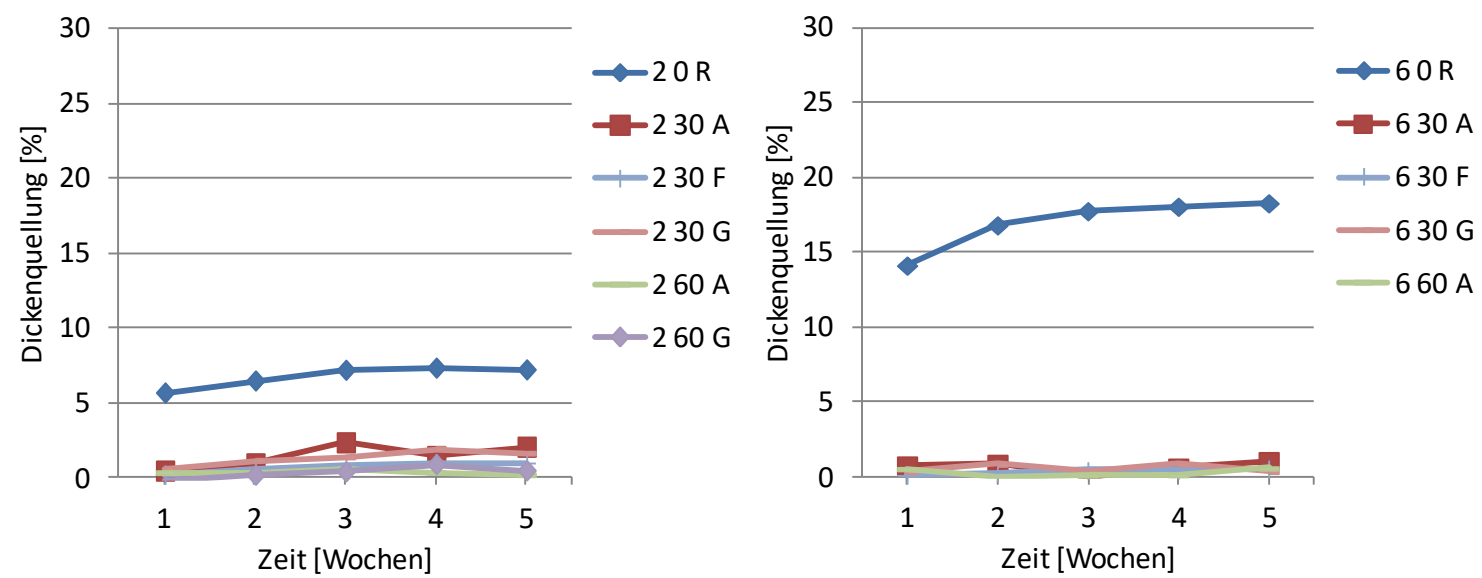

Abbildung 99: Dickenquellung in der Schnellbewitterung von Buchen-LVL $(8 * 3,7 \mathrm{~mm}$, parallel) ohne Sperrlagen, hergestellt bei einem Pressdruck von $2 \mathrm{~N} / \mathrm{mm}^{2}$ (links) und $6 \mathrm{~N} / \mathrm{mm}^{2}$ (rechts) bei WPGs von $30 \%$ und $60 \%$ unter Verwendung verschiedener PF-Harze.

Bei Betrachtung der Dickenquellung von LVL ohne Sperrlagen (Abbildung 99) im Vergleich zum LVL mit Sperrlagen (Abbildung 92 und Abbildung 93) fällt auf, dass alle Werte beim Pressdruck von $2 \mathrm{~N} / \mathrm{mm}^{2}$ (links) und von $6 \mathrm{~N} / \mathrm{mm}^{2}$ (rechts) niedriger ausfielen. Für den Harztyp A konnte hier bei einem WPG von 30\% und $2 \mathrm{~N} / \mathrm{mm}^{2}$ maximal 2,3\% gemessen werden und für die Harze $\mathrm{F}$ und $\mathrm{G}$ lag bei den gleichen Herstellungsparametern die Dickenquellung bei maximal 1,9\% - im Vergleich zu maximal 6,1\% beim Plattentyp mit Sperrlagen. Anhand der Harztypen A und G ließ sich, wie in der Abbildung 99 (links) gezeigt, zudem eine weitere Reduktion der Dickenquellung auf durchschnittlich 0,3\% feststellen. In Verbindung mit dem höheren Pressdruck von $6 \mathrm{~N} / \mathrm{mm}^{2}$ kam es auch bereits bei einem WPG von 30\% für die untersuchten Harztypen zu einer Reduzierung auf maximal 1,1\% und 0,5\% im Durchschnitt. Auf den Unterschied zwischen den Plattenaufbauten kann über den Harztyp A geschlossen werden, da beim WPG von 30\% bei beiden Pressdrücken die Dickenquellung mit Sperrlagen doppelt so hoch ausfiel (arithmetische Mittel) wie ohne Sperrlagen, d.h. bei ausschließlich paralleler Ausrichtung der Furniere. Wie durch bewusste Wahl der Skalen verdeutlicht werden sollte, zeigten auch die unbehandelten Referenzen mit Sperrlagen eine höhere Dickenquellung. 


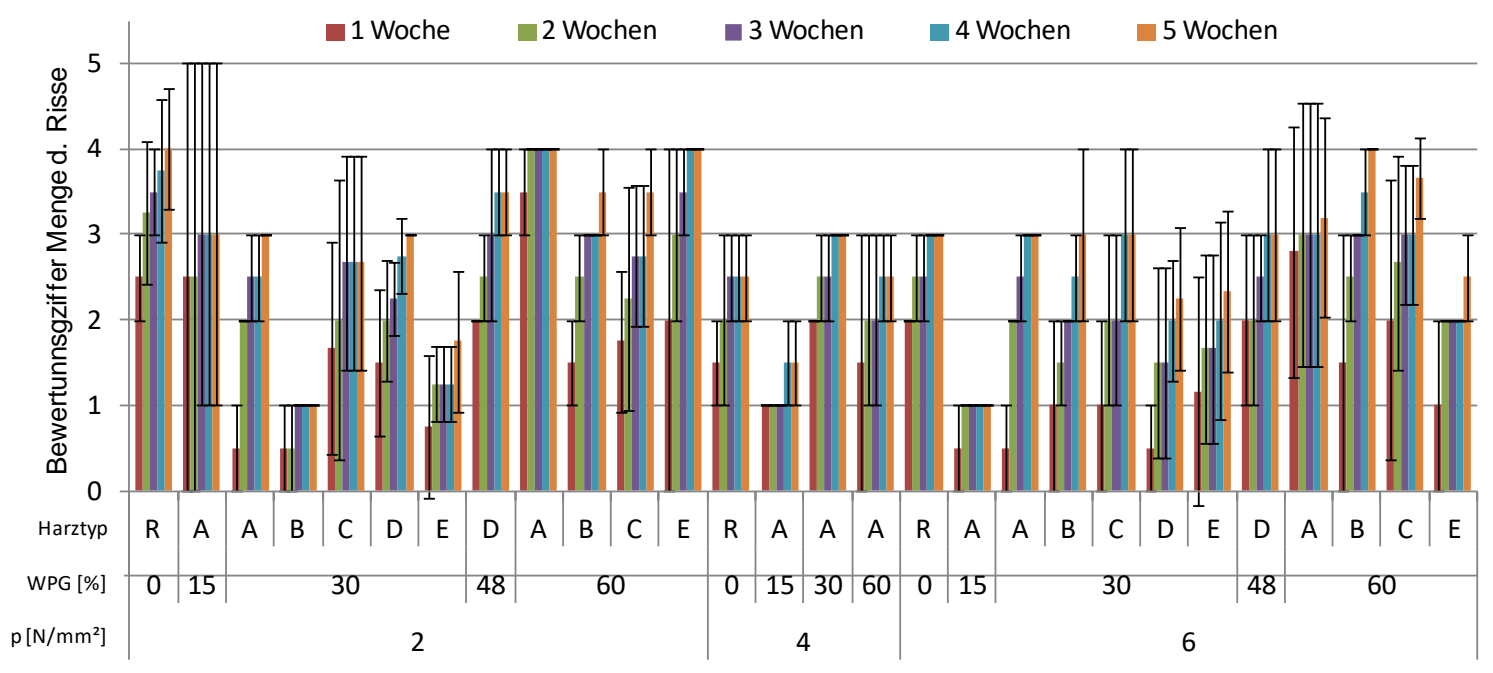

Abbildung 100: Bewertungsziffern (0-5) nach DIN ISO 4628-4 für die Menge der Risse in der Vorderseite von modifizierten Buchen-LVL (8-lagig mit 2 Sperrlagen) nach der Schnellbewitterung in Abhängigkeit von der Dauer, des Harztyps, des WPG und des Pressdruckes (p).

Entsprechend der Wasseraufnahmen und -abgaben sowie der Quell- und Schwindprozesse beim Buchen-LVL in der Schnellbewitterung kam es zur Bildung von Rissen in den Oberflächen bzw. Deckfurnieren der Prüfkörper. In der Abbildung 100 sind Bewertungsziffern für die Menge an Rissen in der Vorderseite (Oberseite) von LVL ohne Sperrlagen dargestellt (Mittelwerte und Standardabweichung). Bei einem Pressdruck von $2 \mathrm{~N} / \mathrm{mm}^{2}$ zeigten die Referenzen einen stetigen Anstieg innerhalb der 5 Wochen auf die Bewertungsziffer 4 (viele Risse). Für den Harztyp A ließ sich feststellen, dass der Mittelwert bei 15\% WPG zwar niedriger, die Streuung jedoch größer war. Bei gleichem Harz und Pressdruck mit einem WPG von 30\% wurde die Menge an Rissen mit der Ziffer 3 (mäßig viele) bewertet, was eine Verbesserung darstellte. Im Gegenteil dazu führte der WPG von 60\% (Harztyp A; $2 \mathrm{~N} / \mathrm{mm}^{2}$ ) zu der gleichen Bewertung wie bei den Referenzen, wobei der Wert nach der zweiten Woche konstant blieb. Bei einem Pressdruck von $4 \mathrm{~N} / \mathrm{mm}^{2}$ (Harztyp A), zeigten sich bei einem WG von $15 \%$ deutlich weniger Risse (2; wenige Risse, aber deutlich erkennbare Menge), während bei 30\% und 60\% WPG die gleiche Menge an Rissen (3) wie bei Referenzen festgestellt wurde. Auch bei einem Pressdruck von $6 \mathrm{~N} / \mathrm{mm}^{2}$ war es die Serie mit dem Harztyp A und einem WPG von 15\%, die die geringste Menge an Rissen aufwies. Bei einem WPG von 30\% hingegen fiel der Wert wieder höher, bzw. gleich den Referenzen, aus (3). Bei $6 \mathrm{~N} / \mathrm{mm}^{2}$ und $60 \%$ WPG wurde im Durchschnitt zwar noch die „3“ vergeben, jedoch war die Standardabweichung sehr hoch. Somit kann durch Betrachtung dieses einen Harztyps für die Menge der Risse festgestellt werden, dass nur der niedrigste WPG von 15\% unabhängig vom Pressdruck zur einer Verbesserung führte, während ein WPG von 30\% und 60\% gleich viele Risse oder sogar mehr Risse als die Referenzen aufwies.

Im Vergleich der Harztypen zueinander fällt in Abbildung 100 auf, dass bei einem WPG von 30\% die Harztypen B und E besser waren als A, C und D, eine Analogie zur Wasseraufnahme und Dickenquellung jedoch nicht bestand. Bei einem WPG von $60 \%$ erreichten alle Harztypen die gleiche Bewertungsziffer (3), jedoch stieg der Wert bei den Harztypen B und C langsamer an als bei den anderen. Bei einem Druck von $6 \mathrm{~N} / \mathrm{mm}^{2}$ und einem WPG von 30\% zeigten die 
Harztypen A, B und C den gleichen Mittelwert, wobei B und C die größere Standardabweichung aufwiesen. Diese war ähnlich hoch bei den Harztypen C und D, jedoch lag hier der Mittelwert nur bei „2“, d.h. wenige Risse, die aber in deutlich erkennbarer Menge festgestellt wurden. Bei einem WPG von $60 \%$ beim Pressdruck von $6 \mathrm{~N} / \mathrm{mm}^{2}$ lagen die Ergebnisse bis zur vierten Messung noch erkennbar unter den anderen Harzen, jedoch stieg die Menge an Rissen in der fünften Woche noch einmal an und reichte damit an eine Bewertungsziffer 3 (mäßig viele) heran. Damit wurden auch die Harztypen A und D im Durchschnitt bei diesen Herstellungsparametern bewertet, jedoch wiesen diese im direkten Vergleich die größere Standardabweichung auf. Die Harztypen B und C erhielten die Bewertungsziffer 4, da sie erkennbar viele Risse nach fünf Wochen aufwiesen.

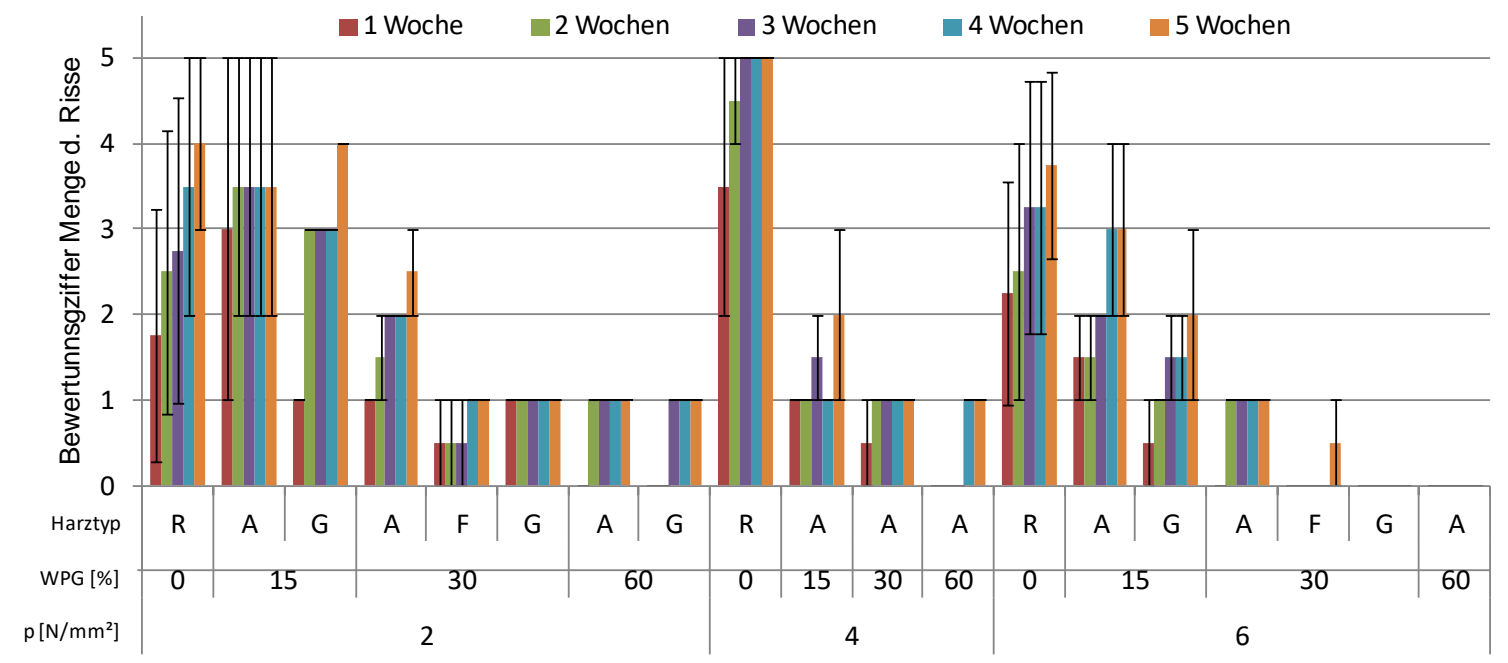

Abbildung 101: Bewertungsziffern (0-5) nach DIN ISO 4628-4 für die Menge der Risse in der Vorderseite von modifizierten Buchen-LVL (8-lagig parallel) nach der Schnellbewitterung in Abhängigkeit von der Dauer, des Harztyps, des WPG und des Pressdruckes (p).

In der Abbildung 101 sind Bewertungsziffern für die Menge an Rissen in der Vorderseite (Oberseite) von LVL ohne Sperrlagen abgebildet. Im Gegensatz zu den Versuchsplatten mit Sperrlagen war es hier die Bewertungsziffer 1, für nur einzelne, sich gerade abzeichnende Risse, die bei den modifizierten Serien am häufigsten vergeben wurde. Erhielten die Harztypen A und G beim Pressdruck von $2 \mathrm{~N} / \mathrm{mm}^{2}$ und einem WPG von 15\% noch die Ziffer 4, für viele Risse, und lagen damit auf dem Niveau der Referenzen, so führte der höhere WPG von $30 \% \mathrm{zu}$ einer deutlichen Abnahme. Die Prüfköper mit dem Harztyp A wiesen, mit einer Zunahme in der letzten Woche des Versuchs, noch mäßig viele Risse (3) auf. Die Harztypen F und G jedoch wurden bereits mit der Ziffer 1 bewertet, wie dies auch für die Harztypen A und G bei einem WPG von $60 \%$ geschah.

Bei einem Pressdruck von $4 \mathrm{~N} / \mathrm{mm}^{2}$ kam es für den Harztyp A mit 15\% zu einer geringeren Menge an Rissen (2) als bei $2 \mathrm{~N} / \mathrm{mm}^{2}$, wohingegen die Referenzen eine Steigerung erfuhren (5) und die größte Menge an Rissen aufwiesen. Bei dem Pressdruck von $6 \mathrm{~N} / \mathrm{mm}^{2}$ wiesen die Referenzen genauso viele Risse auf wie bei $2 \mathrm{~N} / \mathrm{mm}^{2}$. Die Harztypen A und G zeigten bei einem WPG von 15\% etwas weniger Risse auf. Die beste Bewertung im Test erhielten schließlich die Prüfkörper mit den WPGs von 30\% und $60 \%$ bei dem Pressdruck von $6 \mathrm{~N} / \mathrm{mm}^{2}$. Beim Harztyp G mit 30\% sowie beim Harztyp A mit $60 \%$ waren keine Risse nach fünf Wochen 
Schnellbewitterung erkennbar. Im Vergleich der Harze zueinander zeigte der Harztyp A bei allen Kombinationen etwas mehr Risse als die Harztypen F und G. Dieses Ergebnis weist eine Analogie zur den Ergebnissen der Wasseraufnahme auf, jedoch nicht zur Dickenquellung. Da eine höhere Wasseraufnahme durch Risse begünstigt werden kann, ist ein Zusammenhang wahrscheinlich.

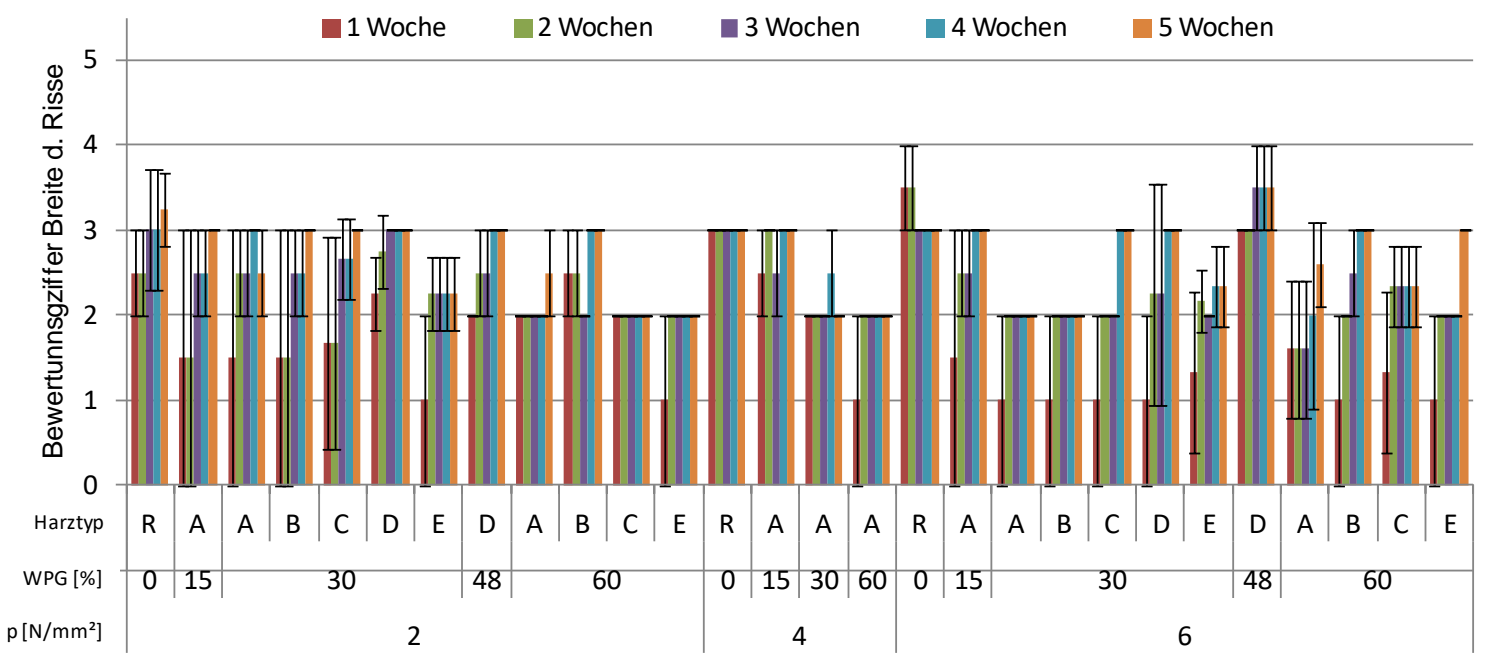

Abbildung 102: Bewertungsziffern (0-5) nach DIN ISO 4628-4 für die Breite der Risse in der Vorderseite von Buchen-LVL (8-lagig mit zwei Sperrlagen), hergestellt mit verschiedenen Drücken, WPG und Harztypen, in Abhängigkeit von der Zeit in der Schnellbewitterung.

Bei der Bewertung der Breite der Risse auf der Oberfläche, bzw. im Deckfurnier des BuchenLVL mit Sperrlagen zeigte sich, dass, wenn Risse vorhanden waren, sie gerade mit bloßen Auge sichtbar (2) oder sie noch klein, aber deutlich sichtbar (3) und nur in Ausnahmen breit (4) waren. Dabei war bei einem Pressdruck von $2 \mathrm{~N} / \mathrm{mm}^{2}$ und einem WPG von $30 \%$ kein Unterschied zwischen den modifizierten Proben und der Referenz feststellbar. Beim geleichen Pressdruck und mit einem WPG von 60\% zeigten die Harztypen C und E, und bis zur vierten Messung auch Harztyp A, schmalere Risse als die Referenz sowie der Harztyp B. Dieser positive Einfluss des WPG zeigte sich auch bei einem Pressdruck von $4 \mathrm{~N} / \mathrm{mm}^{2}$; die Proben mit $30 \%$ und 60\% WPG zeigten hier etwas schmalere Risse als die Referenz und die Variante mit 15\%. Bei einem Pressdruck von $6 \mathrm{~N} / \mathrm{mm}^{2}$ zeigte sich, dass die Risse bei den Harztypen A und B bei einem WPG von 30\% schmaler waren als bei ihren Pendants mit dem Pressdruck von 2 $\mathrm{N} / \mathrm{mm}^{2}$. Die Harztypen C und D hingegen wiesen ein ähnliches Ergebnis auf. Somit kann nur partiell auf einen positiven Einfluss eines höheren Pressdruckes geschlossen werden. 


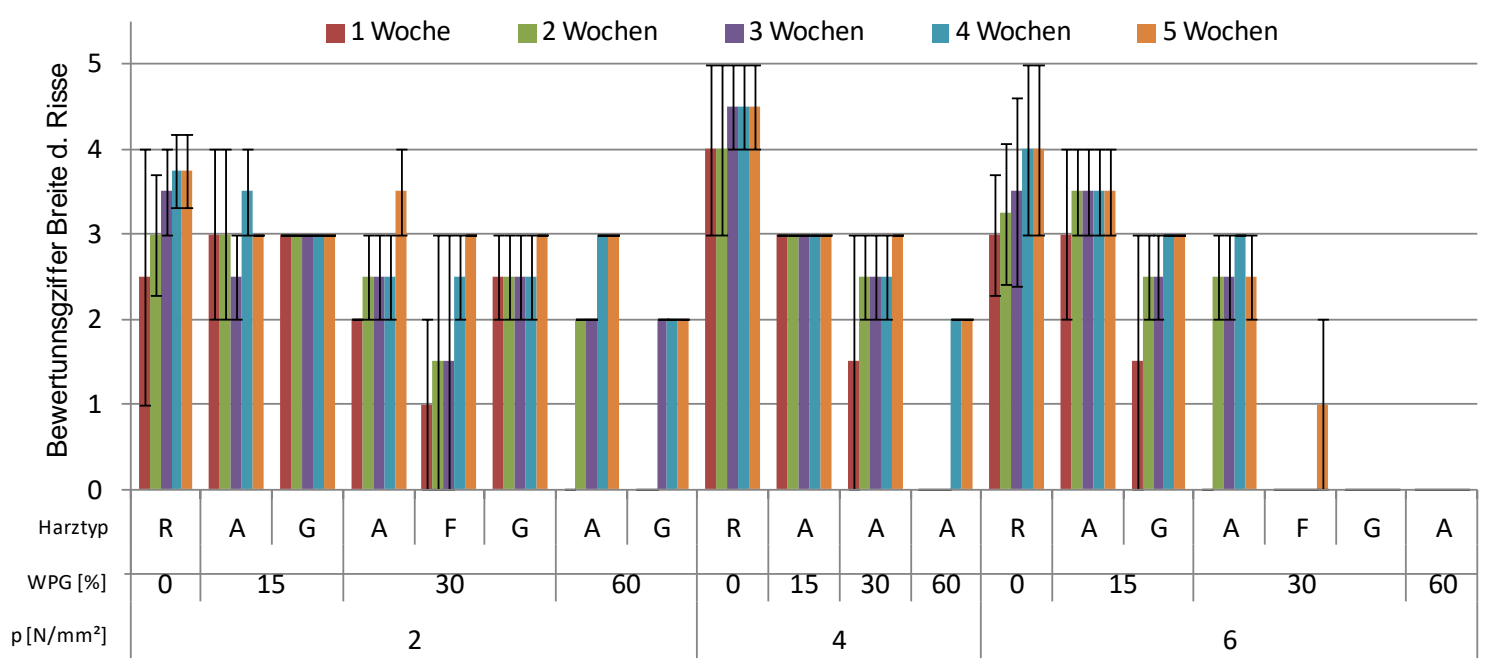

Abbildung 103: Bewertungsziffern (0-5) nach DIN ISO 4628-4 für die Breite der Risse in der Vorderseite von Buchen-LVL (8-lagig parallel), hergestellt ohne Sperrlagen mit verschiedenen Drücken, WPG und Harztypen, in Abhängigkeit von der Zeit in der Schnellbewitterung.

Bei den Versuchsplatten ohne Sperrlagen fällt auf, dass die Referenzen bei diesem Plattentyp breitere Risse aufwiesen, als jene mit Sperrlagen. Ohne Modifizierung hatte die unter dem Deckfurnier in ihrem Faserverlauf um 90 Grad gedrehte Sperrlage somit einen Einfluss auf die Rissbreite. Bei den modifizierten Serien mit dem Harztyp A und im direkten Vergleich der Plattenaufbauten war dies jedoch nicht der Fall. Hier zeigten die Proben des Harztyp A, bei einem Pressdruck von $2 \mathrm{~N} / \mathrm{mm}^{2}$ und $15 \%$ sowie $30 \%$, eine Tendenz zu breiten Rissen mit der Bewertungsziffer 4. Die weniger breiten Risse konnten, wie beim Plattenaufbau mit Sperrlagen, bei diesem Pressdruck bei den Serien mit 60\% WPG festgestellt werden. Auch bei einem Pressdruck von $4 \mathrm{~N} / \mathrm{mm}^{2}$ waren beim höheren WPG geringere Rissbreiten festgestellt worden. Die Rissbreite der Referenzen lag hier, wie auch bei $6 \mathrm{~N} / \mathrm{mm}^{2}$, höher als bei $2 \mathrm{~N} / \mathrm{mm}^{2}$. Bei $6 \mathrm{~N} / \mathrm{mm}^{2}$ wurden, wie sich bei der Menge der Risse bereits zeigte, die besten Ergebnisse hinsichtlich des Rissverhaltens bei den mit den Harztypen F und G modifizierten Buchen-LVL erzielt. Die einzelnen Risse des Harztyp A bei diesen Herstellungsparametern waren jedoch deutlich sichtbar. Wenngleich sich in Bezug auf den Einsatz von Sperrlagen zeigte, dass diese zu einer größeren Menge an Rissen führten, führten sie jedoch nicht zu breiteren Rissen, sondern konnten bei den unbehandelten Referenzen auch zu einer Limitierung führen. Modifizierte Serien waren zusammenfassend beim Einsatz von Sperrlagen nur wenig besser, ohne Sperrlagen wiesen einzelne modifizierte Serien jedoch eine deutlich reduzierte Rissneigung infolge der Schnellbewitterung auf. 
5.3.5.1.2 Beschichtetes modifiziertes LVL

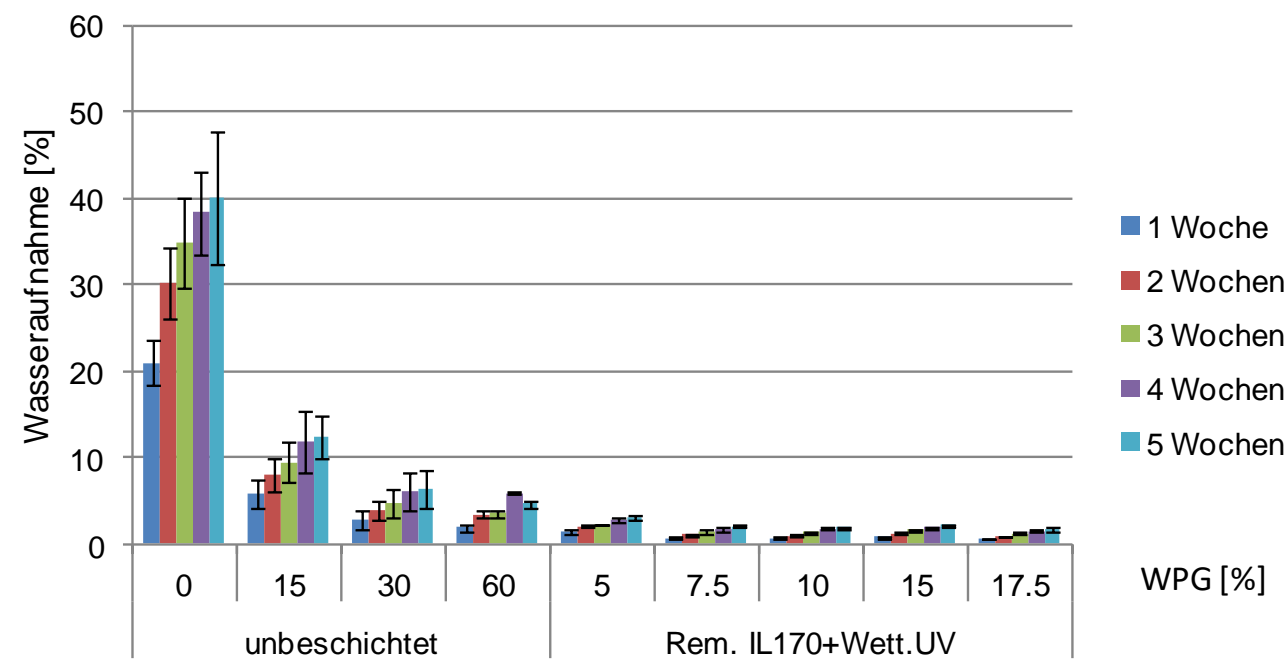

Abbildung 104: Wasseraufnahme von Buchen-LVL (8-lagig parallel) in der Schnellbewitterung; beschichtet und unbeschichtet mit verschiedenen WPGs bei einem Pressdruck von 2 N/mm ${ }^{2}$.

Infolge einer Beschichtung konnte bei modifiziertem Buchen-LVL mit ausschließlich parallelem Aufbau eine deutliche Reduzierung der Wasseraufnahme unabhängig vom WPG erzielt werden. Die Werte der beschichteten Proben lagen sogar unter dem Wert für modifizierte Proben mit einem WPG von 60\%.

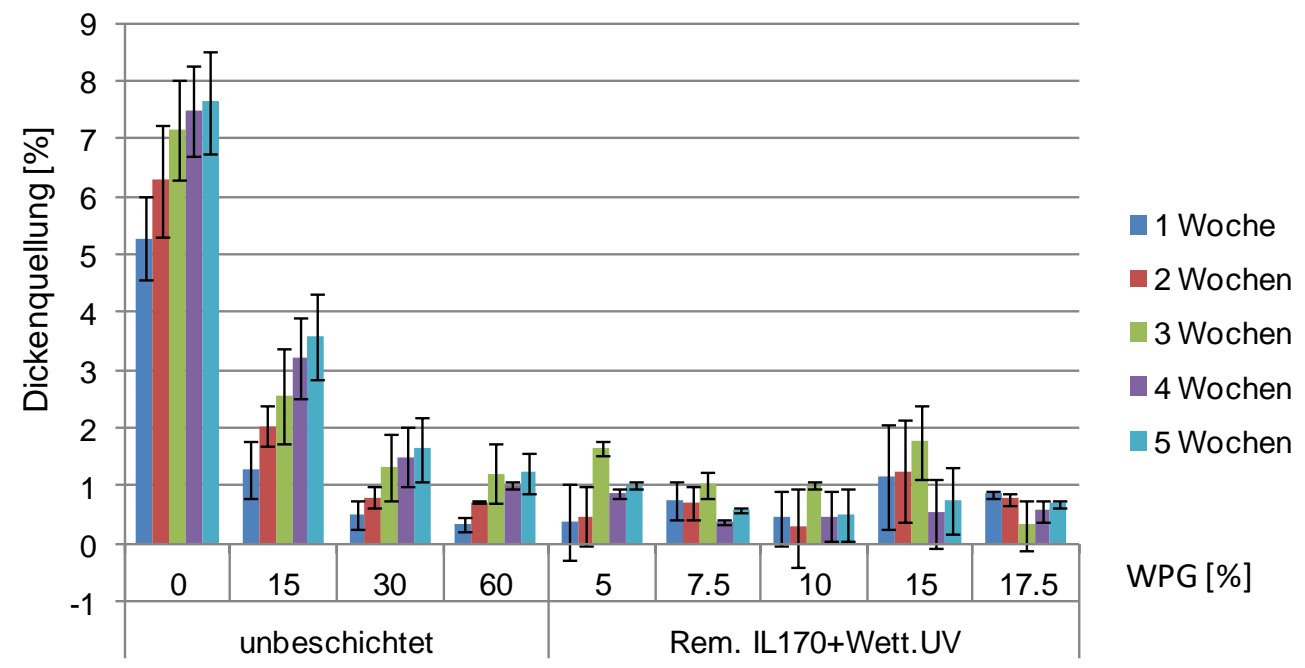

Abbildung 105: Dickenquellung von Buchen-LVL (8-lagig parallel) in der Schnellbewitterung; beschichtet und unbeschichtet mit verschiedenen WPGs bei einem Pressdruck von $2 \mathrm{~N} / \mathrm{mm}^{2}$.

Die beschichteten und modifizierten Buchen-LVL-Prüfkörper wiesen in der Schnellbewitterung die niedrigsten Ergebnisse bezüglich der Dickenquellung auf. Mit einer Ausnahme lag die Dickenquellung nach 5 Wochen bei 1\% und damit auf dem Niveau unbeschichteter Proben bei einem WPG von 60\%. Eine beschichtete Variante mit einem WPG von 15\% lag etwas darüber, aber mit unter 2\% Dickenquellung waren ihre Ergebnisse mit denen der unbeschichteten 30\%-Variante vergleichbar. Es fiel auf, dass, im Gegensatz zu den unbeschichteten Proben, die Höhe der Ergebnisse nicht mit zunehmender Testdauer stieg, sondern auch abfiel. Eine stetige Entwicklung und längerfristige Prognose lässt sich somit nicht 
eindeutig ableiten. Wenngleich die Beschichtung hier im Hinblick auf die Wasseraufnahme und Dickenquellung vorteilhaft zu sein scheint, ist hier aufgrund der geringen Versuchsdauer damit zu rechnen, dass mit einer längeren Bewitterungsdauer weitere Veränderungen der Ergebnisse auftreten würden.

Tabelle 17: Bewertungsziffern (0-5) nach DIN ISO 4628-4 für die Menge der Risse in der Vorderseite von beschichteten und unbeschichteten modifizierten Buchen-LVL (8-lagig, parallel, $2 \mathrm{~N} / \mathrm{mm}^{2}$ Pressdruck) in Abhängigkeit der Testdauer in der Schnellbewitterung. Beschichtung: 1x Remmers Grundierung IL170 (60s getaucht) und $1 \times$ Remmers Wetterschutzlack $U V+(150 \mu \mathrm{m}$ gespritzt).

\begin{tabular}{cccccccc} 
Beschichtung & WPG [\%] & Harz & 1 Woche & 2 Wochen & 3 Wochen & 4 Wochen & 5 Wochen \\
\hline- & 0 & R & $3 \pm 1,4$ & $3 \pm 1,3$ & $4 \pm 1,3$ & $4 \pm 1,1$ & $4 \pm 0,7$ \\
& 15 & $F$ & $2 \pm 1,2$ & $2 \pm 0,9$ & $3 \pm 1,1$ & $3 \pm 1,1$ & $3 \pm 0,7$ \\
& 30 & $F$ & $1 \pm 0,4$ & $2 \pm 1,0$ & $2 \pm 1,2$ & $2 \pm 0,9$ & $2 \pm 0,9$ \\
& 60 & $F$ & $1 \pm 0,0$ & $1 \pm 0,0$ & $2 \pm 0,5$ & $2 \pm 0,5$ & $2 \pm 0,0$ \\
\hline IL170+ Wett.UV+ & 5 & $F$ & $0 \pm 0,0$ & $0 \pm 0,0$ & $1 \pm 0,5$ & $1 \pm 0,5$ & $1 \pm 0,5$ \\
& 7.5 & $F$ & $0 \pm 0,0$ & $0 \pm 0,0$ & $0 \pm 0,0$ & $0 \pm 0,0$ & $0 \pm 0,0$ \\
& 10 & $F$ & $0 \pm 0,0$ & $0 \pm 0,0$ & $0 \pm 0,0$ & $0 \pm 0,0$ & $0 \pm 0,0$ \\
& 15 & $F$ & $0 \pm 0,0$ & $0 \pm 0,0$ & $0 \pm 0,0$ & $0 \pm 0,0$ & $0 \pm 0,0$ \\
& 17.5 & $F$ & $0 \pm 0,0$ & $0 \pm 0,0$ & $0 \pm 0,0$ & $0 \pm 0,0$ & $0 \pm 0,0$
\end{tabular}

Bei Betrachtung der Ergebnisse zur Rissevaluation in der Oberfläche von beschichteten, modifizierten Buchen-LVL (Tabelle 17) fällt auf, dass innerhalb der fünf-wöchigen Versuchsdauer, außer bei der Variante mit einem WPG von 5\%, keine Risse festgestellt werden konnten. Unter Beachtung der Dickenquellung fällt bei der 5\%-Variante auf, dass diese zwar ähnlich quoll wie unbeschichtete Proben mit einem WPG von 60\%, jedoch nur einzelne, sich gerade abzeichnende Risse (1), festgestellt werden konnten, während bei letztgenannter Serie die Risse von geringer, aber deutlich erkennbarer Menge (2), vorlagen.

Aufgrund dessen, dass es, bis auf die eine Ausnahme, nicht zu Rissen in den Oberflächen beschichteter Proben kam, sind die beschichteten Proben mit der Ziffer "0“ zu bewerten gewesen. Bei der beschichteten Variante mit 5\% WPG, waren die Risse gerade mit 10facher Vergrößerung erkennbar und erhielten die Bewertungsziffer „1“. 


\subsubsection{Freilandversuch}

\subsection{Klimadaten}

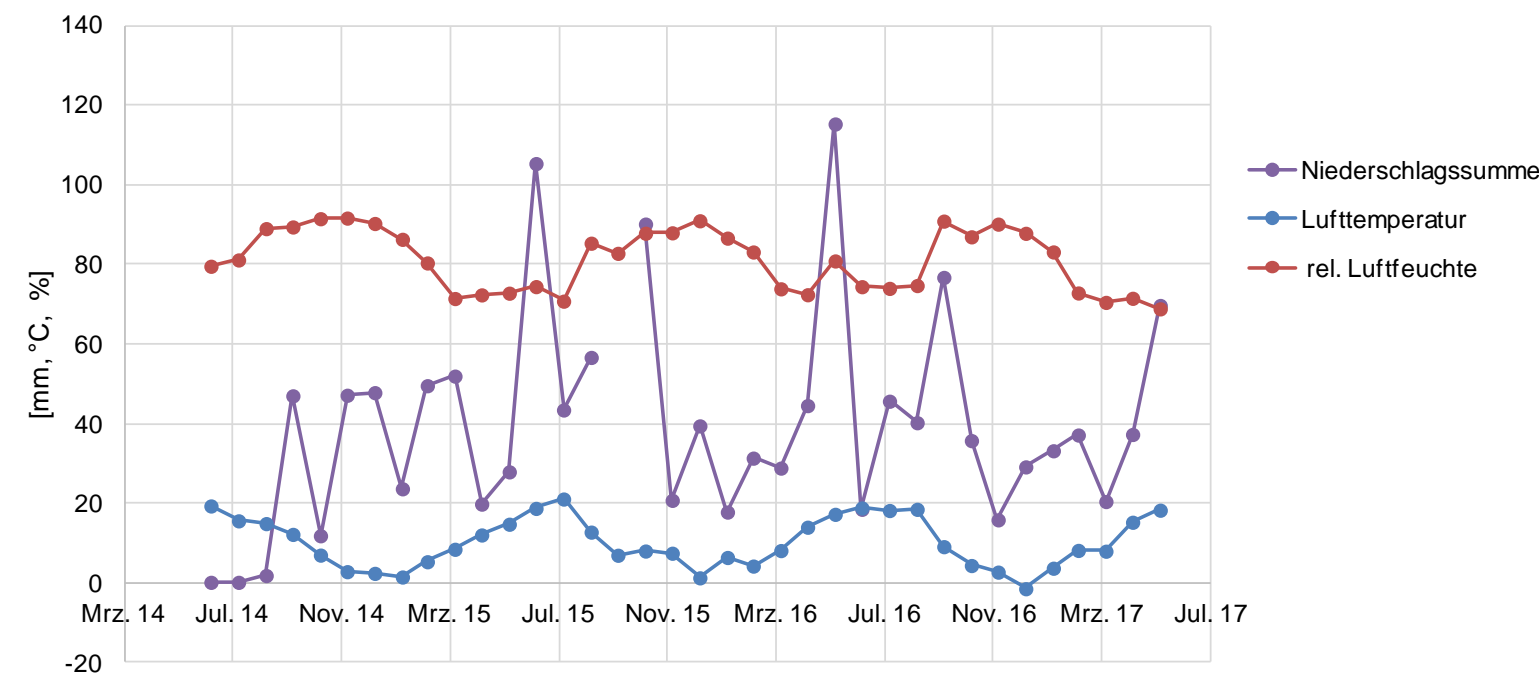

Abbildung 106: Klimadaten zum Niederschlag, der Lufttemperatur und relativen Luftfeuchte in $2 \mathrm{~m}$ Höhe über dem Boden, gemessen an der Messstation im Forstbotanischen Garten in Göttingen.

Die Klimadaten in der Nähe der Versuchsfläche, auf der die Freilandbewitterung stattfand, zeigten die für Göttingen typischen jahreszeitlichen Schwankungen hinsichtlich Niederschlag, Lufttemperatur und Luftfeuchte. Auffällig dabei sind die jährlichen Niederschlagsmaxima zwischen April und Juli in Kombination mit einer, aufgrund der sommerlich hohen Durchschnittstemperaturen, geringen relativen Luftfeuchte. Die zweitgrößten Niederschlagsmengen wurden im Oktober 2015 und September 2016 gemessen, wobei die durchschnittliche relative Luftfeuchte in diesen Zeiträumen aufgrund fallender Temperaturen deutlich (maximal $91 \%$ im Oktober 2016) anstieg.

\subsection{Unbeschichtetes modifiziertes LVL}

Nachfolgend werden die Ergebnisse der Bewitterungsprüfung für unbeschichtete, PFmodifizierte, 8-lagige Buchen-LVL mit zwei Sperrlagen dargestellt.

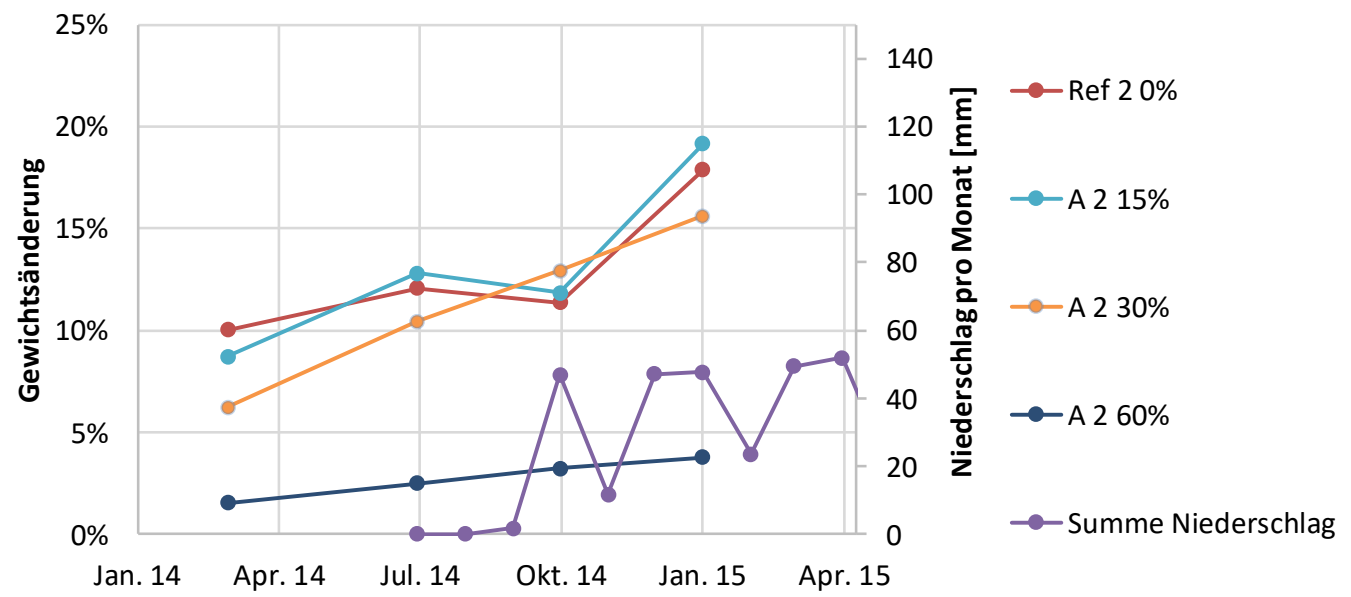

Abbildung 107: Gewichtsänderung unbeschichteter PF-modifizierter Buchen-LVL-Prüfkörper (8-lagig mit Sperrlagen) in Relation zum Ausgangsgewicht sowie monatliche Niederschlagmengen (Sekundärachse). 
In Abbildung 107 ist die relative Veränderung der Probengewichte bezogen auf das Ausgangsgewicht zum jeweiligen Messintervall dargestellt. Es handelte sich dabei um unbeschichtete 8-lagige Buchen-LVL mit zwei Sperrlagen, welche mit dem Harztyp A modifiziert wurden. Die Serienbezeichnung in der Legende gibt den WPG und den Pressdruck wieder. Der Pressdruck der hier abgebildeten Proben war konstant bei $2 \mathrm{~N} / \mathrm{mm}^{2}$ und folglich wiesen die modifizierten Serien nur eine leichte Verdichtung auf - ca. 10\%. Der WPG dieser Serien lag zwischen $15 \%$ und $60 \%$. Infolge von Niederschlag und Wasseraufnahme zeigte sich für alle Proben eine Gewichtszunahme innerhalb von 4 Messintervallen bzw. einem Jahr. Die Prüfkörper der Serien mit einem WPG von $60 \%$ zeigten hier die niedrigste Gewichtszunahme bzw. Wasseraufnahme in Höhe von maximal 4\%. Die Serien mit einem WPG von 15\% und von $30 \%$ nahmen hingegen 19\% bzw. 16\% auf. Sie unterschieden sich somit gravimetrisch betrachtet nicht signifikant von den Referenzen, welche bei $18 \%$ lagen. Auffällig ist jedoch bei den Proben mit einem WPG von 30\%, dass hier die Steigerung der Gewichte zwischen den Messintervallen nahezu konstant blieb, im Gegensatz zu den Proben mit niedrigeren WPG die Schwankung kleiner war und somit auch eine Abtrocknung in Zeiten niedrigeren Niederschlags nur in geringem Maße stattfand. Die Anzahl an Tagen mit erhöhter Probenfeuchte war bei diesen Proben im Betrachtungszeitraum trotzdem geringer, da sie durchschnittlich eine Gewichtszunahme von $11 \%$ aufwiesen, im Gegensatz zu der Variante mit 15\% WPG, die im Durchschnitt bei 13\% lag.

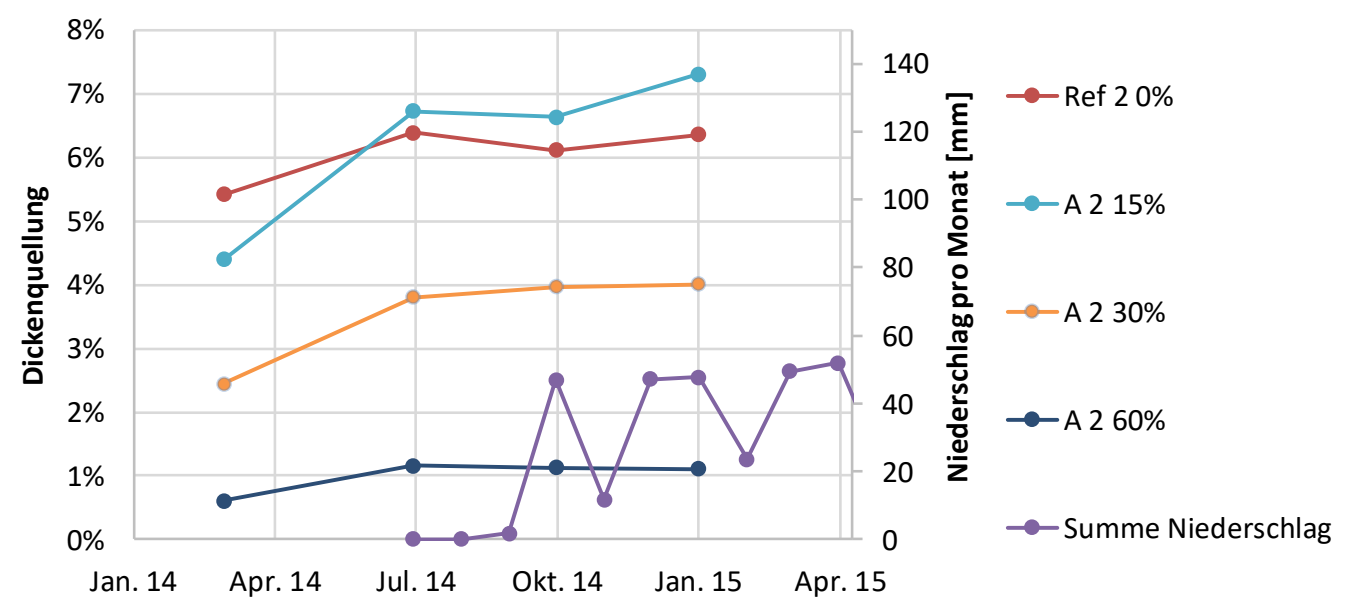

Abbildung 108: Dickenquellung unbeschichteter PF-modifizierter Buchen-LVL-Prüfkörper (8-lagig mit Sperrlagen) in Relation zum Ausgangszustand sowie monatliche Niederschlagmengen (Sekundärachse).

Durch die Abbildung der Dickenquellung unbeschichteter PF-modifizierter Buchen-LVLPrüfkörper und der unbehandelten Referenzprüfkörper wird ersichtlich, dass trotz einer ähnlichen Wasseraufnahme, die Referenzen, die Serie mit einem WPG von 15\% und die Serie mit einem WPG von 30\%, deutliche Unterschiede aufwiesen. Während ein WPG von 30\% nur eine maximale Quellung von 4\% aufwies, lag nach einem Jahr bei den Proben mit nur 15\% WPG die Quellung mit 7\% über dem Wert der Referenzen. Wie im Kap. 5.3.2.1 gezeigt, ist dies auf eine im Vergleich zu den Referenzen höhere Verdichtung der Proben zurückzuführen. Eine weitere Beobachtung ist, dass die meiste Quellung im ersten Messintervall zu verzeichnen war und in allen weiteren Intervallen nur mäßig oder gar nicht mehr anstieg. Die niedrigste Quellung in Höhe von nur 1\% wies die Serie mit einem WPG von 60\% auf. 

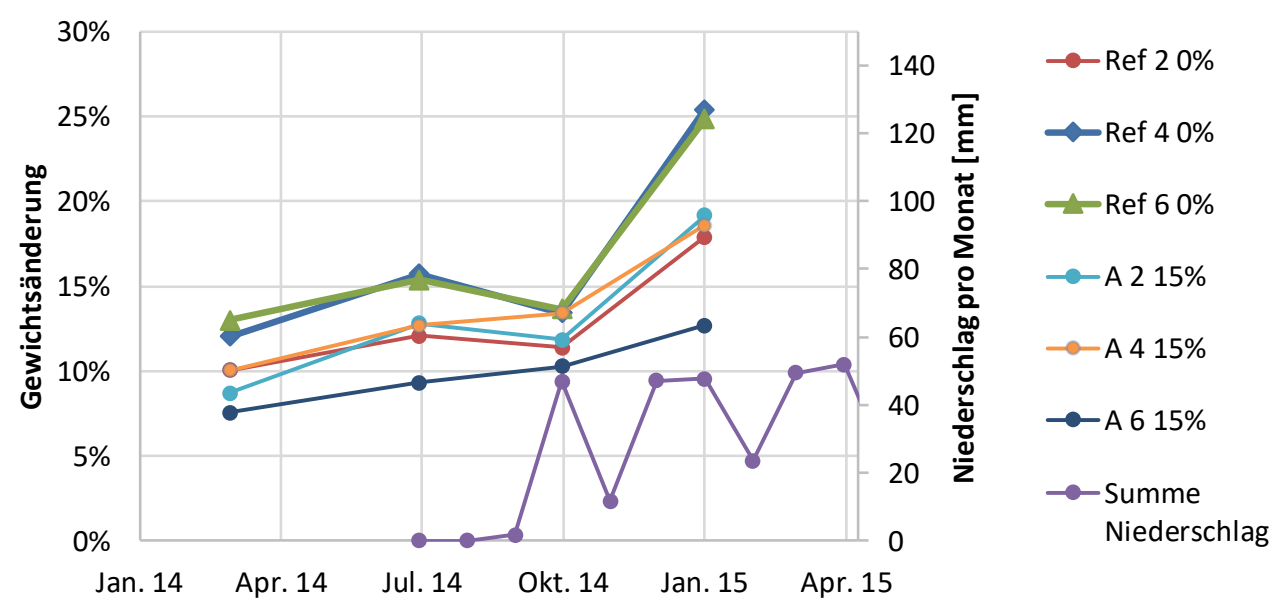

Abbildung 109: Gewichtsänderung unbeschichteter PF-modifizierter Buchen-LVL-Prüfkörper (8-lagig mit Sperrlagen), mit einem WPG von $15 \%$ bei unterschiedlichen Pressdrücken, in Relation zum Ausgangsgewicht sowie monatliche Niederschlagmengen (Sekundärachse).

Um die Auswirkung des Pressdrucks auf die Witterungseigenschaften PF-modifizierten Buchen-LVLs zu veranschaulichen, wurden die Abbildung 109 und die Abbildung 110 erstellt. Dabei wurden für einen WPG von $15 \%$ sowie für die unbehandelten Referenzen die Gewichtsänderung und die Dickenquellung für die Serien mit $2 \mathrm{~N} / \mathrm{mm}^{2}, 4 \mathrm{~N} / \mathrm{mm}^{2}$ und $6 \mathrm{~N} / \mathrm{mm}^{2}$ Pressdruck ausgewählt. Bei der Gewichtsänderung durch Wasseraufnahme zeigen beide Referenzen mit $4 \mathrm{~N} / \mathrm{mm}^{2}$ und $6 \mathrm{~N} / \mathrm{mm}^{2}$ die höchsten Werte, mit maximal 25\% nach einem Jahr. Dabei wiesen sie die größten Schwankungen zwischen den Messintervallen auf. Auf die geringen Niederschlagsmengen zwischen Juli und Oktober 2014 reagieren sie mit einer Gewichtsabnahme infolge von Trocknung. Etwas abgeschwächt zeigt auch die Referenz mit $2 \mathrm{~N} / \mathrm{mm}^{2}$ eine Abtrocknung auf. Sie wies von beiden abweichend jedoch nur eine Gewichtszunahme von 18\% auf. Die modifizierten Serien mit 15\% WPG und einem Pressdruck von $2 \mathrm{~N} / \mathrm{mm}^{2}$ und $4 \mathrm{~N} / \mathrm{mm}^{2}$ wiesen eine ähnliche Wasseraufnahme von $19 \%$ auf. Folgte der Verlauf der modifizierten Serie mit $2 \mathrm{~N} / \mathrm{mm}^{2}$ noch der zugehörigen Referenz, konnte bei 4 $\mathrm{N} / \mathrm{mm}^{2}$ keine Gewichtsabnahme zwischen den Intervallen festgestellt werden. Ganz deutlich unterschiedlich von den vorher genannten Serien wies die modifizierte Serie mit einem Pressdruck von $6 \mathrm{~N} / \mathrm{mm}^{2}$ die niedrigste Wasseraufnahme von nur 13\% nach einem Jahr bei gleichbleibenden Zunahmen zwischen den Intervallen auf. Die Verdichtung hat hier somit bei einem Pressdruck von $4 \mathrm{~N} / \mathrm{mm}^{2}$ bereits die Wasseraufnahme und Abgabe verlangsamt, da hier die Zelllumen bereits reduziert wurden und die Wegsamkeit für Wasser und Wasserdampf herabgesetzt wurde. Dieser Effekt kam bei $6 \mathrm{~N} / \mathrm{mm}^{2}$ noch stärker zum Tragen und so ist es auf die verminderte Wegsamkeit infolge der Verdichtung zurückzuführen, dass nach einem Jahr deutlich weniger Wasser aufgenommen wurde als bei den Referenzen und übrigen Serien mit dem gleichen WPG. 


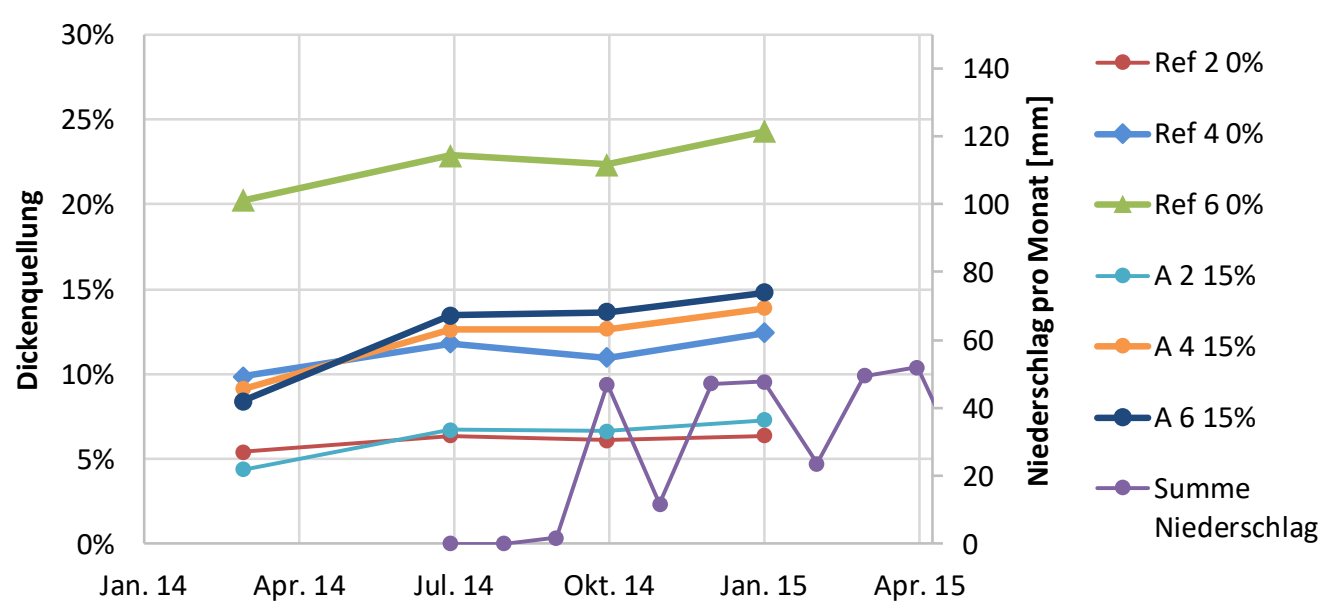

Abbildung 110: Dickenquellung unbeschichteter PF-modifizierter Buchen-LVL-Prüfkörper (8-lagig mit Sperrlagen), mit einem WPG von $15 \%$ bei unterschiedlichen Pressdrücken, in Relation zum Ausgangszustand sowie monatliche Niederschlagmengen (Sekundärachse).

Die in Abbildung 110 dargestellte Dickenquellung für Referenzen und Serien mit einem WPG von 15\% (nur Harztyp A) zeigt, entgegen der bei der Wasseraufnahme beobachteten Schwankungen zwischen den Messintervallen, dass die stärkste Dickenquellung bereits in den ersten 6 Monaten der Bewitterung stattfand und bis zum vierten Messintervall (1 Jahr) nur noch gering anstieg. Die deutliche Gewichtsabnahme bei der dritten Messung, wie oben beschrieben, führte nur bei den Referenzen mit $4 \mathrm{~N} / \mathrm{mm}^{2}$ und $6 \mathrm{~N} / \mathrm{mm}^{2}$ zu einem leichten Rückgang in der Quellung. Die niedrigsten Quellraten wiesen die Referenz und die modifizierte Serie bei einem Pressdruck von $2 \mathrm{~N} / \mathrm{mm}^{2}$ auf. Wie bei der Gewichtsänderung wiesen sie auch hier sehr ähnliche Ergebnisse auf. Die maximale Quellung dieser beiden Serien lag nach einem Jahr bei $6 \%$ bzw. 7\%. Alle übrigen Serien wiesen infolge der Verdichtung bei den höheren Pressdrücken, größere Quellraten auf. Dicht beieinander lagen auch die Ergebnisse der Serien mit einem Pressdruck von $4 \mathrm{~N} / \mathrm{mm}^{2}$, wobei die Referenz bei den letzten drei Messungen niedriger lag, als die modifizierte Serie. Eine nur leicht höhere Quellung mit maximal 15\% wies die modifizierte und bei einem Pressdruck von $6 \mathrm{~N} / \mathrm{mm}^{2}$ verdichtete Serie auf. Von allen modifizierten Serien wies sie damit einerseits die höchste Quellung auf, jedoch im Vergleich zu ihrer bei gleichem Pressdruck hergestellten Referenz, auch eine Reduzierung der Quellung um 38\%. Daraus lässt sich in Bezug auf Verdichtung generell ableiten, dass sie die Quellung stets erhöht. Dies gilt für unmodifizierte und für mit einem WPG von 15\% modifizierte Buchen-LVL gleichfalls.

$\mathrm{Da}$, wie bereits oben für den Pressdruck von $2 \mathrm{~N} / \mathrm{mm}^{2}$ gezeigt, höhere WPGs zu einer geringeren Wasseraufnahme und Dickenquellung führten, werden nachfolgend die Ergebnisse für einem WPG von 30\% bei den drei verwendeten Pressdrücken dargestellt. 

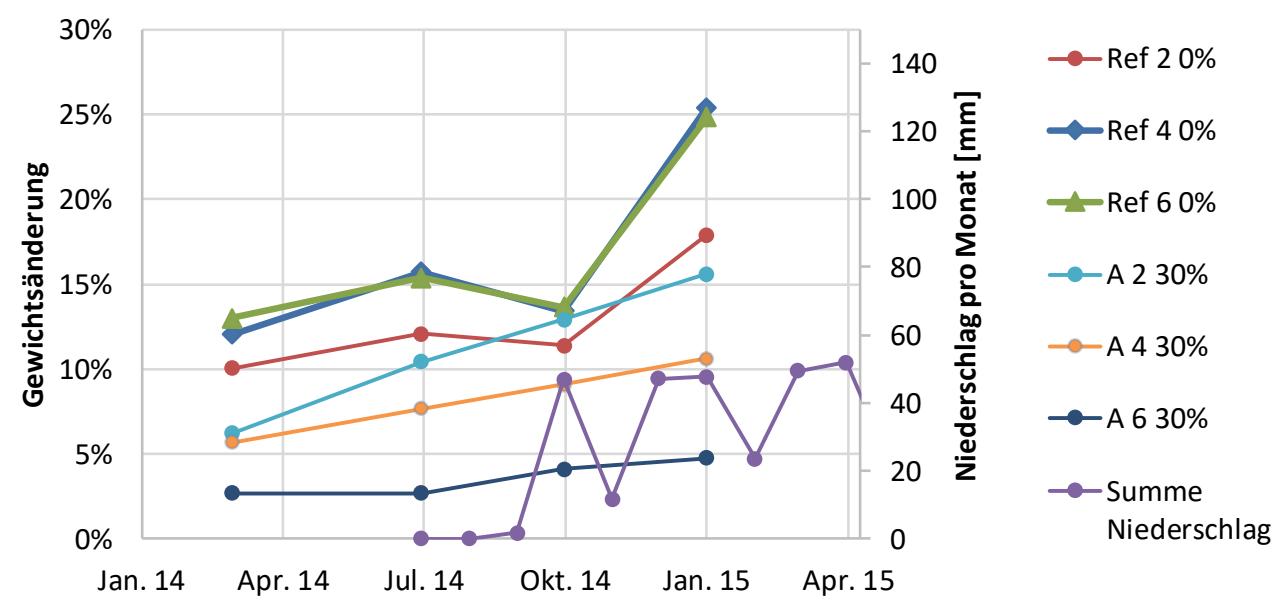

Abbildung 111: Gewichtsänderung unbeschichteter PF-modifizierter Buchen-LVL-Prüfkörper (8-lagig mit Sperrlagen), mit einem WPG von $30 \%$ bei unterschiedlichen Pressdrücken, in Relation zum Ausgangsgewicht sowie monatliche Niederschlagmengen (Sekundärachse).

Abbildung 111 zeigt, dass mit zunehmendem Pressdruck bei einem WPG von 30\% die Gewichtsänderung bzw. Wasseraufnahme abnahm. So unterscheiden sich alle modifizierten Serien deutlich voneinander. Mit einer Wasseraufnahme von $16 \%$ bei einem Pressdruck von $2 \mathrm{~N} / \mathrm{mm}^{2}$ nach einem Jahr Bewitterung, ergaben sich hier im Verhältnis zu den Referenzen mit $2 \mathrm{~N} / \mathrm{mm}, 4 \mathrm{~N} / \mathrm{mm}^{2}$ und $6 \mathrm{~N} / \mathrm{mm}^{2}$ Reduzierungen von 13\%, 39\% und 37\%. Dabei weisen alle modifizierten Serien einen nahezu gleichbleibenden Anstieg der Feuchte auf, während die Referenzen, wie bereits beschrieben, stärkeren Schwankungen unterlagen. Es ist davon auszugehen, dass Gewichtszunahmen in Höhe von $11 \%$ und $5 \%$, wie bei $4 \mathrm{~N} / \mathrm{mm}^{2}$ und 6 $\mathrm{N} / \mathrm{mm}^{2}$, ausgehend von klimatisierten Proben, zu Holzfeuchten unterhalb des Fasersättigungspunktes führten.

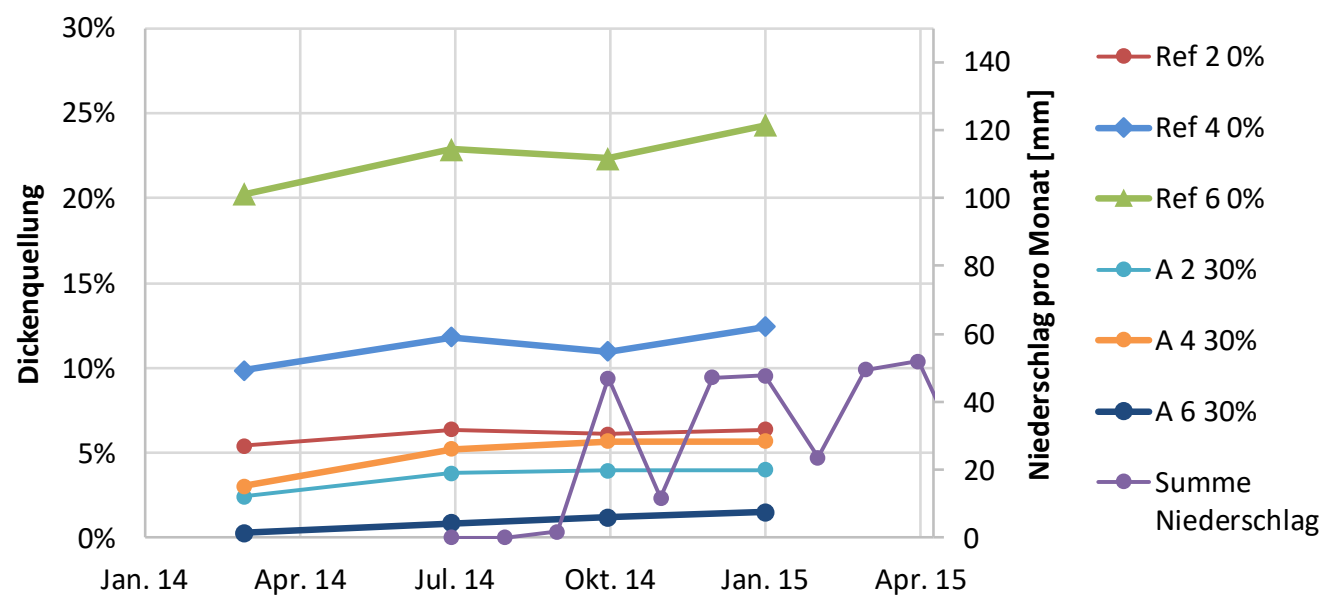

Abbildung 112: Dickenquellung unbeschichteter PF-modifizierter Buchen-LVL-Prüfkörper (8-lagig mit Sperrlagen), mit einem WPG von $30 \%$ bei unterschiedlichen Pressdrücken, in Relation zum Ausgangszustand sowie monatliche Niederschlagmengen (Sekundärachse).

Die Dickenquellung, senkrecht zur Plattenebene gemessen, wie sie in Abbildung 112 dargestellt wird, ist bei allen modifizierten Serien mit 30\% im Vergleich zu den Referenzen, bei allen untersuchten Pressdrücken, niedriger. In Relation zu den jeweiligen Referenzen ergaben sich somit infolge der PF-Modifizierung Reduktionen von 38\%, 54\% und 94\% für die Pressdrücke $2 \mathrm{~N} / \mathrm{mm}^{2}, 4 \mathrm{~N} / \mathrm{mm}^{2}$ und $6 \mathrm{~N} / \mathrm{mm}^{2}$. Dies stellt einen Gegensatz zur den Ergebnissen mit dem WPG von 15\% dar, bei denen modifizierte Serien $\left(2 \mathrm{~N} / \mathrm{mm}^{2}\right.$ und 4 
$\mathrm{N} / \mathrm{mm}^{2}$ ) infolge von Verdichtung stärker quollen, als ihre jeweilige Referenz. Daraus lässt sich bereits schließen, dass für eine dauerhafte Dimensionsstabilisierung ein WPG von 30\% besser ist, als ein WPG von 15\%. Dieses Ergebnis wird gestützt durch die Ergebnisse mit einem WPG von $60 \%$. Bei diesem lagen im ersten Jahr der Bewitterung alle Ergebnisse für die Wasseraufnahme unter $4 \%$ und für die Dickenquellung unter 1,6\%.
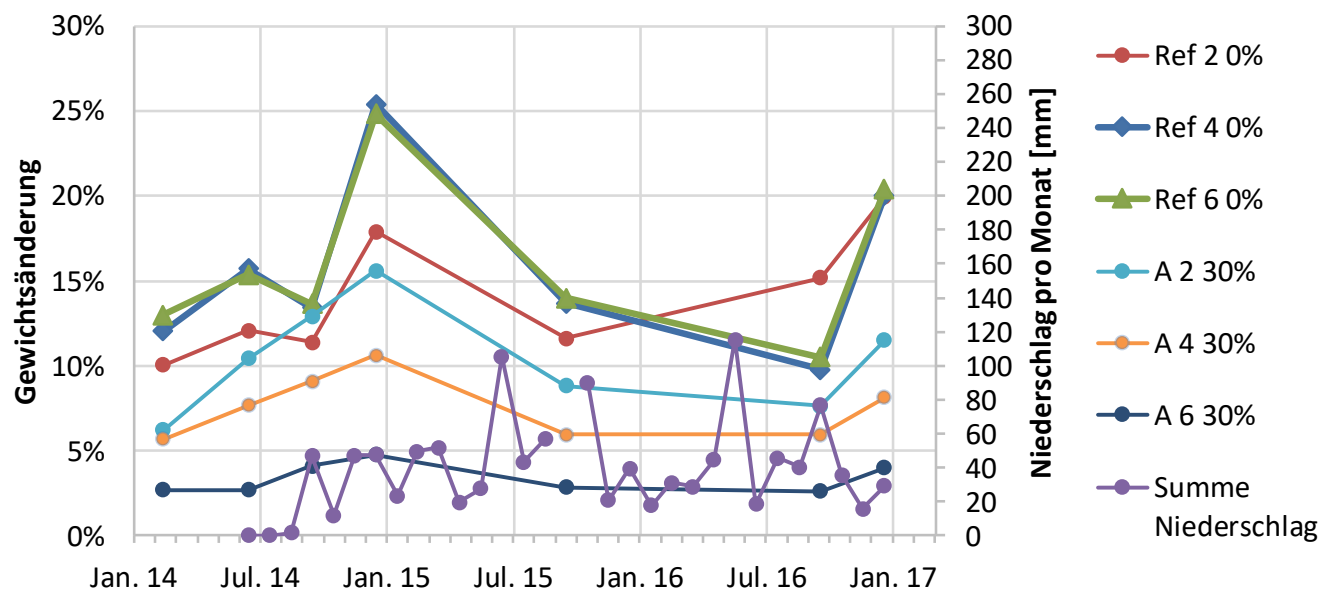

Abbildung 113: Gewichtsänderung unbeschichteter PF-modifizierter Buchen-LVL-Prüfkörper (8-lagig mit Sperrlagen), mit einem WPG von $30 \%$ bei unterschiedlichen Pressdrücken, in Relation zum Ausgangsgewicht sowie monatliche Niederschlagmengen (Sekundärachse).

Wie die Abbildung 113 zeigt, änderte sich bei jährlicher Messung der Gewichte der Bewitterungsproben bei den Referenzen die Reihenfolge für die Höhe des Ergebnisses, da hier zeitweilig die Serie mit $2 \mathrm{~N} / \mathrm{mm}^{2}$ schwerer war und bei der letzten Messung gleichauf lag mit den Referenzen, die einem Pressdruck von $4 \mathrm{~N} / \mathrm{mm}^{2}$ und $6 \mathrm{~N} / \mathrm{mm}^{2}$ unterlagen. Bei den modifizierten Proben war dies nicht der Fall. Auch nach 3 Jahren Bewitterung wiesen die Proben mit einem höheren Pressdruck die niedrigeren Wasseraufnahmen auf. Bei der Interpretation des Diagrammes ist zu beachten, dass im zweiten Jahr der Bewitterung im Oktober 2015 gemessen wurde. Die Probengewichte schienen bei allen Serien im Vergleich zur letzten Messung abgenommen $\mathrm{zu}$ haben, jedoch lagen die der Referenzen und der modifizierten Serie mit $6 \mathrm{~N} / \mathrm{mm}^{2}$ Pressdruck auf dem Niveau vom Oktober des Vorjahres (2014) und ein saisonaler Einfluss wird hier eine Rolle gespielt haben. Dafür spricht auch, dass zum Ende des dritten Bewitterungsjahres, ähnlich zum ersten Jahr, ein starker Anstieg der Probengewichte zwischen Oktober und Januar zu verzeichnen war. Niederschläge in Verbindung mit tieferen Temperaturen und einer höheren relativen Luftfeuchte, wie in Abbildung 106 (S.116) dargestellt, haben in dieser Jahreszeit wahrscheinlich zu höheren Holzfeuchten geführt, welche bei den Referenzen in der Nähe des Fasersättigungspunktes lagen. 

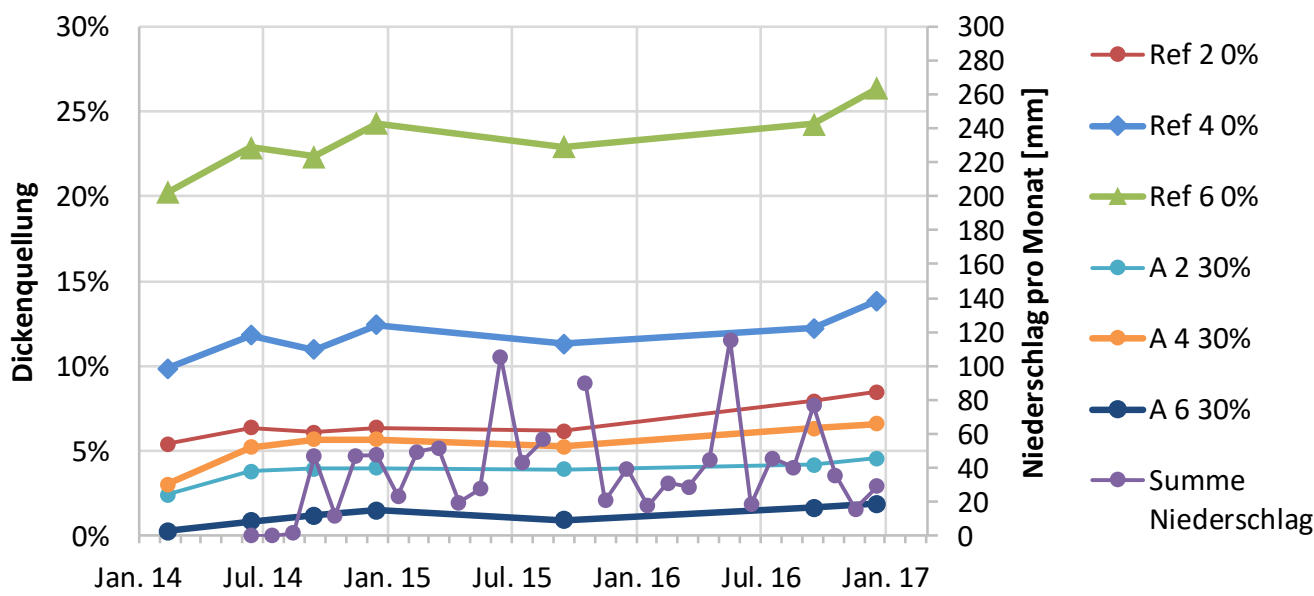

Abbildung 114: Dickenquellung unbeschichteter PF-modifizierter Buchen-LVL-Prüfkörper (8-lagig mit Sperrlagen), mit einem WPG von $30 \%$ bei unterschiedlichen Pressdrücken, in Relation zum Ausgangszustand sowie monatliche Niederschlagmengen (Sekundärachse).

Abbildung 114 veranschaulicht den Verlauf der Dickenquellung über drei Jahre Freilandbewitterung. Dabei fällt auf, dass im Gegensatz zu der Gewichtsänderung, deutlich geringere Schwankungen auftraten und die Ergebnisse der Folgejahre kaum einen Unterschied zum ersten Jahr aufwiesen. Abgesehen von der absoluten Quellung der Proben, lässt sich somit auf eine Sekundär- bzw. Langzeitdimensionsstabilität schließen, welche von den klimatischen Schwankungen weniger abhängig war. Der leichte Anstieg der Quellung von Oktober zu Januar im ersten Jahr, war in gleicher Höhe auch im dritten Jahr ersichtlich und ist wahrscheinlich auf die stärkere Erhöhung der Holzfeuchte im Winter zurückzuführen.

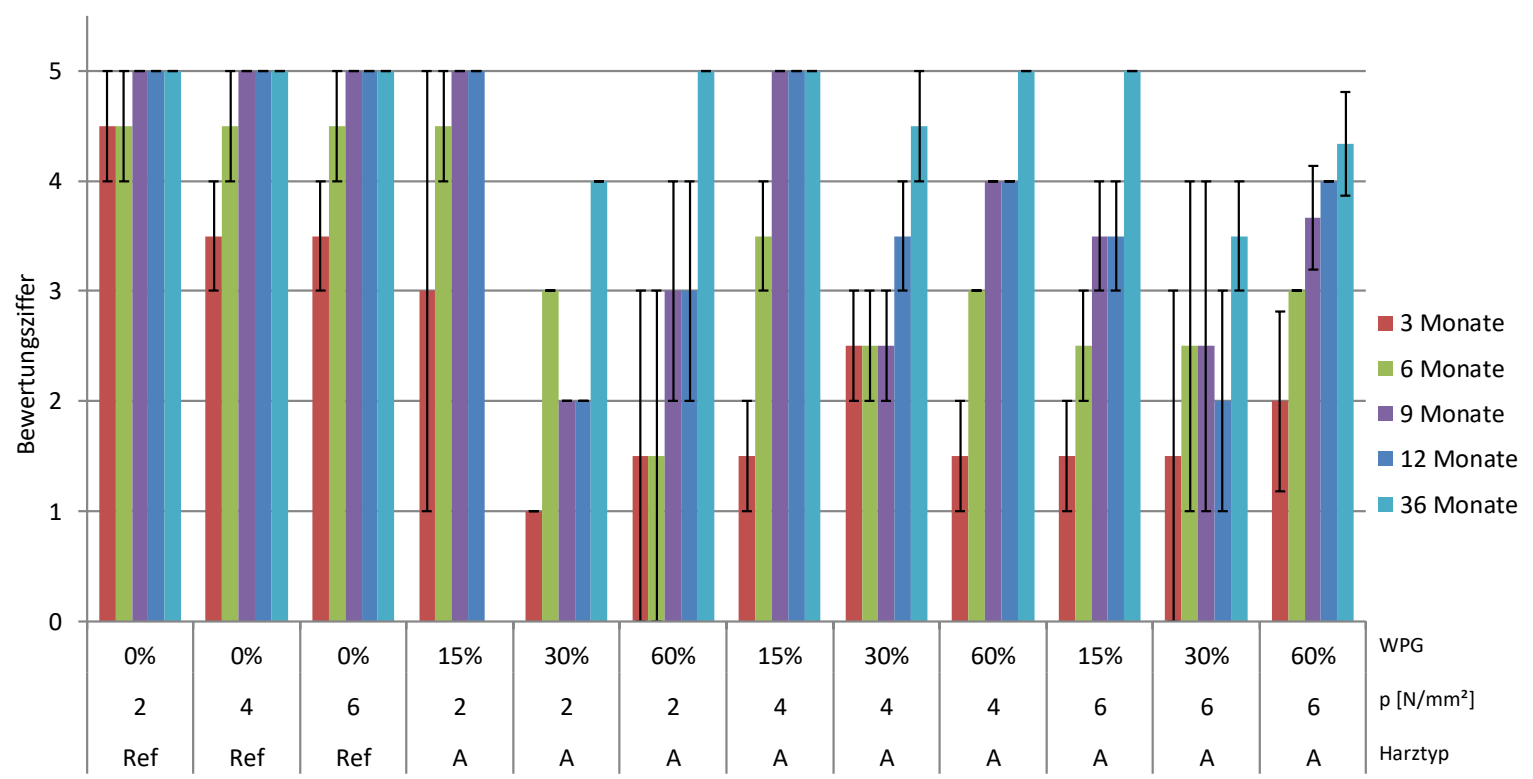

Abbildung 115: Bewertungsziffern (0-5) nach DIN ISO 4628-4 für die Menge der Risse in der Vorderseite von mit dem Harztyp A modifizierten Buchen-LVL (8-lagig mit zwei Sperrlagen) in Abhängigkeit von der Bewitterungsdauer im Freiland.

Bei der Evaluation der Menge der Risse (Abbildung 115) kam für das Buchen-LVL mit zwei Sperrlagen heraus, dass die Referenzen innerhalb des ersten Halbjahres an die maximale Bewertungsziffer (5) heranreichten und damit sehr viele Risse aufwiesen, womit nach einem Jahr keine weitere Steigerung mehr möglich war. Bei den mit dem Harztyp A modifizierten 
Serien erreichten erst nach drei Jahren einige diese höchste Bewertungsziffer und eine langsamere Zunahme lässt sich hier feststellen. Die besten Ergebnisse erzielten die Kombinationen mit 30\% WPG, wobei die Serie mit $2 \mathrm{~N} / \mathrm{mm}^{2}$ zwar das beste Ergebnis erzielte, jedoch auch hier die zweithöchste Menge an Rissen (Bewertungsziffer 4, viele Risse) aufwies.

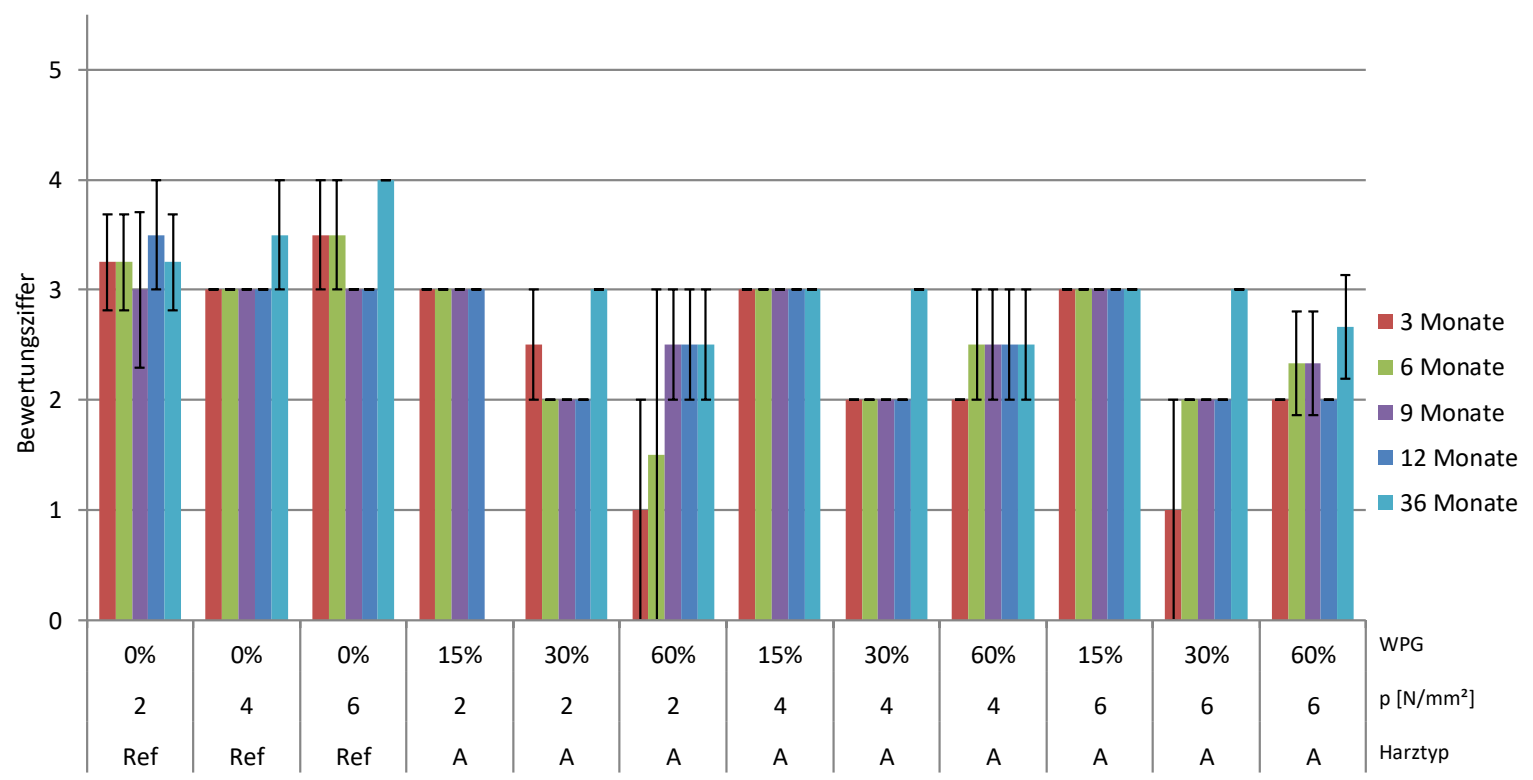

Abbildung 116: Bewertungsziffern (0-5) nach DIN ISO 4628-4 für die Breite der Risse in der Vorderseite von mit dem Harztyp A modifizierten Buchen-LVL (8-lagig mit zwei Sperrlagen) in Abhängigkeit von der Bewitterungsdauer im Freiland.

Die neben der Menge der Risse zusätzlich evaluierte Breite der Risse zeigte die höchsten Werte bei den Referenzen. Diese lagen im Versuchszeitraum zwischen den Bewertungsziffern 3 und 4, d.h., dass deutlich sichtbare bzw. bis $1 \mathrm{~mm}$ breite Risse an der Oberfläche der Proben festgestellt wurden. Die modifizierten Serien wiesen maximal die Bewertungsziffer 3 auf. Bis zu einem Jahr hoben sich, wie auch bei der Menge der Risse, die Serien mit 30\% WPG und Pressdrücken von $2 \mathrm{~N} / \mathrm{mm}^{2}, 4 \mathrm{~N} / \mathrm{mm}^{2}$ und $6 \mathrm{~N} / \mathrm{mm}^{2}$, positiv von den übrigen Serien ab. Beim WPG von 15\% zeigten sich schon ab der ersten Evaluation die deutlich sichtbaren Risse, bei einem WPG von 60\% erfolgte der Anstieg sukzessiv innerhalb der ersten neun Monate. Die Ergebnisse zur Rissevaluation lassen somit darauf schließen, dass die PF-Modifizierung im Wesentlichen zu einer verlangsamten Zunahme der Menge an Rissen führte, welche weniger breit waren als bei den Referenzen. Dabei erwies sich der WPG von 30\% besonders im ersten Jahr als vorteilhaft, wenngleich nach drei Jahren Bewitterung eine Verschlechterung der Bewertung auftrat.

Da neben einem Plattenaufbau mit Sperrlagen auch ein Plattenaufbau ohne Sperrlagen, d.h. mit ausschließlich parallelem Faserverlauf aller acht Furniere, untersucht wurde, sind nachfolgend die Bewitterungsergebnisse für den Harztyp A und die Pressdrücke $2 \mathrm{~N} / \mathrm{mm}^{2}, 4 \mathrm{~N} / \mathrm{mm}^{2}$ und 6 $\mathrm{N} / \mathrm{mm}^{2}$ aufgeführt. 


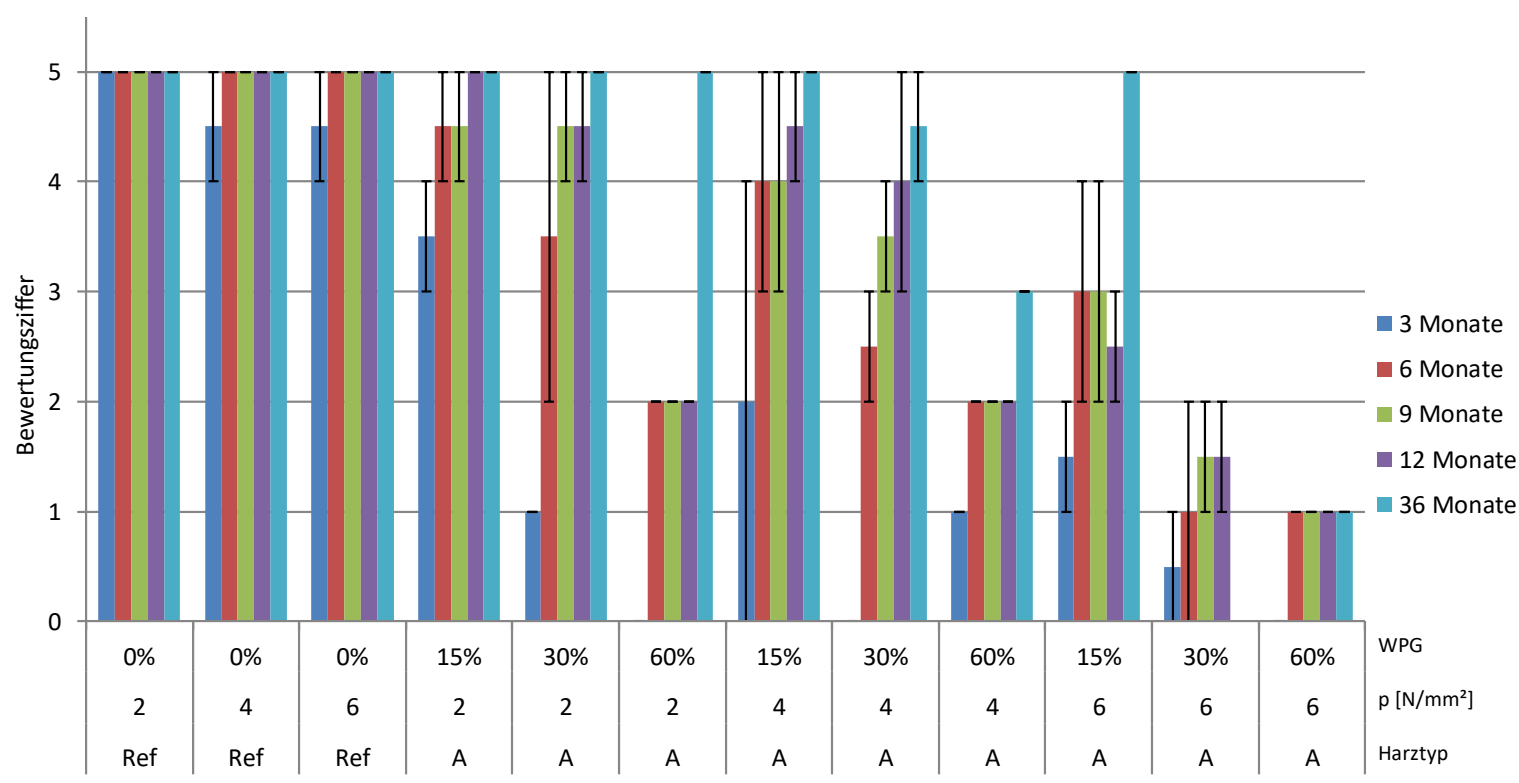

Abbildung 117: Bewertungsziffern (0-5) nach DIN ISO 4628-4 für die Menge der Risse in der Vorderseite von mit dem Harztyp A modifizierten Buchen-LVL (8-lagig parallel) in Abhängigkeit von der Bewitterungsdauer im Freiland.

Wie auch bei dem Plattenaufbau mit Sperrlagen, reichen die Bewertungsziffern für die Menge der Risse bei den Serien mit ausschließlich parallelem Aufbau an die Ziffer "5" heran (Abbildung 117). Bei den modifizierten Serien ließen sich im Vergleich zu den Referenzen im ersten Versuchsjahr deutlich weniger Risse feststellen. Die besten Varianten waren Platten mit $60 \% \mathrm{WPG}$ bei $2 \mathrm{~N} / \mathrm{mm}^{2}, 4 \mathrm{~N} / \mathrm{mm}^{2}$ und $6 \mathrm{~N} / \mathrm{mm}^{2}$ sowie mit $30 \% \mathrm{WPG}$ bei $6 \mathrm{~N} / \mathrm{mm}^{2}$. Letztere ging jedoch verloren und konnte nach drei Jahren nicht mehr gemessen werden. Dennoch wurden nach drei Jahren Freilandbewitterung auch bei den modifizierten Serien sehr viele Risse erkennbar. Ausnahmen stellen die Serien dar, die bei $4 \mathrm{~N} / \mathrm{mm}^{2}$ mit $60 \%$ WPG und bei 6 $\mathrm{N} / \mathrm{mm}^{2}$ mit 30\% und 60\% WPG hergestellt wurden. Beim Vergleich der Ergebnisse zwischen den beiden Plattenaufbautypen, fällt weiterhin auf, dass bei den Referenzen diejenigen mit Sperrlagen erst nach neun Monaten in die höchste Kategorie fielen, diese beim parallelen Aufbau jedoch schon nach 6 Monaten erreicht war. Ebenso war bei diesem Plattentyp bei einem Pressdruck von $2 \mathrm{~N} / \mathrm{mm}^{2}$ bei 30\% WPG nach 9 Monaten die Bewertungsziffer 5 mit einem Mittelwert von 4,5 erreicht, während bei dem Plattentyp mit Sperrlagen und den gleichen Herstellungsparametern nach 12 Monaten noch die Ziffer 2 und nach 36 Monaten dann die Ziffer 4 vergeben wurde. Da jedoch beim Plattenaufbau mit Sperrlagen alle Serien nach 36 Monaten zwischen Bewertungsziffer 4 und 5 lagen, beim parallelen Aufbau jedoch noch die Serien, die bei $4 \mathrm{~N} / \mathrm{mm}^{2}$ mit $60 \%$ WPG und bei $6 \mathrm{~N} / \mathrm{mm}^{2}$ mit $30 \%$ und $60 \% \mathrm{WPG}$ hergestellt wurden, die Bewertungsziffern „1“, „2“, und „3“ erhielten, kann wiederrum nicht eindeutig auf einen positiven Einfluss der Sperrlagen geschlossen werden. 


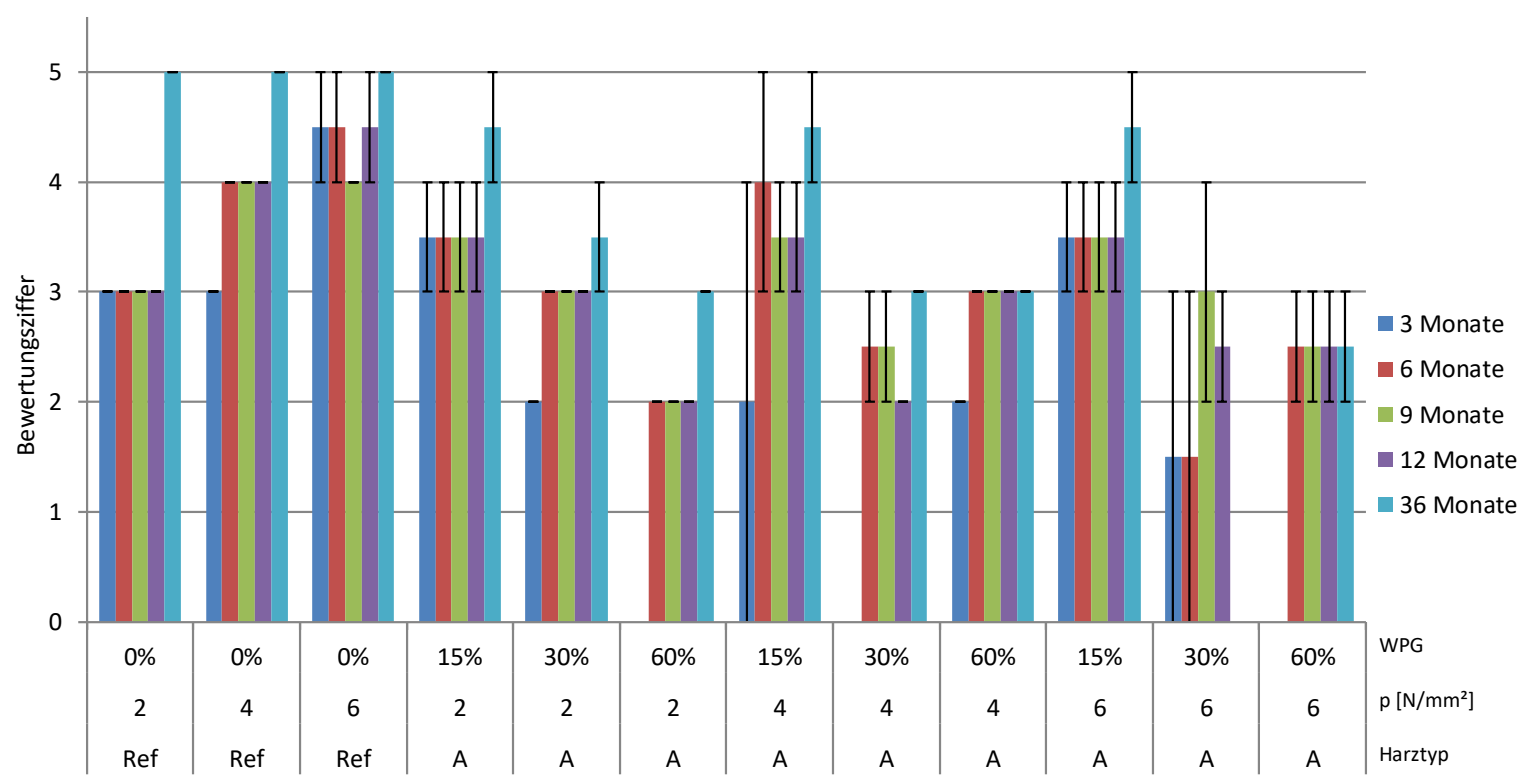

Abbildung 118: Bewertungsziffern (0-5) nach DIN ISO 4628-4 für die Breite der Risse in der Vorderseite von mit dem Harztyp A modifizierten Buchen-LVL (8-lagig parallel) in Abhängigkeit von der Bewitterungsdauer im Freiland.

Bei der Bewertung der Rissbreite an den LVL-Prüfkörpern mit ausschließlich paralleler Anordnung der Furniere (Abbildung 118) zeigte sich, dass bei den Referenzen im ersten Jahr eine größere Rissbreite (4-5) für Platten, die mit dem größeren Pressdruck, als für die die mit dem geringeren Pressdruck, hergestellt worden waren (3). Jedoch wurden nach drei Jahren alle Referenzprüfkörper mit der höchsten Bewertungsziffer (5) beurteilt. Dies stellt bereits einen Unterschied zu den Platten mit Sperrlagen dar, bei denen für die Referenzen auch nach drei Jahren maximal die Bewertungsziffer 4 vergeben wurde. Bei den modifizierten Serien ohne Sperrlagen wurde im ersten Jahr, mit Ausnahme der Serie mit 15\% WPG und $4 \mathrm{~N} / \mathrm{mm}^{2}$, für alle modifizierten Prüfköper maximal die „4“, für Risse bis $1 \mathrm{~mm}$ Breite, vergeben. Hinsichtlich des WPG zeigte sich bei allen untersuchten Pressdrücken eine geringere Rissbreite für höhere WPG und umgekehrt. Dabei war der Unterschied zwischen 15\% WPG und 30\% WPG größer als der Unterschied zwischen 30\% WPG und 60\% WPG. Der WPG hatte demnach einen großen negativen Einfluss auf die Rissbreite. Der Pressdruck hingegen schien einen geringeren Einfluss gehabt zu haben, da bei 15\% WPG alle drei Pressdrücke nahezu die gleichen Ergebnisse über alle Messintervalle hinweg aufwiesen. Bei diesen Serien fällt im Vergleich zu den Platten mit Sperrlagen zusätzlich auf, dass die Risse breiter sind, sie eine Ziffer schlechter abschnitten und statt einer „3“ eine "4“ zugeteilt bekamen. Daraus lässt sich schließen, dass dort wo der WPG niedrig war, die Sperrlagen hinsichtlich der Breite der Risse limitierenden Einfluss ausgeübt haben, bei höherem WPG (30\% und 60\%) jedoch kein Unterschied erkennbar war.

Nachfolgend sind die Gewichtsänderungen und die Dickenquellung nach Freilandbewitterung von Buchen-LVL, achtlagig mit zwei Sperrlagen, dessen Furniere mit unterschiedlichen Harztypen modifiziert wurden, dargestellt. 


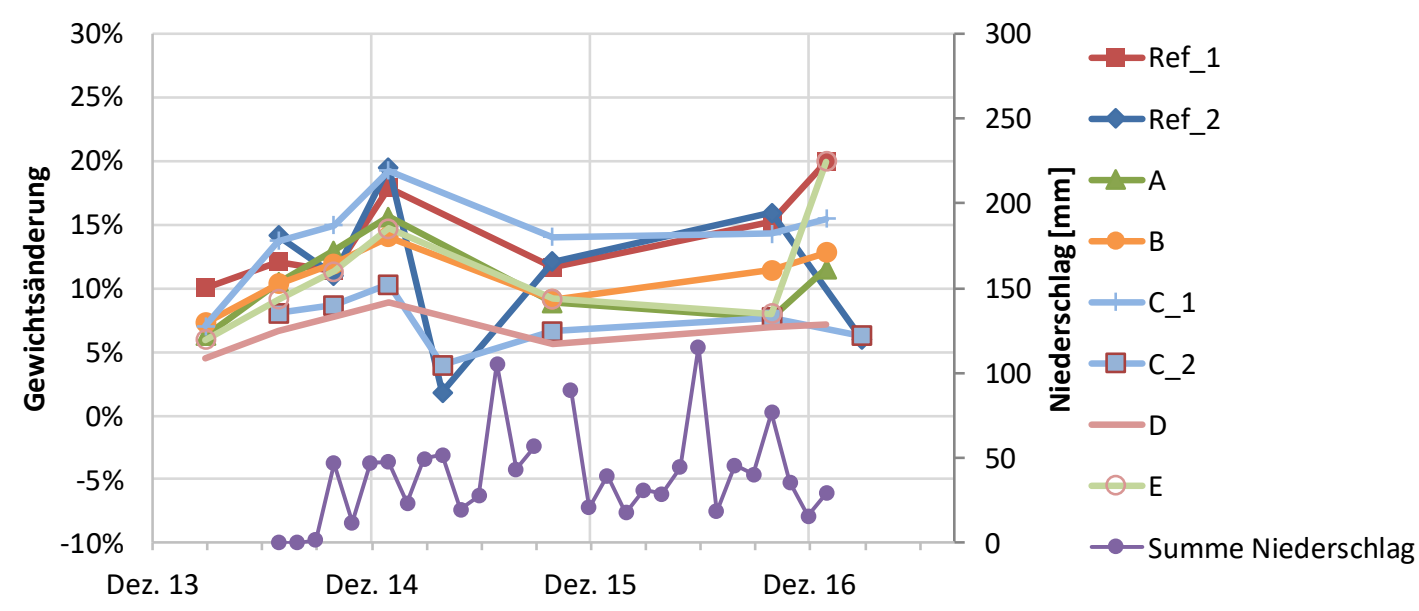

Abbildung 119: Gewichtsänderung unbeschichteter PF-modifizierter Buchen-LVL-Prüfkörper (8-lagig mit Sperrlagen), mit einem WPG von $30 \%$ bei einem Pressdruck von $2 \mathrm{~N} / \mathrm{mm}^{2}$, in Relation zum Ausgangsgewicht sowie monatliche Niederschlagmengen (Sekundärachse).

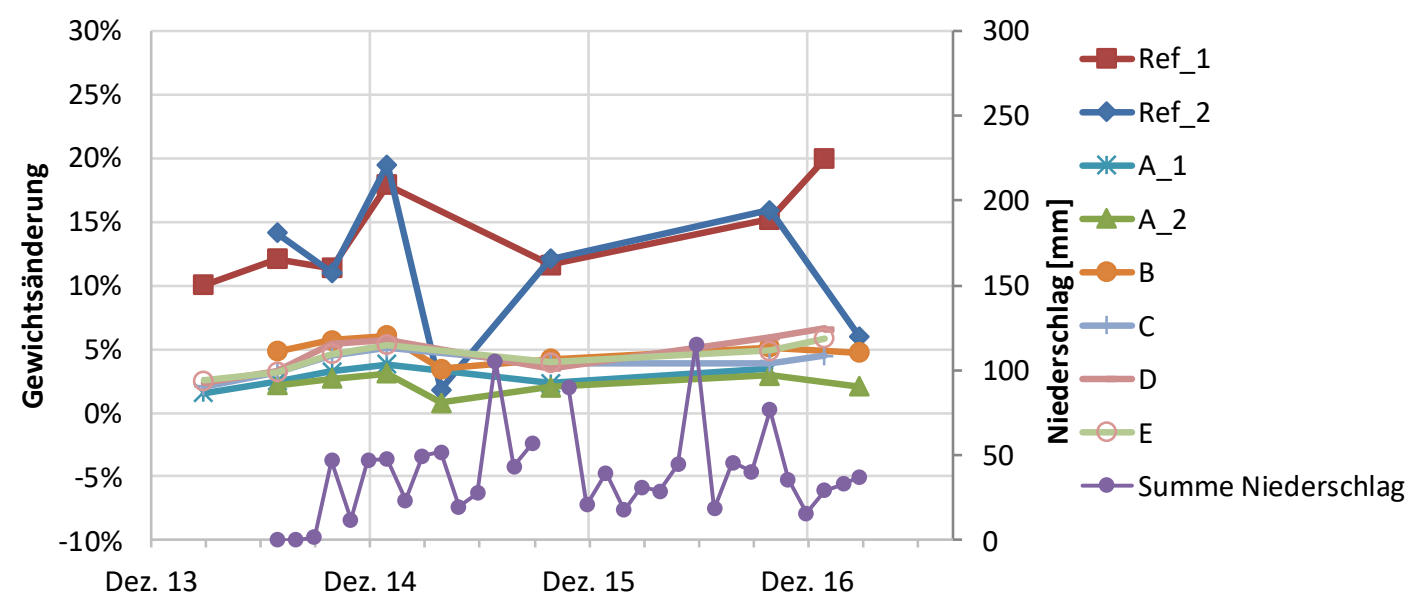

Abbildung 120: Gewichtsänderung unbeschichteter PF-modifizierter Buchen-LVL-Prüfkörper (8-lagig mit Sperrlagen), mit einem WPG von $60 \%$ bei einem Pressdruck von $2 \mathrm{~N} / \mathrm{mm}^{2}$, in Relation zum Ausgangsgewicht sowie monatliche Niederschlagmengen (Sekundärachse).

In Abbildung 119 und Abbildung 120 sind die Gewichtsänderungen von unbeschichteten PFmodifizierten Buchen-LVL-Prüfkörpern (8-lagig mit Sperrlagen), mit einem WPG von 30\% respektive $60 \%$ WPG bei einem Pressdruck von $2 \mathrm{~N} / \mathrm{mm}^{2}$ dargestellt. Da es nicht gelang alle Varianten gleichzeitig herzustellen und im Freilandtest zu starten, wurde sowohl im Dezember 2013 als auch im März 2014 mit dem Versuch gestartet. Aus diesem Grund sind zwei Referenzserien abgebildet, welche im ersten Jahr vier Mal gemessen wurden. Bei der Serie Ref_2 fällt bei der vierten Messung im April 2015 ein deutlich niedrigeres Ergebnis als bei allen anderen Messungen auf, welches für die Serie Ref_1 mit Start im Dezember 2013 nicht aufgezeichnet wurde, da diese bereits im Januar 2015 ein viertes Mal evaluiert wurde. In der Folge kam es bei gleichen Messzeitpunkten wieder zu einer Deckung der Ergebnisse, was zeigt, dass sich die verwendeten Referenzen gleich verhielten und von den vor der Messung herrschenden Klimaverhältnissen mehr beeinflusst waren als von der Dauer der Bewitterung. Bei den modifizierten Serien mit 30\% WPG und einem Pressdruck von $2 \mathrm{~N} / \mathrm{mm}^{2}$ ist ebenso deutlich wie bei den Referenzen im ersten Jahr unabhängig vom Harztyp ein starker Anstieg der Holzfeuchte (Gewichtszunahme) bis Januar 2015 festzustellen gewesen, mit sehr ähnlichen Ergebnissen aller Harze im Vergleich zu den Referenzen. Der Harztyp C war zwar 
aufgrund separater Starts mit zwei Ergebnisreihen in der Abbildung 119 vertreten, jedoch ist durch die Lage der zusammengehörenden Ergebnisse, über und unterhalb der übrigen Serien, keine Abweichung festzustellen. Somit ist für alle Harztypen ohne eine erhöhte Verdichtung bei diesem Pressdruck kein Einfluss auf die Wasseraufnahme deutlich geworden.

Im Unterschied dazu konnten bei einem WPG von 60\% beim gleichen Pressdruck völlig andere Messwerte aufgezeichnet werden. Hier lagen alle Messwerte der modifizierten Proben näher beieinander und maximal lag die Gewichtszunahme im ersten Jahr, wie auch nach 36 Monaten, nur bei $6 \%$, während die der Referenzen bei $18 \%$ und 20\% lag. Somit ist hier durch den erhöhten WPG bei allen Harztypen eine deutliche Reduzierung der Wasseraufnahme in der Freilandbewitterung erzielt worden.

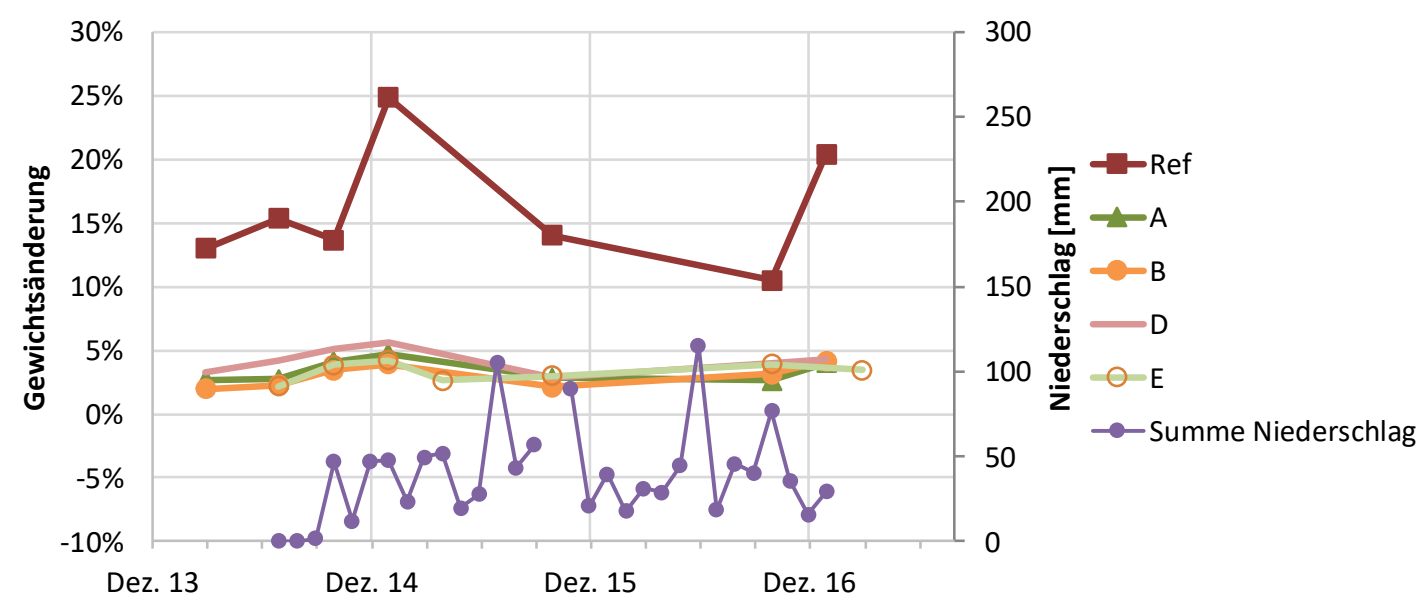

Abbildung 121: Gewichtsänderung unbeschichteter PF-modifizierter Buchen-LVL-Prüfkörper (8-lagig mit Sperrlagen), mit einem WPG von $30 \%$ bei einem Pressdruck von $6 \mathrm{~N} / \mathrm{mm}^{2}$, in Relation zum Ausgangsgewicht sowie monatliche Niederschlagmengen (Sekundärachse).

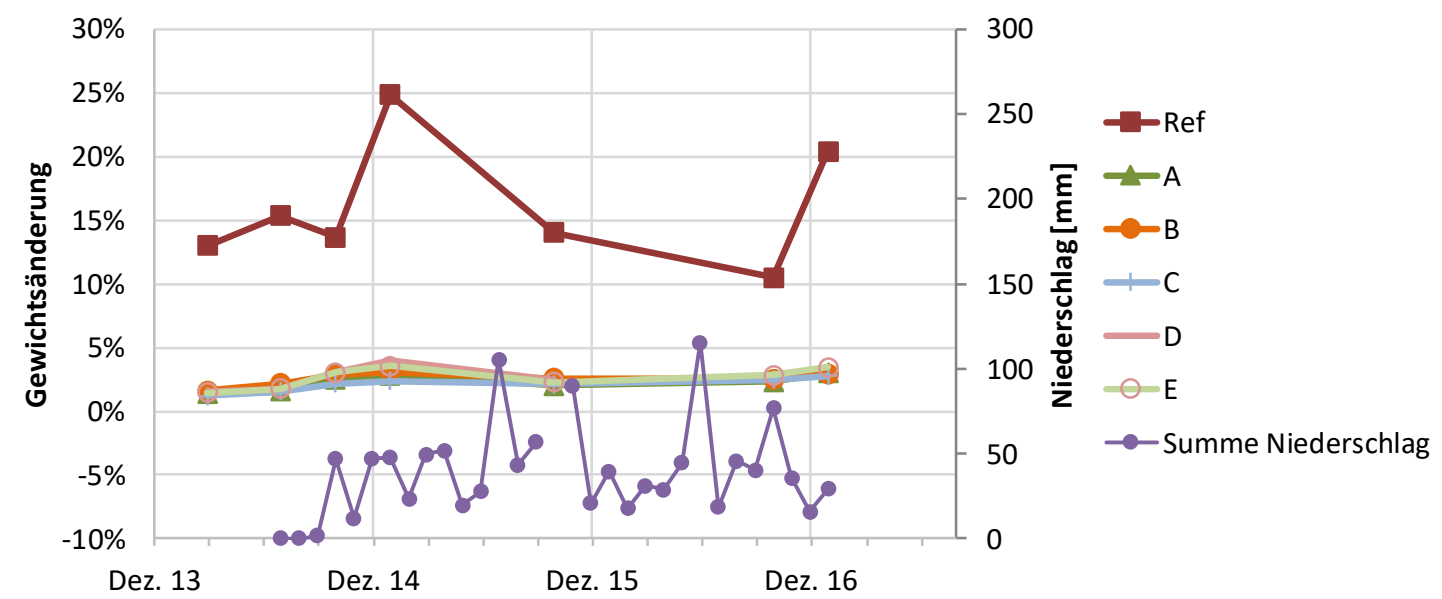

Abbildung 122: Gewichtsänderung unbeschichteter PF-modifizierter Buchen-LVL-Prüfkörper (8-lagig mit Sperrlagen), mit einem WPG von $60 \%$ bei einem Pressdruck von $6 \mathrm{~N} / \mathrm{mm}^{2}$, in Relation zum Ausgangsgewicht sowie monatliche Niederschlagmengen (Sekundärachse).

Die Abbildung 121 und die Abbildung 122 zeigen die Gewichtsänderungen für Buchen-LVL mit einem WPG von 30\% und 60\% mit unterschiedlichen Harztypen, welches bei dem höchsten Pressdruck $\left(6 \mathrm{~N} / \mathrm{mm}^{2}\right)$ hergestellt wurde. Es fällt bei beiden Abbildungen auf, dass die Ergebnisreihen nahezu deckungsgleich verlaufen und deutlich niedriger lagen als die der Referenzen. Der hohe Pressdruck von $6 \mathrm{~N} / \mathrm{mm}^{2}$ hat hier infolge einer sehr hohen Verdichtung 
dazu geführt, dass trotz eines WPG von 30\% deutlich weniger Wasser aufgenommen wurde als beim LVL, welches bei $2 \mathrm{~N} / \mathrm{mm}^{2}$ (Abbildung 119) hergestellt wurde. Bei allen Messintervallen wurden im Unterschied zu den Referenzen, welche noch deutlich von den klimatischen Verhältnissen beeinflusst waren, nur geringe Abweichungen zum vorherigen Ergebnis und damit eine von Klimaschwankungen weitgehende Unabhängigkeit festgestellt. Bei der intensivsten Form der Modifizierung mit einem WPG von 60\% und einem Pressdruck von $6 \mathrm{~N} / \mathrm{mm}^{2}$ kam es zu einem noch flacheren Verlauf der Ergebnisreihen mit einer maximalen Wasseraufnahme von 4\% im ersten Jahr und 3\% nach drei Jahren ohne wesentliche Unterschiede bei den verwendeten Harztypen.

Nachfolgend sind die relativen Änderungen der Plattendicken infolge der Bewitterung und Wasseraufnahme des Buchen-LVL mit zwei Sperrlagen dargestellt.

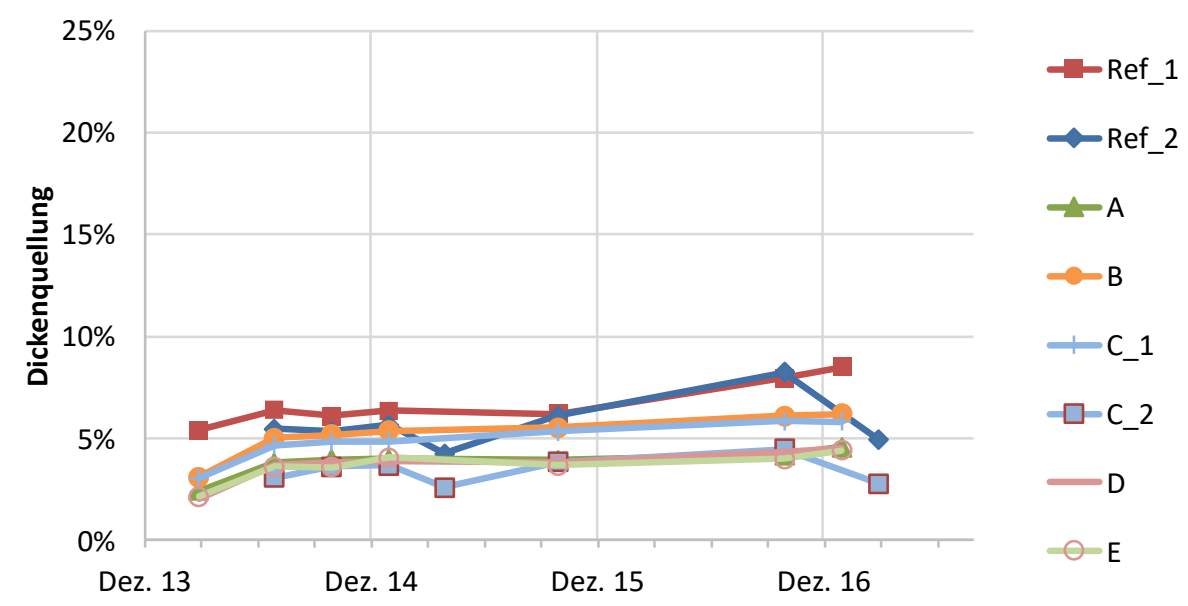

Abbildung 123: Dickenquellung unbeschichteter PF-modifizierter Buchen-LVL-Prüfkörper (8-lagig mit Sperrlagen), mit einem WPG von $30 \%$ bei einem Pressdruck von 2 N/mm ${ }^{2}$, in Relation zur Ausgangsdicke vor Beginn der Freilandbewitterung.

Das unbehandelte Buchen-LVL, Ref_1 und Ref_2, welches mit $2 \mathrm{~N} / \mathrm{mm}^{2}$ hergestellt wurde, zeigte einen flachen Verlauf der Dickenquellung über die Versuchsdauer von drei Jahren mit einem leichten Anstieg von anfänglich 5\% und 6\% (Dezember 2013 und März 2014) auf 8\% (beide) im Oktober 2016. Diese Ergebnisse stehen im Einklang mit der zuvor diskutierten Gewichts- bzw. Wasseraufnahme der Proben, wobei diese stärkeren Schwankungen unterlagen als die hier gezeigte Dickenquellung. Hinzu kam ein weiterer Anstieg der Dickenquellung der ersten Referenz auf 9\% zum Januar 2017, welche auf eine von 15\% auf $20 \%$ gesteigerte Wasseraufnahme in diesem Zeitraum zurückgeführt werden kann. Die modifizierten Serien mit einem WPG von 30\%, welche ebenfalls bei $2 \mathrm{~N} / \mathrm{mm}^{2}$ hergestellt wurden, wiesen einen noch etwas flacheren Verlauf als die Referenzen auf.

Die einzelnen Serien, die mit unterschiedlichen Harzen hergestellt wurden, zeigten unterschiedliche Werte für die Dickenquellung (Abbildung 123). Der Harztyp B zeigte eine geringere Quellung als die Referenzen, jedoch eine höhere als die der Harztypen A, D und E, welche die niedrigsten Quellungen (maximal 5\% nach 36 Monaten) aufwiesen. Unter Bezugnahme auf die Darrdichte ist dieses Ergebnis nicht zu erklären. Eine höhere Darrdichte führt in der Regel zu einem höheren Quellwert als eine niedrigere. Der Harztyp B wies jedoch eine Darrdichte auf, die deutlich kleiner war als die vom Harztyp D. Da Harztyp B jedoch eine 
höhere zahlenmäßig mittlere Molmasse (Mn) und eine höhere Polydispersität aufwies als die anderen Harztypen, kann hier die höhere Dickenquellung mit einen niedrigeren Anteil an Harz, welches in der Lage war in die Zellwand einzudringen, in Verbindung gebracht werden.

Für den Harztyp C ist keine eindeutige Zuordnung möglich, da zwar alle Werte niedriger als die der Referenzen lagen, jedoch die Probe C_1 gleiche Werte wie die Serie B lieferte, während die Probe C_2 näher an den Werten der Harztypen E und D lag.

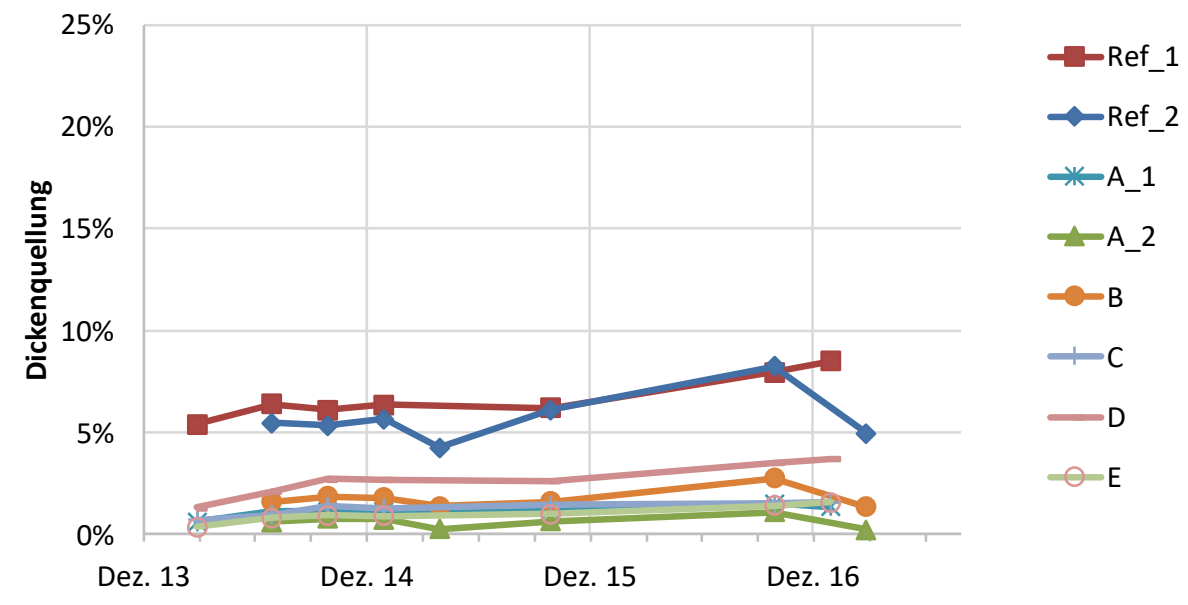

Abbildung 124: Dickenquellung unbeschichteter PF-modifizierter Buchen-LVL-Prüfkörper (8-lagig mit Sperrlagen), mit einem WPG von $60 \%$ bei einem Pressdruck von $2 \mathrm{~N} / \mathrm{mm}^{2}$, in Relation zur Ausgangsdicke vor Beginn der Freilandbewitterung.

Die Abbildung 124 ist insbesondere mit den Ergebnissen der Imprägnierung und der Darrdichte der Proben zu interpretieren. Die modifizierten Serien wiesen hier mit Ausnahme der Serie D einen WPG von 60\% auf. Die Serie D wies aufgrund geringeren Feststoffgehaltes in der Lieferform nur einen WPG von 48\% auf. Somit war die Dickenquellung der Serie D hier höher als die der anderen modifizierten Serien, jedoch auch im Vergleich zu einem WPG von 30\% um einen Prozentpunkt von 5\% auf 6\% nach 36 Monaten herabgesetzt. Das dramatisch schlechte Verhältnis zwischen Verbesserung der Dimensionsstabilität und dem gesteigerten Harzeinsatz muss an dieser Stelle Beachtung finden. Bei genauer Betrachtung der Ergebnisse der übrigen Serien lag die Serie B mit ihren Werten zur Dickenquellung wieder höher als die Serien A, C und E, was eine Übereinstimmung der zuvor diskutierten Ergebnisse bei einem WPG von $30 \%$ bei einem Pressdruck von $2 \mathrm{~N} / \mathrm{mm}^{2}$ darstellt. 


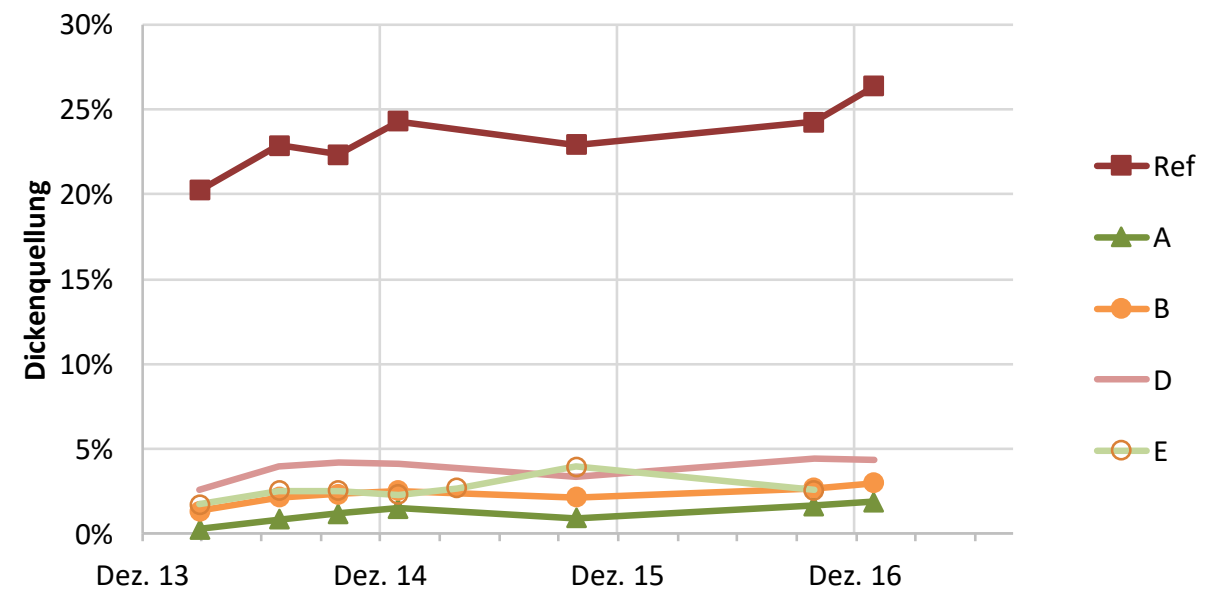

Abbildung 125: Dickenquellung unbeschichteter PF-modifizierter Buchen-LVL-Prüfkörper (8-lagig mit Sperrlagen), mit einem WPG von $30 \%$ bei einem Pressdruck von $6 \mathrm{~N} / \mathrm{mm}^{2}$, in Relation zur Ausgangsdicke vor Beginn der Freilandbewitterung.

Die Anwendung eines Pressdruckes von $6 \mathrm{~N} / \mathrm{mm}^{2}$ bei der Herstellung der LVL führte, wie in 5.1.4.1 gezeigt, bei den modifizierten Serien zu einer stärkeren Erhöhung der Darrdichte als bei den Referenzen. Dennoch sind es gerade die Referenzen, die zeigten (Abbildung 125), dass ohne eine Modifizierung auch eine geringe Verdichtung zu einer Erhöhung der Quellung in der Freilandbewitterung führte. Im Vergleich zu den Referenzen mit $2 \mathrm{~N} / \mathrm{mm}^{2}$ Pressdruck konnte hier nach einem Jahr eine um 20\% bzw. 5-fach erhöhte Dickenquellung gemessen werden. Mit diesem Zusammenhang wird deutlich, welchen Einfluss die Verdichtung auf Holzwerkstoffe haben kann, wenn sie nicht vor der Aufnahme von Wasser und der damit verbundenen Quellung bzw. Rückquellung geschützt werden. Hinsichtlich der modifizierten Serien mit einem WPG von 30\% ließ sich hier feststellen, dass die Verdichtung bei diesem hohen Pressdruck zu einer Reduzierung der Dickenquellung bei allen Serien gegenüber den Varianten mit dem niedrigeren Pressdruck und gleichem WPG führte. Infolge der Verdichtung konnte sogar ein ähnliches Ergebnis wie bei dem WPG von 60\%, nämlich eine maximale Dickenquellung von 4,4\%, erzielt werden. Anders als bei unbehandelten Referenzen konnte durch den höheren Pressdruck die Dickenquellung bei den modifizierten Serien reduziert werden, anstatt sich, wie bei unbehandeltem Holz üblich, infolge von Rückquellung bzw. Deformationsrückgang, zu erhöhen. 


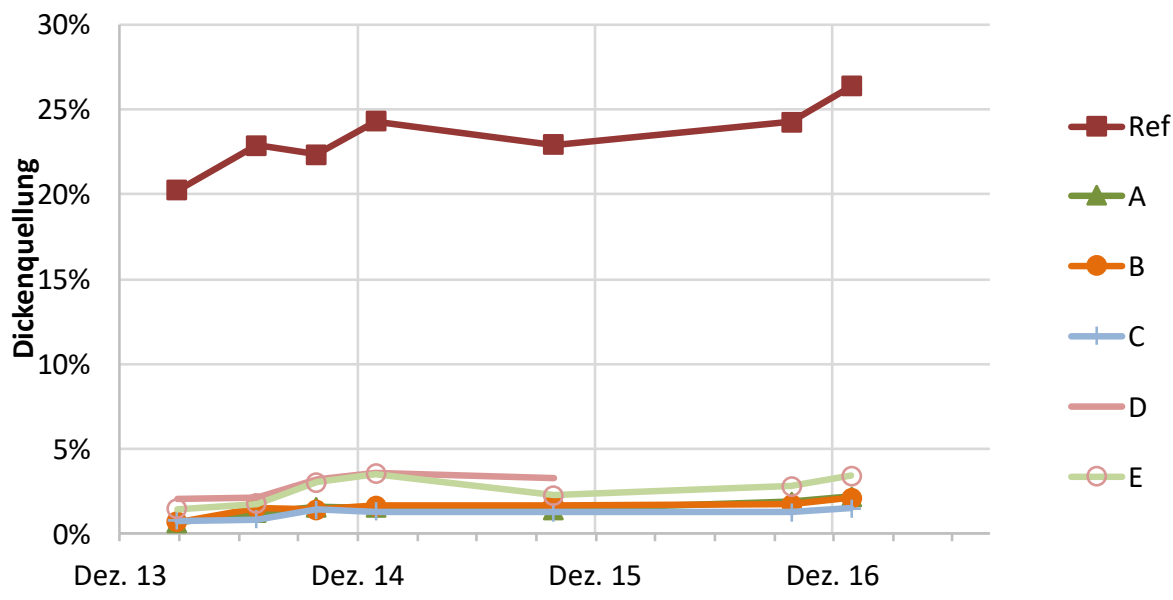

Abbildung 126: Dickenquellung unbeschichteter PF-modifizierter Buchen-LVL-Prüfkörper (8-lagig mit Sperrlagen), mit einem WPG von $60 \%$ bei einem Pressdruck von $6 \mathrm{~N} / \mathrm{mm}^{2}$, in Relation zur Ausgangsdicke vor Beginn der Freilandbewitterung.

Mit der Erhöhung des WPG auf 60\% und der Anwendung des höheren Pressdruckes, wurde, wie in Abbildung 126 ersichtlich, die Dickenquellung für alle Harze außer Harztyp D auf 2,2 \% nach 36 Monaten reduziert. Innerhalb des ersten Jahres lag die Dickenquellung sogar nur bei maximal 1,6\% (Harze A, B, C, E). Für den Harztyp D mit einem WPG von 48\% lag sie jedoch bei 3,3\%. Demnach konnten bei Anwendung eines Pressdruckes von $6 \mathrm{~N} / \mathrm{mm}^{2}$, der höhere WPG von $60 \%$ im Vergleich zu einem WPG von 30\%, nur noch sehr kleine und zum Harzeinsatz unverhältnismäßige Verbesserungen hinsichtlich der Dickenquellung erzielen.

Nachfolgend werden die Ergebnisse zur Rissevaluation der unbeschichteten LVL mit Sperrlagen gezeigt und sich hierbei auf den Zeitraum des ersten Jahres beschränkt, da keine wesentlichen Änderungen danach mehr eintraten und die Übersicht bewahrt werden soll.

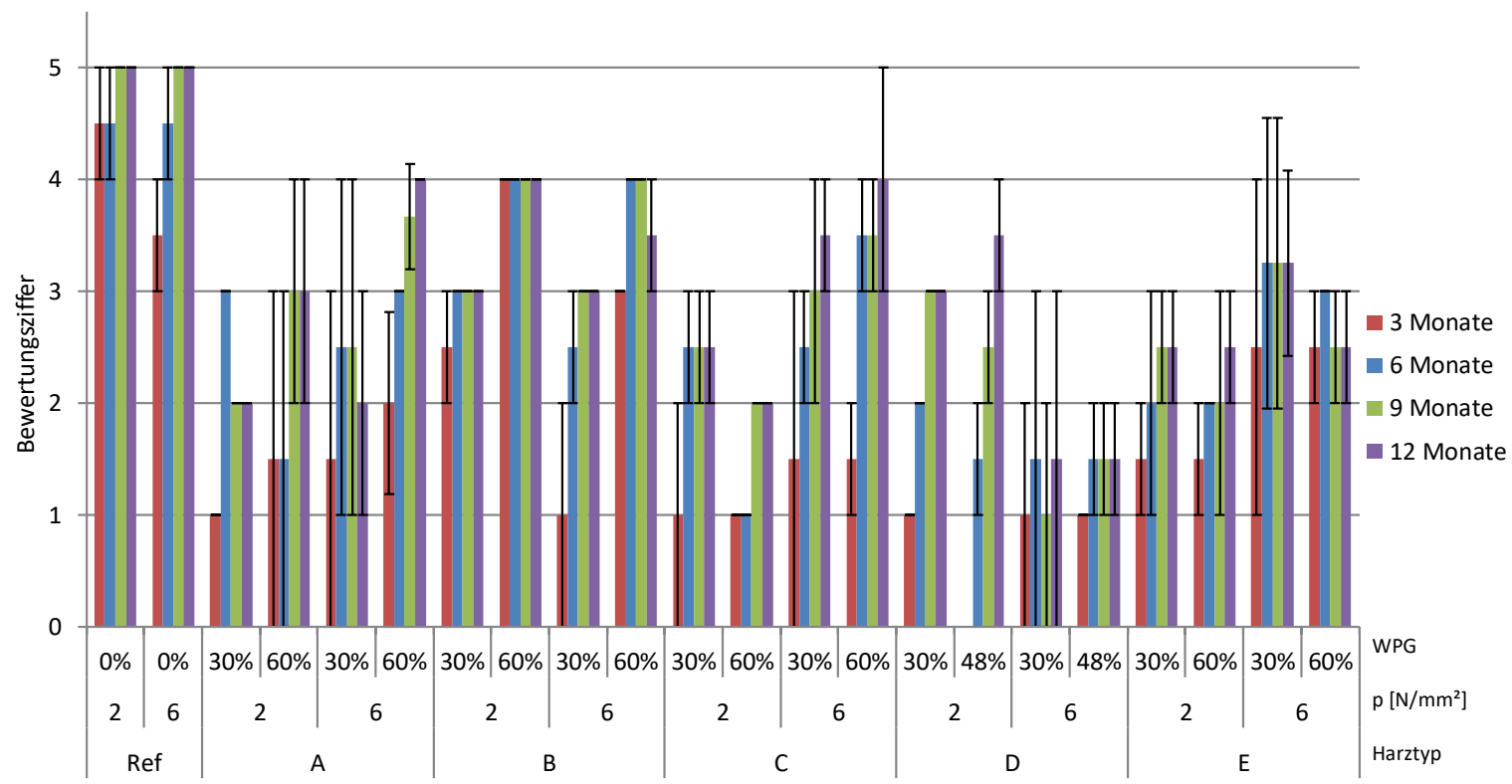

Abbildung 127: Bewertungsziffern (0-5) nach DIN ISO 4628-4 für die Menge der Risse in der Vorderseite von PF-modifiziertem Buchen-LVL (8-lagig) mit zwei Sperrlagen in Abhängigkeit von der Bewitterungsdauer im Freiland. 
Bei unbeschichteten 8-lagigem LVL mit zwei Sperrlagen zeigte sich in den meisten Fällen innerhalb von 12 Monaten eine deutliche Zunahme der Menge an Rissen auf der Oberfläche der Prüfkörper (Abbildung 85). Auffallend war die große Menge an Rissen bei den Referenzen bereits nach drei Monaten bzw. sechs Monaten in der Bewitterung. Sie erhielten, unabhängig vom Pressdruck bzw. der Verdichtung, nach 12 Monaten die höchste Bewertungsziffer (5), da sie sehr viele Risse aufwiesen. Alle modifizierten Serien wiesen im Vergleich dazu weniger Risse auf.

Bei den modifizierten Serien mit Harztyp A und B zeigte sich, dass der höhere WPG bei unverdichteten und verdichteten Platten stärker zu Rissen neigte. Dies konnte beim Harztyp C nur für die verdichteten Platten festgestellt werden. Bei Harztyp D zeigten, im Vergleich zu den verdichteten Platten, die unverdichteten Platten für beide WPGs die stärkere Rissneigung. Aufgrund dieser Befunde kann weder auf einen positiven Einfluss des WPG, noch auf einen positiven Einfluss der Verdichtung bzw. des Pressdruckes, geschlossen werden. Lediglich der Unterschied zwischen modifiziertem und unmodifiziertem LVL kam hier zum Vorschein.

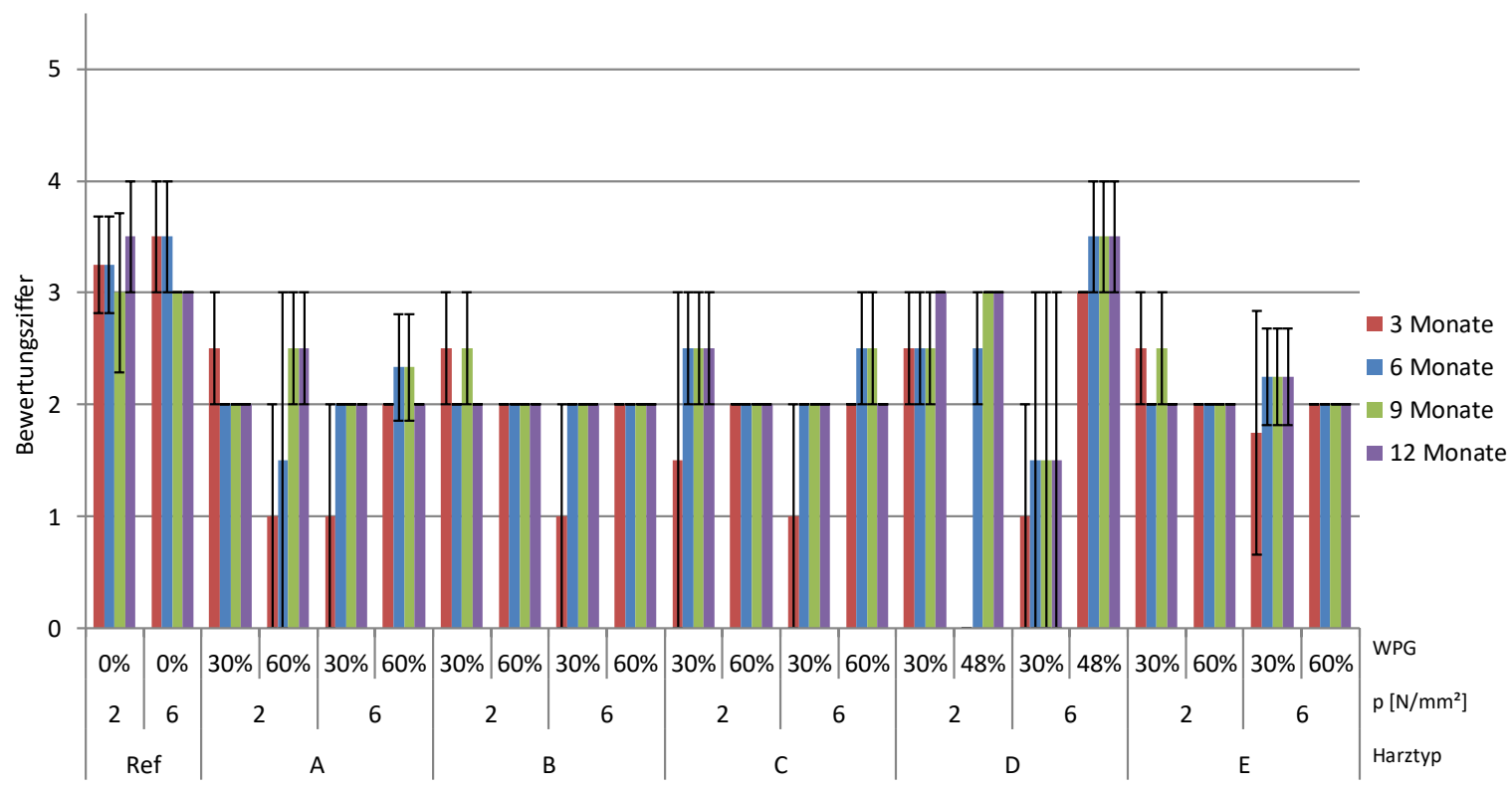

Abbildung 128: Bewertungsziffern (0-5) nach DIN ISO 4628-4 für die Breite der Risse in der Vorderseite von PF-modifiziertem Buchen-LVL (8-lagig) mit zwei Sperrlagen in Abhängigkeit von der Bewitterungsdauer im Freiland.

Die Rissbreite an der Oberfläche der bewitterten LVL war, mit Ausnahme einer Variante, bei den modifizierten Serien geringer als bei den unbehandelten Referenzen, welche von Beginn an im Bereich zwischen 3 und 4 (deutlich sichtbar bzw. bis $1 \mathrm{~mm}$ breit) lagen. Es gilt bei der Betrachtung der Ergebnisse zu beachten, dass aufgrund des Quellens und Schwindens des Holzes, dieser Wert stark von der Feuchte der obersten Furnierlage und damit von den klimatischen Verhältnissen kurz vor der Evaluation abhängt. Dennoch zeigte sich bei den modifizierten Serien häufig eine sehr niedrige Standardabweichung in Kombination mit einer konstant bleibenden Einschätzung der Rissbreite über den gesamten Versuchszeitraum hinweg. In der Regel war das für die Bewertungsziffer 2 der Fall, die zu vergeben war, wenn Risse gerade mit dem bloßen Auge sichtbar waren (DIN ISO 4628-4). Abweichungen von diesem Wert ergaben sich bei jedem Harztyp für unterschiedliche Kombinationen aus Druck 
und WPG, womit kein Zusammenhang der Rissbreite mit diesen Parametern der Herstellung der Platten erkennbar wurde.

Nachfolgend dargestellt sind die Ergebnisse der Freilandbewitterung von unbeschichtetem PFmodifizierten Buchen-LVL mit ausschließlich paralleler Anordnung der Furniere.

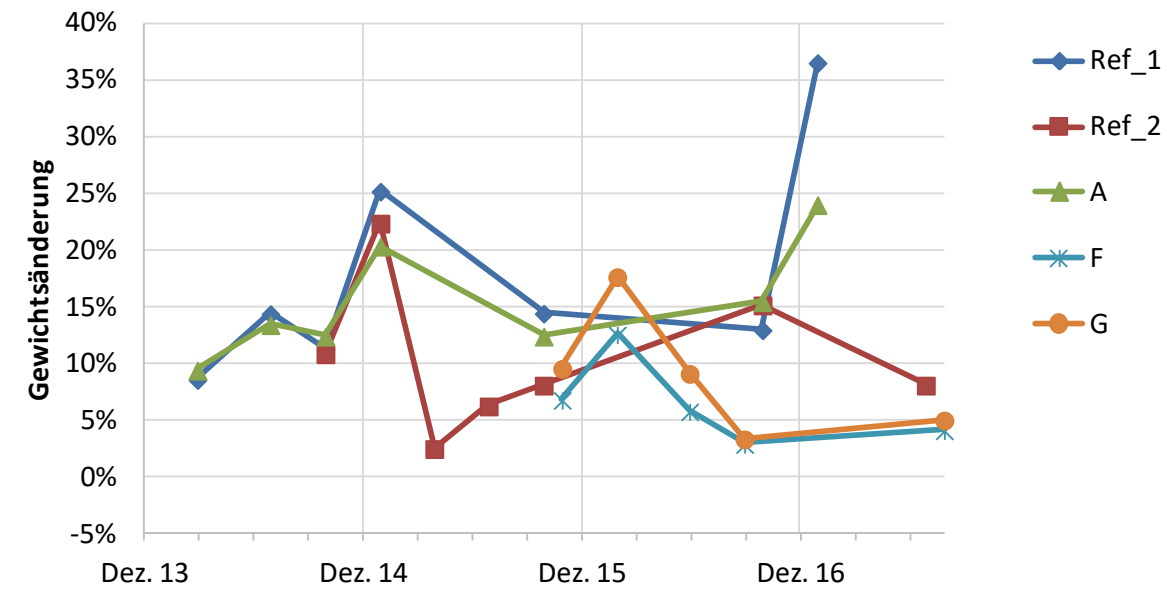

Abbildung 129: Gewichtsänderung unbeschichteter, PF-modifizierter Buchen-LVL-Prüfkörper (8-lagig parallel) mit einem WPG von 15\% bei einem Pressdruck von $2 \mathrm{~N} / \mathrm{mm}^{2}$, in Relation zum Ausgangsgewicht.

Bei einem Pressdruck von 2 N/mm² sind, wie in Abbildung 129, Abbildung 130 und Abbildung 131 dargestellt, unterschiedliche Gewichtsveränderungen in Relation zum Ausgangsgewicht durch die Bewitterung festgestellt worden. Bei einem WPG von 15\% folgte die Variante mit dem Harztyp A im Verlauf der Ergebnisse nahezu der Referenz (Ref_1), die zum gleichen Zeitpunkt in die Bewitterung kam. Erst nach 36 Monaten, im Frühjahr 2017, zeigte sich, dass die modifizierte Variante in der maximalen Gewichtszunahme bzw. Wasseraufnahme von 24\% niedriger lag als die Referenz mit 37\%. Bei der Betrachtung der Harztypen F und G fällt auf, dass diese erst später mit der Freilandbewitterung gestartet wurden. Sie zeigten im Vergleich zum Harztyp A ebenso eine drastische Wasseraufnahme im Winter und eine Abtrocknung im Sommer, die zu Ergebnissen führte, die niedriger Lagen als beim Harztyp A und der ersten Referenz, jedoch keinen Unterschied zum Wertebereich der zweiten Referenzserie aufwiesen. Damit ließ sich für 8-lagiges Buchen-LVL mit paralleler Furnieranordnung beim WPG von 15\%, ebenso wie zuvor beim LVL mit Sperrlagen, für die genannten Harztypen kein Einfluss auf die Wasseraufnahme in der Freilandbewitterung nachweisen. 


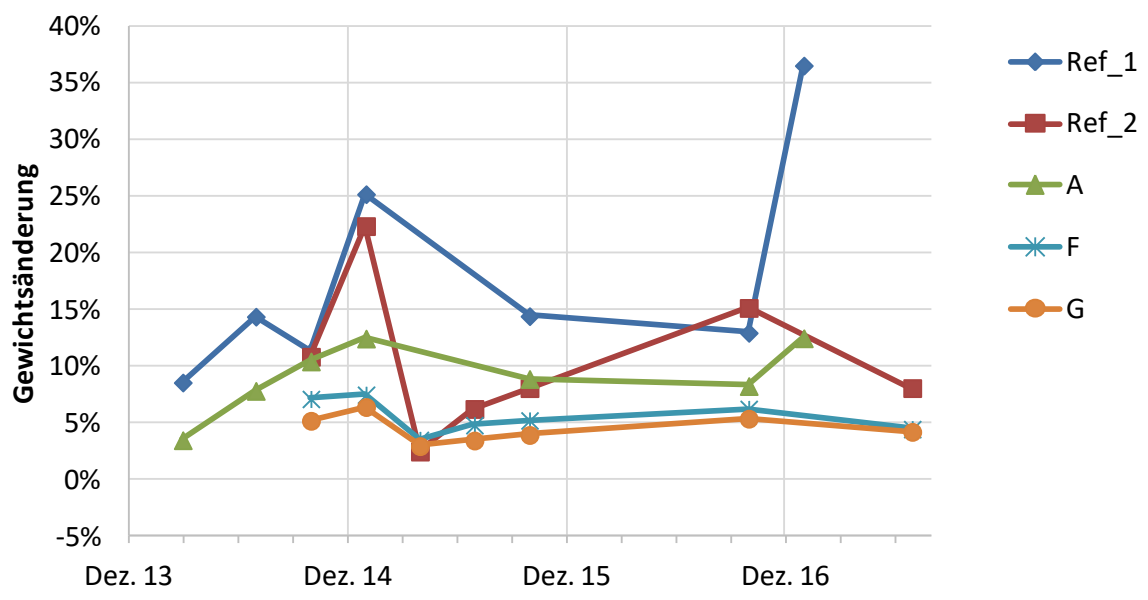

Abbildung 130: Gewichtsänderung unbeschichteter, PF-modifizierter Buchen-LVL-Prüfkörper (8-lagig parallel) mit einem WPG von 30\% bei einem Pressdruck von $2 \mathrm{~N} / \mathrm{mm}^{2}$, in Relation zum Ausgangsgewicht.

In Abbildung 130 sind die Gewichtsänderungen der modifiziertem und unbeschichtetem Buchen-LVL mit einem Pressdruck von 2 N/mm²und einem WPG von 30\% dargestellt. Beim Harztyp A zeigten sich gegenüber der zugehörigen Referenz (Ref_1) niedrigere Werte in Verbindung mit einem flacheren Verlauf und geringeren Schwankungen. Die Harztypen F und G zeigten beide sehr ähnliche Ergebnisse, die deutlich niedriger lagen als beim Harztyp A und, mit einer Ausnahme beim dritten Messintervall (nach 9 Monaten), niedriger lagen als ihre Referenz (Ref_2). Die extrem niedrigen Messergebnisse für die beiden Harztypen und ihre Referenz beim dritten Messintervall traten im Juli 2016 auf, der sich entsprechend der Klimadaten als ein niederschlagsarmer und zugleich als der wärmste Monat in dem Jahr herausstellte.

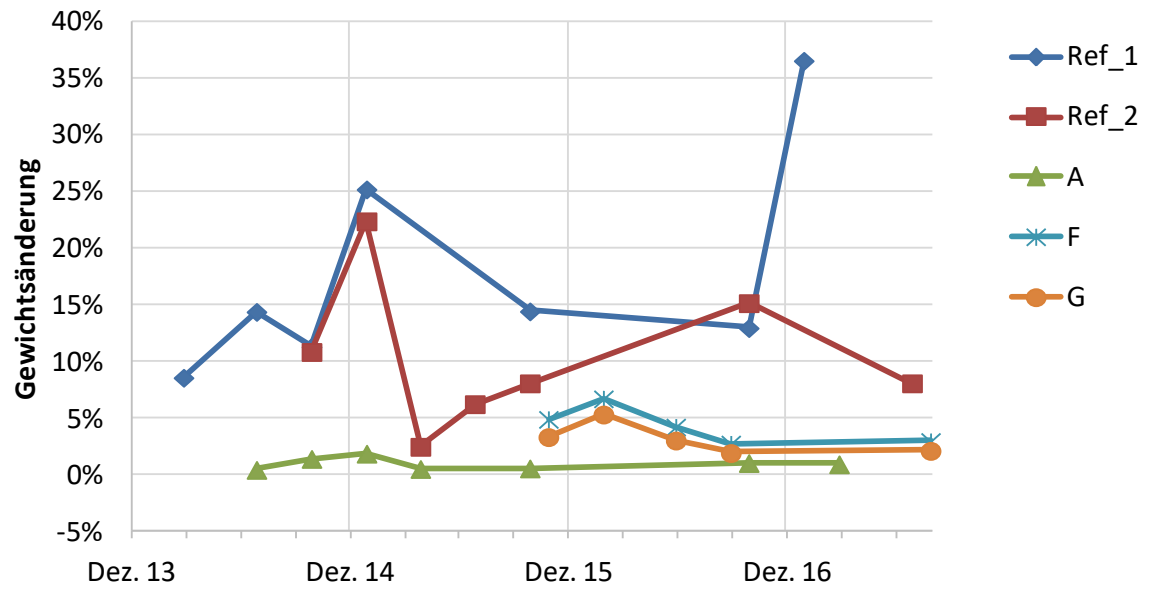

Abbildung 131: Gewichtsänderung unbeschichteter, PF-modifizierter Buchen-LVL-Prüfkörper (8-lagig parallel) mit einem WPG von $60 \%$ bei einem Pressdruck von $2 \mathrm{~N} / \mathrm{mm}^{2}$, in Relation zum Ausgangsgewicht.

Die maximale Wasseraufnahme bei modifizierten LVL ohne Sperrlagen, mit einem WPG von $60 \%$ und einem Pressdruck von $2 \mathrm{~N} / \mathrm{mm}^{2}$, lag bei 7\%. Dabei lagen alle Werte immer unter denen der Referenzen. Im Gegensatz zu den bisherigen Ergebnissen bei diesem Plattentyp lagen die Werte des Harztyps A, mit maximal 2\%, niedriger als bei den Harztypen F und G, welche wieder sehr ähnliche Ergebnisse zeigten. Diese Ergebnisse deuten somit darauf hin, dass der Einfluss des WPG auf die Wasseraufnahme größer war als die Wahl des Harzes. 


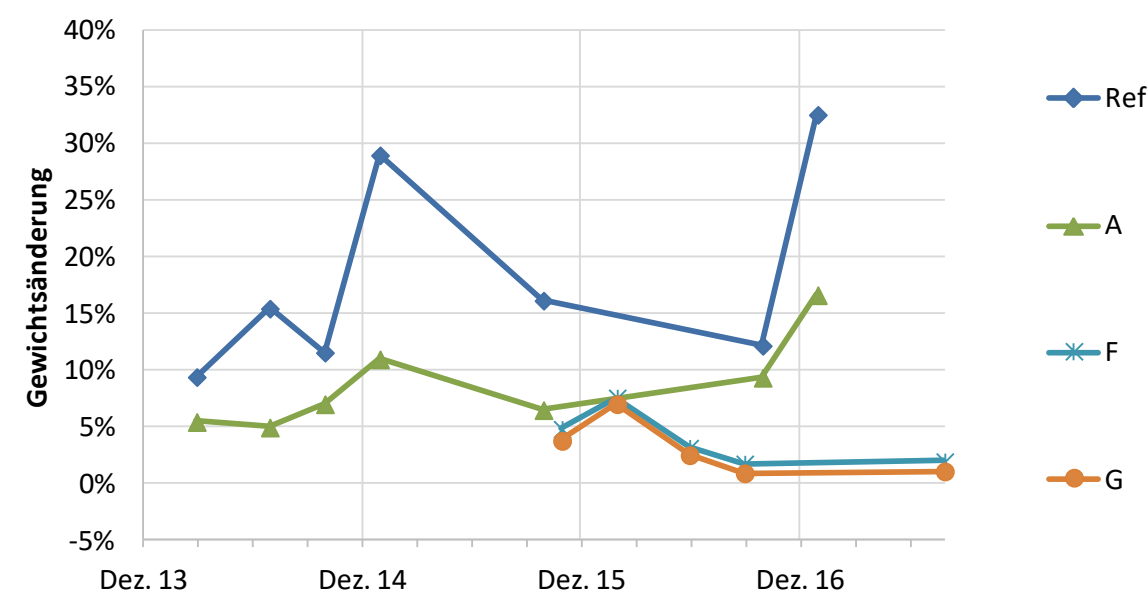

Abbildung 132: Gewichtsänderung unbeschichteter, PF-modifizierter Buchen-LVL-Prüfkörper (8-lagig parallel) mit einem WPG von 15\% bei einem Pressdruck von $6 \mathrm{~N} / \mathrm{mm}^{2}$, in Relation zum Ausgangsgewicht.

Bei einem Pressdruck von 6 N/mm ${ }^{2}$ in Kombination mit einem WPG von 15\% zeigte sich eine verringerte Wasseraufnahme der modifizierten Proben bei allen Harztypen gegenüber der Referenz (Abbildung 132). Gegenüber den modifizierten Serien mit dem gleichen WPG, die bei einem Pressdruck von $2 \mathrm{~N} / \mathrm{mm}^{2}$ hergestellt wurden, betrug die Reduzierung für den Zeitraum von 12 Monaten sogar 50\%. Im Vergleich der Harztypen zueinander zeigte sich wieder, dass die Harztypen F und G niedrigere Werte erzielten als der Harztyp A. Dennoch ist der Einfluss des Pressdruckes hier offensichtlich geworden, da die Wasseraufnahmen ähnlich niedrig wie bei den Serien mit einem WPG von $60 \%$ mit $2 \mathrm{~N} / \mathrm{mm}^{2}$ waren.

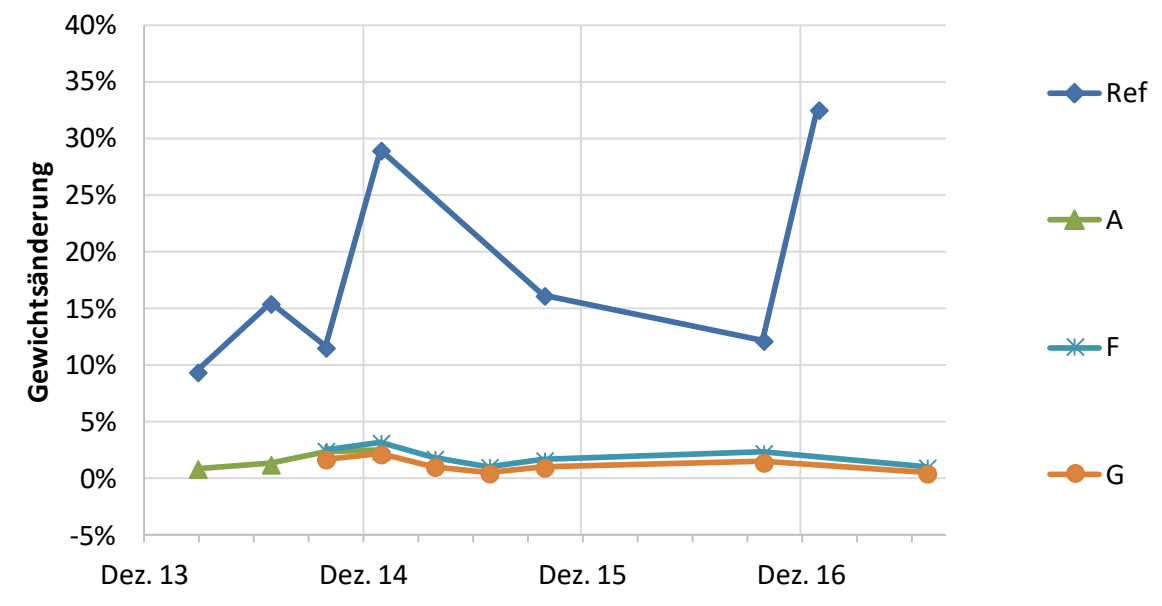

Abbildung 133: Gewichtsänderung unbeschichteter, PF-modifizierter Buchen-LVL-Prüfkörper (8-lagig parallel) mit einem WPG von $30 \%$ bei einem Pressdruck von $6 \mathrm{~N} / \mathrm{mm}^{2}$, in Relation zum Ausgangsgewicht.

Bei einem Pressdruck von $6 \mathrm{~N} / \mathrm{mm}^{2}$ und einem WPG von 30\% waren keine Unterschiede zwischen den Harztypen mehr zu erkennen und alle Ergebnisse der modifizierten Serien lagen unter 3\% Wasseraufnahme, während die Referenzen im Versuchszeitraum von drei Jahren 
stets zwischen 9\% und 33\% lagen (Abbildung 133). Im Vergleich zu den modifizierten Versuchsserien mit Sperrlagen sind die Ergebnisse identisch.

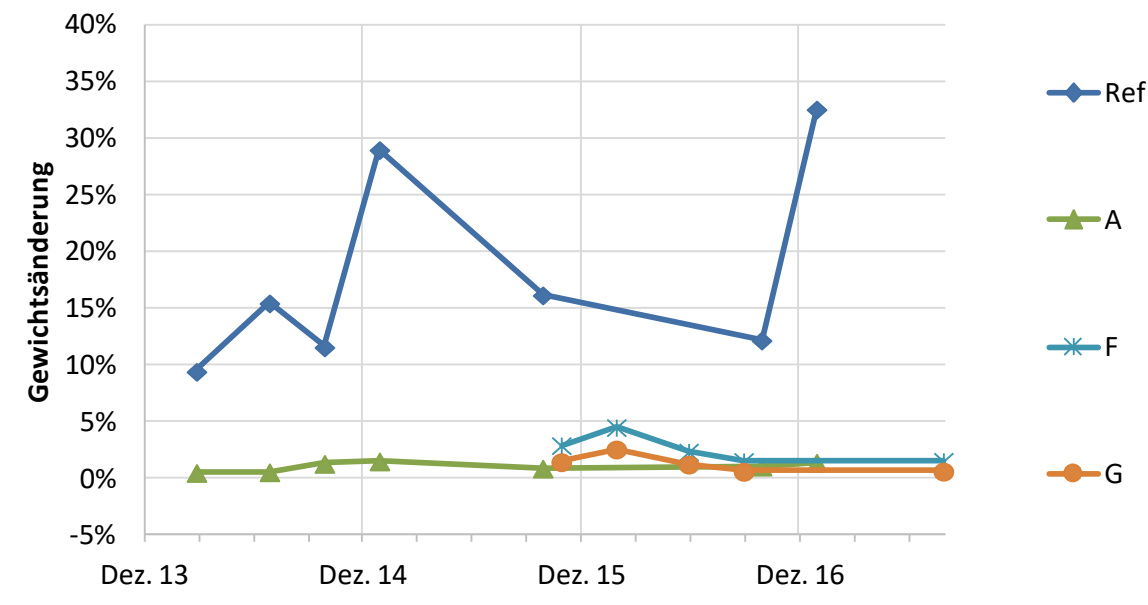

Abbildung 134 Gewichtsänderung unbeschichteter, PF-modifizierter Buchen-LVL-Prüfkörper (8-lagig parallel) mit einem WPG von $60 \%$ bei einem Pressdruck von $6 \mathrm{~N} / \mathrm{mm}^{2}$, in Relation zum Ausgangsgewicht.

Wie Abbildung 134 zeigt, konnte bei einem Pressdruck von $6 \mathrm{~N} / \mathrm{mm}^{2}$ durch Erhöhung des WPG auf $60 \%$ keine weitere Verringerung der Wasseraufnahme, im Vergleich zu einem WPG von $30 \%$ bei gleichem Pressdruck, erzielt werden.

Die Auswirkungen der Gewichtsänderungen von Proben in der Aussenbewitterung, welche im Wesentlichen auf Wasseraufnahme und Wasserabgabe zurückzuführen sind, werden durch die Dickenquellung der LVL-Prüfkörper mit parallelem Aufbau nachfolgend veranschaulicht.

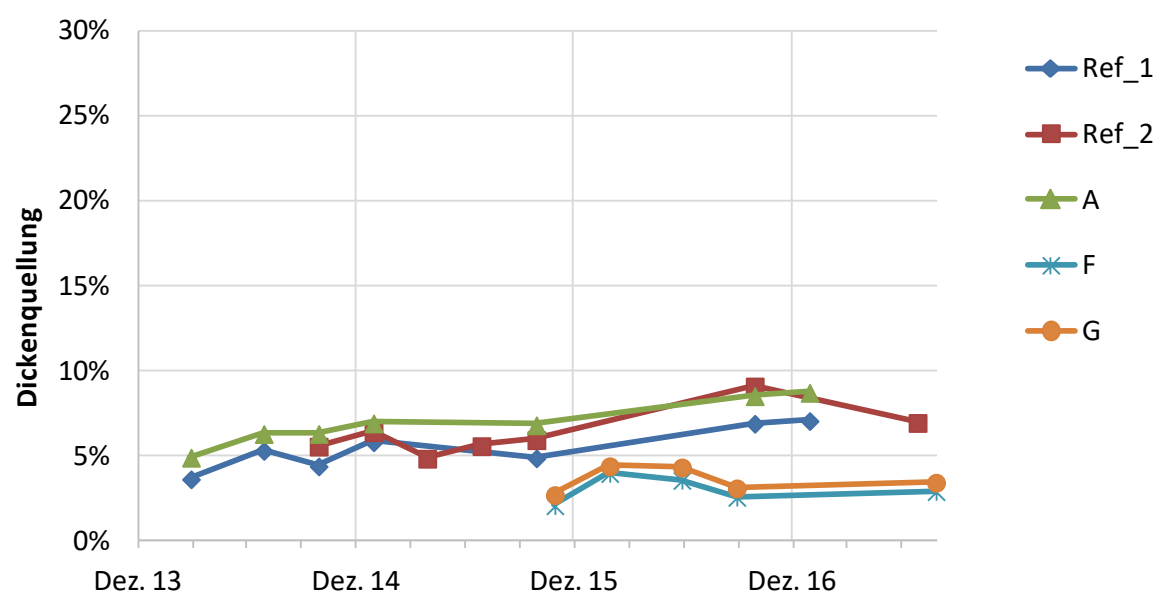

Abbildung 135: Dickenquellung unbeschichteter, PF-modifizierter Buchen-LVL-Prüfkörper (8-lagig parallel) mit einem WPG von 15\% bei einem Pressdruck von 2 N/mm.

Bei einem Pressdruck von $2 \mathrm{~N} / \mathrm{mm}^{2}$ zeigten die Referenzen mit parallelem Aufbau sehr ähnliche Werte für die Dickenquellung wie beim unbehandelten LVL mit Sperrlagen. Da die Dickenquellung senkrecht zur Plattenebene gemessen wurde, ist es plausibel, dass die Orientierung der Furniere bei den beiden Plattentypen keinen Einfluss auf diese Messgröße hatte. Bei den modifizierten Serien mit einem WPG von 15\% konnte für Harztyp A keine Verbesserung festgestellt werden, da die Werte denen der Referenzen glichen oder darüber lagen (Abbildung 135). Für die Harztypen $F$ und $G$ konnte indes eine verringerte Dickenquellung festgestellt werden. Es zeigte sich auch bereits bei der Wasseraufnahme, dass 
diese Harztypen weniger Wasser aufnahmen als die Referenzen und der Harztyp A. Mögliche Einflussfaktoren können die Lage der Prüfkörper in der Freilandbewitterung (Verschattung, o.ä.) oder Schwankungen in der Lösungsaufnahme, dem Feststoffgehalt und dem tatsächlichen WPG, insbesondere bei den äußeren Furnieren, gewesen sein. Beim Vergleich der Darrdichten stellte sich bereits heraus, dass die Prüfkörper des Harztyps A näher an den Referenzen lagen als bei den anderen beiden Harztypen. Dies deutet auf einen etwas geringen WPG als vermutet hin. Ein Grund dafür könnte wiederum in einer beobachteten kritischen Lösungsstabilität der Phenol-Formaldehyde bei niedrigen Konzentrationen liegen.

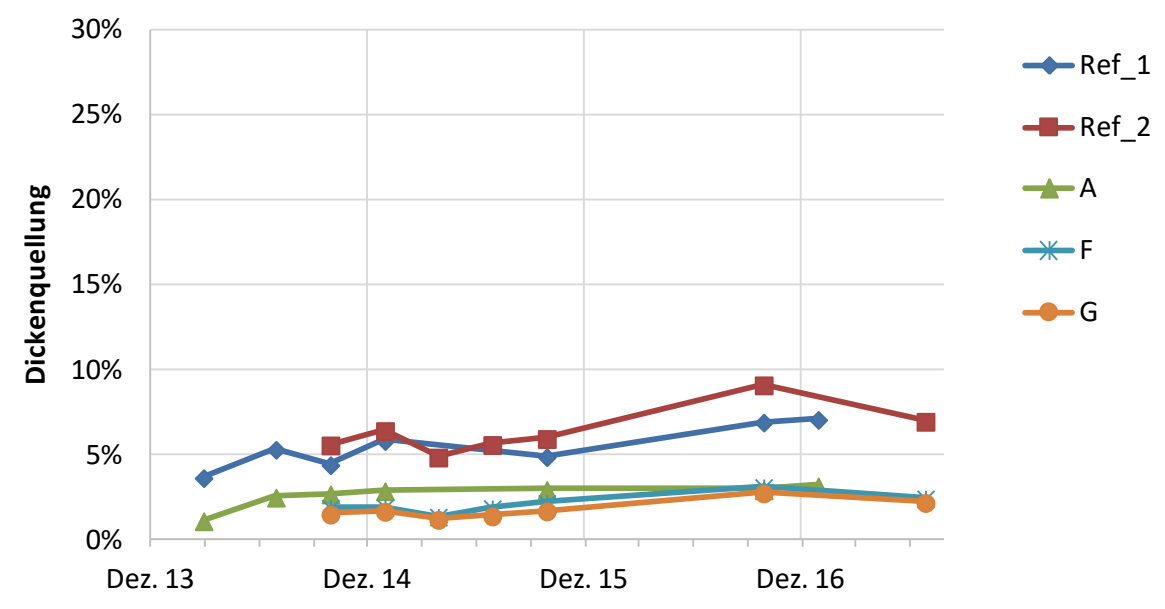

Abbildung 136: Dickenquellung unbeschichteter, PF-modifizierter Buchen-LVL-Prüfkörper (8-lagig parallel) mit einem WPG von 30\% bei einem Pressdruck von 2 N/mm.

Bei einem Pressdruck von $2 \mathrm{~N} / \mathrm{mm}^{2}$ und einem WPG von 30\% wiesen alle drei Harztypen eine ähnliche Dickenquellung auf, welche auch nach 36 Monaten maximal bei 3\% lag und damit erheblich niedriger als bei den Referenzen (Abbildung 136). Hier zeigt sich somit ein Unterschied zu den Beobachtungen der Wasseraufnahme für diese Serien, denn da lag der Harztyp A zwar unterhalb der Referenzen, aber doch über den Werten der Harztypen F und G. Das zeigt, dass die Wasseraufnahme bei modifiziertem Buchen-LVL nicht zwangsläufig zu einer Quellung führen muss. Dies liegt darin begründet, dass das PF-Harz mit dem gewählten Verfahren überwiegend in der Zellwand eingelagert wird, dort die Einlagerung von Wasser und Quellung der Zellwand einschränkt und dennoch ausreichend Raum im Holzkörper für die Aufnahme von Wasser zur Verfügung steht. Es ist anzumerken, dass, wie bereits bei der Schnellbewitterung für den Harztyp A festgestellt, die Dickenquellung dieser Platten ohne Sperrlagen niedriger war als die Dickenquellung der Platten mit Sperrlagen bei gleichem Harztyp, WPG und Pressdruck. 


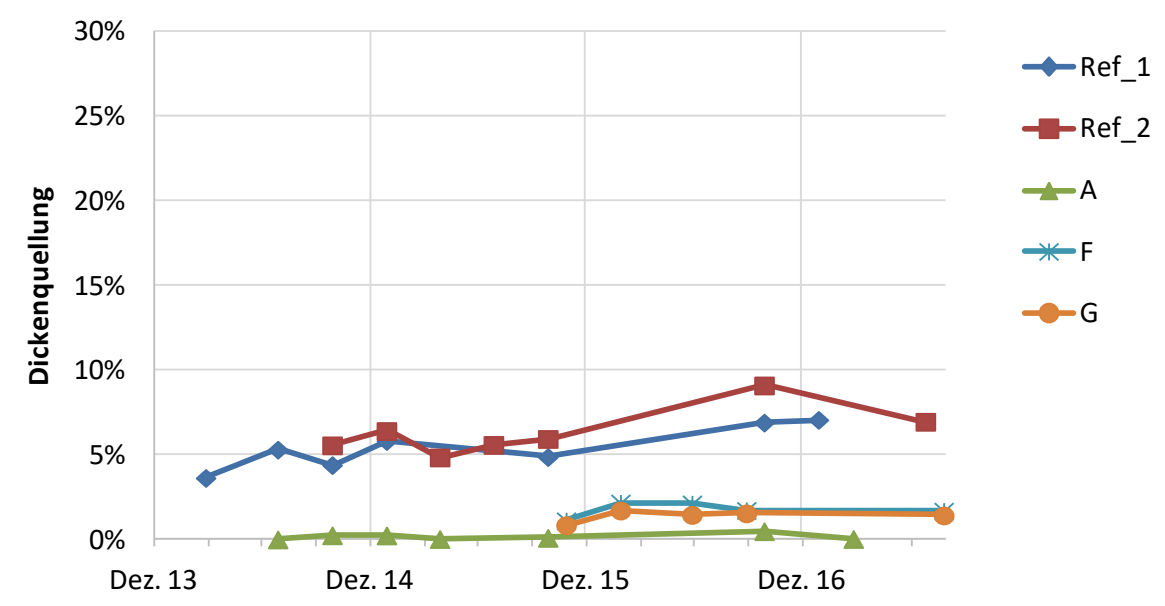

Abbildung 137: Dickenquellung unbeschichteter, PF-modifizierter Buchen-LVL-Prüfkörper (8-lagig parallel) mit einem WPG von $60 \%$ bei einem Pressdruck von $2 \mathrm{~N} / \mathrm{mm}$.

Die Dickenquellung von modifiziertem Buchen-LVL war, wie Abbildung 137 zeigt, infolge eines WPG von $60 \%$ bei den Harztypen G und F weiter herabgesetzt als bei 30\% WPG und sogar fast gänzlich verhindert beim Harztyp A (maximal 1\%). Natürlich sind diese Ergebnisse neben einer Füllung der Zellwände auch auf die Füllung der Zelllumen zurückzuführen, womit die Wegsamkeit für Wasser eingeschränkt wurde.

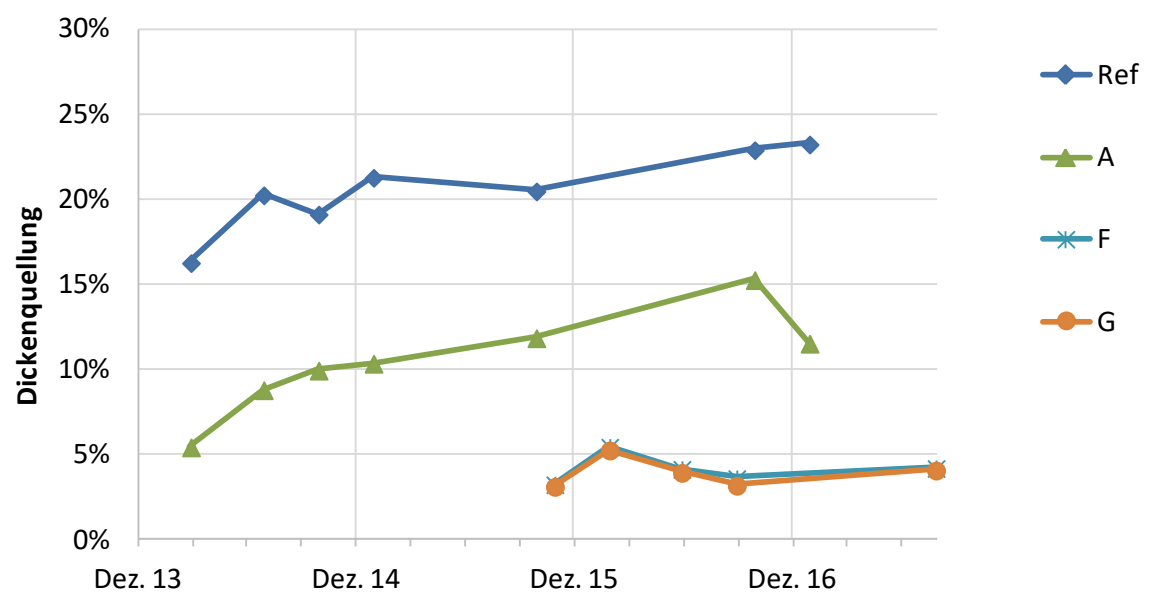

Abbildung 138: Dickenquellung unbeschichteter, PF-modifizierter Buchen-LVL-Prüfkörper (8-lagig parallel) mit einem WPG von 15\% bei einem Pressdruck von 6 N/mm.

Infolge des höheren Pressdruckes von $6 \mathrm{~N} / \mathrm{mm}^{2}$ kam es, wie bereits bei dem Plattentyp mit Sperrlagen gezeigt, bei den Referenzen infolge einer leichten Verdichtung in Kombination mit Rückquellung bei Wasseraufnahme, zu einer starken Erhöhung der gemessenen Dickenquellung. Die größten Steigerungen konnten dabei im ersten Jahr des Versuchszeitraumes gemessen werden. Bei den modifizierten Serien ließ sich, wie bereits beim niedrigeren Pressdruck diskutiert, ein deutlicher Unterschied zwischen dem Harztyp A und den Harztypen $F$ und $G$ feststellen. Auch hier kann ein Zusammenhang mit der Wasseraufnahme festgestellt werden, was auf einen geringeren WPG als beabsichtigt hindeutet. 


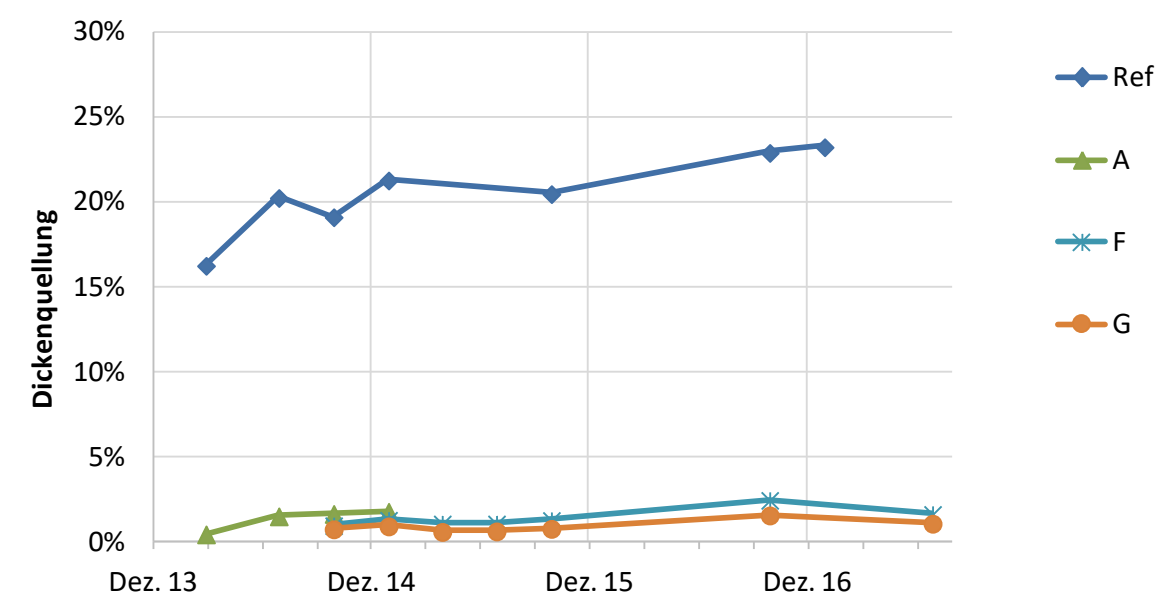

Abbildung 139: Dickenquellung unbeschichteter, PF-modifizierter Buchen-LVL-Prüfkörper (8-lagig parallel) mit einem WPG von $30 \%$ bei einem Pressdruck von $6 \mathrm{~N} / \mathrm{mm}$.

Bei einem WPG von 30\% und einem Pressdruck von 6 N/mm² konnte, wie bei den Platten mit Sperrlagen, die Dickenquellung, nicht nur im Vergleich zu den Referenzen, sondern auch zu den Serien, die bei einem Pressdruck von $2 \mathrm{~N} / \mathrm{mm}^{2}$ hergestellt wurden, drastisch reduziert werden. Die maximale Dickenquellung aller drei Harztypen lag nur noch bei 2\% (Abbildung 139).

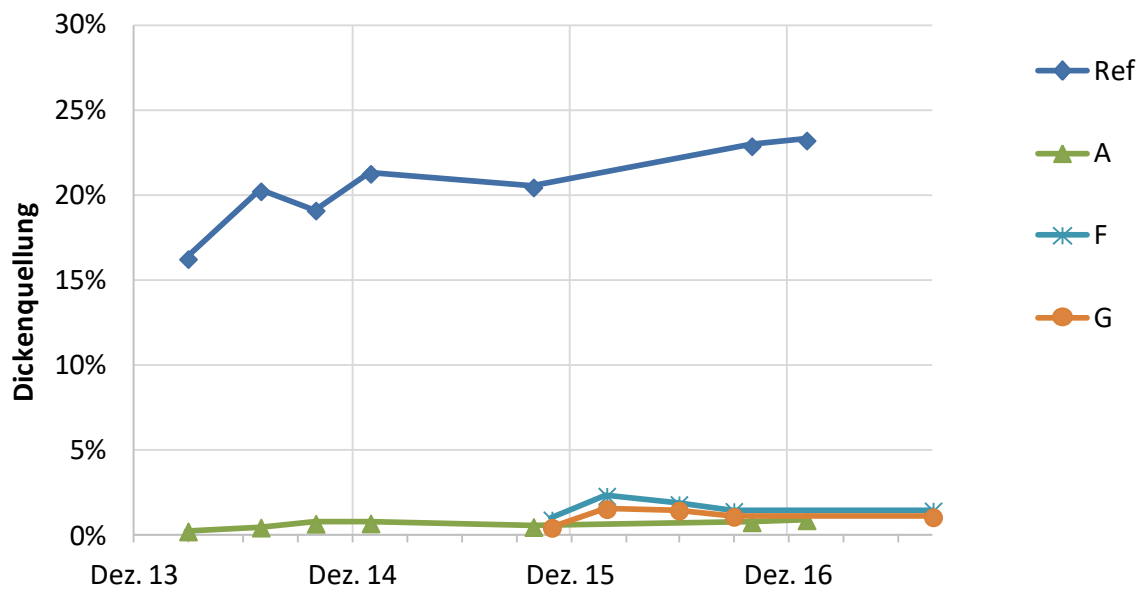

Abbildung 140: Dickenquellung unbeschichteter, PF-modifizierter Buchen-LVL-Prüfkörper (8-lagig parallel) mit einem WPG von $30 \%$ bei einem Pressdruck von $6 \mathrm{~N} / \mathrm{mm}$. 
Im Vergleich zu einem WPG von 30\% konnte, wie Abbildung 140 zeigt, bei einem Pressdruck von $6 \mathrm{~N} / \mathrm{mm}^{2}$ auch mit einem WPG von 60\% keine weitere Verbesserung erzielt werden.

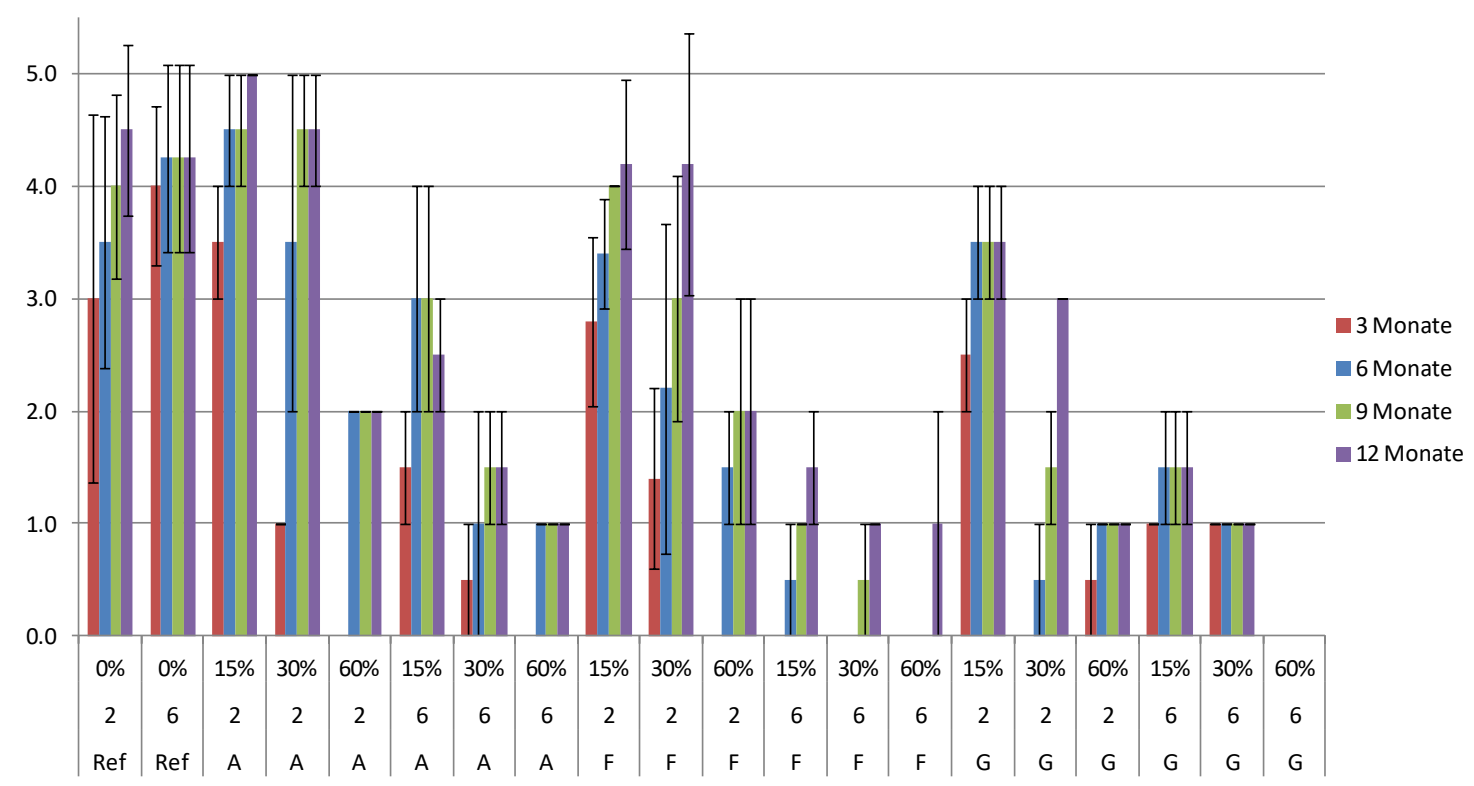

Abbildung 141: Bewertungsziffern (0-5) nach DIN ISO 4628-4 für die Menge der Risse in der Vorderseite von PF-modifiziertem Buchen-LVL mit 8 Lagen (parallel) in Abhängigkeit von der Bewitterungsdauer im Freiland.

Wie in Abbildung 141 dargestellt, konnte in einem Zeitraum von 12 Monaten eine sehr unterschiedliche Anzahl von Rissen zwischen den einzelnen Messintervallen (3 Monate) und bei den Prüfkörperserien mit paralleler Ausrichtung der Furniere festgestellt werden. Völlig unbehandelte Referenzen zeigten den schnellsten Anstieg der Anzahl an Rissen unabhängig von der Verdichtung $\left(2 \mathrm{~N} / \mathrm{mm}^{2}\right.$ oder $6 \mathrm{~N} / \mathrm{mm}^{2}$ ). Bei den modifizierten Serien zeigte sich, dass ein WPG von 15\% keinen ausreichenden Schutz bot, da hier beim Harztyp A die größte (5; sehr viele) und bei den Harztypen F und G die zweitgrößte Anzahl an Rissen (4; viele) beobachtet werden konnte. Insofern zeigte sich hier für die vergleichende Beurteilung der Harze eine Analogie zu den Ergebnissen der Wasseraufnahme und der Dickenquellung. Bei einem WPG von 30\% zeigte sich eine deutlich verlangsamte Zunahme der Risse. Beim Harztyp A lag die Anzahl an Rissen dann nach 12 Monaten jedoch ähnlich hoch wie bei den unbehandelten Proben, während bei Harztyp G eine deutlich geringere Anzahl (3; mäßig viele) festzustellen war. Hinsichtlich des Pressdruckes von $6 \mathrm{~N} / \mathrm{mm}^{2}$, der zu einer starken Verdichtung der Platten in der Herstellung führte, lässt sich feststellen, dass hierdurch die Anzahl Risse im Versuchszeitraum stark reduziert wurde. Der höhere WPG von $60 \%$ reduzierte beim niedrigen Pressdruck die Anzahl an Rissen bereits deutlich. Der Pressdruck von $6 \mathrm{~N} / \mathrm{mm}^{2}$ mit der deutlichen Verdichtung der Proben führte bei einem WPG von 60\% zu den besten Ergebnissen im Versuch, da hier zum Teil keine Risse (0) oder nur einzelne, sich gerade abzeichnende Risse (1) festgestellt wurden.

Im Vergleich zum LVL mit Sperrlagen fällt auf, dass alle Serien, die ohne Sperrlagen hergestellt wurden, wenngleich hier andere Harztypen zum Einsatz kamen, tendenziell besser bewertet wurden. Betrug der Mittelwert modifizierter Proben, über alle Messintervalle und Varianten hinweg, bei den Serien mit ausschließlich parallel orientierten Furnieren 1,7, so betrug er bei 
den Serien mit zwei Sperrlagen 2,7. Im direkten Vergleich der Serien, die mit dem Harztyp A bei gleichem Pressdruck und WPG hergestellt wurden, wurde die Anzahl Risse durchschnittlich mit einer 1,7 für ausschließlich parallele Furniere und mit einer 2,4 für die Serien mit zwei Sperrlagen bewertet. Daraus lässt sich erkennen, dass für die Bewitterungsstabilität der Aufbau der Platten einen Einfluss auf die Anzahl der Risse hatte und Sperrlagen, entgegen der Erwartung, zu mehr Rissen führten. Es ist möglich, dass dafür die in den beiden oberen Furnierlagen unterschiedlich gerichteten Quellkräfte zu Spannungsspitzen führten, welche die Holzfestigkeit überstiegen und so eine Gefügeschädigung nach sich zogen, bzw. das äußere Furnier durch die Verklebung an seiner Unterseite fixiert war und an der Oberseite ungehindert quellen konnte.

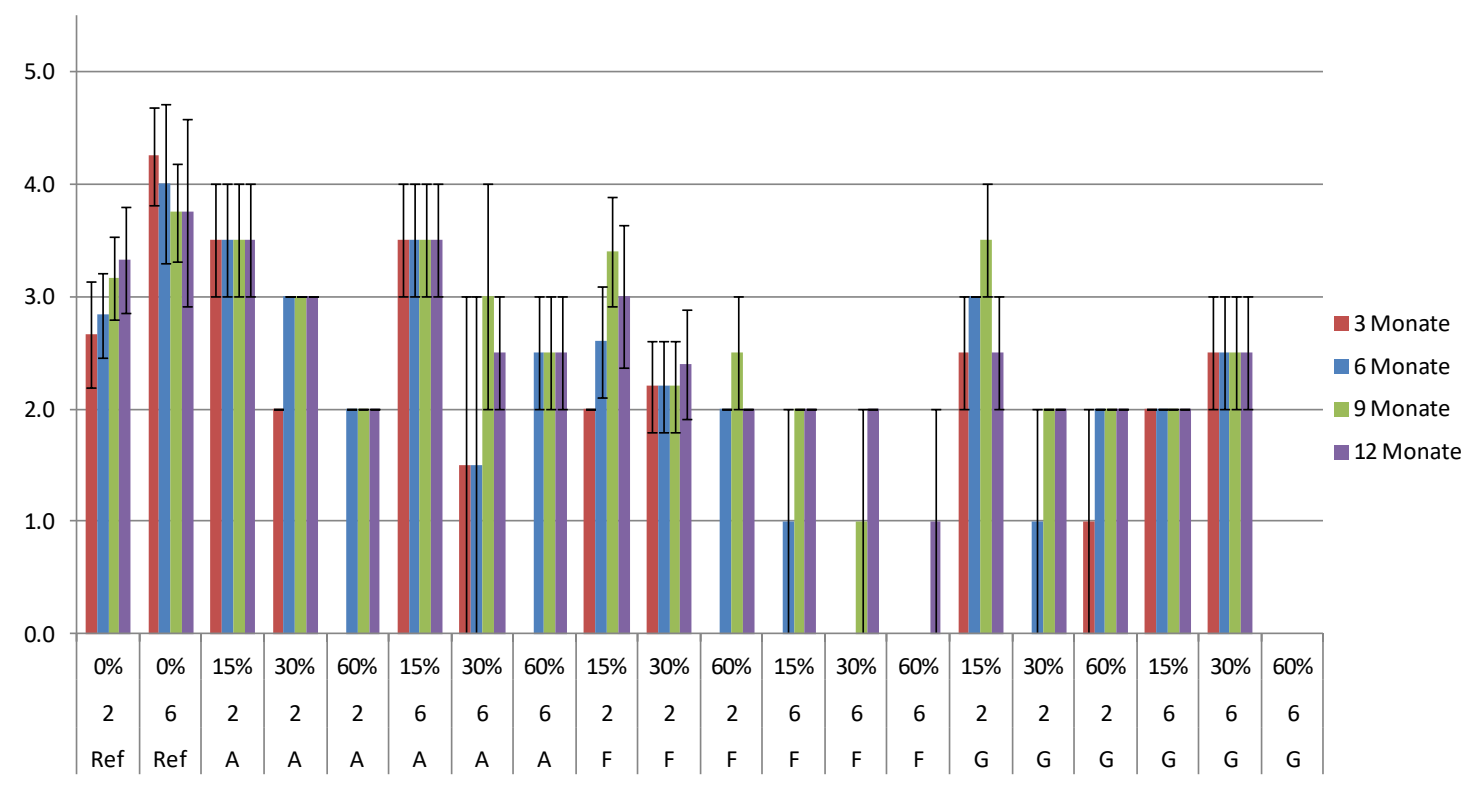

Abbildung 142: Bewertungsziffern (0-5) nach DIN ISO 4628-4 für die Breite der Risse in der Vorderseite von PF-modifiziertem Buchen-LVL mit 8 Lagen (parallel) in Abhängigkeit der Bewitterungsdauer im Freiland.

Wie in Abbildung 142 dargestellt, konnte in einem Zeitraum von 12 Monaten eine sehr unterschiedliche Breite bei Rissen zwischen den einzelnen Prüfkörperserien mit paralleler Ausrichtung der Furniere festgestellt werden. Deutlich sichtbare (3) resp. breite $(4 ;<1 \mathrm{~mm})$ Risse ließen sich bei den Referenzen schon bei der ersten Messung für unverdichtete resp. verdichtete Probe feststellen. Bei den behandelten Serien fallen die Varianten mit 15\% auf, welche auch im Bereich der deutlich sichtbaren Risse lagen (3). Ein höherer WPG und eine höhere Verdichtung wirkten sich in den meisten Fällen positiv aus. Diese Serien wiesen dann, wie auch bei den Serien, welche Sperrlagen enthielten, überwiegend Risse auf, die gerade mit dem bloßen Auge sichtbar waren und für die die Bewertungsziffer 2 (DIN ISO 4628-4) zu vergeben war.

In Bezug auf die Oberflächenqualität bewitterten Buchen-LVLs bedeutet dies, dass ohne eine Beschichtung ein länger währender Schutz vor Schäden nur durch hohe WPG und hohe Pressdrücke erzielbar wäre. 
5.3.5.2.3 Beschichtetes modifiziertes LVL

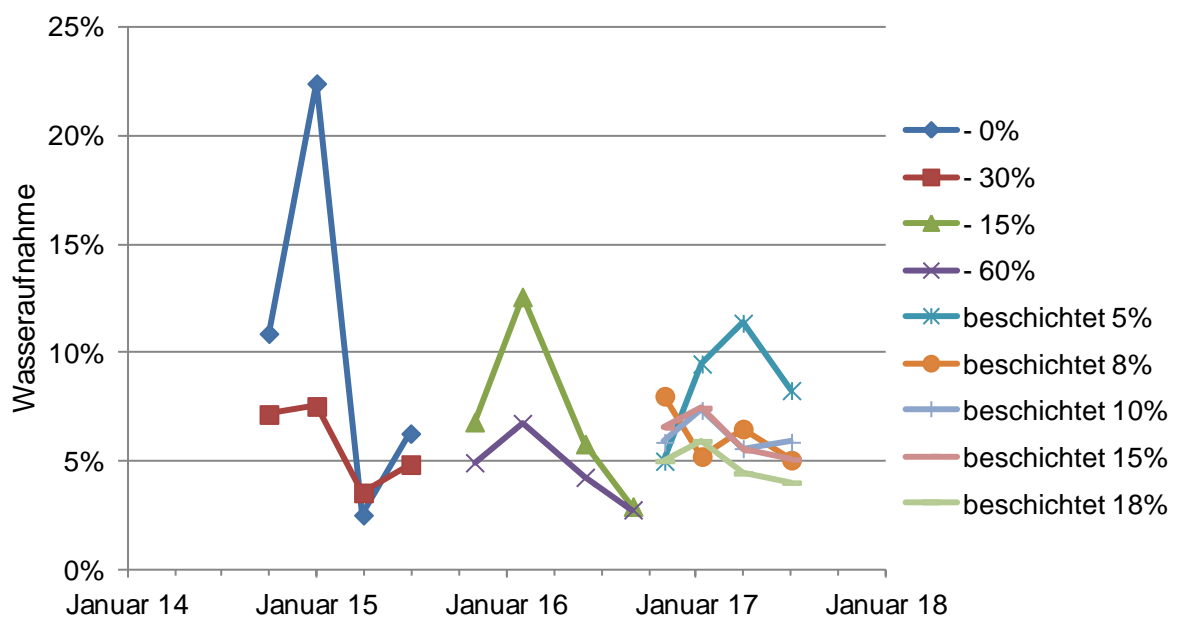

Abbildung 143: Wasseraufnahme von beschichteten und unbeschichteten Buchen-LVL (8-lagig parallel, Pressdruck $2 \mathrm{~N} / \mathrm{mm}^{2}$ ) mit verschiedenen WPGs (Harztyp F) in der Freilandbewitterung über 12 Monate. Beschichtung: 1x Remmers Grundierung IL170 (60s getaucht) und 1 x Remmers Wetterschutzlack UV+

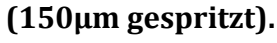

Die Beschichtung von modifiziertem Buchen-LVL führte bei Bewitterung im Freiland zu einer reduzierten Wasseraufnahme und Dickenquellung. Bei Betrachtung der Wasseraufnahme (Abbildung 143) fällt auf, dass alle Werte im Verlauf von 12 Monaten, entsprechend der jahreszeitlichen Klimata, deutlichen Schwankungen unterlagen. Die größten Schwankungen wiesen erwartungsgemäß die Referenzen auf, da sie durchschnittlich 11\% schwerer waren, aber minimal bei 3\% und maximal bei 22\% lagen. Bei den modifizierten Serien mit den WPGs 15\%, 30\% und 60\% waren die Schwankungen umso kleiner je größer der WPG war. Einen ähnlichen Wertebereich wie diese unbeschichteten modifizierten LVL wiesen die modifizierten LVL mit einer Beschichtung auf, obwohl diese nur WPGs zwischen 5\% und 18\% hatten. Bei dem WPG von 5\% waren die Schwankungen sowie die maximale Wasseraufnahme mit 11\% größer als bei den übrigen beschichteten Varianten.

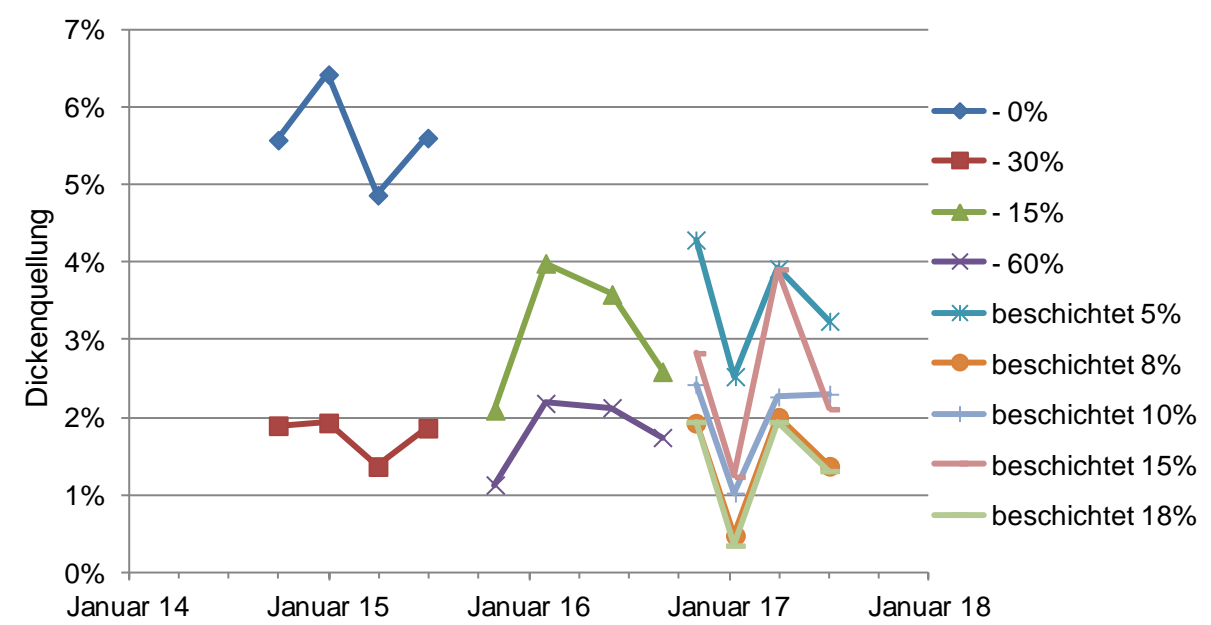

Abbildung 144: Dickenquellung von beschichteten und unbeschichteten Buchen-LVL (8-lagig parallel, Pressdruck $2 \mathrm{~N} / \mathrm{mm}^{2}$ ) mit verschiedenen WPGs (Harztyp F) in der Freilandbewitterung über 12 Monate. Beschichtung: 1x Remmers Grundierung IL170 (60s getaucht) und 1 x Remmers Wetterschutzlack UV+

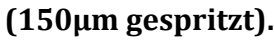


Bei der Dickenquellung in der Freilandbewitterung zeigte sich, dass einige beschichteten Varianten teilweise mehr und andere weniger als die modifizierten unbeschichteten Serien quollen. So erreichten die beschichteten Varianten mit einem WPG von 5\% und mit einem WPG von $15 \%$ eine maximale Quellung von $4 \%$ nach drei Monaten bzw. nach 9 Monaten der Bewitterung und lagen damit auf dem gleichen Niveau wie die unbeschichtete Variante mit 15\% WPG nach 6 Monaten. Während die beschichtete Variante mit einem WPG von 10\% dazwischenlag, zeigten unter den beschichteten Varianten jene mit 8\% WPG und mit 18\% WPG mit durchschnittlich 1\% die niedrigste Quellung, erreichten jedoch auch eine maximale Quellung von 2\%. Im Vergleich dazu lagen die unbeschichteten Varianten mit 30\% WPG und mit 60\% WPG bei durchschnittlich 2\% und maximal bei 2\%, ihre Schwankungen somit kleiner waren. Im Vergleich zu den Ergebnissen der Schnellbewitterung fällt auf, dass bei beiden Versuchen die Schwankungen zwischen den Messintervallen bei den beschichteten Proben relativ groß waren und insbesondere keine stetig steigende Wasseraufnahme und Dickenquellung mit zunehmender Bewitterungsdauer zu verzeichnen war. Bei den unbeschichteten modifizierten Proben war es zwar in der Schnellbewitterung zu einer stetigen Zunahme gekommen, jedoch folgten sie im Freiland auch den saisonalen Klimawechseln und zeigten Abtrocknung und Schwinden.

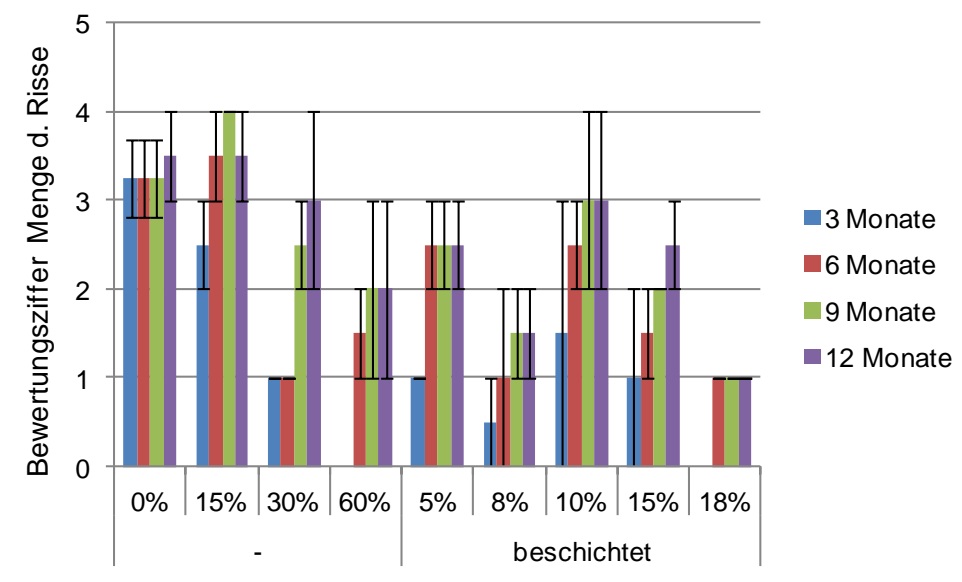

Abbildung 145: Bewertungsziffer (0-5) nach DIN ISO 4628-4 für die Menge der Risse in der Vorderseite von beschichteten und unbeschichteten modifizierten Buchen-LVL (8-lagig, parallel, $2 \mathrm{~N} / \mathrm{mm}^{2}$ Pressdruck) in Abhängigkeit von der Testdauer in der Schnellbewitterung. Beschichtung: 1x Remmers Grundierung IL170 (60s getaucht) und $1 \times$ Remmers Wetterschutzlack $U V+(150 \mu m$ gespritzt $)$.

Das Ausmaß der durch das Quellen und Schwinden des Holzes entstehenden Risse wird durch die Menge und die Breite der Risse in der Oberfläche der bewitterten Proben ersichtlich. Bei den in Abbildung 145 dargestellten Bewertungsziffern für die Menge der Risse wird ersichtlich, dass bei allen Proben zu jeder Zeit mindestens einzelne sich gerade abzeichnende Risse erkennbar waren. Bei den unbeschichteten und den transparent beschichteten Proben bezog sich die Auswertung auf das Deckfurnier, was eine Schädigung der Beschichtung implizierte. Die größte Menge an Rissen zeigten, neben den Referenzen, die unbeschichteten Proben mit den WPGs von 15\% und 30\% sowie die beschichteten Proben mit einem WPG von 10\%. Dabei stieg die Menge an Rissen bei den unbeschichteten mit einem WPG von 15\% schneller an als bei den unbeschichteten mit 30\%. Da bei einem WPG von 60\% die Menge an Rissen niedriger war, konnte in diesem Zusammenhang ein positiver Einfluss des WPG auf die Vermeidung von Rissen festgestellt werden. Der gleiche Einfluss wurde auch für die beschichteten Proben 
erwartet, jedoch bestätigte das nur die Variante mit 18\%, die nur einzelne, sich gerade abzeichnende Risse (1) und damit weniger Risse als die anderen beschichteten und unbeschichteten Proben aufwies. Die beschichtete Variante mit einem WPG von 15\% wies im Gegensatz zu ihrer unbeschichteten Komplementärserie, die mäßig viele (3) bis viele Risse (4) aufwies, weniger Risse (durchschnittlich „2“, maximal „3“) auf.

Die Ergebnisse der Freilandbewitterung in Bezug auf Risse stellen einen deutlichen Unterschied zu den Ergebnissen der Schnellbewitterung dar. Es zeigte sich hier, dass die beschichteten Proben im Freiland unterschiedlich viele Risse zeigten, während in der Schnellbewitterung für die gleichen Serien, mit Ausnahme der Variante mit 5\% WPG, gar keine Risse gefunden werden konnten. Die Ergebnisse beider Versuche für unbeschichtete Varianten zeigten jedoch sehr ähnliche Tendenzen auf.

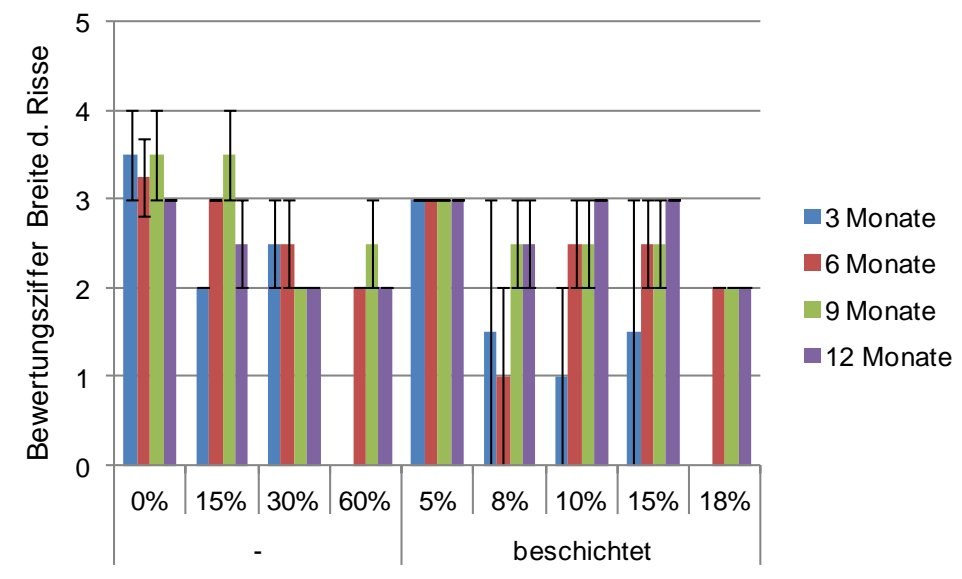

Abbildung 146: Bewertungsziffer (0-5) nach DIN ISO 4628-4 für die Breite der Risse in der Vorderseite von beschichteten und unbeschichteten modifizierten Buchen-LVL (8-lagig, parallel, $2 \mathrm{~N} / \mathrm{mm}^{2}$ Pressdruck) in Abhängigkeit von der Testdauer in der Schnellbewitterung. Beschichtung: 1x Remmers Grundierung IL170 (60s getaucht) und $1 \times$ Remmers Wetterschutzlack $U V+(150 \mu m$ gespritzt $)$.

Entgegen der Beobachtungen in der Schnellbewitterung beschichteter Proben, konnten für die hier aufgetretenen Risse Bewertungsziffern für die Breite vergeben werden (Abbildung 146). Die Ergebnisse zeigen, wenn Risse innerhalb von 12 Monaten auftraten, dass diese überwiegend im Bereich zwischen „2“ und „3“ lagen, d.h. mit bloßem Auge gerade sichtbar bzw. deutlich sichtbar waren. Bei den Referenzen lag die Rissbreite erwartungsgemäß höher, da sie aufgrund von starkem Quellen und Schwinden eine stärkere Rissneigung zeigten. Auch die unbeschichtete Variante mit 15\% WPG zeigte nach 9 Monaten, als sie wie oben gezeigt, stark abgetrocknet war, Risse die deutlich sichtbar (4) waren. Die komplementäre Variante mit Beschichtung schnitt dadurch vergleichsweise besser ab, da die Rissbreite hier maximal mit einer „3“ bewertet wurde. Die konstant niedrigste Rissbreite, mit gerade sichtbaren Rissen (2), wies die beschichtete Variante mit einem WPG von 18\% auf, welche bereits die geringste Anzahl an Rissen und die im Durchschnitt niedrigste Wasseraufnahme und Dickenquellung in diesem Versuch, wie auch in der Schnellbewitterung, zeigte. In dieser Variante wirkte offenbar der positive Einfluss des WPG und einer Beschichtung zusammen und verdeutlicht so die Effizienz einer Kombination aus Modifizierung und nachträglicher Beschichtung. Hinsichtlich der Methode der Auswertung ist bei allen Freilandprüfungen aufgefallen, dass die Höhe der Messwerte zu Wasseraufnahme und Dickenquellung weniger als die Bewertung der Rissmenge 
und Rissbreite von der Dauer der Bewitterung abhingen als von der Jahreszeit und den herrschenden klimatischen Verhältnissen.

\subsubsection{LAB-Auswertung}

Die Bewitterung von unbeschichteten Holzwerkstoffen führt in der Regel schnell zu einer deutlichen Farbveränderung infolge von UV-Abbau, Auswaschung von Abbauprodukten und Kolonisation durch Schimmelpilze, was allgemein als Vergrauung durch den Betrachter wahrgenommen wird (Mai 2009). Nachfolgend dargestellt sind die Veränderungen der Helligkeit (delta $\mathrm{L}, \Delta \mathrm{L}$ ), der Buntheit (Chroma, delta $\mathrm{C}, \Delta \mathrm{C}$ ) und der Gesamtheit aller Werte (delta $\mathrm{E}, \Delta \mathrm{E}$ ) bezogen auf den Ausgangszustand für einen Bewitterungszeitraum von 12 Monaten. Die Beschränkung auf einen festen Zeitraum (12 Monate) ermöglichte bei der Auswertung die vergleichende Betrachtung vieler Serien mit unterschiedlichen WPGs und Pressdrücken. Hinsichtlich der zeitlichen Entwicklung von Farbveränderungen zeigte Mai (2009) in ihrer Dissertationsschrift zu modifiziertem Sperrholz, dass die größten Veränderungen hinsichtlich Helligkeit und Farbe bei unbeschichteten Proben innerhalb von drei bis sechs Monaten eintraten und sich dann deutlich verlangsamt weiterentwickelten.

Tabelle 18: Helligkeit (L) und Farbwerte (a und b) von unbeschichtetem Buchen-LVL mit $2 \mathrm{~N} / \mathrm{mm}^{2}$ Pressdruck zu Beginn und nach 12 Monaten Bewitterung.

\begin{tabular}{|c|c|c|c|c|c|c|c|c|}
\hline Harztyp & $\begin{array}{c}\text { Druck, spez. } \\
{\left[\mathrm{N} / \mathrm{mm}^{2}\right]}\end{array}$ & WPG & LO & L 12 & a 0 & a 12 & b 0 & b 12 \\
\hline$R$ & 2 & $0 \%$ & 60.5 & 45.4 & 10.3 & -0.5 & 31.9 & 13.4 \\
\hline \multirow[t]{3}{*}{ A } & 2 & $15 \%$ & 54.9 & 50.5 & 9.3 & 1.8 & 29.2 & 16.9 \\
\hline & 2 & $30 \%$ & 52.9 & 35.0 & 8.6 & 3.0 & 26.3 & 17.9 \\
\hline & 2 & $60 \%$ & 33.1 & 36.0 & 16.4 & 8.6 & 37.6 & 26.6 \\
\hline \multirow[t]{2}{*}{ B } & 2 & $30 \%$ & 42.3 & 35.2 & 11.3 & 1.7 & 33.1 & 16.0 \\
\hline & 2 & $60 \%$ & 24.9 & 36.9 & 15.2 & 10.7 & 29.9 & 28.7 \\
\hline \multirow[t]{2}{*}{$C$} & 2 & $30 \%$ & 44.1 & 36.4 & 10.6 & 3.7 & 32.2 & 19.9 \\
\hline & 2 & $60 \%$ & 49.7 & 31.0 & 9.4 & 10.5 & 23.7 & 23.3 \\
\hline \multirow[t]{2}{*}{ D } & 2 & $30 \%$ & 41.8 & 34.6 & 13.8 & 5.8 & 40.6 & 20.4 \\
\hline & 2 & $48 \%$ & 58.7 & 30.6 & 10.6 & 7.7 & 28.2 & 20.0 \\
\hline \multirow[t]{2}{*}{$E$} & 2 & $30 \%$ & 44.6 & 32.9 & 12.4 & 3.3 & 33.1 & 17.2 \\
\hline & 2 & $60 \%$ & 40.0 & 33.5 & 15.1 & 7.6 & 39.5 & 22.4 \\
\hline \multirow[t]{3}{*}{$F$} & 2 & $15 \%$ & 55.2 & 35.8 & 11.8 & 1.0 & 31.2 & 14.8 \\
\hline & 2 & $30 \%$ & 51.6 & 35.7 & 12.1 & 5.2 & 30.8 & 20.5 \\
\hline & 2 & $60 \%$ & 41.0 & 36.3 & 10.7 & 3.9 & 24.7 & 17.6 \\
\hline \multirow[t]{3}{*}{ G } & 2 & $15 \%$ & 51.5 & 36.5 & 13.7 & 1.5 & 33.1 & 16.0 \\
\hline & 2 & $30 \%$ & 53.7 & 39.1 & 13.6 & 5.7 & 35.2 & 23.7 \\
\hline & 2 & $60 \%$ & 41.7 & 32.9 & 13.5 & 8.4 & 28.6 & 23.2 \\
\hline
\end{tabular}




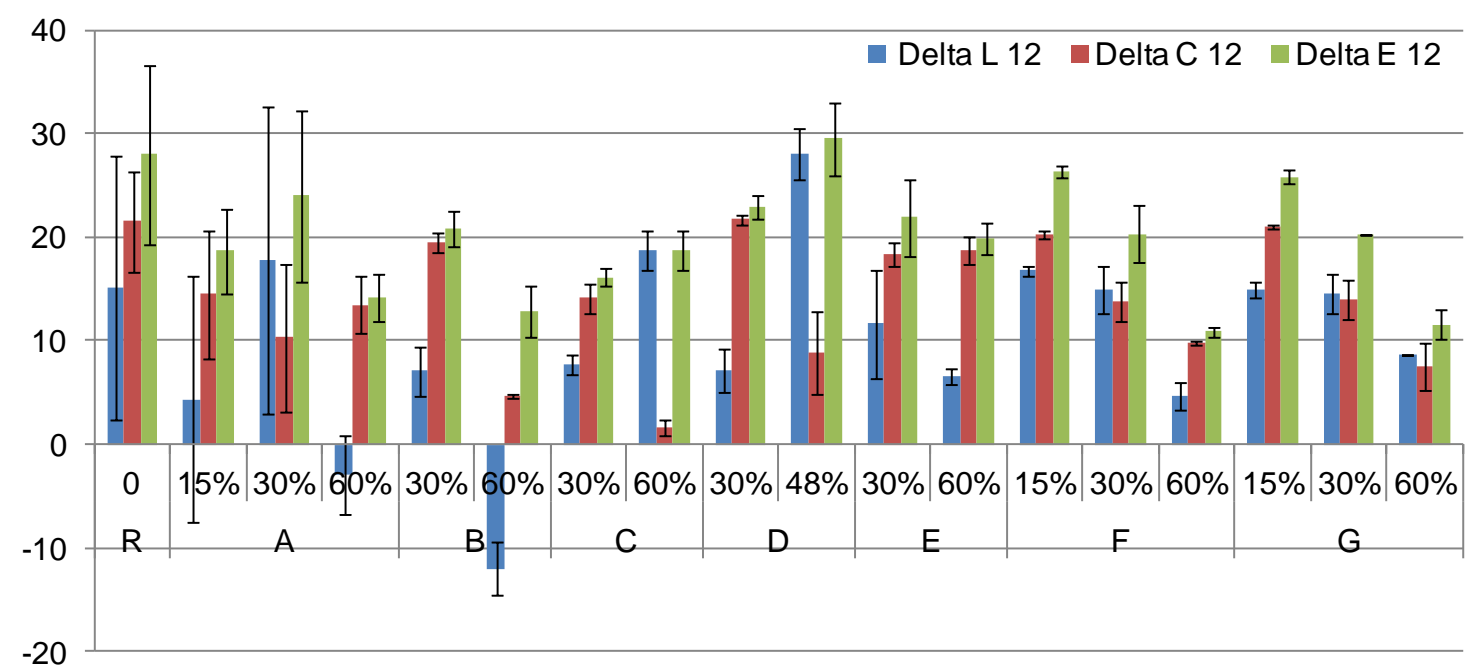

Abbildung 147: Farbwertveränderungen an unbeschichteten Buchen-LVL mit einem Pressdruck von $2 \mathrm{~N} / \mathrm{mm}^{2}$, abgebildet als delta L, delta $\mathrm{C}$ und delta $\mathrm{E}$ nach 12 Monaten Bewitterung auf dem Testfeld.

Wie Abbildung 147 zeigt, waren bei allen untersuchten Serien deutliche Farbveränderungen messbar. Für die meisten Serien äußerte sich dies durch einen positives delta L nach 12 Monaten, was nach Formel 24 gleichsteht mit einer Abnahme der Helligkeit. Die angesprochenen Ausnahmen beziehen sich hier auf Serien mit einem WPG von 60\% (Harztypen A und B). Initial waren deren Prüfkörper infolge des hohen WPGs und der typischen dunkelrotbraunen Eigenfarbe des ausgehärteten PFs extrem dunkel (Abbildung 149 und Abbildungen im Anhang). Infolge von UV-Abbau und Auswaschung von Lignin und PF kam es bei diesen Prüfköpern zu einer relativen Aufhellung. Die absolute Helligkeit dieser Serien unterschied sich nach 12 Monaten jedoch nicht mehr von den Serien mit einem WPG von 30\%, d.h., dass sich die Serien unterschiedlicher WPGs einander angeglichen hatten (Tabelle 18). Bei Betrachtung der Farbwerte a (Rot/Grün) und b (Blau/Gelb) fällt auf, dass infolge der Bewitterung beide Werte in Richtung „null“ verschoben und damit die Farbigkeit in Richtung Unbunt abnahm. 


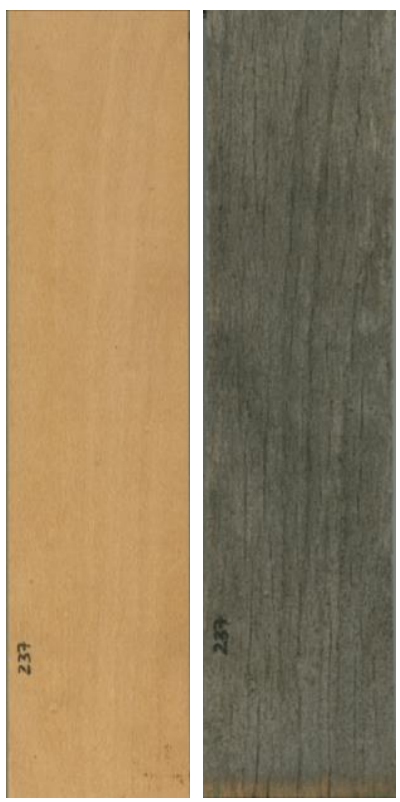

Abbildung 148: Unbehandeltes Buchen-LVL mit $2 \mathrm{~N} / \mathrm{mm}^{2}$ Pressdruck, vor (links) und nach einer 12monatigen Bewitterung (rechts).

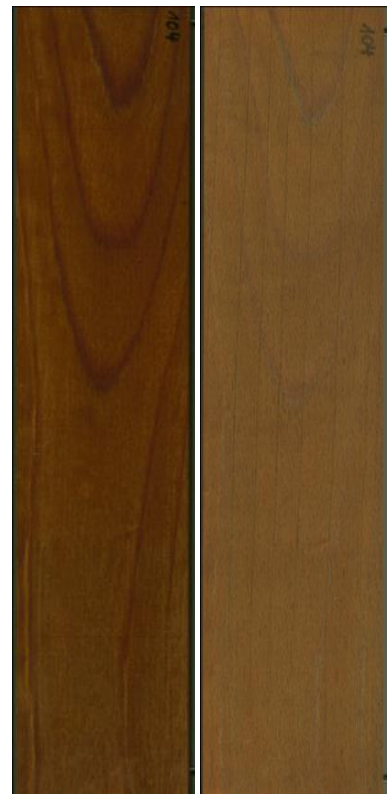

Abbildung 149: Buchen-LVL mit $2 \mathrm{~N} / \mathrm{mm}^{2}$ Pressdruck, modifiziert mit dem Harztyp B bei einem WPG von $60 \%$, vor (links) und nach einer 12-monatigen Bewitterung (rechts).

Delta C zeigt die Höhe der relativen Änderung, welche bei 30\% WPG in einigen Fällen- jedoch nicht in allen Fällen - niedriger ausfiel, als bei den Referenzen. Bei einem WPG von $60 \%$ und $48 \%$ war das delta C immer kleiner als bei den Referenzen, somit fanden weniger Änderungen in der Farbigkeit statt. Der Parameter delta E, welcher sich aus den Änderungen der Farbigkeit und Helligkeit zusammensetzt, ist daher bei einigen Serien, wie z.B. D-48\%, ähnlich hoch wie bei den Referenzen, da die Helligkeit stark abgenommen hatte, während die Farbigkeit gleichblieb. Wie Abbildung 150 vermuten lässt, finden sich hohe Werte für delta $\mathrm{C}$ bei Probeköpern, die auch subjektiv von einem Betrachter als stark in ihrem Erscheinungsbild verändert wahrgenommen werden. 


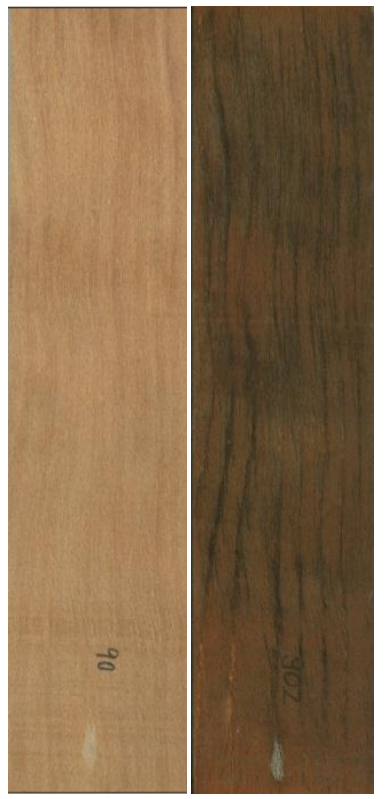

Abbildung 150: Buchen-LVL mit $2 \mathrm{~N} / \mathrm{mm}^{2}$ Pressdruck, modifiziert mit dem Harztyp D bei einem WPG von $48 \%$, vor (links) und nach einer 12-monatigen Bewitterung (rechts).

Neben den Veränderungen der Farb- und Helligkeitswerte der Probe in Abbildung 150 fällt zudem eine Strukturierung auf, welche auf eine Uneinheitlichkeit der Veränderungen schließen lässt. Diese „neue“ Inhomogenität in der Erscheinung geht bei einer Berechnung, die nur auf den Mittelwerten von $\mathrm{L}$, a und b aller aufgenommenen Pixel eines Probenscans basiert, verloren. Jedoch besteht auch die Möglichkeit die Variation aller Messwerte über die Gesamtfläche der Probe abzubilden und deren Veränderung zu vergleichen, indem man die Standardabweichung betrachtet.

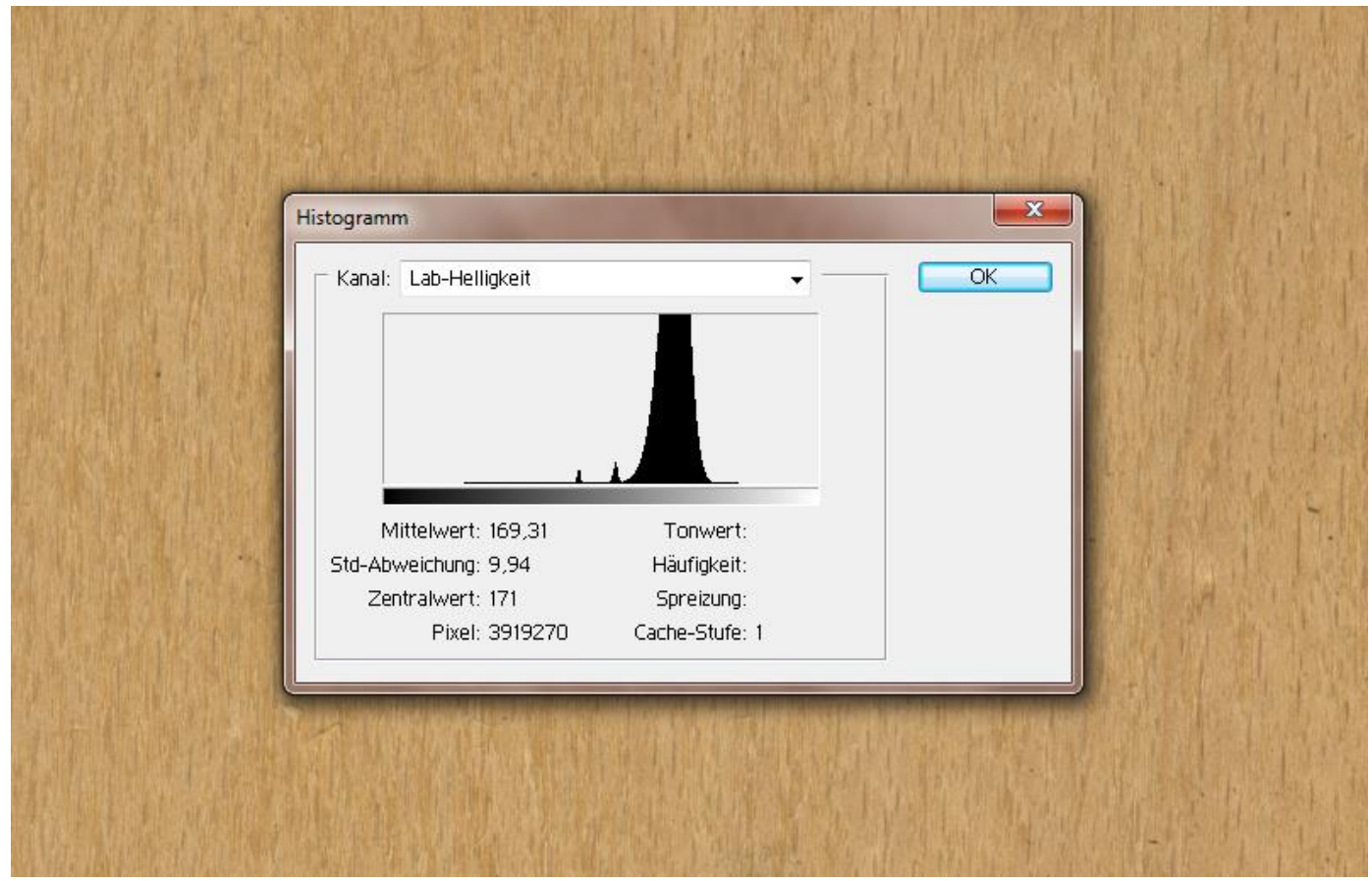

Abbildung 151: Verteilung der Helligkeit über die gesamte Prüfkörperoberfläche bei unbehandeltem Buchen-LVL (Referenz) vor der Bewitterung, gemessen mit Adobe Photoshop im Lab-Modus mit Wertebereich 0-255 vor der rechnerischen Korrektur. 


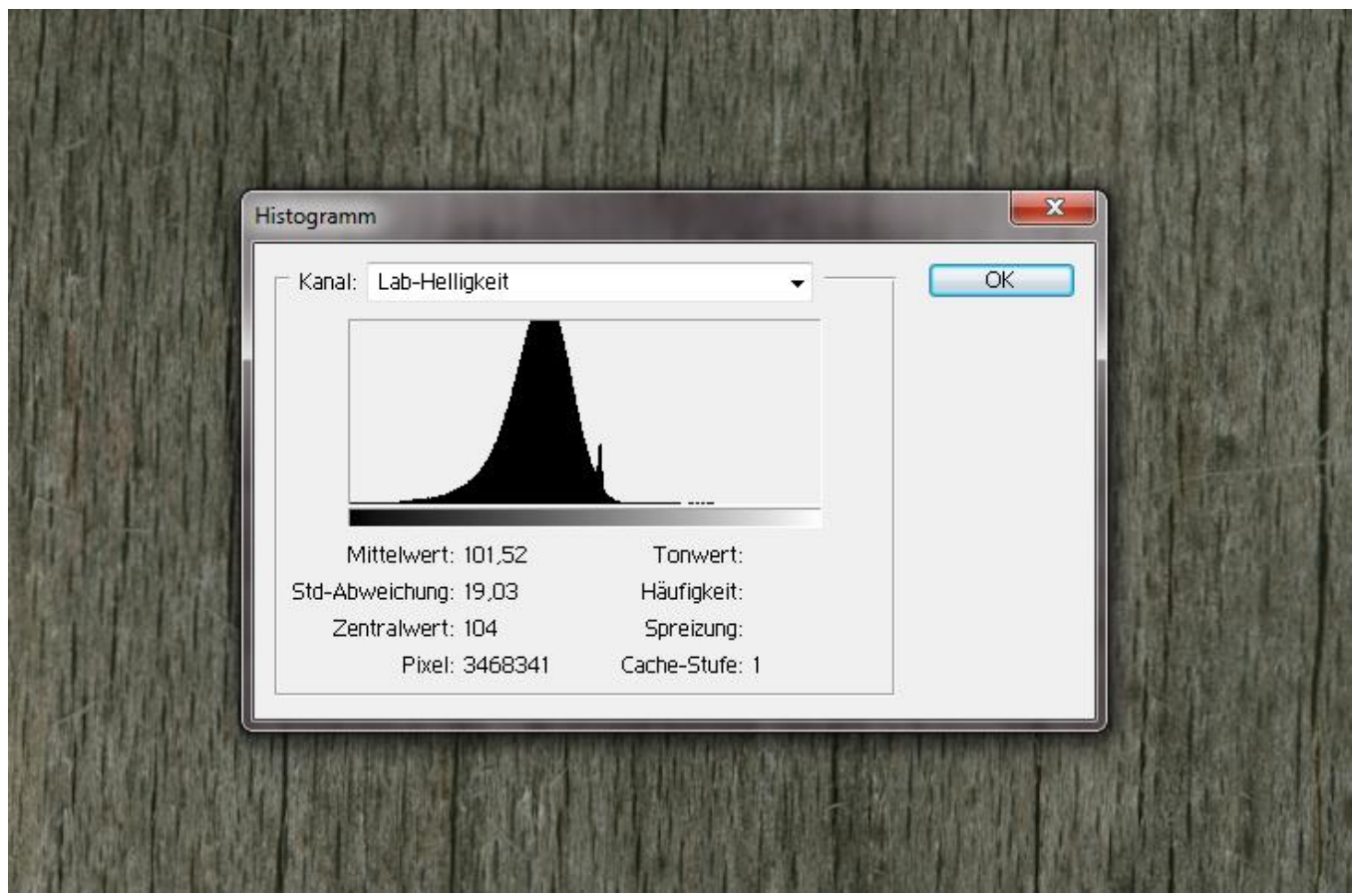

Abbildung 152: Häufigkeitsverteilung der Helligkeit über die gesamte Prüfkörperoberfläche bei unbehandeltem Buchen-LVL (Referenz) nach 12-monatiger Bewitterung, gemessen mit Adobe Photoshop im Lab-Modus mit Wertebereich 0-255 vor der rechnerischen Korrektur.

Die Abbildung 151 und Abbildung 152 verdeutlichen den Einfluss der Bewitterung auf die Häufigkeitsverteilung der Helligkeitswerte auf der gesamten Prüfkörperoberfläche.

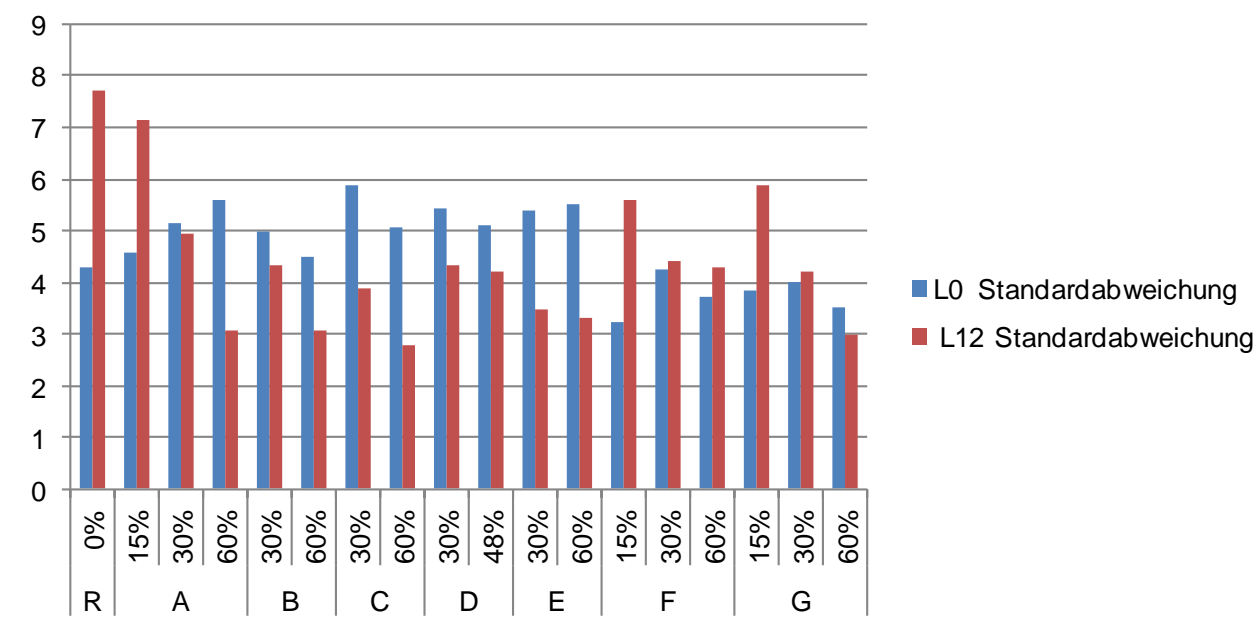

Abbildung 153: Standardabweichungen der Helligkeit L zu Beginn und nach 12-monatiger Bewitterung von Buchen-LVL mit $2 \mathrm{~N} / \mathrm{mm}^{2}$ Pressdruck. 


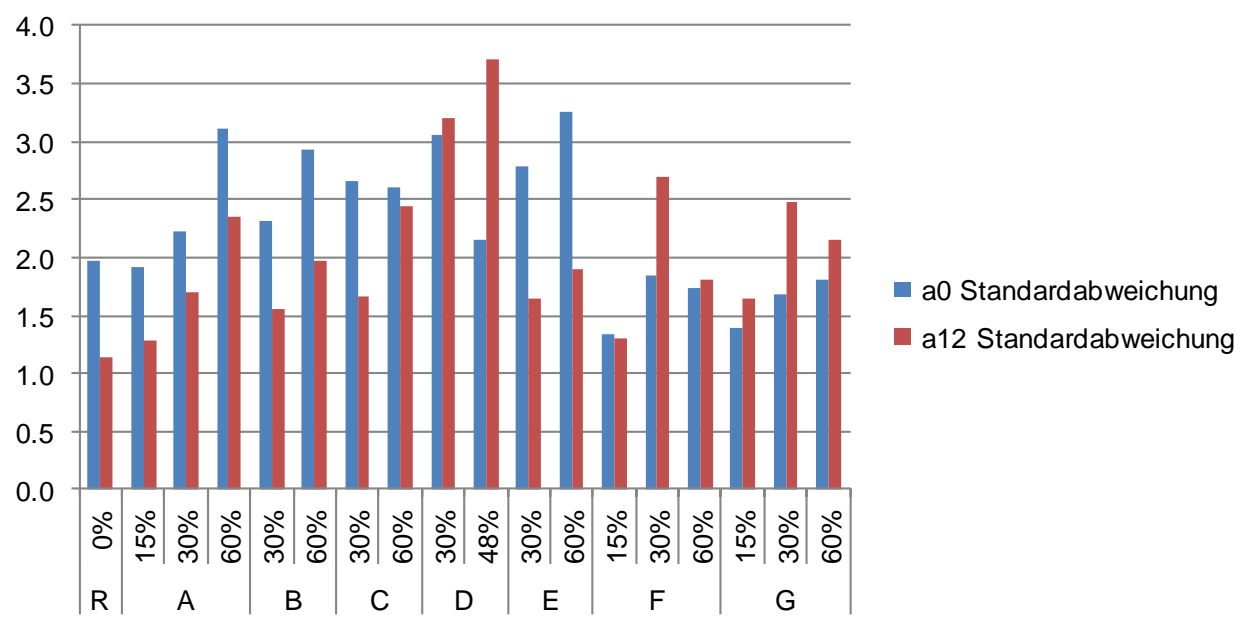

Abbildung 154: Standardabweichungen des Farbwerts a (Grün/Rot) zu Beginn und nach 12-monatiger Bewitterung von Buchen-LVL mit 2 N/mm² Pressdruck.

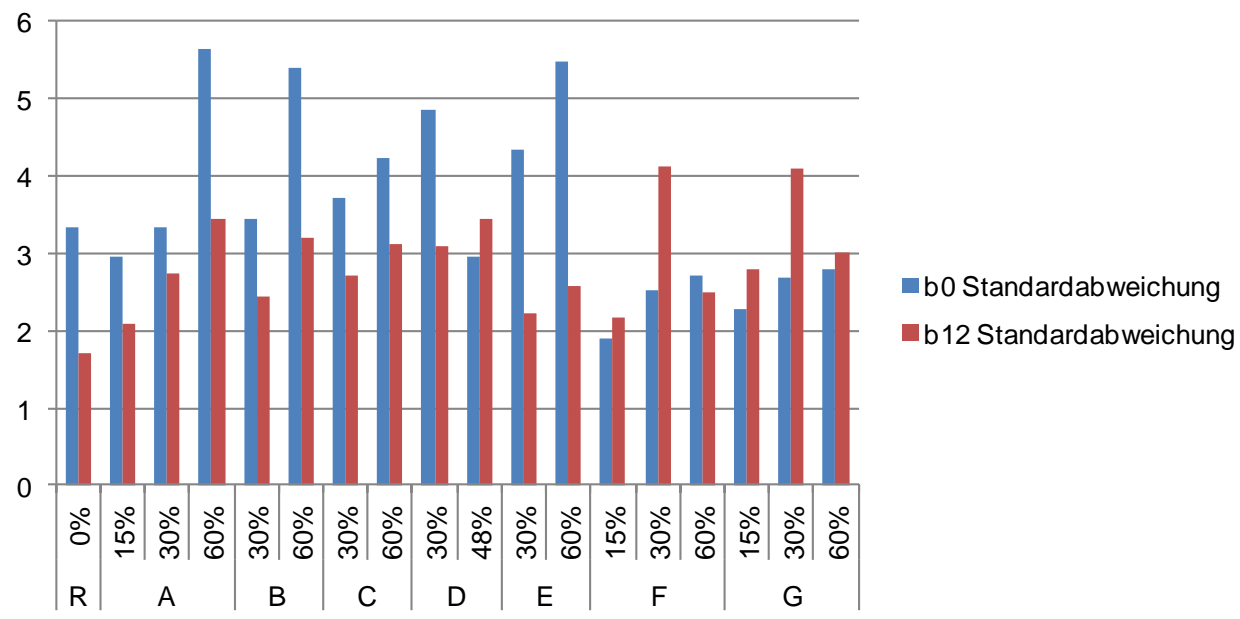

Abbildung 155: Standardabweichungen des Farbwerts b (Blau/Gelb) zu Beginn und nach 12-monatiger Bewitterung von Buchen-LVL mit $2 \mathrm{~N} / \mathrm{mm}^{2}$ Pressdruck.

Wie auch die Abbildung 153 zeigt, nahm die Streuung der Helligkeitswerte von Buchen-LVL, welches einen WPG von 15\% aufwies oder unbehandelt war, deutlich zu. Dies kann einerseits auf die Auswaschung von Lignin und Phenol, verbunden mit dem Freilegen weiß, bzw. hell, reflektierender Zellulose und andererseits auf die Besiedlung schwarzer Schimmelpilze sowie dunkel erscheinender Risse zurückgeführt werden (siehe auch Abbildung 152). Hinsichtlich der Standardabweichung des Farbwertes a (Grün/Rot) trat in den meisten Fällen keine Zunahme auf, jedoch bilden die Serien des Harztyps F und G bei einem WPG von 30\%, insbesondere die oben diskutierte Serie mit dem Harztyp D bei einem WPG von 48\%, Ausnahmen. Deren Standardabweichung, d.h. die Streuung der Messwerte für a hatte infolge der Bewitterung zugenommen. Die Streuung der Messwerte auf der Blau-Gelb-Achse (b) war ebenso bei den Harztypen F und G bei 30\% WPG erhöht, bei den übrigen Serien jedoch deutlich reduziert. Am Beispiel der Serie B-30\% bei $2 \mathrm{~N} / \mathrm{mm}^{2}$, mit einer geringen Standardabweichung für $\mathrm{a}$ und $\mathrm{b}$, sowie einem durchschnittlich hohem delta $\mathrm{E}$, wird deutlich, dass eine Veränderung 
homogen erfolgen kann. Bei subjektiver Betrachtung kann das Erscheinungsbild dann harmonischer erscheinen (Abbildung 156).

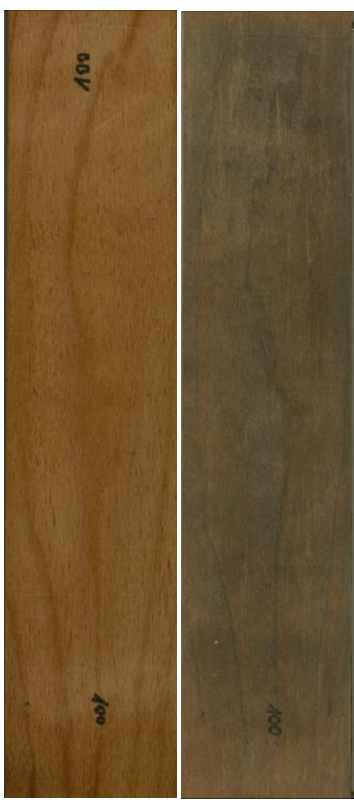

Abbildung 156: Buchen-LVL mit $2 \mathrm{~N} / \mathrm{mm}^{2}$ Pressdruck, modifiziert mit dem Harztyp B bei einem WPG von $30 \%$, vor (links) und nach einer 12-monatigen Bewitterung (rechts).

Tabelle 19: Helligkeit (L) und Farbwerte (a und b) von unbeschichtetem Buchen-LVL mit $6 \mathrm{~N} / \mathrm{mm}^{2}$ Pressdruck zu Beginn und nach 12 Monaten Bewitterung.

$\begin{array}{ccccccccc}\text { Harztyp } & \begin{array}{c}\text { Druck, spez. } \\ \text { [N/mm } \mathbf{2}]\end{array} & \text { WPG } & \text { L0 } & \text { L 12 } & \text { a 0 } & \text { a 12 } & \text { b 0 } & \text { b 12 } \\ \text { R } & 6 & 0 \% & 59.0 & 43.6 & 10.5 & -0.1 & 32.6 & 14.1 \\ \text { B } & 6 & 30 \% & 39.5 & 34.2 & 13.6 & 2.6 & 36.3 & 17.4 \\ & 6 & 60 \% & 17.4 & 31.6 & 12.7 & 8.9 & 21.0 & 21.9 \\ \text { A } & 6 & 15 \% & 51.5 & 38.2 & 10.9 & 0.7 & 30.1 & 14.6 \\ & 6 & 30 \% & 33.8 & 35.7 & 11.7 & 4.5 & 32.0 & 24.5 \\ & 6 & 60 \% & 31.2 & 34.0 & 14.0 & 7.9 & 26.3 & 22.5 \\ \text { C } & 6 & 30 \% & 35.2 & 38.5 & 16.1 & 4.2 & 34.1 & 21.7 \\ & 6 & 60 \% & 51.9 & 33.9 & 10.8 & 8.5 & 23.1 & 22.4 \\ \text { D } & 6 & 30 \% & 44.0 & 34.6 & 12.4 & 4.4 & 30.3 & 18.2 \\ & 6 & 48 \% & 15.6 & 33.8 & 12.3 & 7.3 & 18.6 & 20.4 \\ \text { E } & 6 & 30 \% & 35.7 & 34.5 & 14.7 & 5.6 & 34.7 & 20.9 \\ & 6 & 60 \% & 26.5 & 33.1 & 14.2 & 6.8 & 32.1 & 20.4 \\ \text { F } & 6 & 15 \% & 53.8 & 35.8 & 13.3 & 1.7 & 32.1 & 16.0 \\ & 6 & 30 \% & 47.2 & 40.6 & 15.7 & 9.4 & 35.9 & 28.3 \\ & 6 & 60 \% & 37.4 & 35.4 & 11.3 & 5.8 & 22.5 & 19.6 \\ \text { G } & 6 & 15 \% & 53.1 & 36.8 & 13.5 & 1.2 & 34.0 & 15.3 \\ & 6 & 30 \% & 45.6 & 40.1 & 16.9 & 8.3 & 34.6 & 27.6 \\ & 6 & 60 \% & 40.9 & 36.2 & 14.0 & 7.7 & 27.3 & 22.9\end{array}$




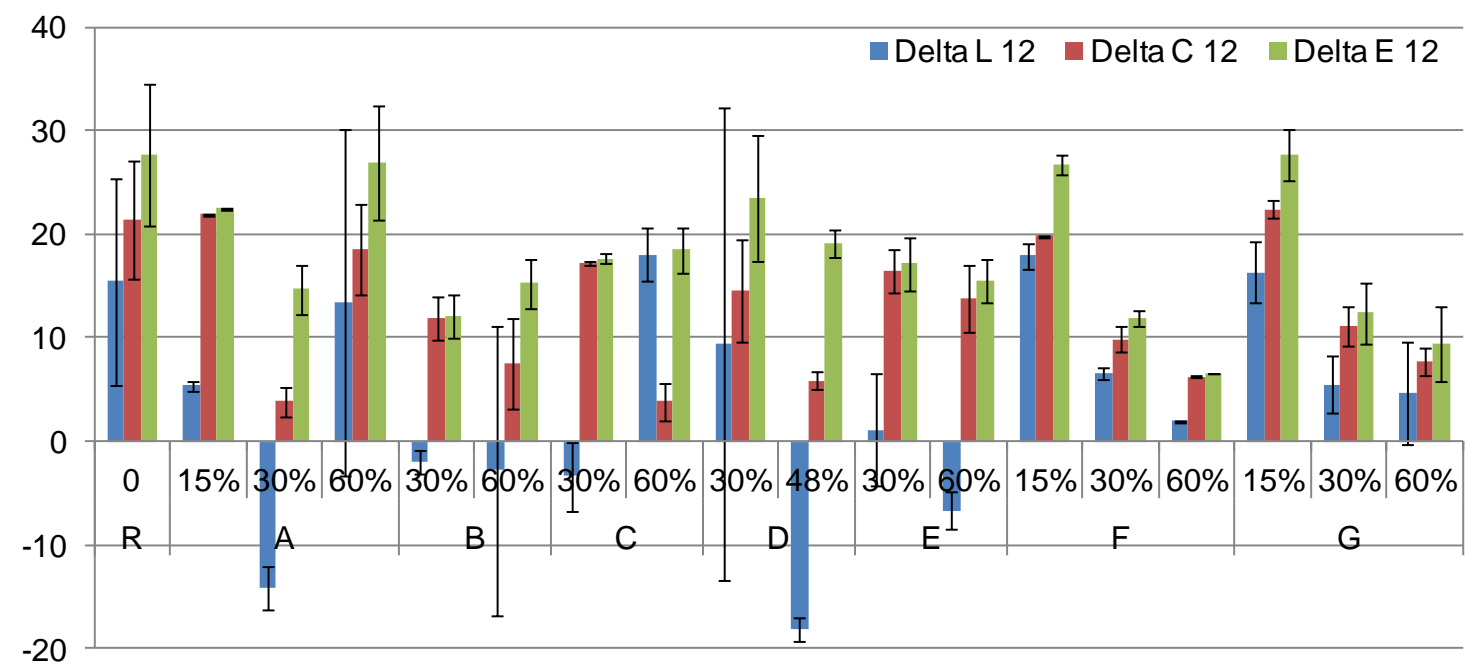

Abbildung 157: Farbwertveränderungen an unbeschichtetem Buchen-LVL mit einem Pressdruck von $6 \mathrm{~N} / \mathrm{mm}^{2}$, abgebildet als delta L, delta C und delta E nach 12 Monaten Bewitterung auf dem Testfeld.

Wie aus Tabelle 19 hervorgeht, waren die Proben aus Platten mit einem Pressdruck von $6 \mathrm{~N} / \mathrm{mm}^{2}$ insgesamt dunkler (niedrigere L-Werte) vor der Bewitterung, als die Proben von Platten mit einem Pressdruck von $2 \mathrm{~N} / \mathrm{mm}^{2}$. Die Verdichtung hatte hier demnach einen Einfluss auf das Erscheinungsbild. Durch die Bewitterung erfuhren hier auch Proben (Harze A, B, C) mit einem WPG von 30\% eine Aufhellung (negatives delta L). Eine Aufhellung beim WPG von 60\% bzw. 48\% fand nur in drei von sieben Fällen statt - eine Verringerung der Helligkeit überwog. Hinsichtlich der Veränderung der Farbigkeit (delta C) zeigten sich, mit Ausnahmen beim Harztyp A, geringere Werte bei höheren WPGs und damit der gleiche Zusammenhang wie beim niedrigeren Pressdruck $\left(2 \mathrm{~N} / \mathrm{mm}^{2}\right)$. Die Variation der Ergebnisse zwischen allen Harztypen war hoch, jedoch ist auch ein unvermeidlich niedriger Stichprobenumfang von nur zwei Prüfköpern pro Serie an der Ergebnislage beteiligt. Ein deutlicher Zusammenhang eines zunehmenden WPGs mit abnehmenden delta L, delta $\mathrm{C}$ und delta $\mathrm{E}$ lässt sich nur bei den Harztypen F und G erkennen und zwar bei beiden Pressdrücken. Im Unterschied zu den übrigen Harztypen wurden sie zu einem späteren Zeitpunkt der Forschungsarbeit hergestellt und, auch wenn ebenso nur zwei Prüfköper pro Serie in der Bewitterung eingesetzt werden konnten, stammten die modifizierten Furniere aus einem Prozess mit deutlich höherem Umfang an Furnieren. Die Trocknung erfolgte dementsprechend nicht in einem Trockenschrank, sondern unter noch besser steuerbaren Bedingungen in einem Versuchstrockner mit größerem Fassungsvermögen. Ein Einfluss der Skalierung auf die Homogenität des Prozesses und der Eigenschaften der Furniere und des LVL lässt sich somit vermuten, jedoch nicht eindeutig beweisen. 


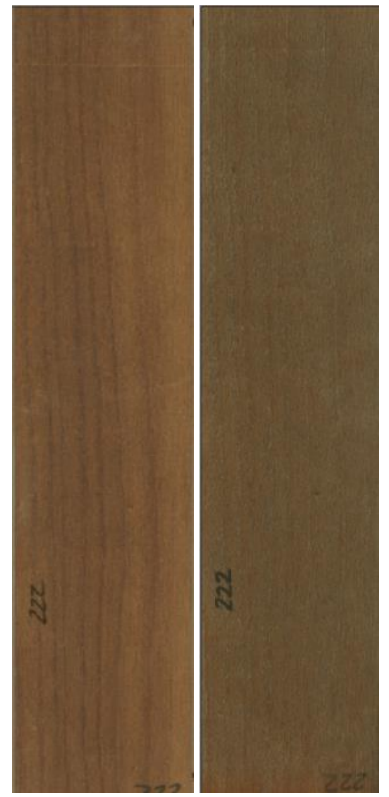

Abbildung 158: Buchen-LVL mit $6 \mathrm{~N} / \mathrm{mm}^{2}$ Pressdruck, modifiziert mit dem Harztyp F bei einem WPG von $60 \%$, vor (links) und nach einer 12-monatigen Bewitterung (rechts).

\subsection{Zusammenfassung}

Die Modifizierung von Buchenschälfurnieren unterschiedlicher Dicke konnte erfolgreich im Vakuum-Verfahren durchgeführt werden. Es gelang dabei durch Auswahl geeigneter niedermolekularer PF-Harze sowie Anpassung der Lösungskonzentration, in Abhängigkeit von der Darrdichte und der Feuchte der Furniere, definiert unterschiedliche WPGs zu erzielen. Durch eine die Aushärtung vermeidende Trocknung und Verlagerung der Aushärtung in die Heißpresse, ließen sich LVL mit unterschiedlichen Dichten bzw. Verdichtungen herstellen. Aufgrund der geringen Vorkondensation der Imprägnierharze musste die Presszeit bei gleicher Temperatur angehoben werden, um eine vollständige Kondensation zu erzielen, was durch die Messung der Formaldehydabgabe validiert wurde.

Dessen mechanische Eigenschaften waren, mit Ausnahme der Bruchschlagarbeit, weitgehend erhalten geblieben. Infolge der Verdichtung konnten hohe Biegefestigkeiten sowie ein höheres Elastizitätsmodul ermittelt werden. Infolge der Modifizierung nahmen die Proben einerseits weniger Wasser auf, was zum Teil auf den WPG und die Verdichtung zurückzuführen war. Wie bereits an Vollholzproben bewiesen, konnte zudem in die Zellwand eingedrungenes und dort fixiertes Phenol-Formaldehyd die Quellung signifikant reduzieren. Dies bewiesen neben einer Langzeitwasserlagerung von Prüfkörpern für den Pilzversuch auch die Verklebungskontrollen in einer vierstündigen Kochprüfung. Über alle Serien hinweg zeigte sich, wie Buchen-LVL mit

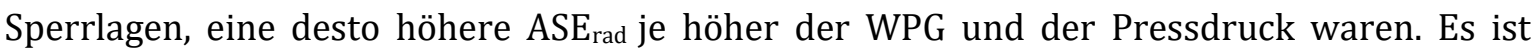
bemerkenswert, dass bei einem WPG von nur 15\%, je nach Harztyp und Pressdruck, eine Verminderung der Dickenquellung in Höhe von $47 \%$ bis $62 \%$ möglich war.

Hinsichtlich der biologischen Dauerhaftigkeit, konnte gezeigt werden, dass die Zellwandeindringung bzw. die Quellvergütung gute Indizien liefern für eine Resistenz gegenüber Basidiomyceten, in diesem Fall Trametes versicolor. In Abhängigkeit des WPG 
konnte, unabhängig von der Verdichtung oder dem Harztyp, eine sehr hohe biologische Dauerhaftigkeit nachgewiesen werden. Im Vergleich zum Vakuumverfahren wies dabei das Tauchverfahren zwar ebenfalls eine Reduzierung des Abbaus auf, jedoch mit einer größeren Streuung der Werte.

In der Freilandbewitterung sowie im Labor konnte die erhöhte Dimensionsstabilität des modifizierten unbeschichteten LVLs über einen Zeitraum von drei Jahren bewiesen werden. Nach einer Phase der Auffeuchtung innerhalb des ersten Jahres blieben die Messwerte stabil auf einem Niveau oder leicht steigend. Im Gegensatz $\mathrm{zu}$ den Referenzen waren die jahreszeitlichen Schwankungen deutlich abgemindert. Diese Verringerung der damit verbundenen Quell- und Schwindbewegungen des Holzes kann als Vorteil bei der Verwendung von Beschichtungen gesehen werden. Auffällig war jedoch eine nur gering verbesserte Rissneigung der modifizierten Prüfkörper, wobei die Bewertung nach den strengen Kriterien einer Beurteilung von Schäden in Beschichtungen durchgeführt wurde.

Da wesentliche Verbesserungen des LVLs hinsichtlich Dimensionsstabilität und biologischer Dauerhaftigkeit bei bereits niedrigen WPGs erzielt wurden, bzw. auch Verschlechterungen wie die Abnahme der Bruchschlagarbeit geringer ausfielen, wurde die Kombination einer Modifizierung mit geringem WPG und einer Beschichtung als vielversprechend erachtet. Die Untersuchungsergebnisse nach einem Jahr Freilandbewitterung zeigten erwartungsgemäß, dass beschichtete Proben mit niedrigeren WPGs gleiche oder bessere Ergebnisse erzielten als unbeschichtete Proben mit höheren WPGs.

PF-modifiziertes LVL im Erdkontakt wies nach drei Jahren keine Ausfälle auf. Nur beim niedrigsten WPG wurden leichte Veränderungen festgestellt, während alle unbehandelten Referenzen so stark abgebaut waren, dass sie in der Erd-Luft-Zone durchbrachen.

Somit lässt sich feststellen, dass es, aus dickeren Buchenfurnieren als es bisher üblich war, durch die Modifizierung im Vakuumverfahren und Auswahl geeigneter Harze möglich ist, dauerhaftes und dimensionsstabiles LVL mit hohen Festigkeiten für die Außenanwendung herzustellen. Dabei ist infolge der erläuterten Zusammenhänge der Prozess entsprechend der Anforderungen an das spätere Produkt anpassbar und damit deutlich effizienter gestaltbar als zuvor. 


\section{Diskussion}

Die Untersuchungen an Vollholz ermöglichten einen Rückschluss auf die Zellwandeindringung von PF in Abhängigkeit des Prozesses und gewählten Harzes. Die Ergebnisse der ASE-Prüfung und die Werte für das Bulking ließen in den ersten Versuchen an Buchenvollholz mit den Harztypen A, B, und C einen Einfluss der Molekülgröße und der Polydispersität vermuten. Bei einem WPG von $15 \%$ zeigte der Harztyp B ein etwas geringeres Bulking als der Harztyp C, wobei B eine etwas höhere Molmasse und eine größere Polydispersität aufwies als C. Der Einfluss des WPG war bei diesen immer noch sehr ähnlichen Harztypen jedoch dominant. Um den Einfluss der Molekülgröße besser zu erfassen, war es somit nötig, Musterharze mit deutlicher verschiedenen Molekülgrößen einzusetzen und eine ASE-Prüfung durchzuführen. Dabei zeigte sich, dass bei zahlenmäßig mittleren Molmassen unter $421 \mathrm{~g} / \mathrm{mol}$ ein identisches Ergebnis vorlag. Bei größeren, zahlenmäßig mittleren Molmassen (655 g/mol und $854 \mathrm{~g} / \mathrm{mol}$ ), nahm die ASE sukzessive ab. Daraus lässt sich schließen, dass es sich bei einer Molmasse im Bereich von $400 \mathrm{~g} / \mathrm{mol}$ um einen kritischen Wert handelt. Das liegt daran, dass verbunden mit der Molmasse bestimmte Größen, resp. bestimmte Volumen und Durchmesser der Moleküle, einhergehen. Die Größe der Moleküle bestimmt, ob sie noch in der Lage sind, durch die Mikroporen der Zellwand in diese einzudringen. HILL (2006) gibt die maximale Größe der Mikroporen verholzter Zellwände im wassergequollenen Zustand mit 2-4 nm an. Ausgehend von dem in ChemSketch (ACD/ChemSketch V.14.01) berechneten hydrodynamischen Volumen von $290 \mathrm{~cm}^{3}$ bei einem Trimer mit einer Molmasse von $412 \mathrm{~g} / \mathrm{mol}$, ergibt sich nach Annahme eines Kugelvolumens ein maximaler Durchmesser des PF-Moleküls von ca. $1 \mathrm{~nm}$. Die dynamische Lichtstreuung ergab bei der Untersuchung des Harztyps A eine Molekülgrößenverteilung in einen Bereich von $1 \mathrm{~nm}$ bis $4 \mathrm{~nm}$, wobei $50 \%$ der Werte unter $2 \mathrm{~nm}$, resp. $3 \mathrm{~nm}$, lagen. Somit steht die Beobachtung, dass Bulking und ASE bis zu einer zahlenmäßig mittleren Molmasse von $440 \mathrm{~g} / \mathrm{mol}$ am höchsten waren, damit in Verbindung, dass der überwiegende Teil dieses Harzes in die Zellwand eindringen konnte, da dessen Moleküle die Mikroporen der Zellwand passieren konnten. Auch FuRUno, IMAMURA ET AL. (2004) konnten für zahlenmäßig mittlere Molmassen bis $480 \mathrm{~g} / \mathrm{mol}$ die Zellwandeindringung nachweisen, während sie feststellten, dass der bei $820 \mathrm{~g} / \mathrm{mol}$ überwiegende Teil des Harzes im Zelllumen verblieb. Die tatsächliche Ausdehnung eines Moleküls weicht jedoch von der Kugelform ab und es könnten somit Moleküle mit einer größeren Molmasse, mit einer stärker linearen, anstelle einer dreidimensionalen, Vernetzung, eine ebenso gleiche Porengröße passieren, wie kleinere, gleichmäßig dreidimensional vernetzte, Moleküle. Aus der Molmassenverteilung lässt sich des Weiteren ableiten, dass auch bei größeren zahlenmäßig mittleren Molmassen ein bestimmter Anteil an Molekülen klein genug ist, um in die Zellwand einzudringen und einer Verminderung der Wasseraufnahme und Quellung beizutragen.

Insbesondere die UMSP konnte den Nachweis der Zellwandeindringung erbringen und so eine positive Korrelation der Absorption mit dem Bulking der Zellwand erstellt werden. Dabei stehen die Ergebnisse im Einklang mit der Untersuchung von HUANG ET AL. (2014). Diese untersuchten die Zellwandeindringung von niedermolekularem basischem PF $\left(\mathrm{M}_{\mathrm{w}}=183-\right.$ $319 \mathrm{~g} / \mathrm{mol}$ ) an Frühholztracheiden der Spießtanne (Cunninghamia lanceolata). Bei einer Wellenlänge von $280 \mathrm{~nm}$ fanden sie in den Frühholztracheiden einen durchschnittlichen Anstieg der Absorption in der Zellwand um 49\% und in der Mittellamelle um 23\%. 
Im Gegensatz zu HUANG ET AL. (2014), welche keine Angaben zum WPG der Proben machten, wurden in dieser Arbeit unterschiedliche WPGs untersucht und während sie zwischen S2-Zellwand und Mittellamelle unterschieden, wurde hier nach den im Laubholz vorkommenden Gefäßtypen differenziert. Dennoch konnten beide Studien eine deutliche Zunahme der Absorption bei $280 \mathrm{~nm}$ bzw. $278 \mathrm{~nm}$ infolge der Imprägnierung mit niedermolekularem PF feststellen. Im Vergleich der Studien bezüglich der Höhe der Zunahme der Absorption fällt auf, dass bei der PF-modifizierten Buche durchschnittlich höhere Werte ermittelt wurden als bei der Spießtanne. Da die Dichte der Spießtanne von YANG ET AL. (2001) mit $273 \mathrm{~kg} / \mathrm{m}^{3}$ bis $310 \mathrm{~kg} / \mathrm{m}^{3}$ angegeben wird, muss der erzielte WPG bei Imprägnierung bis zur vollständigen Sättigung wesentlich höher gelegen haben als bei den eigenen Versuchen. Unter Zuhilfenahme der Formel 27 nach KoLLMAnN (1951) würden sich maximale Feuchten, resp. Lösungsaufnahmen, zwischen 328\% und 284\% ergeben. Unter Abzug der Holzfeuchte von $12 \%$ vor der Imprägnierung und Vernachlässigung der Quellung, wird davon ausgegangen, dass bei der Lösungskonzentration von 25\% ein WPG zwischen 79\% und 68\% erzielt werden konnte. Die Zunahme der Absorption im Verhältnis zum WPG viel bei HUANG ET AL. (2014) somit deutlich geringer aus als bei der Messung der PF-modifizierten Buchenholzproben dieser Arbeit. Unter Berücksichtigung der schwierigeren Differenzierung der Absorptionswerte auf Zellwandebene bei höheren WPGs als 15\%, beispielsweise 30\%, bei welchem nicht nur das Zelllumen, sondern auch Bereiche der Mittellamelle außerhalb des möglichen Messbereichs lagen, wird für zukünftige Untersuchungen der Zellwandeindringung von PhenolFormaldehyden empfohlen, niedrigere WPGs als 30\% einzusetzen. Ebenso sollte für weitergehende Untersuchungen der Einfluss der unterschiedlichen Verhältnisse von Guaiacylund Syringyllignin besser berücksichtigt werden. Nach TAKADA ET AL. (2016) und КосH \& GRÜNWALD (2004) sind die Absorptionskoeffizienten dieser beiden Lignintypen bei $280 \mathrm{~nm}$ sehr unterschiedlich, wobei Guaiacyllignin stärker absorbiert als Syringyllignin. Dies kann dazu führen, dass die UV-Absorption bestimmter Zelltypen überschätzt wird, z.B. in den Zellwänden von Gefäßen, oder unterschätzt wird, z.B. in den Zellwänden von Fasern. Nach TAKADA ET AL. (2016) wäre die Absorption für beide Lignintypen ähnlicher bei $240 \mathrm{~nm}$, jedoch liegt die Absorption von Phenolen, u.a. aufgrund einer bathochromen Verschiebung, bei $284 \mathrm{~nm}$. Hier wurde bei $278 \mathrm{~nm}$ gemessen, da hier bei der Buche für das Lignin insgesamt ein Absorptionsmaximum liegt (КосH \& GRÜNWALD 2004). In diesem Zusammenhang muss bei den behandelten Proben die starke Absorption der Mittellamelle im Verhältnis zur S2-Wand eines Gefäßes bzw. einer Faser, kritisch hinterfragt werden. Die höhere Absorption der Mittellamelle bei unbehandelten Proben erklärt sich durch die generell höhere Ligninkonzentration. HoN UND SHIRAISHI (2000) führen an, dass bei der Weißbirke (Betula papyrifera) je nach Zelltyp Ligninanteile zwischen $36 \%$ und $47 \%$ ermittelt wurden. Dabei verglichen sie auch die Ergebnisse der UV-Spektroskopie mit einer EDXA (Energiedispersive Röntgenspektroskopie, engl. energy dispersive X-ray analysis). Es wird daraus ersichtlich, dass die reine UVSpektroskopie für die Mittellamelle einen deutlich höheren Wert erbrachte als die EDXA. Ein Grund dafür war der höhere Anteil an Guaiacyllignin, welcher bei 80-100\% lag. Wenngleich die Arbeiten von KoCH \& GRÜNWALD (2004), KoCH \& KLEIST (2001) sowie KoCH, RICHTER ET AL. (2006) vermuten lassen, dass die Absorption nach dem Lambert Beer'schen Gesetz direkt proportional zu der Lignin- und Phenolkonzentration ist, so ist für eine exakte quantitative Auswertung der Absorptionen, die in dieser Arbeit gemessen wurden, eine genaue Kenntnis 
des Verhältnisses von Guaiacyl- zu Syringyllignin Voraussetzung. Im direkten Vergleich der Absorption zwischen den Behandlungen, insbesondere beim Vergleich verschiedener Harze und Molmassen, ist dieser Umstand jedoch nicht von Relevanz gewesen.

Eine bei gleichem WPG und Molmasse unterschiedliche Veränderung der Absorption bei den UMSP-Messungen könnte sich aus dem Lösungsalter, bzw. dem Alter des Harzes, d.h. dem Zeitraum zwischen Herstellung und Verwendung, sowie der Lagertemperatur, ergeben.

Die Untersuchung zum Alterungsverhalten von PF-Harzen in Verbindung mit deren Effizienz im Gebrauch als Modifizierungsmittel ergab eine Abnahme des Bulking und der ASE in Abhängig von der Dauer und der Temperatur der Lagerung einer 25\%igen Harzlösung. Die Wasserverdünnbarkeit, welche als bewährtes Mittel anerkannt ist, um die Verwendbarkeit von PF-Harzen zu klären, veränderte sich hingegen so wenig, dass keine erkennbare Dissipation oder kein Farbumschlag von Rot zu Weiß während der Versuchsdauer auftraten. Die Lagerung bzw. das Lösungsalter werden somit als wesentliches Kriterium für den Erfolg der Modifizierung von Holz mit niedermolekularem PF angesehen. Vereinfacht ausgedrückt bedeutet dies, dass ein Harz, dessen Transport und Lagerung nicht nachvollziehbar sind, zu einem ungewissen Ergebnis führt. Die kontrollierte Lagerung eines Harzes, bei z.B. einer Temperatur von $7^{\circ} \mathrm{C}$, ermöglicht eine Abschätzbarkeit des Modifizierungserfolges. Diese Erkenntnisse beruhen jedoch auf Laborversuchen. Im industriellen Maßstab ergeben sich daraus weitere Fragestellungen. Bei industriellen Prozessen werden verbrauchte Volumen mit frischer Lösung aufgefüllt, der Anteil alter Lösung nimmt somit mit jedem Zyklus ab. Es stellt sich somit die Frage, ob durch ausreichenden Verbrauch und entsprechende Auffrischung eine Kühlung der Lösung vermieden werden kann. Im Falle einer Kühlung stellt sich die Frage, ob die damit verbundene höhere Viskosität und geringere Molekularbewegung einen Einfluss auf die Zellwandeindringung haben.

In der industriellen Anwendung kann neben dem Alterungsverhalten eines Harzes auch die Verfügbarkeit von Harzen eine Rolle spielen. Somit war es sinnvoll für die Entwicklung eines dauerhaften LVL auf Basis PF-modifizierter Furniere, die Anzahl der verwendeten Harze nicht zu reduzieren, sondern unter den typischen Umgebungsbedingungen der LVL-Herstellung im Technikumsmaßstab einzusetzen. Da Furniere bei der LVL-Herstellung nach dem Schälen und vor dem Verpressen mit Klebstoff getrocknet werden, wurden getrocknete Furniere als Ausgangsmaterial für die Untersuchungen gewählt. Da die Holzfeuchte einen Einfluss auf die maximale Lösungsaufnahme hat und die Holzfeuchte von Furnieren entsprechend des Klimas, in dem sie gelagert und transportiert werden, unterschiedlich sein kann, war es nötig die Lösungsaufnahmen in einem Vakuum-Imprägnierprozess bei unterschiedlichen Holzfeuchten der Furniere zu bestimmen. Das anhand der gewonnenen Daten erstellte Regressionsmodell ermöglicht nun die Vorhersage der Lösungsaufnahmen und somit des erzielbaren WPG bei bekannter Konzentration und mithin ressourcenschonendem Materialeinsatz. Im Vergleich des auf empirisch ermittelten Werten basierenden Modells zu dem von KollmanN (1951) publizierten Modell zur maximalen Holzfeuchte, stellt sich eine gute Übereinstimmung heraus. Die Anwendung einer Tauchimprägnierung in Verbindung mit einer Reifelagerung, welche die Diffusion in weiter von der Oberfläche entfernte Zellschichten ermöglichen sollte, wurde in geringem Umfang untersucht und ließ auf eine unzureichende Aufnahme der Harzlösung 
schließen. Die Techniken des Tauchens, Streichens oder Walzens sind erfahrungsgemäß für dünnere Furniere geeignet, jedoch wurde für die angestrebte Verwendung von 2,13 mm und 3,7 mm dicken Furnieren die Vakuum-Druck-Imprägnierung als sichereres Verfahren herangezogen um vergleichbare Ergebnisse zu erzielen, wie es sich auch bei den Arbeiten zur Modifikation von Furnieren von DIESTE ET AL. (2008) und MAI (2009) bewährt hat.

Die Untersuchungen an LVL zeigten deutlich den Einfluss des Herstellungsprozesses dieser Holzwerkstoffe auf deren Eigenschaften und damit auf den Erfolg der Modifizierung.

Im Vergleich unterschiedlich langer Presszeiten zeigte sich, dass die Formaldehydemissionen sanken, wenn länger gepresst wurde. Diese Erhöhung der Presszeit widerstrebt dem industriellen Einsatz dieser Modifizierung. Für das Ausbilden einer guten Verklebung reichte bereits eine kürzere Presszeit. Ein Grund für erhöhte Formaldehydabgaben kann ein höherer Gehalt an freiem und gebundenem Formaldehyd sein, welcher, ohne es untersucht zu haben, bei den eingesetzten Harztypen unterschiedlich gewesen sein kann. Daneben lag auch ein Unterschied im Aushärteverhalten zwischen Imprägnierharz und Klebharz vor, da die DSCMessungen zeigten, dass Reaktionsmaximum zu höheren Temperaturen verschoben war. Dieser Unterschied ist wahrscheinlich darin begründet, dass der PF-Klebstoff mit Härter versetzt war, das Imprägnierharz hingegen keinen zusätzlichen Härter enthielt, um eine Erhöhung der Viskosität und verminderte Zellwandeindringung zu verhindern. Wie STEFANOWSKI ET AL. (2018) schrieben, kann die Zugabe von Cresol in Phenol-Formaldehyden eine höhere Reaktivität zur Folge haben. Somit besteht die Möglichkeit, die notwendige Presszeit, um die Emissionsgrenzwerte einzuhalten, durch Zugabe von Cresol zum Imprägnierharz zu verkürzen. Da Cresol teurer ist als Phenol, würden jedoch nur geringe Mengen zum Einsatz kommen und dieser Mehraufwand müsste im Verhältnis zum höheren Produktionsergebnis stehen.

Eine Eigenschaft, die besonderen Einfluss auf viele weitere Eigenschaften mit sich bringt, ist die Dichte. Die Darrdichte sowie die Rohdichte erwiesen sich als besonders offensichtlich beeinflussbar, wenn die Furniere mit PF imprägniert worden waren. Wie auch GABRIELLI \& KAMKE (2010) zeigen konnten, unterliegt Holz, welches mit PF imprägniert ist, einer Plastifizierung solange das Harz nicht vollständig auskondensiert ist. Da die imprägnierten Furniere bei einer Temperatur getrocknet wurden, die unterhalb der Temperatur lag bei der die Kondensation des Harzes vorherrscht, war es möglich bei einem Pressdruck von $6 \mathrm{~N} / \mathrm{mm}^{2}$ eine Darrdichte von $1251 \mathrm{~kg} / \mathrm{m}^{3}$, was dem 1,8-fachen Wert der Darrdichte der Referenzen entsprach, zu erzielen. Um den Gewichtsvorteil von Holz als Baustoff zu erhalten, lag jedoch das Augenmerk dieser Arbeit auf der Entwicklung von LVL, welches nur geringfügig, ähnlich dem unbehandelten Buchen-LVL, beim Verkleben verdichtet wurde. Aufgrund der zuvor beim Vollholz beobachteten dauerhaften Quellung, dem sog. Bulking, konnte bei einem WPG von $30 \%$ und einem Pressdruck von $2 \mathrm{~N} / \mathrm{mm}^{2}$ eine Darrdichte erzielt werden, welche mit $830 \mathrm{~kg} / \mathrm{m}^{3}$ nur 19\% über der Darrdichte der Referenzserie lag. Hierin lässt sich der wesentliche Unterschied $\mathrm{zu}$ bekannten Holzwerkstoffen (HWS) auf Basis von kunstharzimprägnierten Furnieren, sog. Kunstharz-Pressschichthölzer, erkennen, da diese eine wesentlich höhere Dichte von bis zu $1400 \mathrm{~kg} / \mathrm{m}^{3}$ aufweisen (HILl 2006, STAMM \& SEBORG (1955). EHLBECK \& HÄTTICH (1986) gaben für die untersuchten Kunstharz-Pressschichthölzer 
und Kunstharz-Presssperrhölzer in der Verwendung als Verbindungsmittel Rohdichten zwischen $1300 \mathrm{~kg} / \mathrm{m}^{3}$ und $1340 \mathrm{~kg} / \mathrm{m}^{3}$ an. Gründe dafür, dass diese hohen Dichten Anwendung in speziellen Bereichen finden können, liegen in den relativ höheren Festigkeiten als bei unverdichteten Materialien, aber auch in deren geringerer Wasseraufnahme und Dimensionsänderung. Die in dieser Arbeit ermittelten maximalen Feuchten nach Wasserlagerung (EN84) belegen dies auch. Daneben fällt jedoch auf, dass bei nur geringfügig verdichteten Serien, z.B. $30 \% \mathrm{WPG}, 2 \mathrm{~N} / \mathrm{mm}^{2}, \rho=830 \mathrm{~kg} / \mathrm{m}^{3}$, die maximale Wasseraufnahme mehr als halbiert war. Darauf basierend können eine mechanische Blockierung der Eindringwege von Wasser sowie ein zusätzlicher Hydrophobierungseffekt der Modifizierung mit PF vermutet werden. GUZY (2015) konnte diesbezüglich zeigen, dass die kapillaren Wasseraufnahmen an Buchenvollholz (20 mm* $20 \mathrm{~mm} * 230 \mathrm{~mm}$ (rad*tang*long)), welches mit niedermolekularem PF behandelt wurde (WPG 15\% \& 30\%, unverdichtet), auf ein Zehntel bis ein Viertel der Werte unbehandelten Buchenholzes reduziert waren. Physikalische Barrieren aufgrund von Harzeinlagerungen im Lumen konnte er mithilfe der Lichtmikroskopie nicht nachweisen. Die Ausgleichsfeuchten bei Lagerung der Proben im Normalklima $\left(20^{\circ} \mathrm{C} / 65 \% \mathrm{rF}\right)$ waren zudem zwischen $30 \%$ und $60 \%$ herabgesetzt.

Die Dimensionsstabilität von LVL wurde in dieser Arbeit durch den Vergleich der Dickenquellung von Plattenprüfkörpern, der Volumenquellung, sowie durch die Berechnung der relativen Verminderung der Quellung (ASE) untersucht. Über alle Serien hinweg zeigte sich eine umso höhere ASE je höher der WPG und der Pressdruck waren. Dennoch, wie bereits oben gezeigt, sank dabei die Effizienz des Verfahrens, da die Steigerung der ASE im Vergleich zur Steigerung des Harzeinsatzes deutlich unterproportional ausfiel. Für bestimmte Anwendungen von modifizierten Buchen-LVL kann demnach in Abhängigkeit von den gewünschten Eigenschaftsverbesserungen, wie z.B. Dauerhaftigkeit und Dimensionsstabilität, der Prozess entsprechend gewählt und damit so effizient wie möglich gestaltet werden.

Bei den Untersuchungen von DiEsTE ET AL. (2008), in denen Furniere der Birke (Betula sp.) und der Buche (Fagus sylvatica) mit dem Zellulosevernetzer 1,3-Dimethylol-4,5Dihydroxyethylenurea (DMDHEU) modifiziert wurden, zeigten die daraus hergestellten Sperrhölzer ASE-Werte (berechnet aus der Dickenquellung) von max. 35\% resp. 54\%. Die in dieser Arbeit untersuchten Varianten, die dem genannten Wert für Buche ähneln, sind jene LVL, die mit $2 \mathrm{~N} / \mathrm{mm}^{2}$ und 30\% WPG hergestellt wurden und eine $\mathrm{ASE}_{\text {rad }}$ von $57 \%$ aufwiesen. Wie auch bei DIESTE ET AL. (2008), waren dabei die ASE-Werte stets positiv korreliert mit dem WPG. Aufgrund der Anwendung höherer WPGs und höherer Pressdrücke und somit einer starken Verdichtung des Materials, konnten Mittelwerte für die ASE $_{\text {rad }}$ von bis zu $96 \%$ erzielt werden. Bei dem zur Modifizierung mit PF sehr ähnlichen Prozess unter Verwendung von fettsäuremodifiziertem Melamin-Formaldehyd (MF) konnten TRINH ET AL. (2012) an Buchensperrholz, bei einem WPG von nur 5\%, eine ASE $E_{\text {rad }}$ von bis zu $60 \%$ ermitteln. Die eingesetzten Melaminharze hatten jedoch den Nachteil einer vielfach höheren Formaldehydabgabe gegenüber den Referenzen und dem E1-Grenzwert. Diese Betrachtungen lassen somit den Schluss zu, dass unter ähnlichen Herstellungsbedingungen durch die Modifizierung mit PF eine ähnliche Erhöhung der Dimensionsstabilität wie mit DMDHEU zu erzielen ist, wenngleich mit fettsäuremodifiziertem MF noch bessere Werte erzielt wurden. Die 
Modifizierung von PF mit Fettsäuren zum Zweck der Imprägnierung von Holz und Holzwerkstoffen könnte folglich ein lohnenswertes Forschungsgebiet sein.

In der Regel haben Modifizierungen von Holz einen unmittelbaren Einfluss auf dessen Festigkeiten. Am deutlichsten wird dies bei Thermoholz, da die Masseverluste zu Verlusten der Festigkeiten führen. Bei anderen Verfahren wie der Vernetzung kann Versprödung, gemessen anhand einer abnehmenden Biegearbeit, beobachtet werden. Verfahren, bei denen niedrige pH-Werte vorherrschen, können den Polymerisationsgrad der Zellulose herabsetzen und so eine Herabsetzung der Zugfestigkeit bewirken. Bei der Anwendung von PF wurde eine Erhöhung der mechanischen Festigkeiten vermutet, da Kunstharz-Pressschichthölzer (KPSCHH) bekannt sind für ihre hohen Festigkeiten, historisch einst im Flugzeugbau, z.B. für Luftschrauben, Anwendung fanden und bis heute im Werkzeugbau eingesetzt werden (EHLBECK \& HÄтTICH 1986). Hinsichtlich der Biegefestigkeit der Furnierschichthölzer dieser Arbeit zeigte sich jedoch kein signifikanter Einfluss der Modifizierung und der Furnierdicke bei einem durchschnittlichen Wert von $140 \mathrm{~N} / \mathrm{mm}^{2}$. Die absolute Höhe des Ergebnisses erscheint unter Berücksichtigung der Darrdichten plausibel. Beispielsweise fanden AYDIN ET AL. (2004) bei UF-gebundenem LVL aus Fagus orientalis Lipsky mit 2,1 mm dicken Furnieren eine Biegefestigkeit von $118 \mathrm{~N} / \mathrm{mm}^{2}$ bei einer Darrdichte von $695 \mathrm{~kg} / \mathrm{m}^{3}$. Den weitaus größeren Einfluss zeigte der Plattenaufbau, da bei einem 8-lagigem Aufbau bereits zwei Sperrlagen (zweite und siebte Lage um 90 Grad gedreht), einen Festigkeitsrückgang von 29\% hervorriefen. Hieran wird deutlich, welchen Einfluss die Faserrichtung im LVL haben kann, welches Anwendung als tragendes, zum Teil stabförmiges, Bauteil im Ingenieurholzbau findet. Im Vergleich der Festigkeiten mit der kommerziell erhältlichen BauBuche $\left(\rho=830 \mathrm{~kg} / \mathrm{m}^{3}\right)$ fällt auf, dass alle gemessenen Biegefestigkeiten von LVL ohne Sperrlagen den charakteristischen Wert (95\%-Perzentil) von $80 \mathrm{~N} / \mathrm{mm}^{2}$ (PolLmeIER 2014) weit übertrafen. Der Vergleich ist jedoch nur bedingt zulässig, da hier Laborplatten aus fehlerfreiem Material, d.h. ohne Äste oder Löcher, gemessen wurden und bei der industriellen Herstellung von deutlich inhomogenerem Material ausgegangen werden kann.

Die Beobachtung deutlich gegenüber den Referenzen erhöhter Biegefestigkeiten, geht auf eine Verdichtung des LVL unter Anwendung höherer Pressdrücke als $2 \mathrm{~N} / \mathrm{mm}^{2}$, z.B. $4 \mathrm{~N} / \mathrm{mm}^{2}$ oder $6 \mathrm{~N} / \mathrm{mm}^{2}$, zurück. Wie bereits oben erwähnt, war die Modifizierung mit PF dabei Voraussetzung, dass eine höhere Verdichtung bei diesen Pressdrücken eintreten konnte, welche in reduzierten Plattendicken messbar war. Dabei fiel die Korrelation zwischen Verdichtung und Biegefestigkeit stärker aus, als die Korrelation von Darrdichte und Biegefestigkeit. Dies ist darin begründet, dass zum Ergebnis der Darrdichte der WPG beigetragen haben konnte und ohne mechanische Verdichtung dabei keine Steigerung der Biegefestigkeit eintrat. EHLBECK \& HÄTTiCH (1986) geben für $12 \mathrm{~mm}$ dickes KPSCHH $\left(\rho=1300 \mathrm{~kg} / \mathrm{m}^{3}\right)$ eine Biegefestigkeit von $250 \mathrm{~N} / \mathrm{mm}^{2}$ an. Die höchsten Biegefestigkeiten dieser Arbeit beliefen sich auf $230 \mathrm{~N} / \mathrm{mm}^{2}$. Der geringe Unterschied kann jedoch mit einer unterschiedlichen Homogenität des Werkstoffes zusammenhängen, da die Furnierdicken in genannter Studie bei nur 0,25-0,30 mm lagen. Dennoch wird aus diesen Zusammenhängen ersichtlich, welchen Einfluss die Modifizierung mit PF und die Pressparameter bei der Herstellung auf die Biegefestigkeit von Buchen-LVL haben. 
Hinsichtlich des E-Moduls lieferten die Referenzen beider untersuchter Furnierstärken ähnliche Werte und lagen in einem Bereich zwischen $13.000 \mathrm{~N} / \mathrm{mm}^{2}$ und $14.500 \mathrm{~N} / \mathrm{mm}^{2}$. Damit kamen sie dem Literaturwert von $14.000 \mathrm{~N} / \mathrm{mm}^{2}$ (NIEMZ1993) für Buchenvollholz nahe, jedoch lagen sie weit entfernt von den statischen Kennwerten der BauBuche. Diese soll ein mittleres E-Modul von $16.800 \mathrm{~N} / \mathrm{mm}^{2}$ und ein charakteristisches E-Modul (95\%-Perzentil) von $14.900 \mathrm{~N} / \mathrm{mm}^{2}$ aufweisen und damit der Festigkeitsklasse LVL 80 S gemäß DIN EN 14374 (2016) entsprechen. Auch infolge eines höheren Pressdruckes erfuhren die im Labor hergestellten Referenzen analog zur Biegefestigkeit jedoch keine Steigerung. Im Unterschied zum Buchenvollholz und zur BauBuche wird für Nadelholz allgemein ein Wert von $10.000 \mathrm{~N} / \mathrm{mm}^{2}$ und für Nadel-Brettschichtholz ein Wert von $11.000 \mathrm{~N} / \mathrm{mm}^{2}$ angenommen (NIEMZ 1993). Daraus lässt sich auf eine Vorteilhaftigkeit hinsichtlich der Dimensionierung von Bauteilen aus Buchen-LVL gegenüber Nadelholzprodukten schließen.

Bei den modifizierten Serien ohne eine zusätzliche Verdichtung hingegen ließ sich, anders als bei der Biegefestigkeit, eine deutliche Erhöhung des E-Moduls bereits beim niedrigsten WPG feststellen. Die Werte lagen zwischen $14.500 \mathrm{~N} / \mathrm{mm}^{2}$ und $17.000 \mathrm{~N} / \mathrm{mm}^{2}$ bei ausschließlich parallelem Faserverlauf und einer Furnierdicke von $3,7 \mathrm{~mm}$ und damit über den Anforderungen der DIN EN 14374 (2016). Ähnlich hohe Werte für den E-Modul sind bekannt für tropische Harthölzer, wie z.B. Azobé/Bongossi mit einem Wert von $17.000 \mathrm{~N} / \mathrm{mm}^{2}$ (NIEMZ 1993). Hieraus lässt sich ein Zusammenhang mit der Sprödigkeit des eingebrachten $P F$ herstellen. Einen Anhalt diesbezüglich geben KU, JACOBSEN ET AL. (2008), die für reines Phenolformaldehyd einen E-Modul unter Zugbeanspruchung mit $25.100 \mathrm{~N} / \mathrm{mm}^{2}$ ermittelt haben, wohingegen OZYHAR, HERING ET AL. (2012) einen Zug-E-Modul von Buchenvollholz bei einer Feuchte von 5,9\% von $12.020 \mathrm{~N} / \mathrm{mm}^{2}$ parallel zu den Fasern ermittelten. AsHBY (2013) gibt in der Übersicht der Eigenschaften von Bakelit für das E-Modul einen Bereich von $27.600 \mathrm{~N} / \mathrm{mm}^{2}-48.300 \mathrm{~N} / \mathrm{mm}^{2}$ an. In der Kombination der beiden Materialien werden auch beim Biegeverhalten des modifizierten LVL diese Eigenschaften eine Rolle gespielt und zu einem weniger elastischem, resp. spröderem, Komposit geführt haben. Bei einem WPG von $30 \%$ wiesen verschiedene Harztypen zueinander signifikante Unterschiede auf, die jedoch nicht erklärt werden konnten. Mit einem WPG von $60 \%$ zeigten sich ähnliche Werte wie bei 30\%. Bis auf einen Harztyp lagen die Ergebnisse näher beieinander. Die Darrdichten dieser Prüfkörper lagen ebenso nah beieinander, aber auch deutlich höher als bei 30\% WPG. Der Umstand, dass mit Steigerung des WPGs insgesamt keine Steigerung des E-Moduls festgestellt werden konnte, verdeutlicht, dass es keinen linearen Zusammenhang zwischen dem WPG und diesem Kennwert gab.

Die zu beobachtende Steigerung des E-Moduls unter der Anwendung höherer Pressdrücke (z.B. $4 \mathrm{~N} / \mathrm{mm}^{2}$ ) auf bis zu $24.000 \mathrm{~N} / \mathrm{mm}^{2}$ unter Einsatz 2,13mm dicker Furniere und auf bis zu $20.000 \mathrm{~N} / \mathrm{mm}^{2}$ unter Einsatz 3,7 mm dicker Furniere resultierte aus einer signifikanten Verdichtung des Materials. Eine derartige Steigerung des Elastizitätsmoduls infolge von Phenolmodifizierung in Kombination mit Verdichtung geben auch SHAMS ET AL. (2004) an. Sie konnten bei einem WPG von 60\% bei Japanischer Zeder (Cryptomeria japonica) die Darrdichte von $450 \mathrm{~kg} / \mathrm{m}^{3}$ auf $1100 \mathrm{~kg} / \mathrm{m}^{3}$ erhöhen. Dadurch erhöhte sich auch der E-Modul von $10.000 \mathrm{~N} / \mathrm{mm}^{2}$ auf $22.000 \mathrm{~N} / \mathrm{mm}^{2}$. Für Obo-Festholz, welches aus Buchenfurnieren hergestellt wird, wird ein maximaler E-Modul von $24.000 \mathrm{~N} / \mathrm{mm}^{2}$ angegeben (Blomberger Holzindustrie 
2012), EHLBECK \& HÄTTICH (1986) geben für KPSCHH allgemein einen E-Modul von $24.200 \mathrm{~N} / \mathrm{mm}^{2}$ an. Die größere Nähe der Platten, welche aus 2,13mm dicken Furnieren gefertigt wurden, zu diesen Literaturwerten, beruht wahrscheinlich auf der höheren Homogenität des Materials im Vergleich zu den Platten aus 3,7mm dicken Furnieren, welche bei gleicher Plattendicke nur acht statt 15 Lagen aufwiesen.

Da ein zunehmender E-Modul mit einer zunehmenden Sprödigkeit des Materials einhergehen kann, wurde es als bedeutsam erachtet, die Bruchschlagarbeit zu bestimmen. Diese ist, ähnlich der Arbeit, die bei einem statischen Biegeversuch verrichtet wird, maßgeblich vom Weg des Werkzeuges, bzw. von der Durchbiegung des Prüfkörpers, und der dazu aufgebrachten Kraft abhängig. Es ist somit möglich, durch eine stark zunehmende Kraft bei einem höheren E-Modul und geringerer Biegung bzw. Dehnung bis zum Erreichen der Maximalkraft die gleiche Arbeit verrichtet $\mathrm{zu}$ haben wie bei einem Prüfkörper mit niedrigerem E-Modul. Wie aus einer Datensammlung für Kunststoffe ersichtlich, werden für Phenol-Formaldehyd Werte für die Bruchschlagarbeit zwischen $6,75 \mathrm{~kJ} / \mathrm{m}^{2}$ und $7,75 \mathrm{~kJ} / \mathrm{m}^{2}$ angenommen (KERN 2017), was in etwa einem Zehntel der Werte für Buchenholz entspricht.

Die Bestimmung der Bruchschlagarbeit an eigens dafür gefertigten Prüfkörpern aus der mit dem Harztyp A ohne Sperrlagen hergestellten Varianten, deren statisches Biege-E-Modul zuvor gemessen und deren Feuchte sowie Darrdichte nach der Prüfung bestimmt wurden, sollte zur Aufklärung der Zusammenhänge beitragen. Dabei bestätigten sich die in der Biegeprüfung gemachten Beobachtungen, zuvor sei jedoch die als Referenz ermittelte Bruchschlagarbeit an unbehandeltem Buchen-LVL zu betrachten. Diese lag mit nur geringen Unterschieden im Durchschnitt für alle Drücke bei $74 \mathrm{~kJ} / \mathrm{m}^{2}$ bei einer Rohdichte von $786 \mathrm{~kg} / \mathrm{m}^{3}$ und einer Holzfeuchte von $8,7 \%$. Eine vergleichbare Untersuchung der Bruchschlagarbeit von LVL führten BAL \& BEKTAS (2012) durch. Dabei verwendeten sie $3 \mathrm{~mm}$ dicke Furniere u.a. der Orientalischen Rotbuche (Fagus orientalis L.) mit einer Rohdichte von $656 \mathrm{~kg} / \mathrm{m}^{3}$ bei einer Holzfeuchte von 12\%. Bei der Herstellung des siebenlagigen LVL ohne Sperrlagen verwendeten sie einen Standard-PF-Klebstoff. Im Vergleich zum Vollholz des gleichen Stammes fanden sie bei der Orientalischen Rotbuche eine niedrigere Holzfeuchte, welche, auffallend ähnlich zu den dieser Arbeit zugrundeliegenden Referenzen, bei 8,6 \% lag. Bei einer auf $12 \%$ HF korrigierten Rohdichte von $677 \mathrm{~kg} / \mathrm{m}^{3}$ und einer zu dieser Arbeit identischen Versuchsdurchführung, ermittelten sie eine Bruchschlagarbeit von durchschnittlich $51,8 \mathrm{~kJ} / \mathrm{m}^{2}$. Der im Vergleich dazu höhere Wert der Referenzen dieser Arbeit hängt maßgeblich mit ihrer höheren Rohdichte zusammen. Als weitere Einflussfaktoren sind nach KoLLMANN (1951) jedoch auch die Faserorientierungen der einzelnen Furnierlagen zu berücksichtigen, da eine Abweichung von der Längsachse des Prüfkörpers von $5^{\circ}$ bereits eine Reduzierung von $10 \%$ bewirkt. Es wird somit geschlussfolgert, dass das in dieser Arbeit hergestellte unbehandelte Buchen-LVL gut geeignet war als Referenz genutzt zu werden.

Während das E-Modul dieser Referenzen erst beim höchsten Pressdruck eine geringe Steigerung erfuhr, stieg es mit zunehmenden WPG bei den PF-modifizierten Platten bereits beim niedrigsten Pressdruck deutlich an. Infolge eines höheren Pressdruckes und einer stärkeren Verdichtung stiegen die E-Modi dieser modifizierten Serien weiter, wobei jeweils die Variante mit 30\% WPG höher lag als jene mit 15\% WPG. Mit der Erhöhung des E-Moduls ging 
beim unverdichteten LVL die deutlichste Abnahme der Bruchschlagarbeit einher. Im linearen Regressionsmodel wurde die negative Korrelation deutlich. Das ergibt insoweit Sinn, da weniger Verformung bei gleicher Kraft stattfinden und somit deutlich weniger Energie des auftreffenden Hammers in Wärme umgewandelt werden konnte. Im Vergleich zu den Varianten mit der höheren Verdichtung zeigte sich entgegen der Erwartungen jedoch, dass keine weitere Abnahme der Bruchschlagarbeit stattgefunden zu haben schien, was sich darin ausdrückte, dass die negativen Korrelationen flacher verliefen. Es kam sogar zu einer leichten Erholung der Messwerte der Bruchschlagarbeit mit zunehmendem Pressdruck. Es liegt somit der Schluss nahe, dass trotz Zunahme des E-Moduls, die Verdichtung, d.h. die Abnahme des Querschnittes, auf welchen die verrichtete Arbeit bezogen wird, eine Kompensation bewirkte. KoLLMANN (1951) befand ebenfalls, dass beim Schichtholz mit der Einlagerung spröden Kunstharzes eine Reduktion der Bruchschlagarbeit einhergehe, welche sich jedoch durch Anwendung höheren Pressdruckes ausgleichen ließe. Tatsächlich konnte bei identischem WPG mit dem höheren Pressdruck eine Erhöhung der Bruchschlagarbeit verzeichnet werden, welche jedoch nicht über den Werten der jeweiligen unbehandelten Referenzen lag. Die absolut verrichtete Arbeit des Hammers mit zunehmendem WPG, hatte auch bei zunehmender Verdichtung und zunehmendem E-Modul insgesamt abgenommen. Ein auf das Gewicht der Probe oder auf die Darrdichte bezogener Wert - eine spezifische Bruchschlagarbeit, läge somit niedriger, als der nach der üblichen Betrachtungsweise, bzw. Berechnung, bestimmte Wert der Bruchschlagarbeit. Über die unterschiedlichen Stufen der Verdichtung hinweg betrug die durchschnittliche Abnahme der Bruchschlagarbeit des PF-modifizierten Buchen-LVL, gegenüber dem unbehandelten Buchen-LVL, 13\% und $42 \%$ bei WPGs von $15 \%$ und $30 \%$.

MilletT ET AL. (1956) untersuchten die Bruchschlagarbeit an gekerbten und ungekerbten Proben aus Compreg, einem verdichteten Furnierschichtholz aus 1,6 mm dicken Furnieren der Gelb-Birke (Betula alleghaniensis), welche mit verschiedenen PF-Harzen imprägniert wurden. Bei Ihnen sank die gekerbte Bruchschlagarbeit des unverdichteten PF-imprägnierten Materials im Verhältnis zu den unbehandelten, unverdichteten Referenzen um 67\% und 75\%, bei WPGs von $15 \%$ und $30 \%$. Mit zunehmender Verdichtung dieses mit einem Harztyp hergestellten Materials stiegen die Werte der Bruchschlagarbeit exponentiell an und konnten bei bis zu einer maximalen Verdichtung von ca. 36\% um bis zu 250\% gesteigert werden. Jedoch konnte eine zu den unverdichteten Referenzen äquivalente Bruchschlagarbeit bei einem WPG von 15\% erst bei einer Darrdichte von $1280 \mathrm{~kg} / \mathrm{m}^{3}$ und bei einem WPG von $30 \%$ bei einer Darrdichte von $1350 \mathrm{~kg} / \mathrm{m}^{3}$ erzielt werden. Die verschiedenen untersuchten Harze mit unterschiedlichen WPGs im Bereich von 25\%-29\% wiesen zueinander deutliche Unterschiede in ihren Ergebnissen von bis zu 160\% auf. Hier lässt sich ein Bezug zu den in dieser Arbeit im Rahmen der Biegeprüfung ermittelten E-Modi herstellen. Unterschiedliche Harze führten hier zu unterschiedlichen Zunahmen, die nicht über die Darrdichte, Verdichtung oder den WPG erklärt werden konnten. In Verbindung mit der bewiesenen negativen Korrelation des E-Moduls mit der Bruchschlagarbeit und den von MILLETT ET AL. (1956) gemachten Beobachtungen, ist davon auszugehen, dass PF-Harze unterschiedlicher Herkunft, bei durchaus ähnlicher chemischer Zusammensetzung und ähnlicher Molmasse zu unterschiedlich elastischen bzw. spröden Netzwerken polymerisieren können. In diesem Zusammenhang ist auch die Aussage von 
MilletT ET AL. (1956) zu sehen, dass auch die Trocknung der Furniere, Feuchte beim Pressen und die Presstemperatur einen Einfluss auf die Bruchschlagarbeit gezeigt haben.

Die Abnahme der Bruchschlagarbeit bei modifiziertem LVL, mit der auch eine Abnahme der Biegearbeit einhergeht, sollte bei einem Einsatz in tragenden Anwendungen berücksichtigt werden, da diesem Wert bei der Auslegung von Tragwerken keine Beachtung zukommt und stattdessen der E-Modul als wesentliche Eigenschaft eines Material herangezogen wird. Höhere E-Modi würden in der Auslegung jedoch dazu führen, dass Konstruktionen schlanker, d.h. Querschnitte geringer bemessen würden, was wiederrum dazu führt, dass sich das Vermögen einer Konstruktion dynamische Belastungen aufzufangen noch stärker herabsetzen könnte. Schließlich liegt es jedoch an der Anwendung des modifizierten LVLs, in wieweit dynamische Lasteinwirkungen überhaupt auftreten und tatsächlich eine Biegearbeit zu verrichten ist.

Bei der Untersuchung der biologischen Dauerhaftigkeit PF-modifizierten LVLs mittels Abbaubauversuch mit der Weißfäule Trametes versicolor zeigte sich eine deutliche Verminderung des Abbaus gegenüber dem unbehandelten Buchen-LVL. Bei Versuchsserien mit 2,13 mm dicken Furnieren, die im Vakuumprozess imprägniert wurden und einen WPG von 30\% bzw. 60\% aufwiesen, konnte ein Masseverlust unter 1\% festgestellt werden. Dabei konnte weder ein Einfluss der Darrdichte, welche bei einem WPG von 30\% mit 1,5 N/mm 2 Pressdruck bei $0,75 \mathrm{~g} / \mathrm{cm}^{3}$ und bei $3 \mathrm{~N} / \mathrm{mm}^{2}$ Pressdruck bei $0,87 \mathrm{~g} / \mathrm{cm}^{3}$, sowie bei $60 \% \mathrm{WPG}$ und $6 \mathrm{~N} / \mathrm{mm}^{2}$ bei $1,2 \mathrm{~g} / \mathrm{cm}^{3}$ lag, noch ein Einfluss des WPGs und des Pressdruckes gezeigt werden.

Dass ohne eine hohe Verdichtung bei einer moderaten Dichte von $0,78 \mathrm{~g} / \mathrm{cm}^{3}$, welche generell weniger physikalische Barrieren aufweist und kapillare Wasseraufnahme ermöglicht, eine hohe biologische Dauerhaftigkeit durch eine Modifizierung mit niedermolekularem PF-Harz erreicht werden konnte, ist von besonderer Relevanz, da ähnliche kommerzielle Produkte auf Basis von Buchenfurnieren und PF-Harz, wie z. B. das Obo-Festholz der Delignit AG mit einer Rohdichte von $1,3 \mathrm{~g} / \mathrm{cm}^{3}$, in der Regel höhere Dichten aufweisen. Eine Ausnahme stellt die Produktreihe unter dem Namen Lignostone der Firma Röchling dar, die in der Variante mit der leichtesten Verdichtung ab einer Rohdichte von $0,75 \mathrm{~g} / \mathrm{cm}^{3}$ PF-imprägniertes Furnierschichtholz und Sperrholz anbietet (Röchling 2017). Es sind dem Verfasser keine wissenschaftlichen Veröffentlichungen zur biologischen Dauerhaftigkeit dieser Produkte bekannt, jedoch zeigen die Ergebnisse von UNSAL ET AL. (2009) an thermomechanisch verdichteten Vollholzlamellen, dass eine alleinige Verdichtung von Holz den Abbau durch Trametes versicolor nicht vermindern kann. Wenngleich die Verdichtung des unbehandelten Buchen-LVLs gering ausfiel, zeigte sich auch hier, dass ohne die PF-Modifizierung keine Verminderung des Abbaus damit zu erzielen war. Anhand des Vergleiches unverdichteter und verdichteter PF-modifizierter Serien konnte jedoch bei einer Variante (Harztyp D) mit 30\% WPG gezeigt werden, dass die Verdichtung in Kombination mit PF-Harz durchaus einen positiven Effekt haben kann, da bei der unverdichteten Serie ein zu den anderen Harzen signifikant höherer Masseverlust, bei den verdichteten Serien nicht zu erkennen war.

Bei der Untersuchung des Einflusses niedriger WPGs als 30\%, z.B. 15\%, zeigte sich, dass es auch unter Verwendung von 3,7mm dicken Furnieren zu einem deutlich reduzierten Abbau 
kam, welcher maximal bei $7 \%$ lag, die Mediane und die $95 \%$-Perzentile deutlich unter $5 \%$ lagen. Im Gegensatz zu den Serien mit einen WPG von 30\% lagen hier jedoch Einzelwerte über 5\% und bei alleiniger Anwendung der ENV 12038 (2002) wären diese Serien nicht mehr als voll beständig gegen holzerstörende Basidiomyceten zu klassifizieren. Die Berechnung des DSI (Tabelle 16) sollte hier Abhilfe schaffen, um eine einfache Beurteilung des Erfolgs des Modifizierungsprozesses zu ermöglichen. Während die unbehandelten Referenzen demnach in die Dauerhaftigkeitsklasse (Dk) 5 einzuordnen waren, lagen die Varianten mit 15\% WPG in den Dk 1 und 2. Das Ergebnis, dass erst ab einem WPG von 30\% kein Holzabbau mehr stattfand, schließt jedoch nicht aus, dass geringere WPGs zwischen $15 \%$ und $30 \%$ ausgereicht hätten. Bei WEPNER (2006), welcher ein Modifizierungsverfahren für Buchenfurniere auf Basis der zyklischen N-Methylol-Verbindung DMDHEU entwickelte, sank der Masseverlust unter Verwendung des gleichen Prüfpilzes bis zu einem WPG von 20\%. Dabei konnte ein minimaler Masseverlust von $6 \%$ ermittelt werden. Folglich ist unter Verwendung von PF die Erhöhung der Resistenz des LVL gegenüber Trametes versicolor stärker ausgeprägt gewesen.

Dieses Ergebnis steht im Einklang mit ähnlichen Untersuchungen, bei denen Vollholz mit Phenol-Formaldehyd imprägniert und später ausgehärtet und ggf. verdichtet wurde. LEE ET AL. (2015) zeigten, dass der Abbau durch Pycnoporous sanguineus (Weißfäule) an Sesenduk (Endospermum diadenum) und Jelutong (Dyera costulata) von $32 \%$ resp. $42 \%$ auf unter 1\% Masseverlust reduziert wurde. Sie setzten ein PF-Harz ein mit einer zahlenmäßig mittleren Molmasse von $600 \mathrm{~g} / \mathrm{mol}$ und erzielten in den Holzproben unterschiedliche WPGs von 35 \% $81 \%$. TAKAHASHI UND IMAMURA (1990) modifizierten verschiedene Holzarten mit einem wasserlöslichen PF-Harz ( $\mathrm{Mn}=170 \mathrm{~g} / \mathrm{mol}$ ) und einem in Ethanol löslichen PF-Harz (300 $\mathrm{g} / \mathrm{mol}$ ). Im Abbauversuch mit Tyromyces palustris (Braunfäule) und Trametes versicolor (Weißfäule) erzielten sie an unbehandelten Proben von Cryptomeria japonica D. Don, Tsuga heterophylla Sarg. und Fagus crenata Blume Masseverluste von zwischen 32\% und 63\%. Dabei fanden sie heraus, dass mit dem wasserlöslichen PF bei den Nadelhölzern C. japonica und $T$. heterophylla ein WPG von 10\% nötig war, um den Holzabbau vollständig zu unterdrücken, während beim Laubholz F. crenata ein WPG von 20\% nötig war. Dieses Ergebnis deckt sich mit den eigenen Ergebnissen mit dem Buchen-LVL, bei denen ein WPG von 15\% noch einen Abbau zuließ, während er bei 30\% unterdrückt war. TAKAHASHI \& IMAMURA (1990) zeigten dabei auch, dass für das in Ethanol lösliche PF deutlich höhere WPGs nötig waren, um den Abbau zu verhindern. Sie führten dies auf die etwas höhere Molmasse zurück. Die eigenen Untersuchungen sowie die Studien von LEE ET AL. (2015) sowie von TAKAHSHI \& IMAMURA (1990) belegen jedoch, dass zahlenmäßig mittlere Molmassen (Mn), die größer sind als $300 \mathrm{~g} / \mathrm{mol}$ noch in die Zellwand eindringen. Somit ist ein Einfluss des Lösemittels aufgrund der schwächeren Polarität des Ethanols wahrscheinlicher. Dazu passt, dass der Harztyp D, welcher im Vergleich zu den anderen Harztypen keine unterschiedliche Mn hatte, jedoch in Methanol und Ammoniak gelöst vorlag, deutlich höhere Masseverluste aufwies.

Untersuchungen zur Dauerhaftigkeit von LVL im Besonderen, wurden u.a. von NzOKOU, ZYSKоWSKI ET AL. (2005) mit den Holzarten Acer rubrum, Populus tremuloides, Sassafras albidum, Larix decidua und Robinia pseudoacacia durchgeführt. In einem Labortest mit Weißfäulen und Braunfäulen nach dem Standard ASTM D2017 inkubierten sie Vollholz und 5lagiges PF-gebundenes LVL und verglichen die arithmetischen Mittel der Masseverluste nach 
12 Wochen. Da die Vollholzprüfkörper eine Kantenlänge von einem Zentimeter aufwiesen, die LVL-Prüfkörper jedoch in einer Richtung größer $(1,6 \mathrm{~cm})$ waren und Vollholzprüfkörper gleicher Abmessung, wie in dieser Arbeit die Maß-Kontrollprüfkörper, nicht verwendet wurden, ist die Vergleichbarkeit zu hinterfragen. Betrachtet man die dargestellten mittleren Masseverluste, die mit Trametes versicolor erzielt wurden, erkennt man, dass bei Verwendung der natürlich dauerhaften Holzarten R. pseudoacacia und S. albinum der Masseverlust beim LVL mit 7 \% respektive $12 \%$ nominell höher war als beim Vollholz mit $4 \%$ respektive $9 \%$. Sie führen den höheren Abbau beim LVL auf die Auswaschung von Extraktstoffen während des Dämpfens und Heißdampftrocknens zurück. Möglicherweise sind die Einflüsse des Herstellungsprozesses auf das Furnier auch bei der nicht dauerhaften Holzart Buche der Grund, warum die in dieser Arbeit untersuchten LVL-Referenzen einem höheren Abbau unterlagen als die Buchen-Vollholzprüfkörper gleicher Größe (Maß-Kontrollprüfkörper). Unter Verwendung der nicht dauerhaften Holzart A. rubrum konnten NZOKOU, ZYSKOWSKI ET AL. (2005) wiederum eine im Vergleich zum Vollholz geringere Abbaurate beim LVL festgestellt werden - $53 \%$ statt 77\%. Entsprechend ihres verwendeten Standards ordnen sie Masseverluste in vier Klassen ein, wobei die höchste Dauerhaftigkeitsklasse mit einem mittleren Masseverlust von bis zu 10\% erreicht sei. Das wird vom Autor dieser Arbeit als kritisch betrachtet, da Produkte nach dieser Einteilung als sehr dauerhaft beurteilt würden, jedoch bei einem derartigen Masseverlust erhebliche Festigkeitseinbußen zu erwarten wären. Die Folgerung der Autoren, infolge des LVL-Herstellungsprozesses könne mit der Holzart $A$. rubrum ein dauerhaftes Produkt entstehen, ist besonders zu hinterfragen.

$\mathrm{Zu}$ den potentiellen Ursachen einer erhöhten Dauerhaftigkeit PF-modifizierten Buchen-LVLs können einerseits eine verminderte Wasseraufnahme des Holzes allgemein und eine verminderte Wasseraufnahme der Zellwand gezählt werden. Die Wasseraufnahme der Zellwand jedoch konnte nur indirekt beobachtet werden, anhand einer verminderten Quellung bei Wasserlagerung in Verbindung mit einem dauerhaft gequollenen Zustand der trockenen modifizierten Vollholzproben (Bulking). Da Weißfäulen eine Erosionsfäule verursachen und mit ihren Hyphen dabei initial im Zelllumen wachsen, bestand für sie jedoch auch bei geringeren Feuchten der Zellwand die Möglichkeit der Kolonisation der modifizierten Holzkörper, da, wie in dieser Arbeit nachgewiesen, die Zelllumen bis zu einem WPG von 30\% weitgehend frei blieben. Sie konnten somit auch ihre Enzyme und andere extrazelluläre Substanzen absondern, welche potenziell in der Lage gewesen wären, die Zellwandpolymere, insbesondere das Lignin der Zellwände, oxidativ abzubauen. $\mathrm{Zu}$ den bekannten am Ligninabbau beteiligten Enzymen gehören Manganperoxidase, Ligninperoxidase und Laccase. Aufgrund ihrer Molmassen wird davon ausgegangen, dass sie nicht in die Zellwand eindringen können - HOFRICHTER (2002) sowie GOODELL ET AL. (2008) geben für die Manganperoxidase $40 \mathrm{kDa}$ bis $50 \mathrm{kDa}(40000 \mathrm{~g} / \mathrm{mol}-50000 \mathrm{~g} / \mathrm{mol})$ respektive $35 \mathrm{kDa}$ bis $47 \mathrm{kDa}(35000 \mathrm{~g} / \mathrm{mol}$ - $47000 \mathrm{~g} / \mathrm{mol}$ ) an. Damit beim unbehandelten Holz dennoch ein Abbau stattfinden kann, benötigt der Pilz nicht-enzymatische Mediatoren mit niedrigerer Molmasse. Sie sind bekannt dafür, dass sie tief in die Zellwand eindringen können (GOODELL ET AL. 2008). ENOKI ET AL. (1997) untersuchten extrazelluläre Substanzen mit Redoxreaktion katalysierenden Eigenschaften und ermittelten Molmassen von 1000 - 5000 g/mol. Diese extrazellulären Substanzen sind auf Diffusion angewiesen, jedoch ist diese von einem intakten Netzwerk von zellwandgebundenem 
Wasser abhängig, welches wiederum erst ab einer bestimmten Zellwandfeuchte entsteht. Für die Diffusion von Ionen wurde von ZELINKA ET AL. (2008) nachgewiesen, dass es einen Percolationsgrenzwert der Holzfeuchte gibt und bei Unterschreitung die Transportwege von gelösten Substanzen unterbrochen werden.

Die Untersuchungen von HosSEINPOURPIA ET AL. (2016) zur Fenton-Reaktion mit Bezug zur Braunfäule ergaben, dass Eisenionen in die Zellwand PF-modifizierten Kiefernholzes nicht eindringen konnten. Analog dazu könnte beim Abbauversuch des Buchen-LVLs mit Weißfäule das von der Manganperoxidase gebildete $\mathrm{Mn}^{3+}$ an der Diffusion in die Zellwand gehindert und so die Oxidation von Lignin unterbunden worden sein. Es wird daher vermutet, dass eine Hemmung der Diffusion niedermolekularer Komponenten und Metallionen, die von Weißfäulepilzen zum Abbau von Holzpolymeren genutzt werden, infolge der PF-Modifizierung des Buchenholzes eintreten konnte und auf diese Weise die Dauerhaftigkeit erhöht wurde. Diese Annahmen beruhen auf dem derzeitigen Stand des Wissens hinsichtlich der Abbaumechanismen von Holzpolymeren durch Weißfäulen. In den zitierten Quellen wurde jedoch deutlich, dass diese Mechanismen noch nicht vollständig aufgeklärt sind. Einer Aufklärung der Wirkweise der PF-Modifizierung ist somit weitere Grundlagenforschung voranzustellen. Im Ergebnis kann jedoch aus der Untersuchung der Resistenz des PFmodifizierten Buchen-LVL gegenüber dem Weißfäuleerreger Trametes versicolor geschlossen werden, dass die Modifizierung mit basischem Phenol-Formaldehyd, dessen zahlenmäßig mittlere Molmassen kleiner oder gleich $452 \mathrm{~g} / \mathrm{mol}$ betrug, nach dem beschriebenen Verfahren einen wirksamen Schutz gegen Holzabbau ermöglichte.

Diese in den beschriebenen Laborversuchen erhöhte Resistenz gegenüber holzabbauenden Basidiomyceten, insbesondere Trametes versicolor, spiegelte sich auch in den Feldversuchen im Erdkontakt (EN252, 2015) wieder. PF-modifiziertes Buchen-LVL zeigte auch hier eine gegenüber den unbehandelten Referenzen deutlich erhöhte Dauerhaftigkeit. Wenngleich der beobachtete Zeitraum noch zu kurz ist, um eine abschließende Beurteilung vorzunehmen, die einen Vergleich zu klassischen Holzschutzmitteln zuließe. In Bezug dazu empfehlen Nicholas \& MILITZ (2008) einen Prüfzeitraum von mindestens 5 Jahren.

GOODELL ET AL. (2008) geben an, dass die Moderfäule verursachenden Pilze ihr Substrat über die Zellwand erschließen können und, da sie so potenzielle Schutzmittel im Lumen umgehen können, besonders schwer zu unterdrücken sind. Der oben gezeigte verminderte Abbau wird auf die Zellwandeindringung des Phenol-Formaldehyds zurückgeführt. Bei unterschiedlichen Harzen, unterschiedlichen Chargen und unterschiedlichen WPGs kann es jedoch auch zu gewissen Ungleichverteilungen, bzw. zu lokalen Konzentrationsunterschieden gekommen sein. Diese ermöglichen somit auch partiell, bzw. lokal begrenzt, einen Angriff durch holzabbauende Pilze, wie z.B. Weißfäulen oder Moderfäuleerreger. Nach der Norm, bzw. der Prüfanweisung, wurde hier jedoch nicht nach dem Gewichtsverlust oder einer relativen flächen- oder volumenbezogenen Größe ausgewertet. Mit einer einzigen kleinen Stelle, in der eine Veränderung, bzw. ein Weichwerden der Oberfläche, beobachtet wurde, konnte somit eine Bewertungsziffer bestimmt sein. Da Phenol-Formaldehyd im ausgehärteten Zustand keinerlei Toxizität aufweist, verhindert es allgemein nicht das Wachstum von Pilzen. Somit ist bei der Modifizierung insbesondere die Homogenität, d.h. die gleiche Verteilung des Harzes in 
der Zellwand, von großer Bedeutung. Bei Buchenfurnier aus unterschiedlichen Quellen, Sortimenten, Qualitäten und schließlich unterschiedlichen Dichten, kommt es jedoch sehr wahrscheinlich zu unterschiedlichen Lösungsaufnahmen und damit bereits zwischen den Furnieren einer Platte zu unterschiedlichen WPGs. Dies kann zur Folge haben, dass beispielsweise bei höheren Dichten als der Durschnitt, der WPG geringer ausfällt. Damit nimmt die Wahrscheinlichkeit zu, dass partiell die Konzentration in der Zellwand nicht ausreicht. Dem entgegenwirken könnte man nur, wenn man die Lösungskonzentration bei der Imprägnierung an den Furnieren mit höherer Dichte ausrichten würde, was jedoch einen Mehrverbrauch von PF-Harz nach sich zöge. Die Prozessoptimierung bei der Herstellung fängt somit bereits bei der Auswahl der Furniere an, da ähnliche Dichten sowie der Ausschluss von Buchenrotkern zu den geringsten Unterschieden in der Lösungsaufnahme und im WPG führen. Hinsichtlich der Auswahl der Harze ist festzustellen, dass der Harztyp D, korrespondierend mit seinen vergleichsweise niedrigeren ASE-Werten und höheren Masseverlusten im Abbauversuch mit Weißfäule, hier im Feldversuch mit Erdkontakt tendenziell schlechter abschneidet als alle anderen untersuchten Harztypen. Da der Harztyp D nach Herstellerangaben in Lieferform zwischen 20\% und 25\% Methanol sowie 5\% Ammoniak aufwies, wird vermutet, dass die Eindringung in die Zellwand, trotz einer ähnlich niedrigen Molmasse wie die der anderen Harztypen, schlechter war. Hintergrund ist die geringere Polarität vom Methanol-AmmoniakWassergemisch im Vergleich zu dem Wasser-Natriumhydroxid-Gemisch der übrigen Harztypen. Die geringere Polarität kann während der Imprägnierphase vermutlich zu einer geringeren Quellung der Zellwand geführt haben, wie die Arbeiten von MANTANIS ET AL. (1994) nahelegen.

Zum Vergleich der Ergebnisse dieser Arbeit mit anderen Holzmodifizierungsverfahren werden nachfolgend die Ergebnisse von DMDHEU-behandelten Vollholzproben der Kiefer (Pinus sylvestris) und der Buche (Fagus sylvatica), welche ebenfalls im Erdkontakt geprüft wurden, diskutiert. Auf einem Testfeld in Schweden (Stamsmåla) zeigten Kiefernproben der 1.3 M-DMDHEU- und 2.1 M-DMDHEU-Varianten nach drei Jahren der Exposition durchschnittliche Bewertungsziffern von 0,5 und 0,75 (EMMERICH ET AL. 2018). Auf einem Testfeld in Australien (Redlands) verhielten sich die Kiefernproben ähnlich. Die DMDHEU behandelten Buchenholzproben erreichten im gleichen Beobachtungszeitraum mittlere Bewertungsziffern von 3,25 und 0,6 (EMMERICH ET AL. 2018). Die gleichen Bewertungsziffern erhielten PF-modifizierte LVLs, unverdichtet $\left(2 \mathrm{~N} / \mathrm{mm}^{2}\right)$ mit einem WPG von $15 \%$ und verdichtet $\left(6 \mathrm{~N} / \mathrm{mm}^{2}\right)$ mit einem WPG von $30 \%$. Damit wird einerseits, wie schon zuvor, ersichtlich, welchen Einfluss der Prozess der Herstellung des LVL hat. Anderseits kann auch darauf geschlossen werden, dass wahrscheinlich ein höherer WPG und eine höhere Verdichtung nötig sind, um eine ähnliche Dauerhaftigkeit wie DMDHEU behandeltes Buchenholz zu erzielen.

Neben der Dauerhaftigkeit PF-modifizierten LVLs im Erdkontakt, war im Rahmen dieser Arbeit auch die Witterungsstabilität, im Sinne einer Dimensions- und Farbstabilität, bei mehrjähriger Exposition gegenüber Sonneneinstrahlung und Regen von Interesse. Generell unterliegen die Holzoberflächen einem Abbau durch UV-Strahlung und anschließender Auswaschung. Insbesondere die Ligninkomponente wird dabei primär photo-oxidativ angegriffen wobei chromophore Gruppen entstehen, welche die Oberfläche vorrübergehend dunkler werden 
lassen, bevor sie ausgewaschen werden und eine Aufhellung durch das Zutagetreten der übrigen Holzbestandteile Zellulose und Hemizellulose eintritt (FEIST \& HON 1984). Diese werden zusammen mit Extraktstoffen sekundär abgebaut. Dabei besiedeln Schimmelpilze die Oberfläche und führen durch die meist dunkle, schwarze Farbe ihrer Fruchtköper zu einer Vergrauung. Infolge wechselnder Feuchten und dem damit verbundenen Quellen und Schwinden treten zudem strukturelle Schäden, insbesondere Risse, auf (FEIST \& HON 1984). Um Buchenholz vor dieser Verwitterung $\mathrm{zu}$ schützen, wurden bereits einige Modifizierungsverfahren untersucht. MiNH (2007) setzte u.a. Zellulosevernetzer der Textilindustrie ein, deren Hauptkomponenten fettsäure-modifizierte N-Methylverbindungen waren, aber auch als zusätzliches Hydrophobierungsmittel Paraffin aufwiesen. Für Buchenund Kiefernvollholz konnte dabei nachgewiesen werden, dass bei bestimmten Prozessbedingungen während einer künstlichen Bewitterung verringerte Wasseraufnahmen konstant erhalten blieben. Diese Beobachtungen gingen einher mit einer erhöhten Dimensionsstabilität. In einer einjährigen Freilandbewitterung, bei der jedoch nur Kiefernholz eingesetzt wurde, konnten signifikant weniger Risse und Deformation (Schüsseln) gegenüber unbehandelten Referenzen festgestellt werden, wenngleich eine deutliche Vergrauung eintrat. Unter Anwendung von N-Methylol-Melamin (NMM) und fettsäure-modifiziertem Melamin (mNMM) konnte MAI (2009) an Buchensperrholz nachweisen, dass diese Komponenten in die Zellwand eindringen und dort durch Kondensation ein stabiles Netzwerk ausformen, welches ebenfalls die Wasseraufnahme reduziert und die Dimensionsstabilität erhöht. Nach einer 18-monatigen Freilandbewitterung wurde deutlich, dass alle unbeschichteten Varianten einer ausgeprägten Vergrauung unterlagen, jedoch die Breite, Tiefe und Anzahl von Rissen an den modifizierten Sperrhölzern deutlich reduziert waren. Hinsichtlich der, im Abstand von drei Monaten, gemessenen Feuchten, war zu beobachten, dass die Referenzen höhere Feuchten und größere Schwankungen aufwiesen als die modifizierten Serien. Die Evaluation der Lab-Werte ergab, dass bei unbeschichteten Proben, unabhängig von der Behandlung, alle Werte Helligkeit und Farbigkeit - in dieser Zeit abnahmen. Wohingegen die beschichteten Proben, und insbesondere die modifizierten Serien, eine erhöhte Farbstabilität aufwiesen. Ebenso untersuchte auch WEPNER (2006) die Eigenschaften von Buchensperrholz, dessen Furniere er in unterschiedlichen Prozessen mit DMDHEU modifiziert hatte, von denen jedoch nur die Sperrhölzer aus einem Vakuum-Druck-Verfahren einer 9-monatigen Freilandbewitterung ausgesetzt wurden. Die Untersuchung der Witterungsstabilität des PF-modifizierten BuchenLVLs im Rahmen dieser Dissertation erfolgte nach den gleichen Methoden und Bewertungsschemen und am gleichen Standort, jedoch konnte ein längerer Versuchszeitraum realisiert werden.

In der Freilandbewitterung, wie auch im Labor, konnte dabei die erhöhte Dimensionsstabilität des modifizierten unbeschichteten LVLs über einen Zeitraum von drei Jahren bewiesen werden. Nach einer Phase der Auffeuchtung innerhalb des ersten Jahres blieben die Messwerte stabil auf einem Niveau oder nur leicht steigend. Im Gegensatz zu den Referenzen waren die jahreszeitlichen Schwankungen deutlich abgemindert. Diese Verringerung der damit verbundenen Quell- und Schwindbewegungen des Holzes kann als Vorteil bei der Verwendung von Beschichtungen gesehen werden. Insgesamt fiel jedoch eine gegenüber den Referenzen nur gering verbesserte Rissneigung der unbeschichteten modifizierten Prüfkörper auf, wobei 
die Bewertung wie bei WEPNER (2006) und MAI (2009) nach den strengen Kriterien der DIN ISO 4628-4 (1997) zur Beurteilung von Schäden in Beschichtungen durchgeführt wurde. Das hatte zur Folge, dass schon zu Beginn des Untersuchungszeitraumes vielen Prüfköpern hohe Kennwerte bezüglich der Menge und Breite an Rissen zugeteilt wurden und zu späteren Zeitpunkten zum Teil Unterschiede zwischen den Serien teilweise nicht mehr erfassbar waren.

Im genaueren Vergleich der eigenen Ergebnisse mit den Arbeiten von WEPNER (2006) und MAI (2009) zeigte sich hinsichtlich der Feuchteschwankungen innerhalb eines Jahres, dass ähnliche Kurvenverläufe, bei denen behandelte Serien geringeren Schwankungen unterlagen als die Referenzen, ohne eine zusätzliche Verdichtung erst bei einem WPG von 30\% erkennbar waren. Bei WEPNER (2006) hingegen genügte durch die Modifizierung der Buchenfurniere mit DMDHEU im Vakuum-Druck-Verfahren ein WPG von 20\%. Ähnlich sah dies bei MAI (2009) unter Anwendung von NMM aus, jedoch genügte hier unter Anwendung von mNMM, aufgrund der höheren Hydrophobizität, bereits ein WPG von 5\%. Mit einer reduzierten Wasseraufnahme einher ging die Dickenquellung des Buchen-LVL. Es zeigte sich eine deutliche Abnahme mit zunehmendem WPG, wobei erst ab 30\% ein deutlicher Unterschied zu den Referenzen vorlag. Wenngleich WEPNER (2006) diesen Wert nicht beachtete, so ergaben seine Untersuchungen zum Verwerfen, dass ein WPGs von 20\% bereits eine Erhöhung der Dimensionsstabilität brachte, aber höhere WPG auch noch eine Verbesserung bewirkten. MAI (2009) zeigt diesbezüglich keine Messergebnisse, gibt jedoch an, für NMM und mNMM eine deutliche Reduktion des Verwerfens beobachtet zu haben. Beim Buchen-LVL wurde zudem der Einfluss von Pressdruck und Verdichtung untersucht und hier fiel auf, dass die Wasseraufnahme in der Freilandbewitterung nach einem Jahr bei einem WPG von 15\% zwar um die Hälfte reduziert werden konnte, jedoch die Quellung aller Serien dieses Harztyps bei diesem WPG den Referenzen ähnliche Werte ergaben. Erst bei dem höheren WPG von 30\% wurde die Auswirkung höherer Pressdrücke ersichtlich, als dass die Dickenquellung dauerhaft auf max. $2 \%$, im Vergleich von bis zu $25 \%$ bei den Referenzen, reduziert werden konnte. Die Verwendung unterschiedlicher Harztypen hatte auf diese Zusammenhänge keinen Einfluss. Da jedoch weitgehend ähnliche Harze eingesetzt wurden, mit Ausnahme eines lösemittelhaltigen Typs, kann in Anbetracht der Ergebnisse von MAI (2009) eine weitere Optimierung der Harze durch Veresterung mit Fettsäuren zukünftig, für Anwendungen, in denen eine Verdichtung nicht erwünscht ist, angedacht werden. Hinsichtlich der Menge an Rissen in den bewitterten Oberflächen von Buchen-LVL zeigte sich, dass ab einem WPG von 30\% nach einem Jahr deutlich weniger Risse entstanden waren, wenn die Prüfkörper einen WPG von 30\% aufwiesen. Unter Anwendung von NMM und mNMM konnte MAI (2009) nach einem Jahr bei den behandelten Buchensperrhölzern die Bewertungsziffer 3 (mäßig viele, d.h. Risse in mittlerer Menge. DIN ISO 4628-4, 1997) vergeben, im Vergleich zu einer 5 (sehr viele, d.h. Risse in sehr großer Menge) bei den Referenzen. Ähnlich verhielt es sich beim Buchen-LVL. Hier erhielten die Referenzen bereits nach 9 Monaten die Bewertungsziffer 5. Das Auftreten von sehr vielen Rissen in der Oberfläche von unbehandelten Buchenfurnieren kann somit als typische Erscheinung während einer ganzjährigen Bewitterung gewertet werden. Hinsichtlich der Orientierung der Furniere fiel beim Buchen-LVL auf, dass das Verwenden von zwei Sperrlagen nur geringfügig niedrigere Bewertungen ergab. So schien auch die Breite der Risse auf die Bewertungsziffer 3 (deutlich sichtbare Risse mit bloßem Auge) begrenzt, während beim 
LVL ohne Sperrlagen, mit ausschließlich parallelem Aufbau, Risse nach einem Jahr bis zu $1 \mathrm{~mm}$ breit (Bewertungsziffer 4) waren. WEPNER (2006) konnte eine ebenso deutliche Verringerung der Rissneigung an dem mit DMDHEU modifizierten Buchen-Sperrholz nachweisen, jedoch beschränkte sich der Betrachtungszeitraum nur auf 9 Monate. Auch hinsichtlich der Farbwertänderung ist dieser Zeitraum beim Vergleich zur Modifizierung mit PF zu beachten. So gibt er für die Referenzen eine Farbwertveränderung $(\Delta C)$ von durchschnittlich 27,5 an. Bei unbehandeltem, unverdichtetem Buchen-LVL lag $\Delta \mathrm{C}$ nach 12 Monaten bei durchschnittlich 22,0. Während beim DMDHEU dieser Wert, unabhängig vom WPG, zwischen 17,0 und 17,5 lag, ergaben sich für PF-modifiziertes LVL, vom Harztyp abhängig, unterschiedliche Mittelwerte zwischen 10 (Harztyp A) und 21 (Harztyp D) beim WPG von 30\%. Hinsichtlich der Helligkeitsänderung kommt es beim LVL innerhalb von 12 Monaten über alle Serien hinweg, mit Ausnahme derer mit 60\% WPG, zu einer Verdunklung. In Anbetracht des Untersuchungszeitraumes ist die Verdunklung unbeschichteter Oberflächen auf die Besiedlung mit holzverfärbenden Pilzen, nach Auswaschung von abgebautem Lignin, zurückzuführen. Die relative Aufhellung der Serien mit dem WPG von 60\% beruhte auf einem zu Beginn vergleichsweise dunkleren L-Wert aufgrund der dunkelrotbraunen Eigenfarbe des Harzes und einem beginnenden Abbau aromatischer Strukturen. In Ähnlichkeit dazu wiesen auch die Sperrhölzer, welche mit DMDHEU und NMM sowie mNMM behandelt wurden, eine signifikante Verdunkelung auf (WEPNER 2006, MAI 2009). Im Vergleich zueinander war die Abnahme des L-Wertes bei PF-modifiziertem LVL geringer als bei MAI (2009), jedoch stärker als bei WEPNER (2006). Die gleiche Aussage ist für die Gesamtfarbveränderung zu treffen, jedoch mit der Einschränkung, dass für die mit DMDHEU modifizierten Sperrhölzer nur 9Monatsergebnisse vorlagen. In Ergänzung zu diesen makroskopischen Betrachtungen sind die Beobachtungen von Evans ET AL. (2013) hinzuzuziehen, um die Auswirkungen der PFModifizierung auf die Witterungsstabilität von Holz zu verstehen. An 3-lagigen Sperrhölzern bewitterten sie PF-modifizierte Deckfurniere der Radiata-Kiefer (Pinus radiata D.Don) für 2000h im Freiland von Vancouver, Canada. Sie zeigten, dass die Sperrhölzer, welche mit niedermolekularem PF modifiziert waren und WPGs zwischen 10\% und 30\% aufwiesen, bereits vor der Bewitterung dunkler waren als die Referenzen. Des Weiteren konnten sie zeigen, dass die Verdunklung, resp. die Abnahme des L-Wertes, der PF-modifizierten Sperrhölzer vergleichbar mit der Abnahme bei den Referenzen war. Es ist anzunehmen, dass aufgrund der kurzen Bewitterungsdauer diese Verdunklung überwiegend auf den Abbau von aromatischen Strukturen (Lignin und PF) und das Entstehen chromophorer Gruppen, die noch nicht ausgewaschen wurden, zurückzuführen ist. Durch den Zusatz von sterisch gehinderten Aminen (HALS), welche als Radikalfänger fungierten, konnten sie beim WPG von 10\% die Verdunkelung dagegen fast vollständig unterbinden. Mithilfe von Fourier-TransformationsInfrarotspektren (FTIR) und Aufnahmen mit einem Elektronenmikroskop zeigten sie, dass bereits nach 50 Tagen der Freilandbewitterung substantieller Abbau des Lignins und mithin

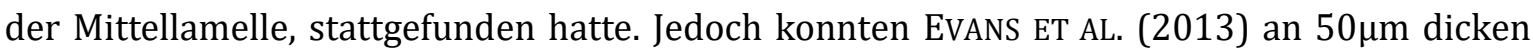
Furnieren der Nootka-Scheinzypresse (Chamaecyparis nootkatensis (D.Don) Spach) in einer Freilandbewitterung feststellen, dass mit zunehmendem WPG eine signifikante Verringerung der Masseverluste durch UV-Abbau stattfand und die Abnahme der Zugfestigkeiten der Furniere deutlich reduziert wurde. Sie kamen somit zu dem Schluss, dass PF, insbesondere aufgrund seiner Eigenschaft die Gefügestabilität zu erhalten, UV-Strahlung zu absorbieren und 
antioxidativ zu wirken, einen positiven Einfluss auf die Bewitterungsstabilität von Holz hat (EVANS, GIBSON ET AL. 2013). Aus ihrer Veröffentlichung wird auch ersichtlich, dass höhere WPGs als 10\%, mindestens 20\%, nötig waren, um eindeutige Verbesserungen zu erzielen und mit 30\%WPG weitere Verbesserungen möglich waren. Hingegen konnte der Einsatz von HALS ähnliche Resultate auch bei niedrigeren WPGs ermöglichen. Passend dazu wurde in dieser Arbeit Buchen-LVL, welches mit WPGs zwischen 5\% und 18\% hergestellt wurde, mit einem transparenten Beschichtungssystem mit UV-Absorbern versehen und ein Bewitterungsversuch im Freiland gestartet. Dabei wurde nach dem ersten Jahr deutlich, dass die Kombination von Beschichtung mit UV-Absorbern und einem WPG von 18\% hinsichtlich der Dimensionsstabilität zu gleichwertigen Ergebnissen führte wie ein WPG von 30\% bei unbeschichteten Proben. Hinsichtlich der Beurteilung von Schäden auf der Oberfläche kam es bei den beschichteten Proben mit einem WPG von 18\% zu weniger Rissen in schwächerer Ausprägung als bei allen anderen beschichteten und unbeschichteten Varianten. Der Einsatz von UV-Absorbern, der hier besonders ressourcenschonend nur in der Beschichtung erfolgte, kann somit die Schutzwirkung der PF-Modifizierung gegen witterungsbedingte Beeinträchtigung von Holzoberflächen verstärken, wie es bereits EVANS ET AL. (2013) schlussfolgerten.

Neuere Veröffentlichungen wie die von PASSAUER, SChUBERT ET AL. (2016) zeigen, dass klassische Kunstharzpresssperrhölzer (KPSP) bei denen „handelsübliche“ PF-Harze im Tauchverfahren eingesetzt wurden, dagegen nur eine geringe Farb- und Witterungsstabilität aufweisen. Bei ihren Untersuchungen führte die Verwendung von dünnen Buchenfurnieren mit einer Dicke von 0,4 mm bis 0,6 mm und einer Harzauftragsmenge von $190 \mathrm{~g} / \mathrm{m}^{2}$, theoretisch zu WPGs im Bereich zwischen $47 \%$ und $70 \%$, welche sich in Verbindung mit einer hohen Verdichtung, nach den hier dargelegten Untersuchungen, als besonders witterungsbeständig hätten erweisen sollen. Eine signifikante Verbesserung in Bezug auf die Verfärbung und das Rissverhalten erzielten sie jedoch durch die Verwendung eines PFimprägnierten transparenten Films eines Herstellers, dessen Harz auch für die Imprägnierung der Buchenfurniere in dieser Arbeit diente. Daraus, sowie aus eigenen Dauerhaftigkeitsuntersuchungen an kommerziell erhältlichen KPSPs, schließe ich, dass die Verwendung von handelsüblichen Harzen, die eine Verklebung und Imprägnierung in einem Schritt erzielen sollen, nicht in einem ausreichenden Maße die erforderliche Eindringung in die Zellwand gewährleistet. Die Bedingungen für eine Zellwandeindringung, niedrige Molmasse sowie niedrige Viskosität, laufen der Ausbildung einer Klebfuge zuwider, während eine höhere Viskosität nicht genügend Mobilität der Moleküle erlaubt. Besonders die Eindringtiefe von Harzen mit höherer Viskosität, welche bereits auf höhere Molmassen hindeutet, ist eingeschränkt. Dennoch konnten im Rahmen dieser Arbeit Prozesse, die eine Tauchtränkung beinhalteten, nah an die Qualität der Vakuum-Druck-Imprägnierung heranreichen, wenn Imprägnierung und Verklebung getrennt durchgeführt wurden. Sie belegen aber mit ihrer höheren Streuung der Ergebnisse auch, dass es bei der zeitabhängigen Diffusion zu Ungleichverteilungen kam, die auch bei WEPNER (2006) beobachtet wurden. 


\section{Fazit}

Die Ergebnisse dieser Arbeit zeigen, dass es möglich ist, die Dimensionsstabilität und Dauerhaftigkeit von Buchen-LVL gegenüber holzabbauenden Pilzen sowie Witterungseinflüssen entscheidend $\mathrm{zu}$ verbessern. Im Vergleich $\mathrm{zu}$ herkömmlichem Kunstharzpressschichtholz kann dies mit deutlich geringeren WPGs und vielfach dickeren Furnieren als üblich besonders ressourcenschonend erfolgen. Es bedarf in diesem Zusammenhang weiterer Studien, um zu zeigen, welche ökonomischen Vorteile mit der Verwendung dickerer Furniere, einem geringeren Harzeinsatz, geringerer Anzahl an Furnierlagen sowie eines niedrigen Standardpressdruckes einhergehen. Dennoch wird vermutet, dass die Modifizierung mit niedermolekularem Harz, welches in der Lage, ist in die Zellwand von Furnieren mit einer Dicke von 3,7 mm einzudringen, in einem zweistufigen Prozess, der Imprägnierung und Verklebung trennt, ressourceneffizienter ist als ein Verfahren, welches Verklebung und Imprägnierung im Tauchprozess vereint.

Das wesentliche Neue bei der Weiterentwicklung der Phenolmodifizierung ist darin zu sehen, dass das Holz (die Furniere) mit Lösungen imprägniert wird, welche maximal nur 30\% Feststoff (PF) enthalten müssen, um die größte Verbesserung der Eigenschaften zu erzielen und eine signifikante Verbesserung der biologischen Dauerhaftigkeit bereits bei einem WPG von 15\% vorlag. Nach der Trocknung verbleibt im Holz entsprechend der Lösungsaufnahme, welche bei der Buche ca. 110\% des absolut-trockenen Eigengewichts entspricht, nur der in der Lösung enthaltene Anteil Harz. Dieser wird überwiegend in der Zellwand eingelagert. Damit unterscheidet sich der beschriebene Prozess als erstes durch die Harzmenge von bisher bzw. derzeit bekannten Verfahren. Des Weiteren unterscheidet sich der Prozess durch die Lokalisation der eingesetzten Harze, da die Trocknung bei einer Temperatur von maximal $55^{\circ} \mathrm{C}$ stattfindet, wird vermieden, dass eine zu frühe Polymerisation der Harze stattfindet. Dies hat zur Folge, dass nur Wasser und Formaldehyd verdunsten und gemäß den bekannten Vorgängen bei der Trocknung von Holz, zuerst aus den Lumen der Holzzellen entweichen. Der sich einstellende Konzentrationsgradient führt dann zu einem in Richtung Zellwandinneres gerichteten Diffusionsstrom. Dieser effiziente Einsatz von Phenol-Formaldehydharz, welcher nicht auf Verklebung, sondern nur auf die Modifizierung der Zellwand abzielt, ist somit eine Neuheit.

Gegenüber dem Stand der Technik bietet der zuvor beschriebene Prozess den Vorteil der optimalen Ressourceneffizienz, da die Gleichverteilung durch die Vollimprägnierung erreicht wird, während nur die für die gewünschte Zellwandmodifizierung benötigte Menge Harz durch Einstellen der Lösungskonzentration verbraucht wird. Die anschließende Niedertemperaturtrocknung führt dann zur Anreicherung in der Zellwand. Dies bietet den wesentlichen Vorteil, dass der spätere Holzwerkstoff eine hohe Dimensionsstabilität sowie eine sehr hohe Resistenz gegenüber holzabbauen Pilzen (Basidiomyceten, Fungi imperfecti und Ascomyceten) erreicht, welches selbst durch den Einsatz von Standardharzen im großen Überschussverfahren (klassisch) nicht bei größeren Furnierdicken als einem Millimeter zu erzielen wäre. Durch die Möglichkeit, Furniere jeder Dicke verwenden zu können, ergibt sich ein neuer Gestaltungsspielraum für den Hersteller der zu mehr Ressourceneffizienz beitragen kann. Diese Form der Holzmodifizierung kann auch zur Kaskadennutzung beitragen, da nach 
Ende der längeren Lebensdauer der Buchenholzprodukte erster Generation, Holzabfälle und ausgediente Holzprodukte nicht verbrannt werden müssen, sondern durch Aufbereitung zu Spänen, z.B. in der Spanplattenindustrie, weiterverwendet werden könnten. Daran kann sich eine erneute Wiederverwendung anschließen, oder die energetische Nutzung. Dies wäre bei Verwendung klassischer Holzschutzmittel nicht möglich.

Die eingesetzten und wirkungsvollsten Phenol-Formaldehyd-Harze waren basische Resole in wässriger Lösung. Sie wiesen in der Ausgangs-/Lieferform zahlenmäßig mittlere Molmassen zwischen $302 \mathrm{~g} / \mathrm{mol}$ und $452 \mathrm{~g} / \mathrm{mol}$ sowie eine Polydispersität von maximal 1,56 und eine dementsprechende Viskosität von durchschnittlich 180 mPas*s auf. Der Anteil an Monomeren lag unter 5\%. Wesentliche Kriterien eines Harzes sind damit die sich ergebene Molmasse und die Verteilung der Molmassen, welche einen Rückschluss auf die chemische Struktur bzw. die Molekülgröße geben. Dieser Eingrenzung liegt die Erkenntnis zugrunde, dass nur Harze eingesetzt werden sollten, deren überwiegender Anteil in der Lage ist, in die Zellwand einzudringen.

In der Praxis lässt sich der aufgezeigte Prozess auf die Furniere aller imprägnierbaren Holzarten anwenden. Eine temperaturindizierte Verklebung (Heißpresse) vorausgesetzt, lassen sich auf diese Weise modifizierte Furniere in unterschiedlicher Weise zusammenfügen und Furnierschichthölzer mit unterschiedlichen Anteilen gleich und ungleich orientierter Furnierrichtungen (Sperrholz) erzeugen. Favorisierte Holzwerkstoffanwendungen sind Sperrholz und Furnierschichtholz (LVL). In dieser Arbeit noch nicht dargestellte Untersuchungen zeigen, dass die dargestellten Ergebnisse insbesondere auch für Birkenholz gültig und anwendbar sind.

Die kontinuierliche industrielle Herstellung von dauerhaftem, witterungsbeständigem Buchenfurnierschichtholz für die Anwendung in tragenden Konstruktionen im Außenbereich stellt eine wesentliche Innovation dar. Im Hinblick auf die Imprägnierung von Buchenfurnieren mit Phenol-Formaldehyd sind positive Wirkungen hoher Beladungen, resp. WPG, zwar hinlänglich bekannt, jedoch ist der Ansatz, besonders niedermolekulare Harze zu verwenden und nicht das Zelllumen, sondern vornehmlich die Zellwand zu modifizieren, gänzlich neu. Dieser Ansatz erreichte, dass die Aufnahme von Wasser in die Zellwand vermindert und damit die Dauerhaftigkeit sowie die Dimensionsstabilität erhöht wurden. Dies führte zu den gewünschten Eigenschaften sowie $\mathrm{zu}$ einer Steigerung der Effizienz des Herstellungsprozesses.

Die Umsetzung der Forschungsergebnisse in Form einer industriellen Herstellung und Vermarktung kann gesellschaftlichen und politischen Zielen dienen, da die stoffliche Nutzung von Buchenholz aus einheimischer nachhaltiger Forstwirtschaft, und damit nichtnahrungsrelevanter Biomasse, in Deutschland vorangetrieben und so der Umstieg von fossilen auf nachwachsende Rohstoffe beschleunigt würde. Dies hätte auch einen unmittelbaren Nutzen für die deutsche Forstwirtschaft, da auf Grund von waldbaulichen Konzepten zukünftig vermehrt Laubholzsortimente, insbesondere der Buche, verfügbar sein werden, die effizient eingesetzt werden müssen. Die der energetischen Nutzung vorgelagerte stoffliche Nutzung der Buche als Bauprodukt, brächte einen volkswirtschaftlichen Gewinn unter anderem dadurch mit sich, dass die für konventionelle Bauprodukte substituierte fossile Ressource für andere 
Nutzungen zu Verfügung stünde. Des Weiteren schüfe die industrielle Fertigung von Holzprodukten, die weltweit exportiert würden, volkswirtschaftliches Einkommen. Eine regionale Produktion hochwertiger Schichtholzprodukte stärke die Konkurrenzfähigkeit gegenüber internationalen Wettbewerbern und schüfe langfristig Arbeitsplätze. Schließlich würde, auch im Hinblick auf aktuelle Klimaziele, der Einsatz eines Baustoffes aus Holz, dessen Lebens- bzw. Nutzungsdauer erhöht ist, eine Senkung des mit dem Bausektor verbundenen $\mathrm{CO}_{2}$-Ausstoßes bewirken. 


\section{Formelverzeichnis}

Formel 1: Berechnung des Weight-Percent-Gain (WPG) ......................................................................16

Formel 2: Berechnung des Bulking (dauerhafte Quellung) der Zellwand ........................................16

Formel 3: Berechnung der maximalen Quellung des Querschnittes ................................................17

Formel 4: Berechnung der Anti-Swell-Effiiency (ASE) ………….....................................................17

Formel 5: Berechnung der Lösungsaufnahme nach dem Imprägnierprozess mit PF .................. 26

Formel 6: Berechnung des Bulking der Querschnittfläche (rad*tang) an Vollholzproben........ 27

Formel 7: Berechnung der Darrdichte......................................................................................... 36

Formel 8: Berechnung der Verdichtung................................................................................ 37

Formel 9: Berechnung der Wasseraufnahme. ................................................................................... 37

Formel 10: Berechnung der Quellrate für eine anatomische Holzrichtung. .................................... 38

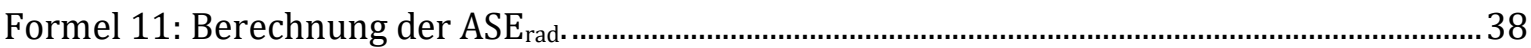

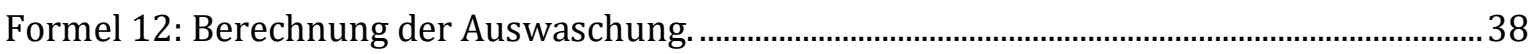

Formel 13: Berechnung der Volumenquellung. ........................................................................... 39

Formel 14: Berechnung der regulären Holzfeuchte............................................................................... 39

Formel 15: Berechnung der korrigierten Holzfeuchte........................................................................... 39

Formel 16: Berechnung des Elastizitätsmodul.................................................................................... 40

Formel 17: Berechnung der Biegefestigkeit ...................................................................................... 40

Formel 18: Berechnung der Bruchschlagarbeit.............................................................................. 41

Formel 19: Berechnung der absorbierten Formaldehydmenge bei der Prüfkammer-Methode

Formel 20: Berechnung des Gasanalysewert Gi für jede Stunde der Extraktion ............................ 43

Formel 21: Berechnung des mittleren Gasanalysewertes $G_{m}$ gemäß EN 717-2 (1994).............. 44

Formel 22: Berechnung des Befallsanfälligkeitsindex (DSI) nach Anhang E in ENV 12038

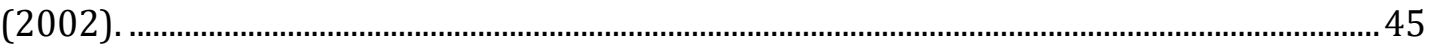

Formel 23: Berechnung der Buntheit C .............................................................................................. 51

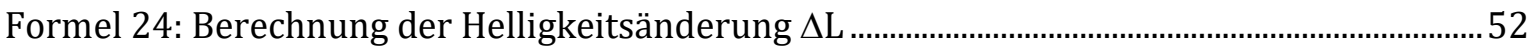

Formel 25: Berechnung der Farbveränderung $\Delta$ C...................................................................... 52

Formel 26: Berechnung der Gesamtveränderung von Farbe und Helligkeit $\Delta \mathrm{E}$.............................52

Formel 27: Maximal möglicher Wassergehalt $u_{\max }$ von Holz in Abhängigkeit vom Darrgewicht

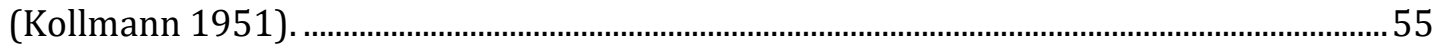

Formel 28: Herleitung der Funktion zur Berechnung der Lösungsaufnahme in Abhängigkeit von Darrdichte und Holzfeuchte............................................................................................56

Formel 29: Funktion zur Berechnung der Lösungsaufnahme in Abhängigkeit von Darrdichte und Holzfeuchte 


\section{Abbildungsverzeichnis}

Abbildung 1: Platten, Träger und Paneele auf Basis von Furnieren - faserparallel oder mit bis zu 20\% Querlagen verklebt (Pollmeier 2014).

Abbildung 2: Verwendung von Furnierschichtholz als Fachwerksträger (Pollmeier 2014).......8

Abbildung 3: Mittlerer relativer Energieverbrauch ( $\mathrm{Holz}=1$ ) ausgewählter Rohstoffe zur Herstellung pro Tonne Fertigprodukt (Lippke, Wilson et al. 2004).

Abbildung 4: Absoluter Energieverbrauch bei Holzwerkstoffherstellung (KT=kammergetrocknet) (Puettmann \& Wilson 2005)

Abbildung 5: Methylolierung von Phenolationen. Gezeichnet in ACD/ChemSketch V.14.01...13

Abbildung 6: Kondensationsreaktion eines Resols. Gezeichnet und berechnet in

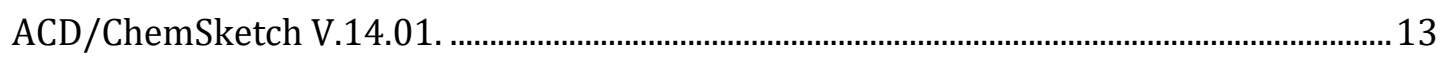

Abbildung 7: Molares Volumen von Phenol berechnet in ACD/ChemSketch V.14.01................14

Abbildung 8: WPG, Bulking und resultierende ASE für modifiziertes Buchenvollholz (Mittelwerte und Standardabweichungen).......................................................................19

Abbildung 9: Zusammenhang von Bulking und ASE für alle Harze, Harzkonzentrationen und Katalysatorzugaben.

Abbildung 10: Ergebnisse der ASE-Prüfung für bis zu sechs Zyklen an PF-modifiziertem Buchenholz

Abbildung 11: Anti-Swell-Efficiency in Abhängigkeit der Molekülgröße an Buchenvollholz... 21

Abbildung 12: UV-Mikrospektrogramm, Häufigkeitsverteilung der Absorptionswerte und 3DLinienscan von Fasern einer unbehandelten Buchenholzprobe.

Abbildung 13: Kumulative Häufigkeit der 14 Klassen (resp. Grundfarben) der Absorption der Fasern einer unbehandelten Buchenholzprobe bei regulären Grenzwerten.

Abbildung 14: Harztyp A, 16\% WPG. Häufigkeitsverteilung der 14 Klassen der Absorption bei regulären Grenzwerten .23

Abbildung 15: Zusammenhang zwischen UV-Absorption bei $278 \mathrm{~nm}$ und dem Bulking. .24

Abbildung 16: Harztyp A, 31\% WPG. Häufigkeitsverteilung der 14 Klassen der Absorption bei regulären Grenzwerten.

Abbildung 17: Harz A, 31\% WPG. Häufigkeitsverteilung der 14 Klassen der Absorption bei veränderten Grenzwerten. .25

Abbildung 18: Abnahme des Weight-Percent-Gain (WPG) in Abhängigkeit von Lösungsalter und Lagerungstemperatur bei Imprägnierung von Buchenvollholz mit PhenolFormaldehyd (Harztyp A, 25\%FS).

Abbildung 19: Bulking (Dauerhafte Quellung der Zellwand) in Abhängigkeit von Lösungsalter und Lagerungstemperatur bei Imprägnierung von Buchenvollholz mit PhenolFormaldehyd (Harztyp A, 25\%FS)......................................................................................... 28

Abbildung 20: Anti-Swell-Efficiency (ASE) in Abhängigkeit von Lösungsalter und Lagerungstemperatur bei Imprägnierung von Buchenvollholz mit Phenol-Formaldehyd (25\%FS).

Abbildung 21: Molekülgrößenverteilung einer 25\%igen Lösung von Typ A in Wasser, gemessen nach der DLS-Methode.

Abbildung 22: Reaktionsenthalpie bei der Aushärtung vom Klebharz 15J173 (Dynea) (blaue Linie) und dem Imprägnierharz TYP A (schwarze Linie) 
Abbildung 23: Trocknungsprozess der PF-Harzimprägnierten Buchenfurniere. 35

Abbildung 24: Buchen-LVL-Prüfkörper während der Inkubation auf Trametes versicolor im Prüfraum bei $22 \pm 1^{\circ} \mathrm{C}$ und $70 \pm 5 \%$ relative Luftfeuchte. 45

Abbildung 25: Freilandprüfung im Erdkontakt (EN 252, 2015) mit PF-modifiziertem BuchenLVL in Göttingen. 46

Abbildung 26: Aufbau der Schnellbewitterungsanlage (links), Prüfkörper unter infrarotem Licht (rechts). 47

Abbildung 27: Prüfkörper unter UV-Licht (links)und während der Beregnung (rechts).......... 48 Abbildung 28: Temperaturverlauf der Buchen-LVL-Oberflächen in der Schnellbewitterungsanlage 48

Abbildung 29: Versuchsaufbau Freilandbewitterung. 50

Abbildung 30: Satellitenbild mit Versuchsfläche der Abt. Holzbiologie und Holzprodukte und der meteorologischen Messstation der Abt. Bioklimatologie der Universität Göttingen.

Abbildung 31: Statistische Angaben zum Wertebereich von Stichprobenergebnissen in Boxdiagrammen durch Symbole

Abbildung 32: Lösungsaufnahme und Darrdichte von absolut trockenen Buchenfurnieren... 54

Abbildung 33: WPG in Abhängigkeit von Konzentration und Lösungsaufnahme, ermittelt an darrtrockenen Furnieren ( $\mathrm{HF}=0 \%$ )... 55

Abbildung 34: Darstellung der Lösungsaufnahmen in Abhängigkeit von der Holzfeuchte....... 56

Abbildung 35: Theoretische Lösungsaufnahmen bei Buchenfurnieren in Abhängigkeit von der Holzfeuchte und der Darrdichte, berechnet nach Formel 17..

Abbildung 36: Darrdichte des 10-lagigen Buchen-LVL-Prüfkörper ohne Sperrlagen $(10 * 2,13 \mathrm{~mm})$, modifiziert mit dem Harztyp A. Pressdrücke: $1,5 \mathrm{~N} / \mathrm{mm}^{2}, 3 \mathrm{~N} / \mathrm{mm}^{2}$ und 6 $\mathrm{N} / \mathrm{mm}^{2}$. WPG: 0\%, 23\%, 30\%, 60\% 58

Abbildung 37: Darrdichte von Buchen-LVL ohne Sperrlagen $(8 * 3,7 \mathrm{~mm}$ parallel) bei Verwendung der Imprägnierharze A, F und G, mit WPGs von $15 \%, 30 \%$ und $60 \%$, bei Pressdrücken von $2 \mathrm{~N} / \mathrm{mm}^{2}$ (a), $4 \mathrm{~N} / \mathrm{mm}^{2}$ (b) und $6 \mathrm{~N} / \mathrm{mm}^{2}$ (c) $\left(\mathrm{t}=45 \mathrm{Min}\right.$., $\left.\mathrm{T}=140^{\circ} \mathrm{C}\right) .59$

Abbildung 38: Darrdichten des Buchen-LVL ohne Sperrlagen mit 3,7 mm (8-lagig) und 2,13 mm (15-lagig) dicken Furnieren mit WPGs von 15\%, 30\% und 60\% des Harztyps A und den Pressdrücken $2 \mathrm{~N} / \mathrm{mm}^{2}, 4 \mathrm{~N} / \mathrm{mm}^{2}$ und $6 \mathrm{~N} / \mathrm{mm}^{2}$.

Abbildung 39: Darrdichte von Buchen-LVL mit 2 Sperrlagen $\left(8^{*} 3,7 \mathrm{~mm}\right)$ bei Verwendung der Imprägnierharze A, B. C, D und E, bei WPGs von 15\%, 30\% und 60\%, bei Pressdrücken von $2 \mathrm{~N} / \mathrm{mm}^{2}$ (a) und $6 \mathrm{~N} / \mathrm{mm}^{2}$ (b)

Abbildung 40: Oberflächendiagramm zur Darstellung der erzielten Darrdichte $\left[\mathrm{kg} / \mathrm{m}^{3}\right]$ bei unterschiedlichen Kombinationen aus Pressdruck und WPG. Ausgangsdaten: 4 mal 4 Wertepaare (WPG/Druck) mit Mittelwerten für die Darrdichte über alle Harze; In Matrix umgewandelt und interpoliert auf 9*11 Gitterpunkte nach dem Renka-Cline Verfahren.

Abbildung 41: 8-lagiges Buchen-LVL ohne Sperrlagen. Untersuchung der Korrelation zwischen spezifischem Pressdruck und Verdichtung für unterschiedliche WPGs des Harztyps A durch lineare Regressionsmodelle.

Abbildung 42: Verdichtung von Buchen-LVL in Abhängigkeit von Pressdruck und WPG......... 63 
Abbildung 43: Feuchte des 10-lagigen Buchen-LVL-Prüfkörper ohne Sperrlagen $\left(10^{*} 2,13 \mathrm{~mm}\right)$ nach Wasserlagerung gemäß EN84

Abbildung 44: Korrelation der maximalen Feuchte nach Wasserlagerung (EN84) und der Darrdichte von modifiziertem Buchen-LVL $(10 * 2,13 \mathrm{~mm})$

Abbildung 45: Feuchte der Buchen-LVL Prüfkörper ohne Sperrlagen (8-lagig) nach Wasserlagerung gemäß EN84- a) $2 \mathrm{~N} / \mathrm{mm}^{2}$ Pressdruck; b) $6 \mathrm{~N} / \mathrm{mm}^{2}$ Pressdruck

Abbildung 46: Feuchte der Buchen-LVL Prüfkörper mit Sperrlagen (8-lagig) nach Wasserlagerung gemäß EN84.

Abbildung 47: Korrelation der maximalen Feuchte nach Wasserlagerung (EN84) und der Darrdichte von modifiziertem Buchen-LVL $(8 * 3,7 \mathrm{~mm})$.

Abbildung 48: Dickenquellung des 10-lagigen Buchen-LVL-Prüfkörper ohne Sperrlagen $(10 * 2,13 \mathrm{~mm})$ nach Wasserlagerung gemäß EN84.

Abbildung 49: Dickenquellung der Buchen-LVL-Prüfkörper ohne Sperrlagen (8-lagig) nach Wasserlagerung gemäß EN84- a) $2 \mathrm{~N} / \mathrm{mm}^{2}$ Pressdruck; b) $6 \mathrm{~N} / \mathrm{mm}^{2}$ Pressdruck.......... 70

Abbildung 50: Dickenquellung der Buchen-LVL-Prüfkörper mit Sperrlagen (8-lagig) nach Wasserlagerung gemäß EN84- a) $2 \mathrm{~N} / \mathrm{mm}^{2}$ Pressdruck; b) $6 \mathrm{~N} / \mathrm{mm}^{2}$ Pressdruck........... 71

Abbildung 51: Volumenquellung der Buchen-LVL ohne Sperrlagen nach Wasserlagerung gemäß EN84- a) $2 \mathrm{~N} / \mathrm{mm}^{2}$ Pressdruck; b) $6 \mathrm{~N} / \mathrm{mm}^{2}$ Pressdruck.

Abbildung 52: Verringerung der Dickenquellung ausgedrückt als ASE ${ }_{\text {rad }}$ von Buchen-LVL mit Sperrlagen (8-lagig) nach Wasserlagerung gemäß EN84.

Abbildung 53: Verringerung der Dickenquellung ausgedrückt als ASE $\mathrm{rad}_{\mathrm{d}}$ von Buchen-LVL ohne Sperrlagen (8-lagig, parallel) nach Wasserlagerung gemäß EN84.

Abbildung 54:Volumenquellung nach 4-stündiger Kochung von Buchen-LVL ohne Sperrlagen mit den Furnierstärken 3,7mm (8-lagig) und 2,13mm (15-lagig). Herstellung bei unterschiedlichen Pressdrücken; a) $p=2 \mathrm{~N} / \mathrm{mm}^{2}$, b) $\mathrm{p}=4 \mathrm{~N} / \mathrm{mm}^{2}$ und c) $\mathrm{p}=6 \mathrm{~N} / \mathrm{mm}^{2} \ldots 75$

Abbildung 55: Volumenquellung nach 4-stündiger Kochung von Buchen-LVL ohne Sperrlagen unter Verwendung verschiedener Harze; 8-lagig, Furnierstärke 3,7mm. Hergestellt bei a) $\mathrm{p}=2 \mathrm{~N} / \mathrm{mm}^{2}$, b) $\mathrm{p}=4 \mathrm{~N} / \mathrm{mm}^{2}$ und c) $\mathrm{p}=6 \mathrm{~N} / \mathrm{mm}^{2}$ .76

Abbildung 56: Volumenquellung nach 4-stündiger Kochung von Buchen-LVL mit 2 Sperrlagen unter Verwendung verschiedener Harze; 8-lagig, Furnierstärke 3,7mm. Hergestellt bei a) $\mathrm{p}=2 \mathrm{~N} / \mathrm{mm}^{2}$ und b) $\mathrm{p}=6 \mathrm{~N} / \mathrm{mm}^{2}$.

Abbildung 57: Biegefestigkeit von Buchen-Furnierschichtholz mit 2 Sperrlagen (\#) und ausschließlich parallelem Faserverlauf (II). Variation der Schichtdicke (3,7 mm und 2,13 $\mathrm{mm}$ ) und des WPG bei einem Pressdruck von $2 \mathrm{~N} / \mathrm{mm}^{2}$ unter Verwendung des Harztyp A... .78

Abbildung 58: Biegefestigkeit von Buchen-Furnierschichtholz mit 2 Sperrlagen (\#) und ausschließlich parallelem Faserverlauf (II). Variation der Schichtdicke (3,7 mm und 2,13 $\mathrm{mm}$ ) und des WPG bei einem Pressdruck von $4 \mathrm{~N} / \mathrm{mm}^{2}$ unter Verwendung des Harztyp A. .79

Abbildung 59: Biegefestigkeit von Buchen-Furnierschichtholz mit 2 Sperrlagen (\#) und ausschließlich parallelem Faserverlauf (II). Variation der Schichtdicke (3,7 $\mathrm{mm}$ und 2,13 $\mathrm{mm}$ ) und des WPG bei einem Pressdruck von $6 \mathrm{~N} / \mathrm{mm}^{2}$ unter Verwendung des Harztyp A... 
Abbildung 60: Biegefestigkeit von Buchen-Furnierschichtholz mit parallelem Faserverlauf (II) bei einer Furnierdicke von 3,7 mm unterschieden nach WPG (nur Harztyp A) und Pressdruck

Abbildung 61: Biegefestigkeit von 8-lagigem Buchen-LVL ohne Sperrlagen bei unterschiedlichen WPGS unter Verwendung verschiedener Harze und Pressdrücke $(a=$ $2 \mathrm{~N} / \mathrm{mm}^{2}, \mathrm{~b}=6 \mathrm{~N} / \mathrm{mm}^{2}$ ).

Abbildung 62: Biegefestigkeit von 8-lagigem Buchen-LVL mit zwei Sperrlagen bei unterschiedlichen WPGS unter Verwendung verschiedener Harze und Pressdrücke $(a=$ $2 \mathrm{~N} / \mathrm{mm}^{2}, \mathrm{~b}=6 \mathrm{~N} / \mathrm{mm}^{2}$ ).

Abbildung 63: Korrelation von Biegefestigkeit und Darrdichte bei 8-lagigem Buchen-LVL mit Sperrlagen aus modifizierten und unbehandelten Furnieren. WPG: 0\%-60\%.

Abbildung 64: Korrelation von Biegefestigkeit und Darrdichte bei 8-lagigem Buchen-LVL ohne Sperrlagen aus modifizierten und unbehandelten Furnieren. WPG: 0\%-60\%.

Abbildung 65: Korrelation von Biegefestigkeit und Verdichtung bei 8-lagigem Buchen-LVL mit Sperrlagen aus modifizierten und unbehandelten Furnieren. WPG: 0\%-60\%.

Abbildung 66: Korrelation von Biegefestigkeit und Verdichtung bei 8-lagigem Buchen-LVL ohne Sperrlagen aus modifizierten und unbehandelten Furnieren. WPG: 0\%-60\%...... 85

Abbildung 67: Biege-Elastizitätsmodul von Buchen-Furnierschichtholz mit 2 Sperrlagen (\#) und ausschließlich parallelem Faserverlauf (II). Variation der Schichtdicke $(3,7 \mathrm{~mm}$ und 2,13 $\mathrm{mm}$ ) und des WPG bei einem Pressdruck von $2 \mathrm{~N} / \mathrm{mm}^{2}$ unter Verwendung des Harztyp A.

Abbildung 68: Biege-Elastizitätsmodul von Buchen-Furnierschichtholz mit 2 Sperrlagen (\#) und ausschließlich parallelem Faserverlauf (II). Variation der Schichtdicke (3,7 mm und 2,13 mm) und des WPG bei einem Pressdruck von $4 \mathrm{~N} / \mathrm{mm}^{2}$ unter Verwendung des Harztyp A. .86

Abbildung 69: Biege-Elastizitätsmodul von Buchen-LVL ohne Sperrlagen im 3-PunktBiegeversuch mit 3,7mm und 2,13 mm dicken Furnieren bei unterschiedlichen WPGs und Pressdrücken.

Abbildung 70 Biege-Elastizitätsmodul von 8-lagigem Buchen-LVL mit Sperrlagen (a) und ohne Sperrlagen (b) bei unterschiedlichen WPGs unter Verwendung verschiedener Harze beim Pressdruck von $2 \mathrm{~N} / \mathrm{mm}^{2}$.

Abbildung 71: Verdichtung der Prüfkörper für die Ermittlung der Bruchschlagarbeit. 8-lagiges Buchen-LVL ohne Sperrlagen; Furnierdicke 3,7mm, Harztyp A.

Abbildung 72: Darrdichte der Prüfkörper bei der Ermittlung der Bruchschlagarbeit an 8lagigem Buchen-Furnierschichtholz mit einer Furnierdicke von 3,7mm. Harztyp A......89

Abbildung 73: Biege-Elastizitätsmodul (MOE) der Prüfkörper bei der Ermittlung der Bruchschlagarbeit an 8-lagigem Buchen-Furnierschichtholz mit einer Furnierdicke von 3,7 mm bei unterschiedlichen Pressdrücken

Abbildung 74: Ergebnisse der Bruchschlagarbeitsprüfung an 8-lagigem BuchenFurnierschichtholz mit einer Furnierdicke von $3,7 \mathrm{~mm}$ bei unterschiedlichen Pressdücken und WPGs mit dem Harztyp A.

Abbildung 75: Korrelation zwischen Elastizitätsmodul und Bruchschlagarbeit von BuchenLVL, modifziert mit Harztyp A mit WPGs von $0 \%, 15 \%, 30 \%$ und $60 \%$, bei einem Pressdruck von $2 \mathrm{~N} / \mathrm{mm}^{2}$. 
Abbildung 76: Korrelation zwischen Elastizitätsmodul und Bruchschlagarbeit von verdichtetem Buchen-LVL, modifziert mit Harztyp A mit WPGs von 0\%, 15\%, 30\% und $60 \%$, bei einem Pressdruck von $4 \mathrm{~N} / \mathrm{mm}^{2}$

Abbildung 77: Korrelation zwischen Elastizitätsmodul und Bruchschlagarbeit von verdichtetem Buchen-LVL, modifziert mit Harztyp A mit WPGs von 0\%, 15\%, 30\% und $60 \%$, bei einem Pressdruck von $6 \mathrm{~N} / \mathrm{mm}^{2}$.

Abbildung 78: Formaldehydabgabe (Ausgleichsfunktion und Einzelwerte) von Buchen-LVL $(10 * 2,13 \mathrm{~mm})$ mit einem WPG von $30 \%$ bei zwei unterschiedlichen Presszeiten, gemessen nach Prüfkammer-Methode (DIN EN 717-1: 2005).

Abbildung 79: Temperaturverlauf in der Plattenmitte von Buchen-LVL mit einem WPG 30\% bei einem Pressdruck von $2 \mathrm{~N} / \mathrm{mm}^{2}$ und einer Presstemperatur von $145^{\circ} \mathrm{C}$

Abbildung 80: Formaldehydabgabe von 8-lagigem Buchen-LVL $\left(8^{*} 3,7 \mathrm{~mm}\right)$ unter Verwendung unterschiedlicher Harztypen beim WPG von 30\% und einer Presszeit von $45 \min (\sim 1,5$ min / mm), gemessen nach der Gasanalyse-Methode (DIN EN 717-2: 1994). 95

Abbildung 81: Masseverlust von Buchen-LVL ohne Sperrlagen $(10 * 2,13 \mathrm{~mm})$ im Abbauversuch mit Weißfäule (Trametes versicolor) nach ENV 12038 (2002). Modifizierung mit Harztyp A im Vakuum- und im Tauchverfahren. .96

Abbildung 82: Masseverlust von Buchen-LVL mit Sperrlagen (8-lagig, Furnierdicke 3,7mm) im Abbauversuch mit Weißfäule (Trametes versicolor) nach EN 12038. .97

Abbildung 83: Masseverlust von Buchen-LVL ohne Sperrlagen (8-lagig, parallel, Furnierdicke 3,7 mm) im Abbauversuch mit Weißfäule (Trametes versicolor) nach EN 12038. 98

Abbildung 84: Unbehandelte Referenzprüfkörper (Buchen-LVL) nach 2 Jahren im Erdkontakt (EN 252, 2015). 100

Abbildung 85: Ergebnisse der Evaluierung der Freilandprüfung mit Erdkontakt in Anlehnung an EN252 (2015). Mittlere Bewertungsziffern der modifizierten Buchen-LVL mit dem Harztyp A bei Pressdrücken von $2 \mathrm{~N} / \mathrm{mm}^{2}, 4 \mathrm{~N} / \mathrm{mm}^{2}$ und $6 \mathrm{~N} / \mathrm{mm}^{2}$ und WPGs von 15\%, $30 \%$ und $60 \%(n=9)$

Abbildung 86: Ergebnisse der Evaluierung der Freilandprüfung mit Erdkontakt in Anlehnung an EN252 (2015). Mittlere Bewertungsziffern der modifizierten Buchen-LVL mit den Harztypen A, F und G bei den Pressdrücken $2 \mathrm{~N} / \mathrm{mm}^{2}$ und $6 \mathrm{~N} / \mathrm{mm}^{2}$ beim WPG von 15\% $(n=9)$.

Abbildung 87: Ergebnisse der Evaluierung der Freilandprüfung mit Erdkontakt in Anlehnung an EN252 (2015). Mittlere Bewertungsziffern der modifizierten Buchen-LVL mit den Harztypen A, C, D und E bei den Pressdrücken $2 \mathrm{~N} / \mathrm{mm}^{2}$ und $6 \mathrm{~N} / \mathrm{mm}^{2}$ beim WPG von $30 \%(\mathrm{n}=9)$.

Abbildung 88: Wasseraufnahme bei Buchen-LVL $(8 * 3,7 \mathrm{~mm})$ mit (\#)und ohne Sperrlagen $(/ /)$, hergestellt bei $2 \mathrm{~N} / \mathrm{mm}^{2}$ mit unterschiedlichem WPG (Harztyp A). 103

Abbildung 89: Wasseraufnahme bei Buchen-LVL $(8 * 3,7 \mathrm{~mm})$ mit (\#)und ohne Sperrlagen $(/ /)$, hergestellt bei $4 \mathrm{~N} / \mathrm{mm}^{2}$ mit unterschiedlichem WPG (Harztyp A). 104

Abbildung 90: Wasseraufnahme bei Buchen-LVL $(8 * 3,7 \mathrm{~mm}$ ) mit (\#)und ohne Sperrlagen (//), hergestellt bei $6 \mathrm{~N} / \mathrm{mm}^{2}$ mit unterschiedlichem WPG (Harztyp A) 104

Abbildung 91: Dickenquellung bei Buchen-LVL $(8 * 3,7 \mathrm{~mm})$ mit (\#)und ohne Sperrlagen $(/ /)$, hergestellt bei $2 \mathrm{~N} / \mathrm{mm}^{2}$ mit unterschiedlichem WPG (Harztyp A) 105 
Abbildung 92: Dickenquellung bei Buchen-LVL $(8 * 3,7 \mathrm{~mm})$ mit (\#)und ohne Sperrlagen $(/ /)$, hergestellt bei $4 \mathrm{~N} / \mathrm{mm}^{2}$ mit unterschiedlichem WPG (Harztyp A).

Abbildung 93: Dickenquellung bei Buchen-LVL $(8 * 3,7 \mathrm{~mm})$ mit (\#)und ohne Sperrlagen $(/ /)$, hergestellt bei $6 \mathrm{~N} / \mathrm{mm}^{2}$ mit unterschiedlichem WPG (Harztyp A). 106

Abbildung 94: Wasseraufnahme in der Schnellbewitterung von Buchen-LVL $(8 * 3,7 \mathrm{~mm}) \mathrm{mit}$ Sperrlagen, hergestellt bei einem Pressdruck von $2 \mathrm{~N} / \mathrm{mm}^{2}$ mit einem WPG von 30\% (links) und 60\% (rechts) unter Verwendung verschiedener PF-Harze.

Abbildung 95: Wasseraufnahme in der Schnellbewitterung von Buchen-LVL $(8 * 3,7 \mathrm{~mm}) \mathrm{mit}$ Sperrlagen, hergestellt bei einem Pressdruck von $6 \mathrm{~N} / \mathrm{mm}^{2}$ mit einem WPG von $30 \%$ (links) und 60\% (rechts) unter Verwendung verschiedener PF-Harze. 107

Abbildung 96: Wasseraufnahme in der Schnellbewitterung von Buchen-LVL $(8 * 3,7 \mathrm{~mm}$, parallel) ohne Sperrlagen, hergestellt bei einem Pressdruck von $2 \mathrm{~N} / \mathrm{mm}^{2}$ (links) und 6 $\mathrm{N} / \mathrm{mm}^{2}$ (rechts) bei WPGs von $30 \%$ und $60 \%$ unter Verwendung verschiedener PFHarze. 108

Abbildung 97: Dickenquellung in der Schnellbewitterung von Buchen-LVL $\left(8^{*} 3,7 \mathrm{~mm}\right) \mathrm{mit}$ Sperrlagen, hergestellt bei einem Pressdruck von $2 \mathrm{~N} / \mathrm{mm}^{2}$ mit einem WPG von $30 \%$ (links) und 60\% (rechts) unter Verwendung verschiedener PF-Harze. 108

Abbildung 98: Dickenquellung in der Schnellbewitterung von Buchen-LVL $(8 * 3,7 \mathrm{~mm}) \mathrm{mit}$ Sperrlagen, hergestellt bei einem Pressdruck von $6 \mathrm{~N} / \mathrm{mm}^{2}$ mit einem WPG von 30\% (links) und 60\% (rechts) unter Verwendung verschiedener PF-Harze.

Abbildung 99: Dickenquellung in der Schnellbewitterung von Buchen-LVL ( $8 * 3,7 \mathrm{~mm}$, parallel) ohne Sperrlagen, hergestellt bei einem Pressdruck von $2 \mathrm{~N} / \mathrm{mm}^{2}$ (links) und $6 \mathrm{~N} / \mathrm{mm}^{2}$ (rechts) bei WPGs von 30\% und 60\% unter Verwendung verschiedener PF-Harze.... 110

Abbildung 100: Bewertungsziffern (0-5) nach DIN ISO 4628-4 für die Menge der Risse in der Vorderseite von modifizierten Buchen-LVL (8-lagig mit 2 Sperrlagen) nach der Schnellbewitterung in Abhängigkeit von der Dauer, des Harztyps, des WPG und des Pressdruckes (p).

Abbildung 101: Bewertungsziffern (0-5) nach DIN ISO 4628-4 für die Menge der Risse in der Vorderseite von modifizierten Buchen-LVL (8-lagig parallel) nach der Schnellbewitterung in Abhängigkeit von der Dauer, des Harztyps, des WPG und des Pressdruckes (p). 112

Abbildung 102: Bewertungsziffern (0-5) nach DIN ISO 4628-4 für die Breite der Risse in der Vorderseite von Buchen-LVL (8-lagig mit zwei Sperrlagen), hergestellt mit verschiedenen Drücken, WPG und Harztypen, in Abhängigkeit von der Zeit in der Schnellbewitterung.. 113

Abbildung 103: Bewertungsziffern (0-5) nach DIN ISO 4628-4 für die Breite der Risse in der Vorderseite von Buchen-LVL (8-lagig parallel), hergestellt ohne Sperrlagen mit verschiedenen Drücken, WPG und Harztypen, in Abhängigkeit von der Zeit in der Schnellbewitterung.. 114

Abbildung 104: Wasseraufnahme von Buchen-LVL (8-lagig parallel) in der Schnellbewitterung; beschichtet und unbeschichtet mit verschiedenen WPGs bei einem Pressdruck von 2 $\mathrm{N} / \mathrm{mm}^{2}$. 115 
Abbildung 105: Dickenquellung von Buchen-LVL (8-lagig parallel) in der Schnellbewitterung; beschichtet und unbeschichtet mit verschiedenen WPGs bei einem Pressdruck von 2 $\mathrm{N} / \mathrm{mm}^{2}$.

Abbildung 106: Klimadaten zum Niederschlag, der Lufttemperatur und relativen Luftfeuchte in $2 \mathrm{~m}$ Höhe über dem Boden, gemessen an der Messstation im Forstbotanischen Garten in Göttingen.

Abbildung 107: Gewichtsänderung unbeschichteter PF-modifizierter Buchen-LVL-Prüfkörper (8-lagig mit Sperrlagen) in Relation zum Ausgangsgewicht sowie monatliche Niederschlagmengen (Sekundärachse).

Abbildung 108: Dickenquellung unbeschichteter PF-modifizierter Buchen-LVL-Prüfkörper (8lagig mit Sperrlagen) in Relation zum Ausgangszustand sowie monatliche Niederschlagmengen (Sekundärachse). 118

Abbildung 109: Gewichtsänderung unbeschichteter PF-modifizierter Buchen-LVL-Prüfkörper (8-lagig mit Sperrlagen), mit einem WPG von 15\% bei unterschiedlichen Pressdrücken, in Relation zum Ausgangsgewicht sowie monatliche Niederschlagmengen (Sekundärachse).

Abbildung 110: Dickenquellung unbeschichteter PF-modifizierter Buchen-LVL-Prüfkörper (8lagig mit Sperrlagen), mit einem WPG von 15\% bei unterschiedlichen Pressdrücken, in Relation zum Ausgangszustand sowie monatliche Niederschlagmengen (Sekundärachse)

Abbildung 111: Gewichtsänderung unbeschichteter PF-modifizierter Buchen-LVL-Prüfkörper (8-lagig mit Sperrlagen), mit einem WPG von 30\% bei unterschiedlichen Pressdrücken, in Relation zum Ausgangsgewicht sowie monatliche Niederschlagmengen (Sekundärachse).

Abbildung 112: Dickenquellung unbeschichteter PF-modifizierter Buchen-LVL-Prüfkörper (8lagig mit Sperrlagen), mit einem WPG von 30\% bei unterschiedlichen Pressdrücken, in Relation zum Ausgangszustand sowie monatliche Niederschlagmengen (Sekundärachse). 121

Abbildung 113: Gewichtsänderung unbeschichteter PF-modifizierter Buchen-LVL-Prüfkörper (8-lagig mit Sperrlagen), mit einem WPG von 30\% bei unterschiedlichen Pressdrücken, in Relation zum Ausgangsgewicht sowie monatliche Niederschlagmengen (Sekundärachse).

Abbildung 114: Dickenquellung unbeschichteter PF-modifizierter Buchen-LVL-Prüfkörper (8lagig mit Sperrlagen), mit einem WPG von 30\% bei unterschiedlichen Pressdrücken, in Relation zum Ausgangszustand sowie monatliche Niederschlagmengen (Sekundärachse).

Abbildung 115: Bewertungsziffern (0-5) nach DIN ISO 4628-4 für die Menge der Risse in der Vorderseite von mit dem Harztyp A modifizierten Buchen-LVL (8-lagig mit zwei Sperrlagen) in Abhängigkeit von der Bewitterungsdauer im Freiland.

Abbildung 116: Bewertungsziffern (0-5) nach DIN ISO 4628-4 für die Breite der Risse in der Vorderseite von mit dem Harztyp A modifizierten Buchen-LVL (8-lagig mit zwei Sperrlagen) in Abhängigkeit von der Bewitterungsdauer im Freiland. 124 
Abbildung 117: Bewertungsziffern (0-5) nach DIN ISO 4628-4 für die Menge der Risse in der Vorderseite von mit dem Harztyp A modifizierten Buchen-LVL (8-lagig parallel) in Abhängigkeit von der Bewitterungsdauer im Freiland 125

Abbildung 118: Bewertungsziffern (0-5) nach DIN ISO 4628-4 für die Breite der Risse in der Vorderseite von mit dem Harztyp A modifizierten Buchen-LVL (8-lagig parallel) in Abhängigkeit von der Bewitterungsdauer im Freiland 126

Abbildung 119: Gewichtsänderung unbeschichteter PF-modifizierter Buchen-LVL-Prüfkörper (8-lagig mit Sperrlagen), mit einem WPG von 30\% bei einem Pressdruck von $2 \mathrm{~N} / \mathrm{mm}^{2}$, in Relation zum Ausgangsgewicht sowie monatliche Niederschlagmengen (Sekundärachse). 127

Abbildung 120: Gewichtsänderung unbeschichteter PF-modifizierter Buchen-LVL-Prüfkörper (8-lagig mit Sperrlagen), mit einem WPG von $60 \%$ bei einem Pressdruck von $2 \mathrm{~N} / \mathrm{mm}^{2}$, in Relation zum Ausgangsgewicht sowie monatliche Niederschlagmengen (Sekundärachse).

Abbildung 121: Gewichtsänderung unbeschichteter PF-modifizierter Buchen-LVL-Prüfkörper (8-lagig mit Sperrlagen), mit einem WPG von 30\% bei einem Pressdruck von $6 \mathrm{~N} / \mathrm{mm}^{2}$, in Relation zum Ausgangsgewicht sowie monatliche Niederschlagmengen (Sekundärachse) 128

Abbildung 122: Gewichtsänderung unbeschichteter PF-modifizierter Buchen-LVL-Prüfkörper (8-lagig mit Sperrlagen), mit einem WPG von 60\% bei einem Pressdruck von $6 \mathrm{~N} / \mathrm{mm}^{2}$, in Relation zum Ausgangsgewicht sowie monatliche Niederschlagmengen (Sekundärachse) 128

Abbildung 123: Dickenquellung unbeschichteter PF-modifizierter Buchen-LVL-Prüfkörper (8lagig mit Sperrlagen), mit einem WPG von 30\% bei einem Pressdruck von $2 \mathrm{~N} / \mathrm{mm}^{2}$, in Relation zur Ausgangsdicke vor Beginn der Freilandbewitterung.

Abbildung 124: Dickenquellung unbeschichteter PF-modifizierter Buchen-LVL-Prüfkörper (8lagig mit Sperrlagen), mit einem WPG von $60 \%$ bei einem Pressdruck von $2 \mathrm{~N} / \mathrm{mm}^{2}$, in Relation zur Ausgangsdicke vor Beginn der Freilandbewitterung. 130

Abbildung 125: Dickenquellung unbeschichteter PF-modifizierter Buchen-LVL-Prüfkörper (8lagig mit Sperrlagen), mit einem WPG von 30\% bei einem Pressdruck von $6 \mathrm{~N} / \mathrm{mm}^{2}$, in Relation zur Ausgangsdicke vor Beginn der Freilandbewitterung 131

Abbildung 126: Dickenquellung unbeschichteter PF-modifizierter Buchen-LVL-Prüfkörper (8lagig mit Sperrlagen), mit einem WPG von $60 \%$ bei einem Pressdruck von $6 \mathrm{~N} / \mathrm{mm}^{2}$, in Relation zur Ausgangsdicke vor Beginn der Freilandbewitterung. 132

Abbildung 127: Bewertungsziffern (0-5) nach DIN ISO 4628-4 für die Menge der Risse in der Vorderseite von PF-modifiziertem Buchen-LVL (8-lagig) mit zwei Sperrlagen in Abhängigkeit von der Bewitterungsdauer im Freiland. 132

Abbildung 128: Bewertungsziffern (0-5) nach DIN ISO 4628-4 für die Breite der Risse in der Vorderseite von PF-modifiziertem Buchen-LVL (8-lagig) mit zwei Sperrlagen in Abhängigkeit von der Bewitterungsdauer im Freiland 133

Abbildung 129: Gewichtsänderung unbeschichteter, PF-modifizierter Buchen-LVL-Prüfkörper (8-lagig parallel) mit einem WPG von $15 \%$ bei einem Pressdruck von $2 \mathrm{~N} / \mathrm{mm}^{2}$, in Relation zum Ausgangsgewicht. 134 
Abbildung 130: Gewichtsänderung unbeschichteter, PF-modifizierter Buchen-LVL-Prüfkörper (8-lagig parallel) mit einem WPG von 30\% bei einem Pressdruck von $2 \mathrm{~N} / \mathrm{mm}^{2}$, in Relation zum Ausgangsgewicht. 135

Abbildung 131: Gewichtsänderung unbeschichteter, PF-modifizierter Buchen-LVL-Prüfkörper (8-lagig parallel) mit einem WPG von $60 \%$ bei einem Pressdruck von $2 \mathrm{~N} / \mathrm{mm}^{2}$, in Relation zum Ausgangsgewicht. 135

Abbildung 132: Gewichtsänderung unbeschichteter, PF-modifizierter Buchen-LVL-Prüfkörper (8-lagig parallel) mit einem WPG von $15 \%$ bei einem Pressdruck von $6 \mathrm{~N} / \mathrm{mm}^{2}$, in Relation zum Ausgangsgewicht. 136

Abbildung 133: Gewichtsänderung unbeschichteter, PF-modifizierter Buchen-LVL-Prüfkörper (8-lagig parallel) mit einem WPG von $30 \%$ bei einem Pressdruck von $6 \mathrm{~N} / \mathrm{mm}^{2}$, in Relation zum Ausgangsgewicht. 136

Abbildung 134 Gewichtsänderung unbeschichteter, PF-modifizierter Buchen-LVL-Prüfkörper (8-lagig parallel) mit einem WPG von $60 \%$ bei einem Pressdruck von $6 \mathrm{~N} / \mathrm{mm}^{2}$, in Relation zum Ausgangsgewicht. 137

Abbildung 135: Dickenquellung unbeschichteter, PF-modifizierter Buchen-LVL-Prüfkörper (8lagig parallel) mit einem WPG von 15\% bei einem Pressdruck von $2 \mathrm{~N} / \mathrm{mm}$. 137

Abbildung 136: Dickenquellung unbeschichteter, PF-modifizierter Buchen-LVL-Prüfkörper (8lagig parallel) mit einem WPG von 30\% bei einem Pressdruck von 2 N/mm. 138

Abbildung 137: Dickenquellung unbeschichteter, PF-modifizierter Buchen-LVL-Prüfkörper (8lagig parallel) mit einem WPG von 60\% bei einem Pressdruck von $2 \mathrm{~N} / \mathrm{mm}$. 139

Abbildung 138: Dickenquellung unbeschichteter, PF-modifizierter Buchen-LVL-Prüfkörper (8lagig parallel) mit einem WPG von 15\% bei einem Pressdruck von $6 \mathrm{~N} / \mathrm{mm}$.

Abbildung 139: Dickenquellung unbeschichteter, PF-modifizierter Buchen-LVL-Prüfkörper (8lagig parallel) mit einem WPG von 30\% bei einem Pressdruck von $6 \mathrm{~N} / \mathrm{mm}$. 140

Abbildung 140: Dickenquellung unbeschichteter, PF-modifizierter Buchen-LVL-Prüfkörper (8lagig parallel) mit einem WPG von 30\% bei einem Pressdruck von $6 \mathrm{~N} / \mathrm{mm}$.

Abbildung 141: Bewertungsziffern (0-5) nach DIN ISO 4628-4 für die Menge der Risse in der Vorderseite von PF-modifiziertem Buchen-LVL mit 8 Lagen (parallel) in Abhängigkeit von der Bewitterungsdauer im Freiland.

Abbildung 142: Bewertungsziffern (0-5) nach DIN ISO 4628-4 für die Breite der Risse in der Vorderseite von PF-modifiziertem Buchen-LVL mit 8 Lagen (parallel) in Abhängigkeit der Bewitterungsdauer im Freiland.

Abbildung 143: Wasseraufnahme von beschichteten und unbeschichteten Buchen-LVL (8-lagig parallel, Pressdruck $2 \mathrm{~N} / \mathrm{mm}^{2}$ ) mit verschiedenen WPGs (Harztyp F) in der Freilandbewitterung über 12 Monate. Beschichtung: 1x Remmers Grundierung IL170 (60s getaucht) und $1 \times$ Remmers Wetterschutzlack UV+ (150 $\mu$ m gespritzt). 143

Abbildung 144: Dickenquellung von beschichteten und unbeschichteten Buchen-LVL (8-lagig parallel, Pressdruck $2 \mathrm{~N} / \mathrm{mm}^{2}$ ) mit verschiedenen WPGs (Harztyp F) in der Freilandbewitterung über 12 Monate. Beschichtung: 1x Remmers Grundierung IL170 (60s getaucht) und $1 \times$ Remmers Wetterschutzlack UV+ $(150 \mu \mathrm{m}$ gespritzt) 143

Abbildung 145: Bewertungsziffer (0-5) nach DIN ISO 4628-4 für die Menge der Risse in der Vorderseite von beschichteten und unbeschichteten modifizierten Buchen-LVL (8-lagig, parallel, $2 \mathrm{~N} / \mathrm{mm}^{2}$ Pressdruck) in Abhängigkeit von der Testdauer in der 
Schnellbewitterung. Beschichtung: 1x Remmers Grundierung IL170 (60s getaucht) und $1 \mathrm{x}$ Remmers Wetterschutzlack UV+ $(150 \mu \mathrm{m}$ gespritzt $)$.

Abbildung 146: Bewertungsziffer (0-5) nach DIN ISO 4628-4 für die Breite der Risse in der Vorderseite von beschichteten und unbeschichteten modifizierten Buchen-LVL (8-lagig, parallel, $2 \mathrm{~N} / \mathrm{mm}^{2}$ Pressdruck) in Abhängigkeit von der Testdauer in der Schnellbewitterung. Beschichtung: 1x Remmers Grundierung IL170 (60s getaucht) und $1 \mathrm{x}$ Remmers Wetterschutzlack UV+ $(150 \mu \mathrm{m}$ gespritzt $)$.

Abbildung 147: Farbwertveränderungen an unbeschichteten Buchen-LVL mit einem Pressdruck von $2 \mathrm{~N} / \mathrm{mm}^{2}$, abgebildet als delta L, delta C und delta E nach 12 Monaten Bewitterung auf dem Testfeld. 147

Abbildung 148: Unbehandeltes Buchen-LVL mit $2 \mathrm{~N} / \mathrm{mm}^{2}$ Pressdruck, vor (links) und nach einer 12-monatigen Bewitterung (rechts). 148

Abbildung 149: Buchen-LVL mit $2 \mathrm{~N} / \mathrm{mm}^{2}$ Pressdruck, modifiziert mit dem Harztyp B bei einem WPG von 60\%, vor (links) und nach einer 12-monatigen Bewitterung (rechts). 148

Abbildung 150: Buchen-LVL mit $2 \mathrm{~N} / \mathrm{mm}^{2}$ Pressdruck, modifiziert mit dem Harztyp D bei einem WPG von 48\%, vor (links) und nach einer 12-monatigen Bewitterung (rechts).

Abbildung 151: Verteilung der Helligkeit über die gesamte Prüfkörperoberfläche bei unbehandeltem Buchen-LVL (Referenz) vor der Bewitterung, gemessen mit Adobe Photoshop im Lab-Modus mit Wertebereich 0-255 vor der rechnerischen Korrektur.

Abbildung 152: Häufigkeitsverteilung der Helligkeit über die gesamte Prüfkörperoberfläche bei unbehandeltem Buchen-LVL (Referenz) nach 12-monatiger Bewitterung, gemessen mit Adobe Photoshop im Lab-Modus mit Wertebereich 0-255 vor der rechnerischen Korrektur. 150

Abbildung 153: Standardabweichungen der Helligkeit L zu Beginn und nach 12-monatiger Bewitterung von Buchen-LVL mit $2 \mathrm{~N} / \mathrm{mm}^{2}$ Pressdruck. 150

Abbildung 154: Standardabweichungen des Farbwerts a (Grün/Rot) zu Beginn und nach 12 monatiger Bewitterung von Buchen-LVL mit $2 \mathrm{~N} / \mathrm{mm}^{2}$ Pressdruck.

Abbildung 155: Standardabweichungen des Farbwerts b (Blau/Gelb) zu Beginn und nach 12monatiger Bewitterung von Buchen-LVL mit $2 \mathrm{~N} / \mathrm{mm}^{2}$ Pressdruck.

Abbildung 156: Buchen-LVL mit $2 \mathrm{~N} / \mathrm{mm}^{2}$ Pressdruck, modifiziert mit dem Harztyp B bei einem WPG von 30\%, vor (links) und nach einer 12-monatigen Bewitterung (rechts).

Abbildung 157: Farbwertveränderungen an unbeschichtetem Buchen-LVL mit einem Pressdruck von $6 \mathrm{~N} / \mathrm{mm}^{2}$, abgebildet als delta L, delta C und delta E nach 12 Monaten Bewitterung auf dem Testfeld. 153

Abbildung 158: Buchen-LVL mit $6 \mathrm{~N} / \mathrm{mm}^{2}$ Pressdruck, modifiziert mit dem Harztyp F bei einem WPG von 60\%, vor (links) und nach einer 12-monatigen Bewitterung (rechts). 154

Abbildung 159: UMSP-Messungen bei 278nm an Buche, wasserimpägniert und getrocknet. Fasern (a), Gefäßwand (b), Parenchym (c,d). 198 
Abbildung 160: UMSP-Messungen bei 278nm an Buche, Harztyp B, 12\% WPG. Gefäßwand (a), Gefäßwand und Fasern(b), Fasern (c), Parenchym (e). 198

Abbildung 161: UMSP-Messungen bei 278nm an Buche, Harztyp A, 16\% WPG. Fasern (a,b), Gefäßwand (c), Parenchym (d,e).

Abbildung 162: UMSP-Messungen bei 278nm an Buche, Harztyp A-gealtert, 17\% WPG. Gefäßwand (a), Fasern (b,c), Parenchym (d). 199

Abbildung 163: UMSP-Messungen bei 278nm an Buche, Harztyp A, 31\% WPG. Gefäßwand (a), Fasern (b,c), Parenchym (d,e). 200

Abbildung 164: Oberflächen von unbeschichtetem Buchen-LVL in Abhängigkeit vom WPG und der Bewitterungsdauer im Freiland in Anlehung an EN 927-3 (2006) unter Verwendung des Harztyps A und einem Pressdruck von $2 \mathrm{~N} / \mathrm{mm}^{2}$. 201

Abbildung 165: Oberflächen von unbeschichtetem Buchen-LVL in Abhängigkeit vom WPG und der Bewitterungsdauer im Freiland in Anlehung an EN 927-3 (2006) unter Verwendung des Harztyps A und einem Pressdruck von $2 \mathrm{~N} / \mathrm{mm}^{2}$. 202

Abbildung 166: Oberflächen von unbeschichtetem Buchen-LVL in Abhängigkeit vom WPG und der Bewitterungsdauer im Freiland in Anlehung an EN 927-3 (2006) unter Verwendung des Harztyps A und einem Pressdruck von $6 \mathrm{~N} / \mathrm{mm}^{2}$. 203

Abbildung 167: Oberflächen von unbeschichtetem Buchen-LVL mit einem WPG von 60\% in Abhängigkeit der Bewitterungsdauer im Freiland in Anlehung an EN 927-3 (2006) unter Verwendung des Harztyps A und einem Pressdruck von $6 \mathrm{~N} / \mathrm{mm}^{2}$. 204

Abbildung 168: Oberflächen von Buchen-LVL mit unterschiedlichen Harztypen (A, B, C, D, E, F, G) bei einem WPG von 30\% und einem Pressdruck von $2 \mathrm{~N} / \mathrm{mm}^{2}$ zu Beginn und nach 12 Monaten der Bewitterung in Anlehnung an EN 927-3 (2006) 205

Abbildung 169: Oberflächen von Buchen-LVL mit unterschiedlichen Harztypen (A, B, C, D, E, F, G) bei einem WPG von 30\% und einem Pressdruck von $2 \mathrm{~N} / \mathrm{mm}^{2}$ nach 36 Monaten der Bewitterung in Anlehnung an EN 927-3 (2006). 206 


\section{Quellenverzeichnis}

\subsection{Normen}

DIN CEN/TS 15083-1 (2005): Dauerhaftigkeit von Holz und Holzprodukten - Bestimmung der natürlichen Dauerhaftigkeit von Vollholz gegen holzzerstörende Pilze, Prüfverfahren - Teil 1: Basidiomyceten. Deutsches Institut für Normung e.V., Beuth Verlag GmbH, Berlin.

DIN 52189-1 (1981): Prüfung von Holz; Schlagbiegeversuch; Bestimmung der Bruchschlagarbeit. Deutsches Institut für Normung e.V., Beuth Verlag GmbH, Berlin.

DIN EN 252 (1989): Holzschutzmittel - Freiland-Prüfverfahren zur Bestimmung der relativen Schutzwirkung eines Holzschutzmittels im Erdkontakt. Deutsches Institut für Normung e.V., Berlin.

DIN EN 310 (1993): Holzwerkstoffe; Bestimmung des Biege-Elastizitätsmoduls und der Biegefestigkeit. Deutsches Institut für Normung e.V., Beuth Verlag GmbH, Berlin.

DIN EN 322 (1993): Holzwerkstoffe; Bestimmung des Feuchtegehaltes. Deutsches Institut für Normung e.V., Beuth Verlag GmbH, Berlin.

DIN EN 335 (2013): Dauerhaftigkeit von Holz und Holzprodukten - Gebrauchsklassen: Definitionen, Anwendung bei Vollholz und Holzprodukten. Deutsches Institut für Normung e.V., Beuth Verlag GmbH, Berlin.

DIN EN 350-2 (1994): Natürliche Dauerhaftigkeit von Vollholz. Teil 2: Leitfaden für die natürliche Dauerhaftigkeit und Tränkbarkeit von ausgewählten Holzarten von besonderer Bedeutung in Europa. Deutsches Institut für Normung e.V., Beuth Verlag GmbH, Berlin.

DIN EN 717-1 (2004): Holzwerkstoffe - Bestimmung der Formaldehydabgabe - Teil 1: Formaldehydabgabe nach der Prüfkammer-Methode. Deutsches Institut für Normung e.V., Beuth Verlag GmbH, Berlin.

DIN EN 717-2 (1995): Holzwerkstoffe - Bestimmung der Formaldehydabgabe - Teil 2: Formaldehydabgabe nach der Gasanalysemethode. Deutsches Institut für Normung e.V., Beuth Verlag GmbH, Berlin.

DIN EN 927-3 (2006): Beschichtungsstoffe - Beschichtungsstoffe und Beschichtungssysteme für Holz im Außenbereich - Teil 3: Freibewitterung. Europäisches Komitee für Normung (CEN), Brüssel.

DIN EN 1534 (2000): Parkett und andere Holzfußböden - Bestimmung des Eindruckwiderstandes (Brinell) - Prüfmethode. Deutsches Institut für Normung e.V., Beuth Verlag GmbH, Berlin.

DIN ISO 4628-4 (1997): Beurteilung von Beschichtungsschäden - Bewertung der Menge und Größe von Schäden und der Intensität von Veränderungen. Teil 4: Bewertung des Rissgrades. Deutsches Institut für Normung e.V., Berlin. 
DIN ENv 12038 (2002): Dauerhaftigkeit von Holz und Holzwerkstoffen Holzwerkstoffplatten - Bestimmung der Beständigkeit gegen holzzerstörende Basidiomyceten. Deutsches Institut für Normung e.V., Beuth Verlag GmbH, Berlin.

DIN EN 14374 (2016): Entwurf. Holzbauwerke - Furnierschichtholz (LVL) - Anforderungen. Deutsches Institut für Normung e.V., Beuth Verlag GmbH, Berlin.

EN 391 (2002): Brettschichtholz - Delaminierungsprüfung von Leimfugen. Deutsches Institut für Normung e.V., Beuth Verlag GmbH, Berlin.

EN 84 (1997): Beschleunigte Alterung von behandeltem Holz vor biologischen Prüfungen; Auswaschbeanspruchung. Deutsches Institut für Normung e.V., Beuth Verlag GmbH, Berlin.

EN 314 (2004): Sperrholz - Qualität der Verklebung. Europäisches Komitee für Normung (CEN), Brüssel.

\subsection{Literatur}

Aderhold, J. (2006). Economic production of plywood made of large diameter timber by improved production technologies. Conference proceedings. Cost Action E44 - E49, Valencia.

Ashby, M. F. (2013). Chapter 15 - Material profiles. Materials and the Environment (Second Edition). M. F. Ashby. Boston, Butterworth-Heinemann: 459-595.

Aydın, İ., Çolak, S., Çolakoğlu, G., Salih, E. (2004). "A comparative study on some physical and mechanical properties of Laminated Veneer Lumber (LVL) produced from Beech (Fagus orientalis Lipsky) and Eucalyptus (Eucalyptus camaldulensis Dehn.) veneers." Holz als Roh- und Werkstoff 62(3): 218-220.

Bauhus, J., Rock, J, et al. (2017): Beiträge der Forst- und Holzwirtschaft zum Klimaschutz. AFZDerWald 3/2017, Deutscher Landwirtschaftsverlag, Hannover.

Bal, B. C., Bektaş, İ. (2012). "The effects of some factors on the impact bending strength of laminated veneer lumber." Bioressources 7(4): 5855-5863.

Bicke, S., C. Mai, et al. (2012). Modification of beech veneers with low molecular weight phenol formaldehyde for the production of plywood: Durability and mechanical properties. European Conference on Wood Modification 2012, Ljubljana, Slovenia.

Bicke, S., H. Militz (2014). Modification of beech veneers with low molecular weight phenol formaldehyde for the production of plywood: Comparison of the submersion and vacuum impregnation. European Conference on Wood Modification 2014. Lisbon, Portugal.

Biziks, V., Bicke, S., Militz, H. (2015). Penetration of phenol formaldehyde (PF) resin into beech wood studied by light microscopy. In: Proceedings of 46th IRG Annual Meeting, Chile.

Biziks, V., Bicke, S., Militz, H. (2016). Decay resistance of beech wood and plywood treated with different type of phenol-formaldehyde (PF) resins. In: Proceedings of 47th IRG Annual Meeting, Portugal. 
Bicke, S., Biziks, V., Militz, H. (2016). Neue Holzmodifizierungsverfahren mit Phenolharzen. Deutsche Holzschutztagung, Dresden, Istitut für Holztechnologie Dresden gemeinnützige $\mathrm{GmbH}$.

Bicke, S., Biziks, V., Militz, H. (2017). Dauerhaftigkeit und Dickenquellung von mit Phenolharz modifizierten Laminated Veneer Lumber (LVL). Holztechnologie, 58 (5), 54-60.

Blomberger Holzindustrie (2012). Obo-Festholz in der Automobil- und Luftfahrtindustrie im Anlagen-, Maschinen- und Schiffbau für Kaltwalzwerke und Gießereien. Blomberger Holzindustrie B. Hausmann GmbH \& Co. KG, Blomberg.

BMEL (2012). Der Wald in Deutschland - Ausgewählte Ergebnisse der dritten Bundeswaldinventur. Bundesministerium für Ernährung und Landwirtschaft (BMEL), Berlin.

Bortoletto, G. (2006). Pinus merkusii LVL from Graded Veneer: Effect on Static Bending Properties. Second International Symposium on Veneer Processing Products, Vancouver.

BWI $^{3}$. (2012). Bundeswaldinventur 3. Berlin: Bundesministerium für Ernährung und Landwirtschaft (BMEL).

Deka, M., Saika, C. N. (2000). Chemical modification of wood with thermosetting resin: effect on dimensional stability and strength property. Bioresource Technology 73(2): pp. 179181.

Dieste, A., Krause, A., et al. (2008). Physical and mechanical properties of plywood produced with 1.3-dimethylol-4.5-dihydroxyethyleneurea (DMDHEU)-modified veneers of Betula spec. and Fagus sylvatica. Holz als Roh- und Werkstoff 66(4): pp. 281-287.

Döring, P., Glasenapp, S., Mantau, U. (2016). Energieholzverwendung in privaten Haushalten 2014. Marktvolumen und verwendete Holzsortimente. Hamburg: p. 37.

Dunky, J., Niemz, P. (2002). Holzwerkstoffe und Leime - Technologie und Einflussfaktoren. Springer Verlag Berlin Heidelberg.

Durchschlag, H., Zipper, P. (1994). "Calculation of the partial volume of organic compounds and polymers." Progress in Colloids \& Polymer Science 94: 20-39.

Dworschak, M. (2014). Bautechnik. Wachsende Windmühlen. Der Spiegel 14: pp. 126-127. SPIEGEL-Verlag Rudolf Augstein GmbH \& Co. KG, Hamburg.

Ehlbeck, J., Hättich, R. (1986). Mechanic properties of resin impregnated compressed wood. Holz als Roh- und Werkstoff 44(12): 449-452.

Epmeier, H., M. Westin, et al. (2004). Differently modified wood: Comparison of some selected properties. Scandinavian Journal of Forest Research 19(Suppl. 5): pp. 31-37.

Enoki, A., Itakura, S., Tanaka, H. (1997). The involvement of extracelluar substances for reducing molecular oxygen to hydroxyl radical and ferric iron to ferrous iron in wood degradation by wood decay fungi. Journal of Biotechnology 53 (2-3): pp. 265-272. 
Emmerich, L., Militz, H., Brischke, C. (2018). Long-term performance of DMDHEU-treated wood exposed in ground, above ground and in the maritime environment. The International Research Group on Wood Protection, Section 4 - Process and properties, IRG/WP1840825. Stockholm, Sweden.

Evans, P. D., Gibson, S. K., Cullis, I., Liu, C.L., Sebe, G. (2013). Photostabilization of wood using low molecular weight phenol formaldehyde resin and hindered amine light stabilizer. Polymer Degradation and Stability 98(1): 158-168.

Furuno, T., Y. Imamura, et al. (2004). The modification of wood by treatment with low molecular weight phenol-formaldehyde resin: a properties enhancement with neutralized phenolic-resin and resin penetration into wood cell walls. Wood Science and Technology 37(5): pp. 349-361.

Feist, W. B., Hon, D.N.-S. (1984). Chemistry of weathering and protection. Rowell, R.M. (ed.). The Chemistry of Solid Wood. ACS, Washington, D.C. Chapter 11, pp. 401-454.

Gabrielli, C., Kamke, F. (2010). Phenol-formaldehyde impregnation of densified wood for improved dimensional stability. Wood Science and Technology 44(1): 95-104.

Galperin, A. S., G. G. Kuleshov, et al. (1995). Manufacturing and properties of modified wood: A review of 25 years work. Holzforschung 49(1): pp. 45-50.

Grunwald, D. (2002). Kombinierte analytische Untersuchungen von Klebstoffen für Holzwerkstoffe. Berlin, Mensch \& Buch Verlag.

Guzy, J. (2015). Untersuchung von Phenolformaldehydharz-Isocyanat-modifiziertem Buchenholz. Masterarbeit. Abteilung Holzbiologie und Holzprodukte, Georg-AugustUniversität Göttingen.

Goodell, B., Qian, Y., Jellison, J. (2008). Fungal Decay of Wood: Soft Rot - Brown Rot - White Rot. In: Development of Commercial Wood Preservatives. Efficacy, Enviromental, and Health Issues. American Chemical Society, Washington, DC: pp. 9-31.

Habenicht, G. (2009). Kleben. Grundlagen, Technologien, Anwendungen. 6. aktualisierte Auflage. Springer - Verlag Berlin Heidelberg.

Hafner, A., Rüter, S., Ebert, S., et al. (2017). Treibhausgasbilanzierung von Holzgebäuden Umsetzung neuer Anforderungen an Ökobilanzen und Ermittlung empirischer Substitutionsfaktoren (THG-Holzbau), Fakultät Bau- und Umweltingenieurwissenschaften, Ruhr-Universität Bochum (RUB): 148.

Haupt, R. A., Sellers, T. (1994). Phenolic resin wood interaction. Forest Products Journal 44(2): 69-73.

Hergert, H., Sarkanen, K. Ludwig, C. (1971). Lignins: Occurrence, formation, structure and reactions. Edited by KV Sarkanen and CH Ludwig, Willey-Interscience, New York.

Hesse, M., Meier, H., Zeeh, B. (1991). Spektroskopische Methoden in der organischen Chemie, 4. Aufl., Thieme Verlag/Stuttgart-New York. 
Hill, C. A. S. (2006). Wood Modification - Chemical, Thermal and Other Processes. Weinheim, Wiley-VCH Verlag.

Hofrichter, M. (2002). Review: lignin conversion by manganese peroxidase (MnP). Enzyme and Microbial Technology 30 (4), pp. 454-466.

Hon, D. N.-S., Shiraishi, N. (2000). Wood and cellulosic chemistry, revised, and expanded, CRC Press.

Høiland H. (1986). In: Hinz, H.-J. (ed.). Thermodynamic Data for Biochemistry and Biotechnology. Springer Verlag, Berlin - Heidelberg - Berlin - New York - Tokyo: pp. 1744.

Hosseinpourpia, R., Mai, C. (2016). "Mode of action of brown rot decay resistance in phenolformaldehyde-modified wood: resistance to Fenton's reagent." Holzforschung 70(3): 253259.

Huang, Y., Fei, B., Zhao, R. (2014). "Investigation of Low-molecular Weight Phenol Formaldehyde Distribution in Tracheid Cell Walls of Chinese Fir Wood." BioResources 9(3): 4150-4158.

Jaffé, H., Orchin, M. (1962). Theory and application of UV spectroscopy. J. Wiley and Sons, London.

Knorz, M. (2012). Verklebung von Buchen - und Eschenholz für tragende Zwecke. Band 40: Stoffliche Nutzung von Laubholz. Fachagentur Nachwachsende Rohstoffe e.V. (FNR); Gülzow-Prüzen.

Koch, G., Grünwald, C. (2004). Application of UV microspectrophotometry for the topochemical detection of lignin and phenolic extractives in wood fibre cell walls. Wood fibre cell walls: methods to study their formation, structure and properties. Swedish University of Agricultural Sciences, Uppsala: 119-130.

Koch, G., Kleist, G. (2001). Application of scanning UV microspectrophotometry to localise lignins and phenolic extractives in plant cell walls. Holzforschung 55(6): 563-567.

Koch, G., Richter, H.-G., Schmitt, U. (2006). Topochemical investigation on phenolic deposits in the vessels of afzelia (Afzelia spp.) and merbau (Intsia spp.) heartwood. Holzforschung 60(6): 583-588.

Kollmann, F. (1951). Technologie des Holzes und der Holzwerkstoffe. Berlin-GöttingenHeidelberg, Springer-Verlag.

Ku, H., W. Jacobson, M. Trada, F. Cardona and D. Rogers (2008). Tensile Tests of Phenol Formaldehyde SLG Reinforced Composites: Pilot Study.

Lee, S. H., Ashaari, Z. (2015). Durability of phenolic-resin treated sesenduk (Endospermum diadenum and jelutong (Dyrea costulata) wood against white rot fungus. European Journal of Wood and Wood Products 73 (4): pp. 553-555. 
Lippke, B., Wilson, J., et al. (2004). CORRIM: Life-cycle environmental performance of renewable building materials. Forest Products Journal 54(6): pp. 8-19.

Lohmann, U. (2008). Holz-Lexikon. Nachschlagewerk für die Holz- und Forstwirtschaft. DRWVerlag Weinbrenner, Leinfelden-Echterdingen.

Lukowski, D. (1999). Holzschutz mit Melaminharzen. Dissertation. Fachbereich Biologie. Universität Hamburg, Hamburg.

Mai, T. H. (2009). Veneer Modification for the Production of Exterior Plywood. Dissertation. Faculty of Forest Sciences and Forest Ecology, Georg-August-University, Göttingen, Germany.

Mantanis, G. I., Young, R. A., Rowell, R. M. (1994). Swelling of wood. Part II. Swelling in organic liquids. Holzforschung 48, pp. 480-490

Mantau, U. (2012). Energieholzverwendung in privaten Haushalten: Marktvolumen und verwendete Holzsortimente: Abschlussbericht. Hrsg. von INFRO - Informationssysteme für nachwachsende Rohstoffe und Universität Hamburg, Zentrum für Holzwirtschaft. Hamburg: pp. 13 - 14.

Marutzky, R., Schwab, H. (2008). Sperrholz. Informationsverein Holz e.V., Berlin.

Millett, M. A., Seborg, R. M., Stamm, A. J. (1956). Influence of manufacturing variables on the impact resistance of resin-treated wood, Forest products laboratory.

Militz, H., Schaffert, S., et al. (2011). Termite resistance of DMDHEU-treated wood. Wood Science and Technology 45(3): pp. 547-557.

Minh, N. H. (2007). Wood Modification with Hydrophobation Textile Finishing Agents. GeorgAugust-Universität Göttingen. Dissertation.

Nicolas, D. D., Militz, H. (2008). Conceps in the Development of New Accelarated Test Methods for Wood Decay. In: Development of Commercial Wood Preservatives. Efficacy, Enviromental, and Helath Issues. American Chemical Society, Washington, DC: pp. 142151.

Niemz, P. (1993). Physik des Holzes und der Holzwerkstoffe. Leinfelden-Echterdingen, DRWVerlag Weinbrenner Gmbh \& Co.

Nzokou, P., Zyskowski, J., Boury, S., Kamdem, D. P. (2005). Natural decay resistance of LVL made of veneers from durable and non-durable wood species. Holz als Roh- und Werkstoff 63(3): 173-178.

Oehmichen, K., Demant, B., et al. (2011). Inventurstudie 2008 und Treibhausgasinventar Wald. Landbauforschung, Sonderheft 343, vTI: Braunschweig.

Ozyhar, T., Hering S., Niemz, P. (2012). Moisture-dependent elastic and strength anisotropy of European beech wood in tension. Journal of Materials Science 47(16): 6141-6150. 
Passauer, L., Schubert, J. Flade, P., Peters, J. (2016). Witterungsbeständigkeit transparenter Oberflächen von mit PF-Harz imprägniertem Schichtpressholz. Holztechnologie 57(4): 3442 .

Puettman, M., Wilson, J. (2004): Forest to Product: Cradle-to-Gate. Life Cycle Inventories of Structural Wood Products. Dept. of Wood Science and Engineering, Oregon State University. Corvallis, Oregon

Puettmann, M. E., Wilson, J. (2005). Life-Cycle Analysis of Wood Products: Cradle-to-Gate LCI of Residential Wood Building Materials. Wood and Fiber Science 37: 12.

Rowell, R. M. (1999). Specialty Treatment. Wood Handbook - Wood as an Engineering Material. Madison, Wisconsin, US, FPL-GTR 113.

Rowell, R. M. (2005). Handbook of Wood Chemistry - Wood Composites. CRC Press, Boco Raton: pp. $381-420$.

Seintsch, B., Weimar, H. (2012). Laubholz in Deutschland - Auf welchen Markt trifft das Potenzial? Tagungsbeitrag. Gülzower Fachgespräche - Stoffliche Nutzung von Laubholz, Würzburg. Fachagentur Nachwachsende Rohstoffe e.V. (FNR), Gülzow-Prüzen.

Schlosser, D., Grey, R., Fritsche, W. (1997). Patterns of ligninolytic enzymes in Trametes versicolor. Distribution of extra- and intracellular enzyme activities during cultivation on glucose, wheat straw and beech wood. Applied Microbiology Biotechnology 47(4): pp. 412-418.

Schmidt, M., Knorz, M., Torno, S. (2014). Bauen mit Laubholz. LWF aktuell, Heft 98. Bayerische Landesanstalt für Wald und Forstwirtschaft, Freising.

Schrod, M. (2002). Neue Methoden zur Synthese und Analyse von Phenol-FormaldehydHarzen. Dr.-Ing., Technischen Universität Darmstadt.

Shams, M. I., Yano, H., Endou, K. (2004). Compressive deformation of wood impregnated with low molecular weight phenol formaldehyde (PF) resin I: effects of pressing pressure and pressure holding. Journal of Wood Science 50(4): 337-342.

Stamm, A. J., Seborg, R. M., et al. (1938). The anti-shrink treatment of wood with synthetic resinforming materials and its application in making superior plywood. Madison, Wis., U.S. Dept. of Agriculture, Forest Service, Forest Products Laboratory.

Stamm, A. J. (1939). Resin-Treated Plywood. Industrial \& engineering chemistry 31(7): pp. 897-902.

Stamm, A. J., Seborg, R. M. (1942). Resin-Treated Wood. Forest Products Laboratory Report No. 1380.

Stamm, A. J., Seborg, R. M. (1955). Forest Products Laboratory resin-treated laminated, compressed wood (COMPREG), Forest Products Laboratory, Forest Service, U.S. Dept. of Agriculture. 
Stefanowski, B., Spear, M., Pitman, A. (2018). Review of the use of PF and related resins for modification of solid wood. Spear, M., editor. In Timber 2018. London: pp. 165-179

Sörgel, C., Mantau, U. (2006). Strukturentwicklung der Sägeindustrie in vier Jahren. HolzZentralblatt, Jg. 132, Nr. 21: pp. 651-653

Takada, M., Tanaka, Y., Minami, E., Saka, S. (2016). Comparative study of the topochemistry on delignification of Japanese beech (Fagus crenata) in subcritical phenol and subcritical water. Holzforschung. 70: 1047.

Takahashi, M., Imamura, Y. (1990). Biological resistance of phenol-resin treated wood. International Research Group on Wood Preservation. Rosenheim, Germany, IRG Secretary Stockholm, Sweden. Document No: IRG/WP /3602.

Trinh, H., Militz, H. Mai, C. (2012). Modification of beech veneers with N-methylol-melamine compounds for the production of plywood. European Journal of Wood and Wood Products 70(4): 421-432.

Unsal, O., Kartal, S. N., Candan, et al. (2009). Decay and termite resistance, water absorption and swelling of thermally compressed wood panels. International Biodeterioration \& Biodegradation 63(5): 548-552.

Wepner, F. (2009). Entwicklung eines Modifizierungsverfahrens für Buchenfurniere (Fagus sylvatica L.) auf Basis von zyklischen N-Methylol-Verbindungen. Dissertation. Fakultät für Forstwissenschaften und Waldökologie, Georg-August-Universität, Göttingen.

Wilson, J. (2009). Resins: A Life-Cycle Inventory of Manufacturing Resins. Used in the Wood Composites Industry. CORRIM: Phase II Final Report. Consortium for Research on Renewable Industrial Materials (CORRIM), Seattle (USA).

Wilson, J. B., Dancer, E.R. (2005). Gate-to-gate life-cycle inventory of laminated veneer lumber production. Wood and Fiber Science 37: 114-127.

Xie, Y., A. Krause, et al. (2007). Effect of treatments with 1,3-dimethylol-4,5-dihydroxyethyleneurea (DMDHEU) on the tensile properties of wood. Holzforschung 61(1): pp. 4350.

Yang, J.-C., Chiu, C.-M., Lin, T.-P., Kung, F.-H. (2001). No Clinal Variation in Cunninghamia lanceolata Wood Density Sampled from Thirteen Chinese Provinces. Taiwan Journal of Forest Science 19: 65-80.

Yazaki, Y., Collins, P. J., et al. (1994). Fast-Curing Phenol-Formaldehyde (PF) Resins. Part 1. Molecular Weight Distribution of PF Resins. Holzforschung - International Journal of the Biology, Chemistry, Physics and Technology of Wood. 48: 41.

Yeh, B. (2006). U.S. Adhesive Standards - An Overview and Roadmap to the Future. Second International Symposium on Veneer Processing Products, Vancouver.

Yusuf, S., Y. Sudiyani, et al. (1999). Biological and physical properties of phenolic-resin treated wood before and after natural weathering. International Research Group on Wood 
Preservation. Rosenheim, Germany, IRG Secretary Stockholm, Sweden: IRG/WP 9940132.

Zelinka, S. L., Glass, S. V., Stone, D. S. (2008). A percolation model for electrical conduction in wood with implications for wood-water relations. Wood and Fiber Science 40(4): 544552.

\subsection{Internetquellen}

Delignit (2014). http://www.delignit.de/Delignit/cms/front_content.php?idcat=7, Zugriff am 21.10.14. Blomberger Holzindustrie B. Hausmann GmbH \& Co. KG, Blomberg.

Pollmeier (2014). Ingenious Hardwood -BauBuche. Zugriff am 17.02.2014: http://www.pollmeier.com/relaunch/downloads/dyn/224/baubuche-info-de-small.pdf. Pollmeier Furnierwerkstoffe GmbH \& Co. KG, Creuzburg.

Röchling (2017). https://www.roechling.com/fileadmin/downloads/Roechling_Industrial/Br ochures/EN/Lignostone/Lignostone-Transformerwood-DE-EN.pdf, Zugriff am 01.09.2017. Röchling Engineered Plastics KG, Haren.

Kern (2018). https://www.kern.de/de/technisches-datenblatt/phenolharz-pf-51?n=2706_1, Zugriff am 01.09.2017. Kern GmbH Kunststoffwerke, Großmaischeid. 


\section{Anhang}

11.1 Abbildungen zur Untersuchung der Zellwandeindringung mit UMSP

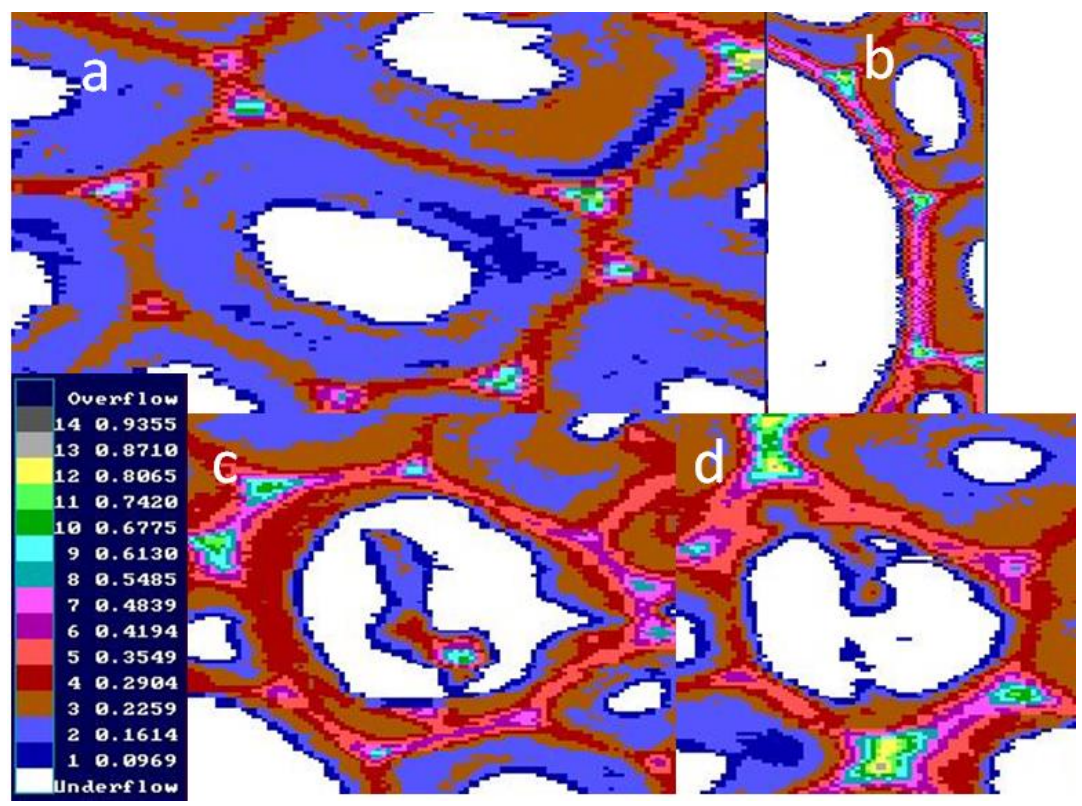

Abbildung 159: UMSP-Messungen bei $278 \mathrm{~nm}$ an Buche, wasserimpägniert und getrocknet. Fasern (a), Gefäßwand (b), Parenchym (c,d).

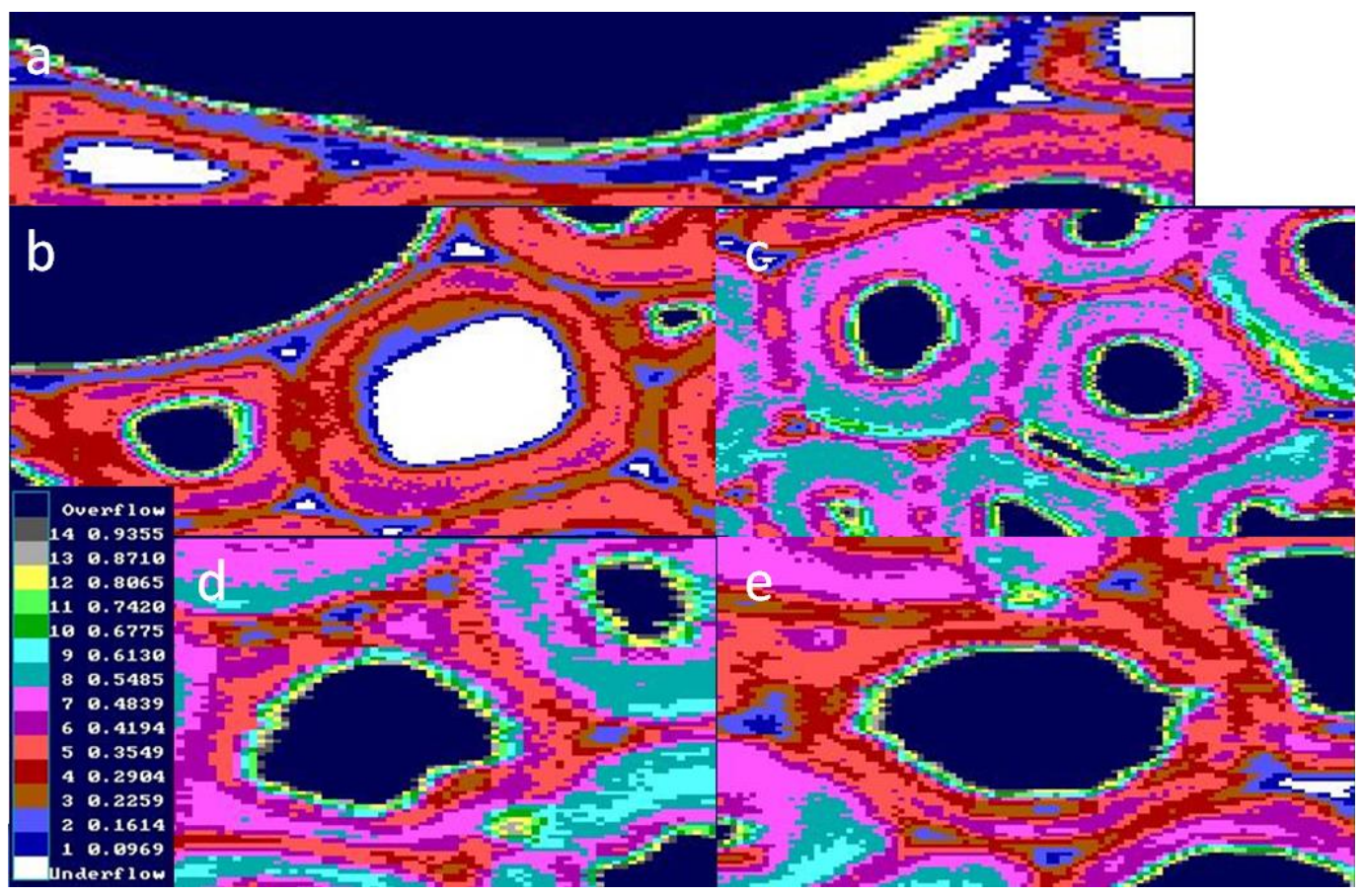

Abbildung 160: UMSP-Messungen bei 278nm an Buche, Harztyp B, 12\% WPG. Gefäßwand (a), Gefäßwand und Fasern(b), Fasern (c), Parenchym (e). 


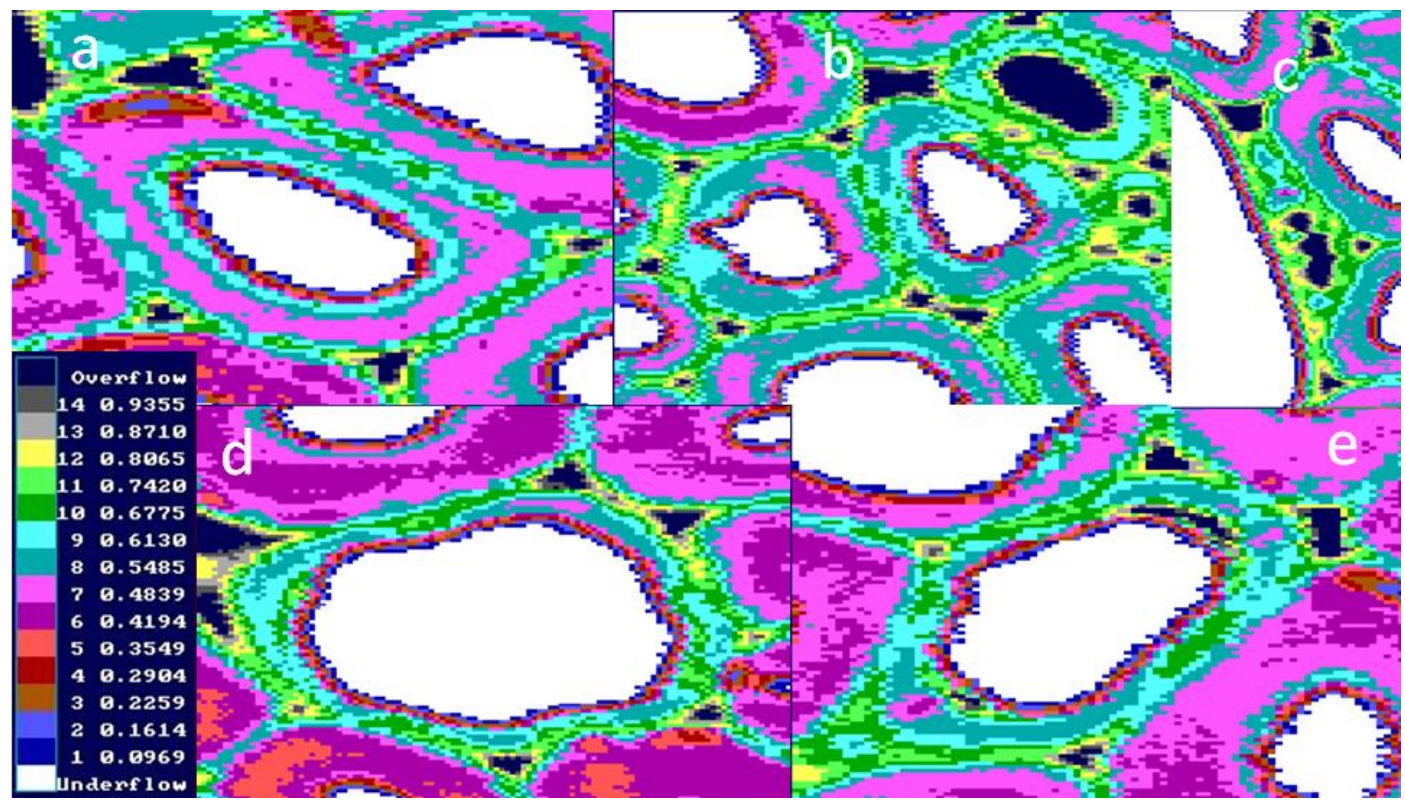

Abbildung 161: UMSP-Messungen bei 278nm an Buche, Harztyp A, 16\% WPG. Fasern (a,b), Gefäßwand (c), Parenchym (d,e).

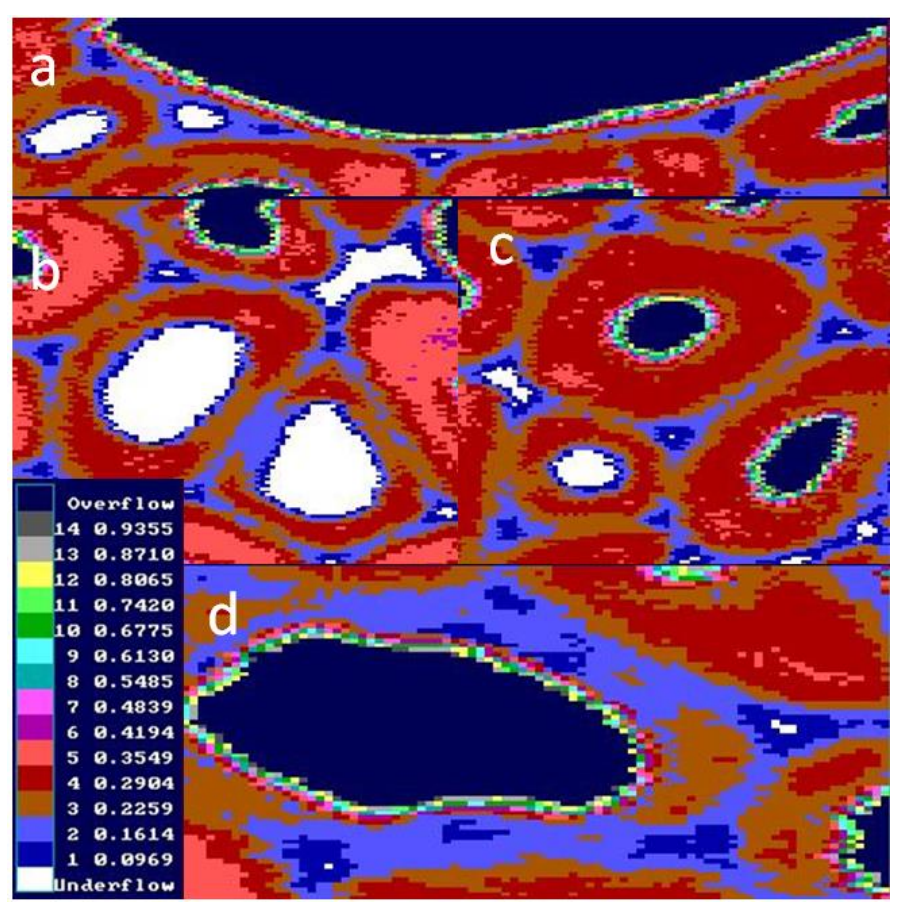

Abbildung 162: UMSP-Messungen bei 278nm an Buche, Harztyp A-gealtert, 17\% WPG. Gefäßwand (a), Fasern (b,c), Parenchym (d). 

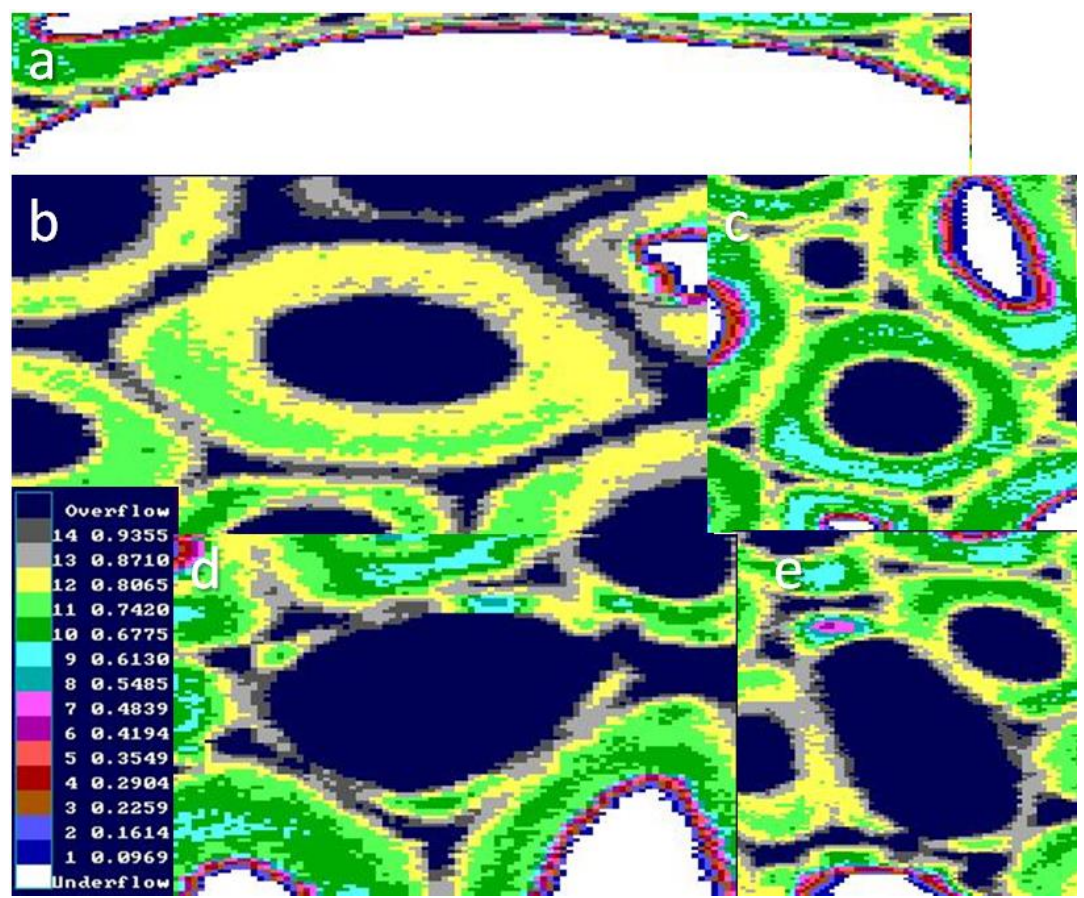

Abbildung 163: UMSP-Messungen bei 278nm an Buche, Harztyp A, 31\% WPG. Gefäßwand (a), Fasern (b,c), Parenchym $(\mathrm{d}, \mathrm{e})$. 
11.2 Abbildungen zur Freilandbewitterung von Buchen-LVL

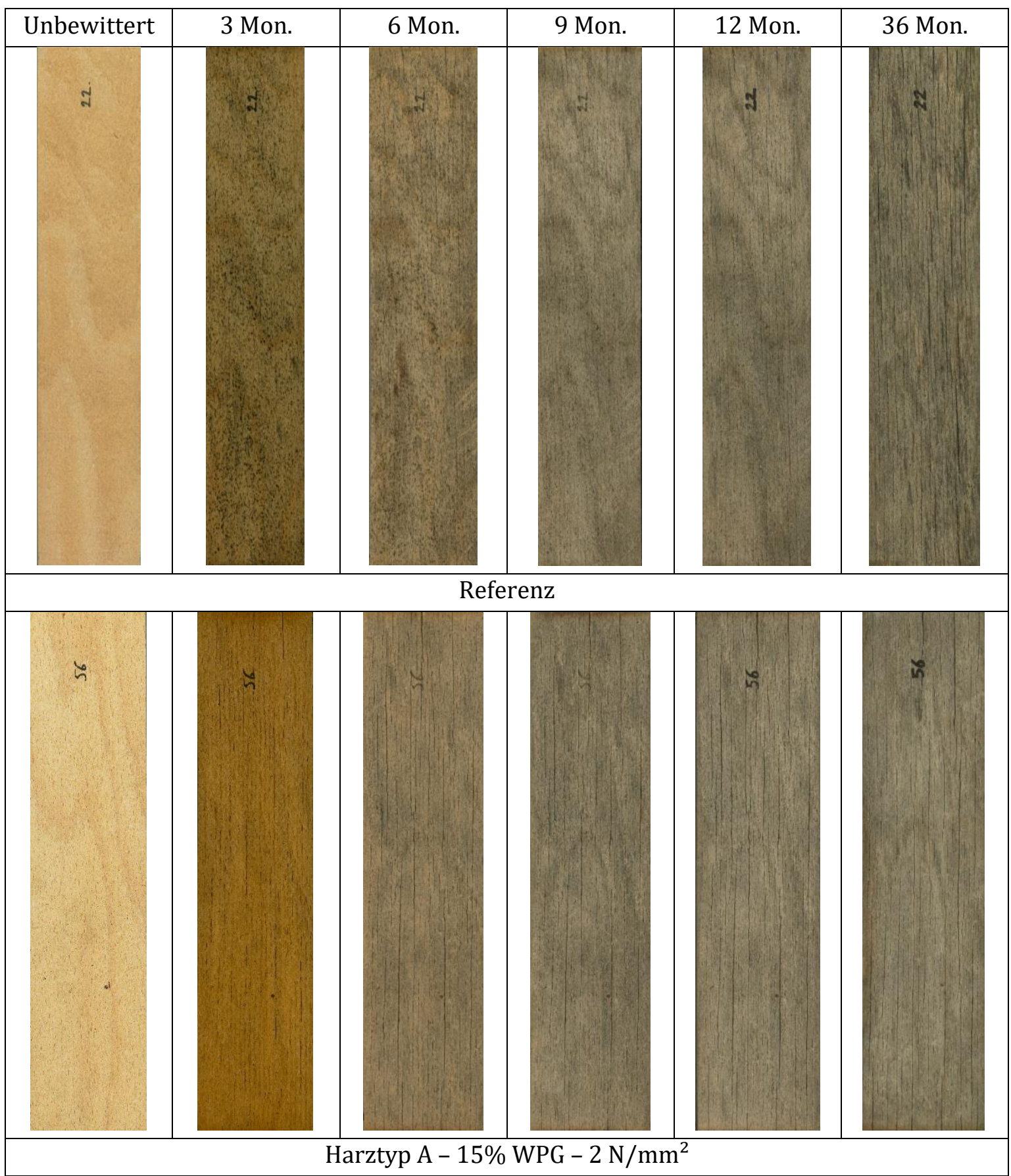

Abbildung 164: Oberflächen von unbeschichtetem Buchen-LVL in Abhängigkeit vom WPG und der Bewitterungsdauer im Freiland in Anlehung an EN 927-3 (2006) unter Verwendung des Harztyps A und einem Pressdruck von $2 \mathrm{~N} / \mathrm{mm}^{2}$. 


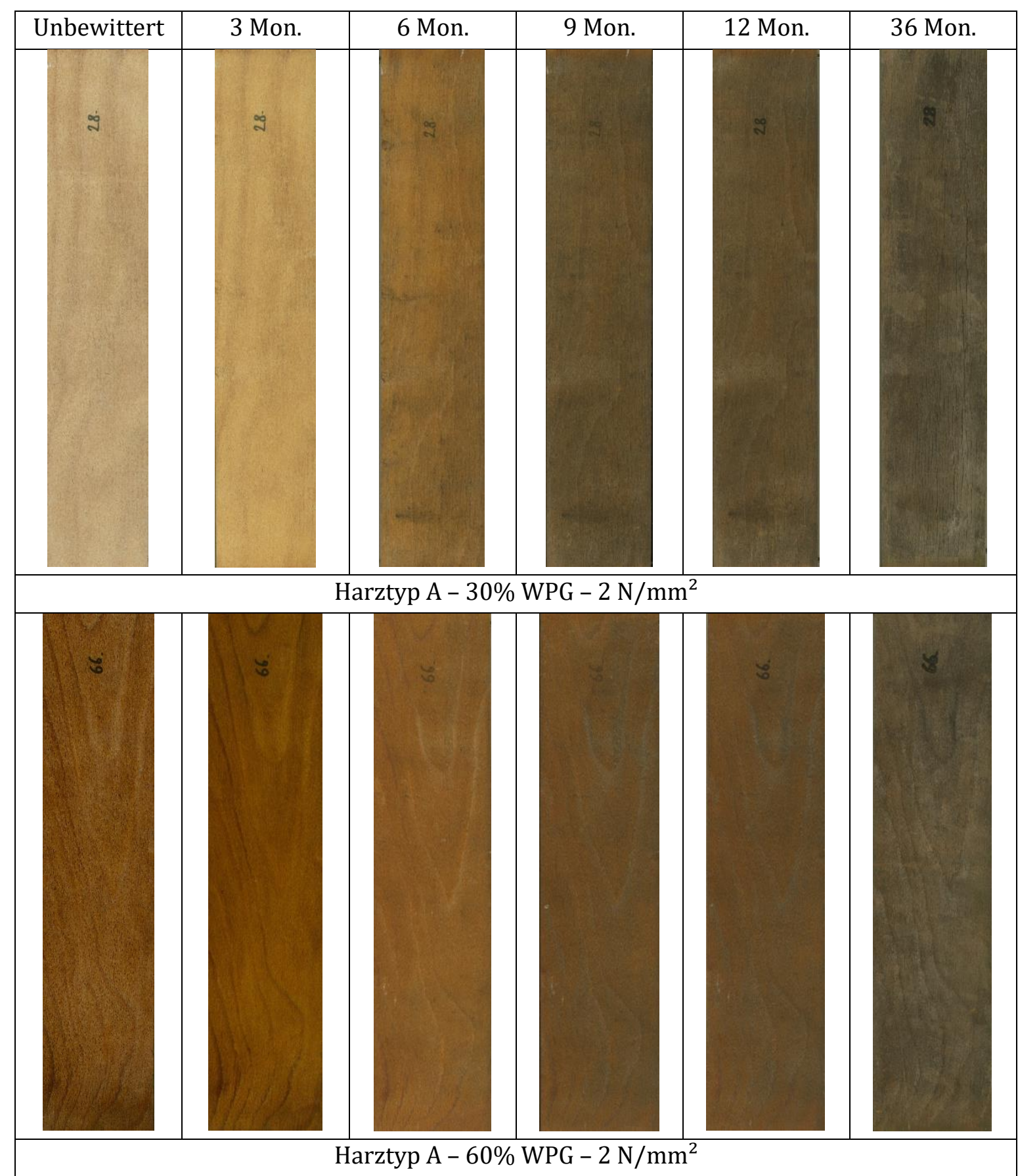

Abbildung 165: Oberflächen von unbeschichtetem Buchen-LVL in Abhängigkeit vom WPG und der Bewitterungsdauer im Freiland in Anlehung an EN 927-3 (2006) unter Verwendung des Harztyps A und einem Pressdruck von $2 \mathrm{~N} / \mathrm{mm}^{2}$. 


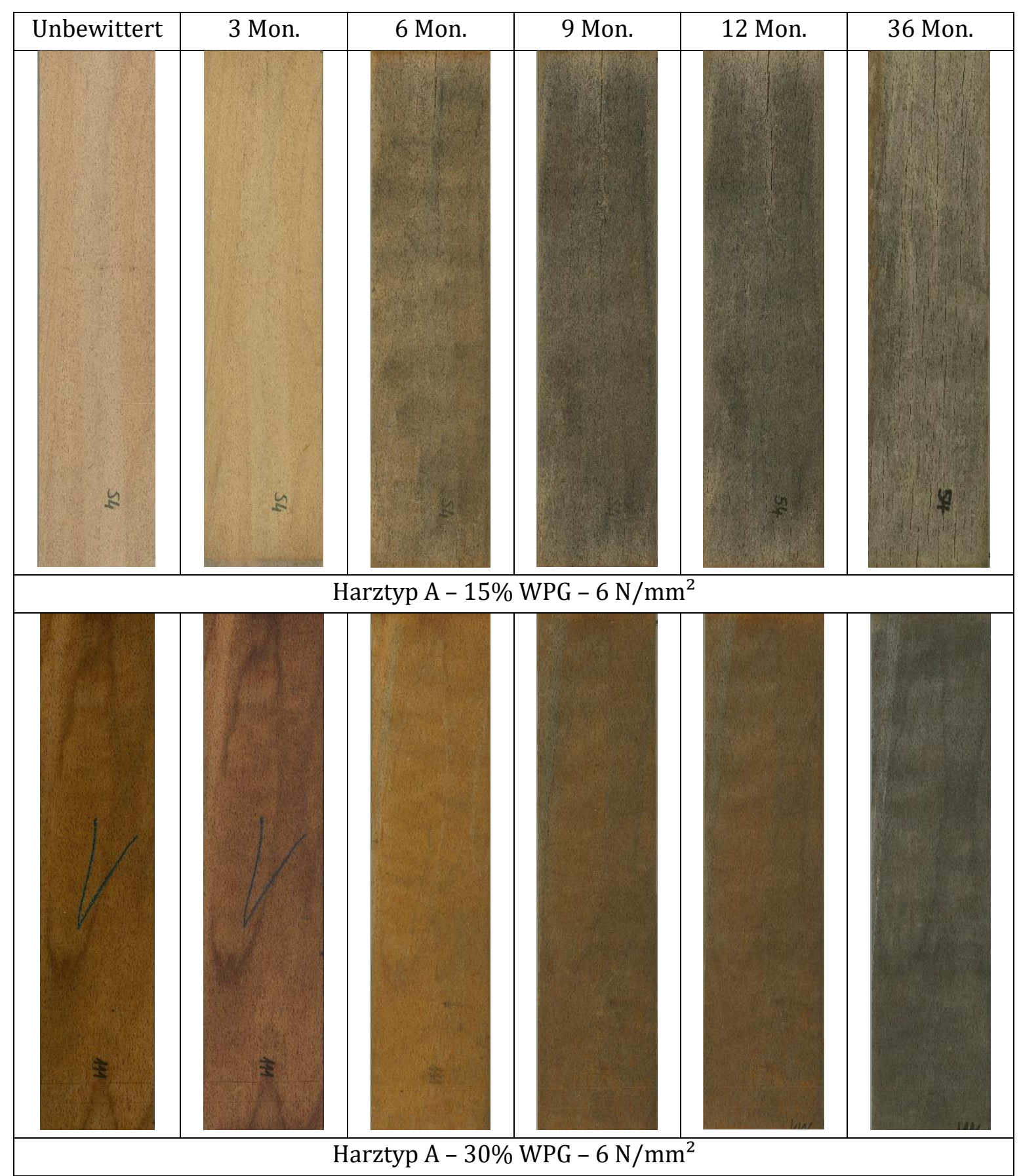

Abbildung 166: Oberflächen von unbeschichtetem Buchen-LVL in Abhängigkeit vom WPG und der Bewitterungsdauer im Freiland in Anlehung an EN 927-3 (2006) unter Verwendung des Harztyps A und einem Pressdruck von $6 \mathrm{~N} / \mathrm{mm}^{2}$. 


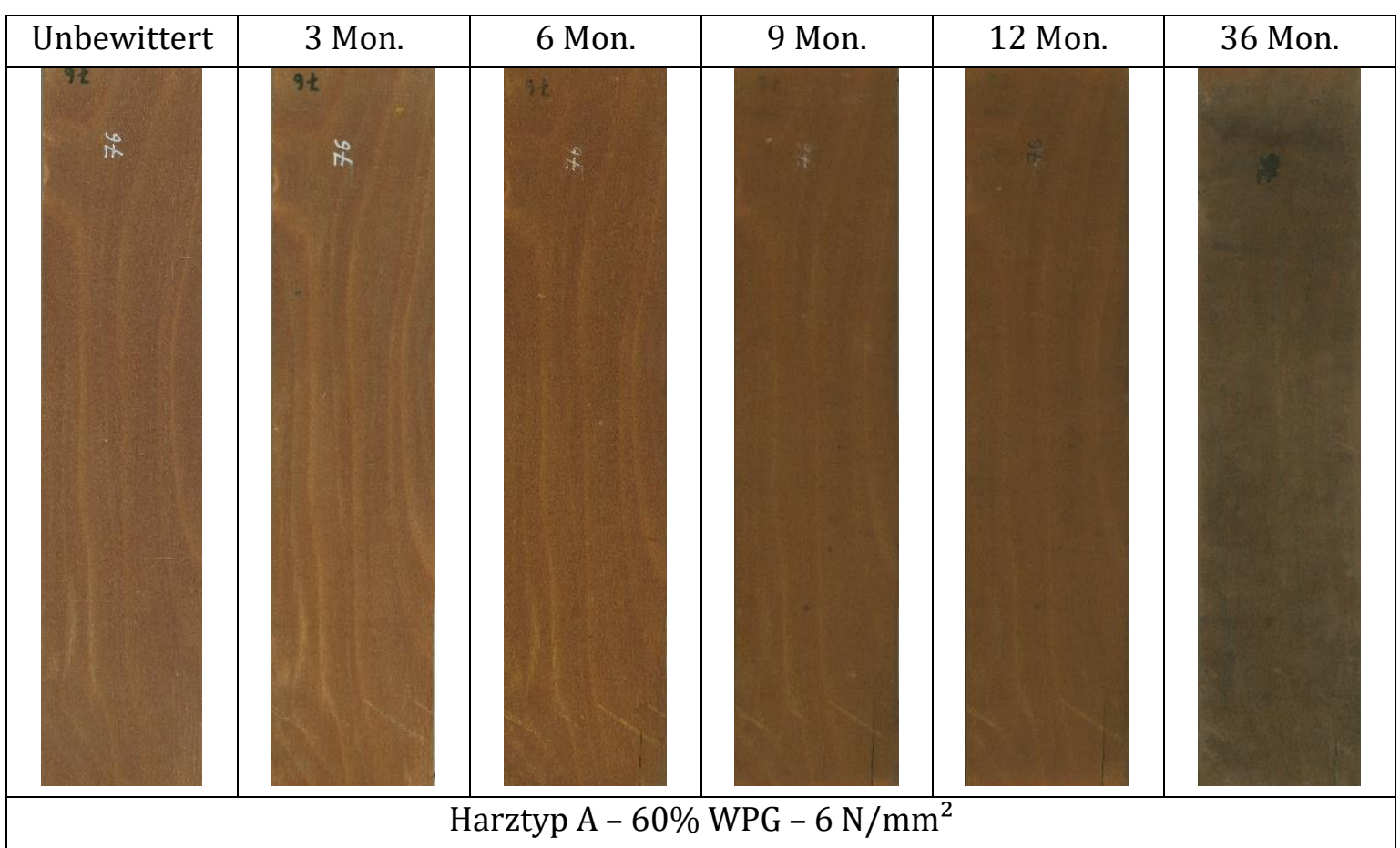

Abbildung 167: Oberflächen von unbeschichtetem Buchen-LVL mit einem WPG von 60\% in Abhängigkeit der Bewitterungsdauer im Freiland in Anlehung an EN 927-3 (2006) unter Verwendung des Harztyps A und einem Pressdruck von $6 \mathrm{~N} / \mathrm{mm}^{2}$. 


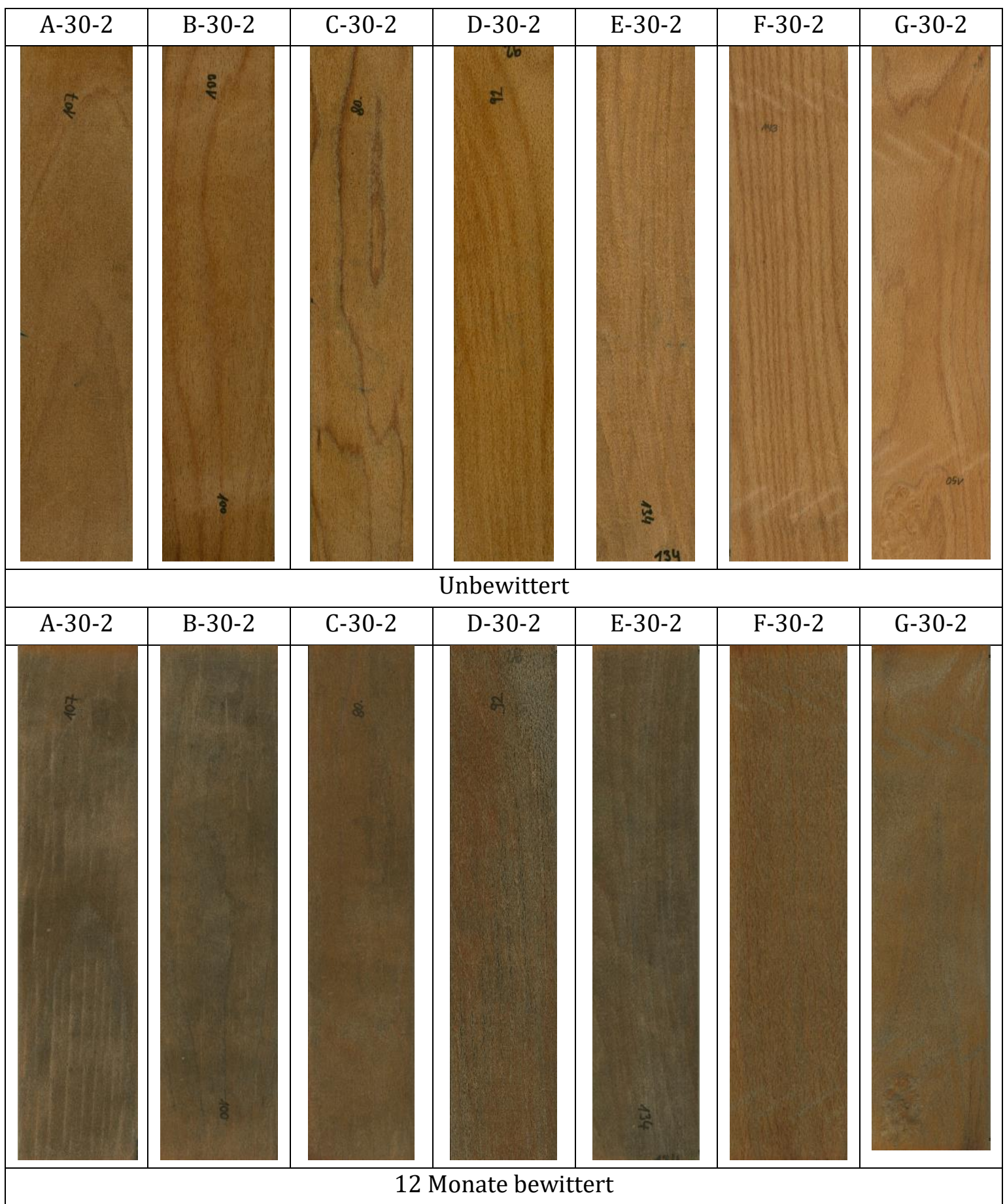

Abbildung 168: Oberflächen von Buchen-LVL mit unterschiedlichen Harztypen (A, B, C, D, E, F, G) bei einem WPG von 30\% und einem Pressdruck von $2 \mathrm{~N} / \mathrm{mm}^{2} \mathrm{zu}$ Beginn und nach 12 Monaten der Bewitterung in Anlehnung an EN 927-3 (2006). 


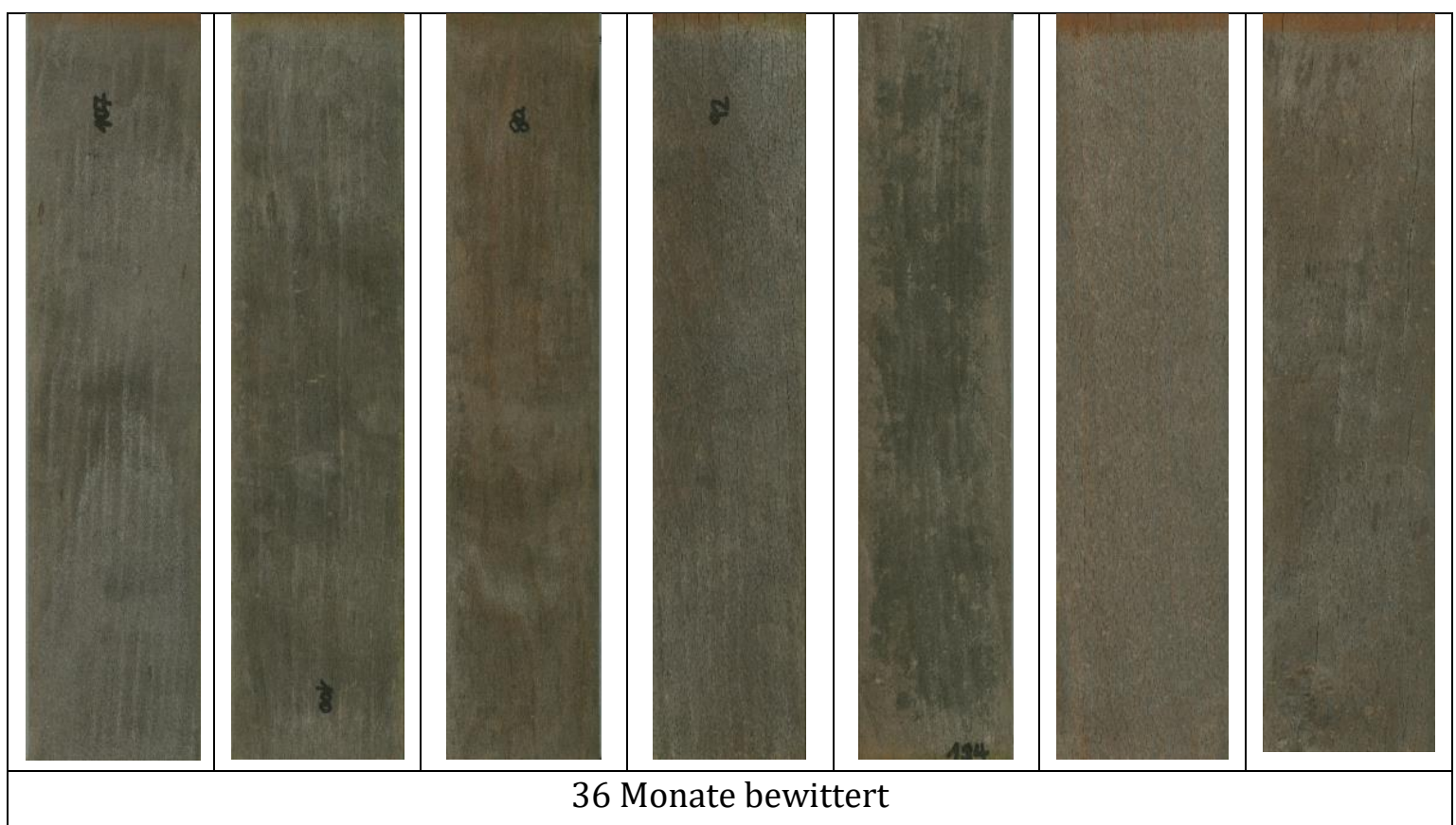

Abbildung 169: Oberflächen von Buchen-LVL mit unterschiedlichen Harztypen (A, B, C, D, E, F, G) bei einem WPG von 30\% und einem Pressdruck von $2 \mathrm{~N} / \mathrm{mm}^{2}$ nach 36 Monaten der Bewitterung in Anlehnung an EN 927-3 (2006). 